Mirko Tillmann

\title{
Risikokapitalbasierte Steuerung in der Schaden- und Unfallversicherung
}

Konzeption einer modellgestützten Risikoanalyse 


\section{Risikokapitalbasierte Steuerung in der Schaden- und Unfallversicherung}

Die ökonomischen Rahmenbedingungen und die Risikolandschaft europäischer Versicherungsunternehmen sind angesichts struktureller Veränderungen einem tiefgreifenden Wandel unterworfen. Ansteigende Schadendurchschnitte, eine zunehmende Belastung durch Naturschadenereignisse, volatile Kapitalmärkte, ruinöse Preiskämpfe in einzelnen Versicherungssparten und nicht zuletzt ambitionierte Renditevorgaben der Anteilseigner lassen die Notwendigkeit einer wertorientierten Unternehmenssteuerung in den Mittelpunkt des Interesses rücken. Diese Untersuchung erschließt die Thematik einer integrierten RenditeRisikosteuerung von Schaden- und Unfallversicherungsunternehmen sowohl aus theoretischer als auch versicherungspraktischer Perspektive. Es wird ein differenzierter Vorschlag zur Ausgestaltung eines risikokapitalbasierten (stochastischen) Steuerungsmodells entwickelt, welches sowohl die ökonomische Risikosicht als auch die bilanzielle Sicht auf ein Versicherungsunternehmen adäquat abbildet. Die Untersuchung erlangt insbesondere durch die exemplarische Anwendung des Modells auf den Datensatz eines deutschen Erstversicherungsunternehmens eine hohe praktisch-normative Relevanz.

Mirko Tillmann absolvierte nach einer Ausbildung zum Bankkaufmann das Studium der Betriebswirtschaftslehre an der Universität in Münster. Nach seinem Abschluss als Diplom-Kaufmann arbeitete er als Assistent des Vorstandsvorsitzenden eines öffentlichen Versicherungsunternehmens und als Wissenschaftlicher Mitarbeiter am Lehrstuhl für Betriebswirtschaftslehre, insbesondere Controlling. Die Promotion erfolgte im Jahr 2005. Seitdem ist der Autor als Referent für Unternehmensentwicklung/M\&A für einen großen Versicherungskonzern in Köln tätig. 
Risikokapitalbasierte Steuerung in der Schaden- und Unfallversicherung 


\section{Beiträge zum Controlling}

Herausgegeben von Wolfgang Berens

\section{Band 8}

\section{(4) \\ $=$

Frankfurt am Main · Berlin · Bern · Bruxelles · New York · Oxford · Wien 


\section{Mirko Tillmann}

\section{Risikokapitalbasierte Steuerung in der Schaden- und Unfallversicherung}

Konzeption einer modellgestützten Risikoanalyse

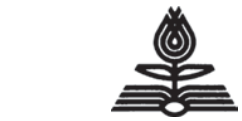

PETER LANG

Europäischer Verlag der Wissenschaften 
Bibliografische Information Der Deutschen Bibliothek

Die Deutsche Bibliothek verzeichnet diese Publikation in der Deutschen Nationalbibliografie; detaillierte bibliografische Daten sind im Internet über <http://dnb.ddb.de> abrufbar.

Open Access: The online version of this publication is published on www.peterlang.com and www.econstor.eu under the international Creative Commons License CC-BY 4.0. Learn more on how you can use and share this work: http://creativecommons. org/licenses/by/4.0.

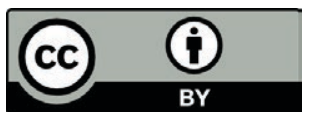

This book is available Open Access thanks to the kind support of ZBW - Leibniz-Informationszentrum Wirtschaft.

Zugl.: Münster (Westfalen), Univ., Diss., 2005

Gedruckt auf alterungsbeståndigem, säurefreiem Papier.

D 6

ISSN 1618-825X

ISBN 3-631-54396-4

ISBN 978-3-631-75327-9 (eBook)

(c) Peter Lang GmbH

Europäischer Verlag der Wissenschaften

Frankfurt am Main 2005

Alle Rechte vorbehalten.

Das Werk einschließlich aller seiner Teile ist urheberrechtlich geschützt. Jede Verwertung außerhalb der engen Grenzen des Urheberrechtsgesetzes ist ohne Zustimmung des Verlages unzulässig und strafbar. Das gilt insbesondere für Vervielfältigungen, Übersetzungen, Mikroverfilmungen und die Einspeicherung und Verarbeitung in elektronischen Systemen.

Printed in Germany $12 \quad 4567$

www.peterlang.de 


\section{Geleitwort}

Die ökonomischen Rahmenbedingungen und die Risikolandschaft europäischer Versicherungsunternehmen sind angesichts struktureller Veränderungen einem tiefgreifenden Wandel unterworfen. Ansteigende Schadendurchschnitte, eine zunehmende Belastung durch Naturschadenereignisse, volatile Kapitalmärkte, ruinöse Preiskämpfe in einzelnen Versicherungssparten und nicht zuletzt ambitionierte Renditevorgaben der Anteilseigner lassen die Notwendigkeit einer wertorientierten Unternehmenssteuerung in den Mittelpunkt des Interesses rücken. Aufgrund der spezifischen Anforderungen und Gegebenheiten kommt einer integrierten Steuerung der Risiko- und Chancenpotenziale gerade in Versicherungsunternehmen besondere Bedeutung zu: Im Gegensatz zu den meisten Produkten anderer Branchen übt das Sicherheitsniveau eines Versicherers einen erheblichen Einfluss auf die Qualität des Produktes (hier: des Versicherungsversprechens) aus. Eine Verbesserung des Sicherheitsniveaus durch eine Erhöhung der Eigenkapitalausstattung ist jedoch mit zusätzlichen Kapitalkosten verbunden und findet daher nicht zwangsläufig die Zustimmung der Anteilseigner. In diesem Spannungsfeld und gerade vor dem Hintergrund der Neuausrichtung der europäischen Solvabilitätsvorschriften (,Solvency II“) können interne Risikomodelle einen wertvollen Beitrag zur Unterstützung strategischer Unternehmensentscheidungen leisten.

Die vorgelegte Arbeit erschließt die Thematik einer integrierten Rendite-Risikosteuerung von Schaden- und Unfallversicherungsunternehmen sowohl aus theoretischer als auch unternehmenspraktischer Sicht. Herr Tillmann entwickelt einen bemerkenswert differenzierten Vorschlag zur Ausgestaltung eines risikokapitalbasierten (stochastischen) Steuerungsmodells, welches als „Referenzmodell“ für die Schaden- und Unfallversicherung herangezogen werden kann. Das Modellkonzept unternimmt den aus der versicherungspraktischen Perspektive motivierten Versuch, einerseits als ökonomisches Steuerungsmodell zu fungieren und gleichzeitig die bilanzielle Sicht auf ein Versicherungsunternehmen adäquat abzubilden. Herrn Tillmann gelingt es in sehr überzeugender Weise, dieses schwierige Unterfangen auch aus dem Blickwinkel der Wissenschaft zu bewältigen. Die Arbeit gewinnt insbesondere durch die exemplarische Anwendung des Modells auf den Datensatz eines deutschen Erstversicherungsunternehmens praktisch-normative Relevanz. Die Beispielrechnungen und Ergebnisse gewähren einen hervorragenden Einblick in die Wirkungsweise des Modellansatzes und ermöglichen zudem die Ableitung wertvoller Handlungsempfehlungen für eine integrierte Steuerung der Rendite-Risiko-Position.

Die durch Herrn Tillmann vorgelegte Dissertation erschließt ein überaus innovatives und hoch aktuelles Forschungsfeld und leistet einen bedeutenden konzeptionellen Beitrag zur strategischen Steuerung in der Schaden- und Unfallversicherung. Wir wünschen ihr daher in Theorie und Praxis eine breite Aufnahme und Resonanz. 
Mirko Tillmann - 978-3-631-75327-9

Downloaded from PubFactory at 01/11/2019 06:10:15AM

via free access 


\section{Vorwort}

Die Anfertigung der vorliegenden Arbeit begleitete meine Tätigkeit als wissenschaftlicher Mitarbeiter am Lehrstuhl für Betriebswirtschaftslehre, insbesondere Controlling der Westfälischen Wilhelms-Universität Münster. Sie wurde im Frühjahr 2005 von der Wirtschaftswissenschaftlichen Fakultät als Dissertation angenommen. Meinem Doktorvater Prof. Dr. WOLFGANG BERENS gilt mein besonders herzlicher Dank für seine uneingeschränkte Unterstützung und die Möglichkeit, mich im Rahmen meines Dissertationsvorhabens der Thematik „Rendite-Risikosteuerung in Versicherungsunternehmen" annehmen zu können. In dem hervorragenden Arbeitsklima, das er am Lehrstuhl zu schaffen verstand, ließ er mir stets die akademischen Freiräume, eigene Wege zu beschreiten und vielfältige persönliche Erfahrungen zu sammeln.

Herrn Prof. Dr. HATO SCHMEISER danke ich sehr herzlich für die Übernahme des Zweitgutachtens sowie für zahlreiche interessante und hilfreiche Diskussionen über den „Sinn und Unsinn der Kapitalallokation“. Seine wertvollen fachlichen Anregungen habe ich immer dankbar aufgenommen.

Die Idee zu dieser Untersuchung entstand während meiner Zeit als Mitarbeiter der Westfälischen Provinzial Versicherungen in Münster. Mein ganz besonderer Dank gebührt an dieser Stelle stellvertretend Herrn Dr. ULRICH LÜXMANN-ELLINGHAUS und Herm Dr. HEIKo WINKLER, die meinen bisherigen beruflichen Werdegang und mein Promotionsvorhaben zu jedem Zeitpunkt sehr konstruktiv und wohlwollend unterstützt haben. Sie vermittelten mir außerdem jenes Praxiswissen, welche die vorliegende Arbeit nicht nur „theoretisch reizvoll“", sondern auch für die Versicherungswirtschaft relevant macht.

Meinem Freund und ehemaligen Arbeitskollegen, Herrn Dr. DIETMAR KOHLRUSS, gilt mein sehr persönlicher Dank für zahlreiche abendliche Diskussionen der in dieser Arbeit entwickelten Ideen. Seine wertvollen Anregungen im Rahmen der stochastischen Modellierung und seine freundschaftliche Aufmunterung haben maßgeblich zur Entstehung der vorliegenden Arbeit beigetragen. Für die Bereitstellung der Modellierungssoftware danke ich der aktuariellen Unternehmensberatung EMB DEUTSCHLAND GMBH. Stellvertretend für das gesamte Team möchte ich mich herzlich bei Herrn Dr. KNUT SCHAEFER und Herrn Dr. GERO NIEßEN für die freundliche und sehr konstruktive Zusammenarbeit bedanken. Besonders verbunden bin ich Herrn Dr. GERO NIEBEN außerdem für sein ausführliches Feedback zu meinen Ausführungen.

Das hervorragende Arbeitsklima und die exzellenten Arbeitsbedingungen am Lehrstuhl für Controlling haben maßgeblich zum Gelingen meiner Arbeit beigetragen. Hierfür möchte ich mich sowohl bei Herrn Prof. Dr. WOLFGANG BERENS als auch bei allen Kolleginnen und Kollegen sehr herzlich bedanken. Ebenso dankbar bin ich für die gewährte Entlastung im Endstadium der Bearbeitung. Namentlich nennen möchte ich an dieser Stelle Frau BeTtina KleINFElder, Frau Gabriele Paning, Herrn Dr. René Bertelsmann, Herrn Dipl.-Kfm. Daniel Bolte, Herrn Dipl.-Kfm. Klaus FlaCKE, Herrn Dipl.-Kfm. Dipl.-Psych. BeRnd HögEMANN, Herm Dr. ANDREAS 
HOFfJan, Herrn Dr. Elmar KaRlowitsch, Herrn Dipl.-Kfm. FloRian Krol, Herm Dipl.-Kfm. Jan-PhilipP MENKE, Herrn Dipl.-Kfm. Pascal NeVRIES, Herrn Dipl.Kfm. ANDREas RÖHRig, Herrn Dipl.-Kfm. KLAUS SegBers, Herrn Dipl.-Kfm. THOMAS TRISKA und Herrn Dipl.-Kfm. ANDREAS WÖMPENER. Hervorheben möchte ich Herm Dipl.-Math. MIRKo KRAFT und Herrn AOR Dr. WALTER SCHMITTING. Beiden bin ich für ihr außerordentliches Engagement, insbesondere für die intensive Durchsicht des Manuskripts sowie für zahlreiche Hinweise und Anregungen in besonderem $\mathrm{Maße}$ verbunden. Für ihre wertvolle Hilfe bei der Beschaffung und Verwaltung der umfangreichen Literatur sowie für sorgfältiges Korrekturlesen möchte ich außerdem meiner studentischen Hilfskraft Frau stud. rer. pol. ALEXANDRA ROHLMANN meinen herzlichen Dank aussprechen.

Abschließend möchte ich meinem privaten Umfeld für die intensive Begleitung und vorbehaltlose Unterstützung des von mir eingeschlagenen Weges danken. Vor allem danke ich meiner Freundin ANNE HOLTKÖTTER für ihre große Geduld und den liebevollen Rückhalt, den sie mir zu jedem Zeitpunkt gegeben hat. Der größte Dank gebührt zweifellos meinen Eltern, MARLENE und WERNER TILLMANN. Durch ihre liebevolle Unterstützung und Aufmunterung haben sie mir in den vergangenen Jahren immer das Gefühl gegeben, den richtigen Weg zu gehen. Ich möchte Ihnen daher diese Arbeit widmen.

MiRKo TILLMANN 


\section{Inhaltsverzeichnis}

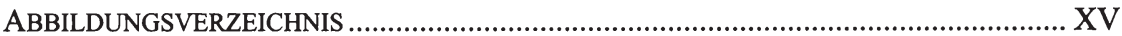

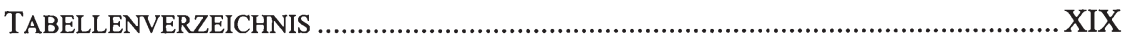

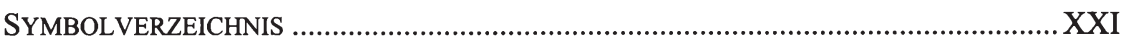

ABKÜRZUNGSVERZEICHNIS ...........................................................................XXII

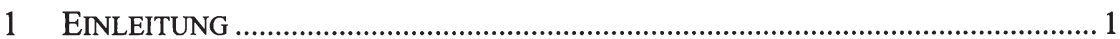

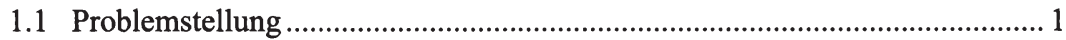

1.2 Zielsetzung der Arbeit ................................................................................ 4

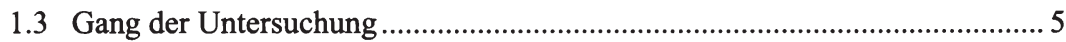

2 SPEZIFIKA DER SCHADEN- UND UNFALLVERSICHERUNG

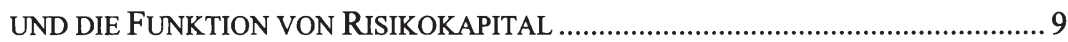

2.1 Begriffliche Abgrenzung der Schaden- und Unfallversicherung .................... 9

2.2 Kategorisierung versicherungsspezifischer Risiken.................................... 10

2.2.1 Definition von Risiko ..................................................................... 10

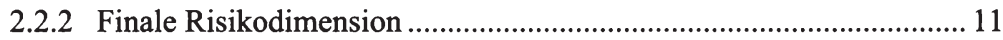

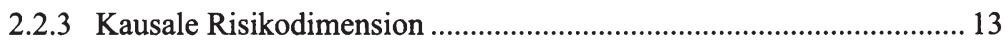

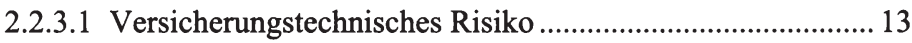

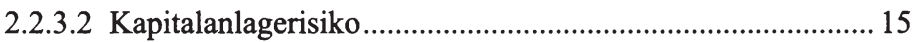

2.2.3.3 Sonstige Unternehmensrisiken ............................................. 17

2.3 Begriff und Funktion von Risikokapital.................................................... 18

3 ANFORDERUNGEN AN DIE STEUERUNG

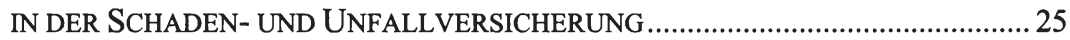

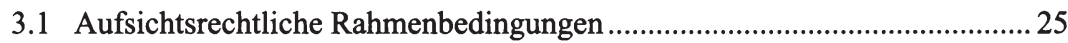

3.1.1 Europäische Solvabilitätsvorschriften de lege lata (Solvency I)........25

3.1.1.1 Ausgestaltung der Soll- und Ist-Solvabilität .........................25

3.1.1.2 Kritische Würdigung ........................................................... 28

3.1.2 Neuausrichtung des europäischen Solvabilitätssystems

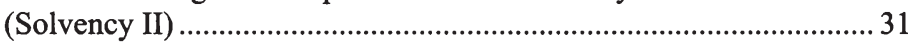

3.1.2.1 Zielsetzungen und allgemeiner Aufbau................................. 31

3.1.2.2 Inhaltliche Ausgestaltung und Implikationen für die Steuerung ...................................................................... 33

3.1.2.2.1 Säule 1: Quantitative Vorgaben ............................. 33

3.1.2.2.2 Säule 2: Qualitative Vorgaben ............................... 39

3.1.2.2.3 Säule 3: Transparenz und Marktdisziplin ............. 41 
3.2 Betriebswirtschaftliche Anforderungen an das Risikomanagement ..............42

3.2.1 Darstellung des betriebswirtschaftlichen Zielsystems........................ 42

3.2.2 Notwendigkeit einer integrierten Rendite-Risikosteuerung ................ 46

3.2.3 Risikomanagementprozess und Instrumente der

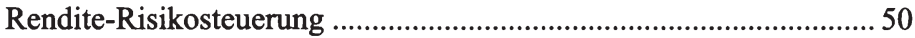

3.2.4 Zentrale Struktursteuerung vs. dezentrale Marktbereichssteuerung .. 55

4 STEUERUNG ÜBER RISIKOKAPITAL

IN DER SCHADEN- UND UNFALLVERSICHERUNG ............................................59

4.1 Diskussion ausgewählter Konzepte zur Risikomessung ………………….......5

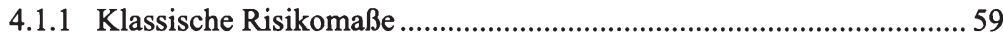

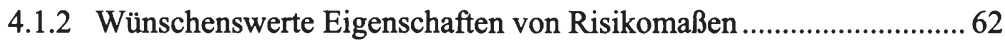

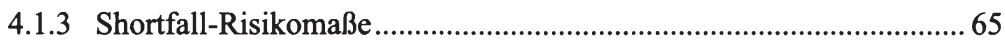

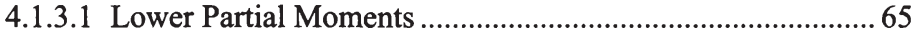

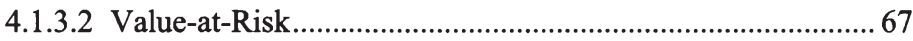

4.1.3.3 Conditional Value-at-Risk ..................................................... 71

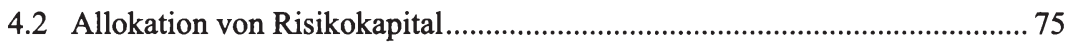

4.2.1 Diversifikationseffekte als Ausgangspunkt der

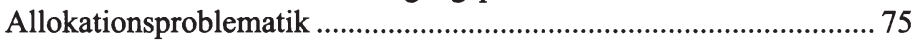

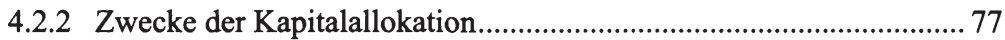

4.2.3 Anforderungen an die Allokation von Risikokapital............................. 83

4.2.4 Methoden der Risikokapitalallokation................................................ 87

4.2.4.1 Standalone-proportionale Allokation .................................... 87

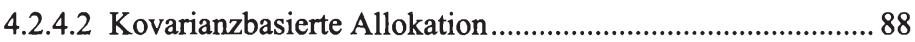

4.2.4.3 Inkrementelle Allokation...................................................... 90

4.2.4.4 Allokation nach dem CVaR-Konzept.................................. 92

5 ENTWICKLUNG EINES PRAXISTAUGLICHEN MODELLANSATZES ......................97

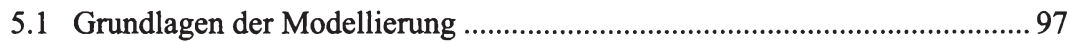

5.1.1 Klassifizierung von Modellen ........................................................... 97

5.1.2 Kategorisierung von stochastischen Modellen................................. 102

5.1.2.1 Modelle auf analytischer Basis........................................ 102

5.1.2.2 Modelle auf der Basis von Simulationen ............................. 104

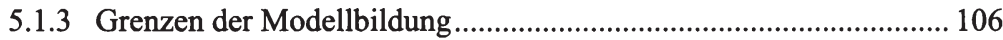

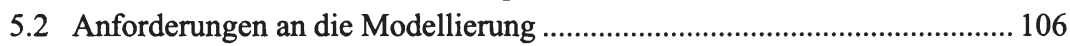

5.3 Rahmenkonzept und Struktur des Modellansatzes ....................................... 111

5.3.1 Modellzweck und Grundannahmen................................................111

5.3.2 Modellarchitektur und Datenbasis.................................................. 113

5.3.3 Zeitliche Struktur .......................................................................... 117 


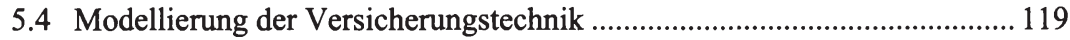

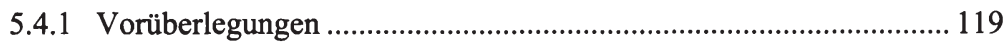

5.4.1.1 Zweidimensionale Modellierung:

Sparten und Geschäftsfelder............................................. 119

5.4.1.2 Differenzierung zwischen Anfalljahren und Geschäftsjahren ......................................................... 121

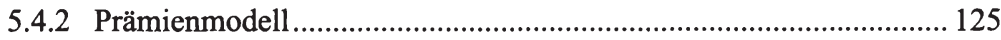

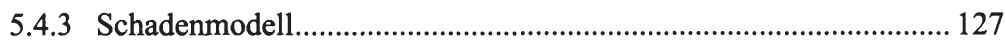

5.4.3.1 Individuelles vs. kollektives Modell der Risikotheorie....... 127

5.4.3.2 Basisschäden..................................................................... 132

5.4.3.2.1 Ermittlung der Gesamtschadenverteilung der Basisschäden ................................................. 132

5.4.3.2.2 Schätzung zentraler Schadenparameter .............. 137

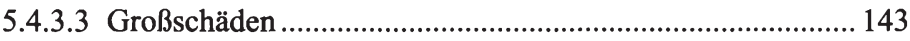

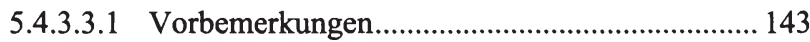

5.4.3.3.2 Ermittlung der Schadenanzahlverteilung ............ 144

5.4.3.3.3 Ermittlung der Schadenhöhenverteilung............. 147

5.4.3.4 Schäden aus Naturgefahren .............................................. 152

5.4.3.4.1 Vorbemerkungen............................................... 152

5.4.3.4.2 Sturmschäden ..................................................... 155

5.4.3.4.3 Hagelschäden ................................................... 158

5.4.3.4.4 Überschwemmungsschäden ............................... 160

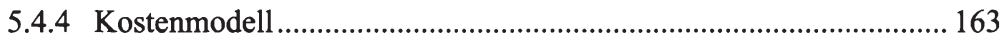

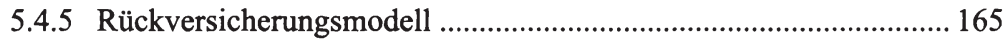

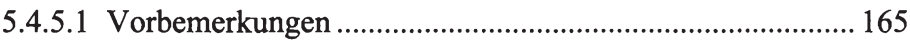

5.4.5.2 Modellierung einzelner Rückversicherungsverträge............ 168

5.4.5.2.1 Quotenvertrag.................................................... 168

5.4.5.2.2 Summenexzedentenvertrag ................................. 169

5.4.5.2.3 Schadenexzedentenvertrag.................................. 171

5.4.5.2.4 Jahresüberschadenexzedentenvertrag (Stop Loss) ......................................................... 172

5.4.5.3 Ermittlung der Preiskomponenten (Pricing).......................... 173

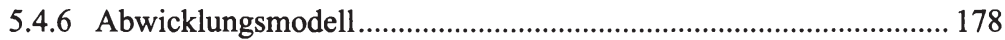

5.4.6.1 Grundlagen zur Abwicklung von Vorjahres- und Geschäftsjahresschäden ......................................................... 178

5.4.6.2 Abwicklung des Bruttogeschäfts......................................... 183

5.4.6.3 Abwicklung des Nettogeschäfts ........................................ 188

5.4.7 Zentrale Ergebnisgrößen des versicherungstechnischen Geschäfts . 189

5.4.7.1 Versicherungstechnisches Ergebnis auf Anfalljahresbasis . 189

5.4.7.2 Versicherungstechnisches Ergebnis auf Geschäftsjahresbasis 


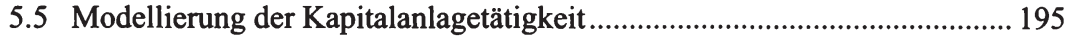

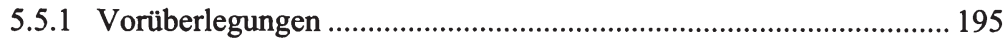

5.5.2 Einperiodige vs. mehrperiodige Investmentmodelle

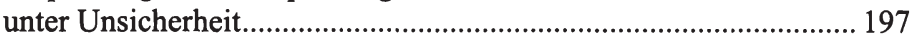

5.5.3 Buchwert- vs. Marktwertmodellierung............................................ 199

5.5.4 Ermittlung des Kapitalanlagevolumens und Integration des versicherungstechnischen Netto Cashflows ..................................... 200

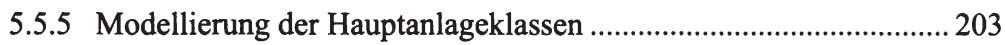

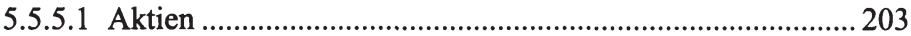

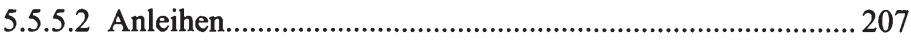

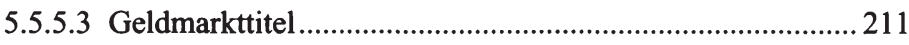

5.5.5.4 Abbildung der Korrelationsstruktur zwischen den

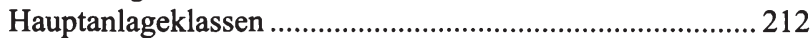

5.5.6 Zentrale Ergebnisgrößen der Kapitalanlagetätigkeit .......................... 214

5.6 Integration der Teilmodelle in ein Gesamtmodell......................................... 216

6 UMSETZUNG DES MODELLANSATZES AM BEISPIEL EINES DEUTSCHEN SCHADEN- UND UNFALLVERSICHERUNGSUNTERNEHMENS ........221

6.1 Strukturierung und Eingrenzung des Analyserahmens ................................221

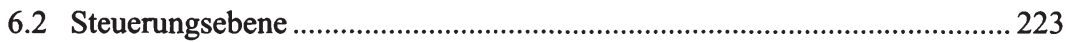

6.2.1 Darstellung und Analyse der Modellergebnisse im Basisszenario .. 223

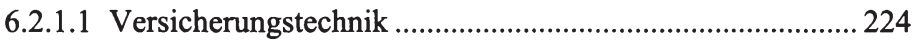

6.2.1.1.1 Darstellung und Analyse des Bruttogeschäfts ... 224

6.2.1.1.2 Darstellung und Analyse des Nettogeschäfts..... 241

6.2.1.2 Integration von Kapitalanlageerträgen ................................254

6.2.2 Einfluss stochastischer Abhängigkeitsstrukturen ........................... 262

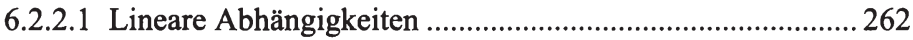

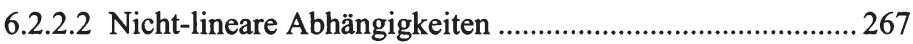

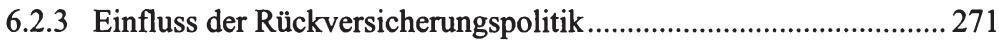

6.2.3.1 Stop-Loss-Deckung für die sturmexponierten Sparten ....... 271

6.2.3.2 XL-Deckung für die Sparte „Kraftfahrt-Haftpflicht““.......... 275

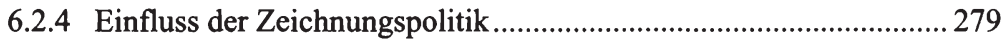

6.2.4.1 Sanierung der Sparte „VGV Leitungswasser“.................... 279

6.2.4.2 Ausbau des industriellen Feuergeschäfts ..............................283

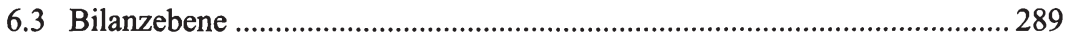

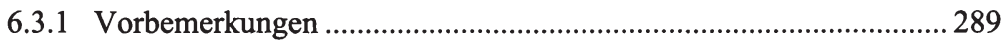

6.3.2 Bilanzielle Darstellung des Basisszenarios ..................................... 293

6.3.3 Effekte ausgewählter Zeichnungs- und

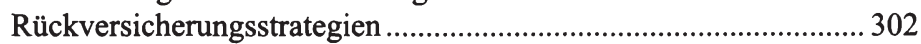

6.4 Implikationen der Modellergebnisse für die risikokapitalbasierte

Steuerung 
Anhang A: Verteilungsannahmen und Parameter der Großschadenmodellierung.... 319 Anhang B: Allokationsergebnisse für unterschiedliche Risikoaggregationsstufen... 320 Anhang C: Übersicht der verwendeten Wahrscheinlichkeitsverteilungen 321

Anhang D: Konvergenzverhalten und Stabilität der Simulationsergebnisse.............. 324 Anhang E: Struktur des Randbereichs der Bruttoergebnisverteilung......................... 326 
Mirko Tillmann - 978-3-631-75327-9

Downloaded from PubFactory at 01/11/2019 06:10:15AM

via free access 


\section{Abbildungsverzeichnis}

Abbildung 2.1: $\quad$ Systematisierung des Risikobegriffs in der Schaden- und

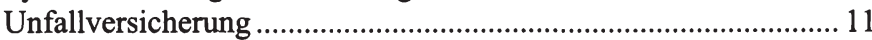

Abbildung 2.2: $\quad$ Einperiodige Ruinwahrscheinlichkeit und Risikokapital................ 22

Abbildung 3.1: Drei-Säulen-Struktur von Solvency II......................................... 33

Abbildung 3.2: Determinanten und Variablen der Rendite-Risikosteuerung ......... 52

Abbildung 3.3: Struktursteuerung vs. Marktbereichssteuerung ................................. 56

Abbildung 4.1: $\quad$ Schematische Darstellung der Value-at-Risk-Konzeption............... 68

Abbildung 4.2: $\quad$ Schematische Darstellung des Conditional Value-at-Risk ............. 73

Abbildung 5.1: Detaillierungsgrad und Kosten der Modellbildung........................ 109

Abbildung 5.2: Struktur des versicherungstechnischen Modellportfolios ............ 120

Abbildung 5.3: Ermittlung des Ergebnisses eines Anfalljahres ............................ 122

Abbildung 5.4: Ermittlung des Geschäftsjahresergebnisses .................................. 124

Abbildung 5.5: Modellparameter der Basisschadenlast ......................................... 136

Abbildung 5.6: Verlaufsmuster auf Incurred-Basis................................................. 139

Abbildung 5.7: Empirische Verteilungsfunktion und Anpassungsgüte ................. 145

Abbildung 5.8: Negative Binomialverteilung vs. Poissonverteilung ..................... 146

Abbildung 5.9: Verteilungsanpassung im Segment „Kraftfahrt-Haftpflicht

Privatkunden“............................................................................. 151

Abbildung 5.10: Verteilungsanpassung des Durchschnittsschadens (Sturm) ......... 156

Abbildung 5.11: Verteilung des Schadenaufwands je Sturmereignis ..................... 157

Abbildung 5.12: Verteilung des Schadenaufwands je Hagelereignis ...................... 160

Abbildung 5.13: Verteilung des Schadenaufwands aus Überschwemmungs-

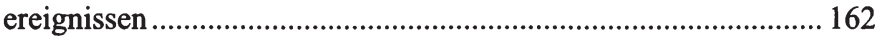

Abbildung 5.14: Systematik zur Abwicklung von Versicherungsfällen .................. 181

Abbildung 5.15: Abwicklung des erwarteten Endschadens in der Sparte „Allgemeine Unfall““.

Abbildung 5.16: Abwicklung des simulierten Endschadens im Geschäftsjahr 2004 .

Abbildung 5.17: Abwicklung der vorangegangenen Anfalljahre im

Geschäftsjahr 2004 
Abbildung 5.18: Dichtefunktionen normalverteilter und lognormalverteilter Aktienkursrenditen 207

Abbildung 5.19: Modellaufbau und Simulationsablauf in der Anfalljahressicht.... 217 Abbildung 5.20: Modellaufbau und Simulationsablauf in der Geschäftsjahressicht

Abbildung 6.1: Zweidimensionaler Untersuchungsaufbau ................................... 222

Abbildung 6.2: Entstehung des Brutto-Anfalljahrergebnisses ............................... 225

Abbildung 6.3: Brutto-Anfalljahrergebnisse und Return on Sales (ROS) nach Versicherungszweigen

Abbildung 6.4: Streuungsverhalten der Schadenkategorien ................................. 228

Abbildung 6.5: Empirische Dichtefunktion des Brutto-Gesamtschadens............. 229

Abbildung 6.6: Streuungsverhalten der Brutto-Anfalljahresergebnisse nach

Versicherungszweigen

Abbildung 6.7: Streuungsverhalten der Brutto-Anfalljahrergebnisse nach Geschäftsfeldern

Abbildung 6.8: Empirische Dichtefunktion des Brutto-Anfalljahrergebnisses..... 234

Abbildung 6.9: Perzentilgraph des Brutto-Anfalljahrergebnisses zwischen $0 \%$ und $5 \%$

Abbildung 6.10: Diversifikationseffekte unterschiedlicher

Portfolio-Aggregationen

Abbildung 6.11: Empirische Dichtefunktionen des Gesamtschadens.

Abbildung 6.12: Streuungsverhalten der Netto-Anfalljahrergebnisse nach

Versicherungszweigen

Abbildung 6.13: Relatives Streuungsverhalten der Spartenergebnisse.

Abbildung 6.14: Perzentilgraph der Anfalljahrergebnisse zwischen

$0 \%$ und $30 \%$

Abbildung 6.15: Allokation des Risikokapitalbedarfs auf Versicherungszweige .. 250

Abbildung 6.16: Streudiagramm des Schadenaufwands aus Sturmschäden in VGV ST (Basisszenario)

Abbildung 6.17: Risikokapitalbedarf des Rückversicherers in der isolierten Betrachtung

Abbildung 6.18: Berücksichtung von Kapitalanlageerträgen im simulierten Anfalljahr.

Abbildung 6.19: Netto-Anfalljahrergebnis unter Berücksichtigung von Kapitalanlageerträgen 
Abbildung 6.20: „Effizienzlinie“" kombinierter Portfolios aus

Versicherungsverträgen und Kapitalanlagen 259

Abbildung 6.21: Lineare Abhängigkeiten im modifizierten Basisszenario 265

Abbildung 6.22: Nicht-lineare Abhängigkeiten im modifizierten Basisszenario (Gumbel-Copula) 270

Abbildung 6.23: Streudiagramm des Schadenaufwands aus Sturmschäden in VGV ST mit Stop-Loss-Deckung 273

Abbildung 6.24: Einfluss der Stop-Loss-Deckung auf den Netto-Risikokapitalbedarf 274

Abbildung 6.25: Streudiagramm der KH-Großschäden im Basisszenario............... 275

Abbildung 6.26: Auswirkungen unterschiedlicher XL-Prioritäten in „Kraftfahrt-Haftpflicht“ 278

Abbildung 6.27: Versicherungstechnische Ergebnisse der Industriellen Feuerversicherung. 284

Abbildung 6.28: Auswirkungen alternativer RV-Strukturen für die Sparte „Feuer Industrie“ 288

Abbildung 6.29: Versicherungstechnisches Ergebnis (Brutto vs. Netto) 294

Abbildung 6.30: Perzentilgraph des versicherungstechnischen Cashflows 296

Abbildung 6.31: Kapitalanlageergebnis vs. Total Return ....................................... 297

Abbildung 6.32: Veränderung der Bewertungsreserven ........................................ 298

Abbildung 6.33: Jahresüberschuss nach Steuern (Basisszenario)............................. 299

Abbildung 6.34: JÜ nach Steuern mit SL-Deckung (VGV ST, AST) und Selbstbeteiligung (VGV LW) 302

Abbildung A.1: Konvergenzverhalten des Brutto-Anfalljahrergebnisses für $\mathrm{n}=5.000$

Abbildung A.2: Konvergenzverhalten des Brutto-Anfalljahrergebnisses für $\mathrm{n}=30.000$

Abbildung A.3: Tailstruktur der Verteilung des Brutto-Anfalljahrergebnisses ..... 326 
Mirko Tillmann - 978-3-631-75327-9

Downloaded from PubFactory at 01/11/2019 06:10:15AM

via free access 


\section{Tabellenverzeichnis}

Tabelle 5.1: Abwicklungsdreieck des kumulierten Schadenaufwands ................... 139

Tabelle 5.2: Individuelle Abwicklungsfaktoren und Chain-Ladder-Faktoren ....... 140

Tabelle 5.3: Vervollständigtes Abwicklungsdreieck mit Endschadenständen........ 141

Tabelle 5.4: Ermittlung der durchschnittlichen Schadenhöhe je Anfalljahr .......... 141

Tabelle 5.5: Aufteilung der Jahreseinheiten auf die PML-Bänder........................... 152

Tabelle 5.6: Verteilung der Naturgefahrschäden auf Versicherungszweige und Geschäftsfelder ......................................................................... 155

Tabelle 5.7: Schadenpotenzial eines einzelnen Sturmereignisses........................... 158

Tabelle 5.8: Schadenpotenzial eines einzelnen Hagelereignisses ............................. 159

Tabelle 5.9: Überschwemmungsszenarien .............................................................. 161

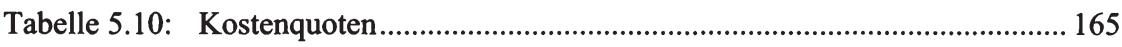

Tabelle 5.11: Bandstruktur der PML-Sparte Feuer Industrie...................................... 170

Tabelle 5.12: Quotierungsverfahren ................................................................... 174

Tabelle 5.13: Haftungsstruktur und Parameter des Rückversicherungsprogramms im Basisszenario.............................................................. 178

Tabelle 5.14: Aufwandsdreieck mit Chain-Ladder-Faktoren und Aufwandsfaktoren

Tabelle 5.15: Zahlungsdreieck mit Chain-Ladder-Faktoren und Zahlungsfaktoren 184

Tabelle 5.16: Berechnungsschema der diskontierten Bedarfsreserve $(\mathrm{i}=2,48 \%) \ldots 190$

Tabelle 5.17: Parameter der Anleihemodellierung................................................. 210

Tabelle 5.18: Korrelationsmatrix der modellierten Assetklassen ............................. 213

Tabelle 5.19: Ausgangsallokation der Kapitalanlagen, Rendite- und

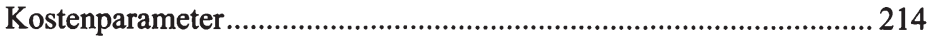

Tabelle 6.1: Prämien- und Geschäftsstruktur im Basisszenario................................ 224

Tabelle 6.2: $\quad$ Struktur und Kennziffern des Brutto-Anfalljahrergebnisses............... 226

Tabelle 6.3: Kennziffern des Brutto-Anfalljahresergebnisses nach Versicherungszweigen

Tabelle 6.4: Kennziffern des Brutto-Anfalljahresergebnisses nach Geschäftsfeldern

Tabelle 6.5: Allokation des Risikokapitalbedarfs (Brutto) ………………………... 237

Tabelle 6.6: Statistische Kenngrößen der Gesamtschadenverteilungen................... 244 
Tabelle 6.7: Kennziffern des Netto-Anfalljahrergebnisses nach

Versicherungszweigen 245

Tabelle 6.8: Ergebnisstruktur, Risikokapitalbedarf und RORAC .......................... 248

Tabelle 6.9: Frequenzrisiko der Naturgefahr Sturm im Basisszenario (Beispiel).. 252

Tabelle 6.10: Rendite-Risiko-Relationen unterschiedlicher Ergebnisstufen

Tabelle 6.11: Risiko-Rendite-Relationen unterschiedlicher Aktien-Geldmarkt-Portfolios.

Tabelle 6.12: Lineare Abhängigkeiten im Basisszenario ........................................... 264

Tabelle 6.13: Nicht-lineare Abhängigkeiten im Basisszenario (Gumbel-Copula) .. 269

Tabelle 6.14: Nettorisikoposition im Basisszenario und bei Verzicht auf XL-Deckung in $\mathrm{KH}$

Tabelle 6.15: Einführung einer Selbstbeteiligung in der Sparte „VGV Leitungswasser“

Tabelle 6.16: Bestandsstruktur „Feuer Industrie“ nach Erhöhung der Zeichnungsquoten

Tabelle 6.17: Auswirkung der erhöhten Zeichnungsquoten auf die

Rendite-Risiko-Position

Tabelle 6.18: Auswirkungen alternativer Rückversicherungsstrukturen

für ,Feuer Industrie“

Tabelle 6.19: Netto-Eingangsreserven nach Versicherungszweigen ......................... 290

Tabelle 6.20: Vereinfachte Eröffnungsbilanz......................................................... 292

Tabelle 6.21: Gewinn- und Verlustrechnung im Basisszenario ................................. 293

Tabelle 6.22: Überleitungsrechnung für das versicherungstechnische Bruttogeschäft

Tabelle 6.23: Vereinfachte Schlussbilanz im Basisszenario ..................................... 300

Tabelle 6.24: Ökonomisches Sicherheitskapital im Basisszenario ............................. 301

Tabelle 6.25: Schlussbilanz mit SL-Deckung und Selbstbeteiligung ........................ 303

Tabelle 6.26: Ökonomisches Sicherheitskapital mit SL-Deckung und

Selbstbeteiligung

Tabelle A.1: Verteilungsannahmen u. Parameter der Großschadenmodellierung .. 319

Tabelle A.2: $\quad$ Allokationsergebnis in der Geschäftsfeld-Betrachtung (3 isolierte Teilunternehmen)

Tabelle A.3: Allokationsergebnis in der Sparten-Betrachtung

(9 isolierte Teilunternehmen)

Tabelle A.4: Allokationsergebnis in der Standalone-Betrachtung

(16 isolierte Teilunternehmen) 


\section{Symbolverzeichnis}

a

$a_{j}$

$a_{k}$

A

$\mathrm{AE}$

AL

AR

$\beta$

b

$B_{n}$

BG

$\mathrm{BI}_{\mathrm{n}}$

BR

BS

c

C

$\mathrm{C}_{\mathrm{i}, \mathrm{k}}$

$\mathrm{C}_{\mathrm{i}, \mathrm{n}}$

$\mathrm{CL}_{\mathrm{k}}^{\mathrm{A}}$

$\mathrm{CL}_{\mathrm{k}}^{\mathrm{Z}}$

Cov

CVaR

d

$\mathrm{D}(\cdot)$

DP

DPML

$\varepsilon$

e

E

E(.)

ER
Priorität

Portfolioanteil der Assetklasse j (Notation ALBRECHT/ZiMMERMANN)

Aufwandsfaktor des Abwicklungsjahres k

Anlagekapital (Notation ALBRECHT/ZIMMERMANN)

Abwicklungsergebnis

Allokationslücke

Ausgangsreserve

Parameter der Gumbel-Copula

sicherer Betrag (Axiomensystem von ARTZNER/DELBEAN/EBER/HEATH)

Bruttoprämien im Geschäftsjahr n

Betroffenheitsgrad

Beitragsindex im Geschäftsjahr $n$

Bedarfsreserve

Basisschaden

sicherer Betrag (Axiomensystem von DENAULT)

Copula

kumulierter Schadenaufwand für die im Anfalljahr i eingetretenen Schäden bis zum Abwicklungsjahr $\mathrm{k}$

geschätzter Endschadenaufwand des Anfalljahres i

Chain-Ladder-Faktor des Abwicklungsjahres k (Schadenaufwand)

Chain-Ladder-Faktor des Abwicklungsjahres k (Schadenzahlungen)

Kovarianz

Conditional Value-at-Risk

Driftparameter eines Random Walk

Diversifikationsmaß

Durchschnittsprämie

durchschnittlicher PML (Probable Maximum Loss)

Ruinwahrscheinlichkeit (auch: Steuerungskriterium)

Handlungsalternative

Menge der möglichen Handlungsalternativen

Erwartungswertoperator (auch: EW, $\mu$ )

Eingangsreserve 
EV Erstversicherer

$\mathrm{f}(\cdot) \quad$ Dichtefunktion

$\mathrm{F}(\cdot) \quad$ Verteilungsfunktion

$\mathrm{F}_{\mathrm{i}, \mathrm{k}} \quad$ individueller Abwicklungsfaktor im Anfalljahr i für das Abwicklungsjahr $\mathrm{k}$

$\gamma \quad$ Schiefekoeffizient

G Gewinn (auch: Unternehmenserfolg, Ergebnis)

GS Großschaden

h „funds generating coefficient“ (Notation ALBRECHT/ZIMMERMANN)

H Haftungslimit

i (quasi) sichere Einperiodenrendite (auch: Geldmarktzins)

I Kapitalanlageergebnis (Notation ALBRECHT/ZIMMERMANN)

JE Jahreseinheiten

K Betriebskosten

$\hat{\mathbf{K}} \quad$ Kuponzahlung einer Standardanleihe

$\mathrm{K}_{0} \quad$ Aktienkurs am Periodenanfang

$\mathrm{K}_{1} \quad$ Aktienkurs am Periodenende

$\mathrm{K}_{\mathrm{RV}} \quad$ Kosten des Rückversicherungsschutzes

$\mathrm{L}_{\mathrm{n}} \quad$ Maximum-Likelihood-Funktion (MLF)

$\hat{\mathrm{L}}_{\mathrm{n}}(\cdot) \quad$ Maximum-Likelihood-Schätzer (MLS)

$\mathrm{LPM}_{\mathrm{n}} \quad$ Lower Partial Moment n-ten Grades

$\mathrm{LPM}_{0} \quad$ Lower Partial Moment nullten Grades (= Shortfall-Wahrscheinlichkeit)

LPM $_{1} \quad$ Lower Partial Moment ersten Grades (= Shortfall-Erwartungswert)

$\mathrm{LPM}_{2} \quad$ Lower Partial Moment zweiten Grades (= Shortfall-Varianz)

$\mu \quad$ Erwartungswert

$\mu_{2} \quad$ zweites Zentralmoment

$\mu_{3} \quad$ drittes Zentralmoment

$\mu_{4} \quad$ viertes Zentralmoment

$\bar{\mu}_{\mathrm{i}} \quad$ (gleitender) Erwartungswert einer Folge mit i Elementen

$\mu_{\log N} \quad$ Erwartungswert der Lognormalverteilung 
$\hat{\mu} \quad$ Driftkoeffizient eines verallgemeinerten Wiener Prozesses

m Durchschnittsschaden

M Maximum

MEL Mean-Excess-Loss

MW Marktwert

$v^{2} \quad$ Varianz der Lognormalverteilung

$\mathrm{N} \quad$ Schadenanzahl

$\pi$

$\mathrm{p}$

$\mathbf{P}$

$\mathbf{P}(\cdot)$

$\mathrm{P}^{\text {Orig }}$

$\mathrm{P}^{\mathrm{Quote}}$

$\mathrm{P}_{\text {eff }}^{\text {Quote }}$

$\mathrm{P}_{\mathrm{RV}, \varepsilon}$ $\mathrm{P}^{\mathrm{RV}}$

Prämienerlöse (Notation ALBRECHT/ZIMMERMANN)

Anteil des Rückversicherers

Prämienvolumen

$(\cdot)$

Wahrscheinlichkeitsoperator

Prämienvolumen des Originalgeschäfts

zediertes Prämienvolumen eines Quotenrückversicherungsvertrages

effektive Rückversicherungsprämie eines Quotenrückversicherungsvertrages

PML Probable Maximum Loss

Prov Rückversicherungsprovision

q

Q

$\rho$

$\rho^{\mathrm{P}}$

$\rho^{s}$

$\mathrm{r}^{*}$

$\mathbf{r}_{1}$

$r_{k}$

$\mathrm{R}(\cdot)$

$\mathbf{R}_{\mathrm{A}}(\cdot)$

$\mathrm{R}$

$\mathrm{R}^{\text {Div }}$

RK

$\Delta \mathrm{RK}$

Schadenfrequenz

Tilgungszahlung einer Standardanleihe

Korrelationskoeffizient

Stichproben-Korrelationskoeffizient nach PEARSON

Rangkorrelationskoeffizient nach SPEARMAN

(isolierte) Renditeerwartung des Rückversicherers

interne Rendite (auch: Yield-to-Maturity)

Reservefaktor im Abwicklungsjahr $\mathrm{k}$

Risikomaß

Allokationsrisikomaß

Einperiodenrendite

Dividendenrendite

Risikokapital (auch: Sicherheitskapital)

Veränderung des Risikokapitalbedarfs 
RORAC Return On Risk Adjusted Capital

RV Rückversicherer

$\sigma \quad$ Standardabweichung

$\bar{\sigma}_{\mathrm{i}} \quad$ (gleitende) Standardabweichung einer Folge mit i Elementen

$\sigma_{\log N}$

$\hat{\sigma}^{2}$

Standardabweichung der Lognormalverteilung

$\mathrm{S}$

Diffusionskoeffizient eines verallgemeinerten Wiener Prozesses

$s^{2}$

Anteil des Schadenaufwands oberhalb der Selbstbeteiligung

$\mathrm{S}$

$S^{\text {Quote }}$

$S^{\text {Orig }}$

$\mathrm{S}_{\mathrm{EV}}$

$\mathrm{S}_{\mathrm{RV}}$

Varianz

Gesamtschaden

zedierter Schadenaufwand eines Quotenversicherungsvertrages

Schadenaufwand des Originalgeschäfts

Schadenbelastung des Erstversicherers

$\mathrm{SA}_{\mathrm{B}}$

Schadenbelastung des Rückversicherers

$\mathrm{SA}_{\mathrm{N}}$

Bruttoaufwendungen für Versicherungsfälle

SAnz

Nettoaufwendungen für Versicherungsfälle

SAufw

Schadenanzahl

SB

Schadenaufwand

$\mathrm{SbQ}$

Selbstbeteiligung (auch: Franchise)

$\mathrm{SD}$

durchschnittliche Selbsthaltsquote der letzten drei Geschäftsjahre

SG

Schadendurchschnitt der letzten drei Geschäftsjahre

$\mathrm{SI}_{\mathrm{n}}$

durchschnittlicher Schadengrad

$\operatorname{SSP}_{\mathrm{n}}$

Schadenindex im Geschäftsjahr $n$

Solvabilitätsspanne im Geschäftsjahr n

$\theta$

Poissonparameter

TP

Teilportfolio

$\mathrm{u}$

Vielfaches des Maximums M

$\mathrm{U}$

Sicherheitskapital (Notation ALBRECHT/ZIMMERMANN)

V Verlustvariable

$V_{0} \quad$ Anfangswert einer Vermögensposition

$V_{1} \quad$ Endwert einer Vermögensposition

$V_{t} \quad$ Wert einer Vermögensposition im Zeitpunkt $t$

$\operatorname{Var}(\cdot) \quad$ Varianz

VaR Value-at Risk 
W Wölbung (auch: Kurtosis)

$\mathrm{WP}_{\mathrm{t}} \quad$ Wiener Prozess

$\mathrm{x}^{\text {Prov }} \quad$ Rückversicherungsprovisionssatz

$\mathrm{x}_{\mathbf{k}} \quad$ Allokationsfaktor des k-ten Teilkollektivs

$\mathrm{z}_{\mathrm{k}} \quad$ Zahlungsfaktor des Abwicklungsjahres $\mathrm{k}$

$Z_{t} \quad$ Zahlungen für Vorjahresschäden im Geschäftsjahr $t$

$\hat{Z}_{\mathrm{t}} \quad$ Zufallsprozess eines Random Walk (mit Drift) 
Mirko Tillmann - 978-3-631-75327-9

Downloaded from PubFactory at 01/11/2019 06:10:15AM

via free access 


\section{Abkürzungsverzeichnis}
Abs.
Absatz
AG
Aktiengesellschaft
AJ
Anfalljahr
AnlV
Kapitalanlageverordnung
Anm. d. Verf.
Anmerkung des Verfassers
APRA
Australian Prudential Regulation Authority
ART
Alternativer Risikotransfer
AST
Allgemeine Sturm
AU
Allgemeine Unfall
BaFin
Bundesanstalt für Finanzdienstleistungsaufsicht
$\mathrm{BC}$
Basis for Conclusions
BW
Buchwert

CAPM

Capital Asset Pricing Model

CF

Cashflow

c.p.

ceteris paribus

CVaR

Conditional Value-at-Risk

disk.

diskontiert

DRS

Deutscher Rechnungslegungsstandard

ED

Exposure Draft

EG

Europäische Gemeinschaft

EK

Eigenkapital

EMB

English Matthews Brockman (aktuarielle Beratungsgesellschaft)

EPD

Expected Policyholder Deficit

ESG

Economic Scenario Generator

EStG

Einkommensteuergesetz

EU

Europäische Union

EW

Erwartungswert

EWG

Europäische Wirtschaftsgemeinschaft

f.

ff.

folgende

FIND

fort folgende

FSA

Feuer Industrie

Financial Services Authority

GDV Gesamtverband der deutschen Versicherungswirtschaft e.V. 


$\begin{array}{ll}\text { GE } & \text { Geldeinheiten } \\ \text { GEW } & \text { Gewerbekunden } \\ \text { GF } & \text { Geschäftsfeld } \\ \text { GJ } & \text { Geschäftsjahr } \\ \text { GK } & \text { Gefährdungsklasse } \\ \text { GNPI } & \text { Gross Net Premium Income } \\ \text { GuV } & \text { Gewinn- und Verlustrechnung } \\ & \\ \text { HUK } & \text { Haftpflicht, Kraftfahrt, Unfall }\end{array}$

IAA International Actuarial Association

IAIS International Association of Insurance Supervisors

IASB International Accounting Standards Board

IFRS International Financial Reporting Standards

IND Industriekunden

JÜ Jahresüberschuss

KA Kapitalanlage

kalk. kalkulatorisch

KapAustV Kapitalausstattungsverordnung

KGS Kreisgemeindeschlüssel

KH Kraftfahrt-Haftpflicht

KStG Körperschaftsteuergesetz

LPM Lower Partial Moment

MEL Mean-Excess-Loss

MLF Maximum-Likelihood-Funktion

MLS Maximum-Likelihood-Schätzer

MPS Mean Preserving Spread

MW Marktwert

o.V. ohne Verfasser

PML Probable Maximum Loss (wahrscheinlicher Höchstschaden)

PRI Privatkunden

RAPM risikoadjustierte Performancemaße

RBF Rentenbarwertfaktor

RechVersV Verordnung über die Rechnungslegung von Versicherungsunternehmen

RLZ Restlaufzeit 


\begin{tabular}{|c|c|}
\hline RORAC & Return On Risk Adjusted Capital \\
\hline ROS & Return on Sales \\
\hline Rückst. & Rückstellung \\
\hline RV & Rückversicherer \\
\hline S. & Seite \\
\hline SimAJ & simuliertes Anfalljahr \\
\hline SimGJ & simuliertes Geschäftsjahr \\
\hline SL & Stop Loss \\
\hline Sp. & Spalte \\
\hline SRK & Schadenregulierungskosten \\
\hline st. $\mathbf{R}$. & stille Reserven \\
\hline Tab. & Tabelle \\
\hline TR & Total Return \\
\hline TVaR & Tail Value-at-Risk \\
\hline Tz. & Textziffer \\
\hline
\end{tabular}

Überschw. Überschwemmung

$\begin{array}{ll}\text { VAG } & \text { Versicherungsaufsichtsgesetz } \\ \text { VaR } & \text { Value-at-Risk } \\ \text { VGV } & \text { Verbundene Wohngebäudeversicherung } \\ \text { VGV EL } & \text { Verbundene Wohngebäudeversicherung Elementar } \\ \text { VGV F } & \text { Verbundene Wohngebäudeversicherung Feuer } \\ \text { VGV LW } & \text { Verbundene Wohngebäudeversicherung Leitungswasser } \\ \text { VGV ST } & \text { Verbundene Wohngebäudeversicherung Sturm } \\ \text { VJ } & \text { Vorjahr } \\ \text { VS } & \text { Versicherungssumme } \\ \text { VT } & \text { Versicherungstechnik } \\ \text { vt. } & \text { versicherungstechnisch }\end{array}$

WA Wiederauffüllung

XL Einzelschadenexzedentenvertrag

YTM Yield-to-Maturity (auch: interne Rendite)

ZÜRS Zonierungssystem für Überschwemmung, Rückstau und Starkregen 
Mirko Tillmann - 978-3-631-75327-9

Downloaded from PubFactory at 01/11/2019 06:10:15AM

via free access 


\section{$1 \quad$ Einleitung}

\subsection{Problemstellung}

Jede unternehmerische Aktivität ist untrennbar mit dem Eingehen von Risiken verbunden, da zukunftgerichtete Entscheidungen stets der Unsicherheit künftiger Entwicklungen unterworfen sind. ${ }^{1}$ Im spezifischen Geschäftsmodell der Schaden- und Unfallversicherung stellt die gezielte Übernahme und das Management von Risiken den Kern jeglicher unternehmerischer Tätigkeit dar. ${ }^{2}$ Im Gegensatz zu anderen Wirtschaftszweigen gilt es zu berücksichtigen, dass die Existenzsicherheit eines Versicherungsunternehmens unmittelbaren Einfluss auf die Qualität des Versicherungsversprechens, mithin die Qualität der angebotenen Produkte ausübt. ${ }^{3}$ Die Kapitalausstattung eines Versicherungsunternehmens stellt daher einen relevanten Wettbewerbsfaktor dar. In Bezug auf die Höhe des vorzuhaltenden Kapitals ist jedoch ein grundlegender Zielkonflikt zu konstatieren. ${ }^{4}$ Neben den originären Wettbewerb um Kunden tritt in zunehmendem Maße der Wettbewerb um potenzielle Investoren, die bereit sind, dem Versicherungsunternehmen Kapital zur Verfügung zu stellen. Die renditeorientierte Erwartungshaltung der Investoren konkurriert mit der Forderung sonstiger Anspruchsgruppen, wie z.B. Versicherungsnehmern, Ratingagenturen oder staatlichen Aufsichtsbehörden, nach einer möglichst hohen, gegebenenfalls jedoch renditeschwächeren Kapitalausstattung. Der beschriebene Zielkonflikt wird dadurch verschärft, dass sich die traditionell sehr komfortable Kapitalisierung des Versicherungssektors in den vergangenen Jahren deutlich verschlechtert hat. ${ }^{5}$ Als Gründe für diese Entwicklung sind insbesondere der Verfall der internationalen Kapitalmärkte in den Jahren 2000 bis 2002 sowie eine signifikante Zunahme von Großschäden aufgrund von Terror- und Naturgefahrereignissen anzuführen. Vor diesem Hintergrund kommt einer effizienten Nutzung der knappen Ressource Kapital sowie der Entwicklung risikoadäquater Steuerungssysteme elementare Bedeutung zu.

Neben der skizzierten Problematik eines veränderten Marktumfeldes ist festzuhalten, dass die Versicherungsbranche derzeitig mit tiefgreifenden institutionellen Veränderungen konfrontiert ist. Ab dem Geschäftsjahr 2005 müssen kapitalmarktorientierte Versicherungsunternehmen in Europa einen Konzernabschluss nach den International

\footnotetext{
Vgl. HÖLSCHER (2002), S. 5.

Vgl. FARNY (2000), S. 22.

3 Vgl. auch Helten (1975), S. 91, AlBRECHT (1994c), S. 3, SCHRADIN (1994), S. 58-59, OleTZKY (1998), S. 6-7, MAURER (2000), S. 149-153 und SCHMEISER (2004b), S. 1.

4 Vgl. im Folgenden OLETZKY (1998), S. 1-2.

5 Die Kapitalausstattung der weltweit tätigen Erst- und Rückversicherer im Bereich Nichtleben reduzierte sich im Zeitraum von 2000 bis 2002 um ca. 25\%. Vgl. SwISS RE (2002b), S. 3.
} 
Financial Reporting Standards (IFRS) aufstellen. ${ }^{6}$ Die IFRS verpflichten Versicherungsunternehmen in einer ersten Phase zu deutlich erhöhten Publizitätsanforderungen in Bezug auf die Risikostruktur und das Verlustpotenzial ihres Gesamtportfolios. Eine weitere wesentliche Änderung betrifft den Wegfall der handelsrechtlichen Schwankungsrückstellungen. In einer zweiten Phase soll die Zeitwertbilanzierung für Vermögensgegenstände und versicherungstechnische Rückstellungen im Sinne des FairValue-Prinzips verbindlich vorgeschrieben werden. Als Folge dieses zeitwertorientierten Ansatzes ist zu vermuten, dass die Eigenkapital- und Ergebnisentwicklung von Versicherungsunternehmen zukünftig einer deutlich erhöhten Volatilität ausgesetzt sein wird. Neben einer veränderten Bilanzierungspraxis sehen sich die europäischen Versicherungsunternehmen auch gestiegenen aufsichtsrechtlichen Anforderungen ausgesetzt. Als Reaktion auf die konzeptionellen Defizite der bestehenden Vorschriften wurde durch die Europäische Kommission ein Projekt zur grundlegenden Neuausrichtung des europäischen Solvabilitätssystems initiiert (,Solvency $\left.\mathrm{II}^{\prime)}\right)^{7}$ Ausgangspunkt der Überlegungen ist ein erweiterter Solvabilitätsbegriff, der eine risikotheoretisch fundierte Abbildung der Gesamtrisikoposition eines Versicherungsunternehmens ermöglichen soll. Im Hinblick auf die Erfassung und Quantifizierung der relevanten Risikokategorien kommt der Entwicklung unternehmensinterner Risikomodelle elementare Bedeutung zu. Nach Prüfung durch die Aufsichtsbehörden sollen diese zukünftig zur Ermittlung der Zielkapitalausstattung eingesetzt werden können.

Die bisherigen Ausführungen verdeutlichen, dass Versicherungsunternehmen in zunehmendem Maße in einem Spannungsfeld zwischen externen Anforderungen und betriebswirtschaftlicher Notwendigkeit agieren. Wenngleich im Rahmen von Solvency II richtige Anreize zur Entwicklung interner Risikomodelle gesetzt werden, erscheint deren Entwicklung bereits aus ökonomischen und unternehmenspolitischen Erwägungen heraus zwingend geboten. Das veränderte Unternehmensumfeld erfordert eine systematische Erfassung der relevanten Unternehmensrisiken (,Risikoinventur") sowie die Etablierung einer konsistenten Bewertungslogik für die unterschiedlichen Risikokategorien. Unter dem Postulat einer wertorientierten Unternehmensführung bedarf es zudem eines Steuerungsinstrumentariums, welches die integrierte Bewertung von Erfolgschancen und korrespondierenden Risikopotenzialen ermöglicht. Im Mittelpunkt einer solchen Bewertung steht immer die grundlegende Frage, ob eine Handlungsal-

6 Vgl. im Folgenden ENGELÄNDER/KÖLSCHBACH (2004), S. 574-579, RITTMANN/ROCKEL (2004), S. 441-446, SWISS RE (2004), S. 3 und ZIELKE (2005).

7 Vgl. hierzu und im Folgenden KPMG (2002) und LONDON GROUP (2002) sowie darüber hinaus SCHRADIN (2003), S. 641-660, HARTUNG/HELTEN (2004), S. 293-303, SCHUBERT/GRIEBMANN (2004a), S. 470-472 und SCHUBERT/GRIEBMANN (2004b), S. 738-739. 
ternative den Wert des investierten Kapitals erhöht, mithin eine Rendite erwirtschaftet, welche die Opportunitätskosten des Kapitaleinsatzes übersteigt. ${ }^{8}$

Das erklärte Ziel der Versicherungsunternehmen muss daher sein, mit Hilfe interner Risikomodelle eine risikogerechte Kapitalisierung zu ermitteln, die einerseits aufsichtsrechtlichen Ansprüchen genügt und andererseits eine für den Kapitalmarkt attraktive Renditeerzielung ermöglicht. Vor diesem Hintergrund erscheint es geboten, interne Risikomodelle als unverzichtbaren Bestandteil der Unternehmenssteuerung aufzufassen und in den Risikomanagementprozess zur integrieren. ${ }^{9}$ Sowohl die Notwendigkeit als auch die Vorzüge einer risikokapitalbasierten Steuerung sind in der Versicherungsbranche zweifellos erkannt. Die marktweite Etablierung interner Modelle wird jedoch derzeitig noch durch die Tatsache erschwert, dass kein anerkannter Standard für deren konzeptionelle Umsetzung existiert. Diese Einschätzung wird durch folgendes Zitat von NIKOLAUS vON BOMHARD, Vorstandsvorsitzender der MünchenerRück-Gruppe, untermauert:

„Im nächsten Jahr wollen wir zwölf Prozent Rentabilität auf das Eigenkapital nach Internationalen Rechnungslegungsstandards erreichen. [...] allerdings schwankt das Eigenkapital durch die Rechnungslegung sehr stark. Deshalb sind wir über die jetzige Steuerungsgröße nicht sehr glücklich und würden lieber heute als morgen auf eine risikobasierte Steuerung umstellen. [...] Derzeit gibt es eine Reihe von Kapitalmodellen am Markt, und die konvergieren auch allmählich, aber es gibt noch keinen verbindlichen Standard. “10

Die Motivation der vorliegenden Arbeit leitet sich unmittelbar aus der oben beschriebenen Problematik ab. In den folgenden Abschnitten wird zunächst die Zielsetzung der Arbeit in den Forschungskontext eingebettet und weiter konkretisiert. Hierauf aufbauend wird der Untersuchungsverlauf im Überblick dargestellt.

8 Vgl. RaPpaPORT (1986), S. 100-134, COPELAND/KOlLER/MuRRIN (1998), S. 53-61, OletZKY (1998), S. 1 und OLETZKY/SCHULENBURG, GRAF VON DER (1998), S. 66.

9 Vgl. vertiefend zum Risikomanagementprozess ReICHMANN (2001), S. 610, GABRIEL/ROCKEL (2001), S. 103, SPELLMANN (2002), S. 28-30 sowie die Ausführungen in Kapitel 3.2.3.

10 BOMHARD, VON (2004), S. 22. 


\subsection{Zielsetzung der Arbeit}

Die wissenschaftliche Literatur fokussiert bislang insbesondere mathematischanalytische Fragestellungen einer integrierten Rendite-Risikosteuerung und formuliert darüber hinaus konzeptionelle Anforderungen an die Art der Modellierung. ${ }^{11}$ Im Interesse einer analytisch geschlossenen Darstellung müssen diese Arbeiten zumeist vereinfachende Annahmen treffen (z.B. hinsichtlich der gültigen Zufallsgesetzmäßigkeit der Schäden), welche die Realität nicht hinreichend abbilden. Die in der Praxis verwendeten internen Risikomodelle stellen entweder unternehmensspezifische „Insellösungen" dar oder basieren auf komplexen Softwareprodukten, die sich in Bezug auf die modelltechnische Abbildung der einzelnen Risikofaktoren einer tiefergehenden Analyse und Bewertung verschließen (,Black-Box-Problematik“) ${ }^{12}$ Eine offene wissenschaftliche Auseinandersetzung mit der Frage, wie ein internes Risikomodell in der Praxis mit den zur Verfügung stehenden Daten konzipiert sein könnte, findet hingegen nur vereinzelt statt ${ }^{13}$ oder konzentriert sich auf die spezifische Anwendung in Lebensversicherungsunternehmen. ${ }^{14}$

Die Zielsetzung der vorliegenden Arbeit besteht darin, einen Beitrag zur Schließung dieser Lücke zu leisten. Es wird ein Vorschlag zur praxistauglichen Ausgestaltung eines risikokapitalbasierten Steuerungsmodells für die Schaden- und Unfallversicherung entwickelt. Dabei liegt der Schwerpunkt der Betrachtung auf der Steuerung von Erstversicherungsunternehmen. Der gewählte Simulationsansatz stellt einen risikotheoretisch fundierten Bezugsrahmen für die strategische Steuerung der Rendite-RisikoPosition eines Versicherungsunternehmens bereit und dient der Unterstützung zielsetzungsgerechter Entscheidungen der Unternehmensleitung. Im Rahmen einer wahrscheinlichkeitsbasierten Bewertung zuvor definierter Handlungsalternativen und Strategien ist demzufolge ausschließlich die Rendite-Risikopräferenz der Unternehmensleitung bzw. der Kapitalgeber relevant.

11 Vgl. im deutschsprachigen Raum exemplarisch OLETZKY (1998), BROHM (2002), GRÜNDL/ SCHMEISER (2002), DOTTERWEICH (2004), ALBRECHT/KORYCIORZ (2004), KORYCIORZ (2004), BROHM/KÖNIG (2004), SCHMEISER (2004a), SCHMEISER (2004b) sowie darüber hinaus DENAULT (2001), ARTZNER/DELBAEN/Eber/HEATH (2002), YAMAI/YoshibA (2002), ACERBI/TASCHE (2002a) und VENTER (2004).

12 Einen Überblick marktgängiger Modelle vermittelt BROHM (2002), S. 244-273.

13 Vgl. Corell (1998a), CORELl (1998b), Frey/NießEN (2001), SANN/JuSTEN/OTTEN/Fromme (2003) und LEYHERR/SCULLY/SOMMERFELD (2003). Eine Ausnahme bildet diesbezüglich die Untersuchung von SCHMEISER, der unter Einsatz der Latin-Hypercube-Methode ein mehrperiodiges Simulationsmodell entwickelt und auf der Basis eines Originaldatensatzes unterschiedliche (Stress-)Szenarien untersucht. Vgl. hierzu vertiefend SCHMEISER (2004a), S. 41-52 und außerdem OSETROVA/SCHMEISER (2005), S. 18-30.

14 Vgl. zur Anwendung in Lebensversicherungsunternehmen exemplarisch FöRTERER (2000), HEINKE (2002a), HEINKE (2002b) und COTTIN/KURZ (2003). 
Bilanzielle oder aufsichtsrechtliche Anforderungen an die Steuerung finden nur insofern Eingang in das Entscheidungskalkül, als dass sie eine „strenge Nebenbedingung“ für die interne Steuerung darstellen.

Um über den theoretischen Erkenntnisfortschritt der Modellbildung hinaus verwertbare Ergebnisse und Steuerungsimplikationen für die Versicherungspraxis abzuleiten, wird das entwickelte Referenzmodell exemplarisch auf den Datensatz eines deutschen Schaden- und Unfallversicherungsunternehmens angewendet. Um Rückschlüsse auf das Originalunternehmen zu vermeiden, wurden die Portfoliovolumina verändert und die Risikoparameter des Originalunternehmens strukturerhaltend modifiziert. Die verwendete Datenbasis erhebt daher lediglich den Anspruch auf strukturelle Repräsentativität für den deutschen Markt und dient dazu, ausgewählte Aspekte einer risikokapitalbasierten Steuerung zu illustrieren. Wenngleich nicht der vollständige Spartenmix eines Schaden- und Unfallversicherungsnehmens abgebildet wird, können die analysierten Fragestellungen durchaus als praxisrelevant und die Parameterkonstellationen als marktnah eingestuft werden. Im Zuge einer modellgestützten Risikoanalyse ${ }^{15}$ werden schließlich Implikationen und Handlungsempfehlungen für die strategische Steuerung abgeleitet. Der Schwerpunkt der Analyse liegt hierbei klar auf dem versicherungstechnischen Kerngeschäft.

Es wird deutlich, dass die vorliegende Arbeit primär eine handlungs- bzw. entscheidungsorientierte Zielsetzung verfolgt. Aus wissenschaftstheoretischer Perspektive ist sie daher der praktisch-normativen Betriebswirtschaftslehre zuzuordnen. ${ }^{16}$

\subsection{Gang der Untersuchung}

In Kapitel 2 werden zunächst die Charakteristika der Schaden- und Unfallversicherung im Überblick behandelt. Nach einer allgemeinen Definition des zugrunde liegenden Risikobegriffs werden unterschiedliche Risikofaktoren diskutiert, welche auf die Rendite-Risiko-Position eines Schaden- und Unfallversicherungsunternehmens Einfluss nehmen. Hierauf aufbauend wird die Notwendigkeit einer ökonomischen Risikokapitalkonzeption aufgezeigt und diese im Hinblick auf ihre theoretische Fundierung beleuchtet.

15 Vgl. zum Begriff der Risikoanalyse auch WEBER (2004), S. 451-452.

16 Vgl. exemplarisch zur wissenschaftstheoretischen Fundierung der Betriebswirtschaftslehre und zu den Zielsetzungen einer realwissenschaftlichen Forschung ULRICH/HILL (1979), S. 161-190 und RAFFÉE (1995), S. 1-120. Zu den klassischen Erkenntnistheorien der modernen Wissenschaftslehre vgl. außerdem RAFFÉE/ABEL (1979), S. 1-10, KERN (1979), S. 11-27 und POPPER (1994). 
Kapitel 3 behandelt ausfuihrlich die externen und internen Anforderungen an die Rendite-Risikosteuerung eines Schaden- und Unfallversicherungsunternehmens. Ausgehend von der Darstellung und kritischen Würdigung der aktuell gültigen Solvabilitätsvorschriften (Solvency I) werden die Überlegungen der Europäischen Kommission zur Neuausrichtung des europäischen Solvabilitätssystems beschrieben und Implikationen für die Steuerung mittels interner Risikomodelle abgeleitet. Im Anschluss an die aufsichtsrechtlichen Rahmenbedingungen werden betriebswirtschaftliche Anforderungen an die Steuerung formuliert und sowohl die Notwendigkeit als auch die Instrumente einer integrierten Rendite-Risikosteuerung aufgezeigt. In Anlehnung an das „Duale Steuerungsmodell ${ }^{117}$ in Kreditinstituten wird hierbei grundlegend zwischen einer zentralen Struktursteuerung und einer dezentralen Marktbereichsteuerung differenziert.

Gegenstand von Kapitel 4 sind die risikotheoretischen Grundlagen einer integrierten Rendite-Risikosteuerung. Die Darstellung gliedert sich thematisch in zwei aufeinander aufbauende Sachgebiete. Der erste Themenkomplex behandelt die Fragestellung: „Wie kann Risiko gemessen und Risikokapital quantifiziert werden?“" Es werden zunächst klassische Risikomaße vorgestellt und hinsichtlich ihrer Eignung für die Unternehmenssteuerung kritisch hinterfragt. Hierauf aufbauend wird mit dem Axiomensystem von ARTZNER/DELBAEN/EBER/HEATH ein Katalog wünschenswerter Eigenschaften skizziert, über die Risikomaße gemeinhin verfügen sollten. Schließlich werden mit dem Value-at-Risk und Conditional Value-at-Risk zwei so genannte Shortfall-Risikomaße vorgestellt und hinsichtlich ihrer Kompatibilität mit dem zuvor beschriebenen Kriterienkatalog verglichen. Der zweite Themenkomplex setzt sich mit der Allokation, mithin der Verteilung des ermittelten Risikokapitals auf einzelne Steuerungseinheiten auseinander. Ausgangspunkt der Überlegungen ist eine kritische Auseinandersetzung mit der Frage, unter welchen Voraussetzungen und zu welchem Zweck eine Allokation überhaupt sinnvoll erscheint. Schließlich werden vier grundlegende Allokationsverfahren vorgestellt und hinsichtlich ihrer Eignung für die interne Unternehmenssteuerung überprüft.

Im Mittelpunkt des Kapitels 5 steht die Entwicklung eines praxistauglichen Referenzmodells zur risikokapitalbasierten Steuerung. Den Anfang bilden generelle modelltheoretische Grundlagen sowie die Formulierung eines versicherungsspezifischen Anforderungsprofils. Dem Geschäftsmodell der Schaden- und Unfallversicherung Rechnung tragend, zerfällt die modelltechnische Abbildung in die Bereiche der Versicherungstechnik und Kapitalanlagen. Hinsichtlich des versicherungstechnischen Kerngeschäfts zeichnet sich das Modell durch einen hohen Detaillierungsgrad aus. Das Portfolio wird

17 Vgl. zum „Dualen Steuerungsmodell“ insbesondere SCHIERENBECK (2001), S. 87-101, SCHIERENBECK/LISTER (2001), S. 14-18 und SCHIERENBECK (2003a), S. 293-303. 
auf der Grundlage einer Matrixstruktur aus Versicherungszweigen und Kundengruppen (= Geschäftsfeldern) abgebildet. In einem separaten Teilmodul wird die Wirkungsweise klassischer Formen der Rückversicherung berücksichtigt. Die Modellierung der Kapitalanlagetätigkeit erfolgt auf stark aggregierter Basis und unterteilt das Anlageportfolio in drei Hauptanlageklassen. Die notwendigen Kapitalmarktszenarien bzw. Renditeverteilungen werden über einen so genannten „Economic Scenario Generator" erzeugt. Der entwickelte Modellansatz unterstützt sowohl eine risikoorientierte, mithin ökonomische Sicht auf ein Schaden- und Unfallversicherungsunternehmen als auch die Überleitung in eine handelsrechtliche Bilanzperspektive.

Das Referenzmodell wird in Kapitel 6 schließlich auf den modifizierten Datensatz eines deutschen Schaden- und Unfallversicherungsunternehmens angewendet. Nach einer detaillierten Analyse der Ausgangssituation („Basisszenario“) werden exemplarisch unterschiedliche strategische Handlungsalternativen hinsichtlich ihrer Auswirkungen auf die Rendite-Risiko-Position des Modellunternehmens analysiert und Implikationen für die risikokapitalbasierte Unternehmenssteuerung abgeleitet. Im Mittelpunkt dieser modellgestützten Risikoanalyse stehen stochastische Abhängigkeitsstrukturen und die Auswirkungen unterschiedlicher Zeichnungs- und Rückversicherungsstrategien.

In Kapitel 7 werden die zentralen Ergebnisse der Arbeit thesenartig zusammengefasst. Aufbauend auf einer kritischen Reflektion der Modellgrenzen werden im Sinne eines Ausblicks zukünftige Arbeitsfelder und weiterer Forschungsbedarf identifiziert sowie angrenzende Einsatzgebiete für den entwickelten Modellansatz aufgezeigt. 
Mirko Tillmann - 978-3-631-75327-9

Downloaded from PubFactory at 01/11/2019 06:10:15AM

via free access 


\section{Spezifika der Schaden- und Unfallversicherung}

\subsection{Begriffliche Abgrenzung der Schaden- und Unfallversicherung}

Die Vielzahl der in der Praxis betriebenen Versicherungsgeschäfte kann anhand unterschiedlicher Kriterien kategorisiert werden. In grober Einteilung wird im Bereich der Erstversicherung ${ }^{18}$ zwischen Lebensversicherungsunternehmen, substitutiven Krankenversicherungsunternehmen und Schaden- und Unfallversicherungsunternehmen differenziert. Als Folge des in $\S 8$ Abs. 1a VAG kodifizierten Spartentrennungsgebots darf ein Versicherungsunternehmen die genannten Versicherungszweige ${ }^{19}$ jedoch nicht gleichzeitig anbieten. Die aufsichtsrechtlich geforderte Spartentrennung stellt daher in Deutschland das zentrale Motiv zur Bildung von Versicherungsgruppen dar. Die Begründung für eine derartige „Spartentrennung durch Unternehmenstrennung ${ }^{\text {(20 }}$ ergibt sich insbesondere aus dem hoch zu bewertenden Gläubigerschutz der Versicherungsnehmer in der Lebens- und Krankenversicherung und der hieraus erwachsenden Zielsetzung, die Versichertenguthaben vor übermäßigen Schwankungen und möglichen Quersubventionierungen anderer Versicherungszweige zu schützen. Die für diese Versicherungszweige typischen, langfristig angelegten Spar- und Entsparprozesse sind sowohl in sozialer als auch in volkswirtschaftlicher Hinsicht von zentraler Bedeutung. ${ }^{21}$ Die Schaden- und Unfallversicherung umfasst in der aufsichtsrechtlich gewählten Aufteilung nach versicherten Gefahren ${ }^{22}$ die in Teil A des Anhangs zum VAG genannten Versicherungssparten, die sich wiederum in die Bereiche der Sachversicherung und HUK-Versicherung (Haftpflicht, Unfall, Kraftfahrt) unterteilen lassen. ${ }^{23}$ Bis zum 30.06.1990 unterlagen auch die Rechtsschutzversicherung sowie die Kredit- und Kautionsversicherung dem Spartentrennungsgebot. Dieses wurde im Zuge europäischer Rechtsharmonisierung jedoch aufgehoben. ${ }^{24}$ In Deutschland wird die Rechtsschutzversicherung jedoch weiterhin zu einem maßgeblichen Anteil von Spezialversicherern betrieben.

18 Vgl. zur Unterscheidung von Erst- und Rückversicherung FARNY (2000), S. 221-222.

19 Die Begriffe Versicherungssparte und Versicherungszweig werden fortan synonym verwendet.

20

21

22

23 FARNY (2000), S. 114.

Vgl. vertiefend STEINMÜLLER (1988), S. 49-53 und ZWEIFEL/EISEN (2003), S. 8-26.

Als Differenzierungskriterien kommen neben der versicherten Gefahr auch die Art der Versicherungsleistung und die Art der versicherten Objekte bzw. Gegenstände in Betracht. Vgl. hierzu vertiefend KoCH (1988), S. 1252-1254.

Betreibt ein Versicherungsunternehmen die Sach- und HUK-Versicherung gemeinsam, ist in der Praxis auch die Bezeichnung „Kompositversicherer“ geläufig. Vgl. FARNY (2000), S. 114-115.

Um Interessenkonflikte zwischen den Versicherungszweigen Rechtsschutz und Haftpflicht zu vermeiden, müssen Versicherungsunternehmen, welche die Rechtsschutzversicherung betreiben, gemäß § 8a VAG die Leistungsbearbeitung (Schadenregulierung) an ein unabhängiges Unternehmen ausgliedern. 
Die vorliegende Arbeit fokussiert die Belange der Schaden- und Unfallversicherung und nimmt nur für ausgewählte Fragestellungen Bezug auf andere Sparten. Abgesehen von der organisatorischen und sachlogischen Trennung von anderen Versicherungszweigen ist die Schaden- und Unfallversicherung sehr spezifischen Risiken bzw. Risikofaktoren ausgesetzt, die im nachfolgenden Abschnitt systematisiert und erläutert werden sollen.

\subsection{Kategorisierung versicherungsspezifischer Risiken}

\subsubsection{Definition von Risiko}

Risiken sind ein integraler Bestandteil unternehmerischer Tätigkeit. ${ }^{25}$ Sie stellen sowohl eine Gefahr als auch eine notwendige Voraussetzung für den wirtschaftlichen Erfolg dar und lassen sich daher niemals vollständig ausschalten oder gar beherrschen. Im Hinblick auf die Eintrittswahrscheinlichkeit möglicher Umweltzustände wird in der betriebswirtschaftlichen Entscheidungstheorie vielfach zwischen den Begriffen Ungewissheit, Unsicherheit und Risiko differenziert. ${ }^{26}$ Ungewissheit liegt vor, sofern grundsätzlich mehrere Umweltzustände für möglich gehalten werden, die Wahrscheinlichkeiten für deren Eintritt jedoch unbekannt sind. Demgegenüber wird von Unsicherheit gesprochen, wenn der Entscheidungsträger alternativen Umweltzuständen zumindest subjektive Eintrittwahrscheinlichkeiten zuordnen kann. Unsicherheit wird zum Risiko, sofern objektive ${ }^{27}$ (z.B. empirisch geschätzte) Eintrittswahrscheinlichkeiten angegeben werden können und zusätzlich die Gefahr einer Zielverfehlung existiert. In einem weit gefassten Begriffsverständnis beinhaltet Risiko sowohl positive als auch negative Zielverfehlungen, welche der Entscheidungsträger durch die Festlegung eines Referenzwertes voneinander abgrenzen muss. ${ }^{28}$ Im Rahmen dieser Arbeit wird Risiko in einer engeren Begriffsauffassung als die in einem unzureichenden Informationsstand begründete Gefahr einer negativen Abweichung von einem erwarteten Referenzwert verstanden. ${ }^{29}$ Der gewählte Risikobegriff umfasst ausdrücklich nicht nur eine einfache Wahrscheinlichkeitsaussage, sondern erstreckt sich auch auf das Ausmaß der poten-

25 Vgl, im Folgenden auch HöLSCHER (2002), S. 5 und BLEUEL/SCHMITTING (2000), S. 69-71.

26 Vgl. im Folgenden ADAM (1996), S. 224-228 und BERENS/DELFMANN/SCHMiTTING (2004), S. 53. Zur ethymologischen Bedeutung des Risikobegriffs vgl. außerdem ROMEIKE/MÜLLER-REICHARD (2005), S. 47.

Das Phänomen subjektiv unterschiedlicher Wahrnehmung objektiv gleichwertiger Unsicherheitssituationen (,perceived risk") wird nicht thematisiert. Vgl. hierzu detailliert BRACHINGER/WEBER (1997) sowie MÜLLER (2003), S. 655-660. Vgl. SPELlManN (2002), S. 9.

29 Vgl. zu einem vergleichbaren Begriffsverständnis ROMEIKE/MÜLLER-REICHARD (2005), S. 47-52, AlBrecht (2003), S. 3, ALBRECHT (1994b), S. 327, LisTer (1997), S. 6 und SPELLMANN (2002), S. 9. ALBRECHT definiert Risiko sowohl als Ausmaß der Abweichung von einer Zielgröße (Typus I) als auch als notwendiges Kapital zum Ausgleich bereits eingetretener Risiken (Typus II). Vgl. in diesem Zusammenhang vertiefend ALBRECHT (2003), S. 8-9. 
ziellen Negativabweichung. ${ }^{30}$ Im weiteren Verlauf werden ausschließlich so genannte Finanzrisiken betrachtet. Diese konkretisieren sich beispielsweise in dem Auftreten finanzieller Verluste oder auch in der Verfehlung von Mindestrenditen oder Mindestvermögensständen. ${ }^{31}$ Der verwendete Risikobegriff beinhaltet somit einen UrsacheWirkungs-Zusammenhang, der einer ökonomischen Interpretation zugänglich ist. Das oben bereits thematisierte Informationsdefizit hinsichtlich zukünftiger Umweltzustände stellt die kausale Risikodimension dar, während die finale Risikodimension die negativen Auswirkungen auf das Zielsystem des Wirtschaftssubjektes beschreibt. Abbildung 2.1 vermittelt einen schematischen Überblick über die Risikosituation eines Schaden- und Unfallversicherungsunternehmens. Die einzelnen Risikokategorien werden in den nachfolgenden zwei Kapiteln detailliert erläutert.

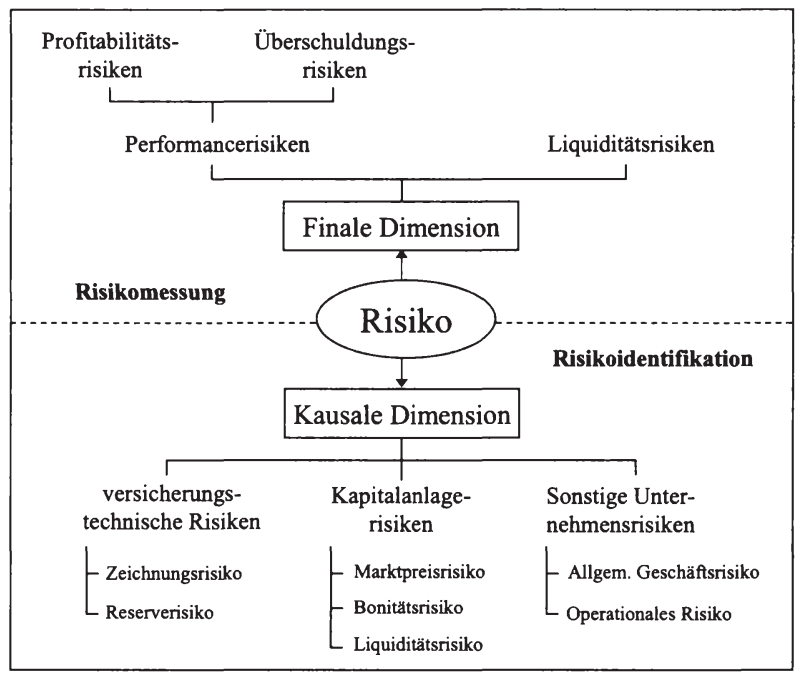

Abbildung 2.1: Systematisierung des Risikobegriffs in der Schaden- und Unfallversicherung Quelle: in Anlehnung an KORYCIORZ (2004), S. 12 und SCHRADIN (1998), S. 102.

\subsubsection{Finale Risikodimension}

Bestandsgefährdende Risiken ergeben sich für Versicherungsunternehmen in Form von Liquiditätsrisiken (Zahlungsebene) und Performancerisiken (Ertragsebene). Liquiditätsrisiken treten auf, sobald das Unternehmen nicht mehr in der Lage ist, jederzeit

30 Vgl. auch AlBrecht (1994b), S. 327-328 und Schradin (1994), S. 39.

31 Die zugrunde liegenden Ergebnis- oder Vermögensgrößen können in einem Modell über verschiedene Zufallsvariablen abgebildet werden. Vgl. zu Beispielen derartiger Zufallsvariablen ALBRECHT (2003), S. 3-5. 
fristgerecht seinen fälligen Zahlungsverpflichtungen nachzukommen. ${ }^{32}$ Die Zahlungsverpflichtungen konkretisieren sich insbesondere in Entschädigungsleistungen an die Versicherungsnehmer und können im Fall von Großschadenereignissen oder Naturkatastrophen ein existenzbedrohendes Ausmaß annehmen, sofern kein hinreichender Rückversicherungsschutz besteht. ${ }^{33}$ Gemäß $\S 88$ Abs. 2 VAG hat der Vorstand eines Versicherungsunternehmens die Zahlungsunfähigkeit unverzüglich der Bundesanstalt für Finanzdienstleistungsaufsicht (BaFin) anzuzeigen. ${ }^{34}$ Unter der Voraussetzung, dass die in $\S 89$ VAG genannten Sanierungsmaßnahmen erfolglos bleiben und das Unternehmen dauerhaft nicht im Stande ist, seine Verpflichtungen zu erfüllen, wird das Insolvenzverfahren auf Antrag der Aufsichtsbehörde eröffnet.

Performancerisiken manifestieren sich im versicherungswirtschaftlichen Umfeld in der Gestalt des Profitabilitätsrisikos und Überschuldungsrisikos. ${ }^{35}$ Profitabilitätsrisiken stellen sich ein, sofern das Versicherungsunternehmen nicht in der Lage ist, nach Erfüllung der Verpflichtungen gegenüber den Versicherungsnehmern und nach Abzug von Provisionen und Betriebskosten eine adäquate Rendite auf das von den Anteilseignern zur Verfügung gestellte Kapital zu erwirtschaften. Eine nachhaltige Unterschreitung der marktseitig geforderten Mindestrendite führt langfristig $\mathrm{zu}$ einer verschlechterten Wettbewerbsposition am Kapitalmarkt, und das Versicherungsunternehmen läuft Gefahr, dass die Anteilseigner ihr Kapital einer profitableren Verwendung zuführen. ${ }^{36}$ Der auf diese Weise induzierte Nachfragerückgang führt typischerweise zu einem Marktwertverfall des emittierten Aktienkapitals. Die unzureichende Profitabilität wird darüber hinaus durch den Kapitalmarkt insofern sanktioniert, dass dieser zusätzliches Eigen- oder Fremdkapital gar nicht oder nur mit hohen Sicherheitsaufschlägen zur Verfügung stellt. ${ }^{37}$ Das Überschuldungsrisiko wird relevant, wenn das Vermögen eines Versicherungsunternehmens nicht mehr ausreicht, die bestehenden Verbindlichkeiten zu decken. Der besonderen Schutzwürdigkeit der Versicherungsnehmer Rechnung tragend, bestehen diesbezüglich aufsichtsrechtliche Solvabilitätsanforderungen, die auf eine Mindestkapitalausstattung und eine als hinreichend erachtete Existenzsicherheit abzielen. ${ }^{38}$ Analog zur Zahlungsunfähigkeit stellt die Überschul-

32

33

35

36

37

38

Vgl. im Folgenden FARNY (2000), S. 805-806.

Vgl. zum Einfluss des Insolvenzrisikos auf Versicherungsunternehmen DOTTERWEICH (2004), S. 31-32.

Die Anzeige ersetzt die dem Vorstand durch andere gesetzliche Vorschriften (Insolvenzordnung) auferlegte Pflicht, die Eröffnung des Insolvenzverfahrens zu beantragen. Vgl. $§ 88$ Abs. 2 VAG.

Vgl. im Folgenden auch KORYCIORZ (2004), S. 9-11.

Vgl. auch ALBRECHT/KORYCIORZ (2000), S. 1106-1107.

Vgl. in diesem Zusammenhang UTECHT (2001), S. 529-530.

Vgl. hierzu detailliert die Ausführungen in Kapitel 3. Vgl. außerdem grundlegend zum Solvabilitätsgedanken SCHMEISER (1997), S. 12-15. 
dung einen Konkurstatbestand dar, welcher der Aufsichtsbehörde unverzüglich anzuzeigen ist. Gemäß $\S 88$ Abs. 1 VAG kann der Antrag auf Eröffnung des Insolvenzverfahrens ausschließlich von der Aufsichtsbehörde gestellt werden.

Im weiteren Verlauf dieser Arbeit beschränkt sich die Betrachtung auf Performancerisiken. Die Vernachlässigung des Liquiditätsrisikos erscheint legitim, da die bisher aufgetretenen Konkurse von Versicherungsunternehmen primär auf Überschuldung und nicht auf Zahlungsunfähigkeit zurückzuführen waren. ${ }^{39}$

\subsubsection{Kausale Risikodimension}

\subsubsection{Versicherungstechnisches Risiko}

Das Risikogeschäft bildet den Kern des Versicherungsgeschäftes. ${ }^{40}$ Als wesentlicher Treiber für die Entstehung von Performance- und Liquiditätsrisiken kann daher das versicherungstechnische Risiko identifiziert werden..$^{41}$ Als branchenspezifisches Risiko tritt es neben diejenigen betrieblichen Risiken, denen auch Nicht-Versicherungsunternehmen ausgesetzt sind. Die in der versicherungswissenschaftlichen Literatur diskutierten Definitionen sind vielfältig und unterscheiden sich oft nur marginal. ${ }^{42}$ Ausgangspunkt sämtlicher Begriffsbestimmungen ist die versicherungsspezifische Problematik, dass mit der Entschädigungsleistung an den Versicherungsnehmer ein wesentlicher Produktbestandteil des Versicherungsschutzes stochastischen Charakter aufweist, mithin ex-ante indeterminiert ist. ${ }^{43} \mathrm{Da}$ die Versicherungsprämie jedoch in der Regel vorschüssig vereinbart wird, läuft das Versicherungsunternehmen Gefahr, dass sich die statistischen Grundlagen der Prämienkalkulation nachträglich als unzutreffend herausstellen. ${ }^{44}$ Neben der Versicherungsprämie muss dann auch verfügbares Sicherheitskapital zur Deckung von Schaden- und Betriebskosten herangezogen werden. ${ }^{45}$ Das versicherungstechnische Gesamtrisiko bezeichnet somit „die Gefahr, dass für einen bestimmten Zeitraum der Gesamtschaden des versicherten Bestandes die Summe der für die reine Risikoübernahme zur Verfügung stehenden Gesamtprämie und des

39 Vgl. übereinstimmend SCHMEISER (1998), S. 97. Vgl. zu empirischen Belegen dieses Sachverhalts Swiss Re (2000), S. 4-7, die auf eine Untersuchung von A.M. Best für den Zeitraum von 1969 bis 1998 verweisen.

40

41

42

Vgl. FARNY (2000), S. 80.

Vgl. FARNY (2000), S. 80 und ALBRECHT/SCHWAKE (1988), S. 651.

Vgl. exemplarisch BRAEß (1960), S. 15-26, HELTEN (1991), S. 135, FARNY (2000), S. 79-94, ALBRECHT/SCHWAKE (1988), S. 651-657 und ALBRECHT (1994b), S. 325-339.

Vgl. AlBRECHT/SCHWAKE (1988), S. 651, SCHMEISER (1997), S. 1 und KORYCIORZ (2004), S. 13. Wird die Versicherungsprämie hingegen erst am Ende der Versicherungsperiode nach dem Umlageverfahren kalkuliert, entsteht naturgemäß kein versicherungstechnisches Risiko. Vgl. ALBRECHT (1992), S. 5.

Vgl. zur Abgrenzung der Begriffe Risikokapital, Sicherheitskapital und Eigenkapital Kapitel 2.3. 
vorhandenen Sicherheitskapitals übersteigt. “46 Zur gedanklichen Präzisierung wird das versicherungstechnische Risiko regelmäßig in verschiedene Komponenten zerlegt. ${ }^{47}$ In diesem Zusammenhang bezeichnet das Zufallsrisiko (,process risk“) die bereits angesprochene Unsicherheit hinsichtlich des zeitlichen Eintritts und der Höhe der einzelnen Versicherungsfälle und zwangsläufig auch der Höhe des Gesamtschadens. Um die Stochastizität des Schadengeschehens im Rahmen der Unternehmenssteuerung angemessen zu berücksichtigen, sollten die Entschädigungsleistungen als Zufallsvariable modelliert werden. ${ }^{48} \mathrm{Da}$ die zukünftige Schadengesetzmäßigkeit jedoch im Betrachtungszeitpunkt unbekannt ist, wird in der Regel auf das Instrumentarium der statistischen Inferenz zurückgegriffen. ${ }^{49}$ Auf der Basis historischer Schadendaten wird versucht, auf die Gestalt der empirischen Schadenverteilung zu schließen. Da im Rahmen der statistischen Analyse einerseits Fehler auftreten und andererseits die verwendeten Daten keine vollständige Charakterisierung der wahren historischen Schadengesetzmäßigkeit erlauben, ist das Versicherungsunternehmen einem Diagnoserisiko ausgesetzt. Im Kontext der Schaden- und Unfallversicherung, insbesondere in großschadenanfälligen Versicherungszweigen, gewinnt das Diagnoserisiko besondere Relevanz, da vereinzelte Groß- und Größtschäden zu erheblichen jährlichen Schwankungen in der Datenbasis führen. ${ }^{50}$ Die zur Datenglättung eingesetzten Verfahren ${ }^{51}$ oder eine Aggregation unternehmensindividueller Schadendaten auf der Verbandsebene können diese Problematik in der Regel nicht vollständig ausräumen. Aber selbst für den unrealistischen Fall, dass die Diagnose fehlerfrei durchgeführt wird, verbleibt die Unsicherheit darüber, ob die ermittelte Gesetzmäßigkeit auch zukünftig Gültigkeit besitzt. Dieses Phänomen wird als Prognoserisiko bezeichnet und zusammen mit dem Diagnoserisiko unter das so genannte Irrtumsrisiko (,parameter risk“) subsumiert. ${ }^{52}$ Die Existenz von Zufalls- und Irttumsrisiken ist jedoch nicht auf das versicherungstechnische Geschäft beschränkt. Die skizzierte Problematik lässt sich beispielsweise analog auf den Kapitalanlagebereich übertragen. ${ }^{53}$

46 ALBRECHT/SCHWAKE (1988), S. 652. Neben dem versicherungstechnischen Gesamtrisiko ist auch die Definition versicherungstechnischer Teilrisiken möglich, sofern einzelne Teilkollektive betrachtet werden. Diese Sichtweise setzt jedoch die Außerachtlassung des Sicherheitskapitalbestandes voraus, da dieser zum Schutz des Gesamtunternehmens vorgehalten wird. Vgl. ALBRECHT/ SCHWAKE (1988), S. 652.

Vgl. zu den folgenden Ausführungen grundlegend ALBRECHT (1992), S. 7-25.

Vgl. ALBRECHT (2003), S. 4 sowie KORYCIORZ (2004), S. 14.

Vgl. ALBRECHT/SCHWAKE (1988), S. 653 und SCHMEISER (1997), S. 3.

Vgl. ALBRECHT (1992), S. 10.

Vgl. hierzu grundlegend BüHLMANN/STRAuB (1970) und SimON/BAILEY (1960).

Vgl. ALBRECHT/SCHWAKE (1988), S. 652-654.

Vgl. hierzu auch die Ausführungen in Kapitel 2.2.3.2. In der Kapitalmarkt- und Risikotransformationstheorie wird typischerweise von gegebenen Wahrscheinlichkeitsverteilungen ausgegangen, insofern also nur Zufalls- und keine Irrtumsrisiken modelliert. Vgl. exemplarisch ALBRECHT (1992), S. 16. 
Neben dieser gedanklichen Unterteilung kann innerhalb der Definition des versicherungstechnischen Gesamtrisikos zwischen den in der Praxis häufig verwendeten Begriffen des Zeichnungsrisikos und des Abwicklungs- bzw. Reserverisikos differenziert werden. ${ }^{54}$ Im Verständnis dieser Arbeit umfasst das Zeichnungsrisiko (,underwriting risk") die Gefahr, dass die kalkulierten Versicherungsprämien nicht ausreichen, um sämtliche finanzielle Risiken (insbesondere Entschädigungsleistungen und Kosten) aus den abgeschlossenen Policen zu decken. Das Zeichnungsrisiko bezieht sich somit auf den noch beeinflussbaren, mithin steuerungsrelevanten Zeithorizont eines zukünftigen Zeichnungs- bzw. Anfalljahres. ${ }^{55}$ Diesem ökonomischen Risiko steht mit dem Reserverisiko (,reserving risk“) ein Risiko bilanzieller Prägung gegenüber. Angesichts der Tatsache, dass die Entschädigungsleistungen eines Geschäftsjahres in Versicherungszweigen mit langer Schadenabwicklungsdauer vielfach nur einen geringen Anteil des erwarteten Gesamtschadens ausmachen, erscheint es ratsam, neben den Schadenzahlungen auch die korrespondierende Entwicklung der Schadenrückstellungen in die Betrachtung einzubeziehen. ${ }^{56}$ Das Reserverisiko ist durch den Sachverhalt charakterisiert, dass die existierenden Schadenrückstellungen nicht ausreichen, um die bekannten und unbekannten Schäden, die in der Vergangenheit eingetreten sind, zu decken. Da die Schäden bereits eingetreten sind, wenn die Schadenrückstellung dotiert wird, trägt das Reserve- bzw. Abwicklungsrisiko im Gegensatz zum Zeichnungsrisiko einen retrospektiven Charakter. Wenngleich beide Risiken sich gegenseitig bedingen ${ }^{57}$ und das Reserverisiko im Falle einer systematischen Unterdotierung der Schadenrückstellungen einen erheblichen Einfluss auf die Ertragslage eines Versicherungsunternehmens ausüben kann, stellt das Zeichnungsrisiko unter der Zielsetzung einer prospektiven, demnach Risiko antizipierenden Unternehmenssteuerung, den ökonomisch relevanteren Teil des versicherungstechnischen Gesamtrisikos dar.

\subsubsection{Kapitalanlagerisiko}

Der unterschiedliche zeitliche Anfall von Versicherungsprämien und Entschädigungsleistungen wird in Schaden- und Unfallversicherungsunternehmen bilanziell durch einen zumeist hohen Bestand versicherungstechnischer Rückstellungen dokumen-

54 Vgl. im Folgenden auch FELdBLUM (1993), S. 370-371.

5s Vgl. zur begrifflichen Differenzierung zwischen Zeichnungs- bzw. Anfalljahren und Geschäftsjahren ausführlich Kapitel 5.4.1.2 sowie DOTTERWEICH (2004), S. 150-153.

56 SCHWAKE weist zutreffend darauf hin, dass sonst „das versicherungstechnische Risiko weitestgehend auf ein Liquiditätsproblem zusammenschrumpfen [würde], und schleppendere Schadenabwicklung [...] vorzüglichstes Instrument zur Risikoreduktion [wäre], was beides wohl den Realitäten wenig gerecht wird." SCHWAKE (1988), S. 72 [Ergänzungen nicht im Original]. Vgl. zur Funktion und Struktur der Schadenrückstellung vertiefend Kapitel 5.4.6.1.

57 In diesem Zusammenhang formuliert PHILBRICK: „[...] the risk load in pricing is inextricably linked to the risk margins in reserving [...]." PHILBRICK (1991), S. 56. 
tiert. ${ }^{58}$ Als weiterer Zinsträger ${ }^{59}$ steht das vorgehaltene Sicherheitskapital zur Verfügung. ${ }^{60}$ Dem erfolgswirtschaftlichen Prinzip folgend, sollte ein Versicherungsunternehmen diesen Zinsträger möglichst rentabel investieren. Während im Bankensektor die Kapitalanlage vordringlich der Erzielung kurzfristiger Erträge aus Eigenhandelsgeschäften dient, erfolgt die Anlagetätigkeit eines Versicherungsunternehmens primär unter der Zielsetzung, die Erfüllbarkeit der versicherungstechnischen Verpflichtungen sicherzustellen. ${ }^{61}$ Aus diesem Grunde sind Versicherungsunternehmen nach $\S 54$ Abs. 1 VAG verpflichtet, ihr Kapital ,unter Berücksichtigung der Art der betriebenen Versicherungsgeschäfte sowie der Unternehmensstruktur so anzulegen, dass möglichst große Sicherheit und Rentabilität bei jederzeitiger Liquidität des Versicherungsunternehmens unter Wahrung angemessener Mischung und Streuung erreicht wird. “62 Die Forderung nach jederzeitiger Erfüllbarkeit der versicherungstechnischen Verpflichtungen impliziert, dass die alleinige Verfolgung rentabilitätsorientierter Zielsetzungen im Rahmen der Kapitalanlage dem Unternehmenszweck nicht angemessen ist. ${ }^{63}$ In Analogie zum versicherungstechnischen Geschäft ist der Kapitalanlagetätigkeit eines Versicherungsunternehmens ebenfalls ein Zufalls- und Irrtumsrisiko immanent. Als konkrete Ausprägungen sind das Marktpreisrisiko, das Bonitätsrisiko und das Liquiditätsrisiko anzuführen. ${ }^{64}$ Das Marktpreisrisiko realisiert sich in der Praxis in Form von Marktzinsänderungen, negativen Kursentwicklungen an den Aktienmärkten und schwankenden Wechselkursen. Darüber hinaus besteht die Gefahr, dass die Schuldner fest- oder variabel verzinslicher Wertpapiere ihren vertraglich vereinbarten Zahlungsverpflichtungen in Form von Zins- und Tilgungsleistungen nicht vollständig nachkommen oder sogar ausfallen. Dem Bonitäts- oder Ausfallrisiko kommt insbesondere auch im versicherungstechnischen Geschäft erhebliche Bedeutung zu, sofern Forderungen gegenüber Rückversicherern uneinbringlich werden. Selbst wenn das Bonitätsrisiko eines Rückversicherers in einer isolierten Betrachtung vernachlässigbar erscheint, können landesspezifische Hemmnisse, wie z.B. politische Unruhen, die ver-

58 Vgl. im Folgenden auch ALBRECHT (1995), S. 35. Neben die versicherungstechnischen Rückstellungen treten beispielsweise mit Pensionsrückstellungen und Rückstellungen für latente Steuern auch Passiva nicht versicherungstechnischer Herkunft. Diese werden im weiteren Verlauf jedoch nicht betrachtet.

Die Begriffe Zinsträger und Kapitalanlagevolumen werden fortan synonym verwendet.

Von integrierten Spar- und Entsparprozessen, wie sie beispielsweise in der privaten Unfallversicherung mit Beitragsrückgewähr auftreten, wird im weiteren Verlauf abstrahiert. Vgl. übereinstimmend ALBRECHT/KORYCIORZ (2000), S. 1108 und FARNY (2000), S. 814-815. $\S 54$ Abs. 1 VAG.

63 Vgl. hierzu vertiefend SCHRADIN (1994), S. 269-279. Dieser formuliert zutreffend: „Bei sorgfältiger Betrachtung des $\S 54$ VAG fordert die Generalnorm bereits ein integriertes Management von Vermögensanlage (Kapitalbindung) und individuellem Risikogeschäft (Kapitalbereitstellung).“ SCHRADIN (1994), S. 271.

Vgl. zur Systematisierung von Kapitalanlagerisiken im Folgenden SPELLMANN (2002), S. 10-28. 
tragsgemäße Erfüllung der Verpflichtungen beeinträchtigen (sog. Länderrisiko). ${ }^{65}$ Auch in Bezug auf das Liquiditätsrisiko ist die Zuordnung auf das versicherungstechnische Geschäft und das Kapitalanlagegeschäft nicht überschneidungsfrei. ${ }^{66}$ Aktivseitig manifestiert sich das Liquiditätsrisiko in der Gefahr, dass gehaltene Anlagen nur unter erheblichen Preiseinbußen veräußerbar sind (sog. Fristigkeitsrisiko). Passivseitig können aufgrund von Groß- oder Naturgefahrereignissen Liquiditätsengpässe auftreten, sofern kein hinreichender Rückversicherungsschutz besteht.

\subsubsection{Sonstige Unternehmensrisiken}

Unter den sonstigen Unternehmensrisiken werden schließlich diejenigen Risikofaktoren subsumiert, die keinen unmittelbaren Bezug zur Bereitstellung des Versicherungsschutzes aufweisen. ${ }^{67}$ Hierzu gehört vorrangig das allgemeine Geschäftsrisiko, welches mit der Erstellung und marktseitigen Verwertung wirtschaftlicher Leistungen untrennbar verbunden ist. Es wird beispielsweise durch Managementfehler, Veränderungen auf den Beschaffungs- und Absatzmärkten sowie sich wandelnde Gesellschafts-, Rechts- und Wirtschaftssysteme hervorgerufen. ${ }^{68}$ Insbesondere sind in diesem $\mathrm{Zu}$ sammenhang konjunkturelle Zyklen, veränderte Verhaltensweisen der Versicherungsnehmer, unvorhergesehene Markteintritte von Wettbewerbern oder auch demographische Entwicklungen zu nennen. In einem marktwirtschaftlichen Umfeld wird die Inkaufnahme des allgemeinen Geschäftsrisikos durch die Chance auf Gewinnerzielung entschädigt. Operationelle Risiken resultieren aus technisch-organisatorischen Unzulänglichkeiten, fehlerbehafteten Geschäftsprozessen und kriminellen Handlungen, wie beispielsweise Betrug oder Untreue von Mitarbeitern, Absatzmittlern oder Versicherungsnehmern. ${ }^{69}$ Im Bereich der betrieblichen Risiken sind die mit der zunehmenden Nutzung moderner Informationstechnologien verbundenen Gefahren zu berücksichtigen. Den an dieser Stelle nur stichwortartig genannten sonstigen Risikofaktoren ist gemein, dass sie zwar benannt, hinsichtlich ihrer finanziellen Auswirkungen aber vielfach nur pauschaliert in ein Risikomodell eingebunden werden können. ${ }^{70}$ In einem ers-

65 Vgl. zum Ausfallrisiko von Rückversicherungsforderungen auch MENTZEL (2004), S. 130-133.

66

67 S. 20-21.

68

69
Vgl. im Folgenden FARNY (2000), S. 819.

Vgl. zu sonstigen Unternehmensrisiken SCHRADIN (1994), S. 35-36 und KORYCIORZ (2004),

Vgl. FARNY (2000), S. 507.

Vgl. im Kontext operationeller Risiken ausführlich ROMEIKE/MÜLLER-REICHARD (2005), S. 103104 und S. 304-317 sowie WINTER (2001), S. $66 \mathrm{ff}$.

Vgl. übereinstimmend BROHM (2002), S. 84 und SCHMEISER (2001), S. 142. Angesichts der zukünftig durch die internationalen Aufsichtsbehörden geforderten Berücksichtung operationeller Risiken ist jedoch eine zunehmende wissenschaftliche Auseinandersetzung mit dieser Thematik zu konstatieren. Vgl. hierzu exemplarisch KING (2001), FAISST/KovACS (2003), HARTUNG (2002) und APARICIO/KESKINER (2004). 
ten Schritt könnte die Modellierung beispielsweise einen funktionalen Zusammenhang zwischen Prämienvolumen und operationellen Risiken vorsehen. ${ }^{71}$ Für eine differenziertere Abbildung in internen Risikomodellen ist jedoch mittelfristig eine präzise Begriffsabgrenzung und die Entwicklung entsprechender Verlustdatenbanken unerlässlich. Die weitere Untersuchung konzentriert sich auf die Abbildung und Quantifizierung von versicherungstechnischen Risiken und Kapitalanlagerisiken. ${ }^{72}$

\subsection{Begriff und Funktion von Risikokapital}

Die im vorangegangenen Abschnitt skizzierten Risiken bewirken in ihrer Gesamtheit, dass sich ein Versicherungsunternehmen grundsätzlich in einer Risiko- bzw. Unsicherheitslage befindet. ${ }^{73}$ Der Natur des Versicherungsgeschäfts entsprechend, muss ein Versicherungsunternehmen jedoch ein hohes $\mathrm{Ma} ß$ an Existenzsicherheit aufweisen, um seine Leistungsversprechen gegenüber den Versicherungsnehmern mit hinreichender Wahrscheinlichkeit erfüllen zu können. ${ }^{74}$ Im versicherungsspezifischen Kontext nimmt somit die Existenzsicherheit des Unternehmens unmittelbaren Einfluss auf die Produktqualität. ${ }^{75}$

Um die Einhaltung dieser wesentlichen Nebenbedingung zu gewährleisten, erscheint die Unterlegung risikobehafteter Geschäfte mit Risiko- bzw. Sicherheitskapital und dem nicht zur Kostendeckung benötigten Anteil der Versicherungsprämien (= Risikoprämie) unverzichtbar. ${ }^{76}$ Im Hinblick auf das Sicherheitsniveau eines Versicherungsunternehmens stellen Risikokapital und Prämien somit substitutive Faktoren dar. ${ }^{77}$

71 Vgl. zu einer derartigen Vorgehensweise exemplarisch OSETROVA/SCHMEISER (2005), S. 14-15.

72 Diese modelltechnische Beschränkung wird durch eine empirische Untersuchung von A.M. Best für den amerikanischen Versicherungsmarkt gestützt. Im Zeitraum von 1969 bis 1998 waren demnach $41 \%$ der Insolvenzen auf versicherungstechnische Risiken zurückzuführen. 13\% der Insolvenzen entfielen auf Risiken der Aktiva, allerdings auch 7\% auf Betrugsfälle. Die verbleibenden Fälle wurden entweder durch sonstige Faktoren (z.B. Insolvenz eines verbundenen Unternehmens oder signifikanter Wechsel des Geschäftsfelds) erklärt oder konnten keiner Ursache unmittelbar zugeordnet werden. Vgl. hierzu SwiSs RE (2000), S. 6. Vgl. FARNY (2000), S. 313.

74 In diesem Kontext formuliert SCHRADIN zutreffend: „Die Bereitstellung eines hinreichend großen Bestandes an Sicherheitskapital ist eine conditio sine qua non für die Erbringung des Versicherungsschutzversprechens." SCHRADIN (1994), S. 196 [Hervorhebung im Original].

75 Vgl. AlBRECHT (1992), S. 19. Zur besonderen Bedeutung des Sicherheitsstrebens in der Versicherungswirtschaft vgl. außerdem SCHRADIN (1994), S. 58-59 und S. 67-76, ALBRECHT (1994c), S. 3, OLETZKY (1998), S. 6-7, FARNY (2000), S. 313-316 sowie die Ausführungen in Kapitel 3.2.1. Die Begriffe Risikokapital und Sicherheitskapital kommen nachfolgend synonym zur Anwendung. Vgl. übereinstimmend ALBRECHT (1992), S. 19 und SCHMEISER (1997), S. 6. 
Es ist zu berücksichtigen, dass der Risikokapitalbegriff im einschlägigen Schriftum sowohl in einer aufsichtsrechtlich motivierten (externen) Perspektive als auch in einer ökonomischen (internen) Perspektive Anwendung findet. ${ }^{78}$ Die aufsichtsrechtlichen Solvabilitätsvorschriften verpflichten das Versicherungsunternehmen, „Zur Sicherstellung der dauernden Erfüllbarkeit der Verträge stets über freie unbelastete Eigenmittel mindestens in Höhe der geforderten Solvabilitätsspanne zu verfügen. ${ }^{\text {" }} 9$ Die erforderliche Solvabilitätsspanne leitet sich aus bilanziellen Kenngrößen ab. ${ }^{80}$ Da bilanziell jedoch lediglich Auszahlungsverpflichtungen für bereits eingetretene Schadenfälle in der Form von versicherungstechnischem Fremdkapital erfasst werden, wird insofern eine retrospektive Sicht eingenommen. ${ }^{81}$ Die ökonomische Kapitalkonzeption basiert hingegen auf risikotheoretischen Überlegungen und versucht, das Gesamtrisiko eines Versicherungsunternehmens prospektiv durch die Bereitstellung von Risiko- bzw. Sicherheitskapital zu berücksichtigen. Im Verständnis dieser Arbeit sei das ökonomisch erforderliche Risikokapital daher definiert als kleinstmöglicher Kapitalbetrag, der im Falle künftig auftretender Risiken ausreicht, um ein negatives Gesamtergebnis mit einer vorgegebenen Wahrscheinlichkeit auszugleichen. ${ }^{82}$ Alternativ formuliert muss im einperiodigen Kontext die Wahrscheinlichkeit, dass das Risikokapital durch ein negatives Gesamtergebnis aufgezehrt wird, kontrolliert klein gehalten werden. Dieses Begriffsverständnis soll nachfolgend anhand eines Ein-Periodenmodells formalisiert werden, welches sowohl das versicherungstechnische Kerngeschäft als auch den Kapitalanlagebereich in die Analyse einbezieht. ${ }^{83}$

Demnach kann der (bilanzielle) Unternehmenserfolg G einer Periode auf der Grundlage des folgenden Schemas ermittelt werden:

$$
\begin{array}{ll}
\mathrm{G}=\pi-\mathrm{S}+\mathrm{I}-\mathrm{K} \\
\text { mit: } \quad \pi=\text { Prämienerlöse } \\
\mathrm{S}=\text { Schadenkosten } \\
\mathrm{I}=\text { Erfolg aus Kapitalanlagen } \\
\mathrm{K}=\text { Betriebskosten }
\end{array}
$$

78 Vgl. auch KORYCIORZ (2004) S. 5-6 und ALBRECHT/ZiMMERMANN (1992), S. 27-41.

$79 \S 53 \mathrm{c}$ Abs. 1 VAG.

80 Vgl. hierzu vertiefend die Ausführungen in Kapitel 3.1.1.1.

81 Vgl. zur Differenzierung zwischen ex-post- und ex-ante-Kapitalbedarf vertiefend SCHRADIN (1994), S. 193-195.

$82 \mathrm{Vgl}$. zu einem korrespondierenden Begriffsverständnis KINDER/STEINER/WILLINSKY (2001), S. 283 und SCHRADIN (1994), S. 210.

83 Die Darstellung und die Notation der nachfolgenden Ausführungen lehnen sich an die Vorgehensweise von AlBrecht/ZimmERMANN an. Vgl. AlbreCHT/ZimMERMANN (1992), S. 27-41. 
Während die Ergebniskomponenten $\pi$ und $\mathrm{K}$ als deterministische Größen in die Berechnung eingehen, sind S und I als zufallsabhängige Größen aufzufassen. Der Kapitalanlageerfolg I ergibt sich als Produkt der Einperiodenrendite R und dem in der Periode durchschnittlich zur Verfügung stehenden Zinsträger A.

$$
\mathrm{I}=\mathrm{A} \cdot \mathbf{R}
$$

Das Anlagekapital A setzt sich wiederum aus dem vorhandenen Sicherheitskapital U und den nicht unmittelbar auszahlungswirksamen Prämien der betrachteten Periode zusammen. Um zu gewährleisten, dass der Verzinsungseffekt der bestehenden versicherungstechnischen Rückstellungen adäquat abgebildet wird, wird der als ,funds generating coefficient" bekannte Parameter $\mathrm{h}$ eingeführt. ${ }^{84}$ Dieser quantifiziert den durchschnittlichen Prämienanteil, der in einer Periode für Kapitalanlagezwecke zur Verfügung steht. Aufgrund des mehrperiodigen Verzinsungseffekts der bestehenden Schadenrückstellungen ist $\mathrm{h}$ regelmäßig größer als $1 .^{85}$

$$
\mathrm{A}=\mathrm{U}+\mathrm{h} \cdot \pi
$$

Aus den Beziehungen (2.1) bis (2.3) ergibt sich der (bilanzielle) Unternehmenserfolg $\mathrm{G}$ einer Periode als

$$
\mathrm{G}=\pi-\mathrm{S}-\mathrm{K}+(\mathrm{U}+\mathrm{h} \cdot \pi) \cdot \mathrm{R}
$$

Beziehung (2.4) beschreibt somit ein formales Modell, welches sowohl die Stochastizität des Schadengeschehens als auch der Kapitalanlageerträge abbildet. Darüber hinaus wird die Abhängigkeit des Kapitalanlagevolumens von den versicherungstechnischen Rückstellungen näherungsweise als Prozentsatz der Prämien erfasst. Das Modell kann nun dahingehend verfeinert werden, dass $\mathrm{n}$ Teilkollektive (z.B. Versicherungszweige) betrachtet werden, und deren spezifischer Beitrag zur Generierung versicherungstechnischen Fremdkapitals in die Analyse einbezogen wird. Das Anlagekapital A umfasst in diesem Fall das Sicherheitskapital U, welches unverändert dem Unternehmen als Ganzes zur Verfügung steht, und die Summe der (approximativ bestimmten) spartenspezifischen Schadenrückstellungen, formal:

$$
\mathrm{A}=\mathrm{U}+\sum_{\mathrm{i}=1}^{\mathrm{n}} \mathrm{h}_{\mathrm{i}} \cdot \pi_{\mathrm{i}}
$$

\footnotetext{
84 Vgl. in diesem Zusammenhang auch SCHMEISER (1997), S. 83.

85 ALBRECHT/ZIMMERMANN folgen hiermit einer Idee von MCCABE/WITT (1980), S. 612-614.
} 
Unter der Annahme, dass das Anlagekapital in unterschiedliche Assetklassen investiert wird, gilt für den Unternehmenserfolg $\mathrm{G}$ nunmehr:

$$
\begin{array}{ll}
G=\sum_{i=1}^{n}\left(\pi_{i}-S_{i}-K_{i}\right)+\sum_{j=1}^{m} a_{j}\left(U+\sum_{i=1}^{n} h_{i} \cdot \pi_{i}\right) R_{j} \\
\text { mit: } \quad K_{i}=\text { Betriebskosten des Kollektivs i mit } i \in\{1, \ldots, n\} \\
& R_{j}=\text { einperiodige Rendite der Assetklasse } j \text { mit } j \in\{1, \ldots, m\} \\
& a_{j}=\text { Portfolioanteil der Assetklasse } j \\
& \text { Ferner gilt: } 0 \leq \mathrm{a}_{j} \leq 1 \text { und } \sum_{j=1}^{m} a_{j}=1
\end{array}
$$

Aus dieser vereinfachten Darstellung wird bereits deutlich, dass eine konsistente Aufspaltung des Gesamtergebnisses $G$ in einzelne Segmentsergebnisse $G_{i}$ nicht möglich ist. Das Sicherheitskapital U dient zur Deckung von Verlusten des Gesamtunternehmens und entzieht sich daher per definitionem einer direkten Zuordnung auf einzelne Teilkollektive. Eine willkürfreie Ermittlung von Segmentergebnissen ist daher nur in Bezug auf das versicherungstechnische Kerngeschäft möglich. Die ZufallsgesetzmäBigkeit der in Beziehung (2.6) formalisierten Ergebnisgröße wird bei ALBRECHT/ ZIMMERMANN durch eine Normalverteilung bzw. eine Normal-Power-Verteilung approximiert. ${ }^{86}$ Die Annahme einer bekannten Ergebnisverteilung impliziert, dass lediglich das Zufallsrisiko betrachtet wird. ${ }^{87}$ Für die Ermittlung des Risikokapitals bedarf es nunmehr zusätzlich der Festlegung einer unternehmensseitig tolerierten Ruinwahrscheinlichkeit $\varepsilon$ respektive der Vorgabe eines Sicherheitsniveaus 1- $\varepsilon$. Die verallgemeinerte Ruinwahrscheinlichkeit $\varepsilon$ bezeichnet „diejenige Wahrscheinlichkeit, mit der es zu einer Aufzehrung des vorhandenen Sicherheitskapitals U durch die im Betrachtungszeitraum entstandenen (aggregierten) Verluste des Unternehmens kommt. “88

$$
\mathrm{P}(\mathrm{G}<-\mathrm{U}) \leq \varepsilon
$$

Das in Beziehung (2.7) definierte Steuerungskriterium kann unter verschiedenen Zielsetzungen Anwendung finden. ALBRECHT/ZiMMERMANN wenden das Modellkonzept insbesondere auf Fragestellungen der Prämienkalkulation, Solvabilität ${ }^{89}$ und Zeichnungskapazität an und diskutieren außerdem Kalküle zur Bestimmung eines „optimalen" Sparten-Anlagen-Mixes. An dieser Stelle soll der Fall betrachtet werden, dass das

Vgl. AlBReCht/ZimMERMANN (1992), S. 30-32.

Vgl. übereinstimmend ALBRECHT (1992), S. 16.

SCHMEISER (1997), S. 85.

Vgl. in diesem Zusammenhang vertiefend SCHMEISER (1997), S. 81-99. 
Prämienniveau durch den Markt determiniert ist. ${ }^{90}$ Der Risikokapitalbedarf U lässt sich dann bei vorgegebener Ruinwahrscheinlichkeit $\varepsilon$ anschaulich anhand der Dichtefunktion des Unternehmenserfolgs $G$ ableiten. Die Dichtefunktion wird als nicht symmetrisch angenommen, da die Gewinnmöglichkeiten für den Fall gänzlich ausbleibender Schäden auf die Prämien beschränkt sind. Dem steht jedoch ein (theoretisch) unbegrenztes Verlustpotenzial gegenüber. ${ }^{91}$

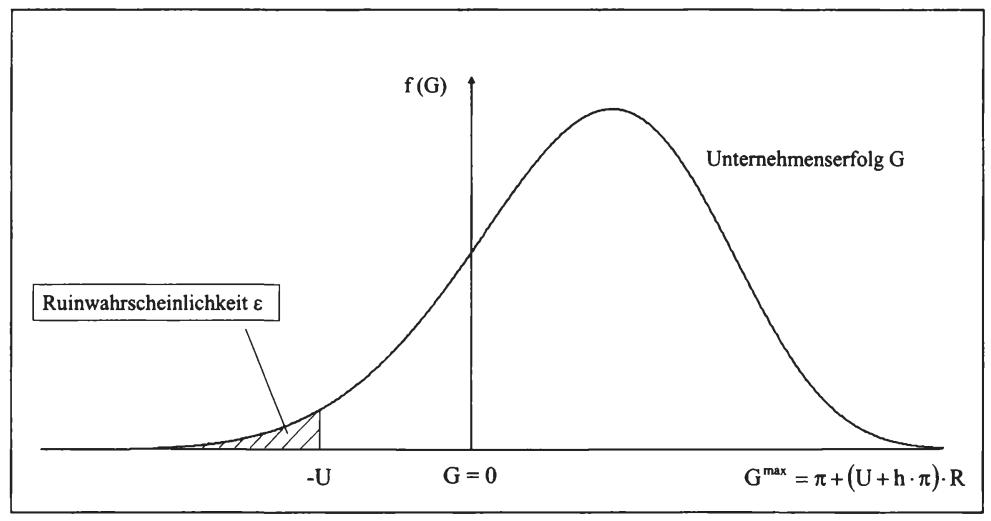

Abbildung 2.2: Einperiodige Ruinwahrscheinlichkeit und Risikokapital Quelle: in Anlehnung an ALBRECHT/ZiMMERMANN (1992), S. 30.

Das in Abbildung 2.2 graphisch ermittelte Risikokapital U stellt den risikotheoretischen Soll-Zustand dar, dem das vorhandene Kapital (Ist-Zustand) gegenüberzustellen ist. Hinsichtlich der Ermittlung des verfügbaren Kapitals kann grundlegend zwischen dem handelsrechtlichen, mithin vergangenheitsbezogenen Eigenkapitalbegriff und dem zukunftorientierten Kapitalbegriff differenziert werden. ${ }^{92}$ In einer ökonomisch motivierten und demnach Risiko antizipierenden Steuerungsperspektive ist dem Risikokapitalbedarf das „ökonomische Eigenkapital“ gegenüberzustellen. Dieses stellt die Differenz zwischen dem Marktwert der Vermögenswerte und dem Marktwert der Verpflichtungen dar. ${ }^{93}$ Während die Marktbewertung der Kapitalanlagen in der Regel unproblematisch ist, werden versicherungstechnische Verpflichtungen nicht auf Finanzmärkten gehandelt. ${ }^{94}$ Ein Marktwert der Verpflichtungen kann aber näherungsweise

90 Bei fixierten Sicherheitskapital U stellt sich im Rahmen der Tarifierung demgegenüber die Frage, in welcher Höhe das Prämienniveau festzulegen ist, um ein vorgegebenes Sicherheitsniveau einzuhalten. Vgl. hierzu vertiefend ALBRECHT/ZiMMERMANN (1992), S. 32-33.

Vgl. hierzu auch FARNY (2000), S. 410-412.

Vgl. im Folgenden auch SAUER (2004), S. 4-5.

Vgl. vertiefend SAUER (2004), S. 14-18 und MUELLER (2003), S. 7-8.

Vgl. auch BROHM (2002), S. 217 und ROCKEL (2004), S. 63. 
als Barwert der aus den abgeschlossenen Policen erwarteten Zahlungsströme zuzüglich eines Risikozuschlags ermittelt werden. ${ }^{95}$ Da die Ermittlung dieses "synthetischen“ Fair Value in der Praxis aber vielfach auf Probleme stößt, wird der Marktwert der Verpflichtungen näherungsweise aus bilanziellen Größen abgeleitet und um außerbilanzielle Haftungsmassen ergänzt, welche im Bedarfsfall neben den vereinnahmten Risikoprämien zur Deckung von Verlusten zur Verfügung stehen. Im Wesentlichen kann das ökonomische Eigenkapital durch die Summe nachstehender Komponenten approximiert werden. ${ }^{96}$

- Bilanzielles Eigenkapital

- Schwankungs- und Großrisikenrückstellungen

- Rückstellung für drohende Verluste

- Bewertungsreserven in den Kapitalanlagen ${ }^{97}$

- $\quad$ Stille Reserven in den Schadenrückstellungen ${ }^{98}$

Die vorliegende Arbeit stellt die ökonomische Perspektive und somit die unternehmensinterne Rendite-Risikosteuerung in den Mittelpunkt des Interesses. Gleichwohl existieren aufsichtsrechtliche Kapitalbildungsnormen in Form von Solvabilitätsvorschriften, die einen unverrückbaren rechtlich-institutionellen Rahmen für die interne Steuerung darstellen. ${ }^{99}$ Vor diesem Hintergrund werden im nachfolgenden Kapitel zunächst die aufsichtsrechtlichen Rahmenbedingungen im Überblick skizziert, bevor auf betriebswirtschaftliche Zielsetzungen und die Notwendigkeit einer integrierten Rendite-Risikosteuerung eingegangen wird.

95 Vgl. übereinstimmend SAUER (2004), S. 15. Zur Ermittlung der Risikoprämie vgl. ROCKEL (2004), S. 63-151.

96 Vgl. auch KorYCIORZ (2004), S. 16 und SCHRADIN (1994), S. 210-211.

97 Vgl. auch Kapitel 5.5.3.

98 Vgl. auch Kapitel 6.3.1.

99 Vgl. SCHRAdIN (1994), S. 196. 
Mirko Tillmann - 978-3-631-75327-9

Downloaded from PubFactory at 01/11/2019 06:10:15AM

via free access 


\section{Anforderungen an die Steuerung in der Schaden- und Unfallversicherung}

\subsection{Aufsichtsrechtliche Rahmenbedingungen}

\subsubsection{Europäische Solvabilitätsvorschriften de lege lata (Solvency I)}

\subsubsection{Ausgestaltung der Soll- und Ist-Solvabilität}

Der Finanzdienstleistungssektor ist wie kaum ein anderer Wirtschaftszweig weitreichenden aufsichtsrechtlichen Regularien unterworfen. ${ }^{100}$ Als Legitimation für derartige staatliche Eingriffe in die unternehmerische Freiheit wird in der Literatur eine Vielzahl von Argumenten angeführt. ${ }^{101}$ In Anbetracht der Zielsetzung dieser Arbeit werden im weiteren Verlauf primär die spezielle Gläubigerstellung und die Schutzwürdigkeit der Versicherungsnehmer in den Mittelpunkt des Interesses gestellt. Aus dem Umstand, dass die Überschuldung eines Versicherungsunternehmens aufgrund eingeschränkter individueller Diversifikations- und Absicherungsmöglichkeiten in der Regel auch eine „persönliche Insolvenz der Versicherungsnehmers" ${ }^{\text {102 }}$ nach sich zieht, wird ein besonderes Schutzinteresse der Versicherungsnehmer abgeleitet. ${ }^{103}$ Darüber hinaus ist zu berücksichtigen, dass die Versicherungsnehmer gegenüber dem Versicherungsunternehmen sowohl eine bedingte Gläubigerposition als auch eine Schuldnerposition einnehmen, welche jeweils unterschiedliche Interessenlagen bedingen. ${ }^{104}$ Der Schutz der Schuldnerinteressen (z.B. niedriges Prämienniveau) steht dem Schutz der Gläubigerinteressen (z.B. risikoadäquates Prämienniveau und hohes Sicherheitskapital) bisweilen diametral entgegen. Ferner ist der einzelne Versicherungsnehmer vielfach nicht in der Lage, risikoerhöhende Aktivitäten des Versicherungsunternehmens ${ }^{105} \mathrm{zu}$ überwachen und über eine entsprechende Anpassung seiner Zahlungsbereitschaft oder die Schaffung von Anreizstrukturen zu sanktionieren. ${ }^{106} \mathrm{Da}$ sich eine Überwachung für jeden einzelnen Versicherungsnehmer als zu aufwändig erweist, wird diese vielfach im Vertrauen auf andere unterlassen und unterbleibt schließlich in Gänze. ${ }^{107}$

$100 \mathrm{Vgl}$. für den Bankensektor im Überblick HARTMANN-WENDELS/PFINGSTEN/WEBER (2004), S. 361-413 und für den Versicherungssektor exemplarisch SCHRADIN (2003), S. 611-664, FARNY (2000), S. 746-768 sowie ZWEIFEL/EISEN (2003), S. 347-377.

101 Vgl. insbesondere FARNY (2000), S. 106-111 und ZWEIFEL/EISEN (2003), S. 348-352.

102 SCHMEISER (2004b), S. 1.

103 Vgl. SCHRADIN (2003), S. 615-616.

104 Vgl. vertiefend FARNY (2000), S. 108 und SCHRADIN (2003), S. 615.

105 Vgl. zur Problematik der Verhaltensunsicherheit (Moral Hazard) vertiefend HARTMANN-WENDELS/PFINGSTEN/WEBER (2004), S. 100-103, ZWEIFEL/EISEN (2003), S. 293-320 und SCHULENBURG, GRAF VON DER (2005), S. 282-296.

$106 \mathrm{Vgl}$. auch SCHMEISER (2004b), S. 1-2.

107 Vgl. zur so genannten Free-Rider-Problematik vertiefend HARTMANN-WENDELS/PFINGSTEN/WEBER (2004), S. 365-366. 
Vor diesem Hintergrund kann die Versicherungsaufsicht als stellvertretende Instanz aller Versicherungsnehmer aufgefasst werden, welche einzelwirtschaftlich nicht sinnvoll herbeizuführende Regelungen zur Vermeidung adversen Verhaltens durch eine staatliche Aufgabenerfüllung substituiert und auf diese Weise eine volkswirtschaftlich erwünschte Stabilisierungsfunktion ausübt. ${ }^{108}$

Die derzeitig auf europäischer Ebene gültigen Solvabilitätsvorschriften fußen auf der Schadenversicherungsrichtlinie 73/239/EWG aus dem Jahre 1973. ${ }^{109}$ Die wenigen durch das Projekt „Solvency I“ initiierten Veränderungen wurden mit Wirkung zum 05.03.2002 durch die Richtlinie 2002/13/EG umgesetzt. Hinsichtlich der Überführung der neuen Richtlinie in nationales Recht wurde den EU-Mitgliedsstaaten eine Frist von 18 Monaten eingeräumt, so dass die neuen Vorschriften frühestens im Geschäftsjahr 2004 Anwendung finden. ${ }^{110}$ In Deutschland sind die Mindestanforderungen an die Eigenkapitalausstattung von Versicherungsunternehmen in § 53c VAG kodifiziert. Demnach setzt sich die Soll-Solvabilität insgesamt aus drei Elementen zusammen, welche jeweils unterschiedliche aufsichtsrechtliche Interventionsniveaus implizieren. ${ }^{111}$

Die Solvabilitätsspanne $\mathrm{SSP}_{\mathrm{n}}$ ergibt sich gemäß $\S 1$ Abs. 1 Nr. 2 Kapitalausstattungsverordnung (KapAustV) als Maximum aus dem Beitragsindex $\mathrm{BI}_{\mathrm{n}}$ bzw. dem Schadenindex $\mathrm{SI}_{\mathrm{n}}$.

$$
\operatorname{SSP}_{n}=\max \left(\mathrm{BI}_{n} ; \mathrm{SI}_{\mathrm{n}}\right)
$$

Wenngleich die Solvabilitätsanforderungen grundsätzlich zu jedem Zeitpunkt ${ }^{112}$ erfüllt sein müssen, wird für die Berechnung beider Indizes auf die Verhältnisse am Bilanzstichtag abgestellt.

${ }_{108}$ Vgl. übereinstimmend SCHRADIN (2003), S. 616.

109 Vgl. zu den rechtlichen Rahmenbedingungen im Folgenden auch MÜLLER (1997a), MÜLLER (1997b), SCHMEISER (1997), S. 12-20, HEISTERMANN (2002a), HEISTERMANN (2002b), HARTUNG/HELTEN (2004), S. 294-295 und ROMEIKE/MÜLLER-REICHARD (2005), S. 111-125.

110 Vgl. Artikel 3 Abs. 1 der Richtlinie 2002/13/EG. Darüber hinaus können die Mitgliedsstaaten den Schadenversicherungsunternehmen gemäß Artikel 2 der Richtlinie eine Übergangsfrist von bis zu 5 Jahren einräumen, um die Eigenkapitalausstattung an die Erfordernisse der neuen Richtlinie anzupassen.

11 Vgl. in diesem Zusammenhang vertiefend SCHMEISER (1997), S. 17-20 und FARNY (2000), S. 749-750. Die weiteren Ausführungen beziehen sich auf die Ermittlung der Soll-Solvabilität einzelner Versicherungsunternehmen (,Solo-Solvabilität“). Zum Begriff der Konzernsolvabilität vgl. FARNY (2000), S. 762-767.

112 Mit dieser in der Richtlinie 2002/13/EG neu eingeführten Verpflichtung sind erhebliche Anforderungen an die Entwicklung unternehmensinterner Informationssysteme verbunden. Vgl. SCHRADIN (2003), S. 633-634. 
Für die Ermittlung beider Kenngrößen sind folgende Schemata vorgeschrieben:

$$
\mathrm{BI}_{n}= \begin{cases}\left(0,18 \cdot B_{n}\right) \cdot S b Q_{n} & \text { für } B_{n} \leq 50 \text { Mio. } € \\ {\left[0,18 \cdot 50 \text { Mio. } €+0,16 \cdot\left(B_{n}-50 \text { Mio. } €\right)\right] \cdot S b Q_{n}} & \text { für } B_{n}>50 \text { Mio. } €\end{cases}
$$

$\mathrm{SI}_{\mathrm{n}}= \begin{cases}\left(0,26 \cdot \mathrm{SD}_{\mathrm{n}}\right) \cdot \mathrm{SbQ}_{\mathrm{n}} & \text { für } \mathrm{SD}_{\mathrm{n}} \leq 35 \text { Mio. } € \\ {\left[0,26 \cdot 35 \text { Mio. } €+0,23 \cdot\left(\mathrm{SD}_{\mathrm{n}}-35 \text { Mio. } €\right)\right] \cdot \mathrm{SbQ}_{\mathrm{n}}} & \text { für } \mathrm{SD}_{\mathrm{n}}>35 \text { Mio. } €\end{cases}$

mit: $\quad \mathrm{n}=$ zuletzt abgeschlossenes Geschäftsjahr (GJ)

$\mathrm{B}_{\mathrm{n}} \quad=$ Bruttoprämien $^{113}$ des direkten und indirekten Geschäfts im GJ n

$\mathrm{SbQ}_{\mathrm{n}}=$ durchschnittliche Selbsthaltsquote ${ }^{114}$ der letzten drei GJe

$\mathrm{SD}_{\mathrm{n}}=$ Schadendurchschnitt der letzten drei GJe

Eine Grenzbetrachtung verdeutlicht, dass der Schadenindex nur zur Anwendung kommt, sofern sich eine Schadenquote (definiert als Verhältnis zwischen Aufwendungen für Versicherungsfälle und Bruttoprämien) oberhalb von ca. $70 \%$ realisiert. Die exakte Grenzschadenquote ${ }^{115}$ ergibt sich jeweils aus den Relationen der Anrechnungssätze in den Beziehungen (3.2) und (3.3), konkret als 0,18/0,26 bzw. als 0,16/0,23.

Für die Ermittlung des Schadendurchschnitts $\mathrm{SD}_{\mathrm{n}}$ wird über einen Zeitraum von drei Geschäftsjahren die Summe aus Bruttozahlungen für Versicherungsfälle und Veränderung der Bruttorückstellung für noch nicht abgewickelte Versicherungsfälle zugrunde gelegt. ${ }^{116}$ Hinsichtlich der Selbstbehaltsquote ist zu beachten, dass diese nach neuer Regelung als Durchschnitt der vergangenen 3 Geschäftsjahre ermittelt wird. Die Anrechenbarkeit der Rückversicherung ist unverändert auf $50 \%$ beschränkt ist.

113 Sofern nicht ausdrücklich erwähnt, wird im weiteren Verlauf dieser Arbeit mit „Brutto“ stets die Darstellung einer versicherungstechnischen Position vor Einfluss der Rückversicherung bezeichnet. „Netto“ bezeichnet hingegen den Wert dieser Position nach Rückversicherungsnahme.

114 Die Selbstbehaltsquote ist definiert als Quotient aus Nettoschadenaufwand und Bruttoschadenaufwand. Vgl. auch SCHMEISER (1997), S. 21.

115 In der versicherungswirtschaftlichen Literatur ist der Begriff der Grenzschadenquote in diesem Kontext geläufig. Vgl. SCHMEISER (1997), S. 22. Formal handelt es ich jedoch ausdrücklich nicht um eine Grenzbetrachtung im Sinne einer mathematischen Differentiation.

116 Vgl. zum mathematischen Ermittlungsschema auch SCHMEISER (1997), S. 21. Sofern das betreffende Versicherungsunternehmen im Wesentlichen nur Kredit-, Sturm-, Hagel- und Frostrisiken übernimmt, bestimmt sich der Schadendurchschnitt gemäß Artikel 16a Abs. 1 Satz 2 der Richtlinie 2002/13/EG als mittlere Schadenbelastung der vergangenen 7 Geschäftsjahre. In den Versicherungszweigen Luftfahrzeughaftpflicht, See-, Binnen-, Flussschifffahrtshaftpflicht und Allgemeine Haftpflicht werden sowohl die Beiträge als auch der Schadendurchschnitt mit dem Faktor 1,5 multipliziert, um der höheren Risikoexponierung dieser Sparten Rechnung zu tragen. Vgl. HEISTERMANN (2002b), S. 16. 
Formal gilt daher:

$$
\mathrm{SbQ}_{\mathrm{n}}=\max \left(\frac{\mathrm{SA}_{\mathrm{N}}}{\mathrm{SA}_{\mathrm{B}}} ; 0,5\right)
$$

mit: $\quad \mathrm{SA}_{\mathrm{N}}=$ Nettoaufwendungen für Versicherungsfälle $\mathrm{SA}_{\mathrm{B}}=$ Bruttoaufwendungen für Versicherungsfälle

Der auf diese Weise quantifizierten Solvabilitätsspanne sind die in § 53c Abs. 3 VAG explizit aufgeführten Bilanzpositionen als anrechnungsfähige Eigenmittel gegenüberzustellen (Ist-Solvabilität). ${ }^{117}$ Bei Unterschreitung der Solvabilitätsspanne muss das betroffene Versicherungsunternehmen der Aufsichtsbehörde einen Solvabilitätsplan vorlegen. Dieser kann Maßnahmen zur Erhöhung der Ist-Solvabilität (z.B. Kapitalerhöhung) oder zur Reduzierung der Solvabilitätsspanne (z.B. durch zusätzliche Rückversicherungsnahme) vorsehen. ${ }^{118}$ Der Garantiefonds stellt eine zusätzliche Interventionsschwelle dar. Er entspricht einem Drittel der Solvabilitätsspanne. Der Mindestgarantiefonds ist als absoluter Betrag definiert und muss zum Zeitpunkt der Erteilung der Geschäftserlaubnis bereitgestellt werden. Seine Höhe bemisst sich anhand der Art und Anzahl der betriebenen Versicherungszweige. ${ }^{119}$ Sollten die anrechnungsfähigen Eigenmittel den Garantiefonds oder sogar den Mindestgarantiefonds unterschreiten, muss das Versicherungsunternehmen der Aufsicht gemäß § 81b Abs. 2 VAG einen kurzfristigen Finanzierungsplan zur Genehmigung vorlegen. Darüber hinaus kann die Aufsichtsbehörde die freie Verfügung über die Vermögenswerte des Unternehmens einschränken oder untersagen.

\subsubsection{Kritische Würdigung}

Die Kritik an den derzeitig gültigen aufsichtsrechtlichen und institutionellen Rahmenbedingungen (Solvency I) macht sich sowohl an den stellenweise unscharf formulierten Zielsetzungen als auch an elementaren konzeptionellen Schwächen fest, die im Zusammenhang mit der Ermittlung der Soll- bzw. Ist-Solvabilität stehen. ${ }^{120}$ Zunächst ist kritisch anzumerken, dass in die Berechnung der Mindestkapitalausstattung aus-

117 Vgl. in diesem Zusammenhang detailliert FARNY (2000), S. 756-759 und SCHMEISER (1997), S. 23-27 und EGBERS (2002), S. $40 \mathrm{ff}$.

118 Vgl. im Folgenden FARNY (2000), S. 750 und SCHMEISER (1997), S. 19. Vgl. detailliert zu den Rechtsfolgen einer Unterschreitung der Eigenmittelanforderungen EGBERS (2002), S. 117-120.

119 Die neue EU-Richtlinie 2002/13/EG sieht in Artikel 17a vor, sowohl den Mindestgarantiefonds als auch die Schwellenwerte des Beitrags- und Schadenindexes an den Europäischen Verbraucherpreisindex zu koppeln. Vgl. hierzu auch HARTUNG/HELTEN (2004), S. 294 und SCHRADIN (2003), S. 636.

120 Vgl. insbesondere FARNY (2000), S. 760 und SCHMEISER (1997), S. 28. 
schließlich bilanzielle, mithin vergangenheitsorientierte Daten Eingang finden. ${ }^{121}$ Potenzielle Verlustrisiken, die mit der Abgabe neuer Zeichnungsversprechen verbunden sind, gehen somit nicht in die Berechnung ein. Darüber hinaus muss lediglich das versicherungstechnische Risiko mit Eigenmitteln unterlegt werden. Wenngleich dieser Risikokomponente im Kontext der Schaden- und Unfallversicherung eine zentrale Bedeutung zukommt, wird der Einfluss von Kapitalanlagerisiken und sonstigen Unternehmensrisiken gänzlich ignoriert. ${ }^{122}$ Ebenfalls finden die zwischen den einzelnen Risiken bestehenden Interdependenzen keinen Eingang in die Berechnung, so dass eine angemessene Quantifizierung der Gesamtrisikoposition nicht gelingen kann. ${ }^{123}$ Es muss allerdings realistischerweise eingeräumt werden, dass die Quantifizierung sämtlicher als relevant erachteter Unternehmensrisiken in der Praxis schnell an Grenzen stößt. ${ }^{124}$

Konzeptionelle Kritik betrifft insbesondere die Verwendung des Beitrags- bzw. Schadenindexes als Risikoindikator und außerdem die Tatsache, dass die numerischen Vorgaben zur Berechnung der Solvabilitätsspanne willkürlich erscheinen und jeglicher risikotheoretischen Fundierung entbehren. ${ }^{125}$ Die Verwendung des Beitragsindexes setzt implizit voraus, dass sich das Bruttoprämienvolumen und das versicherungstechnische Risiko linear-proportional zueinander verhalten. ${ }^{126}$ Dies führt bisweilen zu paradoxen Konsequenzen, denn die kalkulatorische Berücksichtigung höherer Sicherheits- oder Betriebskostenzuschläge induziert automatisch einen unangemessenen Mehrbedarf an Eigenmitteln. ${ }^{127}$ Der Schadenindex kann des Weiteren nur unter der Annahme weitgehend stabiler Umweltbedingungen als akzeptabler Näherungswert für das versicherungstechnische Risiko angesehen werden. SCHMEISER weist darauf hin, dass im Falle stark wachsender Versicherungsbestände „das Verhältnis der beiden In-

121 Vgl. im Folgenden insbesondere SCHMEISER (1997), S. 28-35.

122 Im Hinblick auf die Angemessenheit der Schadenrückstellungen kritisiert SCHRADIN, dass gerade aufgrund der herausragenden Bedeutung des versicherungstechnischen Fremdkapitals die Quantifizierung des Reserve- und Abwicklungsrisikos geboten erscheint. Er formuliert daher: „Es ist betriebswirtschaftlich nicht einzusehen, weshalb prinzipiell quantifizierbare Risikokomponenten in eine Berechnung des Sicherheitskapitalbedarfes nicht eingehen sollten." SCHRADIN (2003), S. 621. Vgl. hierzu ebenfalls kritisch SCHRADIN (2003), S. 620 und SCHMEISER (1997), S. 29.

124 Vgl. übereinstimmend SCHMEISER (1997), S. 29.

125 Vgl. übereinstimmend WAGNER (1992), S. 167-183, SCHMEISER (1997), S. 29-31, FARNY (2000), S. 760-761 und HARTUNG/HELTEN (2004), S. 296-297. Letztgenannter Kritikpunkt wird auch durch die im Zuge von Solvency I erfolgte Anhebung der Schwellenwerte oder die eingeführte Inflationsanpassung nicht entkräftet.

126 SCHMEISER bemerkt zu Recht, dass dieser lineare Zusammenhang „lediglich im irrealen Fall eines nicht ausgleichsfähigen Kollektivs vollständig abhängiger Risiken“ vorliegt. SCHMEISER (1997), S. 31 .

127 Vgl. auch WAGNER (1992), S. 172-177, FARNY (2000), S. 791, HARTUNG/HELTEN (2004), S. 296297 und SCHMEISER (1997), S. 29-30. 
dizes erheblich zu Lasten des Schadenindexes verschoben wird; die Grenzschadenquote kann demnach einen Wert von über $70 \%$ annehmen. “ 128 Ferner ist zu kritisieren, dass im Zuge der Bildung mehrjähriger Schadendurchschnitte zwar der Einfluss von „Ausreißerjahren“ reduziert wird, allerdings durch den Glättungseffekt auch wichtige Informationen über die risikobegründende Volatilität des Schadengeschehens verloren gehen. Ebenfalls bleibt unberücksichtigt, ob der Gesamtschaden in den zurückliegenden Jahren vornehmlich auf eine hohe Frequenz von Kleinschäden oder auf singuläre Groß- oder Katastrophenschäden in Sparten mit starker Exponierung gegenüber Elementargefahren zurückzuführen war. Jedoch übt gerade die Schadenstruktur einen signifikanten Einfluss auf die Schwankungsbreite der versicherungstechnischen Ergebnisse und den Kapitalbedarf des Unternehmens aus. Ein zentraler Mangel ist schließlich darin $\mathrm{zu}$ sehen, dass die Rückversicherungsnahme maximal $\mathrm{zu} 50 \%$ anrechenbar ist. $^{129}$ Gerade in großschadenanfälligen Versicherungszweigen sind Rückversicherungsanteile von über $50 \%$ durchaus üblich. ${ }^{130}$ Eine Außerachtlassung dieses Sachverhalts führt demzufolge zu einer systematischen Überschätzung des Kapitalbedarfs und kann auch nicht durch potenzielle Ausfallrisiken auf Seiten der Rückversicherungsunternehmen hinreichend begründet werden.

Weitere Kritikpunkte betreffen die Ermittlung der Ist-Solvabilität. Zunächst bedürfen die in $\S 53 \mathrm{c}$ Abs. 1 VAG zitierten "freien und unbelasteten Eigenmittel“" einer kritischen Erörterung. Führt man sich vor Augen, dass sämtliche existierenden Eigenkapitalkomponenten in irgendeiner Form in mehr oder weniger risikobehaftete Vermögenswerte investiert werden (Mittelverwendung), so wird deutlich, dass es selbst bei der Haltung liquider Mittel keine gänzlich unbelasteten Eigenmittel geben kann. ${ }^{131}$ Obwohl im Insolvenzfall die kurzfristige Realisierbarkeit dieser Vermögenswerte von substanzieller Bedeutung ist, wird der Liquiditätsaspekt bei der Ermittlung der IstSolvabilität bislang nicht berücksichtigt. ${ }^{132}$ Darüber hinaus finden weder Schwankungsrückstellungen noch Rückstellungen für drohende Verluste Anrechnung, obwohl diese eigenkapitalähnlichen Positionen unter bestimmten Bedingungen zum Verlustausgleich herangezogen werden können. ${ }^{133}$ Die vorangegangenen Ausführungen belegen die grundlegende Problematik, auf europäischer Ebene einheitliche institutionelle Rahmenbedingungen schaffen zu wollen, bevor eine hinreichende Harmonisierung der handelsrechtlichen Bestimmungen stattgefunden hat. Dieser Kritikpunkt gewinnt im

\footnotetext{
128 SCHMEISER (1997), S. 30 bezieht sich hierbei auf KARTEN (1984), S. 346.

129 Vgl. in diesem Zusammenhang vertiefend WAGNER (1992), S. 183-193.

130 Vgl. ebenfalls HARTUNG/HELTEN (2004), S. 297.

131 FARNY bemerkt in diesem Kontext treffend: „Es gibt also bestenfalls mehr oder weniger belastete Eigenmittel." FARNY (2000), S. 761.

132 Vgl. auch SCHMEISER (1997), S. 32-35.

133 Vgl. auch FARNY (2000), S. 762.
} 
Rahmen einer risikoorientierten Solvabilitätspolitik besondere Bedeutung, da abweichende handelsrechtliche Bewertungsvorschriften in den einzelnen Mitgliedsstaaten bereits unterschiedliche Sicherheitsniveaus repräsentieren. ${ }^{134}$

Zusammenfassend ist festzuhalten, dass die aktuell gültigen Solvabilitätsvorschriften auch nach Umsetzung der EU-Richtlinie 2002/13/EG weiterhin umfangreicher konzeptioneller Kritik ausgesetzt sind, die sich primär an einer unzureichenden Berücksichtigung der Gesamtrisikoposition und des zur Verfügung stehenden risikopolitischen Instrumentariums (insbesondere der Rückversicherung) festmacht. Aufbauend auf dieser kritischen Einschätzung werden nachfolgend die Grundzüge der geplanten Neuausrichtung des europäischen Solvabilitätssystems (Solvency II) vorgestellt.

\subsubsection{Neuausrichtung des europäischen Solvabilitätssystems (Solvency II)}

\subsubsection{Zielsetzungen und allgemeiner Aufbau}

Im vorangegangenen Abschnitt ist deutlich geworden, dass die durch Solvency I unlängst initiierten Veränderungen des europäischen Solvabilitätssystems lediglich marginaler und zumeist „technischer" Natur sind. Im Hinblick auf eine risikotheoretisch fundierte Erfassung der Gesamtrisikoposition eines Versicherungsunternehmens sind jedoch keine konzeptionellen Fortschritte gegenüber den seit 1973 gültigen Bestimmungen erkennbar. Vor diesem Hintergrund hat sich die Europäische Kommission bereits im Jahr 2000 entschlossen, die Solvabilitätsvorschriften im Rahmen des Projekts „Solvency II“ grundlegend neu zu ordnen. ${ }^{135}$ Das Projekt ist in zwei Phasen unterteilt. In der ersten Phase erfolgte eine detaillierte Bestandsaufnahme der versicherungsspezifischen Rahmenbedingungen sowie des aufsichtsrechtlichen Instrumentariums. Die Ergebnisse wurden in einer Studie der KPMG und im so genannten „SharmaReport" dokumentiert. ${ }^{136}$ In der seit September 2003 laufenden zweiten Projektphase sollen konkrete Vorschläge zur Neugestaltung und Umsetzung erarbeitet werden.

Die Zielsetzungen von Solvency II leiten sich im Wesentlichen aus den bereits erörterten Unzulänglichkeiten der aktuellen Solvabilitätsvorschriften ab und seien im Folgenden nur schlaglichtartig dargestellt. ${ }^{137}$ Ausgangspunkt der Überlegungen ist ein

\footnotetext{
134 Vgl. SCHMEISER (1997), S. 13 und S. 35.

135 Der Versicherungsausschuss der EU-Kommission steht im Rahmen dieses Projektes in einem engen Dialog mit internationalen Organisationen wie der International Association of Insurance Supervisors (IAIS), der International Actuarial Association (IAA) sowie dem International Accounting Standards Board (IASB). Vgl. auch SCHRADIN (2003), S. 646.

136 Vgl. KPMG (2002) und LONDON GROUP (2002).

137 Vgl. im Folgenden insbesondere KPMG (2002), S. 7-8 sowie HEISTERMANN (2002b), S. 15, KNAUTH/SCHUBERT (2003), S. 902-903 und HARTUNG/HELTEN (2004), S. 298.
} 
erweiterter Solvabilitätsbegriff, der nicht nur Vorschriften zur Ermittlung der Mindestkapitalausstattung umfasst, sondern auch Regelungen zur Bemessung der versicherungstechnischen Rückstellungen sowie Kapitalanlagevorschriften enthält. Ferner sollen im Sinne einer „Gesamtsolvabilität“ auch qualitative Aspekte wie beispielsweise Managementqualität, Wettbewerbslage oder die Ausgestaltung des Risikomanagementprozesses in die Betrachtung einfließen. Die bisher ausschließlich vergangenheitsorientierte Aufsichtsperspektive soll durch eine prospektive und ganzheitlich ausgerichtete Risikobetrachtung ersetzt werden. Dies bedingt insbesondere eine signifikante Ausweitung und Verfeinerung der relevanten Risikoklassen über das versicherungstechnische Risiko hinaus. Wenngleich eine ungerechtfertigte Komplexität der Vorschriften im Grundsatz vermieden werden soll, kommt dennoch einer risikotheoretisch fundierten Quantifizierung der Gesamtrisikoposition beispielsweise durch interne Risikomodelle elementare Bedeutung zu.

Neben einer weitreichenden Harmonisierung der Aufsichtsmethoden wird eine enge Bindung an die Entwicklung internationaler Rechnungslegungsstandards (IFRS) angestrebt. ${ }^{138}$ Durch die Annäherung der Wettbewerbsbedingungen für Versicherungsunternehmen und Kreditinstitute soll die Gefahr regulatorischer Arbitrageeffekte weitgehend vermieden und ein einheitlicher Regulierungsrahmen (,level-playing-field“) geschaffen werden. ${ }^{139}$ Ebenfalls sollte das mittlerweile hoch entwickelte risikopolitische Instrumentarium der Erst- und Rückversicherungsunternehmen bei der Bemessung der Eigenmittelerfordernisse Berücksichtigung finden. Diese Forderung betrifft insbesondere die Fortschritte auf dem Gebiet der Finanzrückversicherung bzw. des Alternativen Risikotransfers (ART). ${ }^{140}$ Die in Aussicht gestellte Anerkennung interner Risikomodelle soll als Anreiz verstanden werden, quantitative Modelle eigenständig zu entwickeln. Die Versicherungsunternehmen sollen auf diese Weise sukzessive an die Nutzung komplexer Risikomanagementtechniken herangeführt werden. ${ }^{141}$ Dies unterstreicht die grundlegende Zielsetzung der Aufsichtsbehörden, im Interesse einer größtmöglichen Wettbewerbsneutralität nur in dem Maße regulierend eingreifen zu wollen, wie dies zur Vermeidung eines Marktversagens notwendig erscheint.

In enger Anlehnung an den Aufbau der neuen Eigenkapitalvorschriften im Bankensektor („Basel II“) liegt dem Projekt „Solvency II“ ebenfalls eine Drei-Säulen-Struktur

\footnotetext{
138 Vgl. insbesondere RITTMANN/ROCKEL (2004), S. 441-475.

139 Vgl. in diesem Kontext kritisch KNAUTH (1996), S. 241-244 und HARTUNG/HelTEN (2004), S. 302.

140 Vgl. auch HARTUNG/HeLTEN (2004), S. 298.

141 Vgl. übereinstimmend KRIELE/LIM/REICH (2004), S. 1052.
} 
zugrunde. ${ }^{142}$ Die erste Säule beinhaltet quantitative Anforderungen an die Finanzausstattung von Versicherungsunternehmen sowie die Forderung nach kohärenten Regelungen zwischen dem Banken- und Versicherungssektor. Die Bestimmungen der zweiten Säule formulieren qualitative Anforderungen an das aufsichtsrechtliche Überprüfungsverfahren, den Risikomanagementprozess der Versicherungsunternehmen und die Ausgestaltung interner Risikomodelle. Erklärtes Ziel ist es demnach, dass die im Zuge der Deregulierung gewährten Freiräume durch die Versicherungsunternehmen verantwortungsvoll genutzt werden. Verschärfte Offenlegungspflichten, ein einheitliches aufsichtsrechtliches Kontrollverfahren sowie ein durch den Markt induzierter Sanktionsmechanismus zur Erhöhung der Marktdisziplin sind schließlich Gegenstand der dritten Säule. Der grundlegende Aufbau von Solvency II wird in Abbildung 3.1 schematisch darstellt und hinsichtlich seiner geplanten inhaltlichen Ausgestaltung im nachfolgenden Kapitel vertieft. ${ }^{143}$

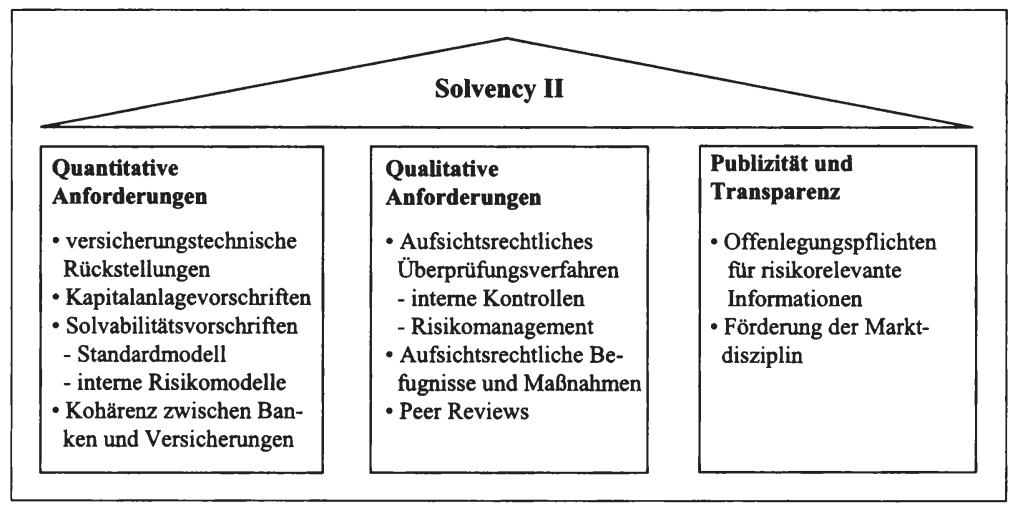

Abbildung 3.1: Drei-Săulen-Struktur von Solvency II

Quelle: in Anlehnung an KPMG (2002), S. 18 und SCHRADIN (2003), S. 649-658.

\subsubsection{Inhaltliche Ausgestaltung und Implikationen für die Steuerung}

\subsection{Säule 1: Quantitative Vorgaben}

Im Gegensatz zu den momentan zur Anwendung kommenden Solvabilitätsvorschriften legen die quantitativen Anforderungen von Solvency II einen differenzierteren Risikobegriff zugrunde. Der KPMG-Bericht nennt als Schlüsselrisiken für die Solvabilität

${ }^{142}$ Vgl. im Folgenden auch KPMG (2002), S. 18, BASLER AUSSCHUSS (2003), SCHRADIN (2003), S. 644-658, RITTMANN/ROCKEL (2004), S. 446-463 und AON RÜCK (2004).

${ }^{143}$ Da sich die Regelungen zur konkreten inhaltlichen Ausgestaltung von Solvency II unverändert in der Konsultationsphase befinden, weisen die weiteren Ausführungen lediglich vorläufigen Charakter auf. 
eines Versicherungsunternehmens das versicherungstechnische Risiko, das Marktrisiko, das Kreditrisiko, das Asset-Liability-Mismatch-Risiko und das operationelle Risiko. ${ }^{144}$ Das versicherungstechnische Risiko umfasst wiederum im Kern die Bereiche Underwriting und Reservierung. Die weiteren Ausführungen konzentrieren sich auf die geplanten Neuerungen in Bezug auf drei Themenfelder, welche jeweils als ,strenge Nebenbedingung “ für die interne Unternehmenssteuerung aufgefasst werden können.

- Versicherungstechnische Rückstellungen

- Kapitalanlagevorschriften

- Solvabilitätsvorschriften

Die Überlegungen der EU-Kommission zu den versicherungstechnischen Rückstellungen stellen im Wesentlichen auf eine verstärkte Harmonisierung der Ansatz- und Bewertungsvorschriften für Schadenrückstellungen (,provisions for claims outstanding") und Ausgleichsrückstellungen (,equalization provisions“) ab. ${ }^{145}$ Neben der Forderung nach angemessenen Methoden zur Schadenerfassung und Schadenregulierung stehen vor allem Vorgaben für eine einheitliche Quantifizierung der Schadenrückstellungen im Mittelpunkt. Es herrscht Einigkeit darüber, dass die Rückstellungshöhe sowohl den Schadenerwartungswert (,best estimate") als auch einen angemessenen Sicherheitszuschlag (,risk margin“) umfassen sollte. In Übereinstimmung mit den derzeit in der Entwicklung befindlichen internationalen Rechnungslegungsstandards (IFRS) für Versicherungsunternehmen wird außerdem gefordert, dass die Reservierung separat für einzelne Teilkollektive mit jeweils vergleichbarer Schaden- und Abwicklungscharakteristik erfolgen sollte. ${ }^{146}$ Für die Bemessung der Sicherheitszuschläge muss zudem ein einheitliches Sicherheitsniveau festgelegt werden, welches sich beispielsweise an den Anforderungen der australischen Aufsichtsbehörde (APRA) orientieren könnte. ${ }^{147}$ Die APRA fordert, dass die Schadenrückstellungen mit einer Wahrscheinlichkeit von $75 \%$ ausreichen müssen, um den erwarteten Endschaden (,central estimate“) zu decken. Angesichts der asymmetrischen Schadenverteilungscharakteristik einiger Versicherungszweige kann sich eine auf diese Weise ermittelte Rückstellungshöhe jedoch als unzureichend herausstellen. Daher sollte der Sicherheitszuschlag mindestens der Hälf-

144 Vgl. vertiefend KPMG (2002), S. 19-29. Die von KPMG gewählte Kategorisierung deckt sich somit weitgehend mit den im DRS 5-20 verankerten Risikoarten. Vgl. DRS 5-20, S. 7-8. Die IAA ergänzt diesen Risikokatalog noch explizit um das Liquiditätsrisiko, welches die KPMG und der DRS 5-20 unter dem Markt- bzw. Kapitalanlagerisiko subsumieren. Vgl. IAA (2004), S. 29-33.

Vgl. im Folgenden RITTMANN/ROCKEL (2004), S. 457-459 und SCHRADIN (2003), S. 649-654.

146 Vgl. EU-KOMMISSION (2004a), S. 26.

147 Der von amerikanischen Aufsichtsbehörden verwendete Risk-based-Capital-Ansatz wird hingegen aufgrund konzeptioneller Mängel als nicht zielführend angesehen. Vgl. EU-KOMMISSION (2002a), S. 18-19. 
te des Variationskoeffizienten entsprechen. ${ }^{148}$ Aufgrund der angestrebten Konvergenz mit den IFRS wird vorgeschlagen, die Quantifizierung der Schadenrückstellungen auf diskontierter Basis vorzunehmen. Dem Vorsichtsgedanken folgend soll hierzu ein risikoloser Zinssatz herangezogen werden, der mit der durchschnittlichen Duration der zugrunde liegenden Verbindlichkeiten korrespondiert. ${ }^{149}$ Die Bemessung und steuerrechtliche Behandlung so genannter Ausgleichsrückstellungen, im deutschen Handelsund Steuerrecht als Schwankungsrückstellung bekannt, weist in den europäischen Mitgliedsstaaten einen sehr geringen Harmonisierungsgrad auf. ${ }^{150}$ Eine Einigung zwischen Ländern mit sehr ausgereiften Ausgleichsmechanismen und solchen, die auf einen bilanziellen Schwankungsausgleich gänzlich verzichten, ist daher nur schwer herbeizuführen. ${ }^{151}$ Darüber hinaus ist zu berücksichtigen, dass Schwankungs- und Katastrophenrückstellungen im Rahmen der IFRS-Einführung wegfallen und dem Eigenkapital zugerechnet werden sollen. ${ }^{152} \mathrm{Da}$ die EU-Kommission jedoch im Grundsatz die Möglichkeit zur steuerbegünstigten Bildung einer Ausgleichsrückstellung befürwortet, wird ein Zwischenschritt vorgeschlagen, der den Anwendungsbereich zunächst auf einige schwankungsanfällige Versicherungszweige beschränkt. ${ }^{153}$ Der Ausgleichsmechanismus muss die Volatilität des Versicherungsgeschäfts sowie den bestehenden Rückversicherungsschutz angemessen berücksichtigen. Im Hinblick auf eine mögliche Anrechnung der Schwankungsrückstellung auf die Solvabilitätsspanne ist außerdem sicherzustellen, dass die Schwankungsrückstellung durch die Unternehmen nicht als Ersatz für den Sicherheitszuschlag in den Schadenrückstellungen fehlinterpretiert wird. ${ }^{154}$ Um zukünftige Solvabilitätsanforderungen und geplante Rechnungslegungsvorschriften besser aufeinander abstimmen zu können, schlägt die EU-Kommission vor, eine endgültige Entscheidung zur Behandlung der Ausgleichsrückstellungen zunächst zu vertagen. ${ }^{155}$

Die in Artikel 20 der dritten EU-Richtlinie kodifizierten Kapitalanlagevorschriften fordern explizit eine Anlagepolitik, die den Zielen Sicherheit und Rentabilität bei jederzeitiger Liquidität und angemessener Mischung und Streuung Rechnung trägt. ${ }^{156}$

\footnotetext{
148 Vgl. vertiefend APRA (2002) Tz. 10-11.

149 Vgl. EU-KOMMISSION (2004a), S. 27.

150 Vgl. zu den länderspezifischen Regelungen KPMG (2002), S. 76-77 und EU-KoMMISSION (2002a), S. 24.

151 Vgl. EU-Kommission (2002a), S. 27-28.

152 Vgl. IFRS 4 BC 87-93 und außerdem ROCKEL/SAUER (2004), S. 4 und SwISS RE (2004), S. 20.

153 Vgl. im Folgenden EU-KOMMISSION (2002a), S. 27.

154 Vgl. EU-KOMMISSION (2002a), S. 28.

155 Vgl. EU-KoMmissiON (2004a), S. 28-29.

156 In Deutschland wurden die Regelungen der dritten EU-Richtlinie in Form der $\S \S 54$ bis 54d VAG sowie der seit 2002 geltenden Kapitalanlageverordnung (AnlV) in nationales Recht umgesetzt.
} 
Sie beziehen sich ferner ausdrücklich nur auf diejenigen Vermögensgegenstände, welche die versicherungstechnischen Rückstellungen bedecken (,gebundenes Vermögen"). Die Kritik an den geltenden Kapitalanlagevorschriften zielt vorwiegend auf diese inhaltliche Beschränkung sowie die fehlende Operationalisierung der oben genannten Anlageziele ab. ${ }^{157}$ Zwar wird der Grundsatz der Mischung und Streuung in den Artikeln 21 und 22 in Form einer Liste zulässiger Anlageformen sowie durch Anlagehöchstgrenzen präzisiert, allerdings schreiben diese keinen operationalisierbaren Grad an Vorsicht vor. Im Rahmen von Solvency II sollen die Kapitalanlagevorschriften sowohl in quantitativer als auch qualitativer Hinsicht überarbeitet werden, um die Kapitalanlagerisiken hinsichtlich ihres spezifischen Beitrags zum Gesamtrisiko transparenter zu machen. ${ }^{158}$ In diesem Kontext kommt einem institutionalisierten Asset-LiabilityManagement verstärkte Bedeutung zu. Offen ist hingegen noch, ob die Kapitalanlagerisiken lediglich durch zusätzliche Eigenmittel zu unterlegen sind oder darüber hinaus noch durch quantitative Anlagegrundsätze limitiert werden sollen. ${ }^{159}$ Um sicherzustellen, dass das Sicherheitsniveau der Kapitalanlagen durch die erwartete Zurechnung der Ausgleichsrückstellungen zum Eigenkapital nicht absinkt, erscheint es aus Sicht der EU-Kommission angezeigt, die Kapitalanlagevorschriften auch auf diejenigen Vermögenswerte auszuweiten, welche die Solvabilitätsspanne bedecken. ${ }^{160}$ Dies würde zukünftig zu einer signifikanten Reduzierung des so genannten „freien Vermögens“ führen und den Handlungsspielraum der Versicherungsunternehmen in Bezug auf ihre Asset Allocation weiter einschränken. ${ }^{161}$

Neben den genannten Bestimmungen zu versicherungstechnischen Rückstellungen und Kapitalanlagegrundsätzen stellen die Solvabilitätsvorschriften den inhaltlichen Kern der ersten Säule dar. Sie basieren auf einem Zwei-Ebenen-Konzept, welches grundlegend zwischen einem absoluten Mindestkapitalniveau (,absolute minimum margin") und einem Zielkapitalniveau (,target capital") differenziert und konkrete Eingriffbefugnisse der Aufsichtsbehörden an diese Interventionsschwellen knüpft. ${ }^{162}$ Eine Unterschreitung der absoluten Mindestspanne zieht sofortige Interventionen der Aufsicht nach sich. Diese konkretisieren sich in der Verpflichtung zur kurzfristigen Wiederherstellung gesunder Finanzverhältnisse und der Aufstellung eines Finanzierungsplans. Vergleichbar dem aus Solvency I bereits bekannten Garantiefonds übt die absolute Mindestspanne somit eine Warnfunktion aus. Um unverzügliche Interventio-

\footnotetext{
157 Vgl. in diesem Zusammenhang vertiefend EU-KOMMISSION (2002b), S. 41-46.

158 Vgl. in diesem Kontext auch GRÜNDL/SCHMEISER (2001), Sp. 2153-2154.

159 Vgl. EU-KOMMISSION (2002b), S. 43.

160 Vgl. EU-KOMMISSION (2002b), S. 45-46.

${ }^{161}$ Vgl. auch HARTUNG/HeLTEN (2004), S. 299. Zu den aufsichtsrechtlichen Begriffen des ,gebundenen Vermögens" und „freien Vermögens" vgl. FARNY (2000), S. 768-770 und S. 823.

162 Vgl. im Folgenden SCHRADIN (2003), S. 652-654 und EU-KOMMISSION (2002b), S. 46-50.
} 
nen der Aufsicht zu ermöglichen, sollten für das Mindestkapital einfache und objektive Berechnungsvorschriften gelten. Hinsichtlich der Frage, wie eine konsistente und dennoch praktikable Verbindung zwischen beiden Kapitalniveaus hergestellt werden könnte, spricht sich die EU-Kommission für eine eigenständige Berechnungsmethodik aus, die sich an die derzeitigen Solvabilitätsvorschriften anlehnt. ${ }^{163}$ Eine prozentuale Koppelung an das Zielkapital wird hingegen nicht angestrebt, da eine derartige Vorgehensweise einerseits dem Anspruch einer einfachen und objektiven Ermittlung zuwider laufen und andererseits die spezifischen Zielsetzungen beider Schwellenwerte vermischen würde. ${ }^{164}$ Die Berücksichtigung von Kapitalanlagerisiken könnte entweder pauschal über Zuschlagssätze erfolgen oder sich an der Höhe der versicherungstechnischen Rückstellungen orientieren.

Das aufsichtsrechtliche Zielkapital soll als neues Aufsichtskonzept weitgehend an die Definition des ökonomisch notwendigen Kapitals angenähert werden. ${ }^{165}$ Eine Unterschreitung des Zielkapitals zieht weniger restriktive Sanktionen nach sich. ${ }^{166}$ Angedacht sind beispielsweise die Aufnahme eines engeren Dialogs zwischen Aufsicht und Versicherungsunternehmen oder die Aufstellung eines Zeitplans zu Wiederherstellung der wünschenswerten Kapitalausstattung. Den Versicherungsunternehmen steht es im Grundsatz frei, für die Ermittlung des Zielkapitals auf ein vorgegebenes (konservatives) Standardmodell ${ }^{167}$ zurückzugreifen, oder aber die Risikoposition des Unternehmens über einen internen Modellansat $\mathrm{z}^{168}$ wesentlich exakter $\mathrm{zu}$ quantifizieren. Bevor interne Risikomodelle im Rahmen der aufsichtsrechtlichen Solvenzkontrolle zur Anwendung kommen können, müssen diese durch die Aufsichtsbehörden hinsichtlich ihrer Eignung geprüft und zertifiziert werden. ${ }^{169}$ Einer Nutzung wird zugestimmt, sofern nachgewiesen werden kann, dass die individuelle Risikolage durch das interne

163 Vgl. EU-Kommission (2004b), S. 11-12.

164 Vgl. EU-Kommission (2002b), S. 47. Zur Diskussion alternativer Vorgehensweisen vgl. EUKOMMISSION (2002b), S. 47-48.

165 Die EU-Kommision wählt für das Zielkapital folgende Definition: „the economic capital that a company would need to operate with a quantified low probability of failure within in given period.“ EU-KoMmiSSION (2003b), S. 34. „In this respect target capital has some things in common with the concept of economic capital [...] as well as with the capital required by rating agencies“. EU-KOMMISSION (2004a), S. 31.

166 Vgl. im Folgenden EU-KOMMISSION (2002b), S. 47.

${ }^{167}$ In Deutschland wurde ein derartiges Standardmodell bereits im Jahr 2002 durch den Gesamtverband der deutschen Versicherungswirtschaft e.V. (GDV) entwickelt. Vgl. GDV (2002a). Dieses wird derzeitig einer grundlegenden Prüfung und Überarbeitung unterzogen. Beispielsweise soll nach Möglichkeit auf unternehmensindividuelle Risikofaktoren zurückgegriffen und der Einfluss von Naturgefahrschäden in das neue GDV-Modell integriert werden. Vgl. GDV (2005), S. 45-54.

168 Für einen konkreten Vorschlag zur Ausgestaltung eines internen Risikomodells auf Simulationsbasis vgl. GRÜNDL/SCHMEISER (2004a), S. 473-474.

169 Die IAA hat in diesem Zusammenhang mögliche Anforderungskriterien zur Bewertung interner Risikomodelle erarbeitet. Vgl. IAA (2004), S. 65. 
Modell zutreffender abgebildet wird und das Modell im Rahmen der operativen und strategischen Unternehmenssteuerung tatsächlich Verwendung findet. ${ }^{170}$ Neben der Ermittlung des aufsichtsrechtlich erwünschten Zielkapitals zielt die Entwicklung interner Risikomodelle somit ausdrücklich darauf ab, die Versicherungsunternehmen sukzessive an die Entwicklung komplexer Steuerungsinstrumente und die Quantifizierung ihrer tatsächlichen Risikoposition heranzuführen (Anreizfunktion). Letztendlich soll der Einsatz unternehmensspezifischer Risikomodelle durch reduzierte Zielkapitalanforderungen honoriert werden. ${ }^{171}$

Hinsichtlich einiger elementarer Rahmenbedingungen hat die EU-Kommission noch nicht abschließend Stellung bezogen und diesbezüglich die beteiligten Organisationen um Stellungnahme gebeten. ${ }^{172}$ In der Diskussion sind weiterhin die Art des RisikomaBes, die Festlegung eines angemessenen Sicherheitsniveaus sowie die Wahl des zeitlichen Modellhorizontes. Seitens der EU-Kommission wird zum jetzigen Zeitpunkt der Vorschlag favorisiert, den Conditional Value-at-Risk (CVaR) insbesondere in denjenigen Versicherungszweigen als Risikomaß einzusetzen, die gegenüber Großschadenereignissen exponiert sind und demzufolge schiefe Schadenverteilungen aufweisen. Hingegen könnte der Value-at-Risk (VaR) in Sparten mit vergleichsweise homogenem Schadenanfall Anwendung finden. ${ }^{173}$ Die Festlegung eines angemessenen Sicherheitsniveaus steht in direkter Verbindung zur Wahl des Risikomaßes. Es kann an dieser Stelle bereits festgehalten werden, dass der CVaR als „vorsichtigeres“ Risikomaß im Grundsatz ein niedrigeres Sicherheitsniveau erfordert als der VaR. ${ }^{174}$ Hinsichtlich des erforderlichen Modellhorizonts sprechen sich sowohl die EU-Kommission als auch die IAA - nicht zuletzt aus Praktikabilitätsgründen - für eine einjährige Betrachtungsweise aus. Dies entbindet die Versicherungsunternehmen jedoch ausdrücklich nicht von der Verpflichtung, für qualitative Risikoeinschätzungen im Sinne der zweiten Säule einen mehrjährigen Zeitraum zugrunde zu legen. ${ }^{175}$ Mögliche Abhängigkeitsstrukturen, bei-

${ }^{170}$ Vgl. im Folgenden EU-KOMMISSION (2002b), S. 48 und KRIELE/LIM/REICH (2004), S. 1052 und HARTUNG/HELTEN (2004), S. 300-301.

171 "Consequently, one way to motivate companies to develop internal models is that the resulting target capital may be lower than in the standard approach." EU-KoMMISSION (2004a), S. 31.

172 Vgl. im Folgenden EU-KOMMISSION (2004a), S. 33-37.

173 Vgl. EU-KoMmISSION (2004a), S. 33. Aus risikotheoretischer Sicht ist dieser Vorschlag als kritisch einzustufen. Er wird daher in Kapitel 4.1.3.3 noch eingehender diskutiert. Vgl. zu den Risikomaßen CVaR und VaR auch die detaillierten Ausführungen in Kapitel 4.1.3.2 und 4.1.3.3.

174 Vgl. übereinstimmend EU-Kommission (2004a), S. 34-35. In Großbritannien, Australien und den Niederlanden wurden bereits die Verwendung des VaR und ein Sicherheitsniveau von $99,5 \%$ gesetzlich verankert. Die IAA schlägt in einem Grundsatzpapier die Verwendung des CVaR und ein Sicherheitsniveau von $99 \%$ vor. Im Falle eines mehrperiodigen Modellhorizonts kann das Sicherheitsniveau nach Ansicht der IAA auf $90 \%$ bis $95 \%$ reduziert werden. Vgl. IAA (2004), S. 22.

$175 \mathrm{Vgl}$. IAA (2004), S. 22. Vgl. übereinstimmend auch EU-KOMMISSION (2004a), S. 34. Zur Vorteilhaftigkeit mehrjähriger Projektionen vgl. KRIELE/LIM/REICH (2004), S. 1049. 
spielsweise die Gefahr des gleichzeitigen Auftretens negativer Schadenszenarien in mehreren Versicherungszweigen, sollten nach Einschätzung der EU-Kommission sowohl innerhalb einer Risikokategorie als auch zwischen den Kategorien berücksichtigt werden. ${ }^{176}$ Es ist jedoch davon auszugehen, dass Standardmodelle in diesem Zusammenhang nur sehr pauschale Vorgaben erlauben werden, um die praktische Umsetzbarkeit zu gewährleisten. ${ }^{177}$ Demgegenüber bieten interne Risikomodelle die Möglichkeit, unter der Zielsetzung einer differenzierten Risikobetrachtung sowohl lineare als auch nicht-lineare Abhängigkeitsstrukturen abzubilden. ${ }^{178}$

\subsection{Säule 2: Qualitative Vorgaben}

Die geplanten Regelungen der zweiten Säule verfolgen die Zielsetzung, ein harmonisiertes aufsichtsrechtliches Überprüfungsverfahren (,supervisory review process") zu initiieren, welches die Versicherungsunternehmen dazu anhält, ihre Risikoposition möglichst umfassend $\mathrm{zu}$ managen und adäquat abzusichern. Die qualitativen Anforderungen zielen somit direkt auf die Erfüllung der in Säule 1 formulierten quantitativen Vorgaben ab. Die seitens der EU-Kommission vorgeschlagenen Grundsätze lehnen sich eng an die diesbezüglichen Empfehlungen des SharmaReports an und beinhalten zentrale Anforderungen an unternehmensinterne Kontrollmechanismen sowie ein umfassendes Risikomanagement. ${ }^{179}$ Durch das Institut der „Peer Reviews" soll eine bestmögliche Harmonisierung der Aufsichtsmethoden in den einzelnen EU-Mitgliedsstaaten sichergestellt werden.

Die in den derzeitig gültigen Versicherungsrichtlinien formulierten Forderungen nach ordnungsgemäßer Verwaltung und angemessener interner Kontrolle bedürfen nach Ansicht der EU-Kommission einer Konkretisierung. ${ }^{180}$ In Anlehnung an den Bankensektor wird gefordert, dass hierarchische Ebenen, Verantwortlichkeiten und Kommunikationswege eindeutig festgelegt sind und für die angemessene Überwachung der Geschäftstätigkeit hinreichende Ressourcen durch die Versicherungsunternehmen bereitgestellt werden. ${ }^{181}$ Die internen Kontrollinstanzen sollten durch ein Risikomanagement flankiert werden, welches die versicherungsspezifischen Kernbereiche Underwriting, Vertrags-, Schaden- und Rückstellungsmanagement, Rückversicherung und

\footnotetext{
176 Vgl. EU-KOMMISSION (2004a), S. 37.

177 Vgl. übereinstimmend HARTUNG/HELTEN (2004), S. 301.

${ }^{178} \mathrm{Vgl}$. zur Definition und zum Einfluss stochastischer Abhängigkeitsstrukturen auch Kapitel 6.2.2.

179 Vgl. im Folgenden vertiefend LONDON GrouP (2002), S. 41 ff., EU-KOMMISSION (2002b), S. 5460, SCHRADIN (2003), S. 654-657 sowie ZIMMERMANN/BACH/RAUB (2004), S. 299-303.

180 Vgl. EU-KOMMISSION (2002b), S. 53-54.

181 Um die Implementierung angemessener Kontrollmechanismen zu forcieren, stellt beispielsweise die britische Aufsichtsbehörde FSA zusätzliche Kapitalanforderungen, sofern die Risikoüberwachung als unzureichend eingeschätzt wird. Vgl. GRÄWERT/STEVENS/TADROS (2003), S. 396-397.
} 
Asset-Liability-Management abdeckt. ${ }^{182}$ Explizit werden eine funktionale Trennung von Schadenmanagement und Zeichnungstätigkeit, ein umfangreiches Reporting mittels Schadenstatistiken sowie eine nachgängige Stichhaltigkeitsüberprüfung der Bewertungsmethodik für versicherungstechnische Rückstellungen gefordert. Im Bereich der Rückversicherung muss sichergestellt werden, dass das Rückversicherungsprogramm hinreichend auf das versicherungstechnische Portfolio abgestimmt ist und die Bonität der Rückversicherungsunternehmen laufend überwacht wird. Das AssetLiability-Management sollte angemessen institutionalisiert sowie im Risikomanagementprozess verankert sein und darüber hinaus gewissen Mindestanforderungen genügen. ${ }^{183}$ Der Einfluss sonstiger Risiken, die sich den vorgenannten Kernbereichen nicht unmittelbar zuordnen lassen (z.B. Konjunkturzyklen oder Naturkatastrophen), sollte mit Hilfe von Szenarioanalysen untersucht werden.

Die nunmehr skizzierten Grundsätze für ein effektives Risikomanagement können nur dann eine sinnvolle Ergänzung der quantitativen Vorschriften aus Säule 1 darstellen, sofern ihre Überprüfung durch die Aufsichtsbehörden einheitlich geregelt ist und für den Fall eines Verstoßes konkrete Eingriffsbefugnisse und Maßnahmen definiert sind. ${ }^{184}$ Als konkrete Ausgestaltungen des aufsichtsrechtlichen Überprüfungsprozesses werden Vor-Ort-Prüfungen, einheitliche Frühwarnindikatoren sowie Stresstests vorgeschlagen. Das Eingriffsniveau der Aufsichtsbehörden ist jeweils so zu definieren, dass ein hinreichender Schutz der Versicherungsnehmer gewährleistet ist. Gleichzeitig ist sicherzustellen, dass durch aufsichtsrechtliche Interventionen oder die Veröffentlichungspflicht kritischer Daten keine prozyklischen Tendenzen hervorgerufen werden (,self-fulfilling prophecy“). ${ }^{185}$ Die EU-Kommission betont außerdem, dass ein einheitlicher Mindeststandard für die statistische Datenerhebung den Informationsaustausch zwischen den Aufsichtsbehörden der Mitgliedsstaaten vereinfachen und die Konvergenz des Aufsichtsprozesses fördern würde. ${ }^{186}$

Die Einführung des Konzepts der „wünschenswerten Kapitalausstattung“ erfordert eine explizite Festlegung möglicher Maßnahmen für den Fall, dass das Zielkapital durch ein Versicherungsunternehmen unterschritten, die absolute Mindestspanne jedoch eingehalten wird. Seitens der Versicherungsunternehmen kommen als gegensteuernde Maßnahmen grundsätzlich eine Veränderung des Risikoprofils (z.B. durch Rückversicherung, Hedging oder alternative Produktgestaltung), Kapital- oder Prä-

\footnotetext{
182 Vgl. im Folgenden EU-KOMMISSION (2002b), S. 55-60.

${ }^{183}$ Vgl. hierzu vertiefend EU-KOMMISSION (2002c), S. 43.

184 Vgl. im Folgenden EU-KOMMISSION (2002b), S. 53.

185 Vgl. EU-Kommission (2003b), S. 41-42 und HARTUNG/HeLTEN (2004), S. 300.

186 Vgl. EU-KOMMISSION (2003a), S. 9-10.
} 
mienerhöhungen sowie eine Reduzierung des Cashouts (z.B. durch eine restriktivere Schadenregulierungs- oder Ausschüttungspolitik) in Betracht. Die Aufgabe der Aufsichtsbehörden besteht darin, die ergriffenen Maßnahmen im Hinblick auf deren Angemessenheit und Durchführbarkeit zu beurteilen und gegebenenfalls frühzeitig gegenzusteuern. ${ }^{187}$ Die Rolle der Aufsicht wandelt sich somit zunehmend von einer Tarife genehmigenden Behörde hin zu einer prozessbegleitenden Instanz. Zusammenfassend zeichnet sich ab, dass die Versicherungsunternehmen im Rahmen von Solvency II in einen deutlich engeren und qualitativeren Dialog mit den Aufsichtsbehörden eintreten werden.

\subsection{Säule 3: Transparenz und Marktdisziplin}

Die dritte Säule beinhaltet verschärfte Publizitätsanforderungen und strebt eine Offenlegung risikorelevanter Informationen zur Förderung der Marktdisziplin an. ${ }^{188}$ Wenngleich inhaltliche Konkretisierungen noch nicht vorliegen, ist es doch das erklärte Ziel der EU-Kommission, durch Veröffentlichungserfordernisse eine weitreichende Transparenz gegenüber Versicherungsnehmern, Kapitalmarktakteuren und Ratingagenturen zu schaffen und die Marktkräfte auf diese Weise gezielt als Korrektiv einzusetzen. Die geplanten Vorschriften erfordern eine enge Abstimmung mit dem IASB, um inhaltliche Überschneidungen und Doppelarbeiten für die Versicherungsunternehmen zu vermeiden. ${ }^{189}$ Mit Wirkung zum 31.03.2004 wurde der „Exposure Draft ED 5 Insurance Contracts“ durch den ersten Teil des Standards „IFRS 4 Insurance Contracts“ ersetzt. Es handelt sich zunächst um eine Übergangsvorschrift, die nach Abschluss einer zweiten Phase endgültig verabschiedet werden soll..$^{190}$ Durch den IFRS 4 werden kapitalmarktorientierte Versicherungsunternehmen zur Veröffentlichung umfangreicher Anhangangaben (,Notes“) verpflichtet. ${ }^{191}$ Vorgesehen ist beispielsweise eine Offenlegung der Bewertungsannahmen und -methoden für die Bemessung von Risikozuschlägen und die Dotierung versicherungstechnischer Rückstellungen. Die den Annahmen inhärente Unsicherheit und die Auswirkungen möglicher Fehleinschätzungen sollen ebenso transparent gemacht werden wie potenzielle Kumul-, Großschaden-, Zins- oder

187 Vgl. EU-KOMMISSION (2003b), S. 41.

${ }^{188}$ Vgl. im Folgenden SCHRADIN (2003), S. 657-658 und RITTMANN/ROCKEL (2004), S. 461-463.

189 Da die IFRS eine weitgehende Orientierung an Zeitwerten vorsehen, kann die angestrebte Konvergenz zwischen Solvenz- und Rechnungslegungsvorschriften dazu führen, dass eine Trennung von Financial Accounting und aufsichtsbezogener Rechnungslegung (Statutory Accounting) erforderlich würde. Diese Vorgehensweise wird in den USA bereits praktiziert. HARTUNG/HELTEN weisen in diesem Zusammenhang kritisch auf die hohen Kosten einer doppelten Rechnungslegung hin. Vgl. HARTUNG/Helten (2004), S. 301-302. Vgl. außerdem RoCKel (2004), S. 193-197.

190 Vgl. RitTMANN/ROCKEL (2004), S. 442-443 und ROCKEL/SAUER (2004), S. 2.

191 Vgl. im Folgenden auch VARAIN (2004) und ROMEIKE/MÜLLER-REICHARD (2005), S. 167-184. 
Kreditrisiken. ${ }^{192}$ Die im ED 5 noch vorgesehene Angabe der Zeitwerte („Fair Value“) für versicherungstechnische Rückstellungen wurde hingegen in der ersten Phase noch nicht im IFRS 4 verankert, sondern ist Gegenstand der zweiten Phase. ${ }^{193}$ Im Rahmen von Sensitivitätsanalysen und Stresstests muss jedoch der Einfluss wesentlicher Risikoparameter auf das Jahresergebnis und Eigenkapital quantifiziert werden. Deutsche Versicherungsunternehmen, welche die Offenlegungs- und Ausweisvorschriften des Deutschen Rechnungslegungsstandards Nr. 5-20 (DRS 5-20) bereits erfüllen, sind vermutlich im europäischen Vergleich gut auf die Anforderungen des IASB vorbereitet. Diejenigen Unternehmen, welche die IFRS aufgrund fehlender Kapitalmarktorientierung nicht anwenden müssen, sollten sich im Hinblick auf Solvency II dennoch frühzeitig auf erhöhte Informationserfordernisse einstellen.

In Abschnitt 3.1 wurden die aufsichtsrechtlichen Anforderungen an den Risikomanagementprozess von Schaden- und Unfallversicherungsunternehmen eingehend diskutiert. Die Aufsichtsperspektive stellte primär den Risikoaspekt des Versicherungsgeschäfts und das Schutzbedürfnis der Versicherungsnehmer in den Mittelpunkt. Im nachfolgenden Abschnitt 3.2 sollen zunächst das betriebswirtschaftliche Zielsystem und daraus abgeleitete Entscheidungskomponenten beleuchtet werden. Darauf aufbauend wird die bisherige Risikosicht um den Erfolgsaspekt ergänzt und die Notwendigkeit einer integrierten Rendite-Risikosteuerung aufgezeigt.

\subsection{Betriebswirtschaftliche Anforderungen an das Risikomanagement}

\subsubsection{Darstellung des betriebswirtschaftlichen Zielsystems}

Im Sinne der betriebswirtschaftlichen Entscheidungstheorie ${ }^{194}$ kann Wirtschaften als Summe von Einzelentscheidungen charakterisiert werden, wobei unter einer Entscheidung „die mehr oder weniger bewusste Auswahl einer von mehreren möglichen Handlungsalternativen “ ${ }^{\text {195 }}$ verstanden wird. Um zudem rationale Entscheidungen treffen $\mathrm{zu}$ können, bedarf es sowohl der Existenz eines adäquaten und hinreichend explizierten Zielsystems als auch der Festlegung eines sachgerechten Entscheidungsprinzips. ${ }^{196}$ Hinsichtlich des Zielsystems sind grundlegend Sach- und Formalziele voneinander zu unterscheiden.

\footnotetext{
192 Vgl. IFRS 4.36-4.39.

193 Vgl. IFRS ED 5.30 i.V.m. IFRS 4 BC 224-226 sowie RITTMANN/ROCKEL (2004), S. 443.

194 Vgl. zur entscheidungsorientierten Versicherungsbetriebslehre FARNY (2000), S. $281 \mathrm{ff}$.

195 SIEBEN/SCHILDBACH (1994), S. 1. Vgl. zur Unterscheidung von deskriptiver und präskriptiver Entscheidungstheorie vertiefend BERENS/DELFMANN/SCHMITTING (2004), S. 49.

196 Vgl. AlBRECHT (1994c), S. 1-2. Vgl. zu den Postulaten rationalen Entscheidens auch BAMBERG/ COENENBERG (2002), S. 33-40. Zum controllingspezifischen Rationalitätsbegriff vgl. insbesondere WEBER (2004), S. 50-61.
} 
Als Sachziel bzw. Unternehmenszweck kann im versicherungswirtschaftlichen Kontext die Bereitstellung von Versicherungsschutz identifiziert werden. ${ }^{197}$ Das Entscheidungsfeld eines Versicherungsunternehmens wird jedoch durch eine Vielzahl von Formalzielen vorgegeben, welche sich wechselseitig beeinflussen und vielfach miteinander konkurrieren. Die Formalziele können gewissermaßen als Motive für die Unternehmensexistenz charakterisiert werden. ${ }^{198}$ Die Heterogenität des Zielsystems wird nachvollziehbar, sofern man ein Versicherungsunternehmen als Koalition unterschiedlicher Anspruchsgruppen auffasst, welche zum Teil divergierende Interessen verfolgen und in unterschiedlichem Ausmaß an Entscheidungen beteiligt sind. ${ }^{199}$ In diesem Zusammenhang kann zwischen internen Anspruchsgruppen (Eigentümer, Management, Mitarbeiter) und externen Anspruchsgruppen (Versicherungsnehmer, Aufsichtsbehörden, Öffentlichkeit) unterschieden werden. ${ }^{200}$ Nachfolgend werden die wesentlichen Formalziele eines Schaden- und Unfallversicherungsunternehmens im Überblick dargestellt.

Gewinn- und Sicherheitsstreben werden in der einschlägigen Literatur als vorrangige Zielsetzungen für Versicherungsunternehmen angesehen. ${ }^{201}$ Darüber hinaus werden gelegentlich auch Bedarfs- und Kostendeckung, Reputation, Macht oder wirtschaftliche Unabhängigkeit angeführt. ${ }^{202} \mathrm{Da}$ diese in der Regel nicht monetären Kategorien jedoch einer modellhaften Abbildung nur schwer zugänglich und zudem nicht versicherungsspezifischer Natur sind, werden sie im weiteren Verlauf nicht mehr betrachtet. ${ }^{203}$ Das Streben nach Gewinn ist als marktwirtschaftliches Prinzip in Theorie und Praxis anerkannt. ${ }^{204}$ Das Gewinnziel ist sowohl für die Eigentümer als auch die Unternehmensleitung von besonderem Interesse. Der Gesamtgewinn eines Schaden- und Unfallversicherungsunternehmens setzt sich im Wesentlichen aus den Teilgewinnen des Risikogeschäfts und des Kapitalanlagegeschäfts zusammen. Als Basis für die Gewinnermittlung können handelsrechtliche, kalkulatorische oder zahlungsorientierte Größen herangezogen werden. ${ }^{205}$ Eine Sonderform des Gewinnziels ist die Maximierung des Marktwertes der Unternehmung, welcher auch als Shareholder Value bekannt ist und als Zielgröße der Steuerung von Versicherungsunternehmen breite Zustim-

\footnotetext{
197 Vgl. übereinstimmend FARNY (1966), S. 135, OLETZKY (1998), S. 4 und KORYCIORZ (2004), S. 1.

198 Vgl. FARNY (1966), S. 135.

199 Vgl. vertiefend OLETZKY (1998), S. 5.

200 Vgl. auch OLETZKY (1998), S. 5-6 und FARNY (1967), S. $53 \mathrm{ff}$.

201 Vgl. GrossmanN (1967), FARNy (1966), S. 138-148 und Albrecht (1994c), S. 2.

202 Eine Übersicht außerökonomischer Zielsetzungen findet man z.B. bei WAGNER (1992), S. 52-55.

203 Vgl. zu einer analogen Vorgehensweise KORYCIORZ (2004), S. 1-2.

204 Vgl. exemplarisch FARNY (1967), S. 57, FARNY (2000), S. 306 und SCHRADIN (1994), S. 60.

205 Vgl. auch WAGNER (1992), S. 49-50 und FARNY (2000), S. 307.
} 
mung findet. ${ }^{206}$ Die Marktwertmaximierung unterstreicht die Langfristigkeit der Gewinnerzielung und formalisiert insofern explizit die Perspektive der Eigentümer. ${ }^{207} \mathrm{Da}$ das Shareholder Value-Konzept in der Regel auf einem zahlungsbasierten Gewinnbegriff (z.B. Free Cashflow) aufbaut, kann die Zielerreichung in der Regel nicht durch handelsrechtliche Ansatz- und Bewertungswahlrechte beeinflusst werden. Wachstum und Marktanteilsgewinne werden in der Versicherungswirtschaft vielfach noch als Oberziele der unternehmerischen Tätigkeit angesehen. ${ }^{208}$ Tatsächlich kann Wachstum jedoch nur als „Mittel zur Erreichung übergeordneter Unternehmensziele“ ${ }^{609}$ verstanden werden, sofern es beispielsweise durch Kostendegressionseffekte oder verbesserte Risikoausgleichseffekte zu einer höheren bzw. konstanteren Gewinnerzielung beiträgt.

Im versicherungsspezifischen Kontext kommt dem Sicherheitsziel elementare Bedeutung zu. ${ }^{210}$ Wie bereits in Kapitel 2.3 beschrieben, liegt die Notwendigkeit des Sicherheitsstrebens in der Natur des Versicherungsgeschäfts begründet, da die Existenzsicherheit des Unternehmens unmittelbaren Einfluss auf die Produktqualität nimmt. Die hohe Relevanz des Sicherheitsziels wird durch eine Reihe verhaltenswissenschaftlicher Untersuchungen zur Zahlungsbereitschaft von Versicherungsnehmern gestützt. ${ }^{211}$ Als wesentliche Erkenntnis dieser Studien ist festzuhalten, dass risikoaverse Individuen (z.B. Versicherungsnehmer) grundsätzlich bereit sind, das abstrakte Schutzversprechen eines Versicherungsunternehmens durch Prämienzahlungen zu honorieren, welche oberhalb des Schadenerwartungswertes liegen. Im Falle ausfallbedrohter Policen sinkt die Zahlungsbereitschaft der Versicherungsnehmer jedoch drastisch ab. Eine reduzierte Erfüllungssicherheit kann darüber hinaus nicht beliebig durch Prämienabschläge kompensiert werden. Die Zahlungsbereitschaft kann sogar auf Null absinken, sofern die Erfüllungssicherheit eine kritische Grenze (z.B. Ratingschwelle) unterschreitet. ${ }^{212}$ Die empirischen Belege zur Zahlungsbereitschaft haben elementare Bedeutung für die strategische Unternehmenssteuerung und stellen zudem eine verhaltenswissenschaftliche Rechtfertigung für die gängige staatliche Regulierungspraxis dar. ${ }^{213} \mathrm{Da}$ die Unterschreitung eines angestrebten Sicherheitsniveaus nicht beliebig durch eine Steigerung

206 Vgl. exemplarisch UTECHT (2001), S. 527-531, FARNY (2000), S. 316-322, OLETZKY (1998), S. 84-87 und MENTZEL (2004), S. 62-72.

207 Vgl. im Folgenden auch OLETZKY (1998), S. 6.

208 Vgl. ebenfalls kritisch hierzu OLETZKY (1998), S. 7 und FARNY (2000), S. 312.

209 SCHRAdIN (1994), S. 28. Vgl. auch WAGNER (1992), S. 47 und BROHM (2002), S. 34-35.

${ }^{210}$ Vgl. im Folgenden SCHRADIN (1994), S. 58-59, FARNY (2000), S. 313-316 und MAURER (2000), S. $108 \mathrm{ff}$.

211 Vgl. im Folgenden WAKKER/THALER/TVERSKY (1997) und MAURER (2000), S. 138-153.

212 Vgl. MAURER (2000), S. 150.

${ }^{213}$ Vgl. übereinstimmend AlBrecht (1994c), S. 3-4, MAURER (2000), S. 151 und KORYCIORZ (2004), S. 4. 
des erwarteten Gewinns kompensiert werden kann, können Gewinnerzielung und Sicherheit nicht als gleichrangige Ziele eingestuft werden. Vielmehr ist dem Sicherheitsziel im Sinne des „Safety-First-Prinzips“ oberste Priorität einzuräumen. HELTEN formuliert in diesem Kontext zutreffend:

\begin{abstract}
„Vom risikotheoretischen Standpunkt aus, und ich meine auch vom versicherungspraktischen Standpunkt, heißt das oberste Ziel: Sicherheit. Über die quantitative Ausprägung dieses Ziels, ob gemessen in Varianz der Schadenverteilung oder Ruinwahrscheinlichkeit [...] lässt sich im Einzelnen streiten. Aber Safety first. Die dann folgenden Ziele Gewinn, Wachstum, Marktanteil, Prestige usw. sind dann immer unter der Bedingung einer bestimmten, fest vorgegebenen Sicherheit zu maximieren.“214
\end{abstract}

Diese Einschätzung unterstreicht, dass das Streben nach Unternehmenssicherheit keinen Selbstzweck darstellt, sondern den Charakter einer notwendigen Nebenbedingung trägt, welche das primäre Ziel der Gewinnerzielung sowohl restringiert als auch stützt. ${ }^{215}$ Das grundlegende Entscheidungskalkül, welches auch dieser Arbeit zugrunde liegt, kann daher durch folgende Zielfunktion formalisiert werden. ${ }^{216}$ Dabei bezeichne $\mathrm{G}(\mathrm{e})$ den in einem noch zu definierenden Zeitraum erwarteten Gewinn einer durch die Unternehmensleitung ergriffenen Politik e, welche der Menge aller möglichen Aktionen $\mathrm{E}$ entstammt.

$$
\mathrm{G}(\mathrm{e}) \rightarrow \max ! \quad \text { mit } \mathrm{e} \in \mathrm{E}
$$

unter der aus Beziehung (2.7) bereits bekannten Nebenbedingung:

$$
\mathrm{P}(\mathrm{G}(\mathrm{e})<-\mathrm{U}) \leq \varepsilon
$$

Unter Fortführung der bisherigen Notation bezeichne U wiederum das verfügbare Sicherheitskapital. Das Sicherheitsniveau sei erneut durch die Festlegung einer unternehmensseitig tolerierten Ruinwahrscheinlichkeit $\varepsilon$ vorgegeben. Die Höhe des Sicherheitsniveaus wird in der Praxis vielfach aus der Vorgabe eines Mindestratings abgeleitet. $^{217}$

214 Helten (1975), S. 91. Vgl. außerdem übereinstimmend MAUER (2000), S. 150-153, OLETZKY (1998), S. 87-90, ALBRECHT (1994c), S. 2-4 und SCHRADIN (1994), S. 58-59.

215 Vgl. SCHRADIN (1994), S. 59 und FARNY (2000), S. 316.

216 Vgl. übereinstimmend SCHRAdIN (1994), S. 72, AlBrECHT (1994a), S. 18, ALBRECHT (1994c), S. 14, OLETZKY (1998), S. 88 und GRAUMANN/BAUM (2003), S. 431.

${ }^{217}$ Vgl. exemplarisch GDV (2002b), S. 25. 
Es sei bereits an dieser Stelle darauf hingewiesen, dass ein auf diese Weise formuliertes Entscheidungskalkül ausdrücklich nicht mehr dem Rationalitätskriterium nach BERNOULLI genügen kann, da eine unbegrenzte Substitution von Gewinn und Risiko (sog. Substitutionsaxiom) im Falle einer bindenden Sicherheitsrestriktion gemäß (3.6) nicht mehr möglich ist. ${ }^{218}$ Für ein risikoscheues Management ist es gerade nicht zielführend, erhöhte Risikoübernahmen in unbegrenztem Maße durch höhere Erfolgschancen zu kompensieren. ${ }^{219}$ Im Hinblick auf die weitere Vorgehensweise dieser Arbeit kann festgehalten werden, dass das Safety-First-Prinzip unmittelbar an die in Kapitel 2.3 vorgestellten Überlegungen zur Funktion und Notwendigkeit von Risikokapital anknüpft und somit ,eine tragfähige Basis zur Konzeptionalisierung und Umsetzung einer erfolgsorientierten Steuerung von Versicherungsunternehmen ${ }^{، 220}$ bietet.

\subsubsection{Notwendigkeit einer integrierten Rendite-Risikosteuerung}

Neben dem Primat des Sicherheitsgedankens wurde im vorangegangenen Abschnitt herausgearbeitet, dass die Beurteilung geschäftspolitischer Entscheidungen stets unter der Fragestellung erfolgen sollte, inwiefern diese zur Erzielung eines angemessenen Gewinns bzw. einer adäquaten Rentabilität des Unternehmens beitragen. ${ }^{221} \mathrm{Da}$ unternehmerische Entscheidungen jedoch in der Regel unter Unsicherheit getroffen werden, müssen im Sinne einer ertragsorientierten Risikopolitik stets die Rentabilitätspotenziale und die korrespondierenden Risiken aufeinander abgestimmt werden. Ziel dieses Abstimmungsprozesses ist es, diejenigen Entscheidungsalternativen zu identifizieren, welche eine lohnenswerte Rendite-Risiko-Relation aufweisen. Im Rahmen dieses Risiko-Chancen-Kalküls stellt die verfügbare Risikodeckungsmasse eines Unternehmens jedoch einen limitierenden Faktor dar. ${ }^{222}$ Selbst wenn der erwartete Gewinn einer geplanten Geschäftspolitik die Übernahme zusätzlicher Risiken als vorteilhaft erscheinen lässt, muss ergänzend geprüft werden, ob das Unternehmen überhaupt die notwendige Risikotragfähigkeit besitzt, um diese Risiken einzugehen. Dem Grundsatz der Risikotragfähigkeit kommt gerade im versicherungsspezifischen Kontext elementare Bedeutung zu, da aufgrund des Safety-First-Prinzips die Einhaltung eines bestimmten Min-

218 Vgl. im Folgenden auch SCHRADIN (1994), S. 67-76, ALBRECHT (1994c) sowie die Ausführungen in Kapitel 4.1.1. Solange die Sicherheitsrestriktion gemäß Beziehung (3.6) nicht bindet, ist das Safety-First-Konzept hingegen ohne weiteres mit dem BERNOULLI-Konzept in Einklang zu bringen.

219 Bezüglich grundlegender, nicht versicherungsspezifischer Kritik am BERNOULLI-Prinzip (z.B. Messproblematik und fragliche Zeitstabilität des Präferenzfunktionals) vgl. BAMBERG/COENENBERG (2002), S. 81-118.

220 ALBRECHT (1994c), S. 15.

221 „Risikoübernahme darf kein Selbstzweck sein und muss dem Rentabilitätsdenken konsequent untergeordnet werden." SCHIERENBECK (1999), S. 718. Vgl. im Folgenden auch SCHIERENBECK (2003a), S. 1-3 und PAUL (2001), S. 74-78.

222 Vgl. übereinstimmend SCHRADIN (1998), S. 205, SCHRADIN/ZONS (2002), S. 3 und GRÜNDL/ SCHMEISER (2004b), S. 4. 
dest-Sicherheitsniveaus stets gewährleistet sein muss. Die Risikotragfähigkeit eines Versicherungsunternehmens ist sichergestellt, sofern folgende Bedingung erfüllt ist:

Gesamtrisikopotenzial $\leq$ verfügbare Risikodeckungsmassen

Risikodeckungsmassen bzw. Sicherheitskapital ${ }^{223}$ stellen in der Unternehmenspraxis regelmäßig einen knappen Faktor dar, den es möglichst effizient zu nutzen gilt. Die Anwendung des Tragfähigkeitskalküls kann zur Folge haben, dass auf den Abschluss lohnenswerter Geschäfte verzichtet werden muss, da die verfügbaren Risikodeckungspotentiale bereits durch Bestandsrisiken ausgelastet sind. ${ }^{224}$ Um eine Überschreitung der Tragfähigkeitspotenziale durch laufende Verluste zu verhindern, kann ein Risikolimitsystem implementiert werden, welches einzelnen Steuerungseinheiten explizit Risikokapital zuteilt und somit deren „Risk Taking Capacity“ auf ein vorgegebenes $\mathrm{Maß}$ begrenzt. ${ }^{225}$ Auf diese Weise soll sowohl das Verlustpotenzial der Teilbereiche als auch des Gesamtunternehmens wirksam begrenzt werden. Bei der Quantifizierung des Gesamtrisikopotenzials sind kollektive Risikoausgleichseffekte zu berücksichtigen. ${ }^{226} \mathrm{Da}$ die betrachteten Einzelrisiken in der Regel nicht vollständig positiv korreliert sind, wird das Risikopotenzial auf der Unternehmensebene (Gesamtrisikopotenzial) kleiner sein als die Summe der Einzelrisiken. Eine einfache Addition der für jede Steuerungseinheit isoliert ermittelten Risikopotenziale würde daher die tatsächliche Risikoposition des Gesamtunternehmens überschätzen. ${ }^{227}$

Die aufgezeigten Zusammenhänge machen die Notwendigkeit einer integrierten Rendite-Risikosteuerung deutlich. Um zu objektivierbaren Aussagen über die relative Ergebnisqualität von Geschäftsaktivitäten bzw. Strategien zu kommen, bedarf es operationaler Steuerungskennziffern, welche Ertrags- und Risikoaspekte geeignet miteinander verknüpfen. In diesem Zusammenhang kommt risikoadjustierten Performancemaßen (RAPM) eine zentrale Bedeutung zu. ${ }^{228}$ Diese stellen Kennzahlen zur Beurteilung der Zielerreichung unter Risiko dar. Sie beziehen ihre theoretische Fundierung aus der klassischen Portfoliotheorie und legen demzufolge auf ein lineares Austauschverhält-

${ }^{223}$ Die Risikodeckungsmasse ist inhaltlich den bereits bekannten Begriffen des (verfügbaren) Sicherheitskapitals bzw. Risikokapitals gleichzusetzen. Vgl. auch die Ausführungen in Kapitel 2.3.

224 Vgl. übereinstimmend PAUL (2001), S. 78.

225 Vgl. vertiefend zum bankspezifischen Aufbau eines Limitsystems LISTER (1997), S. 200-204 und SCHIERENBECK (2003b), S. 39-42.

226 Vgl. im Folgenden auch MEYER (1999), S. 388-397 und DRESEL (2003), S. 87-99.

$227 \mathrm{Vgl}$. in diesem Zusammenhang auch die Ausführungen in Kapitel 4.2.1.

${ }^{228}$ Einen Überblick über die verschiedenen Ausprägungen risikoadjustierter Performancemaße geben beispielsweise MATTEN (2000), S. 146-148 und LEHAR/WELT/WIESMAYR U.A. (1998a). 
nis von Rendite und Risiko zugrunde. ${ }^{229}$ Ausdrücklich sei an dieser Stelle darauf hingewiesen, dass Risiko im kapitalmarkttheoretischen Kontext stets als Volatilität, mithin als symmetrisches Risikomaß in das Kalkül eingeht. Überträgt man den kapitalmarkttheoretischen Grundgedanken auf die Unternehmenssteuerung, so quantifizieren RAPM-Kennziffern genau denjenigen Erfolgsbeitrag, der je eingesetzter Einheit an Risikodeckungsmasse erwirtschaftet wird. Im kapitalmarkttheoretischen Kontext entspräche dieser Erfolgsbeitrag gerade dem Marktpreis des Risikos, mithin der Steigung der so genannten Kapitalmarktlinie. ${ }^{230}$ Im Mittelpunkt der nachfolgenden Betrachtung steht die Kennziffer „Return On Risk Adjusted Capital“ (RORAC). Diese ist definiert als Relation zwischen erwartetem Gewinn und erforderlichem Risikokapital. ${ }^{231}$

$$
\text { RORAC }=\frac{E(G)}{\text { Risikokapital }}
$$

Allein auf der Basis des RORAC kann die Vorteilhaftigkeit einer Investition oder Strategie noch nicht abschließend beurteilt werden, da noch nicht ersichtlich ist, ob überhaupt eine Risikoprämie erwirtschaftet wird, welche dem Risikogehalt des zu beurteilenden Geschäfts angemessenen ist. Hierzu muss zusätzlich eine Mindestvorgabe (,hurdle rate") bekannt sein, beispielsweise in Form einer Renditeforderung der Anteilseigner, welche das Spektrum alternativer Verwendungsmöglichkeiten abbildet. ${ }^{232}$ Erst die resultierende Über- bzw. Unterrendite gibt letztlich Aufschluss über die Vorteilhaftigkeit der untersuchten Strategie. ${ }^{233}$ Eine Alternativenbeurteilung nach dem RORAC-Kriterium entspricht somit einer engpassspezifischen Optimierung der Risikokapitalverwendung. Der RORAC kann als relatives Vorteilhaftigkeitskriterium unter Risiko interpretiert werden.

Die Verwendung risikoadjustierter Performancemaße wird in der Literatur kontrovers diskutiert. ${ }^{234}$ Ein erster Kritikpunkt betrifft die grundlegende Annahme, dass zwischen erwartetem Gewinn und Risiko ein lineares Austauschverhältnis besteht. Ein identi-

Vgl. grundlegend zur kapitalmarkttheoretischen Fundierung risikoadjustierter Performancemaße LISTER (1997), S. 204-212, LEHAR/WELT/WIESMAYR U.A. (1998a) und LEHAR/WELT/WIESMAYR U.A. (1998b).

${ }^{230} \mathrm{Vgl}$. zum kapitalmarkttheoretischen Hintergrund auch STEINER/BRUNS (2002), S. 1-27.

${ }^{231}$ Die Ermittlung des erforderlichen Risikokapitalbedarfs ist Gegenstand von Kapitel 4.

232 Vgl. hierzu vertiefend SPELlMANN (2002), S. 40-41 und BAUMEISTER (2003), S. 224-225.

233 Demgegenüber wird bei der Berechnung des „Risk Adjusted Return On Capital“ (RAROC) die geforderte Mindestverzinsung unmittelbar als Risikoprämie in Abzug gebracht. Ein RAROC gröBer Null deutet somit unmittelbar auf eine Strategie hin, deren risikoadjustierte Rendite die Mindestvorgabe übersteigt. Vgl. hierzu auch SPELLMANN (2002), S. 42-44, LISTER (1997), S. 210-212 und BAUMEISTER (2003), S. 223-225.

${ }^{234}$ Vgl. im Folgenden auch GRÜNDL/SCHMEISER (2002), BAUMEISTER (2003) und ALBACH (2001). 
scher RORAC kann daher durch unterschiedliche Kombinationen von Zähler- und Nennergröße erreicht werden. Angesichts der zentralen Stellung des Sicherheitsziels bildet die Proportionalitätsannahme zwischen Gewinn und Risiko jedoch weder die Risikopräferenz der Anteilseigner noch die der Versicherungsnehmer angemessen ab. Alternativen mit unterschiedlicher Risikostruktur aber identischem RORAC stiften aus Anteilseignersicht nicht notwendigerweise den gleichen Nutzen. ${ }^{235}$ Der Berechnung risikoadjustierter Performancekennzahlen sollte daher im versicherungsspezifischen Kontext stets ein explizites Sicherheitsniveau zugrunde gelegt werden.

Ein weiterer zentraler Kritikpunkt betrifft den Sachverhalt, dass die Verwendung risikoadjustierter Performancemaße nur unter sehr restriktiven Prämissen mit einer postulierten Zielfunktion wie Maximierung des erwarteten Gewinns oder Marktwertmaximierung kompatibel ist. ${ }^{236}$ Eine Maximierung des RORAC (oder auch des RAROC) führt nur dann ebenfalls zu einer Maximierung des Kapitalwertes bzw. Unternehmenswertes, sofern Risikoneutralität vorliegt und das Risikokapital konstant gehalten wird. ${ }^{237}$ Die letztgenannte Anforderung kann jedoch gerade für Finanzdienstleistungsunternehmen als sehr restriktive Prämisse gewertet werden, da deren Risikomanagement-Mix gerade bei mehrjähriger Betrachtung immer wieder zu definierten Zeitpunkten an die aktuelle Risikosituation angepasst wird. ${ }^{238}$ Eine Veränderung des Risikomanagement-Mixes induziert jedoch unmittelbar einen veränderten Risikokapitalbedarf, beispielsweise wenn Risiken an den Rückversicherer oder den Kontrahenten eines aktivseitigen Sicherungsgeschäfts transferiert werden. Die Steuerung über risikoadjustierte Performancemaße stellt einen Spezialfall des Kapitalwertkalküls dar und führt nur dann zu übereinstimmenden Handlungsempfehlungen, sofern zusätzlich zur Annahme eines fixierten Risikokapitalbestands auch Risikoneutralität seitens der Anteilseigner vorausgesetzt wird. ${ }^{239}$ Die Vorgabe einer vom risikolosen Zins abweichenden „hurdle rate" birgt somit die Gefahr von Über- bzw. Unterinvestitionen und kann im Sinne des Kapitalwertkalküls zu suboptimalen Entscheidungen führen. Verlässt man die Steuerungsebene des Gesamtunternehmens und geht über auf eine dezentrale Bereichssteuerung, so ist die grundlegende Frage zu beantworten, auf welche Art und Weise den einzelnen Steuerungseinheiten Risikokapital zugeordnet werden soll. ${ }^{240}$ Die

${ }^{235}$ Vgl. auch BAUMEISTER (2003), S. 225. Dieser Sachverhalt wurde bereits im vorangegangenen Kapitel in Zusammenhang mit dem Rationalitätskriterium nach BERNOULLI kritisch diskutiert.

236 Vgl. übereinstimmend GRÜNDL/SCHMEISER (2002), ALBACH (2001) und FROOT/STEIN (1998).

237 Vgl. hierzu vertiefend GRÜNDL/SCHMEISER (2002), S. 806-808.

238 Vgl. auch GRÜNDL/SCHMEISER (2002), S. 801.

239 Vgl. im Folgenden insbesondere GRÜNDL/SCHMEISER (2002), S. 799-801.

${ }^{240}$ Ebenfalls erweist sich bereits eine eindeutige Gewinnzuweisung auf Geschäftsbereiche als nichttriviales Problem, da zwischen den betrachteten Bereichen oftmals Verbundeffekte existieren, die einer willkürfreien Quantifizierung nicht zugänglich sind. Vgl. hierzu auch GRÜNDL/SCHMEISER (2002), S. 801. 
unterschiedlichen Verfahren zur Kapitalallokation werden daher in Kapitel 4.2 im Überblick dargestellt. Ferner werden die in Versicherungspraxis und -theorie vielfach angeführten Gründe für eine Kapitalallokation einer kritischen Würdigung unterzogen.

Im weiteren Verlauf dieser Arbeit wird die Vorteilhaftigkeit unterschiedlicher Unternehmensstrategien durchgängig anhand des RORAC-Kriteriums beurteilt. Diese Vorgehensweise hat den Vorteil, dass sowohl die Rendite- als auch die Risikodimension einer Entscheidungssituation in einer einzigen, leicht nachvollziehbaren Kennzahl verdichtet werden und die Entscheidungssituationen entsprechend ihrer relativen Vorteilhaftigkeit in eine Rangfolge gebracht werden können. Da ein einperiodiger Modellhorizont zugrunde gelegt wird, erscheint die Annahme eines konstanten Risikokapitalbestandes und eines unveränderten Risikomanagement-Mixes zulässig. Beispielsweise ist es durchaus realistisch, dass der Rückversicherungsschutz bereits zu Beginn der Modellperiode abgeschlossen wird und im Jahresverlauf Gültigkeit behält. Dies vorausgeschickt, stellt der RORAC im Hinblick auf die bereits postulierte Zielfunktion („Maximierung des erwarteten Gewinns unter Einhaltung eines vorgegebenen Sicherheitsniveaus") ein adäquates Entscheidungskriterium dar.

\subsubsection{Risikomanagementprozess und Instrumente der Rendite-Risikosteuerung}

Versicherungsunternehmen agieren in einem Geschäftsumfeld, welches durch eine Vielzahl exogener und endogener Einflussfaktoren gekennzeichnet ist. ${ }^{241}$ Als exogene Faktoren können rechtliche und institutionelle Rahmenbedingungen, konjunkturelle und gesellschaftliche Entwicklungen sowie Veränderungen auf den relevanten Kapitalund Versicherungsmärkten angeführt werden. Im Hinblick auf die Steuerung von Versicherungsunternehmen stellen diese Faktoren wesentliche Nebenbedingungen dar, welche jedoch für alle Marktteilnehmer gleichermaßen gelten. Sie werden daher im weiteren Verlauf nicht explizit als Modellvariable in die Untersuchung einbezogen. Es wird vielmehr davon ausgegangen, dass das Modellunternehmen auf dem Kapital- und Rückversicherungsmarkt als Preisnehmer agiert. ${ }^{242}$ Unternehmensspezifische Entscheidungen und Dispositionen nehmen somit keinen nennenswerten Einfluss auf die Marktpreisbildung. Im Rahmen der Risiko- und Renditesteuerung kommt vielmehr denjenigen Steuerungsparametern besondere Bedeutung zu, die ein Versicherungsunternehmen unmittelbar beeinflussen kann.

${ }^{241}$ Vgl. zu den Rahmenbedingungen des Versicherungsgeschäfts grundlegend FARNY (2000), S. $97-$ 158 und HöLLER (1997), S. 43-44.

242 Vgl. zu dieser Annahme auch BROHM (2002), S. 162-163. 
Eine systematische Rendite-Risikosteuerung (kurz: Steuerung) setzt eine korrekte Identifikation und Bewertung der unternehmerischen Risiken voraus. ${ }^{243}$ Die identifizierten Risiken sollten nach Möglichkeit hinreichend quantifizierbar seien, um im Rahmen des bereits skizzierten Risiko-Chancen-Kalküls eine Risikoselektion zu ermöglichen. Die Risikobewertung ist darüber hinaus Ausgangspunkt für unterschiedliche Strategien der Risikobewältigung bzw. Risikosteuerung, welche nachfolgend diskutiert werden. Ein umfassendes Risikomanagement erfordert zudem eine kontinuierliche Kontrolle der durchgeführten Maßnahmen. Diese genannten Komponenten werden in der Literatur vielfach im Rahmen des Risikomanagementprozesses zusammengefasst. ${ }^{244}$ Dieser beinhaltet somit sämtliche Maßnahmen zur zielgerichteten Analyse, Steuerung und Kontrolle der Rendite-Risiko-Position. Das Risikomanagement sollte sich nicht auf zeitpunktbezogene Aktivitäten beschränken, sondern im Rahmen eines zyklischen Prozesses kontinuierlich ausgeführt werden. ${ }^{245}$ Durch die Realisierung konkreter Maßnahmen soll entweder eine bestehende Rendite-Risiko-Position aufrechterhalten oder eine bestimmte Zielposition erreicht werden. Die angestrebte Rendite-Risiko-Position wird wiederum unter Berücksichtigung der Unternehmensziele und des vorgegebenen Sicherheitsniveaus definiert und umfasst die Gesamtheit aller eingegangenen Risiken.

Hinsichtlich der Instrumente zur Rendite-Risikosteuerung kann grundlegend eine Differenzierung in ursachenbezogene Steuerungsinstrumente und wirkungsbezogene Steuerungsinstrumente vorgenommen werden. ${ }^{246}$ Eine ursachenbezogene Risikopolitik versucht, die Eintrittswahrscheinlichkeit negativer Zielverfehlungen zu reduzieren bzw. das Ausmaß der Zielverfehlungen bereits vor Eintritt der Risiken zu begrenzen. Durch Strategien der Risikovermeidung, Risikominderung oder Risikodiversifikation wird die Intention verfolgt, das bestehende Risikokollektiv strukturell zu verändern. ${ }^{247}$ In der Schaden- und Unfallversicherung sind in diesem Kontext insbesondere Strategien aus den Kernbereichen der Zeichnungs- und Kapitalanlagepolitik zu nennen.

Demgegenüber umfasst eine wirkungsbezogene Risikopolitik sämtliche Aktivitäten, welche auf die Begrenzung der Folgen bereits eingetretener Risiken abstellen. Die Struktur der zugrunde liegenden Originalrisiken, beispielsweise der Vertragsbestand

243 Risikoidentifikation und Risikobewertung werden in der Literatur häufig unter dem Begriff der Risikoanalyse zusammengefasst. Vgl. im Folgenden auch HÖLSCHER (2002), S. 12-16.

244 Vgl. übereinstimmend SCHULTE (1998), S. 13. Zu den einzelnen Phasen des Risikomanagementprozesses vgl. im Folgenden HÖlSCHER (2002), S. 12-16, SPELlMANN (2002), S. 28-30, REICHMANN (2001), S. 610, GABRIEL/ROCKEL (2001), S. 103 und SCHMEISER (2001), S. 141.

$245 \mathrm{Vgl}$. auch BITZ (2000), S. 25.

246 Vgl. vertiefend SPELLMANN (2002), S. 33-35 und FARNY (2000), S. 508-511.

247 Vgl. auch SpELLMANN (2002), S. 34 und SCHIERENBECK/LISTER (2002), S. 189. 
einer Versicherungssparte, bleibt im Gegensatz zur ursachenbezogenen Risikopolitik unverändert. Als wesentliche Strategien können der Risikotransfer und die Risikovorsorge genannt werden. Diese Strategien konkretisieren sich im Anwendungsbereich der Schaden- und Unfallversicherung wiederum in Form der Rückversicherungs-, Schadenregulierungs- und Reservierungspolitik. ${ }^{248}$

Abbildung 3.2 stellt den Versuch dar, das Entscheidungsfeld eines Schaden- und Unfallversicherers in Bezug auf die Rendite-Risikosteuerung zu systematisieren. Im weiteren Verlauf der Ausführungen werden die ursachen- und wirkungsbezogenen Risikosteuerungsstrategien hinsichtlich ihrer Anwendung in der Schaden- und Unfallversicherung konkretisiert.

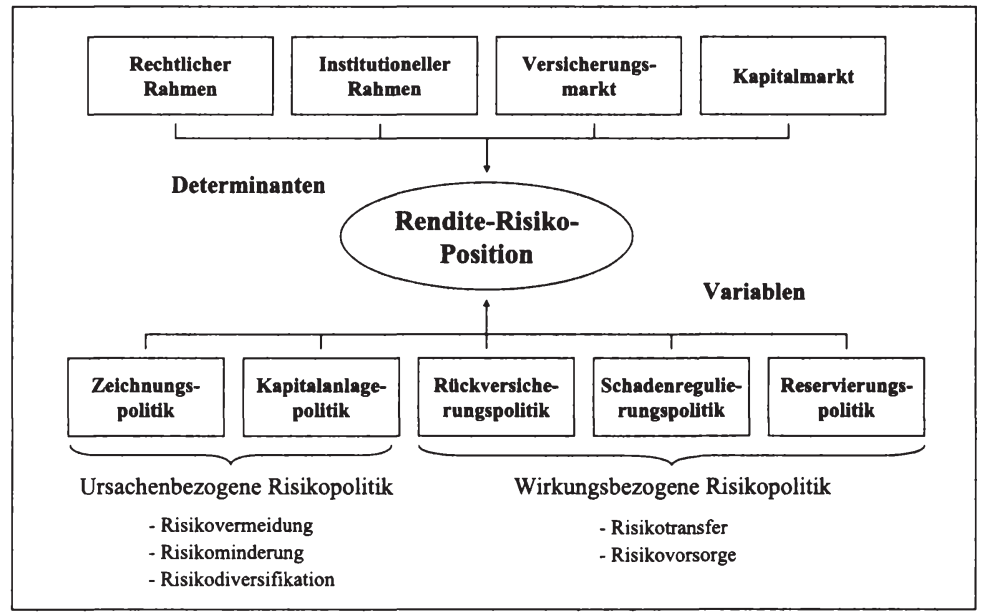

Abbildung 3.2: Determinanten und Variablen der Rendite-Risikosteuerung

Quelle: Erweiterte Darstellung in Anlehnung an HÖLSCHER (2002), S. 14.

Die Zeichnungspolitik stellt einen zentralen versicherungstechnischen Steuerungsparameter dar. Grundlegend kann in diesem Zusammenhang zwischen der Prämien- und Bestandspolitik unterschieden werden. ${ }^{249}$ Im Rahmen der Prämienpolitik versucht das Versicherungsunternehmen, einen über den Erwartungswert der Schäden hinausgehenden Sicherheitszuschlag zu ermitteln und diesen am Markt zu realisieren. ${ }^{250} \mathrm{Zum}$

248 Vgl. ebenfalls HÖLSCHER (2002), S. 15.

249 Vgl. im Folgenden auch FARNY (2000), S. 410-414.

${ }^{250}$ Von der (eher theoretischen) Möglichkeit, die Risikoprämie im Zuge eines Umlageverfahrens nachträglich an den Effektivwert der Schäden anzupassen, wird abstrahiert. Vgl. hierzu FARNY (2000), S. 410. Vgl. vertiefend zu den Bestimmungsgrößen eines unternehmenswertorientierten Versicherungspreises WAGNER/WARMUTH (2005), S. 15-45. 
Zwecke der Tarifierung können unterschiedliche Prämienprinzipien herangezogen werden. ${ }^{251}$ Insbesondere in langfristigen Vertragsbeziehungen stellen so genannte Prämienanpassungsklauseln ein wichtiges Instrument zur Begrenzung des Änderungsrisikos dar. Derartige vertragliche Regelungen sehen eine Anpassung der Risikoprämie an steigende oder auch sinkende Schadenerwartungswerte vor. Um lediglich systematische Änderungen und keine stochastischen Schwankungen zu erfassen, sind Anpassungsklauseln häufig an objektive Indikatoren wie beispielsweise Preisindizes gekoppelt. ${ }^{252}$ Die Bestandspolitik umfasst sämtliche Maßnahmen, die die Größe und Zusammensetzung des versicherungstechnischen Kollektivs beeinflussen und konkretisiert sich in den Bereichen der Produkt-, Programm- und Annahmepolitik. ${ }^{253}$ Es wird die Zielsetzung verfolgt, durch aktive Risikoselektion und Produktgestaltung diejenigen Geschäftsbereiche auszubauen, welche die Streuung der Gesamtschadenverteilung vermindern. ${ }^{254}$ Im Einzelfall können Verträge mit hohem Schadenerwartungswert oder ungünstiger Korrelation zum Gesamtportfolio abgelehnt bzw. gekündigt werden. Des Weiteren können Haftungslimite sowie explizite Sicherheitsvorschriften oder Schadenverhütungsmaßnahmen vereinbart werden, um das Streuungsverhalten der Schäden zu homogenisieren. Die Einführung von Schadenselbstbeteiligungen (Franchisen) nimmt eine Zwitterstellung zwischen Prämien- und Bestandspolitik ein. Franchisen bewirken sowohl eine nennenswerte Eindämmung des moralischen Risikos als auch eine substanzielle Entlastung des Schadenbedarfs. Allerdings wird ein Teil der Entlastungseffekts oftmals in Form von Prämiensenkungen an die Versicherungsnehmer zurückgegeben. ${ }^{255}$

Die Steuerung im Bereich der Kapitalanlagepolitik zielt primär auf die Sicherung der traditionellen Anlageziele Kapitalerhaltung, Rentabilität, Liquidierbarkeit und Diversifikation $a b .{ }^{256}$ Diese Ziele leiten sich wiederum aus betriebswirtschaftlichen Erfordernissen und aufsichtsrechtlichen Mindestvorgaben ab. Als mögliche Instrumente sind hier Verfahren der strategischen, taktischen und operativen Asset Allocation, portfoliotheoretische Optimierungskalküle, derivative Finanzinstrumente, aber auch Szena-

251 Vgl. ausführlich zu klassischen Prämienprinzipien LIPPE (1984), S. 133-156, SCHMIDT (2002), S. 239-269, MACK (2002), S. 121-220, HELBIG (2002), S. 85-88 und DOTTERWEICH (2004), S. 9199. Vgl. außerdem in diesem Zusammenhang die Ausführungen in Kapitel 5.4.2.

252 Vgl. vertiefend FARNY (2000), S. 512-514 und MARLOW (1999), S. 209-218.

253 Vgl. insbesondere SCHRADIN (1994), S. 329-433.

${ }^{254}$ Vgl. FARNY (2000), S. 413-414.

255 Vgl. hierzu vertiefend MACK (2002), S. 322-325 und die Ausführungen in Kapitel 6.2.4.1.

256 Vgl. auch Farny (2000), S. 812-819, GritZMANN (1998), S. 31-37 und SChWEbler (1991), S. 36-46. 
rioanalysen und Stresstests zu nennen. ${ }^{257}$ Darüber hinaus kommt einer integrierten Steuerung von versicherungstechnischem Portfolio und Kapitalanlageportfolio zentrale Bedeutung zu, da sich in der Schaden- und Unfallversicherung die Stochastizität des Schadengeschehens auch aktivisch fortsetzt und entsprechende Dispositionen des Kapitalanlagebereichs erfordert. ${ }^{258}$

Die Rückversicherung kann als elementare Form des versicherungstechnischen Risikotransfers bezeichnet werden. ${ }^{259}$ Das Versicherungsunternehmen zediert einen Teil der gezeichneten Originalrisiken an einen oder mehrere Rückversicherer. Der Entlastungseffekt der Rückversicherung wird wesentlich durch die Auswahl des Rückversicherungsvertrages bestimmt. In diesem Zusammenhang sind die Grundformen der proportionalen und nicht-proportionalen Rückversicherung zu unterscheiden. ${ }^{260}$ In Abhängigkeit von der gewählten Vertragsform sind mit der Rückversicherungsnahme eine spürbare Homogenisierung der Schaden- und Ergebnisentwicklung sowie gegebenenfalls eine Ausweitung der Zeichnungskapazität verbunden. Hochsummige bzw. stark exponierte Risiken, welche das Versicherungsunternehmen nicht in vollem Umfang im Selbstbehalt tragen könnte, werden teilweise auf den Rückversicherer übertragen. Dies verdeutlicht die zentrale Funktion der Rückversicherung als Risikoträger. ${ }^{261}$

Im Rahmen der Schadenregulierungspolitik konkretisiert sich das abstrakte Schutzversprechen des Versicherungsunternehmens gegenüber dem Versicherungsnehmer. Das Schadenmanagement wird daher vielfach als zentraler Leistungsbestandteil gegenüber Kunden und Geschädigten angesehen, welcher einen substanziellen Einfluss auf die Kundenzufriedenheit ausübt. ${ }^{262}$ Die bereits eingetretenen Versicherungsfälle eines Bestandes sind nur in begrenztem Maße einer betriebswirtschaftlichen Steuerung zugänglich, da die vertraglichen Regelungen zumeist die Höhe der Entschädigungsleistung präzise vorschreiben. Dennoch hat das Versicherungsunternehmen in eingeschränktem Umfang die Möglichkeit, durch „aktives Schadenmanagement“ die Höhe der ersatzpflichtigen Schäden zu beeinflussen und auf diese Weise Schadenkosten einzusparen. ${ }^{263}$ Durch eine besonders sorgfältige Schadenprüfung und Schadenregulierung

257 Einen Überblick über das anlagetechnische Risikoinstrumentarium vermitteln JOST (2000), S. 269295, GRITZMANN (1998), S. 62-323 und HöLLER (1997), S. 70-72. Vgl. zum Vergleich zwischen bank- und versicherungsspezifischen Instrumenten auch KRAMER (1991), S. 60-84.

${ }^{258} \mathrm{Vgl}$. vertiefend Kapitel 5.5.4.

259 Vgl. im Folgenden auch FARNY (2000), S. 414-422 und LiEBWEIN (2000), S. 21-50.

${ }^{260}$ Die wesentlichen Vertragsformen der Rückversicherung werden in Kapitel 5.4.5 vorgestellt.

261 Vgl. grundlegend zu den Funktionen der Rückversicherung LiEBWEIN (2000), S. 41-45, SCHENK (1995), S. 363-369 und ZWEIFEL/EISEN (2003), S. 206-207.

${ }^{262}$ Vgl. zur Schadenpolitik vertiefend JARA (2000), S. 5-8 und FARNY (2000), S. 412.

263 Vgl. im Folgenden auch FARNY (2000), S. 624-626. 
können unberechtigte Ansprüche erkannt und auf ein vertragsgemäßes Ausmaß reduziert werden. In diesem Zusammenhang ist jedoch der Einsparungseffekt gegen die erhöhten Regulierungskosten abzuwägen. Ferner kann das Versicherungsunternehmen den Versicherungsnehmer oder den Geschädigten bei der Schadenbehebung unterstützen, indem er beispielsweise eine Vorauswahl möglicher Dienstleister und Lieferanten vornimmt oder die Schadenbeseitigung überwacht. In bestimmten Versicherungssparten (z.B. Kraftfahrt) kann das Versicherungsunternehmen die Schadenbeseitigung auch selbst durch Kooperationspartner oder konzerneigene Unternehmen durchführen. ${ }^{264}$

Die Reservierungspolitik fungiert als Instrument der Risikovorsorge im Zeitverlauf. ${ }^{265}$ Durch den Aufbau bilanzieller Haftungsmasse in Form versicherungstechnischer Rückstellungen bleibt die Verteilung der Gesamtschadens unangetastet, jedoch kann das Versicherungsunternehmen Verluste aus eingetretenen Risiken kompensieren, indem beispielsweise Schadenrückstellungen (im aufsichtsrechtlich zulässigen Umfang) in Form von Abwicklungsgewinnen aufgelöst werden. Die Reservierungspolitik kann insofern als spezieller Anwendungsfall der Sicherheitsmittelpolitik interpretiert werden. ${ }^{266}$ Aus risikotheoretischer Perspektive stellt die Reservierungspolitik ein wichtiges Instrument zur Gestaltung des Risikoausgleichs in der Zeit dar. ${ }^{267}$

\subsubsection{Zentrale Struktursteuerung vs. dezentrale Marktbereichssteuerung}

Im Mittelpunkt einer integrierten Rendite-Risikosteuerung steht immer eine aktive und bewusste Risikoübernahme, welche einerseits an die Erzielung entsprechender Mindestrenditen und andererseits an die Wahrung eines zuvor definierten Sicherheitsniveaus gebunden ist. ${ }^{268} \mathrm{Um}$ eine in diesem Sinne effiziente Rendite-Risiko-Position zu erreichen, bedarf es eines adäquaten Steuerungsprinzips, welches die Einhaltung der Safety-First-Nebenbedingung zu jedem Zeitpunkt gewährleistet und gleichzeitig einer dezentralen Marktbereichssteuerung nicht im Wege steht. In Anlehnung an die Steuerung von Kreditinstituten erscheint es daher sinnvoll, eine zweidimensionale Vorgehensweise zu wählen, welche zwischen einer zentralen Struktursteuerung und einer dezentralen Marktbereichssteuerung differenziert. Dieses Konzept soll nachfolgend in seinen Grundzügen auf den Anwendungskontext der Schaden- und Unfallversicherung übertragen werden. ${ }^{269}$

\footnotetext{
264 Vgl. vertiefend FARNY (2000), S. 623-626.

265 Vgl. auch AlFERMANN/RADTKE/REICH (2004), S. 65-70.

${ }^{266}$ Vgl. zur Sicherheitsmittelpolitik auch FARNY (2000), S. 422-424 und WAGNER (1992), S. 94-97.

267 Vgl. FarNY (2000), S. 424-425 und SCHRADIN (1998), S. 130-134.

268 Vgl. übereinstimmend SPELLMANN (2002), S. 35.

${ }^{269} \mathrm{Vgl}$. im Folgenden grundlegend zur integrierten Rendite-Risikosteuerung im Kontext des „Dualen Steuerungsmodells“" SCHIERENBECK (2001), S. 87-101, SCHIERENBECK (2003a), S. 293-303 und SCHIERENBECK (2003b), S. 505-614.
} 
Der duale Steuerungsansatz basiert auf dem bereits durch SCHMALENBACH formulierten Gedanken der pretialen Lenkung ${ }^{270}$, nach dem dezentrale Steuerungseinheiten eine weitgehende Dispositionsfreiheit erhalten, gleichzeitig jedoch deren Entscheidungen durch ein Verrechnungskonzept bzw. durch adäquate Kontrollrechnungen im Hinblick auf die Zielsetzung der Gesamtunternehmung koordiniert werden. ${ }^{271}$ Die Trennung der Steuerungsbereiche basiert auf einer organisatorischen Zuordnung von Entscheidungskompetenzen und Verantwortlichkeiten. Die zentrale Struktursteuerung umfasst sämtliche gesamtgeschäftsbezogenen Problemkreise und Entscheidungstatbestände, die ausschließlich aus der übergeordneten Perspektive des Gesamtunternehmens beurteilt werden können bzw. nur aus einer Zentralkompetenz heraus verantwortbar sind. Die dezentrale Marktbereichssteuerung hingegen umfasst Sachverhalte und Fragestellungen, die mit der Steuerung von Einzelgeschäften in Zusammenhang stehen. Sie ermöglicht somit eine direkte Aufnahme und Verarbeitung von Marktimpulsen „,vor Ort". Abbildung 3.3 stellt den Versuch dar, beiden Steuerungsbereichen wesentliche versicherungsspezifische Entscheidungstatbestände zuzuordnen.

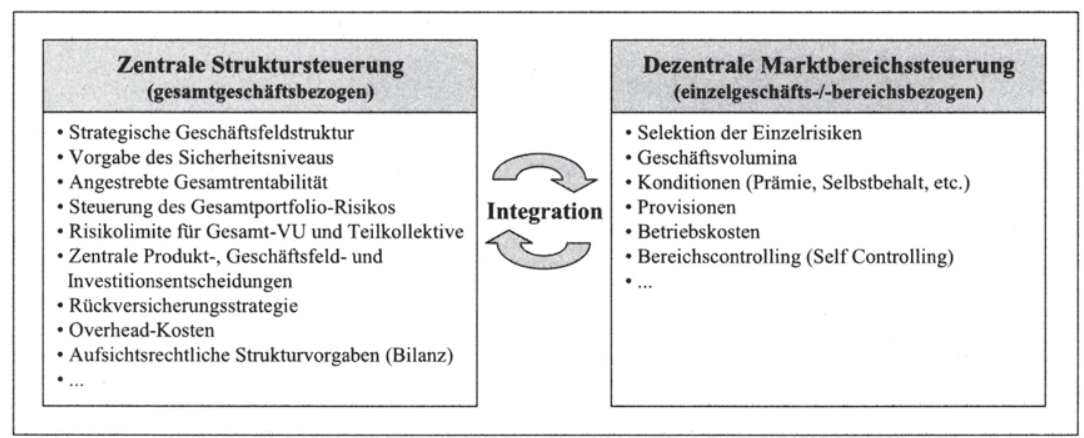

Abbildung 3.3: Struktursteuerung vs. Marktbereichssteuerung Quelle: in Anlehnung an SCHIERENBECK (2003a), S. 301.

Auf der Unternehmensebene besteht eine zentrale Aufgabe darin, die Entscheidungsbereiche der einzelnen Steuerungseinheiten zu fixieren und deren Bereichsziele auf das Unternehmensziel hin zu koordinieren. ${ }^{272}$ Wesentliche Voraussetzung für die Umsetzung eines derartigen Steuerungsmodells ist ein angemessenes und leistungsfähiges Führungsinformationssystem, welches eine eindeutige Abgrenzung bzw. Zurechnung von Ergebnisbeiträgen einzelner Steuerungseinheiten ermöglicht. Im Sinne des klassi-

\footnotetext{
270 Vgl. SChMalenBaCH (1909), S. 165-185.

271 Vgl. auch SPELLMANN (2002), S. 36 und SCHIERENBECK (2003a), S. 301.

272 Vgl. SCHIERENBECK (2001), S. 91-92.
} 
schen Kongruenzprinzips ${ }^{273}$ der Organisationslehre müssen den Steuerungseinheiten neben Ergebnisverantwortung, Akquisitions- und Betreuungsaufgaben auch die erforderlichen Entscheidungskompetenzen zugewiesen werden. ${ }^{274}$

Der Fokus der vorliegenden Arbeit liegt auf der Steuerung der strategischen RenditeRisikoposition eines Versicherungsunternehmens und ist demnach im Bereich der zentralen Struktursteuerung angesiedelt, welche im weiteren Verlauf ausschließlich betrachtet wird. Nachfolgend gilt es daher zu untersuchen, auf welche Weise die Risikokapitalsteuerung zur Erreichung einer effizienten Rendite-Risiko-Position eingesetzt werden sollte. $\mathrm{Zu}$ diesem Zweck wird grundlegend zwischen einer Steuerungsebene und einer Bilanzebene differenziert. ${ }^{275}$ Dieser Unterscheidung liegt die Überlegung zugrunde, dass ein Versicherungsunternehmen in der Lage ist, seine Rendite-RisikoPosition durch die Variation zentraler Risikoparameter und die Steuerung seines Risikomanagement-Mixes zu beeinflussen.

Wie im vorangegangenen Kapitel beschrieben, können diese Parameter im Wesentlichen dem risikopolitischen Instrumentarium der Zeichnungs-, Rückversicherungs- und Kapitalanlagepolitik zugeordnet werden. ${ }^{276}$ Die Bilanzsicht leitet sich aus der ökonomischen Risikosicht ab und stellt eine „strenge Nebenbedingung“ für die Steuerung, jedoch keinen Bestandteil der Zielfunktion dar. Konsequenterweise sollte daher eine Quantifizierung und Beurteilung der Rendite-Risiko-Position ausschließlich auf der Steuerungsebene angesiedelt sein. ${ }^{277}$ In einem weiteren Schritt ist dann zu überprüfen, inwiefern die ergriffenen Steuerungsmaßnahmen und Geschäftsstrategien der bilanziellen bzw. aufsichtsrechtlichen Nebenbedingung genügen und insofern „zulässige“ Handlungsalternativen darstellen.

${ }^{273}$ Der einschlägigen Literatur zur Organisationslehre folgend, soll unter dem Kongruenzprinzip die Übereinstimmung zwischen Aufgabe, Kompetenz und Verantwortung einer Steuerungseinheit verstanden werden. Vgl. vertiefend BÜHNER (2004), S. 63.

274 Vgl. WÖHLE (2003), S. 84.

275 Im analogen Kontext differenzieren BROHM/KÖNIG zwischen einer „Risikosicht“ und einer „Bilanzsicht“". Vgl. BROHM/KÖNIG (2004), S. 10-11.

${ }^{276}$ Aufgrund der in der Regel sehr fallspezifischen Entscheidungstatbestände entzieht sich das Schadenmanagement weitgehend einer angemessenen quantitativen Modellierung. Die Schadenregulierungspolitik wird daher im weiteren Untersuchungsverlauf nicht mehr betrachtet.

277 SPELLMANN formuliert in diesem Kontext zutreffend: „Entscheidungsbasis für alle Geschäftsaktivitäten [...] ist die Rendite-Risiko-Position. Grundlage der Risikoquantifizierung können damit nicht handelsrechtliche Bewertungsvorschriften sein.“ SPELLMANN (2002), S. 30. 
Das nachfolgende Hauptkapitel 4 stellt den zentralen Steuerungsparameter Risikokapital, mithin die Nennergröße des RORAC-Kriteriums in den Mittelpunkt des Interesses. In Kapitel 4.1 werden zunächst ausgewählte Konzepte zur Risikomessung vorgestellt und einer kritischen Würdigung unterzogen. Hierauf aufbauend wird in Kapitel 4.2 die Fragestellung diskutiert, unter welchen Voraussetzungen und zu welchem Zweck eine Zuweisung (Allokation) von Risikokapital auf einzelne Geschäftsbereiche zielführend erscheint. Schließlich werden vier grundlegende Allokationsmethoden erläutert und anhand eines Kriterienkatalogs miteinander verglichen. 


\section{Steuerung über Risikokapital in der Schaden- und Unfallversicherung}

\subsection{Diskussion ausgewählter Konzepte zur Risikomessung}

\subsubsection{Klassische Risikomaße}

Die zentrale Aufgabe von Risikomaßen besteht darin, unsichere Handlungsalternativen hinsichtlich ihres Risikogehaltes zu bewerten und in eine Rangordnung zu bringen. ${ }^{278}$ Als klassische Risikomaße werden diejenigen Messkonzepte zusammengefasst, welche bestimmte Verteilungsmomente zur Quantifizierung des Risikos heranziehen. Im einfachsten Fall wird lediglich der Erwartungswert einer Verteilung als Entscheidungskriterium herangezogen. Aussagen über den Risikogehalt der zugrunde liegenden Finanzposition oder Handlungsalternative lassen sich auf dieser Basis jedoch nur bedingt ableiten. ${ }^{279}$ Volatilitätsbasierte Maße wie die Standardabweichung und Varianz quantifizieren demgegenüber das Ausmaß der Streuung der möglichen Realisationen um den Erwartungswert und beziehen sich demnach auf die gesamte Verteilung. Sie legen ein Risikoverständnis zugrunde, welches sowohl auf positive als auch negative Abweichungen vom Erwartungswert abstellt. ${ }^{280}$ Auf diese Weise wird der Erwartungswert gewissermaßen als Zielgröße unterstellt. Die Unsicherheit besteht darin, dass eben diese Zielgröße verfehlt wird. „Damit wird das fast sichere Ereignis, nämlich die Abweichung der Realisation von ihrem Erwartungswert, zum relevanten Risiko erklärt. “281 Ein symmetrisches Risikoverständnis führt gerade im versicherungsspezifischen Kontext dazu, dass sich interne und externe Mindestkapitalanforderungen einer aktiven Steuerung entziehen und lediglich in Form einer strengen Nebenbedingung in das Kalkül eingehen können. ${ }^{282}$ Über diesen Einwand hinaus ist zu beachten, dass die Annahme einer symmetrischen Schadenverteilung (z.B. der multivariaten Normalverteilung) gerade in der Versicherungswirtschaft oftmals nur eine grobe Approximation des stochastischen Schadengeschehens darstellt. Dieser Kritikpunkt wird in der praktischen Anwendung auch nicht durch die Gültigkeit des Zentralen Grenzwertsatzes vollständig entkräftet, da dieser lediglich besagt, dass der Gesamtschaden (verstanden als standardisierte Summe einer großen Zahl von unabhängigen und reellwertigen Zufallsvariablen) durch die Normalverteilung approximiert werden kann. ${ }^{283}$ Sofern jedoch Schadenverteilungen einzelner Teilkollektive (z.B. Versicherungssparten) betrachtet werden, kann nicht mehr davon ausgegangen werden, dass die model-

\footnotetext{
278 Vgl. übereinstimmend KORYCIORZ (2004), S. 40.

279 Vgl. auch PFINGSTEN/HOMÖLLE/RIESO (2001), S. 1870.

${ }^{280}$ Volatilitätsmaße werden daher auch als symmetrische Risikomaße bezeichnet. Sie legen implizit einen weit gefassten Risikobegriff zugrunde. Vgl. in diesem Zusammenhang auch Kapitel 2.2.1.

${ }^{281}$ SCHRADIN (1998), S. 106.

282 Vgl. ebenso SCHRAdin (1998), S. 106 und PfingSten/Homölle/RIESO (2001), S. 1871.

283 Vgl. auch MACK (2002), S. 111. Zum zentralen Grenzwertsatz vgl. ALBRECHT (1982), S. 501-538.
} 
lierten Vertragsbestände eine hinreichende Kollektivstärke aufweisen. Gerade großschadenanfällige Versicherungszweige sind vielfach durch rechtsschiefe Schadenverteilungen charakterisiert. ${ }^{284}$ Darüber hinaus ist als generelle Problematik parametrischer Verteilungen anzuführen, dass die Verteilungsparameter im Zeitablauf als stabil unterstellt werden ${ }^{285}$ Im Hinblick auf die Verwendung in einem internen Steuerungsmodell bedeutet dies, dass eine sehr lange Datenhistorie zur Verfügung stehen muss, um hinreichend valide Verteilungsparameter abschätzen zu können.

Um der Kritik an der Normalverteilungsannahme zu begegnen und das bestehende Risikopotenzial präziser abschätzen zu können, kann auf statistische Maße höherer Ordnung zurückgegriffen werden. Beispielsweise kann der Schiefekoeffizient $\gamma$ zur Beurteilung asymmetrischer Verteilungen herangezogen werden. Dieser leitet sich aus dem dritten Zentralmoment $\mu_{3}$ ab und ist nach FISHER definiert als: $:^{286}$

$$
\gamma=\frac{\mu_{3}}{\mu_{2}^{3 / 2}}=\frac{\mu_{3}}{\sigma^{3}}
$$

Nimmt der Schiefekoeffizient einen positiven Wert an, so liegt die Mehrzahl der Realisationen unterhalb des Erwartungswertes. ${ }^{287}$ Die Verteilung wird in diesem Fall als rechtsschief bezeichnet. ${ }^{288}$ Für symmetrische Verteilungen nimmt der Schiefekoeffizient den Wert Null an. Die Wölbung (Kurtosis) leitet sich aus dem vierten Zentralmoment $\mu_{4}$ ab und gibt Auskunft über die „Steilheit“ einer Verteilung. Formal ist die Wölbung W definiert als: ${ }^{289}$

$$
\mathrm{W}=\frac{\mu_{4}}{\left(\mu_{2}\right)^{2}}-3
$$

\footnotetext{
284 Vgl. im Folgenden auch LIPPE (1983), S. 50 und ALBRECHT (1992), S. 10. MACK bemerkt hierzu treffend: „Auch die aus dem zentralen Grenzwertsatz abgeleitete Hoffnung, die Verteilung von $\mathrm{S}$ [= Gesamtschaden, Anm. d. Verf.] könne bei wachsendem $\mathrm{E}(\mathrm{N})$ [= erwartete Vertragsanzahl, Anm. d. Verf.] gegen eine Normalverteilung konvergieren, führt nicht zum Ziel, denn die Erfahrung hat gezeigt, dass selbst große Portefeuilles noch eine beträchtliche Schiefe aufweisen, so dass die Normalverteilungsapproximation die Wahrscheinlichkeit für das Eintreten von hohen Gesamtschäden erheblich unterschätzt.“ MACK (2002), S. 111.

${ }^{285} \mathrm{Vgl}$. im Folgenden SPELLMANN (2002), S. 46. Darüber hinaus ist anzumerken, dass das statistische Datenmaterial in nahezu keinem Zeitpunkt eine vollständige Beschreibung der wahren Zufallsgesetzmäßigkeit erlaubt. Folglich ist die statistische Inferenz stets mit Fehlern behaftet. Vgl. KORYCIORZ (2004), S. 14.

286 Vgl. vertiefend zur Definition der Zentralmomente NEUBAUER (1994), S. 98-100.

${ }^{287}$ Modus und Median der Verteilung sind demnach kleiner als der Erwartungswert.

288 Vgl. zum Schiefekoeffizienten auch NEUBAUER (1994), S. 93-100.

289 Vgl. zum Wölbungsmaß im Folgenden NEUBAUER (1994), S. 100-108.
} 
Verteilungen mit einer positiven Wölbung sind schmalgipfliger als die Normalverteilung. Es liegt demzufolge mehr Wahrscheinlichkeitsmasse an den Verteilungsenden. Verteilungen mit negativer Wölbung weisen im Vergleich zur Normalverteilung eine plateauförmige Gestalt auf. Sowohl Schiefe als auch Wölbung sind invariant gegenüber linearen Transformationen der zugrunde liegenden Zufallsvariablen. Jedoch sind beide Risikomaße aufgrund der hohen Exponenten sehr anfällig gegenüber Ausreißerwerten und entziehen sich weitgehend einer intuitiven ökonomischen Interpretation. ${ }^{290}$

Das BERNOULLI-Prinzip hat sich in der normativen Entscheidungstheorie unter Risiko als Alternative zu den genannten parametrischen Risikomaßen etabliert. ${ }^{291}$ Es bewertet und ordnet die Handlungsalternativen anhand ihres erwarteten Nutzens für den Entscheidungsträger („Erwartungsnutzentheorie“). Ausgehend von der Annahme, dass Nutzen kardinal messbar ist, wird die subjektive Risikopräferenz des Entscheidenden in einer übergeordneten Risikonutzenfunktion berücksichtigt, welche jedem Ergebniswert einer Alternative einen Nutzenwert zuordnet. ${ }^{292}$ Anschließend werden die Nutzenwerte mit ihren Eintrittswahrscheinlichkeiten gewichtet und zum Erwartungswert des Nutzens einer Alternative summiert. Die Erwartungsnutzentheorie ermöglicht rationale Entscheidungen unter Unsicherheit, sofern fünf Strukturanforderungen, die so genannten „Axiome rationalen Verhaltens“ erfüllt sind. ${ }^{293}$ Insbesondere das Stetigkeitsaxiom und das Substitutionsaxiom unterliegen jedoch im Hinblick auf ihre empirische und entscheidungslogische Gültigkeit der Kritik. ${ }^{294}$ In der praktischen Anwendung besteht die zentrale Problematik darin, die individuelle Risikopräferenz des Entscheidungsträgers in einer Nutzenfunktion zu quantifizieren, denn die Risikoneigung hängt in der Regel von der konkreten Handlungsalternative und den begrenzenden, in der Regel situativen und zeitlich instabilen Umweltbedingungen ab. Es wurde bereits in Kapitel 3.2.1 darauf hingewiesen, dass das BERNOULLI-Prinzip im versicherungsspezifischen Kontext nicht mit der Safety-First-Nebenbedingung kompatibel ist, da Risiko und Gewinn nicht mehr in beliebigem Ausmaß substituierbar sind.

${ }^{290}$ Vgl. übereinstimmend SPELlMANN (2002), S. 47. Vgl. zu weiterer Kritik auch AlBRECHT (2003), S. $20-22$.

291 Vgl. zum BERNOULli-Prinzip im Folgenden NEUMANN/MORGENSTERN (1973), ADAM (1996), S. 241-250, BAMBERG/COENENBERG (2002), S. 81-118 und BERENS/DELFMANN/SCHMITTING (2004), S. 76-83.

292 Vgl. auch PERridon/STEINER (2004), S. 113-119.

293 Vgl. grundlegend zur Axiomatik des BERNOULli-Prinzips NEUMANN/MORGENSTERN (1973) im dortigen Anhang. Vgl. darüber hinaus vertiefend BERENS/DELFMANN/SCHMITTING (2004), S. 77, BAMBerg/COENENBERg (2002), S. 100-102, PERRIDON/STEINER (2004), S. 116-117 und ADAM (1996), S. 245-250.

294 Vgl. in diesem Zusammenhang ADAM (1996), S. 248-249 sowie ALBRECHT (1994c), S. 10 und die dort angegebene Literatur. 
Die vorgestellten klassischen Risikomaße sind offenbar nicht dazu geeignet, das in Kapitel 2.2.1 definierte (asymmetrische) Risikoverständnis angemessen abzubilden. Darüber hinaus ist kritisch zu bewerten, dass stets die gesamte Verteilung einer $\mathrm{Zu}$ fallsvariable zur Risikoabschätzung herangezogen wird. Dies bedingt in der Regel sehr komplexe Berechnungsalgorithmen, selbst wenn die Anzahl der zu bewertenden Handlungsalternativen bereits ex-ante durch Dominanzüberlegungen ${ }^{295}$ reduziert werden kann. Shortfall-Risikomaße basieren hingegen auf einem asymmetrischen Risikoverständnis und reduzieren die Komplexität der Berechnung dadurch, dass sie lediglich den Risiko tragenden Rand einer Verteilung betrachten. ${ }^{296}$

Um geeignete von weniger geeigneten Risikomaßen abgrenzen zu können, hat sich in der einschlägigen Literatur ein Katalog „wünschenswerter Eigenschaften“ etabliert, welcher im nachfolgenden Abschnitt im Überblick skizziert wird. In Kapitel 4.1.3 werden schließlich ausgewählte Shortfallmaße vorgestellt und anhand dieses Kriterienkatalogs einer kritischen Würdigung unterzogen.

\subsubsection{Wünschenswerte Eigenschaften von Risikomaßen}

Grundsätzlich sollte sich die Auswahl eines Risikomaßes an dem zugrunde liegenden Risikoverständnis orientieren und dem postulierten Modellzweck angemessen sein. ${ }^{297}$ Neben einer angemessenen entscheidungstheoretischen Fundierung sollte ein Risiko$\mathrm{ma} ß$ darüber hinaus entweder auf numerische Weise oder sogar in analytischer Form quantifizierbar sein. Um zu gewährleisten, dass die Ergebnisse und Implikationen quantitativer Risikomodelle in der Praxis an Akzeptanz und Relevanz gewinnen, sollte das gewählte Risikomaß zudem einer ökonomischen Interpretation zugänglich sein. ${ }^{298}$ Zwischen den genannten Anforderungen existiert jedoch offensichtlich ein Zielkonflikt. „So konkurrieren typischerweise das Postulat der Praktikabilität mit dem einer möglichst engen Problemorientierung der Modellkonstruktion und Risikodefinition. “299 Neben diesen eher praktisch geprägten Anforderungen sind jedoch auch formale Eignungskriterien erforderlich, um „gute“ und „schlechte“ Risikomaße voneinander abgrenzen zu können. ${ }^{300}$ In der Literatur ist in diesem Zusammenhang das Axiomensystem von ARTZNER/DELBAEN/EBER/HEATH auf breite Akzeptanz gestoßen, welches

\footnotetext{
295 Vgl. zum Prinzip der stochastischen Dominanz auch BAWA (1982), GUTHOFF/PFINGSTEN/WoLF (1998), S. 115-118, MAURER (2000), S. 21-27, BAMBERG/COENENBERG (2002), S. 112-116.

$296 \mathrm{Vgl}$. SPELLMANN (2002), S. 50-51.

297 Vgl. im Folgenden SCHRADIN (1998), S. 105.

298 Vgl. auch MAURER (2000), S. 41-45.

299 SCHRADIN (1998), S. 105.

${ }^{300}$ Für eine komprimierte Darstellung und Beurteilung verschiedener Axiomensysteme vgl. im Folgenden auch MAURER (2000), S. 45-50 und ALBRECHT (2003), S. 11-18.
} 
speziell für die Bestimmung der Kapitalausstattung von Finanzdienstleistungsinstituten entwickelt wurde. ${ }^{301}$ Demnach sollte ein Risikomaß $\mathrm{R}$ über folgende vier konstituierende Eigenschaften verfügen. ${ }^{302}$

(ADEH 1) Translationsinvarianz

(ADEH 2) Positive Homogenität

(ADEH 3) Monotonie

(ADEH 4) Subadditivität

$$
\begin{array}{ll}
R(V+b)=R(V)+b & \text { mit } b \in R \\
R(\lambda \cdot V)=\lambda \cdot R(V) & \text { für alle } \lambda \geq 0 \\
R(V) \geq R(W) & \text { für alle } V \geq W \\
R\left(V_{1}+V_{2}\right) \leq R\left(V_{1}\right)+R\left(V_{2}\right) & \text { für alle } V_{1}, V_{2}
\end{array}
$$

In einem weit gefassten Begriffsverständnis wird unter dem Risikomaß $R: V \rightarrow R(V)$ zunächst eine Funktion verstanden, welche die Zufallsvariable V auf die Menge der reellen Zahlen abbildet. Risikomaße, welche allen vier Axiomen genügen, werden als kohärent bezeichnet. Es gilt zu beachten, dass die Zufallsvariable V in der hier verwendeten Notation eine Verlustgröße bezeichnet, demnach $V=-G$ gilt. Im Vergleich zu der zitierten Literatur resultieren hieraus partielle Unterschiede in der formalen Darstellung der Axiome. ${ }^{303}$

Das Axiom der Translationsinvarianz fordert, dass die Erhöhung der zufallsabhängigen Verlustvariablen $\mathrm{V}$ um den sicheren Betrag b gleichzeitig eine Zunahme des Risikoausmaßes in Höhe von $b$ induziert. Eine negative Ausprägung von $b$, beispielsweise in Form einer deterministischen Prämienerhöhung oder Kapitalzuführung, führt hingegen im Umkehrschluss zu einer Reduzierung des Risikos in gleicher Höhe. Aus dem Axiom der Translationsinvarianz folgt daher unmittelbar die Beziehung $\mathrm{R}(\mathrm{V}-\mathrm{R}(\mathrm{V}))=0 .{ }^{304}$

Die Homogenitätseigenschaft postuliert, dass sich der Risikogehalt einer risikobehafteten Finanzposition proportional zum Volumen der Position verhält. Anschaulich kann dies am Beispiel der Quotenrückversicherung verdeutlicht werden. Aus der Sicht des Rückversicherers kann der Faktor $\lambda(0 \leq \lambda \leq 1)$ dann als derjenige Prozentsatz inter-

\footnotetext{
301 Vgl. im Folgenden grundlegend ARTZNER/DELBAEN/EBER/HEATH (1997) und ARTZNER/DELBAEN/EBER/HEATH (1999). Für eine mehrperiodige Darstellung vgl. auch ARTZNER/DELBAEN/ EBER/HEATH (2002).

${ }^{302}$ Für den in den Originalquellen anzutreffenden risikolosen Aufzinsungsfaktor gelte nachfolgend $r=1$. Zinseffekte werden demnach vernachlässigt. Vgl. zu einer analogen Vorgehensweise KORYCIORZ (2004), S. 41 und ALBRECHT (2003), S. 13.

${ }^{303}$ Die hier gewählte Darstellung orientiert sich im Folgenden an KORYCIORZ (2004), S. 40-47.

${ }^{304}$ Vgl. vertiefend Koryciorz (2004), S. 42 sowie ARTZNER/DELBAEN/EBER/HEATH (1997), S. 69, DELBAEN (2002), S. 5 und ALBRECHT (2003), S. 13.
} 
pretiert werden, zu dem er an sämtlichen durch den Erstversicherer gezeichneten Risiken eines (Teil-) Bestands partizipiert. ${ }^{305}$

Die Monotonieeigenschaft besagt, dass ein sinnvolles Risikomaß die Zufallsvariable V nicht als weniger risikobehaftet klassifizieren darf, sofern sie für jeden Umweltzustand einen Wert annimmt, der mindestens so groß ist wie der korrespondierende Wert einer Zufallsvariablen W. Für den Fall, dass die Verlustvariable V ausschließlich negative Ausprägungen aufweist, mithin durchgängig Gewinne erwirtschaftet werden, nimmt das Risikomaß R einen negativen Wert an. Dieser Effekt ergibt sich aus der kombinierten Betrachtung der Axiome 2 und 3. ${ }^{306}$

Das Axiom der Subadditivität weist einen unmittelbaren Bezug zum so genannten Ausgleich-im-Kollektiv-Effekt auf, welcher im Kontext der Schaden- und Unfallversicherung von zentraler Bedeutung ist. ${ }^{307}$ Demnach darf das Risiko einer Gesamtposition, welche sich aus vielen Einzelrisiken zusammensetzt, nicht größer sein als die Summe der Einzelrisiken. Das Axiom der Subadditivität fordert somit, dass ein Risikomaß in der Lage sein muss, Diversifikationseffekte adäquat abzubilden. Da gerade im Anwendungskontext der Schaden- und Unfallversicherung sowohl im Kapitalanlagebestand als auch im versicherungstechnischen Portfolio erhebliche Risikoausgleichseffekte auftreten können, wird der Subadditivitätseigenschaft in der Literatur außerordentliche Bedeutung beigemessen. ${ }^{308}$ In Verbindung mit der Forderung nach positiver Homogenität kann ferner nachgewiesen werden, dass kohärente Risikomaße ebenfalls konvex sind. ${ }^{309}$

Abschließend ist einschränkend anzumerken, dass nicht jedes Risikomaß, welches dem beschriebenen Axiomensystem genügt, auch gleichzeitig ein sinnvolles Risikomaß bzw. Steuerungskriterium darstellen muss. Es kann gezeigt werden, dass insbesondere die Risikomaße $\mathrm{R}(\mathrm{V})=\mathrm{E}(\mathrm{V})$ und $\mathrm{R}(\mathrm{V})=\max (\mathrm{V})$, mithin der erwartete Verlust und der Maximalverlust, die Eignungskriterien kohärenter Risikomaße erfüllen. ${ }^{310}$ Es ist jedoch unmittelbar einsichtig, dass beispielsweise der Maximalverlust im Rah-

${ }^{305}$ Vgl. übereinstimmend KORYCIORZ (2004), S. 42-43, insbesondere dort die Ausführungen in Fußnote 148. Zur detaillierten Erläuterung der Quotenrückversicherung vgl. Kapitel 5.4.5.2.1.

306 Vgl. zur mathematischen Fundierung ARTZNER/DelbaEN/EBER/HEATH (1997), S. 71, DelbaeN (2002), S. 4, ACERBI/TASCHE (2002b), S. 381 und FISCHER (2003), S. 136 ff.

$307 \mathrm{Vgl}$. hierzu auch die Ausführungen in Kapitel 4.2.1.

308 Vgl. exemplarisch ARTZNER/DELBAEN/EbER/HEATH (1999), S. 209 und ACERBI/TASCHE (2002b), S. 382.

309 Vgl. zum Begriff der Konvexität auch KORYCIORZ (2004), S. 273-274 und PfLUG (2000), S. 275.

${ }^{310} \mathrm{Vgl}$. zu weiterer grundlegender Kritik an kohärenten Risikomaßen GOOVAERTS/DHAENE/KAAS (2001), GoOVAERTS/KAAS/DHAENE (2002) und DHAENE/VANDUFFEL/TANG U.A. (2004). 
men der Tarifierung kein sinnvolles Risikomaß oder Entscheidungskriterium darstellt. „Dies zeigt, dass zwar gegebenenfalls die Kohärenzbedingungen ,vernünftige' Anforderungen an Risikomaße stellen, aber nicht jedes kohärente Risikomaß auch ein ,vernünftiges’ Risikomaß ist.“311

\subsubsection{Shortfall-Risikomaße}

\subsubsection{Lower Partial Moments}

Im Gegensatz zu den skizzierten klassischen Risikomaßen basieren ShortfallRisikomaße auf einem asymmetrischen Risikoverständnis. ${ }^{312}$ Die Güte der Risikoeinschätzung ist daher nicht an die Existenz einer symmetrischen Verteilungscharakteristik (z.B. einer Normalverteilung) gebunden. Shortfall-Risikomaße quantifizieren die Gefahr einer negativen Abweichung von einem beliebig wählbaren Zielwert und fokussieren auf diese Weise den Risiko tragenden Randbereich einer Verteilung. Positive Abweichungen vom Zielwert bleiben hingegen unberücksichtigt, da sie als wünschenswert eingestuft werden. Eine allgemeine Klasse von Shortfall-Risikomaßen sind die Lower Partial Moments (LPM) vom Grad $n \geq 0$. Diese sind in allgemeiner Form definiert als das n-te Moment der Abweichung der zufallsabhängigen Gewinngröße $G$ vom Zielwert z. Im Fall stetiger Verteilungen gilt: ${ }^{313}$

$$
\operatorname{LPM}_{\mathrm{n}}(\mathrm{G}, \mathrm{z})=\mathrm{E}\left[\max (\mathrm{z}-\mathrm{G}, 0)^{\mathrm{n}}\right]=\int_{-\infty}^{z}(z-g)^{\mathrm{n}} f(g) d g
$$

Die Beziehung (4.3) verdeutlicht, dass Zielunterschreitungen mit steigender Anzahl der Momente n, d.h. mit steigendem Exponenten immer stärker gewichtet werden. ${ }^{314}$ Dieser Vorgehensweise liegt die Intention zugrunde, die Nichtlinearität der Risikowahrnehmung eines Entscheidungsträgers geeignet abzubilden. ${ }^{315}$ Hohe Verluste mit nur sehr geringer Eintrittswahrscheinlichkeit werden demnach durch den Entscheidungsträger als größeres Risiko wahrgenommen als kleinere, jedoch mit höherer Wahrscheinlichkeit eintretende Verluste, selbst wenn die Ausfallerwartung beider Ereignisse gleich hoch ist. In Abhängigkeit von der Anzahl der betrachteten Momente, kann zwischen verschiedenen Ausprägungen der LPM unterschieden werden. Sofern

311 Albrecht (2003), S. 14.

312 Vgl. im Folgenden auch Poddig/DichtL/Petersmeyer (2001), S. 137 und PortMANN (1999), S. 14-16.

${ }^{313}$ Vgl. im Folgenden grundlegend FISHBURN (1977), S. 116-126 und außerdem PFINGSTEN/ HOMÖLlE/RIESO (2001), S. 1871-1874, AlBRECHT (2003), S. 22-23, AlBRECHT/MAURER (2002), S. 111-112 und MEYER (1999), S. 45.

314 Vgl. auch PfingSTEN/HOMÖLlE/RIESO (2001), S. 1873 und KORYCIORZ (2004), S. 37.

315 Vgl. im Folgenden PORTMANN (1999), S. 16-24. 
für den Entscheidungsträger das Ausmaß der Zielunterschreitung unerheblich ist, kann das LPM nullten Grades herangezogen werden. Für $\mathrm{n}=0$ vereinfacht sich Beziehung (4.3) zu:

$$
\operatorname{LPM}_{0}(G, z)=\int_{-\infty}^{z} f(g) d g=P(G \leq z)=F(z)
$$

Folglich beziffert das $\mathrm{LPM}_{0}$ die Wahrscheinlichkeit, dass maximal ein Gewinn in Höhe des Zielwertes erzielt wird. Diese Wahrscheinlichkeit entspricht wiederum dem Wert der Verteilungsfunktion F an der Stelle z. Das LPM $_{0}$ wird auch als ShortfallWahrscheinlichkeit bezeichnet und korrespondiert in der Entscheidungstheorie mit der stochastischen Dominanz erster Ordnung. ${ }^{316}$ Demgegenüber berücksichtigt der Shortfall-Erwartungswert $(\mathrm{n}=1)$ auch das durchschnittliche Ausmaß einer Zielunterschreitung. Es gilt zu beachten, dass Unterschreitungen des Zielwertes z nicht durch Ergebnisrealisationen oberhalb des Zielwertes kompensiert werden können. ${ }^{317}$ Formal ist das $\mathrm{LPM}_{1}$ definiert als: ${ }^{318}$

$$
\operatorname{LPM}_{1}(G, z)=E[\max (z-G, 0)]=\int_{-\infty}^{z}(z-g) f(g) d g
$$

Das $\mathrm{LPM}_{2}$ wird schließlich als Shortfall-Varianz bezeichnet. Zielunterschreitungen gehen hier quadratisch in die Berechnung ein und werden auf diese Weise höher gewichtet. ${ }^{319}$ Für den Spezialfall $\mathrm{z}=\mathrm{E}(\mathrm{G})$ entspricht das $\mathrm{LPM}_{2}$ dem Risikomaß der Semivarianz, welche lediglich negative Abweichungen vom Erwartungswert erfasst. ${ }^{320}$

Zusammenfassend kann festgehalten werden, dass risikoaverse Entscheidungsträger ein LPM höherer Ordnung ( $\mathrm{n}>0$ ) wählen sollten, um Fehleinschätzungen in Bezug auf das Risikoausmaß einer Handlungsalternative zu vermeiden. ${ }^{321}$ Je risikoaverser der Entscheidungsträger ist, desto höher sollte $\mathrm{n}$ gewählt werden. Aus der Anwendbarkeit der Lower Partial Moments für beliebige Verteilungsannahmen erwächst deren gene-

\footnotetext{
316 Vgl. auch Meyer (1999), S. 60, SpellmanN (2002), S. 53 und GuthofF/PFingSTEN/Wolf (1998), S. 146.

$317 \mathrm{Vgl}$. Guthoff/PFingSTEN/WOLF (1998), S. 137.

318 Vgl. auch HahN/Pfingsten/WAGNER (2002), S. 4 und Albrecht (2003), S. 23

319 Vgl. vertiefend Albrecht (2003), S. 23 und PfingSTEN/HomÖLle/RieSO (2001), S. 1872-1873 sowie SPELLMANN (2002), S. 56.

320 Vgl. übereinstimmend Albrecht (2003), S. 24, SPELlMANN (2002), S. 52 und MEYER (1999), S. 46-51. Die Semivarianz wird bereits durch MARKowITZ im Kontext der Portfoliotheorie als asymmetrisches Risikomaß diskutiert. Vgl. MARKOWITZ (1959), S. 194.

321 Vgl. im Folgenden SPELlmanN (2002), S. 56.
} 
relle Eignung für versicherungsspezifische Anwendungen, die oftmals auf nicht symmetrischen Verteilungsannahmen fußen. Darüber hinaus bildet die Klasse der LPM die konzeptionelle Grundlage für weitere prominente Risikomaße wie den Value-at-Risk oder Conditional-Value-at-Risk, welche in den nachfolgenden Abschnitten vorgestellt werden.

\subsubsection{Value-at-Risk}

Der Value-at-Risk (VaR) hat sich mittlerweile in Kreditinstituten als Instrument der Marktrisikosteuerung und als aufsichtsrechtliches Risikomaß etabliert. ${ }^{322}$ Er entspricht derjenigen Verlusthöhe, welche innerhalb eines bestimmten Zeitraums lediglich mit einer (sehr geringen) Wahrscheinlichkeit $\varepsilon$ überschritten wird. Mit $\varepsilon$ sei daher im weiteren Verlauf die unternehmensseitig tolerierte Ausfall- bzw. Ruinwahrscheinlichkeit bezeichnet. ${ }^{323}$ In allgemeiner Form ist somit der $\mathrm{VaR}_{\varepsilon}$ zum Konfidenzniveau 1- $\varepsilon$ definiert durch: $:^{324}$

$$
\mathrm{P}\left[\mathrm{V}>\mathrm{VaR}_{\varepsilon}(\mathrm{V})\right]=\varepsilon
$$

Aufbauend auf dieser Definition kann der VaR als spezifisches Quantil der Verteilungsfunktion $\mathrm{F}(\mathrm{V})$ der Verlustvariablen $\mathrm{V}$ aufgefasst werden. Es wird im weiteren Verlauf der Ausführungen vorausgesetzt, dass das Eindeutigkeitskriterium des VaR erfüllt ist. ${ }^{325}$ Dies bedeutet, dass genau eine Realisation der Verlustvariablen $\mathrm{V}$ der Definition des $(1-\varepsilon)$-Quantils genügt. ${ }^{326}$ Bezeichne also $F_{\varepsilon}(V)=F_{V}^{-1}(1-\varepsilon)$ das $(1-\varepsilon)$ Quantil der Verteilung der Verlustvariablen V, so wird die Quantileigenschaft des VaR aus folgender Beziehung deutlich:

$$
\operatorname{VaR}_{\varepsilon}(\mathrm{V})=\mathrm{F}_{\varepsilon}(\mathrm{V})=\mathrm{F}_{\mathrm{V}}^{-1}(1-\varepsilon)
$$

322 Vgl. exemplarisch Albrecht/Maurer (2002), S. 673 und PfingSten/HomÖLle/Rieso (2001), S. 1874 und JOHANNING (1998), S. $20 \mathrm{ff}$.

323 Vgl. übereinstimmend Dresel (2003), S. 23, AlbreCht/KoryciorZ (2000), S. 1111, SPELLMANN (2002), S. 54 und AlBrecht/MAURER (2002), S. 674. Die Gegenwahrscheinlichkeit 1- $\varepsilon$ kann als Konfidenzniveau (synonym: Sicherheitsniveau) interpretiert werden.

324

Als Berechnungszeitpunkt wird fortan das Ende einer Modellperiode zugrunde gelegt. Eine explizite Indizierung des Berechnungszeitpunktes kann daher entfallen. In Kreditinstituten wird der $\mathrm{VaR}$ hingegen primär zur Messung von Marktrisiken von Handelspositionen verwendet, denen eine Haltedauer von nur wenigen (in der Regel 10) Tagen zugrunde liegt. Vgl. hierzu vertiefend JOHANNING (1998), S. 31-32 und BEECK/JOHANNING/RUDOLPH (1999), S. 263. die betrachtete Verteilungsfunktion ein waagerechtes Teilstück aufweist. Die Eindeutigkeit des $(1-\varepsilon)$-Quantils bedingt außerdem, dass $F(V)$ invertierbar ist, mithin die Umkehrfunktion existiert. Vgl. auch KORYCIORZ (2004), S. 26. 
Im stetigen Fall kann der VaR implizit über die zugehörige Dichtefunktion $f(v)$ dargestellt werden.

$$
\int_{-\infty}^{\mathrm{VaR}_{\varepsilon}(\mathrm{V})} \mathrm{f}(\mathrm{v}) \mathrm{dv}=1-\varepsilon
$$

Die Annahme einer stetigen Verlustvariablen V ist zwar aus analytischer Sicht wünschenswert, kann jedoch lediglich als Approximation der Realität aufgefasst werden, da die möglichen Verlustausprägungen in der Praxis auf eine endliche Anzahl beschränkt sind. ${ }^{327}$ Es gilt ferner zu beachten, dass der VaR über die Eigenschaft der Nichtnegativität verfügt. Dies bedeutet, dass der VaR definitionsgemäß den Wert Null annimmt, sofern Verluste nur mit einer Wahrscheinlichkeit auftreten, die kleiner ist als $\varepsilon .{ }^{328}$ Abbildung 4.1 stellt die Konzeption des VaR in graphischer Form dar.

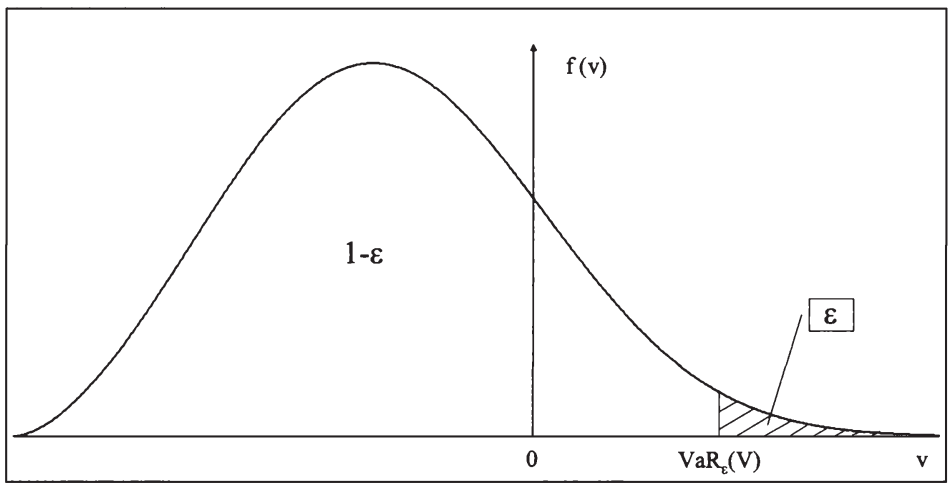

Abbildung 4.1: Schematische Darstellung der Value-at-Risk-Konzeption Quelle: in Anlehnung an KORYCIORZ (2004), S. 27.

Unter Verwendung der bereits in Kapitel 4.1.2 betrachteten Beziehung $V=-G$ kann die Definition des VaR auch anhand der Verteilung der korrespondierenden Gewinnvariablen $\mathrm{G}$ erfolgen. Die Risikobetrachtung verlagert sich in diesem Fall auf den linken Rand der Verteilung. Zwischen dem (1-E)-Quantil der Verlustverteilung und dem $\varepsilon$-Quantil der korrespondierenden Gewinnverteilung besteht folgende Äquivalenzbeziehung: ${ }^{329}$

\footnotetext{
${ }^{327}$ KORYCIORZ weist darauf hin, dass im Falle diskreter Verteilungsfunktionen ,leicht Konstellationen vorstellbar [sind], in denen der Funktionswert 1- $\varepsilon$ schlichtweg übersprungen wird, mithin nicht existiert.“ KORYCIORZ (2004), S. 29 [Ergänzung nicht im Original].

329 Vgl. auch KORYCIORZ (2004), S. 34.
} 


$$
\mathrm{F}_{\mathrm{v}}^{-1}(1-\varepsilon)=-\mathrm{F}_{\mathrm{G}}^{-1}(\varepsilon)
$$

Während beim Lower Partial Moment nullter Ordnung das Shortfall-Risiko durch die Vorgabe eines bestimmten Zielwertes $\mathrm{z}$ ermittelt wird, wird der VaR durch die Höhe des Konfidenzniveaus 1- $\varepsilon$ determiniert. Beide Risikomaße weisen somit vergleichbare Eigenschaften auf und sind grundsätzlich ineinander überführbar. Der VaR kann dann als Inverse des $\mathrm{LPM}_{0}$ dargestellt werden. ${ }^{330}$ Ein wesentlicher Vorteil der Value-atRisk-Konzeption besteht darin, dass die Risikoeinstellung der Unternehmensleitung in einer monetär messbaren Größe verdichtet wird, welche einer ökonomisch intuitiven Interpretation zugänglich ist. ${ }^{331}$ Im Hinblick auf die Akzeptanz quantitativer Risikomodelle bei Entscheidungsträgern stellt dies einen nicht zu unterschätzenden Faktor dar. Darüber hinaus erlaubt der VaR, unterschiedliche Entscheidungsalternativen in eine vollständige Rangordnung zu bringen. ${ }^{332}$ Es kann jedoch gezeigt werden, dass diese Ordnung nicht notwendigerweise mit der Erwartungsnutzentheorie, mithin nicht mit dem Rationalitätskriterium des BERNOULLI-Prinzips vereinbar ist. Eine Risikoanordnung nach dem VaR kann demnach von einer Anordnung nach der stochastischen Dominanz zweiten Grades abweichen. ${ }^{333}$ Dies ist beispielsweise für den Fall eines so genannten „Mean Preserving Spread“ (MPS) gegeben. ${ }^{334}$ Vereinfachend beschrieben, wird durch den MPS eine Dichtefunktion insofern manipuliert, als dass Wahrscheinlichkeitsmasse aus zwei Intervallen der Dichtefunktion entnommen und in zwei anderen, weiter am Verteilungsrand liegenden Intervallen hinzugefügt wird. Der Erwartungswert bleibt unverändert, da sich die gegenläufigen Effekte exakt kompensieren. Die risikoerhöhende Wirkung des MPS wird jedoch durch den VaR nicht erfasst. Es wurde bereits in Kapitel 3.2.1 erläutert, dass die Kompatibilität zwischen Erwartungsnutzenmaximierung und Safety-First-Prinzip im versicherungsspezifischen Kontext in Zweifel zu ziehen ist, da beliebig hohe Risiken nicht mehr in uneingeschränktem Ausmaß durch Gewinne substituiert werden können. Dem oben genannten Kritikpunkt kommt daher hier nur abgeschwächte Bedeutung zu.

${ }^{330}$ Vgl. hierzu insbesondere MEYER (1999), S. 54-55 sowie KORYCIORZ (2004), S. 36-39 und SPELLMANN (2002), S. 53-88. JOHANNING erbringt jedoch den Nachweis, dass beide Risikomaße nicht in jedem Fall zu einer identischen Anordnung von Verteilungen führen müssen. Vgl. JOHANNING (1998), S. 246-249.

331 Vgl. auch ARTZNER/DELBAEN/EBER/HEATH (1999), S. 216 und LOCAREK-JUNGE/STAHL (2001), Sp. 2121.

332 Vgl. im Folgenden GuTHOFF/PfingSTEN/WOLF (1998), S. 126-128.

333 Vgl. zur Konzeption der stochastischen Dominanz FishBURN (1977), S. 116 ff., BAWA (1982), GUTHOFF/PFINGSTEN/WOLF (1998), S. 115-118 und MEYER (1999), S. 64-66.

334 Vgl. hierzu und im Folgenden RothSCHILD/STIGLITZ (1970), GuTHOFF/PFINGSTEN/WOLF (1998), S. 119-120 und KORYCIORZ (2004), S. 54-57. 
Schwerer wiegt hingegen der Einwand, dass der VaR lediglich eine singuläre Bewertung des Risikopotenzials vornimmt, da nur ein Punkt der Wahrscheinlichkeitsverteilung betrachtet wird. ${ }^{335}$ Das Gefährdungspotenzial jenseits der $(1-\varepsilon)$-Quantils bleibt jedoch unberücksichtigt. ${ }^{336}$ Im Anwendungskontext der Schaden- und Unfallversicherung bedeutet dies, dass Szenarien mit sehr geringer Eintrittswahrscheinlichkeit, aber sehr hohem Verlustpotenzial (z.B. im Fall von Naturkatastrophen) nicht in die Quantifizierung des Risikokapitalbedarfs einfließen. Der tatsächliche Kapitalbedarf wird auf diese Weise gegebenenfalls unterschätzt. Es kann außerdem gezeigt werden, dass sich die Risikorelation zwischen zwei Handlungsalternativen durch eine Veränderung des Konfidenzniveaus verändern kann, da die Risikocharakteristik des Tails nicht in die Beurteilung einfließt. ${ }^{337}$

In Bezug auf den in Kapitel 4.1.2 skizzierten Anforderungskatalog für kohärente Risikomaße kann festgehalten werden, dass der VaR den Axiomen der Translationsinvarianz, positiven Homogenität und Monotonie genügt. ${ }^{338}$ Es sind jedoch Konstellationen denkbar, in denen der VaR gegen das Axiom der Subadditivität verstößt und somit Risikoausgleichseffekte im Gesamtportfolio eines Versicherungsunternehmens nicht adäquat abbildet. ${ }^{339}$ Dies ist gerade im versicherungsspezifischen Kontext als elementarer Mangel zu werten. Eine Verletzung der Subadditivitätseigenschaft liegt immer dann vor, wenn die zugrunde liegende Zufallsgesetzmäßigkeit nicht der Familie der elliptischen Verteilung entstammt. ${ }^{340}$ Innerhalb der Klasse elliptischer Verteilungen, z.B. unter der Annahme einer multivariaten Normalverteilung, stellt der VaR jedoch für Konfidenzniveaus $1-\varepsilon>0,5$ ein kohärentes Risikomaß dar. ${ }^{341}$

${ }^{335}$ Vgl. übereinstimmend KORYCIORZ (2004), S. 58-59, HERI/ZIMMERMANN (2001), S. 1005-1006 und GOOVAERTS/DHAENE/KAAS (2001), S. 547. Es sei in diesem Zusammenhang ausdrücklich betont, dass der VaR keinen absoluten Maximalverlust kennzeichnet, sondern lediglich eine Verlustschranke angibt.

336 „Überspitzt könnte man formulieren, dass VaR-Maße genau jenen Teil der Wahrscheinlichkeitsverteilung außer Acht lassen, der für das Risikomanagement am relevantesten wäre. Wahrscheinlichkeiten sind sehr unvollkommene Risikomaße - gerade wenn es um Stressfälle geht.“ HERI/ ZIMMERMANN (2001), S. 1005.

337 Vgl. hierzu vertiefend KORYCIORZ (2004), S. 58-59, ACERBI/TASCHE (2002a) und GuTHOFF/ PFINGSTEN/WOLF (1998), S. 127-128.

338 Vgl. zum formalen Nachweis KORYCIORZ (2004), S. 47-49.

339 Beispiele für Verstöße des VaR gegen das Axiom der Subadditivität werden in der Literatur in großer Zahl angeführt. Vgl. exemplarisch WIRCH/HARDY (1999), S. 339, ACERBI/NORDIO/SIRTORI (2001), S. 5-6, TASCHE (2002), S. 1522, EMBRECHTS/MCNEIL/ STRAUMANN (2002), S. 206 ff., FREY/MCNEIL (2002) und KORYCIORZ (2004), S. 49-51.

${ }^{340}$ Vgl. KORYCIORZ (2004), S. 52. Vgl. zur Klasse elliptischer Verteilungen JORION (2001), S. 147 ff. sowie die Ausführungen in Kapitel 6.2.2.1 mit der dort angegebenen Literatur.

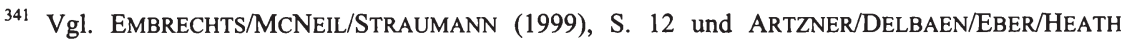
(1999), S. 217. 
In Anbetracht der aufgezeigten Schwächen des VaR wird im nachfolgenden Abschnitt mit dem Conditional Value-at-Risk ein Risikomaß vorgestellt, welches dem Kriterienkatalog von ARTZNER/DELBAEN/EBER/HEATH genügt und zudem das Ausmaß potenzieller Verluste jenseits des VaR explizit berücksichtigt.

\subsubsection{Conditional Value-at-Risk}

Der Conditional Value-at-Risk (CVaR) ist definiert als derjenige Verlustbetrag, der im Falle einer Überschreitung des VaR durchschnittlich zu erwarten ist. ${ }^{342}$ Im Gegensatz zur VaR-Konzeption wird demnach eine explizite Aussage über das potenzielle Verlustausmaß für den Fall getroffen, dass ein zuvor definierter Shortfall eintritt. In allgemeiner Form ist der $\mathrm{CVaR}_{\varepsilon}$ definiert als: ${ }^{343}$

$$
\mathrm{CVaR}_{\varepsilon}(\mathrm{V})=\mathrm{E}\left[\mathrm{V} \mid \mathrm{V} \geq \mathrm{VaR}_{\varepsilon}(\mathrm{V})\right]
$$

Sofern eine Stichprobe von Verlusten betrachtet wird, kann der CVaR anschaulich als Durchschnitt der $100 \cdot \varepsilon \%$ schlechtesten Realisationen interpretiert werden. Im Falle stetiger Zufallsvariablen kann der CVaR unmittelbar aus dem Shortfall-Erwartungswert $\left(\mathrm{LPM}_{1}\right)$ abgeleitet werden, indem der Zielwert des $\mathrm{LPM}_{1}$ mit dem VaR gleichgesetzt wird $\left(\mathrm{z}=\mathrm{VaR}_{\varepsilon}\right){ }^{344}$ Der $\mathrm{CVaR}$ genügt dann uneingeschränkt dem Axiom der Subadditivität, so dass stets gewährleistet ist, dass durch die Addition zweier Risikopositionen kein zusätzliches Risiko entsteht. ${ }^{345}$ Dies entspricht der von ARTZNER/DELBAEN/ EBER/HEATH formulierten Auffassung, dass beispielsweise durch eine Fusion zweier Unternehmen kein zusätzliches Risiko entsteht. ${ }^{346}$ Diesbezüglich sei jedoch einschränkend angemerkt, dass durchaus Umweltzustände oder Präferenzstrukturen denkbar sind, die einer Fusion eine risikoerhöhende Wirkung beimessen.$^{347}$ Die Subadditivitätseigenschaft des CVaR kann formal durch Beziehung (4.11) beschrieben werden.

$$
\mathrm{CVaR}_{\mathrm{\varepsilon}}\left(\mathrm{V}_{1}+\mathrm{V}_{2}\right) \leq \mathrm{CVaR}_{\mathrm{\varepsilon}}\left(\mathrm{V}_{1}\right)+\mathrm{CVaR}_{\mathrm{\varepsilon}}\left(\mathrm{V}_{2}\right)
$$

${ }^{342}$ Synonym ist in der Literatur auch der Begriff des „Tail Value-at-Risk“ (TVaR) geläufig. Vgl. exemplarisch VENTER (2004), S. 97 und ALBRECHT/MAURER (2002), S. 675.

Vgl. im Folgenden auch HÜrLimanN (2002a), S. 2, Albrecht/MAURER (2002), S. 675, ALBRECHT/KORYCIORZ (2003), S. 2, DRESEL (2003), S. 54 und KORYCIORZ (2004), S. 60-63.

Vgl. vertiefend KORYCIORZ (2004), S. 64-65 und DRESEL (2003), S. 54-55.

${ }^{345}$ Der mathematische Beweis ist z.B. bei ACERB//TASCHE (2002a), S. 1490-1491 nachzulesen. Im Falle diskreter Verteilungen ist das Axiom der Subadditivität hingegen nur unter bestimmten Voraussetzungen bzw. Modifikationen erfüllt. Vgl. hierzu auch ACERBI/TASCHE (2002a) und MEYERS (2000), S. 10. Im Verlauf der weiteren Ausführungen werden durchgängig stetige Zufallsvariablen unterstellt.

346 „A merger does not create extra risk.“ ARTZNER/DELBAEN/EBER/HEATH (1999), S. 216.

347 Vgl. übereinstimmend KORYCIORZ (2004), S. 51. 
Die Konzeption des $\mathrm{CVaR}$ zeichnet sich außerdem durch die wünschenswerte Eigenschaft aus, dass der Risikokapitalbedarf nicht mehr auf der Basis einer einfachen „Punktbetrachtung“ ermittelt wird, sondern Informationen über das Verteilungsende in die Risikobewertung einfließen. Auf diese Weise werden auch extreme Verlustszenarien entsprechend ihrer Eintrittswahrscheinlichkeit berücksichtigt. ${ }^{348}$ Aufgrund der Durchschnittsbildung über die „schlechten“ Szenarien jenseits des (1- $\varepsilon$ )-Quantils ergeben sich außerdem durch leichte Variationen des Steuerungsparameters $\varepsilon$ keine substanziellen Auswirkungen auf den $\mathrm{CVaR}^{349} \mathrm{Da}$ die Charakteristik des Verteilungsrandes (zumindest als Erwartungswert) in die Betrachtung eingeht, besteht im Gegensatz zum VaR nicht mehr die Gefahr, dass sich die Risikorelation konkurrierender Handlungsalternativen mit ansteigendem Sicherheitsniveau verändert. ${ }^{350}$

Die Konzeption des CVaR gewinnt zusätzlich an Transparenz, sofern man die Beziehung zwischen VaR und CVaR durch eine formale Zerlegung offen legt. Demnach lässt sich der CVaR in die Komponenten der „Quantilreserve“ und „Excessreserve“ aufspalten: ${ }^{351}$

$$
\begin{aligned}
& \mathrm{CVaR}_{\varepsilon}=\mathrm{VaR}_{\varepsilon}+\underbrace{\mathrm{E}\left[\mathrm{V}-\mathrm{VaR}_{\varepsilon}(\mathrm{V}) \mid \mathrm{V} \geq \mathrm{VaR}_{\varepsilon}(\mathrm{V})\right]} \\
& =\mathrm{VaR}_{\varepsilon}(\mathrm{V})+\mathrm{MEL}_{\mathrm{VaR}_{c}}(\mathrm{~V}) \\
& =\text { Quantilreserve }+ \text { Exzessreserve }
\end{aligned}
$$

Der Mean-Excess-Loss (MEL) stellt den bedingten Shortfall-Erwartungswert dar. Er misst die durchschnittliche Überschreitung des VaR unter der Bedingung, dass ein Shortfall eintritt und kann demzufolge auch als „Worst-Case-Risikomaß“ interpretiert werden. ${ }^{352}$ Die beschriebene Konzeption des CVaR wird in Abbildung 4.2 schematisch dargestellt:

${ }^{348}$ Es ist jedoch ausdrücklich zu betonen, dass der Steuerungsparameter $\varepsilon$ im Anwendungskontext des CVaR nicht mehr anschaulich als Ausfall- bzw. Ruinwahrscheinlichkeit interpretiert werden kann. Eine ökonomisch intuitive Interpretation von $\varepsilon$ ist daher nicht mehr möglich. Vgl. auch KORYCIORZ (2004), S. 79 sowie YAMAI/YoshIBA (2002), S. 61.

349 Unbeschadet dieser Aussage gilt natürlich, dass der CVaR mit zunehmendem Konfidenzniveau monoton ansteigt. Vgl. in diesem Zusammenhang vertiefend ACERBI/TASCHE (2002a), S. 14911496.

350 Vgl. übereinstimmend KORYCIORZ (2004), S. 63 und DRESEL (2003), S. 54.

351 Vgl. im Folgenden KORYCIORZ (2004), S. $62-63$ und ALBRECHT/KORYCIORZ (2003), S. 2-3.

352 Vgl. Albrecht (2003), S. 24 und AlbreCht/KLETt (2004), S. 4. Der MEL entstammt ursprünglich der Extremwerttheorie. Vgl. hierzu vertiefend EMBRECHTS/KLÜPPELBERG/MIKOSCH (2003), S. 161. 


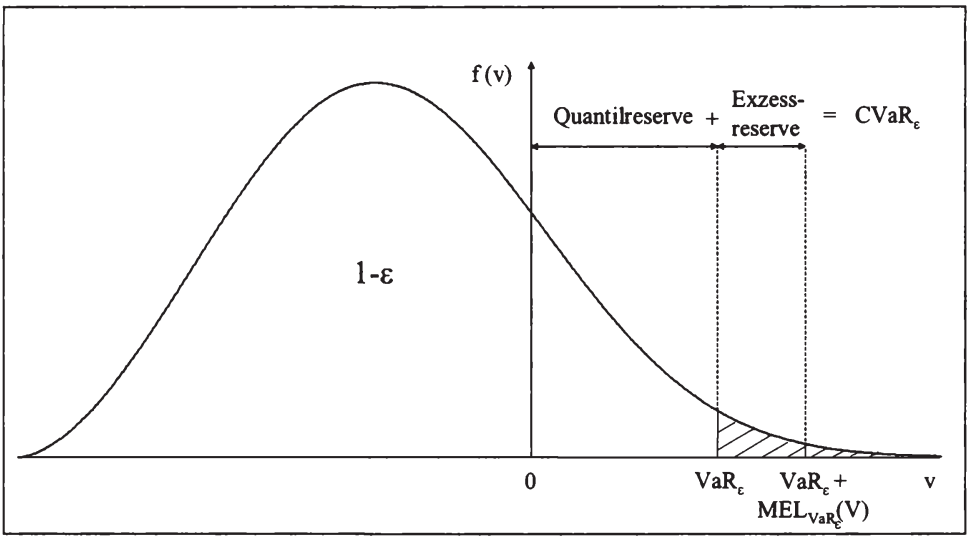

Abbildung 4.2: Schematische Darstellung des Conditional Value-at-Risk Quelle: ALBRECHT/KORYCIORZ (2003a), S. 3.

Da der CVaR die obere Schranke ${ }^{353}$ für den VaR darstellt, nimmt der Mean-ExcessLoss stets nichtnegative Werte an. ${ }^{354}$ Der CVaR kann demzufolge niemals kleiner sein als der VaR. Diese „konservative Eigenschaft“ kann durch folgende Beziehung formalisiert werden. ${ }^{355}$

$$
\mathrm{CVaR}_{\varepsilon}(\mathrm{V}) \geq \operatorname{VaR}_{\varepsilon}(\mathrm{V})
$$

Als Ergebnis der bisherigen Ausführungen ist festzuhalten, dass der CVaR dem VaR in konzeptioneller Hinsicht überlegen ist. ${ }^{356}$ Er kommt daher in den nachfolgenden Untersuchungen durchgängig zur Anwendung. Die Risikoeinschätzung durch den VaR ist der Perspektive eines beschränkt haftenden Eigentümers (z.B. Aktionär) vergleichbar. Sobald das eingebrachte Kapital durch Verluste aufgezehrt ist, spielt es für den Aktionär keine Rolle mehr, in welchem Umfang darüber hinaus weitere Verluste anfallen. Demgegenüber korrespondiert der CVaR mit der Sichtweise der Versicherungsnehmer, für die auch das Ausmaß eines Verlustes von Belang ist. ${ }^{357}$

353 Vgl. zur mathematischen Präzisierung dieser Aussage PFLUG (2000), S. 273.

354 Vgl. auch KORYCIORZ (2004), S. 62 und ALBRECHT/KORYCIORZ (2003), S. 2.

355 Vgl. übereinstimmend YAMAL/YoshiBA (2002), S. 61.

356 Eine vergleichende Darstellung der Stärken und Schwächen findet sich u.a. bei YAMAI/YOSHIBA (2002), S. 81.

357 Der CVaR weist daher einen engen konzeptionellen Bezug zum „Expected Policyholder Defizit“ (EPD) auf. Dieses Risikomaß ergibt sich als Produkt aus Ruinwahrscheinlichkeit und bedingter erwarteter Überschuldung. Das EPD wird insbesondere durch die Aufsichtsbehörden im angloamerikanischen Raum bevorzugt eingesetzt. Vgl. vertiefend BARTH (2000), S. 397-414 und MEYERS (2000), S. 8. 
„And indeed market evidence suggests that shareholders tend to be better when they address policyholder needs. ${ }^{\text {“358 }}$ Vorteile des CVaR bestehen außerdem in der expliziten Abbildung des Tailverhaltens und der adäquateren Abbildung von Risikoausgleichseffekten (Axiom der Subadditivität). Dies hat mittlerweile dazu geführt, dass der CVaR im Zuge der Neuausrichtung des europäischen Solvabilitätssystems (Solvency II) neben dem VaR als mögliches Risikomaß diskutiert wird. ${ }^{359}$ Der durch die EU-Kommission favorisierte Vorschlag, in Abhängigkeit von der Verteilungscharakteristik einzelner Teilkollektive unterschiedliche Risikomaße zu verwenden, ist jedoch aus risikotheoretischer Perspektive abzulehnen. ${ }^{360}$ Einerseits wäre die vorgeschlagene kombinierte Verwendung von VaR und CVaR nur unter sehr restriktiven Anforderungen an die verwendeten Verteilungsklassen zulässig. Andererseits würden Risikoausgleichseffekte nicht mehr angemessen berücksichtigt, da das Risikopotenzial einzelner Teilkollektive auf der Basis unterschiedlicher Risikomesskonzepte und somit auf isolierter Basis quantifiziert würde. Bei der Aggregation der einzelnen Kapitalbeträge zum Risikokapitalbedarf des Gesamtunternehmens wäre jedoch die geforderte kohärente Risikomessung nicht sicherzustellen. Aktuarielle Organisationen und internationale Expertengruppen haben sich vor diesem Hintergrund bereits für den CVaR als wünschenswertes (und ausschließlich anzuwendendes) Risikomaß ausgesprochen. ${ }^{361}$ Abschließend sei jedoch betont, dass die konzeptionelle Überlegenheit des CVaR gegenüber dem VaR in der praktischen Anwendung nur dann zum Tragen kommen kann, sofern hinreichend valide historische Informationen über die Risikostruktur des relevanten Verteilungsendes vorliegen. Nur wenn der Tail der Verteilung in ausreichendem Maße empirisch besetzt ist, kann eine stabile Risikoabschätzung erfolgen. ${ }^{362}$

Nachdem nunmehr die konzeptionellen Grundlagen für die Messung von Risiko bzw. die Quantifizierung von Risikokapital erörtert wurden, wird im nachfolgenden Kapitel der Frage nachgegangen, unter welchen Zielsetzungen eine Zuweisung (= Allokation) von Risikokapital auf bestimmte Geschäftsbereiche sinnvoll erscheint. Die Diskussion um die Allokation von Risikokapital wird bisweilen dadurch erschwert, dass sich in

358 VENTER (2004), S. 99.

359 Vgl. vertiefend EU-KOMMISSION (2004a), S. 33-34.

360 „VaR could be used as a general rule except for low frequency, high severity risks which would be measured with TailVaR or other measures that takes skewness into account appropriately. This is possible only if the sum of risks measured in different ways remains coherent." EU-KOMMISSION (2004a), S. 33-34.

361 Vgl. GCAE (2003), S. 3 und IAA (2004), S. 34-36.

${ }^{362}$ Vgl. vertiefend zur Problematik einer geringen Glaubwürdigkeit der empirischen Datenbasis aufgrund unzureichender Kollektivbesetzung SCHRADIN (1998), S. 122-123. YAMAI/YOSHIBA bemerken in diesem Kontext zutreffend. „From the practical point of view, the effectiveness of expected shortfall depends on the stability of estimation and the choice of efficient backtesting methods." YAMAI/YOSHIBA (2002), S. 80. 
der Literatur eine unübersichtliche Variantenvielfalt herausgebildet hat. ${ }^{363}$ Die Zielsetzung des nachfolgenden Kapitels besteht daher in der Systematisierung und Beschreibung grundlegender Allokationsmethoden. Es werden zunächst generelle Anforderungen an Allokationsverfahren formuliert. Hierauf aufbauend werden ausgewählte Allokationsverfahren im Überblick dargestellt und einer kritischen Würdigung unterzogen.

\subsection{Allokation von Risikokapital}

4.2.1 Diversifikationseffekte als Ausgangspunkt der Allokationsproblematik

Die Möglichkeit des Auftretens kollektiver Risikoausgleichseffekte stellt die fundamentale Geschäftsgrundlage des Versicherungsgeschäfts dar. ${ }^{364} \mathrm{Da}$ die Einzelrisiken eines Portfolios in der Regel nicht vollständig positiv miteinander korreliert sind, können sich auf der Kollektivebene günstige und ungünstige Ergebnisrealisationen der Einzelrisiken ausgleichen. ${ }^{365}$ Dieser Effekt kann sowohl aus der Perspektive des Versicherungsunternehmens als auch aus Sicht der Versicherungsnehmer betrachtet werden. Nach einer auf ALBRECHT zurückgehenden Definition genügt ein Versicherungskollektiv dem Ausgleich-im-Kollektiv-Effekt, sofern entweder die einperiodige Verlustwahrscheinlichkeit mit wachsender Bestandsgröße gegen Null strebt (Typ A), oder aber die durchschnittliche Versicherungsprämie bei wachsendem Bestand absinkt, ohne dass die Verlustwahrscheinlichkeit eine definierte Schranke überschreitet (Typ B). Im ersten Fall profitiert das Versicherungsunternehmen in der Form, dass es mit wachsender Kollektivgröße entweder ein höheres Sicherheitsniveau erreichen kann oder aber zur Einhaltung eines zuvor definierten Sicherheitsniveaus weniger Risikokapital vorhalten muss. Im zweiten Fall wird der Diversifikationseffekt in Form sinkender Prämien an die Versicherungsnehmer weitergegeben, ohne dass sich das Sicherheitsniveau verschlechtert.

Der zentralen Bedeutung des Diversifikationseffekts für das Versicherungsgeschäft Rechnung tragend, soll dieser nachfolgend formalisiert und einer Quantifizierung zugänglich gemacht werden. ${ }^{366} \mathrm{Im}$ vorangegangenen Abschnitt wurde implizit unter-

\footnotetext{
363 Vgl. exemplarisch VENTER (2004) und KORYCIORZ (2004), S. 175-268.

${ }^{364}$ ALBRECHT bezeichnet den Ausgleich im Kollektiv als „Produktionstechnik sui generis des Versicherungsgeschäfts." ALBRECHT (1992), 20. Der Begriff des Diversifikationseffekts wird nachfolgend als Oberbegriff für jegliche Risikoausgleichseffekte verwendet. Auf eine sprachliche Differenzierung zwischen dem versicherungstechnischen Ausgleich-im-Kollektiv-Effekt und finanzwirtschaftlichen Diversifikationseffekten wird verzichtet. Vgl. zur Begriffsabgrenzung auch ALBRECHT (1995), S. 99-105.

${ }^{365}$ Vgl. im Folgenden grundlegend ALBRECHT (1982), S. 522-533 und ALBRECHT (1987b), S. 96-99. Vgl. außerdem MACK (2002), S. 23-28 und KORYCIORZ (2004), S. 86-102.

$366 \mathrm{Vgl}$. hierzu und im Folgenden HÜRLIMANN (2002b), S. 16, ALBRECHT/KORYCIORZ (2004), S. 131 und KORYCIORZ (2004), S. 185.
} 
stellt, dass die Verlustvariable V aus der unternehmerischen Gesamtaktivität eines Versicherungsunternehmens resultiert. Der Risikokapitalbedarf des Gesamtunternehmens ergibt sich in allgemeiner Form als $\mathrm{RK}:=\mathrm{R}(\mathrm{V})$. In der Regel setzt sich das Gesamtergebnis eines Versicherungsunternehmens jedoch aus den Ergebnissen unterschiedlicher Teilkollektive (z.B. Versicherungszweige oder Geschäftsfelder) zusammen. Formal gilt dann: $\mathrm{V}=\mathrm{V}_{1}+\mathrm{V}_{2}+\ldots+\mathrm{V}_{\mathrm{m}}$ mit $\mathrm{i}=1,2, \ldots, \mathrm{m}$. In einer isolierten Betrachtung können - analog zur Vorgehensweise auf der Kollektivebene - Risikokapitalien für die einzelnen $m$ Teilkollektive ermittelt werden. Die Summe der isoliert ermittelten Kapitalbeträge wird im weiteren Verlauf dieser Arbeit als Standalone-Kapital bezeichnet. ${ }^{367}$ Sofern die Einzelrisiken des Gesamtportfolios nicht vollständig positiv korreliert sind und das gewählte Risikomaß R dem Axiom der Subadditivität genügt, ist auf der Kollektivebene weniger Risikokapital vorzuhalten als bei isolierter Betrachtung.

$$
R K=R(V)<\sum_{i=1}^{m} R\left(V_{i}\right)=\sum_{i=1}^{m} R_{i}
$$

Der durch die Zusammenfassung der Teilkollektive entstehende Diversifikationseffekt $D_{R}$ lässt sich in Abhängigkeit der Verlustvariablen $V$ formulieren als:

$$
D_{R}\left(V_{1}, \ldots, V_{m}\right)=\sum_{i=1}^{m} R\left(V_{i}\right)-R\left(\sum_{i=1}^{m} V_{i}\right)
$$

Der mittels Beziehung (4.15) quantifizierte Diversifikationseffekt ist das Ergebnis sämtlicher Risikoausgleichseffekte, die im Gesamtportfolio eines Versicherungsunternehmens wirksam werden. Er kann daher nur auf der Gesellschaftsebene willkürfrei zugerechnet werden. ${ }^{368}$ Im Hinblick auf die in Kapitel 5.4 erfolgende Modellierung eines Bestandes von Versicherungsverträgen gilt es außerdem zu beachten, dass in Abhängigkeit von der Art und Weise, wie Ergebnisverteilungen einzelner Teilkollektive zu übergeordneten Kollektiven zusammengefasst werden, unterschiedlich hohe Diversifikationseffekte auftreten. ${ }^{369}$ Dieser Effekt liegt darin begründet, dass der Diversifikationseffekt in der Regel nicht linearer Natur ${ }^{370}$ ist und darüber hinaus simultan von

\footnotetext{
367 Vgl. auch MerTon/Perold (1993), S. 27 und Cummins (2000), S. 20-21.

368 Vgl. im Folgenden auch ALBRECHT/KORYCIORZ (2004), S. 131-135 und KORYCIORZ (2004), S. 185-189.

$369 \mathrm{Vgl}$. auch LEYHERR/SCULLY/SOMMERFELD (2003), S. 1796 und insbesondere die Ausführungen in Kapitel 6.2.1.1.1.

${ }^{370}$ ALBRECHT/KORYCIORZ zeigen für den Fall normalverteilter Verlustvariablen, dass der Diversifikationseffekt $D_{R}$ für die Risikomaße VaR und CVaR eine Funktion der Quadratwurzel darstellt. Vgl. hierzu und im Folgenden ALBRECHT/KORYCIORZ (2004), S. 133-134.
} 
den Ergebnisverteilungen sämtlicher Teilkollektive abhängt. ${ }^{371}$ Im Hinblick auf die Risikokapitalallokation ist dieser Sachverhalt mit der grundlegenden Problematik verbunden, dass eine willkürfreie Aufspaltung des Diversifikationseffektes auf einzelne Teilkollektive nicht möglich ist. Vor diesem Hintergrund werden im nachfolgenden Kapitel 4.2.2 zunächst unterschiedliche Einsatzgebiete und Zielsetzungen diskutiert, die in der Literatur für eine Allokation von Risikokapital angeführt werden. Für den Fall, dass im Rahmen der Unternehmenssteuerung trotz der geschilderten Grundproblematik eine Allokation durchgeführt werden soll, bedarf es ergänzender (formaler) Gütekriterien, welche die Ermittlung weitgehend risikoadäquater Zuweisungsbeträge gewährleisten. Diese Kriterien sind Gegenstand von Kapitel 4.2.3.

\subsubsection{Zwecke der Kapitalallokation}

In den vorangegangenen Abschnitten wurde gezeigt, dass die Unterlegung risikobehafteter Geschäfte mit Eigen- bzw. Risikokapital sowohl aus aufsichtsrechtlicher als auch betriebswirtschaftlicher Perspektive erforderlich ist, um in extremen Verlustszenarien den Fortbestand des Unternehmens zu gewährleisten. ${ }^{372}$ Aufbauend auf diesen Überlegungen werden in der einschlägigen Literatur unterschiedliche Zielsetzungen diskutiert, warum Risikokapital im Rahmen der Unternehmensteuerung auf verschiedene Geschäftsbereiche aufgeteilt werden sollte. ${ }^{373}$ Folgende Zwecke der Risikokapitalallokation sollen im weiteren Verlauf einer kritischen Würdigung unterzogen werden:

- Risikoadjustierte Performancesteuerung

- Vorgabe von Risikolimiten

- Erzielung von Anreiz- und Verhaltenseffekten

Im Zuge der Auseinandersetzung mit Fragestellungen zur risikoadjustierten Performancesteuerung werden Allokationsmethoden derzeitig intensiv diskutiert. ${ }^{374}$ Ausgangspunkt der Überlegungen ist die Erkenntnis, dass Risikokapital eine knappe Res-

${ }^{371}$ KORYCIORZ formuliert zutreffend: „Im Allgemeinen setzt die Durchführung einer Kapitalallokation demzufolge die Fixierung der Verlustvariablen [...] voraus, denn jegliche Veränderung [...] innerhalb nur eines Geschäftssegments lässt den gesamtkollektiven Sicherheitskapitalbedarf nicht unbeeinflusst, sondern erzwingt in letzter Konsequenz eine Adaption des Allokationsergebnisses." KORYCIORZ (2004), S. 189.

${ }^{372}$ Vgl. übereinstimmend auch KINDER/STEINER/WILLINSKY (2001), S. 282-283.

373 Vgl. exemplarisch GRÜNDL/SCHMEISER (2002), S.809 und VALDEZ/CHERNIH (2003), S. 517-519.

374 Vgl. exemplarisch BAMBERG/Dorfleitner/GLAAB (2005), S. 399-414, MildENHALl (2004), S. 32-44, VENTER (2004), S. 96-107, ALBRECHT/KORYCIORZ (2004), S. 123-159, KORYCIORZ (2004), S. 175-268, VALDEZ/CHeRniH (2003), S. 517-532, GRAUMANN/BAUM (2003), S. 421-457, GRÜNDL/SCHMEISER (2002), S. 797-822 und MYERS/ READ (2001), S. 545-580. 
source darstellt. ${ }^{375}$ Im Rahmen der risikoadjustierten Performancesteuerung werden einzelne Steuerungseinheiten (z.B. Versicherungszweige oder Kundengruppen) dezentral über bereichspezifische Performancekennziffern gesteuert. Die Beurteilung der Bereiche erfolgt über risikoadjustierte Performancemaße (z.B. RORAC), welche das jeweilige Bereichsergebnis auf das allozierte Risikokapital beziehen. ${ }^{376}$ Einer derartigen dezentralen Steuerungsphilosophie liegt die Fiktion eines unternehmensinternen Marktes für Risikokapital zugrunde. ${ }^{377}$ Auf diesem fiktiven Kapitalmarkt konkurrieren die einzelnen Bereiche mit ihren Geschäfts- und Investitionsalternativen um das verfügbare Risikokapital. Dabei wird die generelle Zielsetzung verfolgt, die Allokationswirkung des externen Kapitalmarktes auf das Unternehmen zu übertragen und auf diese Weise eine Geschäftsbereichsstruktur zu induzieren, welche die knappe Ressource Risikokapital einer möglichst effizienten Verwendung zuführt. Angesichts der Tatsache, dass das Risikokapital in Versicherungsunternehmen vorwiegend der Deckung potenzieller Verluste dient und keine maßgebliche Finanzierungsfunktion ausübt, hat die Zurechnung des Kapitals auf einzelne Steuerungseinheiten lediglich kalkulatorischen Charakter. ${ }^{378}$ Ein Vergleich der bereichsspezifischen RORAC-Kennziffern mit der durch die Kapitalgeber geforderten Mindestrendite (,hurdle rate“) gibt Aufschluss darüber, inwieweit ein Geschäftsbereich in der Lage ist, seine Kapitalkosten zu erwirtschaften. ${ }^{379}$ Auf der Grundlage eines „Profit Ranking“ sollen schließlich strategische Entscheidungen über den Auf- und Abbau bestimmter Geschäftsbereiche getroffen und eine unternehmenswert- bzw. gewinnmaximierende Geschäftsbereichsstruktur erreicht werden. ${ }^{380}$

In Kapitel 3.2.2 wurde bereits erläutert, dass die verschiedenen risikoadjustierten Performancemaße (z.B. der RORAC) nur innerhalb eines sehr restriktiven Prämissenrahmens mit dem Marktwert- oder Gewinnmaximierungskalkül kompatibel sind. Neben diesem zentralen Kritikpunkt vernachlässigt die oben skizzierte Steuerungsphilosophie den Sachverhalt, dass das verfügbare Risikokapital stets das Versicherungsunternehmen als Ganzes schützt und somit zum Ausgleich von Verlusten beliebiger Steuerungseinheiten herangezogen werden kann. ${ }^{381}$ Eine direkte Zuweisung von Kapital auf einzelne Teilkollektive wäre nur sachgemäß, sofern diese rechtlich selbständig agieren

\footnotetext{
375 Vgl. im Folgenden auch ALBRECHT/SCHRAdIN (1992), S. 571-596, ALBRECHT (1998), KINDER/ STEINER/WILLINSKY (2001), S. 281-300 und KORYCIORZ (2004), S. 177-181.

$376 \mathrm{Vgl}$. auch die Ausführungen in Kapitel 3.2.2.

377 Vgl. hierzu und im Folgenden DreSEL (2003), S. 75-80.

378 Vgl. vertiefend ALBRECHT (1998), S. 232-233 und MATTEN (2000), S. 74-75.

379 Vgl. auch die Ausführungen in Kapitel 3.2.2.

380 Vgl. exemplarisch GraUMANN/BAUM (2003), S. 431.

381 Vgl. in diesem Kontext auch GRÜNDL/SCHMEISER (2004b), S. 6-7, GRÜNDL/SCHMEISER (2004c), S. 11-14 und KORYCIORZ (2004), S. 182 mit der dort in Fußnote 512 angegebenen Literatur.
} 
und demzufolge auch formalrechtlich in Konkurs fallen können. ${ }^{382}$ Da dies jedoch in der Regel nicht der Fall ist, stellen die Verzinsungsansprüche der Risikokapitalgeber Gemeinkosten dar, die einer willkürfreien und beanspruchungsgerechten Verteilung auf die einzelnen Steuerungseinheiten nicht zugänglich sind. ${ }^{383}$ Durch verschiedene Formen der Schlüsselung (Allokation) können demzufolge unterschiedliche Rangordnungen der Geschäftsbereiche erreicht werden, was diese Vorgehensweise letztlich dem Vorwurf der Willkür aussetzt. ${ }^{384}$

Ein weiterer Problemkreis ist im Kontext dezentraler Bereichssteuerung über risikoadjustierte Performancemaße zu nennen. ${ }^{385}$ Die Ermittlung bereichsbezogener Performance-Kennziffern erfolgt in der Regel unter der Zielsetzung, profitable Geschäftsbereiche zu identifizieren und diese dann im Rahmen der Zeichnungspolitik strategisch auszubauen. GRÜNDL/SCHMEISER weisen jedoch zu Recht darauf hin, dass eine derartige Vorgehensweise das fundamentale Problem beinhaltet, „,von der Performance einer bestehenden Unternehmensstruktur auf die Performance nach erfolgter Umstrukturierung schließen zu wollen. Dies ist jedoch grundsätzlich nicht möglich, da sich nach Umstrukturierung neue Risikoallokationen und Effekte aus der beschränkten Haftung ergeben, die sich auf Basis der Ausgangssituation nicht ersehen lassen. ${ }^{3866} \mathrm{Da}$ der Diversifikationseffekt des Gesamtportfolios simultan von sämtlichen Teilkollektiven abhängt, besteht die Gefahr, dass nach erfolgter Umstrukturierung andere Risikoausgleichseffekte im Gesamtportfolio wirksam werden, weil beispielsweise neu gezeichnete Risiken einer profitablen Sparte eine hohe positive Schadenkorrelation zu Bestandsrisiken aufweisen. Derartige bereichsübergreifende Kumulpotenziale können jedoch nicht ex-ante aus der bestehenden Risikostruktur abgeschätzt werden. Ebenfalls problematisch ist in diesem Kontext der Fall einzuschätzen, dass ein unprofitabler Geschäftsbereich geschlossen wird und auf diese Weise ein bislang unbekannter „natürlicher Hedge" für das Gesamtportfolio verloren geht.

${ }^{382} \mathrm{Vgl}$. in diesem Zusammenhang übereinstimmend SCHRADIN (2000), S. 105, KORYCIORZ (2004), S. 182 und VENTER (2002), S. 5.

383 Vgl. übereinstimmend GRÜNDL/SCHMEISER (2002), S. 798 und GRÜNDL/SCHMEISER (2004b), S. 6-7. Die Problematik der Gemeinkostenschlüsselung ist seit vielen Jahren Gegenstand der betriebswirtschaftlichen Diskussion. Vgl. exemplarisch RIEBEL (1994), S. 269-307 und COENENBERG (2003), S. 73-82.

${ }^{384}$ Ein anschauliches Beispiel auf der Basis des RAROC-Konzepts konstruieren in diesem Zusammenhang GRÜNDL/SCHMEISER (2002), S. 814-815. Vgl. darüber hinaus auch GRÜNDL/SCHMEISER (2004b), S. 6-7.

385 Vgl. im Folgenden GRÜNDL/SCHMEISER (2004b), S. 7 und GRÜNDL/SCHMEISER (2004c), S. 13.

${ }^{386}$ GRÜNDL/SCHMEISER (2004b), S. 8. Diese zeigen darüber hinaus anhand eines vereinfachten Optimierungsansatzes, dass für die Erreichung einer optimalen (d.h. die Gewinnerwartung maximierenden) Geschäftsbereichsstruktur keine Allokation des Risikokapitals notwendig ist. Vgl. GRÜNDL/SCHMEISER (2004b), S. 3-5. 
Abschließend sei bemerkt, dass selbst für den Fall, dass das „Profit Ranking“ einzelner Geschäftsbereiche (zufälligerweise) eine wertsteigernde Strategie nahe legt, dieses noch keinen Anhaltspunkt dafür bietet, in welchem Umfang ein profitabler Geschäftszweig forciert bzw. eine defizitärer Geschäftszweig reduziert werden sollte. ${ }^{387}$ Es bleibt festzuhalten, dass die Steuerung dezentraler Geschäftsbereiche mittels risikoadjustierter Performancemaße mit erheblichen konzeptionellen Problemen verbunden ist, die in der einschlägigen Literatur bislang nur wenig Beachtung finden. ${ }^{388}$

Demgegenüber ist die Allokation von Risikokapital auf einzelne Steuerungseinheiten zum Zwecke der Risikolimitierung positiv zu beurteilen. Diese Form der top-downBudgetierung von ökonomischem Kapital kommt bislang insbesondere bei der Steuerung von Marktrisiken in Kreditinstituten zur Anwendung, kann jedoch in modifizierter Form auch in den versicherungsspezifischen Kontext übertragen werden. ${ }^{389}$ Auf der Grundlage des bereits erörterten Risikotragfähigkeitskalküls ermittelt die Unternehmensleitung zunächst das maximale Risikoexposure, dem sich das Unternehmen in Anbetracht der vorhandenen Risikodeckungsmasse (= Sicherheitskapital) aussetzen kann. Dieses Sicherheitskapital kann dann auf die einzelnen Steuerungseinheiten verteilt werden, indem jedem Geschäftsbereich explizite Risikolimite für einen bestimmten Zeitraum (z.B. für ein Geschäftsjahr) vorgegeben werden. Auf diese Weise werden die Bereiche ex-ante dazu angehalten, keine Geschäfte abschließen, deren mögliche Verluste das zugewiesene Kapital überschreiten. Darüber hinaus ist gewährleistet, dass die vorhandenen Risikodeckungsmassen mit einer zuvor definierten Wahrscheinlichkeit ausreichen, um die Verluste sämtlicher Geschäftsbereiche zu decken. ${ }^{390}$ Die verwendete Allokationsmethode muss in der Lage sein, kollektive Risikoausgleichseffekte geeignet abzubilden. ${ }^{391}$ Anderenfalls würden die Risikolimite der einzelnen Bereiche zu gering ausfallen und deren Handlungsspielraum in unangemessener Form eingeschränkt. Anschaulich lässt sich dieser Sachverhalt am Beispiel der Kapitalanlage eines Versicherungsunternehmens verdeutlichen. Würden bei der Festlegung der Risikolimite für den Kapitalanlagebereich perfekte positive Korrelationen zwischen den Assetklassen unterstellt, so würde das Risikopotenzial der Kapitalanlage substanziell

387 Vgl. GRÜNDL/SCHMEISER (2004b), S. 7.

388 Ausnahmen bilden diesbezüglich die Veröffentlichungen von VENTER (2002), VENTER (2004), GRÜNDL/SCHMEISER (2002), GRÜNDL/SCHMEISER (2004b) und GRÜNDL/SCHMEISER (2004c). Darüber hinaus liegen nach Kenntnis des Verfassers bisher keine empirischen Belege dafür vor, dass eine Steuerung über risikoadjustierte Performancemaße zu einer nachhaltigen Unternehmenswertsteigerung führt.

389 Vgl. hierzu und in Folgenden LISTER (1997), S. 200-204, SAITA (1999), S. 96-111, BEECK/JOHANNING/RUDOLPH (1999), S. 259-286, SCHIERENBECK (2003b), S. 39-42 und DRESEL (2003), S. 56.

390 Vgl. übereinstimmend SCHIERENBECK (2003b), S. 32-39 und GRÜNDL/SCHMEISER (2002), S. 816.

391 Vgl. ausführlich zur Berücksichtigung von Korrelationen im Rahmen der Limitsteuerung in Banken DRESEL (2003), S. 87-160. Vgl. außerdem KINDER/STEINER/WILLINSKY (2001), S. 285-287. 
überschätzt. ${ }^{392}$ Bestimmte Geschäfte, die im Wirkungsgefüge des Gesamtportfolios vorteilhafte Diversifikationseigenschaften aufweisen, würden unter Umständen unterbleiben. Dieser Sachverhalt lässt sich analog auf den versicherungstechnischen Ausgleich im Kollektiv übertragen. Im Gegensatz zur risikoadjustierten Performancesteuerung stellt die Risikolimitierung auch für im Entscheidungszeitpunkt noch unbekannte Risiken eine adäquate Vorgehensweise dar, welche jedoch für sich genommen noch keine integrierte Risiko-Renditesteuerung beinhaltet, sondern lediglich eine Risikobegrenzung vornimmt. ${ }^{393}$ Probleme treten bei der Risikolimitierung jedoch immer dann auf, wenn die Geschäftsbereiche im Rahmen ihrer zugeteilten Limite neue Risiken zeichnen, welche ungünstige Korrelationen zu Risiken anderer Bereiche aufweisen. Dies kann zur Folge haben, dass sämtliche Geschäftsbereiche ihre Risikolimite einhalten und trotzdem das Limit des Gesamtportfolios überschritten wird. Im Rahmen eines revolvierenden Prozesses muss daher neben der Kontrolle der Einzellimite auch stets die Einhaltung des Gesamtrisikolimits überwacht werden. ${ }^{394}$ Gegebenenfalls müssen die Einzellimite neu adjustiert werden.

Wenngleich darauf hingewiesen wurde, dass eine dezentrale Marktbereichssteuerung mittels risikoadjustierter Performancemaße mit konzeptionellen Schwächen behaftet ist, sind für den Fall einer asymmetrischen Informationsverteilung zwischen Unternehmensleitung und Bereichsmanagement durchaus sinnvolle Einsatzfelder für Allokationsverfahren und darauf aufbauende Performancemaße zu vermuten. ${ }^{395}$ Im Kern geht es hierbei um die Erzielung von Anreiz- und Verhaltenseffekten und die Etablierung geeigneter Kooperationsdesigns, welche die Bereichsmanager dazu anhalten, nur solche Geschäfte zu tätigen, die die Rendite-Risiko-Position des Unternehmens verbessern. ${ }^{396}$ In diesem Kontext erscheint es zunächst sinnvoll, über charakteristische Entscheidungssituationen bei asymmetrischer Informationsverteilung Klarheit zu gewinnen. ${ }^{397}$ Diese sind häufig durch das Problem der Verhaltensunsicherheit (Moral Hazard) gekennzeichnet. Verhaltensunsicherheit liegt im Sinne der Prinzipal-AgentenTheorie vor, sofern ein Agent (z.B. Bereichsmanager) einen Verhaltensspielraum besitzt und sein tatsächliches Verhalten durch den Prinzipal (z.B. Unternehmensleitung

\footnotetext{
392 Vgl. in diesem Zusammenhang auch ALBRECHT/BÄHRLE/KÖNIG (1997), S. 90-92.

393 Vgl. GRÜNDL/SCHMEISER (2002), S. 816.

394 Vgl. vertiefend DRESEL (2003), S. 56-68, JOHANNING (1998), S. 104-114 und LISTER (1997), S. 200-204.

395 Vgl. übereinstimmend GRÜNDL/SCHMEISER (2002), S. 816.

396 „Properly designed performance measures and executive incentive compensation schemes are central to the value creation process. Their purpose ist straightforward - to motivate managers to create value by rewarding them for the value created." RAPPAPORT (1986), S. 171. Vgl. außerdem RAPPAPORT (1999) und SAITA (1999), S. 107-109.

397 Vgl. im Folgenden vertiefend HARTMANN-WENDELS/PFingSTEN/WEBER (2004), S. 96-104 und PERRIDON/STEINER (2004), S. 542-545.
} 
oder Eigentümer) nicht beobachtet werden kann. ${ }^{398}$ Unter der Annahme rationalen Verhaltens wird der Agent diesen Spielraum zur Maximierung seines individuellen Nutzens ausschöpfen. Die durch den Agenten getroffenen Entscheidungen müssen jedoch nicht zwangsläufig auch den Nutzen des Prinzipals maximieren. Die Differenz zwischen einer auf diese Weise entstehenden „Second-Best-Lösung“ und der „FirstBest-Lösung" entspricht den Kosten asymmetrischer Information, die auch als Agency-Kosten bezeichnet werden.

Als geeignetes Kooperationsdesign zur Reduzierung von Agency-Kosten können beispielsweise anreizkompatible Entlohnungsschemata implementiert werden. ${ }^{399}$ Die erfolgsabhängige Entlohnung dezentraler Verantwortungsbereiche basiert in der Praxis vielfach auf risikoadjustierten Performancemaßen, die den Bereichserfolg der abgelaufenen Periode messen und somit eine ex-post-Beurteilung des Bereichsmanagements vornehmen. ${ }^{400}$ Erklärtes Ziel ist es, die variable Entlohnung des Managers sowohl an den Risiko- als auch den Wertschöpfungsbeitrag seines Geschäftsbereiches zu koppeln und somit ex-ante eine positive Steuerungswirkung zu entfalten. Im versicherungsspezifischen Kontext könnte dies zur Folge haben, dass die einzelnen Steuerungseinheiten im Rahmen ihrer operativen Zeichnungspolitik eine sorgfältigere Risikoselektion vornehmen und lediglich risikoadäquat bepreiste Risiken in den Bestand aufnehmen. Der Einsatz risikoadjustierter Performancemaße im Rahmen von Anreiz- und Vergütungssystemen setzt jedoch streng genommen ein Prinzipal-Agenten-Modell voraus, welches die Steuerungswirkung von ex-post-Performancemaßen hinreichend konkretisiert und einer Quantifizierung zugänglich macht. ${ }^{401}$

Abschließend bleibt festzuhalten, dass in der betrieblichen Praxis durchaus relevante Anwendungsbereiche für Kapitalallokationsverfahren bestehen. Zwischen den grundlegenden Zielsetzungen einer dezentralen Performancesteuerung und zentralen Risikolimitierung ist ein Zielkonflikt zu konstatieren, der letztlich auf asymmetrischen Informationsstrukturen beruht. ${ }^{402}$ Wenngleich die risikoadjustierte Performancesteuerung einzelner Geschäftsbereiche mit erheblichen konzeptionellen Schwächen behaftet ist, kann diese doch in Kombination mit einem auf die Zielsetzungen des Gesamtunternehmens ausgerichteten Anreizsystem eine wertsteigernde Unternehmenspolitik induzieren. Insofern ist GRÜNDL/SCHMEISER ausdrücklich zuzustimmen, wenn sie in

\footnotetext{
398 Vgl. vertiefend zur Prinzipal-Agenten-Theorie BAMBERg/SPREMANN (1989) und JoST (2001).

399 Vgl. HARTMANN-WENDELS/PFInGSTEN/WEBER (2004), S. 102-103 und JOST (2001), S. 411-426.

400 Vgl. auch PFAFF (2004), S. 2, JosT (2001), S. 359-389 und GöX (2004), S. 27-55.

${ }^{401}$ Vgl. zu einer möglichen Vorgehensweise Pfaff (2004), S. 1-25, Göx (2004), S. 57-55 und STOUGHTON/ZECHNER (2004), S. 9-10.

402 Vgl. auch KINDER/STEINER/WILLINSKY (2001), S. 289.
} 
diesem Zusammenhang formulieren: „nicht das Steuerungssystem per se muss sinnvoll sein, sondern die dadurch in Gang gesetzten Handlungen. “403

\subsubsection{Anforderungen an die Allokation von Risikokapital}

Die im vorangegangenen Abschnitt diskutierten Zielsetzungen stellen jeweils spezifische Anforderungen an die verwendete Allokationsmethodik. ${ }^{404}$ Die in der Literatur diskutierten Allokationsverfahren müssen sich daher grundsätzlich an der Frage messen lassen, inwiefern sie geeignet sind, zur Erreichung der unternehmensseitig formulierten Zielsetzung einen Beitrag zu leisten. ${ }^{405} \mathrm{Im}$ Rahmen der vorliegenden Arbeit erfolgt die Beurteilung strategischer Handlungsoptionen unter der Zielfunktion $\mathrm{Ge}$ winnmaximierung unter Einhaltung eines vorgegebenen Sicherheitsniveaus. Aufgrund der geäußerten konzeptionellen Kritik wird ein dezentraler Steuerungsansatz über risikoadjustierte Performancemaße im weiteren Verlauf nicht mehr betrachtet, sondern die Vorteilhaftigkeit der zu untersuchenden Unternehmensstrategien stets anhand des auf der Unternehmensebene erzielten RORAC beurteilt. Für dieses Entscheidungskriterium ist demnach eine Kapitalallokation auf einzelne Teilkollektive nicht erforderlich. Wie in Kapitel 4.2.2 ausgeführt, gewinnt die Allokation von Risikokapital in der betrieblichen Praxis gleichwohl an Relevanz, sofern einzelnen Geschäftsbereichen explizite Risikolimite vorgegeben werden sollen oder aber asymmetrische Informationsstrukturen vorliegen, welche die Implementierung anreizkompatibler Vergütungssysteme nahe legen.

In Kapitel 4.2.1 wurde zudem deutlich, dass im Rahmen der Allokation eine willkürfreie Vorgehensweise zur Verrechnung kollektiver Risikoausgleichseffekte nicht existieren kann, da der Diversifikationseffekt $D_{R}$ in der Regel nicht linearer Natur ist und außerdem Veränderungen der Geschäftsstruktur simultan auf den gesamtkollektiven Risikoausgleich wirken. ${ }^{406}$ In der Literatur werden daher - dem Kriterienkatalog für kohärente Risikomaße vergleichbar - wünschenswerte Eigenschaften diskutiert, über die Allokationsverfahren gemeinhin verfügen sollten, um eine risikogerechte Verteilung des Risikokapitals sicherzustellen. ${ }^{407}$ Eine Allokationsmethode genügt dem Kriterium der Risikogerechtigkeit, sofern sie auf den spezifischen Beitrag eines Geschäftsbereichs zum Gesamtrisiko des Unternehmens abstellt. ${ }^{408}$

\footnotetext{
403 GRÜNDL/SCHMEISER (2002), S. 816.

404 VENTER formuliert in diesem Kontext: „The allocation method in the end depends on why you are allocating capital." VENTER (2004), S. 102.

405 Vgl. übereinstimmend GRÜNDL/SCHMEISER (2004b), S. 2-3.

406 Vgl. übereinstimmend ALBRECHT/KORYCIORZ (2004), S. 134-135.

407 Vgl. exemplarisch Valdez/CHERNiH (2003), S. 519-522, GRAUMANN/BAUM (2003), S. 421-457 und DENAULT (2001), S. 5-7.

$408 \mathrm{Vgl}$. AlBRECHT/KORYCIORZ (2004), S. 134.
} 
In der Diskussion um wünschenswerte Eigenschaften von Allokationsverfahren wird derzeitig dem Axiomensystem von DENAULT die größte Beachtung geschenkt. ${ }^{409}$ Dieses baut auf spieltheoretischen Überlegungen auf und wird nachfolgend im Überblick dargestellt. Es sei jedoch nachdrücklich betont, dass ein derartiges (mathematisch formuliertes) Axiomensystem für sich genommen noch keine abschließende Beurteilung von Allokationsverfahren erlaubt und hinsichtlich seiner „Risikogerechtigkeit“ immer subjektiver Kritik unterliegen wird. ${ }^{410}$ Es ist daher zusätzlich sicherzustellen, dass die verwendete Allokationsmethode kontextabhängig gewählt wird und einen messbaren Beitrag zur Erreichung übergeordneter Unternehmensziele (z.B. zur Steigerung des Unternehmenswertes) leistet. ${ }^{411}$

Nach DENAULT wird ein Allokationsverfahren als kohärent bezeichnet, sofern folgende vier Eigenschaften erfüllt sind: ${ }^{412}$

(D1) Vollständige Allokation $\sum_{\mathrm{k}=1}^{\mathrm{m}} \mathrm{RK}_{\mathrm{k}}=\sum_{\mathrm{k}=1}^{\mathrm{m}} \mathrm{R}\left(\mathrm{V}_{\mathrm{k}}\right)=\mathrm{R}(\mathrm{V})=\mathrm{RK}$

(D2) ,no undercut“

$$
\sum_{k \in T K} R\left(V_{k}\right) \leq R\left(\sum_{k \in T K} V_{k}\right) \quad \text { für alle } T K \subseteq M
$$

(D3) Symmetrie

$$
\mathrm{R}\left(\mathrm{V}_{\mathrm{k}}\right)=\mathrm{R}\left(\mathrm{V}_{1}\right)
$$

wenn $V_{k}=V_{1}$

(D4) Risikolose Allokation

$$
\mathrm{R}\left(\mathrm{V}_{\mathrm{k}}\right)=\mathrm{R}(\mathrm{c})=\mathrm{c}
$$$$
\text { wenn } V_{k}=c \geq 0
$$

Das Axiom der vollständigen Allokation besagt, dass der für das Gesamtportfolio ermittelte Risikokapitalbedarf in voller Höhe auf die betreffenden Teilkollektive aufgeteilt werden soll. Der Zuweisungsbetrag eines Segmentes k ergibt sich formal als:

$$
\mathrm{RK}_{\mathrm{k}}=\mathrm{x}_{\mathrm{k}} \cdot \mathrm{RK} \quad \text { mit } 0<\mathrm{x}_{\mathrm{k}}<1
$$

Um die Forderung nach vollständiger Allokation zu erfüllen, muss die Summe der ermittelten Allokationsfaktoren $x_{k}$ immer 1 betragen. ${ }^{413}$ Eine vollständige Allokation bedingt daher eine lineare Aufspaltung des in der Regel nicht linearen Diversifika-

\footnotetext{
$409 \mathrm{Vgl}$. Denault (2001) S. 5-7.

410 Vgl. auch KORYCIORZ (2004), S. 189 und ALBRECHT/KORYCIORZ (2004), S. 134.

411 Vgl. VENTER (2004), S. 102 und GRÜNDL/SCHMEISER (2004b), S. 2.

${ }^{412} \mathrm{Vgl}$. im Folgenden grundlegend Denault (2001) sowie HüRLIMANN (2001), S. 12-13, PANJER (2001), S. 4-5, DRESEL (2003), S. 73-75, VALDEZ/CHERNIH (2003), S. 21 und KORYCIORZ (2004), S. 189-193.

${ }^{413}$ Vgl. hierzu und im Folgenden KORYCIORZ (2004), S. 187 und ALBRECHT/KORYCIORZ (2004), S. 134
} 
tionseffektes. In Kapitel 4.2.1 wurde bereits kritisch bemerkt, dass eine derartige Vorgehensweise stets dem Vorwurf der Willkür, z.B. in Bezug auf das verwendete Allokationsrisikomaß, ausgesetzt ist.

Entsprechend dem Axiom des ",no undercut “ darf ein Geschäftsbereich k oder auch ein beliebiges Teilkollektiv TK aus diversen Geschäftsbereichen im Rahmen der Allokation nicht mehr Risikokapital zugeteilt bekommen, als wenn dieser/dieses als eigenständige Einheit betrachtet würde. ${ }^{414}$ Anderenfalls würde ein Geschäftsbereich im Portfolioverbund schlechter gestellt und der ökonomisch unerwünschte Anreiz gesetzt, sich vom Gesamtkollektiv abzuspalten. ${ }^{415}$ Ein derartiger Effekt wäre offensichtlich nicht mit der Forderung nach Risikogerechtigkeit vereinbar, da einer Steuerungseinheit ein Kapitalbetrag zugewiesen würde, welcher durch das originäre Geschäftsrisiko dieser Einheit nicht hinreichend erklärbar ist. Das Axiom des „no undercut" setzt somit voraus, dass das der Allokation zugrunde liegende Risikomaß über die Eigenschaft der Subadditivität verfügt. ${ }^{416}$ An dieser Stelle wird erneut deutlich, dass der CVaR als kohärentes, mithin auch subadditives Risikomaß im Rahmen der Risikosteuerung dem VaR überlegen ist.

Die Forderung nach Symmetrie basiert ursprünglich auf dem spieltheoretischen Begriff der Austauschbarkeit ${ }^{417}$ und zielt auf die Gleichbehandlung identischer Risiken im Rahmen des Allokationsprozesses ab. Demnach muss sichergestellt sein, dass zwei Teilkollektiven $\mathrm{k}$ und $\mathrm{l}$, welche durch identische Verlustverteilungen $\mathrm{V}_{\mathrm{k}}$ und $\mathrm{V}_{1}$ charakterisiert sind und darüber hinaus die gleiche stochastische Abhängigkeitsstruktur zum Restportfolio aufweisen, den gleichen Kapitalbetrag zugewiesen bekommen. Die Allokation darf somit ausschließlich von der Risikostruktur der betrachteten Geschäftsbereiche abhängen. ${ }^{418}$ Ausgeschlossen werden auf diese Weise insbesondere diejenigen Allokationsmethoden, bei denen die Höhe der Zuweisungsbeträge von der

${ }^{414}$ KORYCIORZ unterteilt das Axiom des „no undercut“ anschaulich in das „individuelle Exzessverbot“ und „kollektive Exzessverbot“. Demnach sind „Allokationsexzesse“, verstanden als positive Differenz zwischen alloziertem Risikokapital und Standalone-Kapital eines Geschäftsbereiches (oder einer beliebigen Kombination von Geschäftsbereichen) zu vermeiden. Vgl. KORYCIORZ (2004), S. 190.

415 Eine ökonomische Interpretation liefert HüRLIMANN: „No risk manager, or coalition of risk managers, can argue that it would be better off on its own than with the firm, and as a consequence request a lower risk allocation. For short, all the participants should benefit from a positive diversification effect." HÜRLIMANN (2001), S. 12.

416 Vgl. übereinstimmend DENAULT (2001), S. 4 und KORYCIORZ (2004), S. 191-192.

417 Vgl. zur spieltheoretischen Formulierung DENAULT (2001), S. 7 und darüber hinaus ALBRECHT/ KORYCIORZ (2004), S. 139-140 und MANDL (2004), S. 11-12.

418 „The symmetry property ensures that a portfolio`s allocation depends only on its contribution to risk within the firm, and nothing else." DENAULT (2001), S. 5. Vgl. außerdem KORYCIORZ (2004), S. 192, insbesondere dort Fußnote 544. 
Reihenfolge abhängt, in der die Geschäftsbereiche in das Gesamtportfolio eines Versicherungsunternehmens aufgenommen werden.

Gemäß dem Postulat der risikolosen Allokation muss schließlich gewährleistet sein, dass einem Geschäftsbereich, der einen deterministischen Verlust c erwirtschaftet, genau dieser Betrag als Risikokapital zugewiesen wird. Im Umkehrschluss muss sich ebenfalls das allozierte Kapital entsprechend um den Betrag c reduzieren, sofern ein Bereich in eine sichere Position c investiert. Dieses Begriffsverständnis legt die konzeptionelle Verwandtschaft zwischen der risikolosen Allokation und der in Bezug auf Risikomaße geforderten Translationsinvarianz (ADEH1) offen. ${ }^{419}$

Der besonderen Bedeutung stochastischer Abhängigkeitsstrukturen für die Risikokapitalbestimmung Rechnung tragend, wird in der Literatur außerdem gefordert, dass sich unterschiedliche Abhängigkeitsmuster, beispielsweise eine erhöhte Wahrscheinlichkeit für das gleichzeitige Auftreten von Schadenereignissen in zwei Geschäftsbereichen, in den Allokationsergebnissen widerspiegeln müssen. ${ }^{420}$ Dieser Forderung ist ausdrücklich zuzustimmen, da anderenfalls risikoerhöhende oder auch risikoreduzierende Verbundeffekte eines Bereichs mit dem restlichen Kollektiv im Rahmen der Steuerung gänzlich ignoriert würden.

Die in der Literatur diskutierten und in der Praxis verwendeten Allokationsverfahren sind sehr variantenreich und nur noch schwer zu überblicken. ${ }^{421} \mathrm{Im}$ folgenden $\mathrm{Ab}-$ schnitt sollen vier grundlegende Ansätze beschrieben werden, die letztlich den konzeptionellen Rahmen für zahlreiche weitere Varianten und Modifikationen aufspannen. ${ }^{422}$ Vor dem Hintergrund des nunmehr skizzierten Anforderungskatalogs werden diese Ansätze kritisch gewürdigt und hinsichtlich ihrer Eignung für die risikokapitalbasierte Steuerung beurteilt.

\footnotetext{
419 Vgl. vertiefend KORYCIORZ (2004), S. 193.

420 Vgl. AlbreCHT (1998), S. 245 und KORYCIORZ (2004), S. 197.

${ }^{421}$ Vgl. exemplarisch TiJs/Driessen (1986), MERTON/PEROLD (1993), CUMMINS (2000), KINDER/ STEINER/WILLINSKY (2001), MYERS/READ (2001), URBAN (2002), GRAUMANN/BAUM (2003), VALDEZ/CHERNIH (2003), VENTER (2004), MANDL (2004), ALBRECHT/KORYCIORZ (2004) und KORYCIORZ (2004).

${ }^{422}$ Die Systematisierung der Allokationsmethoden und die Notation ihrer Darstellung orientieren sich im Folgenden an den Arbeiten von ALBRECHT/KORYCIORZ (2004) und KORYCIORZ (2004), S. $175-268$.
} 


\subsubsection{Methoden der Risikokapitalallokation}

\subsubsection{Standalone-proportionale Allokation}

Die große Popularität proportionaler Allokationsverfahren liegt in ihrer einfachen und intuitiven Vorgehensweise begründet. Sämtlichen proportionalen Verfahren ist gemeinsam, dass sie das auf der Gesellschaftsebene erforderliche Risikokapital mit Hilfe von Allokationsfaktoren auf die einzelnen Geschäftsbereiche aufteilen. ${ }^{423}$ Hinsichtlich der verwendeten Risikomesskonzepte muss strikt differenziert werden zwischen dem bereits bekannten Risikomaß $R(V)$, welches den Gesamtkapitalbedarf des Unternehmens quantifiziert und dem Allokationsrisikoma $\beta R_{A}(V)$, welches ausschließlich zur Ermittlung der Allokationsfaktoren dient. Wenngleich $R(V)$ und $R_{A}(V)$ in der Regel übereinstimmen, d.h. sowohl der Gesamtkapitalbedarf als auch die Allokationsfaktoren mit Hilfe des gleichen Risikomaßes (z.B. CVaR) ermittelt werden, ist deren Identität nicht notwendige Voraussetzung für eine proportionale Allokation. ${ }^{424}$ Die Allokationsfaktoren $x_{k}$ entsprechen im Falle einer standalone-proportionalen Allokation dem Quotienten aus dem Standalone-Kapital des betreffenden Segments k und der Summe sämtlicher Standalone-Kapitalbeträge, formal:

$$
\mathrm{x}_{\mathrm{k}}=\frac{\mathrm{R}_{\mathrm{A}}\left(\mathrm{V}_{\mathrm{k}}\right)}{\sum_{\mathrm{l}=1}^{\mathrm{m}} \mathrm{R}_{\mathrm{A}}\left(\mathrm{V}_{1}\right)}
$$

$$
\mathrm{k}=1, \ldots, \mathrm{m}
$$

Die Zuweisungsbeträge je Segment ergeben sich demzufolge als:

$$
\mathrm{R}\left(\mathrm{V}_{\mathrm{k}}\right)=\mathrm{x}_{\mathrm{k}} \cdot \mathrm{R}(\mathrm{V})=\frac{\mathrm{R}_{\mathrm{A}}\left(\mathrm{V}_{\mathrm{k}}\right)}{\sum_{\mathrm{l}=1}^{\mathrm{m}} \mathrm{R}_{\mathrm{A}}\left(\mathrm{V}_{\mathrm{l}}\right)} \cdot \mathrm{R}(\mathrm{V}) \quad \mathrm{k}=1, \ldots, \mathrm{m}
$$

Den proportionalen Allokationsverfahren ist gemein, dass sie stets eine vollständige Allokation gewährleisten und darüber hinaus dem Axiom der Symmetrie genügen. ${ }^{425}$ Der im Portfolioverbund wirksame Diversifikationseffekt wird in vollem Umfang linear auf die Geschäftsbereiche aufgeteilt. Stochastische Abhängigkeiten zwischen den Bereichen werden allerdings nur bei der Bemessung des Gesamtkapitalbedarfs durch das Risikomaß R(V) berücksichtigt. Auf die Höhe der Allokationsfaktoren wirken sie

\footnotetext{
${ }^{423}$ Vgl. im Folgenden auch ALBRECHT (1998), S. 246, SCHRAdIN (2000), S. 108, VALDEZ/CHERNIH (2003), S. 520-522, ALBRECHT/KORYCIORZ (2004), S. 141-143 und insbesondere KORYCIORZ (2004), S. 194-202.

${ }^{424} \mathrm{Vgl}$. KORYCIORZ (2004), S. 195-196.

${ }^{425}$ Vgl. KORYCIORZ (2004), S. 195 und VALDEZ/CHERNIH (2003), S. 520-521.
} 
sich hingegen nicht aus, da diese ausschließlich auf der Basis der isoliert ermittelten Segmentkapitalien bestimmt werden. ${ }^{426}$ Im Hinblick auf die Steuerung bzw. Risikobudgetierung einzelner Geschäftsbereiche hat dies den ökonomisch unerwünschten Effekt zur Folge, dass risikoerhöhende Abhängigkeiten nicht durch verschärfte Kapitalzuweisungen sanktioniert bzw. wünschenswerte negative Abhängigkeiten nicht honoriert werden. ${ }^{427}$ Zudem kann gezeigt werden, dass im Rahmen standaloneproportionaler Allokationsverfahren die Einhaltung der Axiome des ,no undercut“ und der risikolosen Allokation nicht immer gewährleistet ist. ${ }^{428}$ Zusammenfassend bleibt somit festzuhalten, dass standalone-proportionale Allokationsverfahren schwerwiegende konzeptionelle Schwächen aufweisen und daher im Rahmen der Unternehmenssteuerung nicht zur Anwendung kommen sollten.

\subsubsection{Kovarianzbasierte Allokation}

In Bezug auf die Berücksichtigung von Abhängigkeitsstrukturen zwischen verschiedenen Steuerungseinheiten stellt die kovarianzbasierte Allokation („Kovarianzprinzip“) eine substanzielle Verbesserung dar. Das Kovarianzprinzip macht sich den Sachverhalt zunutze, dass die Varianz des Gesamtschadens eines Portfolios auch im Falle abhängiger Geschäftsbereiche linear in die Beiträge der einzelnen Bereiche aufgespalten werden kann. ${ }^{429}$ Die Varianz des Gesamtschadens $\sigma^{2}(\mathrm{~S})$ ergibt sich formal als:

$$
\sigma^{2}(S)=\sum_{k=1}^{m} \sum_{l=1}^{m} \operatorname{Cov}\left(S_{k}, S_{1}\right)=\sum_{k=1}^{m} \operatorname{Cov}\left(S_{k}, S\right)=\sum_{k=1}^{m}\left[\sigma^{2}\left(S_{k}\right)+\sum_{i \neq k} \operatorname{Cov}\left(S_{k}, S_{1}\right)\right]
$$

Dabei bezeichnet $\operatorname{Cov}\left(\mathrm{S}_{\mathrm{k}}, \mathrm{S}\right)$ die Kovarianz des k-ten Segments mit dem Restportfolio. Die letzte Umformung von Beziehung (4.19) legt den jeweiligen Beitrag eines Segments zur Varianz des Gesamtschadens offen. Dieser Beitrag setzt sich einerseits aus der Eigenvarianz des Segments $\sigma^{2}\left(S_{k}\right)$ und andererseits der Summe der Kovarianzen mit den übrigen Segmenten zusammen. Die Allokationsfaktoren $x_{k}$ ergeben sich durch

\footnotetext{
${ }^{426} \mathrm{Vgl}$. Albrecht/KORYCIORZ (2004), S. 142.

${ }^{427}$ Vgl. übereinstimmend StOughton/ZeCHNER (2000), S. 889 und KORYCIORZ (2004), S. 196.

${ }^{428}$ Ein konkretes Zahlenbeispiel zum Verstoß proportionaler Allokationsmaße gegen das kollektive Exzessverbot formulieren ALBRECHT/KORYCIORZ (2004), S. 142-143. KORYCIORZ untersucht außerdem exemplarisch den Einfluss unterschiedlicher Allokationsrisikomaße auf das standaloneproportionale Allokationsergebnis. Vgl. KORYCIORZ (2004), S. 197-202.

${ }^{429}$ Unter der Annahme, dass die Stochastizität der Verlustvariablen V lediglich durch das Schadengeschehen beeinflusst wird, sind die Varianzen der Schaden- bzw. Verlustvariablen identisch, formal: $\sigma^{2}(\mathrm{~S})=\sigma^{2}(\mathrm{~V})$. Vgl. KORYCIORZ (2004), S. 205. Im Hinblick auf die Modellierung würde dies bedeuten, dass sonstige Ergebniskomponenten, wie z.B. Prämien oder Betriebskosten, als deterministisch angenommen werden.
} 
Normierung der einzelnen Segmentbeiträge zur Varianz des Gesamtschadens und sind offenbar unabhängig von der Wahl eines konkreten Risikomaßes R. ${ }^{430}$

$$
\mathrm{x}_{\mathrm{k}}=\frac{\sum_{\mathrm{l}=1}^{\mathrm{m}} \operatorname{Cov}\left(\mathrm{S}_{\mathrm{k}}, \mathrm{S}_{1}\right)}{\sigma^{2}(\mathrm{~S})}=\frac{\operatorname{Cov}\left(\mathrm{S}_{\mathrm{k}}, \mathrm{S}\right)}{\sigma^{2}(\mathrm{~S})} \quad \mathrm{k}=1, \ldots, \mathrm{m}
$$

Die Allokationsfaktoren weisen eine enge konzeptionelle Verwandtschaft zum Betafaktor des Capital Asset Pricing Model (CAPM) auf, weshalb in der Literatur auch die Bezeichnung „interne Betas“ einschlägig ist. ${ }^{431}$ Im Falle einer negativen Abhängigkeit zwischen Geschäftsbereich und Restportfolio nimmt der Allokationsfaktor einen negativen Wert an. ${ }^{432}$ Zur Vermeidung negativer Zuweisungsbeträge wird das Kovarianzprinzip bisweilen dahingehend modifiziert, dass den Steuerungseinheiten zunächst deren erwarteter Segmentverlust $E\left(V_{k}\right)$ zugewiesen wird und in einem zweiten Schritt der über den erwarteten Gesamtverlust hinausgehende Kapitalbedarf anhand der Allokationsfaktoren aus Beziehung (4.20) verteilt wird. ${ }^{433}$

$$
\mathrm{R}\left(\mathrm{V}_{\mathrm{k}}\right)=\mathrm{E}\left(\mathrm{V}_{\mathrm{k}}\right)+\mathrm{x}_{\mathrm{k}} \cdot[\mathrm{R}(\mathrm{V})-\mathrm{E}(\mathrm{V})] \quad \mathrm{k}=1, \ldots, \mathrm{m}
$$

Während die Axiome der vollständigen Allokation und Symmetrie als erfüllt angesehen werden können, verstößt das Kovarianzprinzip gegen das Postulat des „,no undercut" und stellt darüber hinaus keine risikolose Allokation sicher. ${ }^{434}$ Letztgenannter Verstoß wird evident, sofern man sich vor Augen führt, dass der Allokationsfaktor für deterministische Verluste immer den Wert Null annimmt. Kritisch ist außerdem festzuhalten, dass die Allokationsfaktoren im Zeitablauf nicht als stabil angenommen werden können, da sie vom Volumen des einzelnen Segments im Verhältnis zum Gesamtkollektiv abhängen. ${ }^{435}$ Überproportionales Wachstum einzelner Geschäftsbereiche

\footnotetext{
${ }^{430}$ Vgl. Hesselager/ANDERSSON (2002), S. 10, VALDEZ/CHERNiH (2003), S. 8, AlBRECHT/KoRYCIORZ (2004), S. 143 und KORYCIORZ (2004), S. 206.

431 Vgl. exemplarisch KORYCIORZ (2004), S. 206 und STOUghtON/ZECHNER (2000), S. 889-892.

${ }^{432}$ Das Auftreten negativer Zuweisungsbeträge ist gleichwohl nur als theoretischer Grenzfall einzustufen. In der Praxis sind vielmehr positive Abhängigkeiten zwischen einzelnen Versicherungsbeständen (oder allenfalls Unabhängigkeit der Einzelrisiken) anzutreffen. Vgl. übereinstimmend MACK (2002), S. 32.

433 Vgl. hierzu AlBreCHT/KORYCIORZ (2004), S. 143 und KORYCIORZ (2004), S. 213. Eine derartige Vorgehensweise setzt ein Risikomaß voraus, dass die Erfüllung der Bedingung $R(V)>E(V)$ gewährleistet.

$434 \mathrm{Vgl}$. im Folgenden KORYCIORZ (2004), S. 207.

$435 \mathrm{Vgl}$. ALBRECHT (1998), S. 248.
} 
nimmt daher unmittelbar Einfluss auf die Kapitalzuweisungen sämtlicher Bereiche. ${ }^{436}$ Abschließend sei in Erinnerung gerufen, dass die durch das Kovarianzprinzip erfassten linearen Abhängigkeiten nur unter der Annahme elliptischer Verteilungen eine vollständige Abbildung der Risikostruktur erlauben. ${ }^{437}$ Die Eingrenzung auf die elliptische Verteilungsklasse (z.B. die Normalverteilung) kann jedoch gerade im versicherungsspezifischen Kontext als sehr restriktive Prämisse gewertet werden.

\subsubsection{Inkrementelle Allokation}

Die Grundidee der standalone-proportionalen und kovarianzbasierten Allokationsverfahren bestand darin, das auf der Unternehmensebene notwendige Risikokapital anhand von Allokationsfaktoren $\mathrm{x}_{\mathrm{k}}$ auf die Geschäftsbereiche zu verteilen. Die inkrementelle Allokation basiert auf einem grundlegend anderen methodischen Ansatz, der auf die Ermittlung von Allokationsfaktoren gänzlich verzichtet. Vielmehr werden die einzelnen Allokationsbeträge durch Differenzbildung ermittelt. Der Zuweisungsbetrag eines Geschäftsbereichs ergibt sich, indem von der Höhe des gesamtkollektiven Risikokapitals derjenige Kapitalbedarf abgezogen wird, welcher für das Kollektiv unter Ausschluss des betreffenden Segments resultieren würde. ${ }^{438}$

$$
\mathrm{R}\left(\mathrm{V}_{\mathrm{k}}\right)=\mathrm{R}(\mathrm{V})-\mathrm{R}\left(\mathrm{V}-\mathrm{V}_{\mathrm{k}}\right)
$$

Es sei an dieser Stelle angemerkt, dass in der Literatur zwei methodische Herangehensweisen anzutreffen sind, die jedoch beide auf der oben beschriebenen Grundidee beruhen. ${ }^{439}$ MERTON/PEROLD legen ihrer With-Without-Betrachtung Portfolioveränderungen zugrunde, die ganze Geschäftsbereiche betreffen. Demgegenüber analysieren MYERS/READ lediglich infinitesimale Veränderungen der Portfoliostruktur. ${ }^{440}$ Während in der englischsprachigen Literatur vielfach beide Methoden unter dem Stichwort der ,marginal capital allocation“ subsumiert werden, soll hier der Terminologie von ALBRECHT/KORYCIORZ bZw. VENTER gefolgt werden, die zwischen inkrementeller

${ }^{436}$ Ein anschauliches Zahlenbeispiel zu diesem Sachverhalt findet sich bei KORYCIORZ (2004), S. 209-210.

${ }^{437} \mathrm{Vgl}$. in diesem Zusammenhang insbesondere die Ausführungen in Kapitel 6.2.2.

438 Vgl. auch CuMmins (2000), S. 12-13, STOUGHTON/ZECHNER (2000), S. 892-894, URBAN (2002), S. 38, GRAUMANN/BAUM (2003), S. 437, ALBRECHT/KORYCIORZ (2004), S. 136 und KORYCIORZ (2004), S. 219.

439 Vgl. im Folgenden MERTON/PEROLD (1993) und MYERS/READ (2001).

440 Beide Methoden basieren in ihrer Originalfassung auf dem optionspreistheoretischen Risikomaß der ,insolvency put option“, können jedoch auch auf andere Risikomesskonzepte angewendet werden. Vgl. in diesem Zusammenhang vertiefend VENTER (2004), S. 103-106 und CUMMINS (2000), S. 12-13. 
und marginaler Allokation unterscheiden. ${ }^{441}$ Letztgenannte Methode stellt eine Grenzbetrachtung im Sinne einer mathematischen Differentiation dar und entspricht daher eher einer Sensitivitätsanalyse als einer Allokationsmethode. ${ }^{442}$ Die Ausführungen dieses Abschnitts beziehen sich daher ausschließlich auf die inkrementelle Allokation von Risikokapital. Im Sinne des Kriterienkatalogs von DENAULT ist zu konstatieren, dass die inkrementelle Methode gegen das Axiom der vollständigen Allokation verstößt. Die Summe der allozierten Risikokapitalbeträge entspricht in der Regel nicht dem Gesamtkapitalbedarf. ${ }^{443}$ Für den realistischen Fall nicht perfekt positiv korrelierter Geschäftsbereiche entsteht daher eine Allokationslücke, die sich mathematisch formulieren lässt als:

$$
\mathrm{AL}(\mathrm{V})=\mathrm{R}(\mathrm{V})-\sum_{\mathrm{k}=1}^{\mathrm{m}} \mathrm{R}\left(\mathrm{V}_{\mathrm{k}}\right)>0
$$

Konzeptionell lässt sich die Allokationslücke dadurch erklären, dass im Rahmen der inkrementellen Allokation dem jeweils betrachteten Geschäftsbereich der gesamte durch dessen Beitritt induzierte Diversifikationseffekt zugeordnet wird. ${ }^{444}$ Über sämtliche Geschäftsbereiche hinweg führt dieses Vorgehen folglich zu einer mehrfachen Verrechnung des Diversifikationseffektes. ${ }^{445}$ Zur Vermeidung bzw. zur Schließung der Allokationslücke wird in der einschlägigen Literatur eine Vielzahl von Verfahren diskutiert, auf die an dieser Stelle jedoch nicht im Detail eingegangen werden soll. ${ }^{446}$ Beispielsweise wird das inkrementelle Verfahren dahingehend modifiziert, dass eine schrittweise Ausweitung des Gesamtportfolios angenommen wird. Jedem neu hinzukommenden Geschäftsbereich wird jeweils das zusätzlich erforderliche Kapital zugewiesen. Zwar kann auf diese Weise eine vollständige Allokation gewährleistet werden, jedoch ist das Allokationsergebnis in hohem Maße von der Reihenfolge abhängig, in der die einzelnen Teilkollektive in das Portfolio aufgenommen werden. Eine willkürfreie Allokation ist demzufolge nicht gewährleistet. Außerdem kann dasjenige Teilkollektiv, welches sich als erstes im Portfolio befindet, nicht an möglichen Risikoausgleicheffekten partizipieren, da es stets sein Standalone-Kapital zugewiesen be-

\footnotetext{
441 Vgl. Albrecht/KORYCIORZ (2004), S. 136-138 und VENTER (2004), S. 98.

442 Vgl. übereinstimmend ALBRECHT/KORYCIORZ (2004), S. 138.

443 Vgl. MERTON/PEROLd (1993), S. 28, STOUGHTON/ZECHNER (2000), S. 893, GRÜNDL/SCHMEISER (2002), S. 810 und GraumanN/BAUm (2003), S. 437.

444 Vgl. übereinstimmend StOughton/ZeCHNER (2000), S. 893, AlbreCHT/KoRYCIORZ (2004), S. 137 und KORYCIORZ (2004), S. 221.

$445 \mathrm{Vgl}$. zu einem einfachen Rechenbeispiel ALBRECHT/KORYCIORZ (2004), S. 136-137.

446 Vgl. hierzu insbesondere KORYCIORZ (2004), S. 223-245 sowie TIJS/DRIESSEN (1986), KINDER/ STEINER/WILLINSKY (2001), URBAN (2002) und MANDL (2004).
} 
kommt. ${ }^{447}$ Als prominente Erweiterung dieses Ansatzes ist der so genannte ShapleyWert zu nennen. ${ }^{448}$ Dieses spieltheoretisch fundierte Verfahren berücksichtigt - in verkürzter Darstellung - nicht nur eine, sondern sämtliche möglichen Beitrittsreihenfolgen und berechnet die Zuweisungsbeträge als Erwartungswert über die $m$ ! denkbaren Kombinationen von $\mathrm{m}$ Geschäftsbereichen.

Für operative Anwendungsgebiete, wie z.B. die Performancesteuerung, ist das inkrementelle Allokationsverfahren aufgrund der unvollständigen Zuweisung des Gesamtkapitalbedarfs ungeeignet, da durchaus Fälle auftreten können, in denen jede Steuerungseinheit ihre Renditevorgabe erfüllt und trotzdem die seitens der Kapitalgeber geforderte Mindestrendite (auf das Gesamtkapital) verfehlt wird. ${ }^{449}$ Darüber hinaus sind auch negative Zuweisungsbeträge nicht gänzlich auszuschließen. ${ }^{450}$ Für strategische Fragestellungen wird die inkrementelle Allokation hingegen in der Literatur durchgängig als risikoadäquat eingestuft. ${ }^{451}$ GRÜNDL/SCHMEISER weisen sogar nach, dass Allokationsverfahren, die stets eine vollständige Allokation sicherstellen, zu systematischen strategischen Fehlentscheidungen über den Auf- bzw. Abbau von Geschäftsbereichen führen können. ${ }^{452}$ Abschließend bleibt festzuhalten, dass inkrementelle Allokationsverfahren im Kontext strategischer Entscheidungskalküle (,Soll das Geschäftssegment $\mathrm{X}$ ausgeweitet oder das Segment $\mathrm{Y}$ veräußert werden?") durchaus mit der übergeordneten Zielsetzung der Unternehmenswertsteigerung kompatibel sind. Im Hinblick auf die in Kapitel 4.2.2 diskutierten Zielsetzungen, wie z.B. die Vergabe von Risikolimiten, sind inkrementelle Verfahren hingegen ungeeignet, da sie keine vollständige Allokation sicherstellen. Vor diesem Hintergrund wird im folgenden Kapitel ein Allokationsverfahren vorgestellt, welches sowohl für operative als auch strategische Fragestellungen eine konsistente Entscheidungsgrundlage darstellt.

\subsubsection{Allokation nach dem CVaR-Konzept}

Eine Allokation von Risikokapital auf der Grundlage des CVaR fußt auf einer ausschließlich risikotheoretisch motivierten Vorgehensweise. In Kapitel 4.1.3.3 wurde gezeigt, dass der CVaR als Anwendungsfall des Konzepts bedingter Erwartungswerte

\footnotetext{
447 Vgl. hierzu vertiefend ALBRECHT/KORYCIORZ (2004), S. 137 und KORYCIORZ (2004), S. 238-239.

448 Vgl. im Folgenden grundlegend SHAPLEY (1971) und im versicherungsspezifischen Kontext Philbrick/PAINTER (2001), S. 125, URBAN (2002), S. 40-68, MANDL (2004), S. 23-24, ALBRECHT/KORYCIORZ (2004), S. 152 und KORYCIORZ (2004), S. 240-245.

$449 \mathrm{Vgl}$. auch KORYCIORZ (2004), S. 222.

450 Beispiele hierzu sind u.a. bei PHILBRICK/PAINTER (2001), S. 130 und KORYCIORZ (2004), S. 222$223 \mathrm{zu}$ finden.

451 Vgl. Merton/Perold (1993), S. 28-30, Cummins (2000), S. 24-25, Stoughton/Zechner (2000), S. 892-894, VENTER (2004), S. 102 und KORYCIORZ (2004), S. 221.

452 Vgl. GRÜNDL/SCHMEISER (2002), S. 809-815.
} 
aufgefasst werden kann. Erwartungswerte weisen die statistisch wünschenswerte Eigenschaft der Additivität auf, die eine lineare Zerlegung in einzelne Summanden erlaubt. ${ }^{453}$ Aufgrund der Linearitätseigenschaft bedingter Erwartungswerte lässt sich der CVaR ebenfalls additiv aufspalten. Formal ist der CVaR definiert als: ${ }^{454}$

$$
\mathrm{CVaR}_{\varepsilon}(\mathrm{V})=\mathrm{E}\left[\mathrm{V} \mid \mathrm{V} \geq \operatorname{VaR}_{\varepsilon}(\mathrm{V})\right]=\sum_{\mathrm{k}=1}^{\mathrm{m}} \mathrm{E}\left[\mathrm{V}_{\mathrm{k}} \mid \mathrm{V} \geq \operatorname{VaR}_{\varepsilon}(\mathrm{V})\right]
$$

Aus der letzten Umformung von Beziehung (4.24) wird ersichtlich, in welcher Höhe die einzelnen Geschäftsbereiche im Falle einer Überschreitung des VaR durchschnittlich zum Gesamtverlust des Unternehmens beitragen. ${ }^{455}$ Die jeweiligen Summanden $\mathrm{E}\left[\mathrm{V}_{\mathrm{k}} \mid \mathrm{V} \geq \mathrm{VaR}_{\varepsilon}(\mathrm{V})\right]$ können unmittelbar als Risikokapitalbetrag jedes einzelnen Geschäftsbereichs aufgefasst werden. Dies lässt die intuitive Interpretation zu, dass jedem Bereich genau soviel Kapital zugewiesen wird, wie dieser im Erwartungswert zu Verlusten jenseits des VaR beiträgt. ${ }^{456}$ Aus der linearen Zerlegung des Gesamtkapitalbedarfs in einzelne Summanden resultieren folgende Allokationsfaktoren: ${ }^{457}$

$$
\mathrm{x}_{\mathrm{k}}=\frac{\mathrm{E}\left[\mathrm{V}_{\mathrm{k}} \mid \mathrm{V} \geq \mathrm{VaR}_{\varepsilon}(\mathrm{V})\right]}{\mathrm{E}\left[\mathrm{V} \mid \mathrm{V} \geq \operatorname{VaR}_{\varepsilon}(\mathrm{V})\right]} \quad \mathrm{k}=1, \ldots, \mathrm{m}
$$

Mit Blick auf die Axiomatik kohärenter Kapitalallokation ist bereits aus Beziehung (4.24) erkennbar, dass sowohl die Forderung nach vollständiger und risikoloser Allokation als auch die Symmetrieeigenschaft jederzeit erfüllt sind. ${ }^{458}$ Darüber hinaus kann der Nachweis erbracht werden, dass das CVaR-Konzept dem Axiom des ,no undercut" sowohl in seiner schwachen Formulierung (individuelles Exzessverbot) als auch in Bezug auf beliebige Kombinationen von Geschäftsbereichen (kollektives Exzessverbot) genügt. ${ }^{459}$ Als kohärentes Allokationsverfahren ist das CVaR-Konzept demzufolge den bislang vorstellten Verfahren überlegen.

Aufgrund der Additivitätseigenschaft von (bedingten) Erwartungswerten stellt das CVaR-Konzept außerdem sicher, dass das Allokationsergebnis von der hierarchischen

\footnotetext{
${ }^{453}$ Vgl. auch BoHLEY (2000), S. 122-131 und SCHMIDT (2002), S. 78-79.

454 Vgl. im Folgenden ALBRECHT/KORYCIORZ (2004), S. 146 und KORYCIORZ (2004), S. 249.

455 Vgl. übereinstimmend KORYCIORZ (2004), S. 250 und VENTER (2004), S. 102.

456 PANJER formuliert in diesem Kontext zutreffend: „This formula ist not only simple, it is also intuitive. The capital required for each risk is precisely the expected contribution to the shortfall when a shortfall occurs." PANJER (2001), S. 5. Vgl. darüber hinaus OVERBECK (2000), S. 10.

457 Vgl. Albrecht/Koryciorz (2004), S. 147 und KORYCIORZ (2004), S. 249.

458 Vgl. hierzu und im Folgenden KORYCIORZ (2004), S. 247 und S. 251.

459 Vgl. zum formalen Nachweis der Kohärenz ALBRECHT/KORYCIORZ (2004), S. 154-155.
} 
Organisationsstruktur eines Versicherungsunternehmens, mithin auch von der Art und Reihenfolge der Kollektivbildung unberührt bleibt. ${ }^{460}$ Dieses Gütekriterium wird in der Literatur auch als Aggregationskonsistenz (,consistency“) bezeichnet und geht im Ursprung auf HESSELAGER/ANDERSSON zurück. ${ }^{461}$ Gerade im Kontext einer konzernweiten Rendite-Risikosteuerung kommt dieser Eigenschaft elementare Bedeutung zu.

Als Schwachpunkt der bisherigen Allokationsverfahren wurde herausgestellt, dass stochastische Abhängigkeitsstrukturen gar nicht oder nur unzureichend erfasst werden können. Auch unter diesem Aspekt ist das CVaR-Konzept als überlegen einzustufen. Neben linearen Abhängigkeiten gehen erstmals auch nicht-lineare (Tail-) Abhängigkeiten in das Allokationsergebnis ein. Sofern also eine erhöhte Wahrscheinlichkeit besteht, dass in zwei Geschäftsbereichen simultan extreme Schadenrealisationen auftreten, wird diesen Bereichen durch die CVaR-basierte Allokationsmethode ein erhöhter Kapitalbedarf zugeordnet. ${ }^{462}$

Zusammenfassend ist festzuhalten, dass das CVaR-Konzept aufgrund seiner konzeptionellen Überlegenheit und intuitiven Interpretationsfähigkeit uneingeschränkt als $\mathrm{Al}$ lokationsmethode befürwortet werden kann. ${ }^{463}$ Wenngleich die in Beziehung (4.25) formulierten Allokationsfaktoren im Grundsatz auf unterschiedliche Risikomesskonzepte angewendet werden können, ist die Erfüllung der skizzierten Güteeigenschaften an die Voraussetzung geknüpft, dass die Risikokapitalbestimmung für das Gesamtunternehmen auf der Basis des CVaR erfolgt. Im weiteren Verlauf dieser Arbeit kommt daher durchgängig eine CVaR-basierte Allokationsmethodik zum Einsatz.

In dem nunmehr abgeschlossenen Kapitel 4 wurden die theoretischen Grundlagen der Risikomessung, Risikokapitalermittlung und Risikokapitalallokation erarbeitet. Die beschriebenen Wirkungszusammenhänge bilden das notwendige Fundament für die Entwicklung interner Unternehmensmodelle zur Rendite-Risikosteuerung. Im Mittelpunkt des nachfolgenden Hauptkapitels steht die Konzeption und praxistaugliche Ausgestaltung eines derartigen Modellansatzes für den Anwendungskontext der Schadenund Unfallversicherung. Den Ausgangspunkt der Überlegungen bilden zunächst modelltheoretische Grundlagen sowie Anforderungen an die Modellierung. Die darauf

\footnotetext{
460 Vgl. übereinstimmend KORYCIORZ (2004), S. 251-252.

461 Vgl. HeSSELAGER/ANDERSSON (2002), S. 11. Die Einhaltung der Aggregationskonsistenz kann auch für das kovarianzbasierte Allokationsverfahren nachgewiesen werden. Vgl. VALDEZ/CHERNIH (2003), S. 9-10.

${ }^{462}$ Konkrete Zahlenbeispiele zum Einfluss linearer und nicht-linearer Abhängigkeitsstrukturen auf das Allokationsergebnis sind bei KORYCIORZ (2004), S. 253-255 und in Kapitel 6.2.2 dieser Arbeit zu finden.

${ }^{463}$ Vgl. übereinstimmend PANJER (2001), S. 5 und insbesondere KORYCIORZ (2004), S. 257.
} 
folgenden Ausführungen lassen sich in zwei Hauptteile aufgliedern. Der Schwerpunkt der Darstellung liegt zunächst auf der Modellierung des versicherungstechnischen Kerngeschäfts. Der hohen Bedeutung der Versicherungstechnik Rechnung tragend, wird diesem Abschnitt die größte Aufmerksamkeit gewidmet. Im Anschluss daran wird die Modellierung des Kapitalanlagegeschäfts beschrieben. 
Mirko Tillmann - 978-3-631-75327-9

Downloaded from PubFactory at 01/11/2019 06:10:15AM

via free access 


\section{Entwicklung eines praxistauglichen Modellansatzes}

\subsection{Grundlagen der Modellierung}

\subsubsection{Klassifizierung von Modellen}

Betriebswirtschaftliches Denken und Handeln beruhen vielfach auf Modellvorstellungen, die als adäquate Abbilder einer ökonomisch relevanten Problemsituation aufgefasst werden können. ${ }^{464}$ Bevor im Folgenden betriebswirtschaftliche Modelle anhand eines Kriterienrasters klassifiziert werden, erscheint es zweckmäßig, zunächst über den Begriff des „Modells“ Klarheit zu gewinnen und ihn in der Weise zu definieren, wie er im Rahmen dieser Arbeit verwendet werden soll.

Ein Modell stellt die strukturerhaltende Abbildung eines realen Sachverhaltes dar, wobei sich die Abbildung sowohl auf die Elemente und Eigenschaften des realen Systems als auch auf die Beziehungen der Elemente untereinander bezieht. ${ }^{465}$ Die Auswahl der explizit modellierten Sachverhalte hängt zudem entscheidend von der Zwecksetzung der Modellanalyse ab und determiniert auf diese Weise den Abstraktionsgrad des Modells. Neben dem Anspruch auf Vereinfachung wird an ein wissenschaftlich nutzbares Modell zudem die Forderung gestellt, dass dieses die realen Gegebenheiten strukturgleich oder zumindest strukturähnlich abbildet. ${ }^{466}$ Nur so kann sichergestellt werden, dass sich aus den Modellergebnissen auch Rückschlüsse auf die Wirklichkeit ableiten lassen. Die in der Literatur ${ }^{467}$ vereinzelt noch geforderte Isomorphie, d.h. eine eineindeutige Äquivalenzrelation zwischen Modell und Originaldaten, ist einer praxistauglichen Modellierung jedoch abträglich. Ein zentraler Nutzen des Modellbildungsprozesses besteht gerade darin, durch die Reduzierung auf zentrale Ursache-WirkungsBeziehungen Erkenntnisse zu vermitteln, zu denen man bei der Analyse des komplexen Realsystems nicht gelangt wäre. ${ }^{468}$ Aus diesem Grunde werden Modelle in dieser Arbeit als homomorphe, d.h. mehreindeutige und strukturerhaltende Abbilder der Realität verstanden. ${ }^{469}$

${ }^{464}$ Vgl. im Folgenden zum Modellbegriff der betriebswirtschaftlichen Entscheidungslehre BERENS/ DELFMANN/SCHMitTING (2004), S. 21-27, BAMBERG/COENENBERG (2002), S. 13 ff., ADAM (1996), S. 60, RiEPER (1992), S. 17 ff., SCHNEEWEIB (1984), S. 480 ff. und EICHHORN (1979), S. 64-75. Zur branchenspezifischen Anwendung auf Finanzdienstleistungsunternehmen vgl. KOPP (1992), S. $88 \mathrm{ff}$. und BROHM (2002), S. $15 \mathrm{ff}$.

${ }^{465}$ Unter einem System wird grundsätzlich die Gesamtheit von Elementen verstanden, deren Beziehungen untereinander durch eine Menge von Relationen dargestellt werden können. Die Beziehungen zwischen den Elementen stellen die Struktur eines Systems dar. Vgl. BERENS/DELFMANN/ SCHMITTING (2004), S. 21-22. Vgl. vertiefend zur Systemtheorie und Systemforschung HaNSSMANN (1987) und MEYER (1996).

466 Vgl. exemplarisch ADAM (1996), S. 60-64 und RIEPER (1992), S. 19-22.

467 Vgl. DINKELBACH (1977).

468 Vgl. zur „Reduktionskette der Modellbildung“ vertiefend SCHNEEWEIB (1984), S. 480.

469 Vgl. zu einem analogen Modellverständnis ADAM (1996), S. 60 und RIEPER (1992), S. 19-22. 
Betriebswirtschaftliche Modelle dienen unterschiedlichen Zwecken und Zielsetzungen. Entsprechend kann grundlegend zwischen folgenden Modelltypen unterschieden werden: $:^{470}$

- Beschreibungsmodelle

- Erklärungsmodelle

- Prognosemodelle

- Entscheidungsmodelle

Beschreibungsmodelle können in der Betriebswirtschaftslehre auf eine lange Geschichte zurückblicken. Sie werden auch als Erfassungs- oder Ermittlungsmodelle bezeichnet. ${ }^{471}$ Ein populäres Anwendungsbeispiel für Beschreibungsmodelle ist das Rechnungswesen, mit dem quantitative Größen zunächst erfasst und dann mittels arithmetischer Operationen in eine andere Form transformiert werden. Dieses Beispiel verdeutlicht bereits, dass Beschreibungsmodelle lediglich komplexe Sachverhalte in handhabbarere Formen überführen, ohne jedoch Hypothesen über Wirkungszusammenhänge zu postulieren. Sie liefern darüber hinaus erst dann verwertbare Erkenntnisse, wenn ihre Ergebnisse im Zeitablauf oder zwischenbetrieblich verglichen werden. ${ }^{472}$ Die Beschreibung eines Problems hat nur dann ökonomischen Erkenntniswert, wenn sie valide und reliabel ist. ${ }^{473}$ Reliabilität kann in diesem Kontext als Maßstab für die Zuverlässigkeit des Beschreibungsmodells interpretiert werden, d.h. dass Wiederholungen eines Abbildungsvorgangs den gleichen Wert liefern. Ein Modell ist hingegen valide, sofern die Modellergebnisse die zuvor definierten Merkmale hinreichend genau repräsentieren.

Erklärungsmodelle greifen vielfach auf eine empirische Datenbasis zurück und versuchen, Hypothesen über Wirkungszusammenhänge und Gesetzmäßigkeiten eines modellierten Systems zu formulieren. ${ }^{474}$ Die formale Struktur von Erklärungsmodellen

470 Vgl. zu einer zweckorientierten Kategorisierung von Modellen im Folgenden HELTEN (1985), S. 118 ff., REICHMANN (2001), S. 56 ff., BROHM (2002), S. 15 ff. und BAMBERG/COENENBERG (2002), S. 15.

471 Vgl. im Folgenden auch RIEPER (1992), S. 88-89.

472 Vgl. hierzu vertiefend REICHMANN (2001), S. 59 f.

473 Vgl. im Folgenden HAAS (1991), S. 119-132, LIEBL (1995), S. 203-214 und HILLIER/LIEBERMAN (1997), S. 20-22 und S. 724-741.

474 „Unter Erklärung im klassischen Sinne versteht man die Deduktion einer Aussage aus singulären und universellen Sätzen, die den zu klärenden Tatbestand abbilden." REICHMANN (2001), S. 61. Es sei jedoch angemerkt, dass neben der hier genannten deduktiven Vorgehensweise in der wissenschaftlichen Praxis vielfach auch eine induktive Schlussweise anzutreffen ist. Die Induktion versucht, durch Extrapolation von beobachteten Einzelfällen auf allgemeine Zusammenhänge zu schließen. Diese Vorgehensweise kann jedoch nur der Entdeckung von Hypothesen dienen und ist daher zur wissenschaftstheoretischen Rechtfertigung ungeeignet. Vgl. hierzu auch KERN (1979). 
ergibt sich daher durch die Verknüpfung von zu erklärenden Größen (Explanandum) und erklärenden Größen (Explanans). ${ }^{475}$

Prognosemodelle hingegen bauen auf den Ergebnissen der Erklärungsmodelle auf und nutzen die ermittelten Zusammenhänge, um zukünftige Entwicklungen vorherzusagen. ${ }^{476}$ Sie können auch als Erklärungsmodelle aufgefasst werden, wobei sich zumindest die zu erklärende Variable auf einen zukünftigen Zeitpunkt oder Zeitraum bezieht. Eine belastbare Prognose setzt daher hinreichende Kenntnisse über die den Sachverhalt beeinflussenden Größen voraus.

Beschreibungs-, Erklärungs- und Prognosemodelle werden in der Literatur häufig zur Gruppe der deskriptiven Modelle zusammengefasst. ${ }^{477}$ Sie sind eine notwendige Voraussetzung für die praktische Anwendung von Entscheidungsmodellen. ${ }^{478}$ Bei Akzep$\operatorname{tanz}$ der zugrunde liegenden Modellwelt und adäquater deskriptiver Modellierung können aus ihren Ergebnissen konkrete Handlungsempfehlungen abgeleitet werden. Entscheidungsmodelle werden daher auch als praktisch-normativ bezeichnet und zeichnen sich aufgrund des häufig implementierten Optimierungsmoduls durch einen erhöhten Komplexitätsgrad aus. ${ }^{479}$ Die Ermittlung der jeweils günstigsten Handlungsalternative wird maßgeblich durch das Zielsystem bzw. die Präferenzen des Entscheiders beeinflusst. ${ }^{480}$ Wie bereits in Kapitel 3.2.1 dargelegt, kann der Zielkonflikt zwischen Risiko und Chance formal durch ein Präferenzfunktional dargestellt werden. Neben dem Zielsystem muss die relevante Umgebung des Entscheidungsträgers, d.h. das Entscheidungsfeld angemessen abgebildet werden. Dieses umfasst sowohl Entscheidungsvariablen als auch Restriktionen, denen die Entscheidungsvariablen unterliegen. $^{481}$

\footnotetext{
475 Vgl. hierzu auch RIEPER (1992), S. 87 ff. und BAMBERG/COENENBERG (2002), S. 4-7.

476 Vgl. im Folgenden RIEPER (1992), S. 91-92.

477 Vgl. exemplarisch BROHM (2002), S. 16.

478 Vgl. im Folgenden auch RiEPER (1992), S. 93 und HOMBURG (1991), S. 287-301.

${ }^{479}$ Im Verständnis dieser Arbeit ist die Existenz eines (zumeist mathematisch formulierten) Optimierungsmoduls nicht zwingend erforderlich, um ein Modell als Entscheidungsmodell zu klassifizieren. Ein Entscheidungsmodell muss vielmehr dem Entscheider die Möglichkeit bieten, aus einer Menge zulässiger Alternativen eine zielsetzungsgerechte Alternative auszuwählen. Vgl. zu einem analogen Modellverständnis beispielsweise RIEPER (1992), S. 17-18.

480 Vgl. ausführlich zum Begriff des Zielsystems und zur Bewertung von Entscheidungsalternativen ADAM (1996), S. 99-163 und BAMBERG/COENENBERG (2002), S. 28-33.

481 Vgl. zum Entscheidungsfeld des in dieser Arbeit vorgestellten Modells insbesondere Kapitel 3. Vgl. zum Grundmodell der betriebswirtschaftlichen Entscheidungslehre ausführlich BAMBERG/ COENENBERG (2002), S. 13-42.
} 
Im Verlauf des Modellierungsprozesses ${ }^{482}$ sollte im Sinne einer zeitlichen Priorisierung darauf geachtet werden, zunächst angemessene deskriptive Modelle zu entwickeln, auf deren Basis in einem zweiten Schritt explikative oder praktisch-normative Modelle formuliert werden. Unabhängig von der Zielsetzung können Modelle auch anhand ihrer modelltheoretischen Eigenschaften eingeordnet werden. In der Literatur finden sich zahlreiche Klassifizierungskriterien, von denen an dieser Stelle jedoch nur folgende - im versicherungsspezifischen Kontext als wesentlich erachtete - behandelt werden sollen. ${ }^{483}$

- Darstellung des Zeitablaufs

- Abbildung von pfadabhängigen Entscheidungsmechanismen

- Art der Modellparameter

Die Unterscheidung zwischen statischen und dynamischen Modellen ist an die Frage geknüpft, ob im Modell der Zeitablauf abgebildet wird. ${ }^{484}$ Angesichts der Tatsache, dass nahezu alle Realsysteme im Zeitablauf existieren, sollte dieses Merkmal grundsätzlich in einem Modell abgebildet werden. Die Berücksichtigung des Zeitablaufs kann in diskreter Form über Zeitreihen oder über stetige Zeitfunktionen erfolgen. In der praktischen Modellierung wird meistens eine Diskretisierung der Zeit vorgenommen, d.h. Änderungen von Systemmerkmalen werden von einem Zeitpunkt zum nächsten erfasst. Der dynamische Charakter derartiger Modelle macht sich an der Tatsache fest, dass die Ergebnisse einer Modellperiode wiederum die Ausgangsposition der Folgeperiode darstellen und auf diese Weise zeitübergreifende Ursache-WirkungsBeziehungen abgebildet werden können. ${ }^{485}$

Ein weiteres Klassifizierungskriterium betrifft die Fragestellung, inwiefern Modelle in der Lage sind, pfadabhängige - oftmals heuristische - Entscheidungsmechanismen abbilden zu können, die zu gewissen Zeitpunkten in den Modellablauf eingreifen. ${ }^{486}$

482 Vgl. zum Prozess der Modellentwicklung ADAM (1996), S. 64-69, HOMBURG (1991), S. 269-273, RIEPER (1992), S. 22-23 und SCHNEEWEIB (1984), S. 501.

483 Vgl. zu anderen Modellkategorisierungen z.B. RIEPER (1992), S. 87-105, ADAM (1996), S. 81, HOMBURG (1991), S. 266 f., BAMBERG/COENENBERG (2002), S. 40-42 oder KOPP (1992), S. 8890. Explizit erwähnt seien an dieser Stelle die Klassifizierungskriterien „Art der Zielfunktion“ (lineares vs. nicht-lineares Modell), „Anzahl der Zielfunktionen“ (Ein-Ziel-Optimierung vs. MehrZiel-Optimierung) und „Sachumfang des Modells“ (Total- vs. Partialmodell). BROHM unterscheidet darüber hinaus zwischen Cashflow-Modellen und Performancemodellen. Vgl. BROHM (2002), S. 95-98.

484 Vgl. hierzu und im Folgenden ADAM (1996), S. 88-91.

${ }^{485}$ Das Begriffsverständnis hinsichtlich dynamischer und statischer Modelle ist in der Literatur nicht einheitlich. Beispielsweise spricht BROHM dynamischen Modellen bereits die Fähigkeit zu, pfadabhängige Entscheidungen im Modellverlauf zu berücksichtigen. Vgl. BROHM (2002), S. 92.

486 Vgl. im Folgenden auch HOMBURG (1991), S. 352-357 und BROHM (2002), S. 136-140. 
Als Entscheidungspunkte werden somit Zeitpunkte im Modellverlauf bezeichnet, zu denen der Entscheidungsträger in Abhängigkeit von den bisher erzeugten Modellergebnissen eine Veränderung der variablen Modellparameter vornehmen kann. Als mögliche Beispiele aus der Versicherungswirtschaft seien veränderte Zeichnungsstrategien, Anpassungen im Rückversicherungsprogramm oder eine modifizierte Asset Allocation zu nennen. Um die Komplexität derartiger Entscheidungsmechanismen modelltechnisch beherrschbar zu machen, orientieren sich die Auswahlentscheidungen häufig an Strategien oder Managementregeln, die bereits zu Modellbeginn explizit festgelegt werden ${ }^{487}$ Handlungserfordernisse und Handlungsalternativen, die im Zeitpunkt der Modellbildung noch nicht bekannt oder unvorstellbar waren, können demzufolge nicht berücksichtigt werden. Im Hinblick auf die Abbildung pfadabhängiger Entscheidungen soll auf eine weitere Problematik hingewiesen werden. Quantifiziert man beispielsweise den Risikokapitalbedarf eines Unternehmens mit Hilfe eines Modells, welches über pfadabhängige Managementregeln verfügt, so ist die Höhe des Risikokapitals nur unter der Voraussetzung valide, dass sich das Management des Unternehmens strikt an die im Modell verankerten Regeln hält. Werden in der Realität jedoch andere Entscheidungen getroffen als im Modell vorgesehen, kann es modellseitig zu substanziellen Fehleinschätzungen der tatsächlichen Risikoposition kommen. Mit Blick auf die Unternehmenspraxis ist jedoch in Frage zu stellen, ob sich die Unternehmensleitung durch derartige ex-ante definierte Entscheidungspfade „entmündigen“ lassen wird.

Die Art der verwendeten Modellparameter führt zu der Unterscheidung zwischen deterministischen und stochastischen Modellen. ${ }^{488}$ In deterministischen Modellen werden zufällige Veränderungen der Modellparameter oder ihrer Wirkungsbeziehungen untereinander ex-ante ausgeschlossen. Da die Unsicherheit und zufällige Abhängigkeiten nicht abgebildet werden, sind deterministische Modelle für die Risikomessung und Risikosteuerung ungeeignet. ${ }^{489}$ Vielfach wird in diesem Zusammenhang auf Szenarioanalysen zurückgegriffen, und es werden singuläre Szenarien im Sinne eines „worst case“, „most likely case“ oder „,best case“ erzeugt. Derartige deterministische Analysen vermitteln jedoch nur einen Eindruck der Bandbreite möglicher Ereignisse, ohne diesen explizit eine Eintrittswahrscheinlichkeit zuordnen zu können.

In stochastischen Modellen stellen hingegen einige Modellparameter unsichere Größen dar, die durch Verteilungsfunktionen charakterisiert werden können. Auf diese Weise

487 Vgl. zur Unterscheidung von Modellen mit expliziter und impliziter Erfassung von Strategien vertiefend ADAM (1996), S. 84-86.

488 Vgl. hierzu und im Folgenden auch BROHM (2002), S. 93 und Bossel (1992), S. 38.

489 Zur Abgrenzung der Begriffe Unsicherheit und Risiko vgl. BERENS/DELFMANN/SCHMitTiNG (2004), S. 53, BAMBERG/COENENBERG (2002), S. 19 und Kapitel 2.2.1 dieser Arbeit. 
werden Aussagen über die Eintrittswahrscheinlichkeit zukünftiger Umweltzustände ermöglicht. ${ }^{490} \mathrm{Da}$ für einige Inputparameter nur ein Wertebereich angegeben werden kann, innerhalb dessen sich die Realisationen der jeweiligen Zufallsvariablen bewegen, müssen die Modellergebnisse bzw. Outputparameter zwangsläufig auch stochastischer Natur sein. Wie bereits in Kapitel 2.2.3 beschrieben, wirken auf die Geschäftstätigkeit eines Schaden- und Unfallversicherungsunternehmens eine Vielzahl unsicherer Ereignisse, die sich insbesondere im versicherungstechnischen Risiko und Kapitalanlagerisiko manifestieren. Angesichts der unsicheren Inputparameter ist es nahe liegend, für die Ermittlung und Steuerung des Risikokapitalbedarfs ein stochastisches Modell zu verwenden. ${ }^{491}$ Allen stochastischen Modellen ist gemeinsam, dass die Realisationen zufallsabhängiger Parameter numerisch ermittelbar sind und über (nicht notwendigerweise stetige) Verteilungsfunktionen dargestellt werden können. ${ }^{492}$ Die Ermittlung der zugrunde liegenden Verteilungen kann entweder analytisch oder mit Hilfe von Simulationen erfolgen. Die nachfolgenden Ausführungen widmen sich der Darstellung stochastischer Modelle und stellen die Abgrenzung zwischen analytischen und simulativen Konzepten in den Mittelpunkt.

\subsubsection{Kategorisierung von stochastischen Modellen}

\subsubsection{Modelle auf analytischer Basis}

Im Rahmen analytischer Modelle wird die gesuchte Ergebnisverteilung auf der Basis mathematisch-statistischer Gesetzmäßigkeiten hergeleitet, und die durch das Modell postulierten Verteilungsannahmen werden analytisch durchdrungen. ${ }^{493}$ Die Parameter der auf diese Weise ermittelten Verteilung der Ergebnisgröße können somit rechnerisch ermittelt werden. Die mathematisch geschlossene Darstellung eines Problems ermöglicht es zudem, ausgehend von akzeptierten Inputparametern eine explizite Lösung $\mathrm{zu}$ formulieren, die jederzeit replizierbar ist. Analytische Modelle eignen sich daher in besonderem Maße, die Grundstruktur eines Problems und seiner Bewältigung mit Hilfe einer geschlossenen Lösung aufzuzeigen. ${ }^{494}$ Sensitivitätsanalysen lassen sich innerhalb derselben Wahrscheinlichkeitsverteilung durch Variation der Inputparameter (z.B. des Durchschnittsschadens oder der Schadenfrequenz) relativ einfach durchfüh-

490 Vgl. im Folgenden RIEPER (1995), S. 99-102 und BROHM (2002), S. 98-104. SCHENK weist darauf hin, dass stochastische Modelle auch deterministische Inputparameter aufweisen können und bezeichnet derartige Abbildungsstrukturen als ,hybride Modelle“. Vgl. SCHENK (1998), S. 15.

491 ALBRECHT formuliert in diesem Zusammenhang bereits im Jahr 1985: „Hinsichtlich der den einzelnen Partialbereichen inhärenten Stochastizität muss gefordert werden, dass zumindest die Schwankungen der Schäden und des Kapitalanlageergebnisses in das Modell zu integrieren sind.“ ALBRECHT (1985), S. 204.

${ }^{492}$ Vgl. BROHM (2002), S. 98.

493 Vgl. im Folgenden BROHM (2002), S. 98-99.

494 Vgl. vertiefend ALBRECHT (1985), S. 209. 
ren. Da derartige Modellkonzepte dem Entscheidungsträger mathematisch fundierte Einblicke in die Ursache-Wirkungs-Zusammenhänge ermöglichen, sind sie aus wissenschaftstheoretischer Sicht den simulativen Modellkonzepten überlegen.

Allerdings stößt die analytische Vorgehensweise recht schnell an die Grenzen mathematischer Handhabbarkeit, wenn das zugrunde liegende Problem - wie in der Realität überwiegend anzutreffen - eine hohe Komplexität aufweist und die Inputparameter nicht-linearen Verknüpfungen unterliegen. Die Nebenbedingung der mathematischen Handhabbarkeit schränkt zudem die Anzahl der in Frage kommenden Verteilungsfunktionen auf einen sehr überschaubaren Kreis ein. ${ }^{495}$ Diese zumeist symmetrischen Verteilungstypen bilden jedoch die Realität des Versicherungsgeschäfts nur unzureichend ab. ${ }^{496}$ Vielmehr kann davon ausgegangen werden, dass in den meisten Versicherungssparten der Schadenaufwand besser durch rechtsschiefe Verteilungen approximiert wird, da die Mehrzahl der Realisationen unterhalb des Erwartungswertes liegt und extreme Schadenrealisationen nur mit einer geringen Wahrscheinlichkeit eintreten. ${ }^{497}$ Soll die Summe zweier Zufallsvariablen, z.B. die gemeinsame Schadenhöhenverteilung zweier Versicherungssparten, bestimmt werden, so geschieht dies im Rahmen analytischer Modelle durch den mathematischen Vorgang der Faltung. ${ }^{498}$ Um jedoch die Verteilung der Summe zweier Zufallsvariablen $\mathrm{X}$ und $\mathrm{Y}$, die ihrerseits den Verteilungen $\mathrm{X} \sim \mathrm{P}$ und $\mathrm{Y} \sim \mathrm{Q}$ genügen, explizit in der Form $\mathrm{X}+\mathrm{Y} \sim \mathrm{P} * \mathrm{Q}$ angeben $\mathrm{zu}$ können, müssen $\mathrm{X}$ und $\mathrm{Y}$ voneinander stochastisch unabhängig sein. In der Praxis ist hingegen zu vermuten, dass das Postulat der stochastischen Unabhängigkeit häufig nicht erfuillt ist. ${ }^{499}$ So treten beispielsweise im Falle von Katastrophen- oder Elementarereignissen meistens gleichzeitig Schäden in mehreren Versicherungssparten auf. ${ }^{500}$

Obwohl die praktischen Schwierigkeiten des Einsatzes analytischer Modelle hier nur kurz skizziert werden konnten, wird dennoch deutlich, dass analytische Konzepte bei der Lösung praxisnaher Problemstellungen schnell an ihre Grenzen stoßen. Daher sollte insbesondere dann auf simulationsgestützte Verfahren zurückgegriffen werden, wenn die Lösungsstruktur eines Sachverhalts bereits im Wesentlichen bekannt ist, die

\footnotetext{
495 Vgl. MACK (2002), S. 111.

496 Dieser Sachverhalt wird in Kapitel 5.4.3 detailliert behandelt.

497 Vgl. auch LiPPE (1983), S. 50 und ALBRECHT (1992), S. 10.

498 Vgl. im Folgenden auch HIPP (2001a), S. 17-23 und BROHM (2002), S. 99.

499 Vgl. übereinstimmend KORYCIORZ (2004), S. 130.

500 Als Beispiel für dieses Phänomen sei hier stellvertretend das Schadenereignis des 11. September 2001 genannt. Vgl. hierzu exemplarisch MÜNCHENER RÜCK (2001) und NÖHRBASS (2001).
} 
oben formulierten Anforderungen an die Einfachheit der Modellstruktur und die restriktiven Verteilungsannahmen jedoch nicht erfüllt sind. ${ }^{501}$

\subsubsection{Modelle auf der Basis von Simulationen}

Stochastische Simulationsmodelle greifen nicht auf eine analytisch geschlossene Herleitung der Ergebnisverteilung zurück, sondern erzeugen konkrete Realisationen der zugrunde liegenden Eingangsverteilungen durch Ziehung von Zufallszahlen. ${ }^{502}$ Diese Vorgehensweise wird auch als Monte-Carlo-Simulation bezeichnet. ${ }^{503}$ Jede einzelne Realisation für sich allein kann auch als (deterministisches) Szenario aufgefasst werden, das unter Berücksichtigung der im Modell unterstellten Interdependenzen eine Ergebnisgröße produziert. Die auf diese Weise generierten Ergebnisrealisationen können jeweils als Stichprobe der Eingangsverteilungen interpretiert werden. Wird der beschriebene Ablauf hinreichend oft wiederholt, erhält man schließlich eine empirische Häufigkeitsverteilung der Ergebnisgröße. Mit wachsendem Stichprobenumfang konvergiert diese empirische Häufigkeitsverteilung unter bestimmten Annahmen gegen die zugrunde liegende theoretische Verteilung. ${ }^{504}$ In diesem Zusammenhang spielt die Konvergenzgeschwindigkeit des Simulationsmodells ein wichtige Rolle, da diese die Anzahl der erforderlichen Simulationsläufe determiniert, die notwendig ist, um eine möglichst genaue Annäherung an die theoretische Verteilung und somit stabile Ergebnisse zu erhalten. ${ }^{505}$ Insbesondere in den Randbereichen der Verteilung, den so genannten Tails, stabilisieren sich die Ergebnisse (in Abhängigkeit von der Varianz der betrachteten Verteilung) erst bei einer sehr großen Simulationsanzahl. Diese Problematik soll am Beispiel des in Kapitel 4.1.3.3 bereits vorgestellten Risikomaßes $\mathrm{CVaR}$ verdeutlicht werden. Hierzu sei unterstellt, dass der CVaR für ein Sicherheitsniveau 1- $\varepsilon$ von $99,5 \%$ bestimmt werden soll. Die Anzahl der Simulationen betrage $\mathrm{n}=1000$. Es ist unmittelbar einleuchtend, dass die Aussagekraft des Simulationsmodells unter diesen Voraussetzungen als gering einzuschätzen ist, da sich der CVaR als Erwartungswert über lediglich fünf Ergebnisrealisationen errechnet.

501 ALBRECHT spricht sich in diesem Zusammenhang dafür aus, vor dem Einsatz von Simulationsmodellen ,ein gegebenes Problem auf der Grundlage eines möglichst einfach strukturierten Modells, unter Beschränkung auf die wesentlichen Variablen und deren Interrelationen, anzugehen [...].“ ALBRECHT (1985), S. 209.

${ }^{502}$ Vgl. im Folgenden auch BROHM (2002), S. 100-104, LIEBL (1995), S. 63-65 und HILLIER/LIEBERMAN (1997), S. 773-805.

503 In der Literatur wird bisweilen noch zwischen deterministischen, hybriden und Monte-CarloSimulationen unterschieden. Vgl. stellvertretend BRAMMERTZ (1991), S. 271. Vgl. zur versicherungsspezifischen und praxisorientierten Anwendung der Monte-Carlo-Simulation insbesondere FREY/NIEBEN (2001).

$504 \mathrm{Vgl}$. zu den Konvergenzannahmen vertiefend LeHN/WEGMANN (1992), S. $94 \mathrm{ff}$.

$505 \mathrm{Vgl}$. auch BROHM (2002), S. 102 und die Ausfuhrungen in Anhang D. 
Ein wesentlicher Vorzug der Monte-Carlo-Simulation besteht darin, dass sie sehr flexibel an den zu modellierenden Sachverhalt angepasst werden kann. ${ }^{506}$ Auswirkungen unterschiedlicher risikopolitischer Maßnahmen, z.B. der Rückversicherungspolitik, lassen sich somit geeignet abbilden. Darüber hinaus können die Parameter der verwendeten Eingangsverteilungen individuell gewählt und empirisch gewonnene Verteilungen in einfacher Weise in das Verfahren eingebracht werden. Den beschriebenen Vorteilen stehen jedoch auch Nachteile gegenüber. Grundsätzlich lassen sich in Modellen, die auf Monte-Carlo-Simulationen basieren, eindeutige Ursache-WirkungsBeziehungen nicht in der geschlossenen Form abbilden wie in analytischen Modellen. Die in der Praxis anzutreffende Komplexität der Problemstellungen kann dann häufig nur über einen (heuristischen) Simulationsansatz bewältigt werden ${ }^{507}$ Wie jede auf Stichproben aufbauende Methode liefert auch die Monte-Carlo-Simulation nur Ergebnisse, die mit einer gewissen Wahrscheinlichkeit zutreffend sind. ${ }^{508}$ Diese Wahrscheinlichkeit wiederum wird stark durch den Stichprobenumfang beeinflusst. Der für die Einhaltung eines bestimmten Sicherheitsniveaus notwendige Simulationsumfang ist jedoch nur für wenige Verteilungsklassen eindeutig bestimmbar. ${ }^{509}$ Darüber hinaus ist $\mathrm{zu}$ beachten, dass die durch einen bestimmten Zufallszahlengenerator erzeugten Simulationen immer nur auf so genannten „Pseudo-Zufallszahlen“ basieren, die sich hinsichtlich ihrer stochastischen Eigenschaften nur annähernd wie echte Zufallszahlen verhalten. ${ }^{510}$ Tatsächlich sind die erzeugten Realisationen jedoch vorhersehbar und darüber hinaus reproduzierbar. ${ }^{511}$

Nachdem nunmehr die spezifischen Vor- und Nachteile der Monte-Carlo-Methode herausgestellt wurden, sollen im nachfolgenden Kapitel grundlegende Problemkreise und Grenzen der Modellbildung diskutiert werden.

\footnotetext{
506 Vgl. im Folgenden auch FEILMEIER/BERTRAM (1987), S. 55.

507 Vgl. übereinstimmend HillieR/LIEBERMAN (1997), S. 773 und BRAMMERTZ (1991), S. 272.

508 Vgl. im Folgenden FEILMEIER/BERTRAM (1987), S. 55.

509 Vgl. FeILMEIER/BerTRAM (1987), S. 55. Zur Dimensionierung des Simulationsumfangs vgl. KOCH (1994).

510 Vgl. vertiefend zur Erzeugung von Zufallszahlen und zu geeigneten Kriterien für „gute“ Zufallszahlengeneratoren LiEBL (1995), S. 23-49 und SOBOL (1991), S. 32-36.

511 Vgl. HiLlier/Lieberman (1997), S. 781. Die Reproduzierbarkeit von Pseudozufallszahlen kann gleichwohl auch als Vorteil aufgefasst werden. Sofern im Rahmen von Modellrechnungen der gleiche Zufallszahlensatz verwendet wird, ist aufgrund der deterministischen Erzeugungsregel gleichzeitig eine Reproduzierbarkeit der Simulationsergebnisse gewährleistet. Vgl. auch LIEBL (1995), S. 24.
} 


\subsubsection{Grenzen der Modellbildung}

Wie bereits in Kapitel 5.1.1 thematisiert, können und sollen Modelle immer nur ein vereinfachtes Abbild der Realität darstellen und komplexe Sachverhalte in ihrer wesentlichen Struktur beschreiben. Das den meisten Problemstellungen zugrunde liegende Phänomen der Unsicherheit wird durch Modelle lediglich transparent und quantifizierbar gemacht, jedoch niemals aufgelöst. ${ }^{512}$ Durch die Reduzierung auf wesentliche Kausalitätsstrukturen sollte jedoch zumindest eine gewisse Rationalitätssicherung erreicht werden. ${ }^{513}$ Darüber hinaus muss betont werden, dass die aus dem Modell abgeleiteten Aussagen und Handlungsempfehlungen immer nur vor dem Hintergrund der zugrunde liegenden Modellwelt Gültigkeit besitzen. Ob eine Übertragung auf die Realität überhaupt zulässig ist, hängt von der Angemessenheit und Güte der Modellierung ab. Die Modellergebnisse müssen daher in einem laufenden Prozess empirisch validiert werden. „Erst recht gilt beim Einsatz von Entscheidungsmodellen für das tatsächliche Handeln im Betrieb, dass ihre errechneten Lösungen nicht verabsolutiert werden dürfen. ${ }^{\text {(514 }}$

Ein grundsätzliches Risiko der Modellbildung besteht schließlich darin, dass bereits bei der Konzeptionierung, der Erfassung der Ursache-Wirkungs-Beziehungen oder bei der Parameterschätzung Fehler auftreten. Diese grundlegende Problematik kann als Modellrisiko verstanden werden. ${ }^{515}$ Es muss an dieser Stelle nicht mehr ausführlich hervorgehoben werden, dass die Modellergebnisse trotz vielfach anspruchsvoller mathematischer Modellierung immer durch den Umfang und die Qualität der zugrunde liegenden Inputparameter determiniert werden. Die verwendeten Daten und Parameter sollten daher niemals als "sicher“ vorausgesetzt, sondern laufend anhand von Erfahrungswerten und Expertenwissen an der Realität gespiegelt und einer kritischen Würdigung unterzogen werden. ${ }^{516}$

\subsection{Anforderungen an die Modellierung}

Grundsätzlich sollte die Modellierung den individuellen Bedürfnissen und Informationsanforderungen der Modelladressaten Rechnung tragen. Die Anforderungen an Modelle stellen sich in Abhängigkeit vom verfolgten Modellzweck daher sehr unterschiedlich dar.

\footnotetext{
512 Vgl. im Folgenden HERI/ZiMMERMANN (2001) und BROHM (2002), S. 94-95.

${ }^{513}$ Vgl. in diesem Kontext auch WEBER, der Rationalität als ,herrschende Meinung von Fachleuten hinsichtlich einer bestimmten Zweck-Mittel-Relation“" versteht. Vgl. WEBER (2004), S. 51.

514 KARTEN (1983), S. 214-215.

515 Vgl. in diesem Zusammenhang insbesondere HERI/ZIMMERMANN (2001), S. 1008-1009.

516 Vgl. auch MACK (2002), S. 22.
} 
In diesem Zusammenhang kann zwischen dem strategischen und operativen Einsatzzweck der Modelle differenziert werden. ${ }^{517}$ Strategische Modelle sollten Prognosen über die zukünftige Geschäftsentwicklung und Risikoexponierung eines Unternehmens ermöglichen und als Entscheidungsgrundlage für mittelfristige Steuerungsprozesse und Managementstrategien dienen können. Bei operativen Modellen hingegen stehen kurzfristige Fragestellungen, wie z.B. die Beurteilung der Vorteilhaftigkeit eines neu abzuschließenden Vertrags im Vordergrund. Im Folgenden soll auf die Differenzierung zwischen strategischen und operativen Modellanforderungen nicht detailliert eingegangen werden. Hierzu wird auf die einschlägige Literatur zur Modellbildung verwiesen. ${ }^{518}$ Es wird vielmehr eine Unterscheidung in generelle und versicherungsspezifische Anforderungen vorgenommen.

Als generelle Anforderungen sind in diesem Zusammenhang zu nennen: ${ }^{519}$
a) Adäquanz
b) Konsistenz
c) Erweiterbarkeit
d) Validierbarkeit
e) Wirtschaftlichkeit

zu a) Modelle sollten problemadäquat, d.h. zur Bewältigung einer konkreten Problemoder Fragestellung geeignet sein. ${ }^{520}$ Eine zweckmäßige Abbildung bedeutet jedoch nicht, dass das Modell eine möglichst genaue Repräsentation des zu untersuchenden Realsystems darstellt. Vielmehr kommt es darauf an, einen angemessenen Kompromiss zwischen Realitätstreue und Problemreduktion zu finden. „Ein Modell ist geeignet, wenn es die für die Fragestellung wesentlichen Aspekte der Realität möglichst genau abbildet und dabei noch so einfach bleibt, dass die Fragestellung mit erträglichem Aufwand innerhalb des Modells beantwortet werden kann. Wenn das Modell geeignet ist, ist seine Antwort auch relevant für die Praxis. “521

zu b) Modelle sollten des Weiteren konsistent sein, d.h. sie sollten widerspruchsfreie Ergebnisse erzeugen, die sich anhand von Erfahrungswerten intersubjektiv überprüfen lassen. ${ }^{522}$ In diesem Zusammenhang ist zu beachten, dass ein Modell durchaus Ergeb-

\footnotetext{
517 Vgl. im Folgenden RIEPER (1992), S. 93-96.

518 Vgl. stellvertretend HOMBURG (1991), S. 17-21 und RIEPER (1992), S. 93-96.

519 Vgl. im Folgenden auch BABBEL/MERRIL (1998), S. 3-4 und BROHM (2002), S. 17-19.

520 Vgl. vertiefend LiEBL (1995), S. 195-198.

${ }^{521}$ MACK (2002), S. 22. In diesem Zusammenhang bemerken BABBEL/MERRILL: „The generality of the model must be tempered by our ability to implement it.“ BABBEL/MERRILL (1998), S. 3.

522 Vgl. auch BALCI (1994), S. 134.
} 
nisrealisationen erzeugen kann, die aus der Historie bislang unbekannt sind. Allerdings müssen diese Realisationen im Sinne eines „Worst-Case-Szenarios“ zumindest „vorstellbar" bzw. „erlebbar“ sein.

zu c) Im Verlauf der Modellbildung und -anwendung treten häufig erweiterte oder sogar neue Fragestellungen auf, die in das Modell integriert werden müssen. Der Modellansatz sollte daher so flexibel gewählt werden, dass Erweiterungen mit vertretbarem Aufwand möglich sind. Es soll an dieser Stelle aber auch darauf hingewiesen werden, dass derartigen Erweiterungen in der Praxis sowohl wirtschaftliche als auch modellierungstechnische Grenzen gesetzt sind. Einer sorgfältigen Formulierung der unternehmensspezifischen Anforderungen im Vorfeld der Modellbildung kommt daher zentrale Bedeutung zu.

zu d) Der Prozess der Validierung stellt einen essentiellen Bestandteil der Konstruktion und des Einsatzes von Modellen dar. ${ }^{523} \mathrm{Da}$ die Validierungskriterien und ihre Gewichtungsverhältnisse in Abhängigkeit von der Problemstellung unterschiedlich ausfallen, sollte die Validierung modellindividuell erfolgen und die jeweilige Zweckbestimmung des Modells berücksichtigen. Darüber hinaus kann der Nutzen eines Modells maßgeblich daran festgemacht werden, ob es seitens der Entscheidungsträger auf Akzeptanz stößt und zur Unterstützung von Managemententscheidungen tatsächlich angewendet wird. In der Praxis ist die Validität eines Modells somit häufig das Ergebnis eines „Verhandlungsprozesses“. ${ }^{524}$ Dies mag zunächst den Eindruck der Manipulation erwecken, jedoch werden auf diese Weise die Fähigkeiten und Kenntnisse möglichst vieler Experten in den Validierungsprozess einbezogen und somit erst die notwendige Glaubwürdigkeit des Modells sichergestellt. Nicht zuletzt sollte die Validierung iterativ erfolgen und sich auf den gesamten Prozess der Modellbildung erstrecken, um auftretende Fehler und Inkonsistenzen frühzeitig aufdecken zu können. Im Hinblick auf die Akzeptanz des Modells ist es außerdem erforderlich, dass dieses eine bereits bekannte Entscheidungssituation im Rahmen eines „Backtesting“ möglichst genau abzubilden vermag. Das Modell sollte daher stets an der Realität kalibriert werden können. ${ }^{525}$

zu e) Die Ergebnisse von Modellen müssen den damit verbundenen Forschungs- und Entwicklungsaufwand rechtfertigen und daher eine angemessene Kosten-NutzenRelation aufweisen. ${ }^{526}$ Dabei geht ein wachsender Informationsnutzen aufgrund eines

523 Vgl. im Folgenden HillieR/Lieberman (1997), S. 20-22, Liebl (1995), S. 203-214, BaLCI (1994), S. 121-173 und LEYHERR/SCULLY/SOMMERFELD (2003), S. 1796.

524 Vgl. vertiefend LIEBL (1995), S. 204-205.

525 Vgl. übereinstimmend auch BABBEL/MERRILL (1998), S. 3 und BROHM (2002), S. 18.

526 Vgl. im Folgenden LiEBL (1995), S. 198-199. 
steigenden Detaillierungsgrads in der Regel mit erhöhten Entwicklungskosten einher. ${ }^{527}$ Während hinsichtlich des Detaillierungsgrads ein abnehmender Grenznutzen zu konstatieren ist, nehmen die Kosten der Modellbildung mit wachsendem Detaillierungsgrad überproportional zu. Dieser Zielkonflikt wird durch Abbildung 5.1 illustriert:

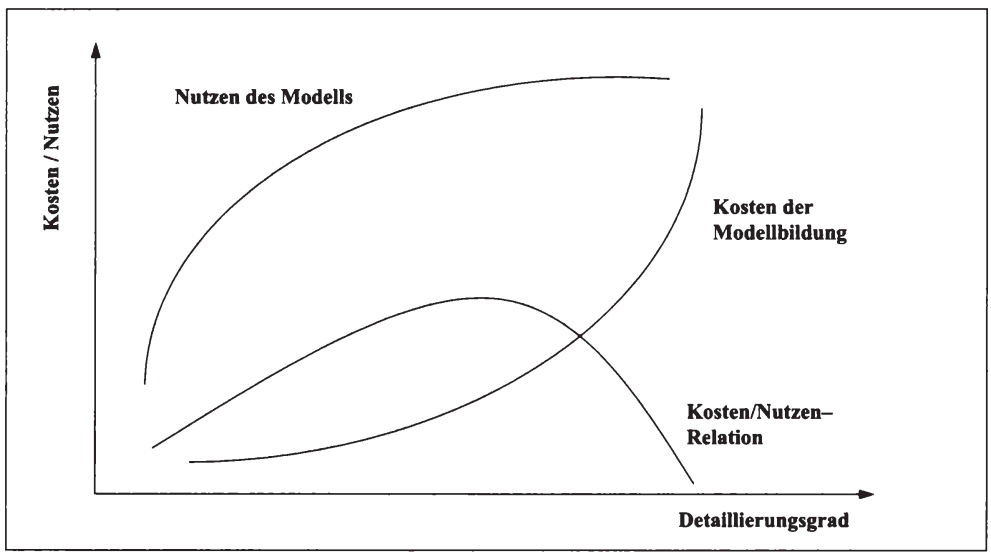

Abbildung 5.1: Detaillierungsgrad und Kosten der Modellbildung Quelle: in Anlehnung an LIEBL (1995), S. 204.

Der Detaillierungsgrad ist von den Zielvorstellungen der Modelladressaten und den verfügbaren finanziellen Ressourcen abhängig. Im Rahmen der Wirtschaftlichkeitsanalyse gilt es außerdem zu beachten, dass qualitative Nebeneffekte, wie z.B. der $\mathrm{Zu}$ wachs an methodischem Know-how oder vertiefte Kenntnisse des Realsystems, nur schwer einer Kosten-Nutzen-Betrachtung zugänglich sind.

Neben den generellen Modellanforderungen sollten im Anwendungskontext der Schaden- und Unfallversicherung zusätzliche spezifische Anforderungen formuliert werden. ${ }^{528}$ Folgende Aspekte, die für das Geschäftsmodell der Schaden- und Unfallversicherung charakteristisch sind, sollten im Rahmen der Modellierung Berücksichtigung finden:

f) Stochastizität der versicherungsspezifischen Risikofaktoren

g) Interdependenzen zwischen einzelnen Risikofaktoren und Teilkollektiven

h) Instrumente der aktiv- und passivseitigen Risikosteuerung

527 Vgl. zum Detaillierungsgrad von Modellen auch LIEBL (1995), S. 118-119.

${ }^{528}$ Vgl. im Folgenden BROHM (2002), S. 134-135. 
zu f) Der Kern des Versicherungsgeschäfts ist das Risikogeschäft. ${ }^{529}$ Im Rahmen dessen überwälzen die Versicherungsnehmer die finanziellen Auswirkungen unsicherer Schadenereignisse ganz oder teilweise auf den Erstversicherer. Die durch den Versicherer zu erbringende Entschädigungsleistung ist daher a priori indeterminiert und zufallsabhängig und sollte als Zufallsvariable modelliert werden. ${ }^{530}$ Neben der Schadenlast sind in der Regel auch die Kapitalanlageergebnisse unsicherheitsbehaftet und sollten vor diesem Hintergrund ebenfalls stochastisch abgebildet werden. ${ }^{531}$

zu g) Die Risikoposition eines Schaden- und Unfallversicherers würde signifikant falsch eingeschätzt, falls man im Zeitpunkt der Modellierung davon ausginge, dass sich die einzelnen Risikofaktoren und Teilkollektive stochastisch unabhängig voneinander entwickeln. ${ }^{532}$ Als weitgehend homogene Teilkollektive können einzelne Versicherungssparten oder auch Kundengruppen in Betracht kommen. So ist es beispielsweise unrealistisch anzunehmen, dass durch ein Naturgefahrenereignis lediglich eine einzige Versicherungssparte betroffen ist oder ausschließlich die Privatkunden eines Versicherers durch dieses Ereignis geschädigt werden, während die Firmenkunden keine Schäden zu verzeichnen haben. Die stochastischen Abhängigkeitsstrukturen zwischen den genannten Teilkollektiven können linearer und gegebenenfalls auch nicht-linearer Natur sein. ${ }^{533}$ Eine praxisrelevante Modellierung sollte daher die Abbildung beider Abhängigkeitstypen unterstützen. Darüber hinaus können versicherungstechnisches Geschäft und Kapitalanlagetätigkeit nicht isoliert voneinander im Modell abgebildet werden, da sich die Stochastizität des versicherungstechnischen Cashflows unmittelbar auf die Höhe des investierten Zinsträgers auswirkt. Die Kapitalanlagetätigkeit ist somit untrennbar mit der Versicherungstechnik verbunden. ${ }^{534}$

zu h) Neben der Berücksichtigung von Stochastizität und Abhängigkeitsstrukturen sollte ein praxistaugliches Modell die Möglichkeit bieten, das risikopolitische Steuerungsinstrumentarium eines Versicherungsunternehmens geeignet $\mathrm{zu}$ integrieren. In Kapitel 3.2 wurde das betriebswirtschaftliche Entscheidungsfeld eines Schaden- und Unfallversicherers bereits skizziert und die Kapitalanlage- und Rückversicherungspoli-

529 Vgl. auch FARNY (2000), S. 22 und die Ausführungen in Kapitel 2.2.3.1.

$530 \mathrm{Vgl}$. zum so genannten Schadenrisiko ausführlich ALBRECHT (1992), S. 14 f. und DAYKIN/PENTIKÄINEN/PESONEN (1996), S. i. Zum Oberbegriff des versicherungstechnischen Risikos vgl. auch Kapitel 2.2.3.1.

531 Vgl. übereinstimmend KORYCIORZ (2004), S. 162. Zu den Einflussfaktoren des Kapitalanlagerisikos vgl. ausführlich BÄHRLE (1997), S. 17-27 und BROHM (2002), S. 74-76.

532 Vgl. auch KORYCIORZ (2004), S. 112-115.

533 Vgl. KORYCIORZ (2004), S. 115. Die Auswirkungen linearer und nicht-linearer Abhängigkeitsstrukturen auf den Risikokapitalbedarf werden in Kapitel 6.2.2 detailliert untersucht.

534 Vgl. exemplarisch SCHMEISER (1999), S. 91, Heinke (2000), S. 630 und die Ausführungen in Kapitel 5.5.4. 
tik als zentrale Steuerungsinstrumente identifiziert. Ein Modell sollte daher in der Lage sein, sowohl die gängigen aktivseitigen Sicherungsgeschäfte als auch das Instrumentarium der proportionalen und nicht-proportionalen Rückversicherung abzubilden. Im Rahmen dieser Arbeit liegt der Schwerpunkt der Betrachtung auf dem Einsatz versicherungstechnischer Sicherungsinstrumente, insbesondere der Rückversicherung. ${ }^{535}$ Hierzu sei auf die Ausführungen in Kapitel 5.4.5 verwiesen.

\subsection{Rahmenkonzept und Struktur des Modellansatzes}

\subsubsection{Modellzweck und Grundannahmen}

Im Folgenden soll ein simulationsgestützter Modellansatz entwickelt werden, der dem in Kapitel 5.2 formulierten Anforderungskatalog genügt und zum Zwecke der strategischen Steuerung der Rendite-Risiko-Position eines Schaden- und Unfallversicherers eingesetzt werden kann. ${ }^{536}$ Das Management eines Versicherungsunternehmens soll in die Lage versetzt werden, auf der Basis risikotheoretisch fundierter Modellaussagen die Auswirkungen unterschiedlicher Geschäftsstrategien auf die Rendite-RisikoPosition beurteilen zu können und hierauf aufbauend zielsetzungsgerechte Entscheidungen treffen zu können. Es handelt sich somit um ein explikativ-normatives Modell, das die Zufallsabhängigkeit der versicherungstechnischen Risikoposition und der Kapitalanlagetätigkeit abbildet. In Kapitel 3.2.4 wurde bereits grundlegend zwischen einer Steuerungsebene und einer Bilanzebene unterschieden. Die weiteren Ausführungen thematisieren vorrangig die betriebswirtschaftliche Steuerungsebene im Sinne einer integrierten Rendite-Risikosteuerung, lassen jedoch die bilanziellen Auswirkungen strategischer Entscheidungen nicht außer Acht. Schließlich stellt die Möglichkeit zur Aufstellung eines erfolgreichen und unter dem Aspekt der Risikotragfähigkeit zulässigen Jahresabschlusses insbesondere für kapitalmarktorientierte Unternehmen einen wichtigen Wettbewerbsfaktor und somit eine „strenge Nebenbedingung“ für die Steuerung dar. ${ }^{537}$ Als Zielgrößen des Modells können folgende Ergebnisverteilungen identifiziert werden:

- Verteilung des versicherungstechnischen Ergebnisses

- Verteilung des Ergebnisses aus der Kapitalanlagetätigkeit

- Verteilung des Gesamtergebnisses

535 Die Wirkung derivativer Finanzinstrumente für die Aktivseite wird lediglich in der Hauptanlageklasse Aktien über einen pauschalen Schwankungs- und Renditeabschlag berücksichtigt. Vgl. hierzu Kapitel 5.5.5.1. Siehe zum Einsatz derivativer Finanzinstrumente in der Kapitalanlage von Versicherungsunternehmen vertiefend BÄHRLE (1997) und SCHENK (1998).

$536 \mathrm{Vgl}$. grundlegend zur Notwendigkeit einer integrierten Rendite-Risikosteuerung auf der Basis von so genannten Risiko-Wert-Modellen MAURER (2000) sowie die Ausführungen in Kapitel 3.2.2.

537 Vgl. übereinstimmend WÖHLE (2003), S. 82-83 und S. 116. 
Es sei an dieser Stelle darauf hingewiesen, dass der entwickelte Modellansatz keine pfadabhängigen Entscheidungsmechanismen bzw. Managementregeln berücksichtigt. Ebenfalls wird kein mathematischer Optimierungsalgorithmus implementiert, so dass durch das Modell selbst keine „optimale“ Geschäftsstrategie vorgeschlagen wird. Nichtsdestotrotz bietet das Modell dem Management die Möglichkeit, diejenige Strategie auszuwählen, die in Abhängigkeit von dem gewählten Sicherheitsniveau die attraktivste Rendite-Risiko-Relation aufweist. Im Begriffsverständnis dieser Arbeit liegt also auch ein Entscheidungsmodell vor.

Bevor im nachfolgenden Abschnitt die Modellarchitektur im Überblick vorgestellt wird, sollen zunächst einige Grundannahmen formuliert werden, die sowohl die Modellstruktur als auch die Vorgehensweise der stochastischen Modellierung betreffen.

- Das Modellunternehmen fungiert sowohl im versicherungstechnischen Geschäft als auch am Kapitalmarkt als Preisnehmer. Dies bedeutet, dass durch Marktaktivitäten, wie z.B. den Kauf von zusätzlichem Rückversicherungsschutz oder den Verkauf von Aktienbeständen, keine Rückwirkungen auf die Marktpreise oder die zugrunde liegenden Zufallsgesetzmäßigkeiten ausgehen. ${ }^{538}$

- Die versicherungstechnische Bruttorisikoposition unterliegt im Modellverlauf keiner strukturellen Dynamik. Da keine pfad- bzw. zwischenergebnisabhängigen Entscheidungsmechanismen implementiert sind, ist die Struktur des versicherungstechnischen Portfolios (Spartenmix) sowie die Preispolitik (Prämienniveau) somit zu Modellbeginn exogen vorgegeben. ${ }^{539}$ Diese Annahme ermöglicht es, verschiedene Strategien und Risikosteuerungsinstrumente auf ein identisches Risikokollektiv anzuwenden und in Form von ceteris-paribus-Analysen vergleichen zu können.

- Die Gesamtschadenverteilungen der betrachteten Risikokollektive können durch das kollektive Modell der Risikotheorie, d.h. durch eine geeignete Verknüpfung einer separat modellierten Schadenanzahl- und Schadenhöhenverteilung dargestellt werden. $^{540}$

- Dem Modellunternehmen stehen zu Beginn der Modellperiode genügend Eigenmittel zur Verfügung, um die aufsichtsrechtlichen Solvabilitätsanforderungen (gemäß „Solvency I“) zu erfüllen.

\footnotetext{
$538 \mathrm{Vgl}$. zu dieser zentralen Annahme auch BROHM (2002), S. 162-163.

539 Vgl. vertiefend BROHM (2002), S. 163-166.

540 Siehe hierzu Kapitel 5.4.3.1 und die dort angegebene Literatur.
} 


\subsubsection{Modellarchitektur und Datenbasis}

Im Folgenden soll die Architektur des Modellansatzes im Überblick beschrieben werden. Die Modellarchitektur gibt gleichzeitig auch die weitere Gliederung des Hauptkapitels 5 vor. Wie bereits erwähnt, stellen die Verteilungen des versicherungstechnischen Ergebnisses und des Kapitalanlageergebnisses zentrale Zielgrößen des Modells dar. Das Gesamtergebnis eines Schaden- und Unfallversicherungsunternehmens setzt sich vereinfachend aus folgenden Komponenten zusammen: ${ }^{541}$

Verdiente Bruttoprämien ${ }^{542}$

- Aufwand für Versicherungsfälle

- Aufwand für den Versicherungsbetrieb

+/- Rückversicherungsergebnis

+/- Abwicklungsergebnis

$=$ Versicherungstechnisches Nettoergebnis $^{543}$

+ - Ergebnis aus Kapitalanlagen

$=$ Jahresüberschuss vor Steuern

- Steuern $^{544}$

$=$ Jahresüberschuss nach Steuern

Sämtliche Komponenten dieser Ergebnisrechnung können durch eigenständige Module abgebildet werden. Die Module werden in den Kapiteln 5.4 und 5.5 zunächst separat erläutert und in Kapitel 5.6 schließlich zu einem Gesamtmodell zusammengeführt. Sowohl die Bruttoprämien als auch der Betriebsaufwand werden im Modell als deterministisch angenommen. Der Betriebsaufwand enthält sowohl die an die Absatzorgane zu zahlenden Provisionen als auch Betriebskosten in Form von Verwaltungskosten und

${ }^{541}$ An dieser Stelle wird zunächst die in der Praxis geläufigere bilanzielle Terminologie und Darstellungsweise gewählt. Auf die Unterscheidung von (bilanziellem) Geschäftsjahresergebnis und Anfalljahresergebnis wird in den Kapiteln 5.4.1.2 und 5.4.7 detailliert eingegangen.

${ }^{542}$ Unter der Bruttoprämie wird im Folgenden die Summe aus Risikoprämie und Kosten- und Gewinnzuschlag (vor Rückversicherung) verstanden. Vgl. auch Kapitel 5.4.2. Des weiteren wird von der Prämisse ausgegangen, dass die Prämien (synonym: Beiträge) in voller Höhe am Anfang eines Geschäftsjahres zahlungswirksam vereinnahmt werden. Es wird daher von der Existenz von Beitragsüberträgen abstrahiert.

${ }^{543}$ Das versicherungstechnische Ergebnis versteht sich in diesem Kontext ohne Berücksichtigung der Schwankungsrückstellung und ist im Sinne der RechVersV mit dem so genannten ,Zwischenergebnis“ (Position I.9 gemäß Formblatt 2 der RechVersV) vergleichbar. Auf eine Erfassung der Schwankungsrückstellung wird aus Gründen der Übersichtlichkeit verzichtet. Aufgrund des in $\S 29$ sowie Anlage zu $\S 29$ RechVersV klar formulierten mathematischen Algorithmus ist eine Implementierung jedoch mit vertretbarem Aufwand möglich. Vgl. in diesem Kontext auch KARTEN (1988), S. 763-765.

544 Im Rahmen dieser Arbeit bleiben vereinfachend steuerbilanzielle Bewertungsdifferenzen unberücksichtigt. 
internen Schadenregulierungskosten. Dem Charakter des Versicherungsgeschäfts entsprechend wird die Gesamtschadenlast, die annahmegemäß neben den Entschädigungsleistungen an die Versicherungsnehmer auch die externen Schadenregulierungskosten (z.B. für externe Sachverständige) umfasst, stochastisch im Schadenmodell abgebildet. Um die Schadencharakteristik jedes Teilkollektivs hinreichend genau abbilden zu können, werden mit Basisschäden, Großschäden und Schäden aus Naturgefahren drei unterschiedliche Schadenkategorien modelliert, die sich additiv zum Gesamtschaden eines jeden Teilkollektivs zusammensetzen. ${ }^{545}$

Im Rückversicherungsmodell werden die gängigen Vertragsformen der proportionalen Rückversicherung (Quotenvertrag, Summenexzedentenvertrag) und der nichtproportionalen Rückversicherung (Einzelschadenexzedentenvertrag, Kumulschadenexzedentenvertrag und Stop Loss) abgebildet. Der Schadenaufwand wird vertragsspezifisch zwischen dem Modellunternehmen (= Erstversicherer) und dem Rückversicherer aufgeteilt. $^{546}$

Neben den aktuell bestehenden Vertragsverhältnissen eines Schaden- und Unfallversicherers müssen auch diejenigen „Altverträge“ im Modell abgebildet werden, deren Garantiezeitraum zwar bereits beendet ist, die jedoch nach wie vor ein Schadenpotenzial tragen, da sie entweder noch nicht bekannt oder noch nicht vollständig abgewickelt sind. ${ }^{547}$ Dem Versicherungsunternehmen bereits gemeldete, jedoch noch nicht zur Auszahlung gelangte Schäden müssen durch eine angemessene Dotierung der Rückstellung für noch nicht abgewickelte Versicherungsfälle (Einzelfallreserven) berücksichtigt werden. ${ }^{548}$ Die bereits eingetretenen, jedoch noch nicht gemeldeten Schä$\operatorname{den}^{549}$ müssen mittels geeigneter Schätzverfahren approximativ bestimmt und durch eine pauschale Dotierung der so genannten Spätschadenrückstellung berücksichtigt werden. Sowohl bei der Spätschadenrückstellung als auch bei den Einzelfallreserven

545 Die Differenzierung zwischen Basisschadenlast und einzelnen Großschäden ist erforderlich, da einige Rückversicherungsformen, insbesondere der (Einzel-) Schadenexzedentenvertrag, auf Einzelschadenbasis ansetzen. Vgl. vertiefend Kapitel 5.4.5.2.

${ }^{546}$ Vgl. zu den Vertragsformen der Rückversicherung auch LIEBWEIN (2000) und Kapitel 5.4.5.2.

547 Vgl. im Folgenden auch BROHM (2002), S. 221-222, OECKING/SANNER (2004), S. 26-30, KORYCIORZ (2004), S. 157-159 und die Ausführungen in Kapitel 5.4.6.

$548 \mathrm{Vgl}$. zur handelsrechtlich vorgeschriebenen Dotierung der Rückstellung für noch nicht abgewickelte Versicherungsfälle auch $\S 341$ e Abs. 1 Satz 1 HGB. Im Aufsichtsrecht wird die Adäquanz der Dotierung durch $\S 81$ Abs. 1 Satz 5 VAG konkretisiert.

549 Diese Schäden werden auch als Spätschäden oder IBNR-Schäden (incurred but not reported) bezeichnet. Darüber hinaus ist es auch denkbar, dass ein Schaden zwar gemeldet ist, dessen Schadenhöhe jedoch noch nicht bestimmt werden kann. Unter Umständen ist die gebildete Einzelschadenreserve daher zu gering. Diese Art der Spätschäden wird auch als IBNER-Schäden bezeichnet (incurred but not enough reserved). Vgl. zur Reservierung und bilanziellen Behandlung von Spätschäden ausführlich SCHMIDT (2001), S. 269-296. 
kommt es regelmäßig zu Abweichungen zwischen der reservierten Schadenhöhe und der tatsächlich zu leistenden Schadenzahlung. Diese Abweichung führt zu einem Abwicklungsergebnis, welches entweder positiv (Abwicklungsgewinn) oder negativ (Abwicklungsverlust) ausfallen kann. Der Barwert der Summe der zukünftig erwarteten Abwicklungsergebnisse stellt im Wesentlichen den Marktwert eines Schadenportfolios dar. Eine differenzierte Berücksichtung von zahlungswirksamen und reservierten Schäden erfolgt im so genannten Abwicklungsmodell, welches zu diesem Zweck auf Methoden der aktuariellen Reservebewertung zurückgreift. ${ }^{550}$

Im Kapitalanlagemodell wird die Verteilung des Kapitalanlageergebnisses simuliert. Neben den vorschüssig vereinnahmten Prämien setzt sich der Zinsträger zu Beginn eines Geschäftsjahres aus den Bestandsgrößen des bilanziellen Eigenkapitals und den versicherungstechnischen Nettorückstellungen zusammen. Der Anfangsbestand der Kapitalanlagen wird in drei Hauptanlageklassen (Geldmarkt, Aktien, Anleihen mit unterschiedlichen Restlaufzeiten) investiert, welche empirisch ermittelten Renditeverteilungen unterliegen. Die zur Simulation des Kapitalanlageergebnisses erforderlichen Kapitalmarktszenarien bzw. Renditeverteilungen werden über einen so genannten „Economic Scenario Generator" erzeugt. Im Verlauf des Geschäftsjahres wird der Anfangsbestand der Kapitalanlagen durch den aus dem Versicherungsgeschäft resultierenden Cashflow erhöht bzw. reduziert. Die Modellierung des versicherungstechnischen Cashflows erfolgt bereits im Abwicklungsmodell mit Hilfe empirisch geschätzter Zahlungsmuster.

Die Implementierung der beschriebenen Teilmodelle und des Gesamtmodells erfolgt mit dem Softwaretool IGLOO Professional ${ }^{\mathrm{TM}}$, welches von der aktuariellen Unternehmensberatung EMB (benannt nach den Gründungspartnern ENGLISH, MATTHEWS, BROCKMAN) entwickelt wurde. ${ }^{551}$ IGLOO Professional ${ }^{\mathrm{TM}}$ ist eine Software zur Finanzund Risikosimulation, die sich im Kontext quantitativer Risikoanalysen durch ihre hohe Leistungsfähigkeit und Flexibilität auszeichnet, jedoch lediglich den Anwendungsrahmen für eine benutzerindividuelle Modellierung zur Verfügung stellt. Die erforderlichen Inputdaten des Modells können durch eine Schnittstelle zu Microsoft Excel ${ }^{\mathrm{TM}}$ bereitgestellt werden. Zusätzlich zu den in IGLOO Professional ${ }^{\mathrm{TM}}$ bereits enthaltenen (graphischen) Auswertungsmöglichkeiten ist ebenfalls ein Datenexport zu gängigen Softwareanwendungen möglich. Die verwendete Software ist nicht dem Vorwurf einer „Black-Box-Modellierung“ ausgesetzt, da die mathematisch-stochastische Struktur des Gesamtmodells für jedes Teilmodul und für jeden Simulationsschritt einsehbar ist.

550 Vgl. hierzu Kapitel 5.4.6.

551 EMB ist eine der führenden (unabhängigen) aktuariellen Beratungsgesellschaften in Großbritannien und hat sich auf die Beratung von Schaden- und Unfallversicherungsunternehmens sowie Rückversicherungsunternehmen spezialisiert. 
Darüber hinaus kann das Gesamtergebnis jedes Simulationslaufs retrograd in seine Einzelkomponenten zerlegt werden. Die verwendeten Wahrscheinlichkeitsverteilungen werden durch einen bereits integrierten Zufallszahlengenerator erzeugt. ${ }^{552}$

Sämtliche modellgestützten Analysen dieser Arbeit basieren auf einer Simulationsanzahl von $\mathrm{n}=30.000$. Dieser Simulationsumfang erscheint zur Erreichung stabiler Modellergebnisse hinreichend und gewährleistet zudem eine befriedigende Beurteilung des Tailverhaltens. Zur visuellen Abschätzung des erforderlichen Simulationsumfangs wird auf die Methodik rollierender Erwartungswerte bzw. Standardabweichungen zurückgegriffen. ${ }^{553}$ Das Konvergenzverhalten der Modellergebnisse wird in Anhang D exemplarisch für $\mathrm{n}=5.000$ und $\mathrm{n}=30.000$ untersucht.

Der nachfolgenden Untersuchung liegt der Datensatz eines deutschen Schaden- und Unfallversicherungsunternehmens in der Rechtsform einer Aktiengesellschaft zugrunde. Das Originalunternehmen agiert primär im Privatkundensegment. Gleichwohl entfällt ein signifikanter Anteil des Bruttoprämienvolumens auf das Geschäft mit Gewerbe- und Industriekunden. Das Unternehmen verfügt darüber hinaus traditionell über einen hohen regionalen Marktanteil in der Verbundenen Gebäudeversicherung und Kraftfahrtversicherung. Aufgrund nur beschränkter Möglichkeiten zur regionalen Diversifizierung impliziert der hohe Marktanteil in der Gebäudeversicherung ein beträchtliches Kumulpotenzial in Bezug auf Naturgefahrenereignisse, wie beispielsweise Sturm, Hagel oder Überschwemmung.

Um Rückschlüsse auf das Originalunternehmen auszuschließen, wurden die Größenverhältnisse (Portfoliovolumina) verändert und die Risikoparameter strukturerhaltend modifiziert. Die Datenbasis erhebt daher lediglich den Anspruch auf strukturelle Repräsentativität und dient dazu, ausgewählte Fragestellungen der Versicherungspraxis und exemplarische Effekte einer risikokapitalbasierten Steuerung zu illustrieren. Wenngleich die zugrunde liegenden Parameterkonstellationen hinsichtlich ihrer Struktur als marktnah eingestuft werden können, triff dies aufgrund der bewussten Beschränkung auf ausgewählte Versicherungszweige auf den Spartenmix des „Modellunternehmens" ausdrücklich nicht zu.

${ }^{552}$ Die Periodenlänge des verwendeten Zufallszahlengenerators beträgt $2^{32}=4.294 .967 .296$. Die Dichte- und Verteilungsfunktionen der im Rahmen dieser Arbeit verwendeten Wahrscheinlichkeitsverteilungen sind in Anhang $\mathrm{C}$ aufgeführt.

553 Vgl. vertiefend zur Anlaufphase und zum Einschwingprozess von stochastischen Simulationen LIEBL (1995), S. 156-166. Zur Dimensionierung des erforderlichen Simulationsumfangs vgl. auch KoCH (1994), S. 29-110. 
Um auch in bilanzieller Hinsicht Rückschlüsse auf das Originalunternehmen zu vermeiden, wurden die in Kapitel 6.3 verwendeten bilanziellen Bestandsgrößen, insbesondere die aus der vereinfachten Eröffnungsbilanz ersichtlichen Schadenrückstellungen (Eingangsreserven) auf ein reduziertes Niveau skaliert. Wie für die Schaden- und Unfallversicherung charakteristisch, entfällt jedoch unverändert ein Großteil der Schadenrückstellungen auf die HUK-Sparten. Die Aufteilung der Kapitalanlagen auf die Hauptanlageklassen kann näherungsweise als marktüblich bezeichnet werden, wurde jedoch zum Zwecke dieser Arbeit exogen vorgegeben. Im Verlauf der weiteren Modellbeschreibung werden die verwendeten Inputparameter für jedes Teilmodell schrittweise offen gelegt und erläutert.

\subsubsection{Zeitliche Struktur}

Aus dem eingangs formulierten Modellzweck der strategischen Rendite-Risikosteuerung ergibt sich grundsätzlich die Notwendigkeit, einen oder mehrere Zeitpunkte im Modellansatz zu verankern, in denen das Management ex-ante eine Unternehmensstrategie vorgibt bzw. steuernd in den Modellverlauf eingreift. In der einschlägigen Literatur wird daher berechtigterweise der Einsatz mehrperiodiger Modelle gefordert. ${ }^{554}$ Die Implementierung pfadabhängiger Entscheidungsmechanismen gestaltet sich jedoch aufgrund des stochastischen Charakters des Schaden- und Unfallversicherungsgeschäfts in der Regel sehr schwierig und birgt auch die Gefahr von Fehlsteuerungen. ${ }^{55}$ Diese Gefahr resultiert aus der grundlegenden Problematik, dass zum Zeitpunkt der Modellierung lediglich die aus der Vergangenheit bekannten Wirkungszusammenhänge abgebildet werden können. Die Interdependenzen zwischen den empirischen Gegebenheiten und den zukünftigen, mithin unbekannten Entwicklungen können nicht erfasst werden. ${ }^{556}$ Bleibt dieser Sachverhalt unbeachtet, läuft man in mehrperiodigen Modellen und bei der Implementierung pfadabhängiger Entscheidungsmechanismen jedoch Gefahr, von einer bekannten Portfolio- und Risikostruktur auf eine zukünftige Struktur schließen zu wollen. Im Kontext der Risikokapitalallokation wurde bereits darauf hingewiesen, dass dieser Rückschluss jedoch in der Regel nicht möglich ist, da sich beispielsweise nach einem Strategiewechsel neue Ausgleichseffekte und Risikoallokationen ergeben, die sich aus der Ausgangssituation nicht abschätzen lassen. ${ }^{557}$

\footnotetext{
554 Vgl. stellvertretend BROHM (2002), S. 137. KRIELE/LIM/REICH befürworten den Einsatz mehrperiodiger Modelle u.a. unter der Zielsetzung, nachhaltige negative Trends abbilden zu können und auf diese Weise die Gefahr eines verfrühten „prozyklischen Reagierens" auf bestimmte Umweltzustände und insbesondere die Kapitalmarktentwicklung zu verringern. Vgl. KRIELE/LIM/REICH (2004), S. 1049.

555 Vgl. übereinstimmend BROHM (2002), S. 137-140 und SCHRADIN (1998), S. 113.

556 Vgl. vertiefend ADAM (1996), S. 91.

557 Vgl. die Ausführungen in Kapitel 4.2.2 und GRÜNDL/SCHMEISER (2004b), S. 7.
} 
Es wurde bereits in Kapitel 5.1.3 darauf hingewiesen, dass der Informationswert eines Modells durch die Qualität der zugrunde liegenden Annahmen und Inputparameter determiniert wird. Mit zunehmender Länge des Prognosehorizonts nimmt die Güte der Parameterschätzungen jedoch in der Regel deutlich $a b .{ }^{558}$ Insbesondere bei der Modellierung und Interpretation der Randbereiche von Verteilungen können in diesem $\mathrm{Zu}$ sammenhang substanzielle Fehleinschätzungen auftreten. Als besonders problematisch ist außerdem die Vorgehensweise einzuschätzen, mehrere einperiodische Modelle ohne verlaufsabhängige Reaktionsmöglichkeiten aneinander zu reihen und auf diese Weise eine Pfadabhängigkeit zu suggerieren. ${ }^{559}$

Im Rahmen dieser Arbeit wird ein einperiodiger Modellhorizont zugrunde gelegt. Die Komplexität der Darstellung wird durch diese Vorgehensweise signifikant reduziert. Gleichwohl ist die einperiodige Betrachtung mit der Einschränkung verbunden, dass lediglich Risikoausgleichseffekte im Kollektiv und keine zeitlichen Ausgleichseffekte, z.B. die Risikovorsorge für Großschäden durch mehrjährigen Reserveaufbau, untersucht werden können. ${ }^{560}$ Es ist daher im Einzelfall zu entscheiden, ob eine reduzierte Qualität der Parameterschätzung durch den vermuteten Zusatznutzen einer mehrperiodigen Modellierung gerechtfertigt werden kann. Da die Entwicklung interner Risikomodelle in der Praxis vielfach noch in den Anfängen begriffen ist, wird an dieser Stelle die Ansicht vertreten, dass den Ergebnissen eines hinreichend validierten Einperiodenmodells durchaus ein höherer Informationswert beizumessen ist als den Ergebnissen mehrperiodiger Modelle, in denen sich gegebenenfalls die verfälschenden Effekte nicht zutreffender Modellannahmen oder Inputparameter über mehrere Perioden verstärken. ${ }^{561}$ Von dieser grundsätzlichen Einschätzung unberührt bleibt die ökonomische Notwendigkeit, die zeitliche Struktur des Schadenanfalls geeignet im Modell abzubilden. Die Tatsache, dass der erwartete Endschadenaufwand eines Anfalljahres ${ }^{562}$ teilweise erst in späteren Geschäftsjahren zur Auszahlungen gelangt und die vorschüssig vereinnahmten Prämien daher für mehrere Perioden als Anlagekapital zur Verfügung stehen, wird im vorliegenden Modellansatz durch einen Diskontierungsmechanismus berücksichtigt. ${ }^{563}$

\footnotetext{
558 Vgl. im Folgenden auch HERI/ZimMERMANN (2001), S. 1004-1009 und BROHM (2002), S. 137.

559 Vgl. hierzu und im Folgenden BROHM (2002), S. 137. Dieser weist außerdem auf die Gefahr hin, dass „ein sich am Ende einer Periode im Ruin befindliches Versicherungsunternehmen am Ende der nächsten Periode wieder ein positives Eigenkapital erreicht.“ BROHM (2002), S. 137.

560 Vgl. zur zeitlichen Dimension des Risikoausgleichs vertiefend SCHRADIN (1998), S. 130-134.

561 Vor diesem Hintergrund ist ebenfalls zu vermuten, dass im Rahmen von Solvency II zunächst einperiodige Risikomodelle zur Bemessung des Zielkapitals herangezogen werden können. Vgl. auch KRIELE/LIM/REICH (2004), S. 1049.

${ }^{562}$ Eine Erläuterung der Begriffe „Anfalljahr“ und „Geschäftsjahr“" erfolgt in Kapitel 5.4.1.2.

$563 \mathrm{Vgl}$. hierzu die Ausführungen in Kapitel 5.4.7.1.
} 
Die Verwendung einperiodiger Modelle wird auch in der Literatur als zulässig erachtet. Im Anwendungskontext der Rückversicherung formuliert SCHRADIN:

\begin{abstract}
„Relativ zum Mehrperiodenmodell führt die Verwendung eines einperiodischen Ansatzes nicht systematisch zu Erkenntnisverlusten, insoweit die strukturelle risikotheoretische Analyse der Elemente der Rückversicherungstätigkeit und ihres Zusammenwirkens möglich bleibt, ein zeitliches Hintereinanderschalten mehrerer Einperiodenmodelle die Analyse periodenübergreifender Effekte gestattet und insoweit ein hohes Maß an Konsistenz zu den aufsichtsrechtlichen Solvabilitätsmodellen [...] erreicht wird. “564
\end{abstract}

Dieser Einschätzung soll hier mit der Einschränkung gefolgt werden, dass ein zeitliches Hintereinanderschalten mehrerer Einperiodenmodelle aus o.g. Gründen ausdrücklich nicht befürwortet wird. Im Interesse einer übersichtlicheren Darstellung wird im weiteren Untersuchungsverlauf vereinzelt auf konkrete Jahreszahlen zurückgegriffen. Dabei bezeichnet 2004 immer das simulierte Anfalljahr bzw. simulierte Geschäftsjahr.

\title{
5.4 Modellierung der Versicherungstechnik
}

\subsubsection{Vorüberlegungen}

\subsubsection{Zweidimensionale Modellierung: Sparten und Geschäftsfelder}

Die strategische Steuerung eines Versicherungsunternehmens basiert in der Regel auf einer Segmentierung des Gesamtportfolios in strategische Geschäftsfelder bzw. Steuerungseinheiten. ${ }^{565}$ In vielen Gesellschaften herrscht diesbezüglich noch die funktionsbzw. spartenspezifische Aufbauorganisation vor. ${ }^{566}$ Im Kontext strategischer Entscheidungen ist dieses Organisationsprinzip jedoch nicht problemadäquat, da ein expliziter Markt- und Wettbewerbsbezug nicht gewährleistet ist und demzufolge spartenübergreifende Cross-Selling- oder Cross-Storno-Effekte nicht erfasst werden. Demgegenüber kann durch eine kundengruppenspezifische Abgrenzung strategischer Geschäftsfelder vielfach eine eigenständige und bedürfnisgerechtere Marktbearbeitung erreicht werden. ${ }^{567}$ Ein Modellansatz zur strategischen Steuerung der Rendite-Risiko-Position sollte daher neben der handelsrechtlich geforderten und organisatorisch bisweilen noch verankerten Spartensicht auch eine differenziertere Darstellung nach Geschäftsfeldern, z.B. nach Kundengruppen, unterstützen. Die Entscheidung, in welcher Form das versicherungstechnische Portfolio in einzelne Steuerungseinheiten aufgeteilt werden soll,

\footnotetext{
564 SCHRADIN (1998), S. 113.

${ }^{565}$ Vgl. zur strategischen Geschäftsbereichssteuerung in Finanzdienstleistungsunternehmen grundlegend GRAUMANN/HellMAYR (1998) und GRÜNDL/SCHMEISER (2002). Zur Abgrenzung strategischer Geschäftsfelder in Versicherungsunternehmen vgl. SCHRADIN (1994), S. 82-93.

566 Vgl. vertiefend EURICH/HÄUSELE (2001), S. 105-109.

567 Vgl. im Folgenden auch SCHRADIN (1994), S. 87.
} 
übt einen determinierenden Einfluss auf die Datenstruktur des Modells aus und sollte daher das Ergebnis eines umfangreichen Abstimmungsprozesses mit den Modelladressaten sein. Im Hinblick auf die Güte und Steuerungsrelevanz der Modellergebnisse ist ferner zu berücksichtigen, dass für die modellierten Teilkollektive eine hinreichend valide Datenbasis zur Verfügung steht. ${ }^{568}$ Abgesehen von dem nur noch schwer handhabbaren Komplexitätsgrad hoch detaillierter Modelle, bergen diese auch immer die Gefahr, dem Entscheidungsträger eine gewisse Scheingenauigkeit zu suggerieren. ${ }^{569}$

Im Rahmen dieser Arbeit wird exemplarisch davon ausgegangen, dass das Modellunternehmen einen Steuerungsansatz verfolgt, dem eine kundengruppenspezifische Definition strategischer Geschäftsfelder zugrunde liegt. Es wird exemplarisch zwischen Privatkunden, Gewerbekunden und Industriekunden ${ }^{570}$ differenziert. Darüber hinaus werden nur ausgewählte Versicherungszweige betrachtet. Die Beschränkung auf insgesamt neun Versicherungszweige und drei Geschäftsfelder dient einer übersichtlicheren Modellbeschreibung und einer transparenteren Interpretation der Modellergebnisse. Die generelle Systematik des Modellansatzes kann jedoch - unter Inkaufnahme einer erhöhten Komplexität und Datenfülle - auch auf größere Portfolios angewendet werden. Die versicherungstechnische Portfoliostruktur des Modellunternehmens kann durch folgende Sparte-Geschäftsfeld-Matrix verdeutlicht werden. ${ }^{571}$

\begin{tabular}{|c|c|c|c|c|}
\hline Sparte & Geschäftsfeld & Privatkunden & Gewerbekunden & Industriekunden \\
\hline Allgemeine Unfall & $\overline{[\mathrm{AU}]}$ & & & \\
\hline Kraftfahrt-Haftpflicht & {$[\mathrm{KH}]$} & & & \\
\hline Kraftfahrt-Kasko & [Kasko] & & & \\
\hline Feuer Industrie & [FIND] & & & \\
\hline Allgemeine Sturm & [AST] & & & \\
\hline VGV Feuer & [VGV F] & & & \\
\hline VGV Leitungswasser & [VGV LW] & & & \\
\hline VGV Sturm & [VGV ST] & & & \\
\hline VGV Elementar & [VGV EL] & & & \\
\hline
\end{tabular}

Abbildung 5.2: Struktur des versicherungstechnischen Modellportfolios

568 Vgl. übereinstimmend WAGNER/WARMUTH (2005), S. 56-62 und darüber hinaus die Ausführungen zum „Modellrisiko“ in Kapitel 5.1.3.

Vgl. zur Wahl des angemessenen Abstraktionsgrads im Modell und zur Problematik der Scheingenauigkeit auch LIEBL (1995), S. 216-219 und S. 226.

570 Unter Industriekunden werden Unternehmen verstanden, die mehr als 50 Mitarbeiter beschäftigen oder deren Jahresumsatz die Grenze von 5 Mio. GE übersteigt. Es handelt sich daher ausdrücklich nicht ausschließlich um so genannte „Großkunden“.

571 Das Feuerrisiko wird annahmegemäß von Industriekunden über die (Industrielle) Feuerversicherung, von Privatkunden über die „Verbundene Wohngebäudeversicherung“ (VGV) abgedeckt. Das Sturmrisiko wird von Gewerbe- und Industriekunden durch den Versicherungszweig „Allgemeine Sturm" versichert. Darüber hinaus wird angenommen, dass die Sparte VGV ausschließlich von Privatkunden gezeichnet wird. Die Anzahl der zu modellierenden Segmente - in Abbildung 5.2 schraffiert dargestellt - reduziert sich somit von 27 auf 16 . 
Die Zusammenstellung der Versicherungszweige erfolgte unter der Zielsetzung, ausgewählte Effekte und Charakteristika des versicherungstechnischen Geschäfts analysieren zu können. Dabei wurde eine marktübliche Relation zwischen HUK-Sparten und Sachversicherungssparten zugrunde gelegt. ${ }^{572}$ Neben der im Marktdurchschnitt sehr profitabel verlaufenden Sparte „Allgemeine Unfall“ ${ }^{\text {‘573 }}$ wurde mit „KraftfahrtHaftpflicht" exemplarisch eine Sparte mit langer Abwicklungsdauer in das Portfolio aufgenommen (sog. Longtail-Sparte). Die Auswahl der Sparte „Kraftfahrt-Kasko“ erfolgte insbesondere mit der Zielsetzung, die Auswirkungen von Hagelereignissen untersuchen zu können.

Im Bereich der Sachversicherung wurde mit der Industriellen Feuerversicherung bewusst eine Sparte mit ausgeprägter Großschadenanfälligkeit und entsprechend hoher Schadenvolatilität in das Portfolio aufgenommen. Das Sachversicherungsportfolio enthält ferner die Sparten „Allgemeine Sturm“ und „Verbundene Wohngebäudeversicherung" mit den versicherten Gefahren Feuer, Leitungswasser, Sturm und Überschwemmung. Die Betrachtung dieser Sparten ermöglicht die Einbeziehung weiterer Naturbzw. Kumulgefahren ${ }^{574}$, insbesondere die Analyse von Sturm- und Überschwemmungsschäden über unterschiedliche Versicherungszweige hinweg. Insgesamt ergeben sich für das Modellunternehmen 16 versicherungstechnische Risikokollektive, deren charakteristische Prämien-, Schaden- und Kostenparameter erfasst werden müssen.

\subsubsection{Differenzierung zwischen Anfalljahren und Geschäftsjahren}

Für die weiteren Ausführungen zur Schadenmodellierung und Schadenprognose ist die Unterscheidung von Anfall- bzw. Zeichnungsjahren und Geschäftsjahren ${ }^{575}$ von grundlegender Bedeutung zu. Die Anfalljahressicht und Geschäftsjahressicht stellen letztlich zwei unterschiedlich motivierte Betrachtungsweisen des versicherungstechnischen Kerngeschäfts dar, die ineinander überführbar sind. Im weiteren Verlauf dieser Arbeit wird vereinfachend angenommen, dass alle Policen eines Zeichnungsjahres genau zu dessen Beginn gezeichnet werden und außerdem eine Vertragslaufzeit von ei-

572 Vgl. hierzu auch die Ausführungen in Kapitel 6.2.1.1.1.

573 Die bilanzielle Schadenquote der im Gesamtverband der deutschen Versicherungswirtschaft e.V. (GDV) organisierten Schaden- und Unfallversicherungsunternehmen lag im Zeitraum von 1993 bis 2003 stabil zwischen $51,0 \%$ und $57,4 \%$. Vgl. GDV (2004), S. 101. Die bilanzielle Schadenquote ist definiert als Bruttoaufwendungen für Versicherungsfälle in Relation zu den verdienten Bruttobeiträgen.

574 Als Ereignis- bzw. Kumulschaden wird die Summe von Einzelschäden bezeichnet, die innerhalb eines festgelegten Zeitraums (z.B. 72 Stunden) aus einem Schadenereignis resultieren. Vgl. FARNY (2000), S. 86.

575 Es sei unterstellt, dass das Geschäftsjahr mit dem Kalenderjahr übereinstimmt. Beide Begriffe werden daher im Folgenden synonym verwendet. 
nem Jahr aufweisen. Unter diesen Prämissen kann jedes betrachtete Zeichnungsjahr auch als Anfalljahr interpretiert werden („Vereinfachtes Zeichnungsjahrkonzept“ $).{ }^{576}$

Die Anfalljahressicht stellt auf die Frage ab, welche finanzielle Belastung zukünftig aus sämtlichen Schäden zu erwarten ist, welche innerhalb des betrachteten Anfalljahres eingetreten sind. Dabei ist es unerheblich, ob diese Schäden bereits zur Auszahlung gelangt sind. Für die Zuordnung eines Schadens zu einer bestimmten Periode ist demzufolge der wirtschaftliche Anfall ausschlaggebend. Das Ergebnis eines Anfalljahres (AJ) ergibt sich als Differenz zwischen den verdienten Prämien und den bereits gezahlten bzw. den in zukünftigen Geschäftsjahren (GJ) aus dem betrachteten Anfalljahr erwarteten Schäden und Kosten. Diese prospektive Sichtweise wird durch Abbildung 5.3 graphisch verdeutlicht:

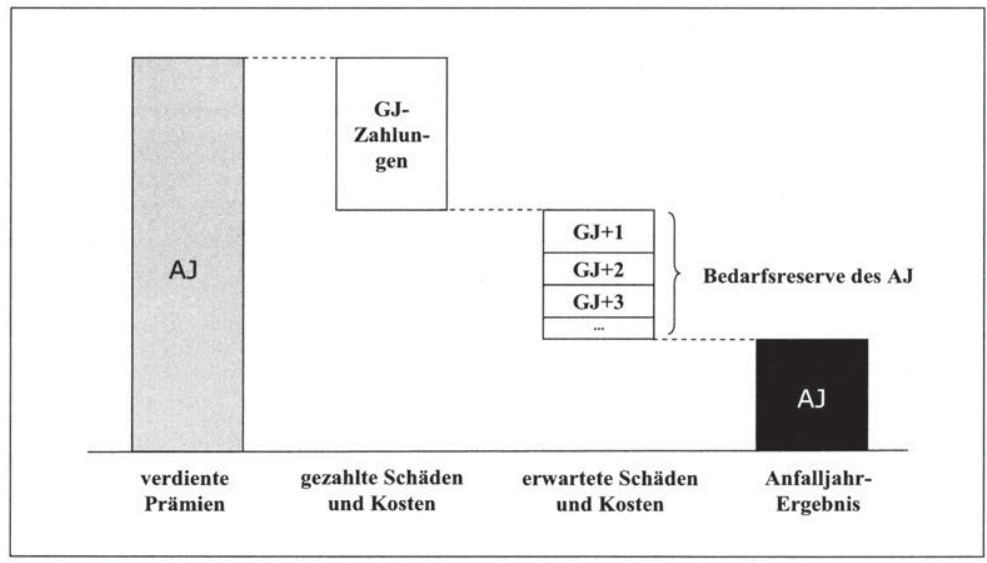

Abbildung 5.3: Ermittlung des Ergebnisses eines Anfalljahres

Den im Rahmen der Schadenprognose verwendeten mathematischen Schätzverfahren „ist gemeinsam, dass sie versuchen, die Erfahrungen früherer Anfalljahre (= Schadeneintrittsjahre) auf spätere Anfalljahre zu übertragen. “577 Die Höhe des Schadenbedarfs, der mittels aktuarieller Verfahren für ein gesamtes Anfalljahr prognostiziert werden kann, wird als Endschadenbedarf oder Ultimate bezeichnet. ${ }^{578}$ Die Differenz zwischen

576 Die Begriffe Anfalljahr und Zeichnungsjahr finden daher im weiteren Verlauf synonym Verwendung. Vgl. hierzu und zu den grundlegenden Zusammenhängen zwischen Anfall-, Abwicklungsund Zeichnungsjahren DOTTERWEICH (2004), S. 150-153.

577 MACK (2002), S. 223 [Hervorhebung im Original].

578 Vgl. zur Anwendung mathematischer Schätzverfahren beispielsweise MACK (2002), S. 221-320, SCHMIDT (2001) S. 269-296 oder HIPP (2001b), S. 117-130. Bezüglich der modellspezifischen Anwendung wird auf Kapitel 5.4.3.2.2 verwiesen. 
dem prognostizierten Ultimate und den bisher im Geschäftsjahr geleisteten Schadenzahlungen ist als „Bedarfsreserve“ geläufig. Diese stellt den minimal erforderlichen Zuführungsbetrag zur bilanziellen Schadenrückstellung dar (,best estimate“). ${ }^{579} \mathrm{Um}$ die dauerhafte Erfüllung der Verpflichtungen gegenüber den Versicherungsnehmern sicherzustellen und dem handelsrechtlichen Vorsichtsprinzip Rechnung zu tragen, wird über diese technische Rückstellung hinaus in der Regel noch ein Sicherheitsaufschlag (,risk margin“) passiviert. ${ }^{500}$ In Kapitel 3.1.2 wurde bereits darauf hingewiesen, dass die Überlegungen zur Neugestaltung des europäischen Solvabilitätssystems (Solvency II) vergleichbare Sicherheitszuschläge vorsehen. ${ }^{581}$

Der Differenzbetrag zwischen handelsrechtlich bilanzierter Schadenrückstellung und Bedarfsreserve gibt letztlich Aufschluss über die Höhe zukünftiger Abwicklungsgewinne oder den erforderlichen Nachreservierungsbedarf. Aus Abbildung 5.3 wird ersichtlich, dass in der Anfalljahrbetrachtung weder die Zahlungen für Schäden vorangegangener Anfalljahre noch die Veränderungen der bilanziellen Schadenrückstellungen Berücksichtigung finden. Die Anfalljahressicht wird somit nicht durch Rechnungslegungserfordernisse oder unternehmens- und fiskalpolitische Überlegungen beeinflusst. Im Vergleich zur Geschäftsjahressicht ermöglicht sie eine verursachungsgerechtere und risikoadäquatere Abbildung des Schadengeschehens und somit der versicherungstechnischen Risikoposition. ${ }^{582}$ Im Hinblick auf die Zeichnungs- und Rückversicherungspolitik eines Schaden- und Unfallversicherungsunternehmens stellt sie daher die entscheidungsrelevante Steuerungsperspektive dar. ${ }^{583}$

Das Geschäftsjahresergebnis leitet sich aus dem aktuellen Anfalljahr und sämtlichen vorangegangenen und noch nicht vollständig abgewickelten Anfalljahren ab. Sowohl die verdienten Prämien als auch die GJ-Zahlungen für Schäden und Kosten stimmen mit der Anfalljahressicht überein. An die Stelle der Bedarfsreserve tritt die Dotierung

579 Da die Bedarfsreserve in Abhängigkeit des zugrunde liegenden Abwicklungsmusters erst in den nachfolgenden Geschäftsjahren zur Auszahlung kommt, sollte diese unter dem ökonomischen Postulat der Marktpreisbewertung auf diskontierter Basis in die Ergebnisermittlung eingehen. Vgl. zum Berechnungsschema detailliert Kapitel 5.4.7.1. Zum handelsrechtlichen Abzinsungsverbot der Rückstellung für noch nicht abgewickelte Versicherungsfälle vgl. auch ANGERER (1994), S. 35-44 und HIPP/MiCHEL (1990), S. 124-134.

580 Vgl. auch OECKING/SANNER (2004), S. 26-27 und BoETIUS (1996), Rn. 102 sowie zum handelsrechtlichen Vorsichtsprinzip § 341 e Abs. 1 Satz 1 HGB.

581 „However, technical provisions can be defined [...] as a risk adjusted estimate of the present value of all future cash-flows arising from the commitments under examination. This can be split further in two parts: expected present values and risk margins." EU-KOMMISSION (2004a), S. 25.

582 „Die Anfalljahresbetrachtung überwindet dabei die Trägheit der Kalenderjahrbetrachtung, in der sich sämtliche Abwicklungseffekte vorangegangener Anfalljahre mischen und deshalb Trends und Strukturbrüche nur schwer zu erkennen sind.“ ALFERMANN/RADTKE/REICH (2004), S. 68.

Vgl. übereinstimmend ALFERMANN/RADTKE/REICH (2004), S. 68. 
der GJ-Schadenrückstellung (auch: GJ-Reserve), welche durch das Unternehmen im Rahmen der Bilanzgestaltung festgelegt wird. Wie bereits erläutert, übersteigen die bilanziellen Schadenrückstellungen den korrespondierenden „best estimate“ in einigen Versicherungszweigen erheblich. Dieser Sachverhalt wird als Überreservierung bezeichnet. Auf das Ergebnis eines Geschäftsjahres wirken außerdem die Abwicklungsgewinne und Abwicklungsverluste ${ }^{584}$ vorangegangener Zeichnungsjahre, die kumuliert betrachtet das Abwicklungsergebnis des Geschäftsjahres darstellen.

Abbildung 5.4 stellt die beschriebenen Zusammenhänge graphisch dar und veranschaulicht somit den retrospektiven Charakter der bilanziellen Geschäftsjahressicht.

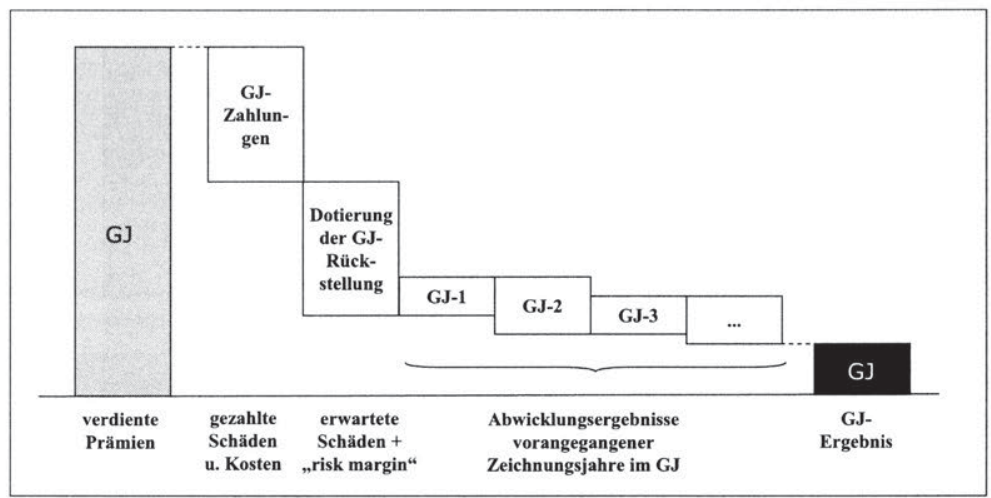

Abbildung 5.4: Ermittlung des Geschäftsjahresergebnisses

Die Modellierung sollte im Sinne eines zweidimensionalen Steuerungsansatzes sowohl die risikoorientierte Anfalljahressicht als auch die bilanzielle Geschäftsjahressicht abbilden können. ${ }^{585}$ Während die Anfalljahressicht für die Beurteilung von Zeichnungsund Rückversicherungsstrategien die entscheidungsrelevante Perspektive darstellt, kommt der bilanziellen Geschäftsjahressicht insbesondere dann Bedeutung zu, wenn das Modell zum Zwecke der Solvabilitätssteuerung eingesetzt werden soll, da sich die aufsichtsrechtlichen Solvabilitätserfordernisse derzeit noch an bilanziellen Stichtagsbetrachtungen festmachen ${ }^{586}$ Vor diesem Hintergrund stellt die bilanzielle Perspektive eine strenge Nebenbedingung für die betriebswirtschaftliche Steuerung dar. Darüber hinaus wurde bereits erörtert, dass der in einer Modellperiode für Kapitalanlagezwecke zur Verfügung stehende Zinsträger durch den versicherungstechnischen Netto Cashflow beeinflusst wird und sich die Stochastizität des Schadengeschehens somit auch

\footnotetext{
${ }^{584}$ Vgl. zur rechnerischen Ermittlung des Abwicklungsergebnisses auch Kapitel 5.4.6.

${ }^{585} \mathrm{Vgl}$. zum Bezugsrahmen eines zweistufigen Steuerungsmodells insbesondere Kapitel 3.2.4.

586 Vgl. hierzu ausführlich Kapitel 3.1.1.
} 
aktivisch fortsetzt. Im Sinne einer sowohl aktiv- als auch passivseitigen RenditeRisikosteuerung erscheint daher eine Aufspaltung des simulierten Gesamtschadens in zahlungswirksame und nicht-zahlungswirksame Komponenten unverzichtbar. ${ }^{587}$

In den vorangegangenen Abschnitten wurden sowohl die matrixartige Struktur des versicherungstechnischen Portfolios als auch die grundlegende Differenzierung zwischen risikoorientierter Anfalljahrperspektive und handelsrechtlicher Bilanzsicht vorgestellt. Auf dieser Grundlage wird in den nachfolgenden Kapiteln schrittweise die Modellierung der Versicherungstechnik erläutert. Der Systematik der versicherungstechnischen Ergebnisermittlung folgend, bildet die Modellierung der Versicherungsprämien den Ausgangspunkt der Beschreibung.

\subsubsection{Prämienmodell}

Die Versicherungsprämie stellt den Preis für das Wirtschaftsgut Versicherungsschutz dar. ${ }^{588}$ Im Folgenden soll unter Prämie (synonym: Beitrag) immer die so genannte Bruttoprämie verstanden werden, welche sich in der Schaden- und Unfallversicherung aus einer Risikoprämie und einem Kosten- und Gewinnzuschlag zusammensetzt. ${ }^{589} \mathrm{Im}$ Rahmen der Tarifierung wird versucht, für die Risiken eines möglichst homogenen Teilbestandes eine risikogerechte Prämie festzulegen. Zu diesem Zweck können unterschiedliche Prämienprinzipien herangezogen werden, auf welche in dieser Arbeit jedoch nicht eingegangen werden soll. ${ }^{590}$ Unter der Voraussetzung, dass die Schadenhöhen eines Teilbestands der gleichen Verteilung genügen, wird jedem Einzelrisiko aus diesem Teilbestand dieselbe Prämie zugeordnet. ${ }^{591}$ Ist zudem die Homogenitätseigenschaft der Einzelrisiken erfüllt, kann für den betrachteten Teilbestand ebenfalls eine risikogerechte Prämie ermittelt werden. Im vorliegenden Modell wird die Prämie in eine Preis- und eine Mengenkomponente unterteilt. ${ }^{592}$ Die Preiskomponente kann im Rahmen der Tarifkalkulation ermittelt werden und lässt sich als Prämie pro Vertrag bzw. Durchschnittsprämie eines Teilbestands interpretieren. Das Prämienvolumen eines Teilbestandes wird durch Multiplikation der Durchschnittsprämie (DP) mit der Anzahl der Einzelrisiken bzw. Jahreseinheiten (JE) ermittelt.

587 Vgl. übereinstimmend HOFFMANN/SANN (2003b), S. 7. Zur Ermittlung des versicherungstechnischen Cashflows vgl. auch Kapitel 5.5.4.

588 Vgl. FarnY (2000), S. 58.

589 Die Risikoprämie wiederum umfasst den Schadenerwartungswert (= Netto- bzw. Bedarfsprämie) und einen Sicherheitszuschlag. Vgl. im Folgenden SCHMIDT (2002), S. 239 und FARNY (2000), S. 60-61.

590 Vgl. zu den klassischen Prämienprinzipien LiPPE (1984), S. 133-156, SCHMIDT (2002), S. 239269, MACK (2002), S. 121-220, HELBIG (2002), S. 85-88 und DOTTERWEICH (2004), S. 91-99.

591 Vgl. im Folgenden SCHMIDT (2002), S. 239-268 und MACK (2002), S. 121-122.

592 Vgl. hierzu und im Folgenden DOTTERWEICH (2004), S. 89-99. 
Für ein Gesamtportfolio, das sich aus $\mathrm{i} \in\{1, \ldots, \mathrm{n}\}$ Teilbeständen zusammensetzt, ergibt sich das Prämienvolumen $P$ folglich als Summe über die Prämienvolumina sämtlicher Teilbestände.

$$
P=\sum_{i=1}^{n} D P_{i} \cdot J E_{i}
$$

In der Praxis besteht ein wesentlicher Vorteil dieses Ansatzes darin, dass die Portfoliogröße unabhängig vom jeweiligen Preis- bzw. Tarifniveau variiert werden kann. Auf die Weise können unterschiedliche Zeichnungsstrategien und Veränderungen des Portfoliovolumens relativ einfach abgebildet werden. Darüber hinaus können marktoder spartenspezifische Besonderheiten des Pricings in die Modellierung einbezogen werden. Es ist jedoch einschränkend anzumerken, dass aufgrund des Ausgleich-imKollektiv-Effekts grundsätzlich Portfoliogröße und Tarifniveau nicht losgelöst voneinander betrachtet werden können. ${ }^{593}$

Darüber hinaus müssten streng genommen die zwischen Absatzmenge und Preisniveau bestehenden Interdependenzen durch die Formulierung einer Preis-Absatz-Funktion berücksichtigt werden. ${ }^{594}$ Letztgenannte Überlegung gewinnt insbesondere in mehrperiodigen Modellen an Bedeutung. Da dem hier vorgestellten Ansatz lediglich eine Modellperiode zugrunde liegt, wird auf die explizite Modellierung des Nachfrageverhaltens der Versicherungsnehmer verzichtet und die Höhe der Durchschnittsprämie je Teilbestand als bekannt vorausgesetzt. Dennoch bietet der Modellansatz die Möglichkeit, die Auswirkungen unterschiedlicher Prämienniveaus auf die Rendite-RisikoPosition zu simulieren. Aus den Simulationsergebnissen können dann ex-ante Erkenntnisse über die Risikoadäquanz und Auskömmlichkeit des Prämienniveaus einzelner Teilbestände gewonnen werden.

Die Modellierung der Parameter Durchschnittsprämie und Jahreseinheiten kann sowohl deterministisch als auch stochastisch erfolgen. Im Rahmen dieser Arbeit wird aus Vereinfachungsgründen eine deterministische Abbildung vorgenommen. ${ }^{595}$ Dieses Vorgehen impliziert, dass die versicherungstechnische Bruttorisikoposition im Mo-

593 Vgl. DOTTERWEICH (2004), S. 90.

594 Eine mögliche Vorgehensweise zur absatzmarktorientierten Planung von Wachstum und Prämienentwicklung unter Berücksichtigung der Kundenzufriedenheit formuliert DOTTERWEICH (2004), S. 116-122.

595 Sofern hinreichend valide Daten zur Formulierung entsprechender Verteilungsannahmen vorliegen, ist eine stochastische Modellierung des Prämienvolumens allerdings mit vertretbarem Aufwand möglich. Vgl. hierzu vertiefend TAYLOR (1991), S. 3 ff. und CUMMINS/HARRINGTON/KLEIN (1991), S. $50 \mathrm{ff}$. 
dellverlauf exogen vorgegeben ist und keiner strukturellen Dynamik hinsichtlich des Spartenmixes und der Preispolitik unterliegt. ${ }^{596}$ Darüber hinaus eröffnet dies die Möglichkeit, verschiedene Strategien und Risikosteuerungsinstrumente auf ein identisches Risikokollektiv anzuwenden und auf diese Weise vergleichbar zu machen.

\subsubsection{Schadenmodell}

\subsubsection{Individuelles vs. kollektives Modell der Risikotheorie}

Die Abbildung der Schadenentwicklung mittels stochastischer Simulation stellt einen zentralen Bestandteil der versicherungstechnischen Modellierung dar und soll daher an dieser Stelle detailliert beschrieben werden. Die Schadenmodellierung erfolgt auf Anfalljahresbasis. Es wird demzufolge der erwartete Endschaden (Ultimate) eines Anfalljahres simuliert. In der Risikotheorie existieren mit dem individuellen und dem kollektiven Modell zwei Grundkonzepte, mit denen die Gesamtschadenverteilung ermittelt werden kann. ${ }^{597}$

Ausgangspunkt der individuellen Risikotheorie ist der einzelne Versicherungsvertrag als „Produzent“ eines Schadens. ${ }^{598}$ Ausgehend von der Prämisse, dass sich das versicherungstechnische Gesamtportfolio in homogene Risikogruppen aufteilen lässt, ergibt sich die Gesamtschadenverteilung durch Faltung der separat geschätzten Schadenverteilungen sämtlicher Einzelrisiken in einer homogenen Risikogruppe. Dieser Faltungsprozess ist zwar in der Regel nicht analytisch durchführbar, durch leistungsfähige Datenverarbeitungssysteme und effiziente Lösungsalgorithmen ${ }^{599}$ wird jedoch die numerische Aggregation der einzelnen Verteilungen in erheblichem Maße begünstigt. Das individuelle Modell wird daher zunehmend dazu verwendet, im Rahmen der Tarifkalkulation Erwartungswert und Varianz der Schadenverteilung einzelner Risikokollektive zu ermitteln. Allerdings weist das individuelle Modell im Kontext der Risikokapitalsteuerung eine wesentliche Schwäche auf. Aufgrund der Homogenitätsbedingung kann die Datenbasis einzelner Teilkollektive bisweilen so klein ausfallen, dass der Tail der Schadenverteilung wegen des zu geringen Stichprobenumfangs entweder nur unzureichend modelliert oder die Angemessenheit der Modellierung mangels Schadener-

\footnotetext{
596 Vgl. auch BROHM (2002), S. 162-163.

597 Vgl. grundlegend zu mathematischen Methoden der Risikotheorie BEARD/PENTIKÄINEN/PESONEN (1977), BÜHLMANN (1970), GERBER (1979), DAYKIn/PENTIKÄINEN/PESONEN (1996) und KlugMAN/PANJER/WILLMOT (1998).

598 Vgl. im Folgenden Hipp/Michel (1990), S. 9-33, FEILMEIER/BERTRAM (1987), S. 9-11, MACK (2002), S. 37-76 und SCHMIDT (2002), S. 141-162.
}

599 Hier sind insbesondere die Rekursionsformel von PANJER und der Fast-Fourier-Algorithmus zu nennen. Diese Lösungsalgorithmen sind jedoch auf bestimmte Verteilungstypen beschränkt Vgl. vertiefend Klugman/PANJER/Willmot (1998), S. 309-322, MACK (2002), S. 111-116 und SCHMIDT (2002), S. 177-181. 
fahrung erst gar nicht überprüft werden kann. ${ }^{600}$ Wie noch gezeigt werden wird, üben jedoch gerade die seltenen Ereignisse mit hohem Schadenpotenzial einen maßgeblichen Einfluss auf die Schadenverteilung des Gesamtportfolios aus.

Das kollektive Modell vernachlässigt die Information, welches Schadenereignis von welchem Vertrag ausgelöst wurde. Vielmehr wird das gesamte (heterogene) Portfolio als „Produzent“ der Schäden betrachtet. ${ }^{601}$ Die Vernachlässigung des direkten Verursachungszusammenhangs erfordert es, die Gesamtschadenverteilung in eine Schadenanzahl- und eine Schadenhöhenverteilung aufzuteilen. Die Gesamtschadenverteilung im kollektiven Modell setzt sich somit zusammen aus einer zufälligen Anzahl von Schäden, deren Schadenhöhe ebenfalls zufällig ist. Diese Ausgangsverteilungen können getrennt voneinander modelliert und ihre Parameter erheblich zuverlässiger geschätzt werden als die einzelnen Schadenverteilungen homogener Risikogruppen im individuellen Modell. Die Trennung der Zufallsvariablen „Schadenanzahl“ und „Schadenhöhe pro Schadenfall" bietet darüber hinaus den Vorteil, den Einfluss externer Modelleinflüsse gezielter abbilden zu können. ${ }^{602}$ So kann beispielsweise in Prognosemodellen sichergestellt werden, dass eine erwartete Inflation der Schadenkosten ausschließlich auf die Parameter der Schadenhöhenverteilung Einfluss nimmt, während eine tendenziell steigende Schadenfrequenz nur Auswirkungen auf die Schadenanzahlverteilung hat. Das kollektive Modell unterstellt konsequenterweise, dass die „Schadenhöhen pro Schadenfall“ untereinander stochastisch unabhängig und identisch verteilt sind und außerdem die „Schadenanzahl“ unabhängig von der „Schadenhöhe pro Schadenfall" ist. ${ }^{603}$ Diese Annahme kann in der Praxis bis auf wenige Ausnahmefälle als erfüllt angesehen werden. Als mögliche Ausnahme sind diejenigen Versicherungssparten zu nennen, die auch Elementargefahren abdecken (z.B. die Verbundene Wohngebäudeversicherung oder Kasko). Hier ist die Forderung nach Unabhängigkeit von Schadenanzahl und Schadenhöhe in der Regel nicht mehr erfüllt. Diese Sparten decken jedoch zumeist unter einer Police mehrere Einzelgefahren (z.B. Sturm, Hagel oder Überschwemmung) ab, so dass „,bei getrennter Betrachtung der Gefahren die Unabhängigkeit von Schadenanzahl und Schadenhöhe fast immer gegeben“" ${ }^{604}$ ist.

${ }^{600}$ Vgl. übereinstimmend MACK (2002), S. 77. MACK weist auch darauf hin, dass empirische Verteilungen keine höheren Schadenrealisationen als die bisher beobachteten zulassen. Vgl. MACK (2002), S. 88.

601 Vgl. im Folgenden auch FeILMEIER/BERTRAM (1987), S. 11, MACK (2002), S. 77-120, SCHMIDT (2004c), S. 111-114 und DOTTERWEICH (2004), S. 46.

$602 \mathrm{Vgl}$. in diesem Zusammenhang KLUGMAN/PANJER/WiLlmot (1998), S. 10.

${ }^{603}$ Diese Annahme wird in der versicherungsmathematischen Literatur auch als „i.i.d.-Bedingung“ (independent identically distributed) bezeichnet. Vgl. hierzu und im Folgenden auch SCHRÖTER (1995), S. 108 und MACK (2002), S. 108-109.

${ }^{604}$ MACK (2002), S. 109. Siehe zur praktischen Erfüllbarkeit der i.i.d.-Bedingung auch SCHMIDT (2002), S. 164. 
Dies unterstreicht die Notwendigkeit, die Modellierung von Naturereignisschäden je Einzelgefahr vorzunehmen und diese darüber hinaus getrennt von der übrigen Schadenlast (z.B. Basis- und Großschäden) abzubilden. ${ }^{605}$

Für die Modellierung wird nachfolgend auf das kollektive Modell der Risikotheorie zurückgegriffen. Bezeichne demnach N die stochastische Anzahl der Schäden, die innerhalb eines Jahres von den Risiken (z.B. Verträgen) eines Bestands verursacht werden, und seien $X_{i}$ die zugehörigen (unabhängig und identisch verteilten) Schadenhöhen pro Schadenfall, so ergibt sich der jährliche Gesamtschaden $\mathrm{S}_{\mathrm{N}}$ dieses Portfolios als „zufällige“ Summe der Form:

$$
S_{N}=\sum_{i=1}^{N} X_{i}
$$

Die ersten beiden Momente der Gesamtschadenverteilung lassen sich unmittelbar aus den Momenten der Schadenanzahl- und Schadenhöhenverteilung ableiten. Unter Beachtung der Regeln für das Rechnen mit bedingten Erwartungswerten gelten folgende Zusammenhänge: ${ }^{606}$

$$
\begin{aligned}
& \mathrm{E}\left(\mathrm{S}_{\mathrm{N}}\right)=\mathrm{E}_{\mathrm{N}}\left(\mathrm{E}\left(\mathrm{S}_{\mathrm{N}} \mid \mathrm{N}\right)\right)=\mathrm{E}_{\mathrm{N}}\left(\mathrm{N} \cdot \mathrm{E}\left(\mathrm{X}_{1}\right)\right)=\mathrm{E}(\mathrm{N}) \cdot \mathrm{E}\left(\mathrm{X}_{1}\right) \\
& \operatorname{Var}\left(\mathrm{S}_{\mathrm{N}}\right)=\mathrm{E}_{\mathrm{N}}\left(\operatorname{Var}\left(\mathrm{S}_{\mathrm{N}} \mid \mathrm{N}\right)\right)+\operatorname{Var}_{\mathrm{N}}\left(\mathrm{E}\left(\mathrm{S}_{\mathrm{N}} \mid \mathrm{N}\right)\right) \\
& =\mathrm{E}_{\mathrm{N}}\left(\mathrm{N} \cdot \operatorname{Var}\left(\mathrm{X}_{1}\right)\right)+\operatorname{Var}_{\mathrm{N}}\left(\mathrm{N} \cdot \mathrm{E}\left(\mathrm{X}_{1}\right)\right) \\
& =\mathrm{E}(\mathrm{N}) \cdot \operatorname{Var}\left(\mathrm{X}_{1}\right)+\operatorname{Var}(\mathrm{N}) \cdot\left(\mathrm{E}\left(\mathrm{X}_{1}\right)\right)^{2}
\end{aligned}
$$

Die Schadenmodellierung mit Hilfe des kollektiven Modells erfordert ein zweistufiges Vorgehen. Im ersten Schritt muss eine angemessene Verteilung für die Schadenanzahl ermittelt werden. Vielfach wird in diesem Kontext auf die Poissonverteilung zurückgegriffen, sofern folgende Voraussetzungen erfüllt sind: $:^{607}$

- Die Schäden aus zwei disjunkten Zeitintervallen sind voneinander unabhängig.

- Zwei oder mehrere Schäden treten nicht exakt zum gleichen Zeitpunkt ein.

- Die Schäden treten nicht bevorzugt zu bestimmten Zeitpunkten ein.

${ }^{605}$ Bezüglich der separaten Modellierung von Ereignis- bzw. Kumulschäden wird auf die Ausführungen in Kapitel 5.4.3.4 verwiesen.

${ }^{606}$ Vgl. hierzu vertiefend MACK (2002), S. 109 und SCHRÖTER (1995), S. 108-109. Der mathematische Beweis dieser Beziehungen geht auf WALD zurück und ist z.B. bei SCHMIDT (2002), S. 166167 nachzulesen.

607 Vgl. auch MACK (2002), S. 78 und DAYKIN/PENTIKÄINEN/PESONEN (1996), S. 452-458 sowie DOTTERWEICH (2004), S. 47. Alternativ kommt oft auch die Negative Binomialverteilung zur Anwendung. 
Die genannten Voraussetzungen können in der Praxis mit Ausnahme der Sachversicherung gegen Elementargefahren als erfüllt angesehen werden. ${ }^{608}$ Die Zähldichte der Poissonverteilung weist folgende Gestalt auf: ${ }^{609}$

$$
\mathrm{P}(\mathrm{N}=\mathrm{n})=\mathrm{e}^{-\theta} \cdot \frac{\theta^{\mathrm{n}}}{\mathrm{n} !} \quad \text { mit } \mathrm{n}=1,2, \ldots
$$

Die Zufallsvariable $\mathrm{N}$ bezeichne hierbei die Anzahl der in einem bestimmten Zeitraum (i. A. dem nächsten Kalender- oder Anfalljahr) eingetretenen Schadenereignisse des modellierten (Teil-) Portfolios. Für den Verteilungsparameter $\theta$ gilt:

$$
\theta=\mathrm{E}(\mathrm{N})=\operatorname{Var}(\mathrm{N}) \quad \text { mit } \theta>0
$$

Diese Beziehung verdeutlicht, dass die Poissonverteilung durch die Abhängigkeit von lediglich einem Verteilungsparameter zwar wenig anpassungsfähig, aber rechnerisch leicht zu handhaben ist. ${ }^{610}$ Die Poissonverteilung zeichnet sich darüber hinaus durch die wünschenswerte statistische Eigenschaft der Volumenproportionalität bzw. Additivität im Parameter $\theta$ aus. ${ }^{611}$ Im Falle eines inhomogenen Schadenportfolios und gegebener Unabhängigkeit der Zufallsvariablen bedeutet dies, dass sich sowohl der Erwartungswert als auch die Varianz dieses Portfolios als Summe aller Erwartungswerte bzw. Varianzen der zugrunde liegenden Einzelrisiken ausdrücken lassen. Bezeichne TP ein bestimmtes Teilportfolio mit JE inhomogenen Einzelrisiken, so gilt: ${ }^{612}$

$$
\theta_{\mathrm{TP}}=\sum_{\mathrm{i}=1}^{\mathrm{JE}} \theta_{\mathrm{i}}
$$

Kann jedoch angenommen werden, dass das betrachtete Portfolio aus JE weitgehend homogenen Einzelrisiken besteht, dass also $\theta_{\mathrm{i}}=\theta_{1}$ gilt, so vereinfacht sich Beziehung (5.8) zu:

\footnotetext{
608 Vgl. hierzu MACK (2002), S. 79 und die Ausführungen in Kapitel 5.4.3.1.

609 Vgl. PANJeR/Willmot (1992), S. 63-69.

610 MACK weist kritisch auf die Instabilität des Parameters $\theta$ im Zeitablauf hin. Selbst für den Fall, dass die Einzelrisiken eines Portfolios als unverändert angenommen werden, üben z.B. Wetterbedingungen einen signifikanten Einfluss auf die Schadeneintrittswahrscheinlichkeit aus. Er schlägt daher vor, diesen oszillatorischen Effekt durch eine Zufallsvariable Q (Qualität) zu modellieren. Unter der Annahme, dass $\mathrm{Q}$ einer Gammaverteilung genügt, kann gezeigt werden, dass $\mathrm{N}$ in eine Negative Binomialverteilung übergeht. Vgl. hierzu im Einzelnen MACK (2002), S. 78-86 und DOTTERWEICH (2004), S. 48-49.

${ }^{611}$ Vgl. im Folgenden DOTTERWEICH (2004), S. 47 und MACK (2002), S. 79.

${ }^{612}$ Die Darstellung erfolgt in Anlehnung an DOTTERWEICH (2004), S. 47.
} 


$$
\theta_{\mathrm{TP}}=\sum_{\mathrm{i}=1}^{\mathrm{JE}} \theta_{\mathrm{i}}=\mathrm{JE} \cdot \theta_{1}
$$

Die erwartete Schadenanzahl pro Einzelrisiko $q:=\theta_{\mathrm{TP}} / \mathrm{JE}$ wird als Schadenfrequenz bezeichnet. ${ }^{613}$ Die Volumenproportionalität weitgehend homogener Risikogruppen kann man sich im Rahmen der Schadenmodellierung zu Nutze machen, „da die Schadenfrequenz bei gleich bleibenden äußeren Bedingungen in der Regel über mehrere Perioden konstant bleibt, während sich die Größe (Volumen) der Gruppe von Periode zu Periode ändert. “614

Nachdem die Schadenanzahlverteilung ermittelt wurde, muss in einem zweiten Schritt eine angemessene Verteilung der „Schadenhöhen pro Schadenfall“ gefunden und deren Parameter so geschätzt werden, dass die empirische Verteilung der Schadenhöhen durch die ermittelte parametrische Verteilung möglichst gut approximiert wird. Bei der Schadenmodellierung einzelner Risikokollektive wird im Folgenden zwischen Basisschäden, Großschäden ${ }^{615}$ und Ereignis- bzw. Kumulschäden unterschieden. Eine Differenzierung zwischen Basisschadenlast und einzelnen Großschäden erscheint aus verschiedenen Gründen zweckmäßig. Wie noch gezeigt werden wird, üben Großschäden einen signifikanten Einfluss auf den Risikokapitalbedarf eines Schaden- und Unfallversicherungsunternehmens aus. Sie wirken aufgrund ihrer geringen Eintrittswahrscheinlichkeit im Tail der Ergebnisverteilung, während sich die kleinen, jedoch hochfrequenten Basisschäden am linken Rand der Gesamtschadenverteilung wiederfinden und den Risikokapitalbedarf in der Regel nur marginal beeinflussen. ${ }^{616}$ Diesem Aspekt sollte durch eine gesonderte Parametrisierung der Schadenanzahl- und Schadenhöhenverteilung für Großschäden Rechnung getragen werden. Großschäden weisen außerdem oft eine längere Abwicklungsdauer auf als Basisschäden. Will man den versicherungstechnischen Cashflow und demzufolge auch das Kapitalanlageergebnis realistisch darstellen, ist eine separate Modellierung von Großschadenereignissen erforderlich. Eine Differenzierung ist darüber hinaus unverzichtbar, um die Wirkungsweise der Rückversicherung explizit in das Modell einbinden zu können. Im Rahmen be-

${ }^{613}$ Vgl. MACK (2002), S. 79-80.

${ }^{614}$ MACK (2002), S. 80. Die Forderung nach Homogenität der Schadenfrequenz einer Risikogruppe ist sogar unter bestimmten Annahmen entbehrlich. Vgl. hierzu MACK (2002), S. 80 und SCHMIDT (2002), S. 163.

${ }^{615}$ Als „Großschäden“ werden diejenigen Schäden definiert, deren Höhe eine (für jedes Teilkollektiv individuell definierte) Großschadengrenze übersteigen. Eine Übersicht der Großschadengrenzen und der verwendeten Schadenhöhenverteilungen ist dem Anhang A zu entnehmen.

${ }^{616}$ In der versicherungswirtschaftlichen Praxis sind aufgrund der unterschiedlichen Eintrittswahrscheinlichkeiten von Basis- und Großschäden daher vielfach rechtsschiefe Schadenverteilungen anzutreffen. Ein Beispiel für dieses „Phänomen abnehmender Schadendichtigkeit“ ist bei MACK (2002), S. 86-89 zu finden. 
stimmter Rückversicherungsformen, wie z.B. dem Schadenexzedentenvertrag, entlastet der Rückversicherer den Erstversicherer, indem er bei Einzelschäden erst ab einem vereinbarten Selbstbehalt die Schadenlast übernimmt. ${ }^{617}$ Daher ist die Modellierung einzelner Großschäden zwingend geboten. In einem weiteren Teilmodul werden schließlich die Schadenverteilungen singulärer Naturgefahrenereignisse durch geeignete Simulationen erzeugt. Konkret werden für das Modellunternehmen Sturm-, Hagel- und Überschwemmungsschäden modelliert und anschließend prozentual auf die betroffenen Versicherungssparten und Geschäftsfelder verteilt. ${ }^{618}$

\subsubsection{Basisschäden}

\subsection{Ermittlung der Gesamtschadenverteilung der Basisschäden}

Im Gegensatz zu Großschäden oder Schäden aus Naturgefahren zeichnen sich Basisschäden durch ein vergleichbar homogenes Anfallverhalten aus. Wenngleich die Ergebnisbelastung durch Basisschäden aufgrund einer bisweilen sehr hohen Schadenfrequenz erheblich sein kann, kann diese "Grundlast" vergleichsweise stabil aus empirischen Daten abgeschätzt werden. Bei der Modellierung der Basisschäden wird davon ausgegangen, dass deren Gesamtschadenverteilung durch das kollektive Modell beschrieben werden kann. Es wird darüber hinaus angenommen, dass die Gesamtverteilung der Basisschäden BS einer Lognormalverteilung genügt. Durch diese Annahme wird dem Umstand Rechnung getragen, dass auch innerhalb der Basisschadenlast vielfach ein Frequenzbereich im linken Bereich der Verteilung zu beobachten ist, der sich durch eine Häufung kleiner Basisschäden auszeichnet. ${ }^{619}$ Für die Gesamtschadenverteilung der Basisschäden $\mathrm{P}^{\mathrm{BS}}$ soll daher gelten:

$$
\mathrm{P}^{\mathrm{BS}} \sim \operatorname{LogNormal}\left(\mu_{\log \mathrm{N}}, \sigma_{\log N}\right)
$$

Unter der Voraussetzung, dass diese Gesamtschadenverteilung im Sinne des kollektiven Modells durch eine Schadenanzahlverteilung $\mathrm{N}$ und eine Schadenhöhenverteilung $\mathrm{X}_{1}$ dargestellt werden kann, lassen sich die Verteilungsparameter der Lognormalverteilung unter Rückgriff auf ihre ersten beiden Momente E(BS) und $\operatorname{Var}(\mathrm{BS})$ wie folgt bestimmen: ${ }^{620}$

\footnotetext{
617 Vgl. vertiefend die Ausführungen in Kapitel 5.4.5.2.3.

${ }^{618}$ Vgl. hierzu im Einzelnen Kapitel 5.4.3.4.

619 Vgl. übereinstimmend LiPPE (1983), S. 50 und BAMBERG/DORFLEITNER/GLAAB (2005), S. 407 ff.

${ }^{620} \mathrm{Vgl}$. auch AlBrechT/MAURER (2002), S. 96.
} 


$$
\begin{aligned}
& \mu_{\log N}=\ln \left[\frac{[\mathrm{E}(\mathrm{BS})]^{2}}{\sqrt{[\mathrm{E}(\mathrm{BS})]^{2}+\operatorname{Var}(\mathrm{BS})}}\right] \\
& \sigma_{\log N}=\sqrt{\ln \left[\frac{\mathrm{Var}(\mathrm{BS})}{[\mathrm{E}(\mathrm{BS})]^{2}}+1\right]}
\end{aligned}
$$

Aus den Beziehungen (5.4) und (5.5) sind im kollektiven Modell die die ersten beiden Momente der Gesamtschadenverteilung bereits bekannt als: ${ }^{61}$

$$
\begin{aligned}
& \mathrm{E}(\mathrm{BS})=\mathrm{E}(\mathrm{N}) \cdot \mathrm{E}\left(\mathrm{X}_{1}\right) \\
& \operatorname{Var}(\mathrm{BS})=\mathrm{E}(\mathrm{N}) \cdot \operatorname{Var}\left(\mathrm{X}_{1}\right)+\operatorname{Var}(\mathrm{N}) \cdot\left(\mathrm{E}\left(\mathrm{X}_{1}\right)\right)^{2}
\end{aligned}
$$

Unter der Annahme, dass die Verteilung der Schadenanzahl einer Poissonverteilung mit dem Parameter $\theta$ genügt und demzufolge $\theta=\mathrm{E}(\mathrm{N})=\operatorname{Var}(\mathrm{N})$ gilt, lassen sich $\mathrm{E}(\mathrm{BS})$ und $\operatorname{Var}(\mathrm{BS})$ nun durch Parameter beschreiben, welche aus den Bestandsdaten eines Versicherungsunternehmens bzw. durch aktuarielle Schätzverfahren zu ermitteln sind. Die ersten beiden Momente der Gesamtschadenverteilung können ausgedrückt werden als:

$$
\begin{aligned}
\mathrm{E}(\mathrm{BS})=\mathrm{JE} \cdot \mathrm{q} \cdot \mathrm{m} \\
\begin{aligned}
\operatorname{Var}(\mathrm{BS}) & =\mathrm{E}(\mathrm{N}) \cdot \operatorname{Var}\left(\mathrm{X}_{1}\right)+\operatorname{Var}(\mathrm{N}) \cdot\left(\mathrm{E}\left(\mathrm{X}_{1}\right)\right)^{2} \\
& =\mathrm{E}(\mathrm{N}) \cdot\left[\operatorname{Var}\left(\mathrm{X}_{1}\right)+\left(\mathrm{E}\left(\mathrm{X}_{1}\right)\right)^{2}\right] \quad \text { wegen } \theta=\mathrm{E}(\mathrm{N})=\operatorname{Var}(\mathrm{N}) \\
& =\mathrm{JE} \cdot \mathrm{q} \cdot\left(\mathrm{s}^{2}+\mathrm{m}^{2}\right) \\
\text { mit: } \quad \mathrm{JE} & =\mathrm{Jahreseinheiten} \\
\mathrm{q} & =\frac{\text { SAnz }}{\mathrm{JE}}=\frac{\theta}{\mathrm{JE}}=\text { Schadenfrequenz } \\
\mathrm{m} & =\mathrm{E}\left(\mathrm{X}_{1}\right)=\frac{\text { SAufw }}{\text { SAnz }}=\text { Durchschnittsschaden } \\
\mathrm{s}^{2} & =\operatorname{Var}\left(\mathrm{X}_{1}\right)=\text { Varianz der Gesamtschadenverteilung (Basisschäden) }
\end{aligned}
\end{aligned}
$$

Unter Ausnutzung der Beziehungen (5.15) und (5.16) können schließlich die Parameter der Lognormalverteilung ermittelt werden als:

${ }^{621}$ Vgl. hierzu auch Kapitel 5.4.3.1. 


$$
\begin{aligned}
& \mu_{\log N}=\ln \left[\frac{(\mathrm{JE} \cdot \mathrm{q} \cdot \mathrm{m})^{2}}{\sqrt{(\mathrm{JE} \cdot \mathrm{q} \cdot \mathrm{m})^{2}+\mathrm{JE} \cdot \mathrm{q} \cdot\left(\mathrm{s}^{2}+\mathrm{m}^{2}\right)}}\right] \\
& \sigma_{\log N}=\sqrt{\ln \left[\frac{\mathrm{JE} \cdot \mathrm{q} \cdot\left(\mathrm{s}^{2}+\mathrm{m}^{2}\right)}{(\mathrm{JE} \cdot \mathrm{q} \cdot \mathrm{m})^{2}}+1\right]}
\end{aligned}
$$

An dieser Stelle muss im Rahmen der Modellierung bereits berücksichtigt werden, ob ein Versicherungszweig über einen Summenexzedentenvertrag rückversichert ist oder die Wirkungsweise eines derartigen Rückversicherungsvertrags gegebenenfalls im weiteren Verlauf analysiert werden soll. Ohne den Ausführungen zur Rückversicherung in Kapitel 5.4.5 vorzugreifen, soll bereits hier auf einen wesentlichen Aspekt hingewiesen werden, der Einfluss auf die versicherungstechnische Bruttomodellierung nimmt. Die proportionale Rückversicherung zeichnet sich dadurch aus, dass der Rückversicherer in einem bestimmten prozentualen Verhältnis am Originalrisiko des Erstversicherers beteiligt ist. ${ }^{622}$ Im einfachen Fall eines Quotenvertrages gilt dieses Verhältnis für sämtliche Risiken eines rückversicherten Portfolios. Im Rahmen eines Summenexzedentenvertrages wird das Verhältnis der Risikoteilung hingegen einzelvertraglich ermittelt. Der Erstversicherer gibt eine einheitliche Schadenhöhe vor, die er pro Einzelrisiko maximal im Eigenbehalt tragen möchte. Diese Grenze wird als Maximum (M) bezeichnet. Erst wenn die Versicherungssumme bzw. der wahrscheinliche Höchstschaden $\left(\mathrm{PML}_{\mathrm{i}}\right)$ eines Einzelrisikos das Maximum übersteigt, gelangt dieses Risiko in die Zession an den Rückversicherer. ${ }^{623}$ Das Verhältnis der Risikoteilung $p_{i}$ wird für jedes Einzelrisiko i separat ermittelt und bemisst sich anhand der Beziehung:

$$
\mathrm{p}_{\mathrm{i}}=\frac{\mathrm{PML}_{\mathrm{i}}-\mathrm{M}}{\mathrm{PML}_{\mathrm{i}}}=1-\frac{\mathrm{M}}{\mathrm{PML}_{\mathrm{i}}} \quad \mathrm{PML}_{\mathrm{i}}>\mathrm{M}
$$

Im Interesse der Problemadäquanz und Wirtschaftlichkeit eines internen Risikomodells sollte das versicherungstechnische Portfolio jedoch nicht auf der Einzelvertragsebene abgebildet werden. Um die Wirkungsweise eines Summenexzedentenvertrages

${ }^{622} \mathrm{Vgl}$. zur Unterscheidung von proportionaler und nichtproportionaler Rückversicherung detailliert LIEBWEIN (2000), S. 61-62 und S. 151-153, SwISS RE (1997a) und Kapitel 5.4.5.1.

${ }^{623}$ Die Versicherungssumme entspricht der maximalen Entschädigungsleistung des Erstversicherers im Schadenfall, erlaubt jedoch keine Aussage über dessen Eintrittswahrscheinlichkeit. In der Praxis ist daher die Aufteilung nach dem wahrscheinlichen Höchstschaden (Probable Maximum Loss) verbreitet. Auf der Grundlage des PML lässt sich der Schadenerwartungswert eines Einzelrisikos sicherer schätzen. Vgl. zur Bestimmung des PML exemplarisch KÜHL (1994), S. 102-115. Im Rahmen dieser Arbeit wird von der Problematik so genannter PML-Verschätzer abstrahiert, d.h. der PML kann annahmegemäß nicht größer sein als die zugehörige Versicherungssumme. Vgl. hierzu auch LIEBWEIN (2000), S. 164-165 und S. 254-256. 
dennoch geeignet darstellen zu können, bedarf es in den betroffenen Segmenten einer von der oben beschriebenen Vorgehensweise abweichenden Modellierung der Basisschäden. Hierzu wird der Vertragsbestand eines Teilkollektivs (z.B. einer Versicherungssparte), das über einen Summenexzedentenvertrag rückversichert ist, in so genannte PML-Bänder aufgeteilt. ${ }^{624}$ Beispielsweise werden innerhalb des PML-Bandes [1-3] sämtliche Verträge modelliert, deren wahrscheinlicher Höchstschaden zwischen 1 Mio. GE und 3 Mio. GE liegt. Folglich weisen alle Verträge dieses PML-Bandes in der Modellwelt das gleiche Risikoteilungsverhältnis $\mathrm{p}$ auf. Im betrachteten Modellunternehmen trifft diese Vorgehensweise auf die Versicherungssparten „Feuer Industrie“ und „VGV Feuer“ zu, da diese durch einen Summenexzedentenvertrag rückversichert sind. Diese Sparten werden im Folgenden als PML-Sparten bezeichnet.

Die Modellierung der Schadenhöhe von PML-Sparten kann über die Parameter „durchschnittlicher PML“625 und „durchschnittlicher Schadengrad“626 erfolgen, während für die sonstigen Sparten die durchschnittliche Schadenhöhe $(\mathrm{m})$ und die Schadenfrequenz (q) herangezogen wird. Aus Beziehung (5.15) ist bekannt, dass der erwartete Schadenaufwand aus Basisschäden dargestellt werden kann als $\mathrm{E}(\mathrm{BS})=\mathrm{JE} \cdot \mathrm{q} \cdot \mathrm{m}$. Überträgt man diesen Zusammenhang auf den erwarteten Basisschadenaufwand eines PML-Bandes i, so gilt:

$$
\begin{aligned}
\mathrm{E}\left(\mathrm{BS}_{\mathrm{i}}\right)=\mathrm{JE}_{\mathrm{i}} \cdot \mathrm{q}_{\mathrm{i}} \cdot \mathrm{m}_{\mathrm{i}} \\
=\mathrm{JE}_{\mathrm{i}} \cdot \mathrm{q}_{\mathrm{i}} \cdot \underbrace{\mathrm{DPML}_{\mathrm{i}} \cdot \mathrm{SG}_{\mathrm{i}}}_{\substack{\text { Durchschnitsschaden } \\
\text { im PML-Bandi }}} \\
\mathrm{mit:} \quad \mathrm{JE}_{\mathrm{i}}=\text { Summe der Jahreseinheiten im PML-Band } \mathrm{i} \\
\mathrm{q}_{\mathrm{i}}=\text { Schadenfrequenz im PML-Band } \mathrm{i} \\
\mathrm{DPML}_{\mathrm{i}}=\text { durchschnittlicher PML im PML-Band i } \\
\mathrm{SG}_{\mathrm{i}}=\text { durchschnittlicher Schadengrad im PML-Band } \mathrm{i}
\end{aligned}
$$

Unter Berücksichtigung der Beziehungen (5.13) und (5.14) und der Annahme, dass die Schadenanzahlen einer Poissonverteilung genügen, können die ersten beiden Momente der Gesamtschadenverteilung von PML-Sparten wie folgt ausgedrückt werden: ${ }^{627}$

\footnotetext{
${ }^{624}$ Vgl. zu einer analogen Vorgehensweise SANN (2001), S. 12.

${ }^{625}$ Der durchschnittliche PML ist definiert als Quotient aus der Summe sämtlicher PML eines PMLBandes i und der Summe der Einzelrisiken (bzw. Jahreseinheiten) im PML-Band i.

${ }^{626}$ Der durchschnittliche Schadengrad ist definiert als Quotient aus dem Schadenaufwand eines PMLBandes i und der PML-Summe der durch einen Schaden betroffenen Einzelrisiken (Jahreseinheiten) im PML-Band i.

${ }^{627}$ Auf die Angabe des Bandindexes i wird fortan verzichtet.
} 


$$
\begin{aligned}
& \mathrm{E}(\mathrm{BS})=\mathrm{JE} \cdot \mathrm{q} \cdot \mathrm{DPML} \cdot \mathrm{SG} \\
& \operatorname{Var}(\mathrm{BS})=\mathrm{JE} \cdot \mathrm{q} \cdot \mathrm{DPML} \cdot\left({\sigma_{\mathrm{SG}}}^{2}+{\mu_{\mathrm{SG}}}^{2}\right) \\
& \text { mit: } \quad \mu_{\mathrm{SG}}=\text { Erwartungswert des durchschnittlichen Schadengrades } \\
& \sigma_{\mathrm{SG}}=\text { Standardabweichung des durchschnittlichen Schadengrades }
\end{aligned}
$$

Unter Ausnutzung der Beziehungen (5.21) und (5.22) gilt schließlich für die gesuchten Parameter der Lognormalverteilung:

$$
\begin{aligned}
& \mu_{\log N}=\ln \left[\frac{(\mathrm{JE} \cdot \mathrm{q} \cdot \mathrm{DPML} \cdot \mathrm{SG})^{2}}{\sqrt{(\mathrm{JE} \cdot \mathrm{q} \cdot \mathrm{DPML} \cdot \mathrm{SG})^{2}+\mathrm{JE} \cdot \mathrm{q} \cdot \mathrm{DPML}^{2}\left(\mu_{\mathrm{SG}}^{2}+\sigma_{\mathrm{SG}}^{2}\right)}}\right] \\
& \sigma_{\mathrm{LogN}}=\sqrt{\ln \left[\frac{\mathrm{JE} \cdot \mathrm{DPML}{ }^{2} \cdot\left(\mu_{\mathrm{SG}}^{2}+\sigma_{\mathrm{SG}}^{2}\right)}{(\mathrm{JE} \cdot \mathrm{DPML} \cdot \mathrm{SG})^{2}}+1\right]}
\end{aligned}
$$

Hinsichtlich der zur Modellierung von Basisschäden erforderlichen Parameter kann zwischen originären und abgeleiteten Parametern differenziert werden. Die originären Parameter der Basisschadenmodellierung können durch Analysen des Vertragsbestandes oder mittels aktuarieller Schätzverfahren ermittelt werden. ${ }^{628}$ Dieser Zusammenhang wird durch Abbildung 5.5 überblicksartig dargestellt.

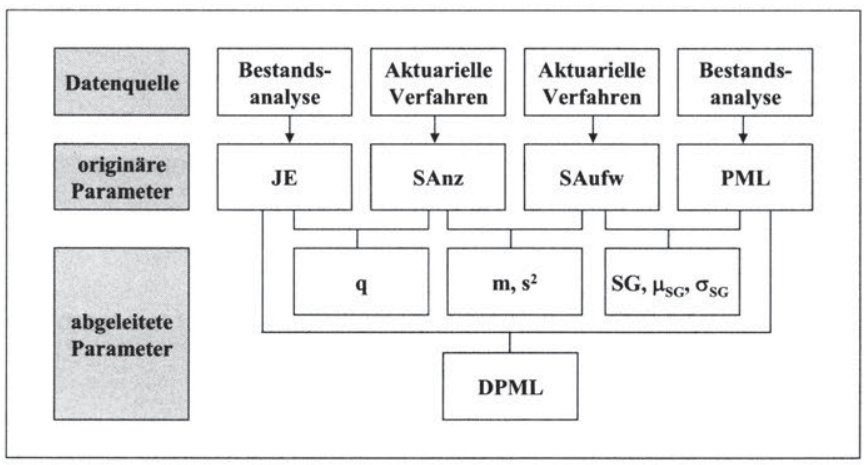

Abbildung 5.5: Modellparameter der Basisschadenlast

Die aufgeführten Parameter müssen für jedes modellierte Teilportfolio, im Falle von PML-Sparten für jedes einzelne PML-Band ermittelt werden. In Abhängigkeit des Modellumfangs kann daher die Datenbereitstellung sehr aufwändig sein.

${ }^{628} \mathrm{Vgl}$. zu aktuariellen Schätzverfahren auch die Ausführungen in Kapitel 5.4.3.2.2. 
Abschließend ist zudem festzuhalten, dass die beschriebene Modellierung von Basisschäden nicht den Anspruch einer allgemeingültigen oder gar optimalen Vorgehensweise erhebt. Es wird lediglich eine Möglichkeit beschrieben, die im Hinblick auf die in der Praxis verfügbare Datenbasis durchführbar erscheint und darüber hinaus die (asymmetrische) Verteilungscharakteristik von Basisschäden angemessen abbildet.

\subsection{Schätzung zentraler Schadenparameter}

Das Management eines Versicherungsunternehmens sollte mittels interner Risikomodelle in die Lage versetzt werden, auf der Basis von Szenarioanalysen die Auswirkungen unterschiedlicher Geschäftsstrategien auf die zukünftige Rendite-Risiko-Position beurteilen zu können. Im Hinblick auf die Prognosekraft eines Modells kommt daher der Ermittlung der erwarteten Schadenanzahl und des erwarteten Schadenaufwands besondere Bedeutung zu. Beide Parameter können entweder anhand von Expertenmeinungen oder mit Hilfe aktuarieller Schätzverfahren bestimmt werden. Im Folgenden wird mit dem Chain-Ladder-Verfahren eine mögliche aktuarielle Vorgehensweise zur Ermittlung dieser zentralen Parameter vorgestellt. Der hier vorgestellte Modellansatz ist jedoch nicht an die Anwendung des Chain-Ladder-Verfahrens gebunden. Die Schätzung der Schadenparameter kann wahlweise auch mit Hilfe sonstiger aktuarieller Verfahren durchgeführt werden. ${ }^{629}$

Das Chain-Ladder-Verfahren schätzt aus empirisch beobachteten Schadenverläufen die zukünftig erwarteten Schadenabwicklungsmuster. Mit Hilfe dieser geschätzten Abwicklungsmuster wird der erwartete Endschadenbedarf eines Anfalljahres prognostiziert. ${ }^{630}$ Für die Endschadenschätzung eines Anfalljahres kann entweder auf die Abwicklungsmuster der Schadenzahlungen (Paid) oder des Schadenaufwands (Incurred) zurückgegriffen werden. Der Schadenaufwand beinhaltet neben den Schadenzahlungen auch die Veränderung der Schadenrückstellungen im betreffenden Jahr. Für die Verwendung von Schadenzahlungen spricht die Tatsache, dass die Datengrundlage noch keinerlei Schätzungen enthält und demzufolge noch nicht durch unternehmenspolitische Überlegungen beeinflusst wurde. Zahlungsbasierte Schätzungen (ohne Tailfaktor) führen jedoch gerade in Versicherungssparten mit langer Abwicklungsdauer zu einer Unterschätzung des Endschadenbedarfs, da in diesen Sparten das Verhältnis zwischen beobachteter und zu schätzender Schadenentwicklung ungünstig ist. Die Einbeziehung der Schadenrückstellungen (Einzelfallreserven) bewirkt in diesen Sparten,

${ }^{629} \mathrm{Vgl}$. grundlegend zu aktuariellen Schätzverfahren RADTKE/SCHMIDT (2004), MACK (2002), S. 221-320, HIPP (2001b), S. 117-129, SCHMIDT (2002), S. 267-296 und DOTTERWEICH (2004), S. $146-180$.

${ }^{630}$ Vgl. im Folgenden SCHMIDT (2002), S. 274, MACK (2002), S. 244-272 und HEEP-ALTINER/ KLEMMSTEIN (2001), S. 29-35. 
dass sich die Höhe des Endschadens bereits in früheren Abwicklungsperioden abschätzen lässt und die Schätzungen ein stabileres Streuverhalten aufweisen. In Versicherungssparten mit konservativer Reservierungspolitik wird der auf Aufwandsbasis ermittelte Schadenendstand in der Regel zunächst überschätzt. In der Theorie konvergieren die Schätzung auf der Basis von Zahlungen und die Schätzung auf Aufwandsbasis gegen den gleichen Schadenendwert. ${ }^{631}$ In der versicherungswirtschaftlichen Praxis ist jedoch festzustellen, dass am Ende des Abwicklungszeitraums vielfach eine gewisse Überreservierung in den Schadenrückstellungen verbleibt, welche in den Folgejahren gegebenenfalls als Abwicklungsgewinn realisiert wird.

Für die Anwendung des Chain-Ladder-Verfahrens müssen folgende Prämissen erfüllt sein: ${ }^{632}$

- Die beobachteten Schadenstände sind strikt positiv und besitzen einen endlichen Erwartungswert.

- Die verwendete Schadenhistorie weist keine Trend- oder Strukturbrüche auf.

- Der Informationsgehalt des jeweils aktuellsten Schadenstandes kann nicht durch die Betrachtung früherer Schadenstände verbessert werden.

- Die erwartete Schadenentwicklung in den einzelnen Anfalljahren ist stochastisch unabhängig voneinander.

- Die Aufteilung des (für jedes Anfalljahr individuell ermittelten) Endschadens auf die einzelnen Abwicklungsjahre ist für alle Anfalljahre gleich.

Die Vorgehensweise des Chain-Ladder-Verfahrens soll nachfolgend am Beispiel der Sparte „Allgemeine Unfall“ illustriert werden. Als Basis für die Schätzung des erwarteten Endschadens dient das Abwicklungsdreieck des kumulierten Schadenaufwandes. ${ }^{633}$ Es wird ein Zeitraum von $n=7$ Kalenderjahren betrachtet und davon ausgegangen, dass das älteste Anfalljahr 1997 vollständig abgewickelt ist. ${ }^{634}$

631 Vgl. HEEP-ALTINER/KLEMMSTEIN (2001), S. 34.

${ }^{632} \mathrm{Vgl}$. zu den Anwendungsvoraussetzungen des Chain-Ladder-Verfahrens im Einzelnen MACK (2002), S. 244-248 und SCHMIDT (2002), S. 274.

${ }^{633}$ Vgl. im Folgenden auch DotTERWEICH (2004), S. 162-166, RADTKE (2004), S. 183-188 und MACK (2002), S. 227-228. Zur Struktur von Abwicklungsdreiecken vgl. vertiefend SCHMIDT (2002), S. 270-274.

${ }^{634}$ Es wird somit vorausgesetzt, dass in der Sparte „Allgemeine Unfall“ keine Möglichkeit zur Verrentung von Entschädigungsleistungen besteht. 


\begin{tabular}{|c|ccccccc|}
\hline Anfalljahr & \multicolumn{7}{|c|}{ Abwicklungsjahr k } \\
$\mathbf{i}$ & $\mathbf{1}$ & $\mathbf{2}$ & $\mathbf{3}$ & $\mathbf{4}$ & $\mathbf{5}$ & $\mathbf{6}$ & $\mathbf{7}$ \\
\hline $\mathbf{1 9 9 7}(\mathrm{i}=1)$ & 13.043 & 10.940 & 9.806 & 9.618 & 9.606 & 9.546 & 9.526 \\
$\mathbf{1 9 9 8}(\mathrm{i}=2)$ & 12.708 & 10.385 & 9.413 & 9.088 & 9.107 & 8.811 & \\
$\mathbf{1 9 9 9}(\mathrm{i}=3)$ & 14.390 & 12.307 & 11.225 & 10.936 & 10.984 & & \\
$\mathbf{2 0 0 0}(\mathrm{i}=4)$ & 16.275 & 13.347 & 12.095 & 10.859 & & & \\
$\mathbf{2 0 0 1}(\mathrm{i}=5)$ & 19.652 & 15.854 & 12.039 & & & & \\
$\mathbf{2 0 0 2}(\mathrm{i}=6)$ & 16.219 & 13.002 & & & & & \\
$\mathbf{2 0 0 3}(\mathrm{i}=7)$ & 18.394 & & & & & & \\
\hline
\end{tabular}

Tabelle 5.1: Abwicklungsdreieck des kumulierten Schadenaufwands [Tsd. GE]

Formal bezeichne $\mathrm{C}_{\mathrm{i}, \mathrm{k}}$ den bis zum Abwicklungsjahr $\mathrm{k}$ angefallenen Schadenaufwand für die im Anfalljahr i eingetretenen Schäden. Dieser Aufwand setzt sich einerseits aus den bislang geleisteten Schadenzahlungen und den bestehenden Schadenrückstellungen zusammen. Die Reduzierung der Aufwandsstände $\mathrm{C}_{\mathrm{i}, \mathrm{k}}$ in Tabelle 5.1 erklärt sich durch den sukzessiven Abbau der Überreservierung in den Schadenrückstellungen im Zeitverlauf.

Für das Chain-Ladder-Verfahren spielt die absolute Höhe des Schadenaufwandes der einzelnen Anfalljahre nur eine untergeordnete Rolle. Für die Prognosegüte ist vielmehr die Homogenität der betrachteten Schadenverläufe von entscheidender Bedeutung. ${ }^{635}$ Das vergleichsweise homogene Abwicklungsverhalten der einzelnen Anfalljahre (AJ) in der Beispielsparte „Allgemeine Unfall“ kann durch folgende Verlaufsmuster visualisiert werden:

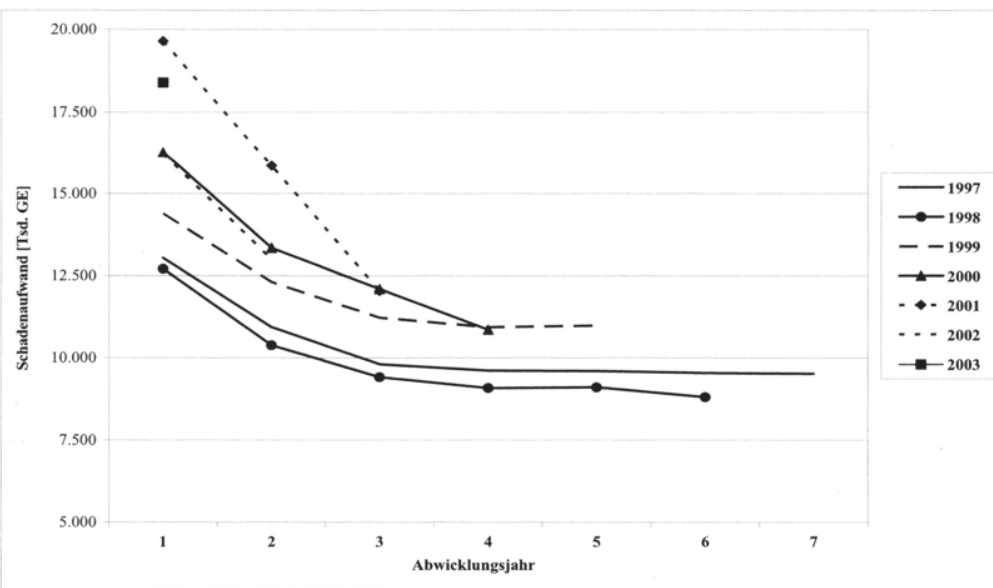

Abbildung 5.6: Verlaufsmuster auf Incurred-Basis

${ }^{635}$ Vgl. hierzu auch MACK (2002), S.223-231. 
In einem ersten Schritt erhält man für jedes Anfalljahr $\mathrm{i}$ und jedes Abwicklungsjahr $\mathrm{k}$ die individuellen Abwicklungsfaktoren $\mathrm{F}_{\mathrm{i}, \mathrm{k}}$. Diese geben Auskunft darüber, wie sich der Schadenaufwand eines Anfalljahres von einem Abwicklungsjahr zum nächsten verändert. ${ }^{636}$

$$
F_{i, k}=\frac{C_{i, k+1}}{C_{i, k}}
$$

mit $i \in\{1, \ldots, n\}$ und $k \in\{1, \ldots, n-i+1\}$

Exemplarisch ergibt sich der individuelle Abwicklungsfaktor für das Anfalljahr 3 und das Abwicklungsjahr 2 als 12.307/14.390 =0,8552 (vgl. Tabelle 5.1 und Tabelle 5.2). In einem zweiten Schritt wird für jedes Abwicklungsjahr $\mathrm{k} \in\{1, \ldots, \mathrm{n}-1\}$ ein ChainLadder-Faktor $\mathrm{CL}_{\mathrm{k}}^{\mathrm{A}}$ ermittelt. Dieser entspricht jeweils dem mit dem Schadenaufwand gewichteten Durchschnitt der individuellen Abwicklungsfaktoren $\mathrm{F}_{\mathrm{i}, \mathrm{k} \cdot \mathrm{r}}{ }^{637}$

$$
C L_{k}^{A}=\frac{\sum_{i=1}^{n-k} C_{i, k+1}}{\sum_{i=1}^{n-k} C_{i, k}}=\sum_{i=1}^{n-k} F_{i, k} \cdot \frac{C_{i, k}}{\sum_{i=1}^{n-k} C_{i, k}}
$$

mit $\mathrm{k} \in\{1, \ldots, \mathrm{n}-1\}$

Als Ergebnis einer sukzessiven Berechungsweise der einzelnen Chain-Ladder-Faktoren erhält man folgendes Tableau:

\begin{tabular}{|c|cccccc|}
\hline $\begin{array}{c}\text { Anfalljahr } \\
\mathbf{i}\end{array}$ & $\mathbf{6} \rightarrow \mathbf{2}$ & $\mathbf{2} \rightarrow \mathbf{3}$ & $\mathbf{3} \rightarrow \mathbf{4}$ & $\mathbf{4} \rightarrow \mathbf{5}$ & $\mathbf{5} \rightarrow \mathbf{6}$ & $\mathbf{6} \rightarrow \mathbf{7}$ \\
\hline $\mathbf{1 9 9 7}(\mathrm{i}=1)$ & 0,8387 & 0,8963 & 0,9808 & 0,9988 & 0,9938 & 0,9979 \\
$\mathbf{1 9 9 8}(\mathrm{i}=2)$ & 0,8172 & 0,9064 & 0,9655 & 1,0021 & 0,9675 & \\
$\mathbf{1 9 9 9}(\mathrm{i}=3)$ & 0,8552 & 0,9121 & 0,9742 & 1,0044 & & \\
$\mathbf{2 0 0 0}(\mathrm{i}=4)$ & 0,8201 & 0,9062 & 0,8978 & & & \\
$\mathbf{2 0 0 1}(\mathrm{i}=5)$ & 0,8068 & 0,7594 & & & & \\
$\mathbf{2 0 0 2}(\mathrm{i}=6)$ & 0,8016 & & & & & \\
\hline $\mathrm{CL}_{\mathrm{k}}^{\mathrm{A}}$ & $\mathbf{0 , 8 2 1 7}$ & $\mathbf{0 , 8 6 8 6}$ & $\mathbf{0 , 9 5 2 1}$ & $\mathbf{1 , 0 0 1 9}$ & $\mathbf{0 , 9 8 1 0}$ & $\mathbf{0 , 9 9 7 9}$ \\
\hline
\end{tabular}

Tabelle 5.2: Individuelle Abwicklungsfaktoren und Chain-Ladder-Faktoren

Im dritten Schritt werden durch Multiplikation der Chain-Ladder-Faktoren mit dem jeweils letzten beobachteten Schadenstand die Chain-Ladder-Schätzer $C_{i, k}^{C L}$ ermittelt und somit das Abwicklungsdreieck sukzessive zu einem Viereck vervollständigt.

${ }^{636}$ Vgl. vertiefend auch SCHMIDT (2002), S. 274-275, SCHMIDT (2004b), S. 56-57 und MACK (2002), S. 244-248.

${ }^{637}$ Vgl. zur Herleitung von Beziehung (5.25) SCHMIDT (2002), S. 276. Neben einer volumengewichteten Durchschnittsbildung kann es unter Umständen auch sinnvoll sein, die individuellen Abwicklungsfaktoren jüngerer Anfalljahre stärker zu gewichten als diejenigen älterer Anfalljahre. Vgl. SCHMIDT (2002), S. 295. 
Exemplarisch ergibt sich der kumulierte Schadenaufwand des sechsten Anfalljahres im dritten Abwicklungsjahr als 13.002 0,8686 =11.293. Die Endschadenstände (Ultimates) der einzelnen Anfalljahre sind der letzten Spalte zu entnehmen.

\begin{tabular}{|c|c|c|c|c|c|c|c|}
\hline \multirow{2}{*}{$\begin{array}{c}\text { Anfalljahr } \\
\mathbf{i}\end{array}$} & \multicolumn{7}{|c|}{ Abwicklungsjahr k } \\
\hline & 1 & 2 & 3 & 4 & 5 & 6 & 7 \\
\hline $1997(\mathrm{i}=1)$ & 13.043 & 10.940 & 9.806 & 9.618 & 9.606 & 9.546 & 9.526 \\
\hline $1998(i=2)$ & 12.708 & 10.385 & 9.413 & 9.088 & 9.107 & 8.811 & 8.792 \\
\hline $1999(\mathrm{i}=3)$ & 14.390 & & 11.225 & 10.936 & 10.984 & 10.775 & 10.752 \\
\hline $2000(\mathrm{i}=4)$ & 16.275 & 13.347 & 12.095 & 10.859 & 10.879 & 10.672 & 10.649 \\
\hline $2001(i=5)$ & 19.652 & 15.854 & 12.039 & 11.462 & 11.484 & 11.265 & 11.241 \\
\hline $2002(i=6)$ & 16.219 & 13.002 & 11.293 & 10.752 & 10.772 & 10.567 & 10.545 \\
\hline $2003(i=7)$ & 18.394 & 15.115 & 13.129 & 12.500 & 12.523 & 12.285 & 12.259 \\
\hline
\end{tabular}

Tabelle 5.3: Vervollständigtes Abwicklungsdreieck mit Endschadenständen [Tsd. GE]

Die Ultimates für die erwartete Schadenanzahl verschiedener Anfalljahre werden in analoger Vorgehensweise ermittelt, indem das Chain-Ladder-Verfahren auf Abwicklungsdreiecke für die Schadenanzahl angewendet wird. Der Modellparameter m (durchschnittliche Schadenhöhe) ergibt sich letztlich als Quotient aus erwartetem Schadenaufwand und erwarteter Schadenanzahl. Die Entwicklung der durchschnittlichen Schadenhöhen je Anfalljahr kann Tabelle 5.4 entnommen werden.

\begin{tabular}{|c|c|c|c|}
\hline $\begin{array}{c}\text { Anfalljahr } \\
\mathbf{i}\end{array}$ & $\begin{array}{c}\text { Ultimate } \\
\text { Incurred }\end{array}$ & $\begin{array}{c}\text { Ultimate } \\
\text { Anzahl }\end{array}$ & $\mathbf{~ m}$ \\
\hline $\mathbf{1 9 9 7}(\mathrm{i}=1)$ & 9.526 & 2.824 & 3,37 \\
$\mathbf{1 9 9 8}(\mathrm{i}=2)$ & 8.792 & 2.838 & 3,10 \\
$\mathbf{1 9 9 9}(\mathrm{i}=3)$ & 10.752 & 3.122 & 3,44 \\
$\mathbf{2 0 0 0}(\mathrm{i}=4)$ & 10.649 & 3.196 & 3,33 \\
$\mathbf{2 0 0 1}(\mathrm{i}=5)$ & 11.241 & 3.249 & 3,46 \\
$\mathbf{2 0 0 2}(\mathrm{i}=6)$ & 10.545 & 3.244 & 3,25 \\
$\mathbf{2 0 0 3}(\mathrm{i}=7)$ & 12.259 & 3.403 & 3,60 \\
\hline
\end{tabular}

Tabelle 5.4: Ermittlung der durchschnittlichen Schadenhöhe je Anfalljahr [Tsd. GE]

Einschränkend ist an dieser Stelle anzumerken, dass die zur Schätzung der Ultimates verwendeten empirischen Daten (Schadenzahlungen, Schadenaufwand, Schadenanzahl) im Zeitverlauf durch exogene Einflüsse wie Inflation, Bestandswachstum oder auch eine veränderte Schadenfrequenz beeinflusst werden. Vor diesem Hintergrund wäre es sinnvoll, die historischen Daten beispielsweise durch eine Indizierung vergleichbar zu machen und die Chain-Ladder-Schätzung dann auf der Basis dieser As-IfParameter durchzuführen. ${ }^{638}$ In Ermangelung eines geeigneten Preisindexes wird in diesem Beispiel auf eine Inflationierung des empirischen Datenmaterials verzichtet. ${ }^{639}$

638 Vgl. in diesem Kontext auch DOTTERWEICH (2004), S. 162-166 und MACK (2002), S. 227-228.

${ }^{639}$ Unter der Prämisse, dass der Schadenaufwand in der Sparte „Allgemeine Unfall“ ausschließlich aus Personenschäden resultiert, könnte beispielsweise ein Heilkostenindex zur Inflationierung herangezogen werden. 
Um die Schadenentwicklung des Anfalljahres 2004 prognostizieren zu können, muss unter Berücksichtigung der bisherigen zeitlichen Entwicklung und gegebenenfalls erkennbarer Trends ein „sinnvoller“ Durchschnittsschaden für das Anfalljahr 2004 festgelegt werden. Spätestens an dieser Stelle sollte die mathematische Parameterschätzung durch Expertenmeinungen untermauert werden. Darüber hinaus ist zu berücksichtigen, inwieweit für die Parameterschätzung separate Abwicklungsdreiecke für Basisschäden und Großschäden zur Verfügung stehen. Für den Fall, dass lediglich statistisches Datenmaterial verfügbar ist, welche beide Schadenkategorien enthält, sollte der verzerrende Einfluss von Großschäden beispielsweise durch Kappungen reduziert werden. ${ }^{640}$ Für die hier betrachtete Beispielsparte „Allgemeine Unfall“ wird - nach Bereinigung des Großschadeneinflusses - für die Simulation der Basisschadenlast eine durchschnittliche Schadenhöhe von 3,2 Tsd. GE zugrunde gelegt.

Das Chain-Ladder-Verfahren ist in der Praxis aufgrund seiner intuitiven Vorgehensweise sehr verbreitet. Ein wesentlicher Vorteil des Verfahrens besteht darin, dass die Abwicklungsfaktoren unabhängig von der Annahme eines bestimmten Verteilungstyps ermittelt werden. ${ }^{641}$ Es beruht in seiner ursprünglichen Form auf einer deterministischen Modellwelt, wurde jedoch von MACK auf einen stochastischen Kontext übertragen. ${ }^{642}$ Diese Erweiterung ermöglicht somit auch Aussagen über die Höhe des Zufallsund Schätzfehlers und die Erwartungstreue der Prognosen. Ein Nachteil des ChainLadder-Verfahrens ist darin zu sehen, dass sowohl der Abwicklungsfaktor des letzten Abwicklungsjahres als auch des jüngsten Anfalljahres lediglich auf einem einzigen Beobachtungswert beruhen. ${ }^{643}$ Sollten diese Werte eine untypische Entwicklung aufweisen, wird diese durch die multiplikative Vorgehensweise des Verfahrens auf sämtliche Schätzwerte übertragen. Aus der hohen Abhängigkeit von den Diagonalelementen können somit erhebliche Verzerrungen der Schätzergebnisse resultieren. ${ }^{644}$ Darüber hinaus weichen die aufwandsbasierten Ultimate-Schätzungen bisweilen signifikant von den zahlungsbasierten Ultimates ab. Dieser Problematik Rechnung tragend hat die MÜNCHENER-RÜCK-Gruppe einen Ansatz entwickelt, der diese Abweichung durch eine integrierte Verwendung von Aufwands- und Zahlungsmustern reduziert. ${ }^{645}$ Die im weiteren Verlauf der Untersuchung verwendeten Ultimate-Schätzungen wur-

\footnotetext{
${ }^{640}$ Vgl. vertiefend HeEP-ALTiNER/KLEMMSTEIN (2001), S. 15-28 und MACK (2002), S. 218-220.

${ }^{641}$ Vgl. im Folgenden MACK (2002), S. 267-272 und SCHMIDT (2002), S. 294-296.

${ }^{642}$ Vgl. ausführlich MACK (2002), S. 228-231 und S. 244-264.

${ }^{643} \mathrm{Vgl}$. zu weiterer Kritik am Chain-Ladder-Verfahren auch RocKEL (004), S. 115-116.

644 Vgl. zu dieser Problematik und zur Möglichkeit einer „Robustifizierung“ durch Diagonalbereinigung oder das Cape-Cod-Verfahren RADTKE (2004), S. 47-53, DOTTERWEICH (2004), S. 160-161, MACK (2002), S. 248-257 und HIPP/MORLOCK (2001).

${ }^{645}$ Dieser Ansatz wird als „Munich Chain Ladder“ bezeichnet. Vgl. QUARG (2003).
} 
den mit Hilfe der aktuariellen Reservierungssoftware Res $Q^{\mathrm{TM}}$ durchgeführt. ${ }^{646}$ Diese ermöglicht ebenfalls eine integrierte Schätzung auf der Basis von Zahlungs- und Aufwandsdreiecken.

Hiermit ist die Beschreibung der Basisschadenmodellierung abgeschlossen. Über die vergleichsweise homogene "Grundlast“ der Basisschäden hinaus wird der Schadenaufwand eines Schaden- und Unfallversicherungsunternehmen substanziell durch Großschäden und Schäden aus Naturgefahrereignissen beeinflusst. Im nachfolgenden Kapitel wird zunächst die Modellierung von Großschäden detailliert erläutert.

\subsubsection{Großschäden}

\subsection{Vorbemerkungen}

Als Großschäden sollen im Folgenden Einzelschäden verstanden werden, die eine bestimmte (spartenspezifisch festzulegende) Großschadengrenze überschreiten. An diese Definition anknüpfend sei darauf hingewiesen, dass sich die Modellierung von Großschäden somit signifikant von der im vorangegangenen Kapitel beschriebenen Basisschadenmodellierung unterscheidet. ${ }^{647}$ Die Modellierung von Großschäden ist hinsichtlich der Parametrisierung von einem Dilemma gekennzeichnet. Einerseits werden die Ergebnissituation und der Risikokapitalbedarf eines Schaden- und Unfallversicherungsunternehmens maßgeblich durch Großschäden beeinflusst, andererseits ist die verfügbare Datenbasis für eine adäquate Abbildung des Großschadenpotenzials häufig sehr dünn. ${ }^{648}$ Bei der Festlegung der spartenspezifischen Großschadengrenzen sollten daher unterschiedliche Gesichtspunkte berücksichtigt werden. ${ }^{649}$ Abgesehen von Erfordernissen, die sich insbesondere aus der nicht-proportionalen Rückversicherung ergeben, dient die separate Modellierung von Großschäden auch dem Ziel, eine zweckmäßige Differenzierung zwischen weitgehend homogener Basisschadenlast und einzelnen Großschäden vornehmen zu können. Auf diese Weise sollte sichergestellt werden, dass die Parameter der Basisschadenverteilung nicht durch „Ausreißer“ verzerrt werden. Die Großschadengrenze sollte darüber hinaus aber auch so bemessen sein, dass die verbleibende Datenbasis eine hinreichend valide Abschätzung des Gefährdungspotenzials durch Großschäden zulässt. Unter der Voraussetzung, dass die historischen Daten keine nennenswerten Strukturbrüche aufweisen, sollte sich der Beobachtungszeitraum daher über möglichst viele Jahre erstrecken.

\footnotetext{
${ }^{646} \operatorname{Res}^{\mathrm{TM}}$ ist ebenfalls ein Produkt der aktuariellen Beratungsgesellschaft EMB.

${ }^{647}$ Zur Begründung dieses Vorgehens siehe auch Kapitel 5.4.3.1.

${ }^{648}$ Vgl. zur Problematik geringer Kollektivbesetzung bereits BRAEB (1969), S. 211-224, HAX (1969), S. 187-210 und BEARD/PENTIKÄINEN/PESONEN (1977), S. 29 sowie MACK (2002), S. 88-91 und SCHRADIN (1998), S. 122-123.

649 Vgl. im Folgenden auch PFEIFER (2003), S. 675.
} 
Im Rahmen dieser Arbeit werden Großschäden in den Sparten „Allgemeine Unfall“, „Kraftfahrt-Haftpflicht“, „Kasko“, „Feuer Industrie“ und „VGV Feuer“ modelliert. Die Schadenentwicklung der Versicherungszweige „Allgemeine Sturm“, „VGV Sturm" und „VGV Elementar" wird in der Regel stark durch Ereignisschäden, z.B. durch Stürme, Hagelereignisse oder Überschwemmungen, beeinflusst. Die Modellierung von Schäden aus Naturgefahren wird in Kapitel 5.4.3.4 erläutert. Die Schadenentwicklung in der Sparte „VGV Leitungswasser" wird vorwiegend durch eine hohe Frequenz von Kleinschäden bestimmt und weniger durch die Schadenhöhe. ${ }^{650}$ Auf eine separate Großschadenmodellierung wird daher verzichtet.

Während das kollektive Modell im Bereich der Basisschäden lediglich dazu herangezogen wurde, die Gesamtschadenverteilung anhand ihrer ersten beiden Momente zu approximieren, folgt die Modellierung der Großschäden der „klassischen“ Vorgehensweise des kollektiven Modells. Die Gesamtschadenverteilung der Großschäden setzt sich demzufolge aus einer Schadenanzahlverteilung und einer Schadenhöhenverteilung zusammen. Da die Schadenhöhenverteilung in der Regel einen deutlich stärkeren Einfluss auf die Gesamtschadenverteilung nimmt, wird diese in den nachfolgenden Ausführungen detaillierter beschrieben als die Schadenanzahlverteilung. ${ }^{651}$

\subsection{Ermittlung der Schadenanzahlverteilung}

Für die Modellierung der Schadenanzahl wird in der Praxis vielfach auf die Poissonverteilung oder die Negative Binomialverteilung zurückgegriffen. ${ }^{652}$ Beide Verteilungen generieren diskrete Zufallsvariablen und gehören zur so genannten PANJERKlasse. ${ }^{653}$ Auf ihre spezifischen Unterschiede soll an dieser Stelle nicht detailliert eingegangen werden. ${ }^{654}$ Aufgrund der Tatsache, dass bei der Poissonverteilung Erwartungswert und Varianz übereinstimmen, erzeugt diese in Portfolios mit einer konstant hohen Schadenanzahl auch eine hohe Streuung der Realisationen der Zufallsvariablen. In diesen Fällen wird in der Regel die Negative Binomialverteilung verwendet. ${ }^{655}$ Vielfach reicht die statistische Datenbasis im Großschadenbereich jedoch nicht aus, um eine hinreichend genaue Anpassung der Schadenanzahlverteilung durchzuführen. Diese Problematik soll am Beispiel des Modellsegmentes „Kraftfahrt-Haftpflicht Privatkunden" verdeutlicht werden. Die Großschadengrenze dieses Segments beträgt

\footnotetext{
${ }^{650} \mathrm{Vgl}$. hierzu vertiefend die Ausführungen in Kapitel 6.2.4.1.

651 Vgl. auch FREY/NIEBEN (2001), S. 73 und S. 86.

${ }^{652}$ Vgl. FREY/NiEBEN (2001), S. 73-79, SANN (2001), S. 12 und MACK (2002), S. 86.

653 Vgl. SCHMIDT (2002), S. 169-176 und HiPp (2001a), S. 163 und S. 165.

654 Vgl. hierzu stellvertretend PANJER/Willmot (1992), S. 85-87 und S. 90-92 sowie KLUGMAN/ PANJER/WILLMOT (1998), S. 202-217.

655 Vgl. hierzu vertiefend SCHMIDT (2002), S. 181 und PANJER/WILLMOT (1992), S. 91.
} 
250.000 GE und wurde als Ergebnis einer Analyse der historischen Schadenentwicklung sowie unter Berücksichtigung der Bestandsstruktur im Zeitverlauf festgelegt. Die Anzahl der Großschäden für dieses Segment wird für einen Zeitraum von sieben Kalenderjahren als bekannt vorausgesetzt. In Abbildung 5.7 wird die empirische Verteilungsfunktion der angepassten Poissonverteilung bzw. Negativen Binomialverteilung gegenübergestellt. ${ }^{656}$ Die graphische Darstellung ermöglicht erste Aussagen über die Güte der Verteilungsanpassung für die Schadenanzahl.

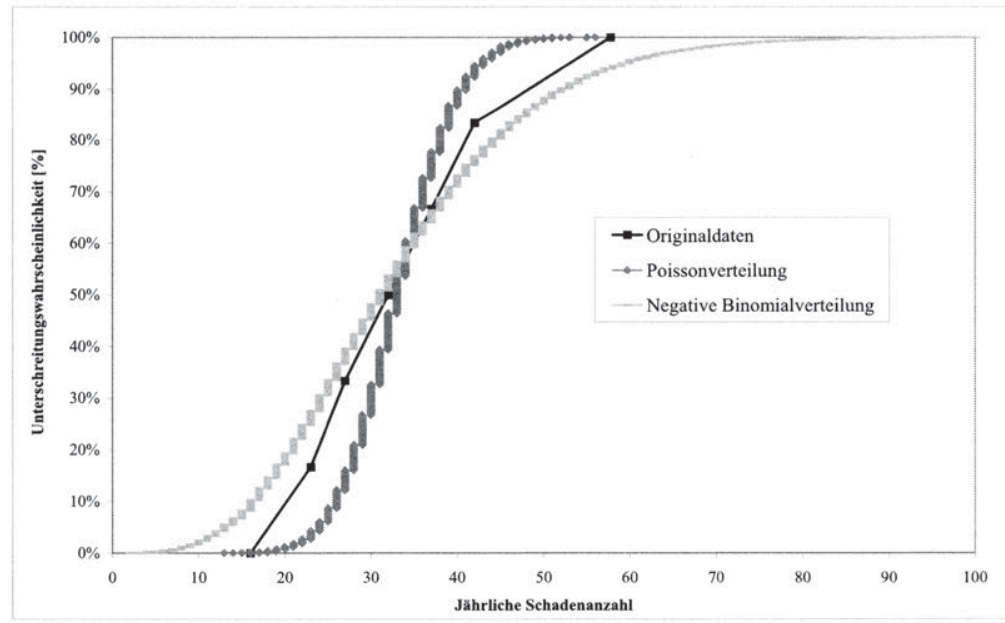

Abbildung 5.7: Empirische Verteilungsfunktion und Anpassungsgüte

Die Güte der Verteilungsanpassung durch die Poissonverteilung erscheint auf den ersten Blick unbefriedigend. Hingegen approximiert die Negative Binomialverteilung die empirisch beobachteten Werte deutlich besser. Die unterschiedliche Anpassungsgüte kann auf die geringe Anzahl von Beobachtungspunkten zurückzuführen sein. Die empirische Verteilungsfunktion (der Originalwerte) könnte in dem kurzen Zeitraum von 7 Kalenderjahren starken Zufallsschwankungen unterworfen sein. Eine alternative Interpretation könnte die Schlussfolgerung nahe legen, dass die Poissonverteilung grundsätzlich die Zufallsgesetzmäßigkeit der Schadenanzahl im betrachteten Segment nicht angemessen approximiert. Die hiermit beschriebene Problematik ist in der Praxis bei der Modellierung von Großschäden häufig anzutreffen. ${ }^{657}$ Es ist daher immer im Ein-

${ }^{656}$ Die graphische Darstellung der Verteilungsanpassung erfolgt ausschließlich zum Zwecke der Anschaulichkeit (,visuelle Intuition“). Hinsichtlich der zur Parameterschätzung verwendeten Momentenmethode wird auf die Literatur verwiesen. Vgl. beispielsweise KLUGMAN/PANJER/WILLMOT (1998), S. 46-47, MACK (2002), S. 100-103 und HIPP (2001a), S. 102. 
zelfall zu entscheiden, ob die angepasste Verteilungsfunktion der Poissonverteilung oder der Negativen Binomialverteilung eine hinreichend genaue Modellierung erlaubt. Darüber hinaus ist ebenfalls zu berücksichtigen, in welchem Ausmaß die durch die angepassten Verteilungen erzeugten Zufallsvariablen streuen. Die in Abbildung 5.8 dargestellten Dichtefunktionen verdeutlichen, dass die Negative Binomialverteilung in der betrachteten Beispielsituation eine signifikant höhere Streuung aufweist als die Poissonverteilung. Während die Poissonverteilung im Maximum nur ca. 70 Großschäden je Anfalljahr generiert, werden durch die Negative Binomialverteilung im Extremfall bis zu 100 Großschäden erzeugt.

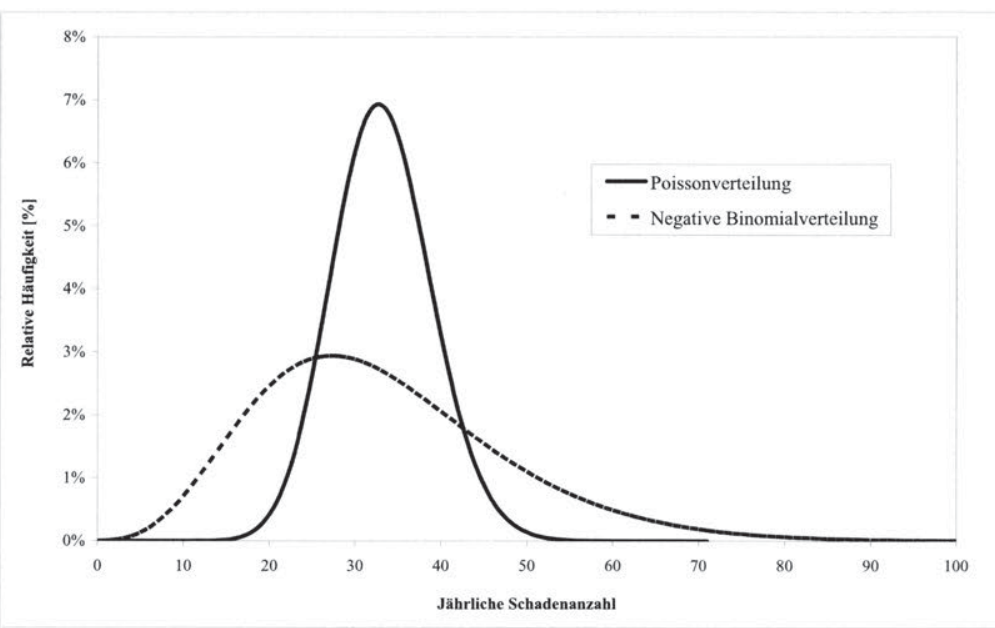

Abbildung 5.8: Negative Binomialverteilung vs. Poissonverteilung (Dichtefunktionen)

Bei der Auswahl der Schadenanzahlverteilung ist ferner zu beachten, dass die einparametrische Poissonverteilung das Streuungsverhalten stark volatiler Stichproben bisweilen unterschätzt. ${ }^{658} \mathrm{Im}$ Rahmen dieser Arbeit wird für die Modellierung der Schadenanzahl jedoch durchgängig die Poissonverteilung verwendet, da die zugrunde liegenden historischen Daten vergleichsweise moderate Streuungen aufweisen. Der Verteilungsparameter $\theta$ wird anhand der empirischen Daten als Erwartungswert geschätzt und gegebenenfalls unter Berücksichtigung segmentspezifischer Trends angepasst. ${ }^{659}$

${ }^{658}$ Diese Problematik tritt naturgemäß nur dann auf, sofern der Verteilungsparameter $\theta$ als Erwartungswert (und nicht als Varianz) geschätzt wird.

${ }^{659}$ Analog zur Modellierung der Basisschadenlast sollten die (mathematischen) Parameterschätzungen auf der Basis historischer Daten ebenfalls durch Expertenschätzungen (z.B. bezüglich der zunehmenden Konzentration von Sachwerten in Ballungsgebieten) untermauert werden. Eine tabellarische Darstellung der im weiteren Verlauf verwendeten Großschadenparameter ist dem Anhang A zu entnehmen. 


\subsection{Ermittlung der Schadenhöhenverteilung}

Die Gesamtschadenverteilung der Großschäden wird maßgeblich durch die Wahl einer angemessenen Schadenhöhenverteilung sowie deren Parametrisierung beeinflusst. In diesem Zusammenhang soll eingangs die Frage diskutiert werden, ob zur Modellierung der Großschadenhöhen auf (beobachtete) empirische Verteilungen oder (angepasste) parametrische Verteilungen zurückgegriffen werden sollte. Infolge des vielfach geringen Stichprobenumfangs weisen empirische Verteilungen den wesentlichen Nachteil auf, dass sie den ökonomisch relevanten Bereich der Groß- und Größtschäden nur unzureichend abbilden. ${ }^{660}$ Darüber hinaus generieren empirische Verteilungsfunktionen per definitionem keine Schäden, die größer sind als der größte bisher beobachtete Schaden. ${ }^{661}$ MACK weist außerdem im rückversicherungsspezifischen Kontext darauf hin, dass eine empirische Verteilungsfunktion „bei gruppierten Daten [...] keinerlei Auskunft über den Verlauf der Verteilung innerhalb der Gruppen gibt. Man müsste in vielen Fällen interpolieren und kann dann gleich ein stetiges Verteilungsmodell benutzen. “662 Es bleibt daher festzuhalten, dass in Segmenten mit dünner Datenhistorie die Verwendung einer parametrischen Verteilung anzuraten ist. Diese Tatsache trifft im Bereich der Großschäden auf sämtliche Risikokollektive des Modellunternehmens zu. Nachfolgend soll mit der Maximum-Likelihood-Methode ein Verfahren beschrieben werden, das zur Berechnung ,guter“ Schätzer in parametrischen Verteilungen eingesetzt werden kann. ${ }^{663}$ Die Maximum-Likelihood-Methode „basiert auf dem heuristischen Prinzip, dass nur ein Parameter als plausibel erscheint, unter dem die beobachteten Daten mit einer hohen Wahrscheinlichkeit auftreten können. “664

Bezeichne $\mathrm{x}_{\mathrm{i}}$ mit $\mathrm{i} \in\{1, \ldots, \mathrm{n}\}$ die Realisationen der stochastisch unabhängigen $\mathrm{Zu}$ fallsvariablen $X_{i}$ (Schadenhöhe) in einer Stichprobe. Die Verteilung $P^{\mathbf{x}_{i}}$ sei durch einen Parametervektor $\lambda \in\left\{\lambda_{1}, \ldots, \lambda_{k}\right\}$ charakterisiert. Im Fall einer diskreten Verteilung ist die Wahrscheinlichkeit, eine Stichprobe zu erhalten, die gerade aus den obigen Werten $\mathrm{x}=\left\{\mathrm{x}_{\mathrm{i}}, \ldots, \mathrm{x}_{\mathrm{n}}\right\}$ besteht, durch folgendes Produkt gegeben:

${ }^{660}$ Vgl. im Folgenden auch MACK (2002), S. 88.

661 Vgl. MACK (2002), S. 88. FREY/NIEBEN weisen darauf hin, dass eine um virtuelle Großschäden erweiterte empirische Verteilungsfunktion, die zwischen den beobachteten Schäden interpoliert, vielfach realistischere Ergebnisse liefert und zudem besser kommunizierbar ist. Vgl. FREY/NIEBEN (2001), S. 86-88.

${ }^{662}$ MACK (2002), S. 88. Die Problematik gruppierter Daten ist hingegen bei Erstversichern nur von untergeordneter Bedeutung, da diese in der Regel über detaillierte (nicht gruppierte) Bestandsdaten verfügen.

${ }^{663}$ Vgl. im Folgenden VoSE (1998), S. 111-117, NEUSSER (2000) und NEUBAUER (1994), S. 393-398.

${ }^{664}$ HIPP (2001b), S. 34. 


$$
\mathrm{L}_{\mathrm{n}}=\mathrm{L}_{\mathrm{n}}(\mathrm{x}, \lambda)=\prod_{\mathrm{i}=1}^{\mathrm{n}} \mathrm{P}\left(\mathrm{X}_{\mathrm{i}}=\mathrm{x}_{\mathrm{i}} \mid \lambda\right) \quad \text { mit } \lambda \in\left\{\lambda_{1}, \ldots, \lambda_{\mathrm{k}}\right\}
$$

Die Funktion $L_{n}$ wird als Maximum-Likelihood-Funktion (MLF) bezeichnet. Derjenige Wert von $\lambda$, der die Funktion $L_{n}$ maximiert, heißt Maximum-Likelihood-Schätzer (MLS) von $\lambda$. Da im Fall einer stetigen Verteilung $P\left(X_{i}=x_{i} \mid \lambda\right)=0$ gilt, wird in der Definition des MLS $P\left(X_{i}=x_{i} \mid \lambda\right)$ durch die Dichtefunktion $f\left(x_{i} \mid \lambda\right)$ ersetzt. Ist diese Dichtefunktion wiederum durch einen Parametervektor $\lambda$ gekennzeichnet, ergibt sich die MLF formal als Produkt der Dichtefunktionen.

$$
\mathrm{L}_{\mathrm{n}}=\mathrm{L}_{\mathrm{n}}(\mathrm{x}, \lambda)=\prod_{\mathrm{i}=1}^{\mathrm{n}} \mathrm{f}\left(\mathrm{x}_{\mathrm{i}} \mid \lambda\right)
$$

Ziel der Maximum-Likelihood-Methode ist es nun, denjenigen Parametervektor $\lambda \mathrm{zu}$ ermitteln, der die Funktion $L_{n}$ maximiert, d.h. bei dem die Dichtefunktion ihren maximalen Wert annimmt. Das Maximum der Funktion $L_{n}$ kann für einige Verteilungstypen analytisch bestimmt werden. ${ }^{665} \mathrm{Zu}$ diesem Zweck wird die MLF nach dem gesuchten Parameter $\lambda_{\mathrm{i}}$ differenziert und die partielle Ableitung gleich Null gesetzt:

$$
\frac{\partial \mathrm{L}_{\mathrm{n}}\left(\mathbf{x}_{\mathrm{i}}, \lambda\right)}{\partial \lambda_{\mathrm{i}}}=0
$$

Als Lösung dieser Gleichung erhält man schließlich den Maximum-LikelihoodSchätzer $\hat{L}_{n}(X) .{ }^{666}$ Da die Differentiation des Produktes $L_{n}$ jedoch häufig an mathematische Grenzen stößt, wird vielfach der natürliche Logarithmus von $L_{n}$ betrachtet, da durch die Logarithmierung das Produkt aus (5.28) in eine Summe logarithmierter Dichtewerte übergeht. ${ }^{667}$

$$
\ln \left(L_{n}\right)=\ln \left[\prod_{i=1}^{n} f\left(x_{i} \mid \lambda\right)\right]=\sum_{i=1}^{n} \ln \left[f\left(x_{i} \mid \lambda\right)\right]
$$

${ }^{665}$ Vgl. zur analytischen Bestimmung der Maximum-Likelihood-Schätzer für die Exponential-, Normal-, Poisson- oder Binomialverteilung auch VosE (1998), S. 113-115 und LEHMANN (2002), S. 222-225.

${ }^{666}$ Im Falle eines k-dimensionalen Parametervektors ist ein Gleichungssystem mit k Gleichungen zu lösen.

${ }^{667} \mathrm{Da}$ der Logarithmus eine (streng) monotone Transformation darstellt, liegt das Maximum von L und von $\ln (\mathrm{L})$ an der gleichen Stelle. Vgl. LeHMANN (2002), S. 221 und NEUSSER (2000), S. 2. 
In vielen Fällen muss an dieser Stelle auf numerische Maximierungsverfahren zurückgegriffen werden. Das Maximum von $\ln \left(\mathrm{L}_{\mathrm{n}}\right)$ kann dann mit Hilfe von Iterationsverfahren, wie beispielsweise dem NEWTON-RAPHSON-Verfahren ${ }^{668}$ oder alternativ auch mit Tabellenkalkulationsprogrammen ${ }^{669}$ näherungsweise bestimmt werden.

Die Güte einer Parameterschätzung wird in der Literatur vielfach anhand der Kriterien Konsistenz, Erwartungstreue, Effizienz bzw. Wirksamkeit und Suffizienz beurteilt. ${ }^{670}$ Auf die mathematische Herleitung dieser Kriterien wird hier verzichtet und auf die Literatur verwiesen. ${ }^{671}$ Der Maximum-Likelihood-Schätzer $\hat{L}_{n}\left(X_{i}\right)$ weist im Hinblick auf diesen Kriterienkatalog eine Reihe wünschenswerter Eigenschaften auf, die nachfolgend skizziert werden sollen.

(MLS 1) $\quad \hat{\mathrm{L}}_{\mathrm{n}}\left(\mathrm{X}_{\mathrm{i}}\right)$ ist konsistent, d.h. er konvergiert im Sinne der Konvergenz nach Wahrscheinlichkeit mit wachsendem Stichprobenumfang gegen den $\mathrm{Pa}$ rameter $\lambda_{\mathrm{i}}{ }^{672}$

$$
\lim _{n \rightarrow \infty} P\left(\hat{L}_{n}\left(X_{i}\right)-\lambda_{i} \mid>\varepsilon\right)=0 \quad \text { für jedes } \varepsilon>0
$$

(MLS 2) $\quad \hat{\mathrm{L}}_{\mathrm{n}}\left(\mathrm{X}_{\mathrm{i}}\right)$ ist asymptotisch erwartungstreu, d.h. der Erwartungswert von $\hat{\mathrm{L}}_{\mathrm{n}}\left(\mathrm{X}_{\mathrm{i}}\right)$ stimmt bei großen Stichproben näherungsweise mit dem Parameter $\lambda_{i}$ überein.

(MLS 3) $\quad \hat{\mathrm{L}}_{\mathrm{n}}\left(\mathrm{X}_{\mathrm{i}}\right)$ ist asymptotisch effizient, d.h. er weist die kleinstmögliche Varianz eines erwartungstreuen Schätzers auf. ${ }^{673}$ Die asymptotische Varianz des Schätzers kann zudem explizit berechnet werden. ${ }^{674}$

(MLS 4) $\quad \hat{\mathrm{L}}_{\mathrm{n}}\left(\mathrm{X}_{\mathrm{i}}\right)$ ist invariant gegenüber Parametertransformationen. ${ }^{675}$

\footnotetext{
668 Vgl. hierzu vertiefend STOER (1993), S. 241-245 und HERZBERGER (1997), S. 197-249.

${ }^{669}$ Vgl. zur Funktionsweise der Solverfunktion von Microsoft Excel ${ }^{\mathrm{TM}}$ und den verwendeten Näherungsverfahren insbesondere FYLSTRA/LASDON/WATSON/WAREN (1998), S. 29-55. Ein anschauliches Beispiel für die Verwendung von Tabellenkalkulationsprogrammen wird z.B. bei VOSE (1998), S. 115-117 beschrieben.

${ }^{670}$ Vgl. exemplarisch NEUBAUER (1994), S. 386-391.

671 Vgl. insbesondere LEHMANN (2002), S. 226-231.

${ }^{672}$ Vgl. NeUSSER (2000), S. 4.

673 Vgl. Klugman/PANJER/Willmot (1998), S. 56 und NeUSSER (2000), S. 5.

674 Vgl. MACK (2002), S. 103 und KLUGMAN/PANJER/WiLLMOT (1998), S. 57.

${ }^{675}$ Vgl. MaCK (2002), S. 103-104 und KLugMan/PANJeR/Willmot (1998), S. 56.
} 
Allerdings muss ebenfalls festgehalten werden, dass alle genannten Eigenschaften asymptotischer Natur sind. Bei sehr kleinen Stichproben können gegebenenfalls andere Parameterschätzer über bessere Eigenschaften verfügen. Darüber hinaus sollte betont werden, dass der MLS immer auf einer Annahme über eine bestimmte Verteilung fußt. Sofern diese Verteilungsannahme nicht zutrifft, liefert der MLS nur unzureichende Ergebnisse. Es ist daher empfehlenswert, die Güte der Verteilungsanpassung beispielsweise anhand graphischer Auswertungen (sog. „goodness-of-fit-plots") zu plausibilisieren ${ }^{676}$ Darüber hinaus existiert eine Vielzahl statistischer Anpassungstests, die an dieser Stelle jedoch nicht thematisiert werden. ${ }^{677}$

Im Rahmen dieser Arbeit wird zur Anpassung der Schadenhöhenverteilungen der Großschäden durchgängig die Maximum-Likelihood-Methode verwendet. Das Maximum von $\ln \left(\mathrm{L}_{\mathrm{n}}\right)$ wird mit Hilfe der Solverfunktion von Microsoft Excel ${ }^{\mathrm{TM}}$ näherungsweise bestimmt. ${ }^{678}$ Im Beispielsegment „Kraftfahrt-Haftpflicht Privatkunden“ erfolgt die Schätzung der Schadenhöhenverteilung auf der Basis des Schadenaufwandes, d.h. es werden sowohl die geleisteten Schadenzahlungen als auch die zugehörigen Einzelfallreserven der beobachteten Großschäden betrachtet. ${ }^{679}$ Die Güte der Verteilungsanpassung kann beispielsweise durch einen Vergleich der empirischen Verteilungsfunktion mit den angepassten Verteilungsfunktionen visualisiert werden. ${ }^{680}$ Dies soll im vorliegenden Fall am Beispiel der verallgemeinerten Pareto A Verteilung und der Normalverteilung verdeutlicht werden. In Abbildung 5.9 werden die empirisch beobachteten Schadenhöhen (Originaldaten) durch den schwarzen Linienzug dargestellt.

${ }^{676}$ Einen Überblick möglicher Darstellungsweisen vermittelt z.B. VOSE (1998), S. 134-135.

${ }^{677}$ Exemplarisch seien hier der KolMOGOROV-SMIRNOFF-Test, ANDERSON-DARLING-Test und ChiQuadrat-Anpassungstest genannt. Vgl. vertiefend VOSE (1998), S. 118-134.

678 Vgl. hierzu vertiefend FYLSTRA/LASDON/WATSON/WAREN (1998), S. 29-55.

${ }^{679}$ Diese Vorgehensweise setzt implizit voraus, dass die erwartete Endschadenbelastung eines Großschadens in der Longtail-Sparte „Kraftfahrt Haftpflicht“ angemessener durch den Schadenaufwand approximiert wird.

680 „By far the most intuitive measure of goodness-of-fit is a visual comparison of probability distributions [...]." VOSE (1998), S. 118. 


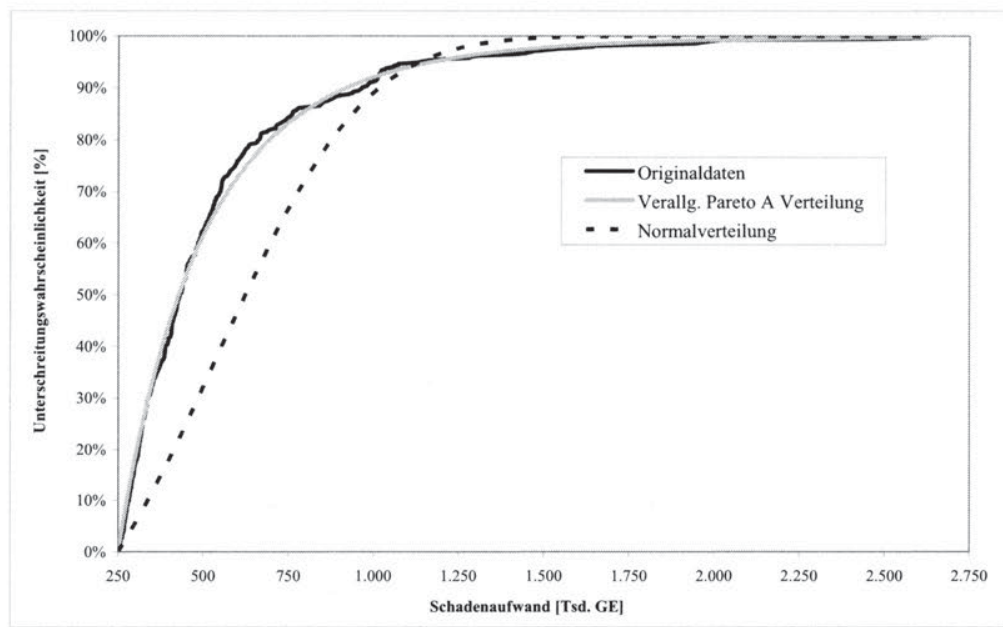

Abbildung 5.9: Verteilungsanpassung im Segment „Kraftfahrt-Haftpflicht Privatkunden“

Offenbar wird die Verteilung der Schadenhöhen im Segment „Kraftfahrt-Haftpflicht Privatkunden" durch die verallgemeinerte Paretoverteilung wesentlich besser approximiert als durch die Normalverteilung. Bis zu einer Schadenhöhe von ca. 1,14 Mio. GE wird die beobachtete Schadenentwicklung durch die Normalverteilung überschätzt, ab diesem kritischen Wert tendenziell unterschätzt. Die verallgemeinerte Paretoverteilung hingegen approximiert die Großschadenentwicklung über das gesamte beobachtete Intervall sehr genau. Sie ist daher der Normalverteilung vorzuziehen.

Zum Abschluss dieses Abschnitts soll noch auf eine Besonderheit der Modellierung von PML-Sparten hingewiesen werden. Wie bereits erläutert, erfolgt die Schadenmodellierung in diesen Sparten über so genannte PML-Bänder, um die Wirkungsweise der Rückversicherung über Summenexzedentenverträge geeignet abbilden zu können. Mit Blick auf die Großschadenmodellierung ist es daher erforderlich, einen Algorithmus oder zumindest eine zweckmäßige Heuristik im Modell zu verankern, mit deren Hilfe die simulierten Großschäden den einzelnen PML-Bändern zugeordnet werden können. ${ }^{681}$ Die im Rahmen dieser Arbeit gewählte (heuristische) Vorgehensweise wird nachfolgend anhand eines fiktiven Beispiels erläutert.

Gegeben sei eine PML-Sparte mit folgender Aufteilung der Jahreseinheiten auf insgesamt vier PML-Bänder.

${ }^{681}$ In der versicherungswirtschaftlichen Praxis kann hingegen in der Regel jeder Großschaden unmittelbar einem Versicherungsvertrag bzw. PML zugeordnet werden. 


\begin{tabular}{|c|c|c|}
\hline $\begin{array}{c}\text { Ifd. } \\
\text { Band-Nr. }\end{array}$ & $\begin{array}{c}\text { Bandgrenzen } \\
\text { [Tsd. GE] }\end{array}$ & $\begin{array}{c}\text { Jahreseinheiten } \\
\text { [Stück] }\end{array}$ \\
\hline 1 & $0-1.500$ & 4.000 \\
2 & $1.501-7.500$ & 3.000 \\
3 & $7.501-14.500$ & 2.000 \\
4 & $14.501-30.000$ & 1.000 \\
\hline
\end{tabular}

Tabelle 5.5: Aufteilung der Jahreseinheiten auf die PML-Bänder

Beispielhaft wird davon ausgegangen, dass das Schadenmodell in dieser PML-Sparte einen Großschaden in Höhe von 3.000 Tsd. GE generiert. Da Überschätzungen bzw. Unterschätzungen des PML annahmegemäß ausgeschlossen sind, kann dieser Großschaden nur die Bänder 2, 3 oder 4 betreffen, in denen insgesamt 6.000 Jahreseinheiten modelliert sind. Die Heuristik geht davon aus, dass sich die Eintrittwahrscheinlichkeit der Großschäden in einem bestimmten Band als Funktion der Jahreseinheiten ausdrücken lässt. Folglich beträgt die Wahrscheinlichkeit, dass der simulierte Großschaden aus dem zweiten PML-Band stammt 3.000/(3.000+2.000+1.000) $=50 \%$. Für das dritte und vierte Band ergeben sich in analoger Vorgehensweise Wahrscheinlichkeiten von 33,33\% und 16,67\%. Anhand dieser Wahrscheinlichkeiten werden die simulierten Großschäden auf die einzelnen PML-Bänder „,zugewürfelt“.

\subsubsection{Schäden aus Naturgefahren}

\subsection{Vorbemerkungen}

Wie bereits in Kapitel 5.4.3.1 angesprochen, sollten Schäden aus Naturgefahrereignissen separat im Schadenmodell berücksichtigt werden. ${ }^{682}$ Diese Vorgehensweise ist einerseits darin begründet, dass die für die Anwendung des kollektiven Modells notwendige Unabhängigkeit von Schadenanzahl und Schadenhöhe bei Ereignisschäden vielfach nicht erfüllt ist, da mehrere Gefahren durch dieselbe Police gedeckt sind. ${ }^{683}$ Dieser Problematik kann jedoch durch die getrennte Betrachtung der Einzelgefahren Rechnung getragen werden. ${ }^{684}$ Als Beispiel kann in dieser Arbeit die getrennte Modellierung der Gefahren Feuer, Sturm, Leitungswasser und Elementar in der Verbundenen Wohngebäudeversicherung herangezogen werden. Schwerer wiegt hingegen die Tatsache, dass die in Kapitel 5.4.3.1 formulierten Anwendungsvoraussetzungen für die

${ }^{682}$ In dieser Arbeit werden die Begriffe Naturgefahrereignis und Kumulereignis synonym verwendet. Gleiches gilt für die Begriffsfamilie der Ereignis- und Kumulschäden. Nach BoETIUS liegt ein Kumulrisiko vor, „wenn eine Vielzahl von Risikoobjekten derselben objekttypischen Gefahr ausgesetzt ist und der Schaden an den einzelnen Risikoobjekten stets durch ein einziges Ereignis verursacht wird, das eine sehr große Zahl der bedrohten Objekte zur gleichen Zeit erfasst und dadurch zu einem Größtschaden führt.“ BOETIUS (1996), S. 266. Vgl. zur zeitlichen und räumlichen Präzisierung des Ereignisbegriffs LIEBWEIN (2000), S. 162.

683

Als Beispiel seien an dieser Stelle Überschwemmungsschäden genannt. In der Regel erhöhen sich sowohl die Schadenanzahl als auch der Durchschnittsschaden mit ansteigendem Wasserstand.

${ }^{684}$ Vgl. auch MACK (2002), S. 109. 
Poissonverteilung als Schadenanzahlverteilung nicht erfüllt sind. Insbesondere die Forderung nach Regularität der Intensitätsfunktion, d.h. dass mehrere Schäden nicht exakt zum gleichen Zeitpunkt eintreten dürfen, ist im Fall von Ereignisschäden per definitionem verletzt. ${ }^{685}$ Gleichwohl kann jedoch davon ausgegangen werden, dass die Anzahl der Naturereignisse selbst durch eine Poissonverteilung approximiert werden kann. ${ }^{686}$ Unabhängig von dieser risikotheoretischen Problematik ist es aus Sicht des Versicherungsunternehmens ebenfalls sinnvoll, Kumulschäden separat zu modellieren, da sie sich ökonomisch betrachtet wie ein einziger großer Schaden auswirken ${ }^{67}$ Weil in kumulgefährdeten Beständen der versicherungstechnische Ausgleich im Kollektiv nicht greift, kann der Ausgleich bestenfalls über die Zeit erfolgen. ${ }^{688}$ Zur Finanzierung dieses zeitlichen Ausgleichs kann entweder auf Rückversicherungsschutz oder zusätzliches Risikokapital zurückgegriffen werden. ${ }^{689}$

Grundsätzlich existieren zwei mögliche Vorgehensweisen, Schadenpotenziale aus Naturgefahrereignissen und deren Eintrittswahrscheinlichkeiten $\mathrm{zu}$ approximieren und modelltechnisch abzubilden. ${ }^{690}$ Mathematisch-statistische Ansätze treffen anhand der beobachteten Schadenhistorie Aussagen über den zukünftig erwarteten Schadenverlauf und greifen zu diesem Zweck auf Informationen über die historische Portfoliostruktur zurück. Physikalisch-technische Modelle bieten die Möglichkeit, durch Auswertung meteorologischer Daten und unter Rückgriff auf naturwissenschaftliche Zusammenhänge (z.B. zwischen Windgeschwindigkeit und Schadengrad) auf das Schadenpotenzial zu schließen. ${ }^{691}$ Die Ergebnisse derartiger Modelle können sowohl deterministischer als auch probabilistischer Natur sein. Mit Hilfe eines deterministischen Ansatzes können die Auswirkungen historischer Schadenereignisse auf die aktuelle Portfoliostruktur untersucht und beispielsweise „Worst-Case-Szenarien“ formuliert werden. Der stochastische Ansatz ermöglicht die Quantifizierung von Eintrittswahrscheinlichkeiten bzw. Wiederkehrperioden ${ }^{692}$ einzelner Schadenereignisse. Zu diesem Zweck

685 Vgl. übereinstimmend MACK (2002), S. 79.

686 Vgl. MACK (2002), S. 116-117 und PFEIFER (2004).

${ }^{687}$ Vgl. FARNY (2000), S. 86, ZWEIFEL/EISEN (2003), S. 452 und BERZ (1999), S. 427.

${ }^{688}$ Vgl. hierzu BoETIUS (1996), S. 277 und insbesondere SCHRADIN (1998), S. 121-134.

${ }^{689}$ Allerdings ist kritisch anzumerken, dass die Absicherung über Rückversicherungsschutz im Falle von Kumulschäden vielfach nicht fristenkongruent ist. Vgl. hierzu auch POHLHAUSEN (1999), S. 457-460.

${ }^{690}$ Vgl. im Folgenden auch FrIEDMAN (1972), PFEIFER (2000), HIPP (1999) und BERZ (1999).

${ }^{691}$ Prominente Vertreter geophysikalisch-meteorologischer Modelle sind beispielsweise „RMS“ (Risk Management Solutions) und „EQECAT“. Vgl. auch PFEIFER (2000), S. 771-772.

${ }^{692}$ Als Wiederkehrperiode $\mathrm{T}$ wird im Folgenden derjenige Zeitraum bezeichnet, innerhalb dessen durchschnittlich eine Überschreitung des Probable Maximum Loss (PML) eintritt. Vgl. PFEIFER (2000), S. 772. 
werden real eingetretene Stürme durch künstliche Sturmereignisse ergänzt und zu einem stochastischen Ereigniskatalog (,,event set“) zusammengefasst. ${ }^{693}$

Die mathematisch-statistischen Ansätze werden häufig dahingehend kritisiert, dass sie die historische Schadenentwicklung als hinreichenden Schätzer für die zukünftige Schadenentwicklung ansehen. ${ }^{694}$ Dieser Einwand zielt insbesondere auf den Bereich bislang nicht beobachteter Schadenhöhen bzw. langer Wiederkehrperioden ab. Er trifft jedoch auch auf physikalisch-technische Modellen zu, „da die [...] verwendeten Parameter ebenfalls einer historischen Quelle entstammen und darüber hinaus die der Wiederkehrperiode zugrunde liegende Philosophie davon ausgeht, dass die Schadensituation in der Zukunft vergleichbar der heutigen bleibt. “695

Im Rahmen dieser Arbeit werden die Naturgefahren Sturm, Hagel und Überschwemmung abgebildet. Den weiteren Ausführungen zur Modellierung von Naturgefahrschäden liegt eine Schadenhistorie zugrunde, die sich auf den Zeitraum von 1972 bis 2003 erstreckt. Es erscheint daher vertretbar, die Prognose von Sturm- und Hagelereignissen zum Zwecke dieser Arbeit anhand historischer Daten vorzunehmen. Im Rahmen der Modellierung von Naturgefahren sollte in der versicherungswirtschaftlichen Praxis jedoch stets eine Plausibilisierung des empirischen Datenmaterials mit sonstigen Informationsquellen, wie z.B. unternehmensspezifischen Studien von Rückversicherungsunternehmen oder Rückversicherungsmaklern erfolgen. Für die Modellierung von Sturm- und Hagelereignissen wird nachfolgend erneut auf das kollektive Modell der Risikotheorie zurückgegriffen. Der aus einer Naturgefahr (z.B. Sturm) resultierende Gesamtaufwand kann approximiert werden, sofern einerseits die Verteilung der Ereignisanzahl und andererseits die Verteilung des Schadenaufwands je Ereignis bekannt sind. Zur Abschätzung des Überschwemmungsrisikos wird auf modifizierte Parameter einer hydrologischen Studie zurückgegriffen, die das Schadenpotenzial unter Berücksichtigung der unternehmensindividuellen Bestandsstruktur anhand unterschiedlicher Überschwemmungsszenarien und deren Wiederkehrperioden abschätzt. ${ }^{696}$ Der simulierte Gesamtschaden aus Naturgefahrereignissen kann beispielsweise anhand von Erfahrungswerten prozentual auf die betroffenen Segmente verteilt werden. Durch diese heuristische Vorgehensweise wird sichergestellt, dass ein Elementarereignis gleichzeitig, d.h. modelltechnisch innerhalb desselben Simulationslaufs, auf mehrere Teilkollektive wirkt. Hinsichtlich des Aufwandes aus Ereignisschäden entstehen somit „natürliche“ Abhängigkeitsstrukturen zwischen den betroffenen Sparte-Geschäftsfeld-

\footnotetext{
${ }^{693}$ Vgl. AON RÜCK (2004), S. 12.

${ }^{694}$ Vgl. exemplarisch JAHN (2001), S. 393.

695 PFEIFER (2000), S. 772.

${ }^{696} \mathrm{Vgl}$. hierzu die Ausführungen in Kapitel 5.4.3.4.4 und dort insbesondere Tabelle 5.9.
} 
Kombinationen. ${ }^{697}$ Es sei an dieser Stelle daran erinnert, dass der Schadenaufwand aus Basis- und Großschäden hingegen zunächst zwischen den einzelnen Risikokollektiven als unabhängig unterstellt wird. ${ }^{698}$ Die spezifischen Aufteilungsverhältnisse der Naturereignisschäden auf die einzelnen Sparte-Geschäftsfeld-Kombinationen können aus Tabelle 5.6 abgelesen werden.

\begin{tabular}{|l|r|r|r|}
\hline Sturm & PRI & GEW & IND \\
\hline Kasko & 0 & 0 & 0 \\
\hline AST & 0 & 0,23 & 0,02 \\
\hline VGV ST & 0,75 & 0 & 0 \\
\hline VGV EL & 0 & 0 & 0 \\
\hline
\end{tabular}

\begin{tabular}{|l|r|r|r|}
\hline Hagel & PRI & GEW & IND \\
\hline Kasko & 0,8 & 0,17 & 0,03 \\
\hline AST & 0 & 0 & 0 \\
\hline VGV ST & 0 & 0 & 0 \\
\hline VGV EL & 0 & 0 & 0 \\
\hline
\end{tabular}

\begin{tabular}{|l|r|r|r|}
\hline Überschw. & PRI & GEW & IND \\
\hline Kasko & 0,15 & 0,04 & 0,02 \\
\hline AST & 0 & 0,22 & 0,02 \\
\hline VGV ST & 0 & 0 & 0 \\
\hline VGV EL & 0,55 & 0 & 0 \\
\hline
\end{tabular}

Tabelle 5.6: Verteilung der Naturgefahrschäden auf Versicherungszweige und Geschäftsfelder

Exemplarisch entfallen demnach von dem Schadenaufwand eines simulierten Sturmereignisses $75 \%$ auf das Modellsegment „VGV Sturm Privatkunden“. Die aus Tabelle 5.6 ersichtlichen Aufteilungsquoten wurden auf der Grundlage der oben genannten Beobachtungshistorie als volumengewichteter Durchschnittswert ermittelt.

\subsection{Sturmschäden}

Sturmereignisse stellen in Deutschland die beherrschende Naturgefahr dar. Im Zeitraum von 1978 bis 1998 entfielen $65 \%$ aller Naturereignisse, $75 \%$ der volkswirtschaftlichen und $86 \%$ der versicherten Schäden auf Stürme. ${ }^{699}$ Allein $64 \%$ der versicherten Schäden wurden durch so genannte Winterstürme verursacht. Diese zeichnen sich hinsichtlich ihres Schadenprofils durch eine Vielzahl kleiner Schäden und wenige Großschäden aus. Für das Modellunternehmen liegen für den Beobachtungszeitraum von 1972 bis 2003 Informationen über den Gesamtaufwand pro Sturmereignis sowie die Anzahl der jeweils betroffenen Policen vor. Darüber hinaus ist in den sturmexponierten Sparten „Allgemeine Sturm“ und „VGV Sturm“ für jedes Beobachtungsjahr die Gesamtzahl der abgeschlossenen Policen bekannt. ${ }^{700}$

Es wird davon ausgegangen, dass die jährliche Anzahl der Sturmereignisse einer Poissonverteilung genügt. Der Verteilungsparameter $\theta$ wird auf der Basis des historischen Datensets ermittelt und beträgt $\theta=0,75$. Wie bereits erläutert, kann die mathematischstatistische Methode nur zu sinnvollen Ergebnissen führen, sofern die zur Schätzung der Schadenhöhe verwendeten Daten repräsentativ für das heutige Portfolio sind. ${ }^{701}$

\footnotetext{
697 Vgl. auch FARNY (2000), S. 86 und SCHMEISER (1999), S. 91.

${ }^{698}$ Diese zentrale Annahme wird erst in Kapitel 6.2.2 schrittweise aufgehoben.

699 Vgl. im Folgenden MÜNCHENER RÜCK (1999), S. 7 und BOETIUS (1996), S. 274-278.

700 Vereinfachend wird angenommen, dass Kaskoverträge durch Sturmereignisse nicht betroffen sind.

701 Vgl. im Folgenden auch AON RÜCK (2004), S. 15.
} 
Diese Forderung impliziert, dass die empirischen Schadendaten mittels eines geeigneten Indexes auf das gegenwärtige Preisniveau inflationiert werden und außerdem die historische Bestandsstruktur im Rahmen der Schätzung berücksichtigt wird. Der hier verwendete Modellierungsansatz zieht daher zur Abschätzung der Schadenhöhenverteilung die Größen „Durchschnittsschaden“ und „Betroffenheitsgrad“ heran, die für jedes beobachtete Sturmereignis ermittelt werden. ${ }^{702}$ Der Durchschnittsschaden ist definiert als Quotient aus dem indizierten Gesamtschadenaufwand je Ereignis und der Anzahl der von diesem Ereignis betroffenen Policen. Zum Zwecke des Inflationsausgleichs wird der Baukostenindex herangezogen. ${ }^{703}$ Der Betroffenheitsgrad stellt das Verhältnis der von einem Sturmereignis betroffenen Policen zur Gesamtzahl der im jeweiligen Anfalljahr abgeschlossenen Policen dar. Sowohl an den Durchschnittsschaden als auch an den Betroffenheitsgrad werden mit Hilfe der Maximum-LikelihoodMethode Verteilungen angepasst. Abbildung 5.10 zeigt beispielhaft die empirische Verteilung des Durchschnittsschadens und die angepasste Verteilungsfunktion, die einer Lognormalverteilung genügt.

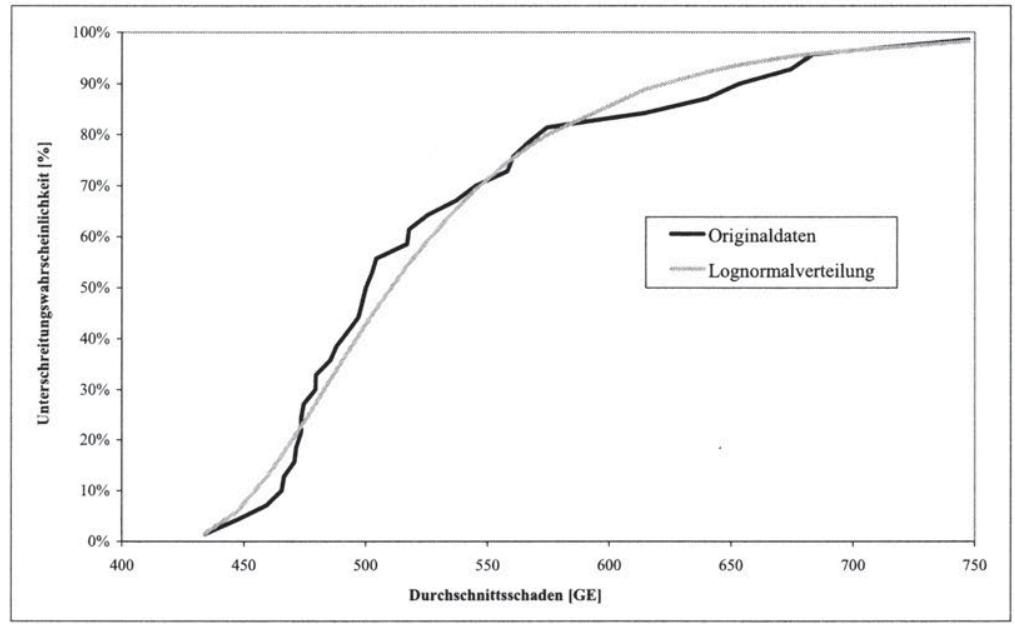

Abbildung 5.10: Verteilungsanpassung des Durchschnittsschadens (Sturm)

702 Im Hinblick auf die Anwendung des kollektiven Modells müssen die Parameter „Betroffenheitsgrad" und „Durchschnittsschaden" streng genommen unabhängig und identisch verteilt sein. Vgl. hierzu vertiefend auch Kapitel 5.4.3.1. Ob die ,i.i.d.-Bedingung“ im Fall von Sturmschäden erfüllt ist, kann zumindest in Frage gestellt werden. Allerdings eröffnet diese Vorgehensweise die Möglichkeit, die Bestandsgröße und Bestandsstruktur des zugrunde liegenden Portfolios explizit in die Modellierung einfließen zu lassen.

${ }^{703}$ Alternativ kann auch der gleitende Neuwertindex verwendet werden. Vgl. PRÖHLSS (1988), S. 215-216. 
In analoger Vorgehensweise kann an die empirische Verteilung des Betroffenheitsgrads eine Verteilungsfunktion angepasst werden. Diese genügt ebenfalls einer Lognormalverteilung. Der Gesamtaufwand je Sturmereignis ergibt sich schließlich als Produkt aus Durchschnittsschaden, Betroffenheitsgrad ${ }^{704}$ und Anzahl der Policen in den sturmexponierten Sparten „Allgemeine Sturm“ und „VGV Sturm“ im simulierten Anfalljahr. Während die beiden erstgenannten Größen stochastisch modelliert werden, stellt die Policenanzahl einen deterministischen Bestandsparameter dar. In Abbildung 5.11 wird der beobachtete Schadenaufwand aus Sturmereignissen dem simulierten Aufwand je Sturmereignis gegenübergestellt. Ein Vergleich der beiden Linienzüge erlaubt erste Aussagen darüber, wie gut der gewählte Modellierungsansatz den empirischen Schadenaufwand je Sturmereignis approximiert.

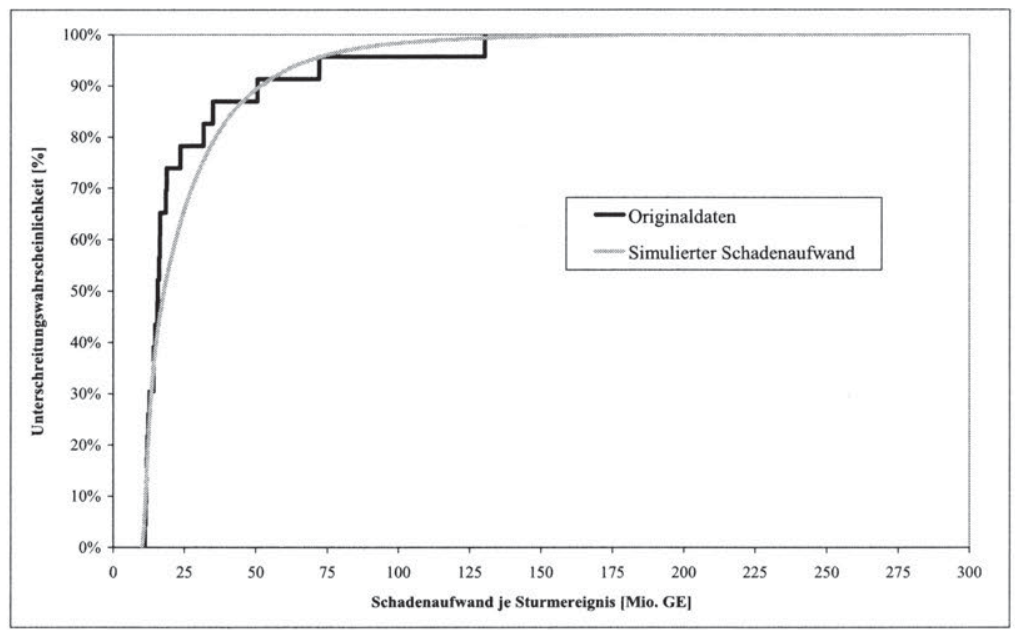

Abbildung 5.11: Verteilung des Schadenaufwands je Sturmereignis

Im Bereich der Schadenhöhen bis ca. 20 Mio. GE ist die Anpassungsgüte erwartungsgemäß hoch, da in diesem Bereich die meisten bekannten Schäden liegen. Im Intervall zwischen 20 und 45 Mio. GE wird die beobachtete Schadenentwicklung überschätzt, während sie im Bereich zwischen 45 und 135 Mio. GE tendenziell unterschätzt wird. Bei der Interpretation ist jedoch zu berücksichtigen, dass das empirische Datenset lediglich 3 Sturmereignisse enthält, deren indizierter Schadenaufwand größer als 45 Mio. GE ist. Daher sollten die Ergebnisse der mathematisch-statistischen Methoden

${ }^{704}$ Hinsichtlich des Betroffenheitsgrads wird vorausgesetzt, dass bei Eintritt eines Sturmereignisses mindestens $2 \%$ der abgeschlossenen Policen in „Allgemeine Sturm“ und „VGV Sturm“ betroffen sind (untere Kappungsgrenze). Als obere Kappungsgrenze wurde ein Anteil von $40 \%$ der abgeschlossenen Policen festgelegt. 
immer auch durch physikalisch-technische Modellergebnisse und Expertenmeinungen plausibilisiert werden. Im Tailbereich, d.h. für lange Wiederkehrperioden, stellt sich darüber hinaus die grundsätzliche Frage, ob überhaupt in Wertebereiche extrapoliert werden darf, für die nur wenige oder gar keine historischen Daten bekannt sind. In der Extremwerttheorie ${ }^{705}$ wird diese Frage unter Bezugnahme auf den Grenzwertsatz von PICKANDS bejaht, sofern die beobachteten Größen stationär sind, d.h. keinen zeitlichen Trends unterliegen und eine „gutartige“ Verteilung besitzen. ${ }^{706}$ Hinsichtlich des Tailverhaltens ist darüber hinaus sicherzustellen, dass die gewählte Verteilungsfunktion auch im Bereich großer Wiederkehrperioden keine Werte „produziert", die unter Berücksichtigung der aktuellen Portfoliostruktur abwegig erscheinen. Der Schadenaufwand je Einzelereignis wird vor diesem Hintergrund bei 200 Mio. GE gekappt. ${ }^{707}$ Das simulierte Schadenpotenzial eines einzelnen Sturmereignisses kann Tabelle 5.7 entnommen werden.

\begin{tabular}{|c|c|c|}
\hline Wiederkehrperiode & Perzentil & Schadenaufwand \\
\hline 10 Jahre & 0,90 & 51,8 \\
\hline 20 Jahre & 0,95 & 68,8 \\
\hline 50 Jahre & 0,98 & 94,5 \\
\hline 100 Jahre & 0,99 & 118,1 \\
\hline 200 Jahre & 0,995 & 139,8 \\
\hline 500 Jahre & 0,998 & 164,8 \\
\hline 1.000 Jahre & 0,999 & 180,4 \\
\hline
\end{tabular}

Tabelle 5.7: Schadenpotenzial eines einzelnen Sturmereignisses [Mio. GE]

\subsection{Hagelschäden}

Starke Hagelunwetter werden vielfach durch großräumige Kaltfronten ausgelöst und treten im Gefolge intensiver Gewittersysteme auf. ${ }^{708}$ Hinsichtlich ihres Schadenpotenzials sind Hagelunwetter gekennzeichnet durch geringe lokale Eintrittswahrscheinlichkeiten bei gleichzeitig sehr hoher Schadendichte. Aufgrund des Hageleinschlusses im Massengeschäft vieler Erstversicherer können einzelne Hagelschläge mit geringer räumlicher Ausdehnung bereits hohe Schäden im Sach-, Kraftfahrt- und Landwirtschaftsgeschäft verursachen. ${ }^{709}$ Im Rahmen dieser Arbeit wird vereinfachend davon ausgegangen, dass ausschließlich die Sparte „Kasko“ von Hagelschäden betroffen ist.

\footnotetext{
705 Vgl. zur Extremwerttheorie EMBRECHTS/KLÜPPELBERG/MIKOSCH (2003).

706 Vgl. PICKANDS (1975), S. 119-131. Eine weit verbreitete Methode zur Tailapproximation ist die Peaks-Over-Threshold-Methode. Vgl. hierzu vertiefend MCNEIL/SALADIN (1997) und MCNEIL/ SALADIN (2000).

707 Die Kappung wird im Simulationsmodell dadurch sichergestellt, dass Realisationen größer 200 Mio. GE sofort verworfen und erneut simuliert werden (sog. „Accept-Bedingung“).

708 Vgl. im Folgenden MüNCHENER RÜCK (1999), S. 21.

709 Der bislang schwerwiegendste deutsche Hagelsturm verursachte am 12.07.1984 in München versicherte Schäden in Höhe von ca. 0,7 Mrd. €. Vgl. MüNCHENER RüCK (1999), S. 58.
} 
Zur Abschätzung des Kumulrisikos aus Hagelschäden wird daher ausschließlich auf die Schadenerfahrung in der Sparte „Kasko“ sowie die zugehörigen Bestandsdaten zurückgegriffen. ${ }^{710}$

Die Modellierung erfolgt analog zur Vorgehensweise bei Sturmereignissen. Die durchschnittliche jährliche Anzahl von Hagelereignissen wurde auf der Basis empirischer Schadendaten ermittelt und beträgt $\theta=6,5$. Die Modellierung der Ereignisanzahl erfolgt erneut über die Poissonverteilung. Sowohl der erwartete Durchschnittsschaden als auch der Betroffenheitsgrad werden aus der Schaden- und Bestandshistorie für jedes Ereignis ermittelt. Anschließend wird mit Hilfe der Maximum-LikelihoodMethode eine Verteilung an die historischen Daten angepasst. Als Ergebnis der Verteilungsanpassung wird der Durchschnittsschaden durch die Inverse Burr-Verteilung, der Betroffenheitsgrad durch die verallgemeinerte Pareto A Verteilung approximiert. ${ }^{711}$ Die Kappungsgrenze für die Schadenhöhe eines einzelnen Hagelereignisses wird auf 100 Mio. GE festgelegt. Für Wiederkehrperioden zwischen 10 und 1.000 Jahren ergeben sich folgende Schadenpotenziale eines einzelnen Hagelereignisses.

\begin{tabular}{|c|c|c|}
\hline Wiederkehrperiode & Perzentil & Schadenaufwand \\
\hline 10 Jahre & 0,90 & 1,0 \\
\hline 20 Jahre & 0,95 & 1,7 \\
\hline 50 Jahre & 0,98 & 3,2 \\
\hline 100 Jahre & 0,99 & 4,9 \\
\hline 200 Jahre & 0,995 & 8,0 \\
\hline 500 Jahre & 0,998 & 13,7 \\
\hline 1.000 Jahre & 0,999 & 22,5 \\
\hline
\end{tabular}

Tabelle 5.8: Schadenpotenzial eines einzelnen Hagelereignisses [Mio. GE]

Das eigentliche Gefährdungspotenzial wird jedoch erst bei Betrachtung der Verteilungsfunktion deutlich, welche in Abbildung 5.12 für den Wertebereich oberhalb des 90\%-Perzentils dargestellt ist. Im Tail der Verteilung kann der Schadenaufwand eines einzelnen Hagelereignisses durchaus die Größenordnung von 60 Mio. GE erreichen. Das ausgeprägte Schadenpotenzial im Randbereich der Verteilung ist auch darauf zurückzuführen, dass die Anzahl der erwarteten Hagelereignisse mit $\theta=6,5$ deutlich höher ausfällt als beispielsweise die erwartete Anzahl von Sturmereignissen. Die verwendete Poissonverteilung weist demzufolge für Hagelereignisse eine erheblich stärkere Streuung auf. ${ }^{712}$

\footnotetext{
${ }^{710}$ In der Realität ist hingegen eine breite Schadenstreuung über sämtliche Sachversicherungssparten festzustellen. Vgl. MÜNCHENER RÜCK (1999), S. 21.

711 Die betreffenden Verteilungs- und Dichtefunktionen sind dem Anhang $\mathrm{C}$ zu entnehmen.

712 Vgl. in diesem Kontext auch die Ausführungen in Kapitel 5.4.3.3.2.
} 


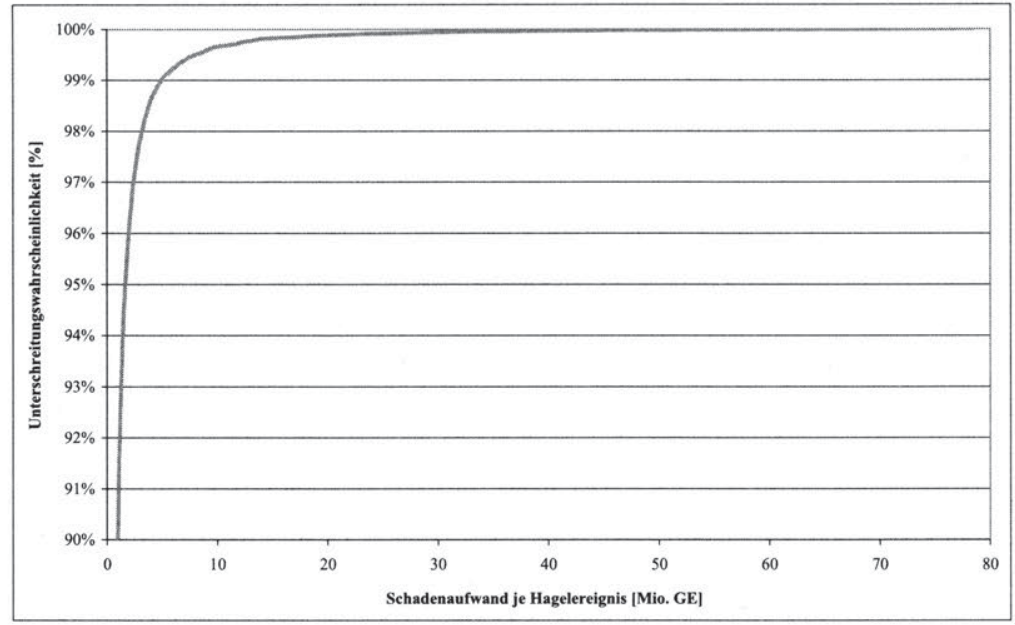

Abbildung 5.12: Verteilung des Schadenaufwands je Hagelereignis

\subsubsection{4 Überschwemmungsschäden}

Im Rahmen dieser Arbeit werden ausschließlich so genannte Flussüberschwemmungsund Sturzflutrisiken betrachtet. Diese werden durch lang anhaltende Niederschläge bzw. lokale Starkregenereignisse verursacht. ${ }^{713}$ Die Deckung von Überschwemmungsrisiken erfolgt in der Regel über die erweiterte Elementarschadendeckung, welche als Zusatzdeckung zur Verbundenen Gebäude- und Hausratversicherung angeboten wird. ${ }^{714}$ Es wird im weiteren Verlauf der Ausführungen davon ausgegangen, dass neben dem Versicherungszweig „VGV Elementar“ auch die Sparten „Kasko“ und „Allgemeine Sturm" von Überschwemmungsschäden betroffen sind. Die Modellierung von Überschwemmungsschäden ist von der generellen Problematik geprägt, dass für den deutschen Versicherungsmarkt keine flächendeckenden Schadenstatistiken vorliegen. ${ }^{715}$ Die Schadenerfahrung beschränkt sich lediglich auf die Analyse punktueller Hochwasserereignisse. Vor diesem Hintergrund wurde auf Initiative des Gesamtverbandes der deutschen Versicherungswirtschaft e.V. das Zonierungssystem für Überschwemmung, Rückstau und Starkregen (ZÜRS) entwickelt. ${ }^{716}$ ZÜRS ist ein flächendeckendes Geoinformationssystem, welches die Klassifizierung einzelner Risiken nach ihrem individuellen Gefährdungsgrad ermöglicht. Die Grundlage des Systems stellt ein

713 Vgl. zur Definition sowie zur zeitlichen und geographischen Abgrenzung eines Flutereignisses MÜNCHENER RÜCK (2004), S. 28-29.

${ }^{714}$ Im Jahr 2002 waren lediglich 3-4\% aller Wohngebäude in Deutschland gegen Elementargefahren versichert. Vgl. SiMON/GROLLMANN (2002), S. 689.

715 Vgl. POHLHaUSEN (1999), S. 461.

716 Vgl. im Folgenden POHLHAUSEN (1999), S. 462-466 und SiMON (2004), S. 1356-1357. 
digitales Landschaftsmodell dar, welches sowohl ein Geländehöhen- als auch ein Flussnetzmodell umfasst und die Simulation von Hochwasserereignissen erlaubt. Die Einzelrisiken eines Versicherungsunternehmens werden in vier Gefährdungsklassen eingeteilt. ${ }^{717}$ Durch Verschneidung der simulierten Überschwemmungsflächen mit den Bestandsdaten eines Versicherers können die Einzelrisiken den Gefährdungsklassen zugeordnet und ihr Gefährdungspotenzial quantifiziert werden. ${ }^{718}$

In Anlehnung an das skizzierte Zonierungsmodell wird das Geschäftsgebiet des Modellunternehmens in vier Gefährdungsklassen unterteilt, denen in grober Näherung jeweils ihre Wiederkehrperiode sowie der erwartete Durchschnittsschaden und Betroffenheitsgrad zugeordnet werden können. Die Wiederkehrperioden der gewählten Gefährdungsklassen sind nicht mit den in ZÜRS zugrunde gelegten Wiederkehrperioden identisch. Um diesen Unterschied zu verdeutlichen, werden die Gefährdungsklassen mit A, B, C und D bezeichnet. Tabelle 5.9 stellt die zugrunde liegende Parameterkonstellation im Überblick dar.

\begin{tabular}{|c|c|c|c|}
\hline Gefahrdungsklasse & Wiederkehrperiode & Durchschnittsschaden & Betroffenheitsgrad \\
\hline A & 50 Jahre & $5.000 \mathrm{GE}$ & $0,22 \%$ \\
\hline B & 100 Jahre & $7.000 \mathrm{GE}$ & $0,43 \%$ \\
\hline C & 200 Jahre & $15.000 \mathrm{GE}$ & $0,65 \%$ \\
\hline D & 500 Jahre & $35.000 \mathrm{GE}$ & $1,30 \%$ \\
\hline
\end{tabular}

Tabelle 5.9: Überschwemmungsszenarien

Mit diesen Informationen sind 4 Punkte der Verteilungsfunktion für Überschwemmungsschäden definiert. Die Gefährdungspotenziale zwischen diesen Punkten werden unter der Annahme einer Gleichverteilung interpoliert. Da die Punkte nicht als deterministisch angenommen werden können, wird vereinfachend unterstellt, dass sowohl der Durchschnittsschaden als auch der Betroffenheitsgrad einer Normalverteilung genügen und mit einer Standardabweichung von $20 \%$ um ihren Erwartungswert (siehe Tabelle 5.9) schwanken. Die aus Abbildung 5.13 ersichtliche Verteilungsfunktion vermittelt einen Eindruck desjenigen Schadenpotenzials, welches im simulierten Anfalljahr aus Überschwemmungsschäden resultiert. Die Kappungsgrenze für den Anfall-

717 Als Reaktion auf das Elbe-Hochwasser im August 2002 wurde die bislang vorherrschende Einteilung in drei Gefährdungsklassen (GK) im Jahr 2004 um eine zusätzliche Klasse erweitert. Die neue GK 2 wird auf der Grundlage eines Hochwasserereignisses mit einer Wiederkehrperiode zwischen 50 und 200 Jahren berechnet. Diese Klasse bildet nun auch das Risikopotenzial von Versicherungspolicen ab, die durch einen Deichbruch gefährdet sind. Vgl. auch SiMON/GrolLMANN (2002), S. 682-689.

718 Die Zuordnung der Einzelrisiken erfolgt mit Hilfe des so genannten Kreisgemeindeschlüssels KGS16, welcher unterhalb der Postleitzahlenebene angesiedelt ist und auf einzelne Straßenzige innerhalb eines Stadtteils abstellt. Vgl. POHLHAUSEN (1999), S. 464 und MÜNCHENER RÜCK (2004), S. 26-31. 
jahresschaden wurde auf 200 Mio. GE festgelegt. Dargestellt ist lediglich der Wertebereich oberhalb des $98 \%$-Perzentils.

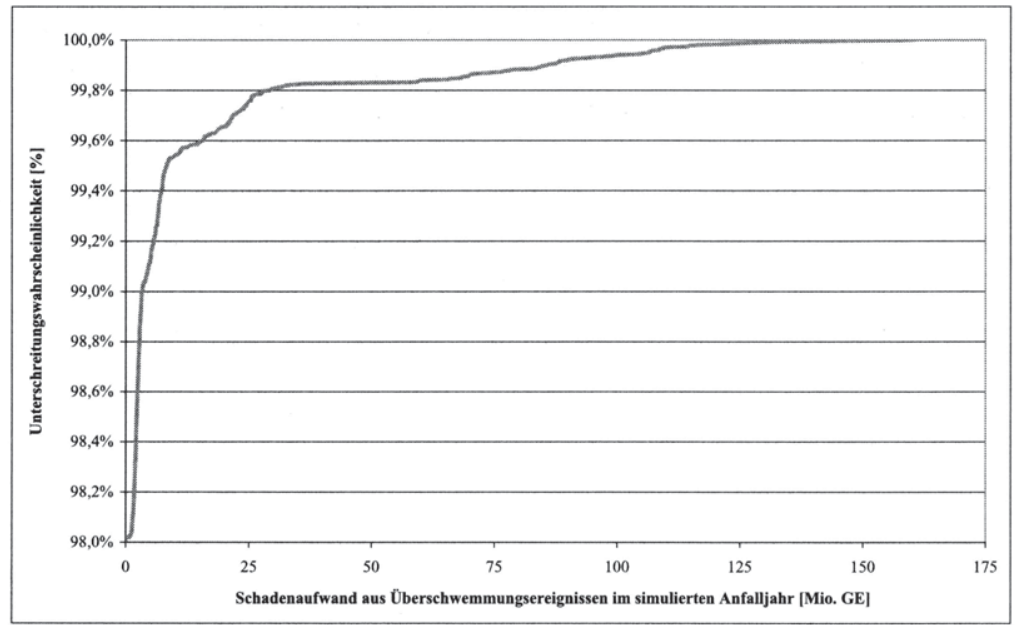

Abbildung 5.13: Verteilung des Schadenaufwands aus Überschwemmungsereignissen

Angesichts der geringen lokalen Eintrittswahrscheinlichkeit von Überschwemmungsschäden manifestiert sich ihr beträchtliches Gefährdungspotenzial lediglich im Tail der Verteilung. In analoger Vorgehensweise zu Sturm- und Hagelschäden wird der ermittelte Jahresschaden aus Überschwemmungen anhand der aus Tabelle 5.6 bekannten Aufteilungsverhältnisse prozentual auf die betroffenen Sparte-Geschäftsfeld-Kombinationen verteilt.

Die Darstellung der Schadenmodellierung ist hiermit abgeschlossen. Der hohen Bedeutung des stochastischen Schadeneintritts Rechnung tragend, wurde in den vorangegangenen Abschnitten die Modellierung der unterschiedlichen Komponenten der Gesamtschadenlast sukzessive erläutert. Der Gesamtschaden eines Risikokollektivs setzt sich grundsätzlich additiv aus Basisschäden, Großschäden und gegebenenfalls Schäden aus Naturgefahrereignissen zusammen. Während die Schadensumme aus Basisund Großschäden zwischen den modellierten Sparte-Geschäftsfeld-Kombinationen zunächst als unabhängig abgenommen wird, entstehen durch die simultane Wirkung von Naturgefahrschäden auf mehrere Risikokollektive bereits „natürliche“ Abhängigkeitsstrukturen. 


\subsubsection{Kostenmodell}

Neben den in Kapitel 5.4.3 ausführlich beschriebenen und ex-ante unbestimmten Schadenkosten stellen die betrieblichen Kosten zur Erbringung der Versicherungsleistung eine wesentliche Ergebnisgröße dar. Im versicherungsspezifischen Kontext lassen sich folgende Kostenarten unterscheiden: ${ }^{719}$

- Provisionen

- Verwaltungskosten

- Schadenregulierungskosten

Die Provisionen umfassen im Verständnis dieser Arbeit sämtliche an die Absatzorgane zu zahlenden Vergütungen. ${ }^{720}$ Obwohl sich die Ermittlung der Provisionshöhe in der Praxis an unterschiedlichen Bezugsgrößen, wie beispielsweise Zeichnungs- oder Bestandsvolumen festmacht, können die Provisionen als weitgehend beitragsproportional aufgefasst werden. Die Modellierung erfolgt daher über Provisionsquoten, d.h. in Relation zum Bruttoprämienvolumen des jeweiligen Teilbestands im simulierten Anfalljahr. Die Provisionsquoten werden sowohl sparten- als auch geschäftsfeldspezifisch ermittelt und deterministisch im Modell abgebildet.

Die Verwaltungskosten eines Versicherungsunternehmens setzen sich in der Regel aus fixen und variablen Bestandteilen zusammen. ${ }^{721}$ Als Fixkosten können insbesondere die Kosten für die Bereitstellung der notwendigen Infrastruktur (z.B. Bestandsführungssysteme) und die Kosten der Zentralbereiche des Unternehmens klassifiziert werden. Demgegenüber haben beispielsweise die Kosten der Rechnungserstellung variablen Charakter und könnten demnach in Abhängigkeit von der Portfoliogröße (gemessen in Policen bzw. Jahreseinheiten) modelliert werden. Aus Vereinfachungsgründen erfolgt die Implementierung der Verwaltungskosten im vorliegenden Modell proportional zum Bruttoprämienvolumen eines jeden Modellsegments. Die Ermittlung der Verwaltungskostensätze kann in einem ersten Schritt in Anlehnung an bilanzielle Kostenquoten erfolgen. ${ }^{722}$

719 Die Summe aus Provisionen und Verwaltungskosten ist in der externen Rechnungslegung den „Aufwendungen für den Versicherungsbetrieb“ (GuV-Position I. 7a gemäß Formblatt 2 RechVersV) vergleichbar. Die Schadenregulierungskosten werden hingegen bilanziell als „Aufwendungen für Versicherungsfälle“ ausgewiesen.

${ }^{720}$ In diesem Zusammenhang sind insbesondere Abschluss-, Verlängerungs- und Folgeprovisionen zu nennen. Vgl. im Folgenden zu den Elementen eines Vergütungssystems für Absatzorgane auch FARNY (2000), S. 712-720.

721 Vgl. im Folgenden FARNY (2000), S. 573-574.

722 Vgl. übereinstimmend BACH (2003), S. 644. 
Unter der Zielsetzung einer beanspruchungsgerechteren Kostenbelastung der modellierten Teilkollektive erscheint es jedoch empfehlenswert, beispielsweise auf das Instrument der Prozesskostenrechnung zurückzugreifen. ${ }^{723}$

Die Schadenregulierung umfasst alle Leistungsprozesse nach dem Eingang von Schadenmeldungen und konkretisiert somit das abstrakte Schutzversprechen des Versicherungsunternehmens. ${ }^{724}$ Schadenregulierungskosten können für externe Dienstleister (z.B. Sachverständige oder Rechtsanwälte) oder für unternehmensinterne Leistungsprozesse (z.B. Prüfung des Leistungsanspruchs oder Schadenbewertung) anfallen. Im Zuge der Modellierung gilt es zu beachten, dass die externen Schadenregulierungskosten für das Versicherungsunternehmen nicht beeinflussbare Schadenaufwendungen darstellen. Sie werden daher konsequenterweise im Rahmen des Schadenaufwands berücksichtigt. Hinsichtlich der internen Schadenregulierungskosten stellt sich die Frage, anhand welcher Bezugsgröße bzw. welches Kostentreibers diese angemessen im Modell berücksichtigt werden können. Eine mögliche Herangehensweise besteht darin, die Entwicklung der internen Schadenregulierungskosten proportional an den erwarteten Schadenaufwand zu koppeln. ${ }^{725}$ Diese Vorgehensweise bewirkt, dass die Schadenregulierungskosten aufgrund der Stochastizität des Schadengeschehens stark schwanken und in Sparten mit hoher Großschadenbelastung tendenziell überschätzt werden. Vielmehr ist in der Realität jedoch zu vermuten, dass die internen Schadenregulierungskosten trotz volatilem Schadenverlauf nur in geringem Ausmaß schwanken. Vor diesem Hintergrund erfolgt die Berücksichtigung im vorliegenden Modellansatz ebenfalls in Relation zum Bruttoprämienvolumen des betrachteten Segmentes.

Die für das Modellunternehmen unterstellten Kostenquoten können Tabelle 5.10 entnommen werden. Die Verwaltungskosten und internen Schadenregulierungskosten werden aus Vereinfachungsgründen unter dem Obergriff „Betriebskosten“ zusammengefasst, gehen jedoch separat in die Modellierung ein.

${ }^{723}$ Zur spezifischen Ausgestaltung der Prozesskostenrechnung in Versicherungsunternehmen vgl. WIESEHAHN (2001), S. 397-404.

${ }^{724}$ Vgl. im Folgenden auch FARNY (2000), S. 623.

${ }^{725}$ Hiervon abweichend schlägt DOTTERWEICH vor, die Schadenregulierungskosten an die Anzahl der betroffenen Policen zu koppeln. Unter der Annahme eines mittleren (und von der Schadenhöhe unabhängigen) Bearbeitungsaufwands pro Schadenfall können die Schadenregulierungskosten durch Multiplikation mit der erwarteten Anzahl betroffener Policen ermittelt werden. Vgl. DOTTERWEICH (2004), S. 206. 


\begin{tabular}{|c|c|c|c|c|}
\hline & & Privatkunden & Gewerbekunden & Industriekunden \\
\hline \multirow{3}{*}{$\begin{array}{c}\text { Allgemeine } \\
\text { Unfall }\end{array}$} & Provisionen & 18,9 & 17,8 & 17,6 \\
\hline & Betriebskosten & 21,2 & 13,8 & 8,1 \\
\hline & Gesamtkosten & 40,1 & 31,6 & 25,7 \\
\hline \multirow{3}{*}{$\begin{array}{l}\text { Kraftfahrt- } \\
\text { Haftpflicht }\end{array}$} & Provisionen & 5,5 & 5,3 & 5,2 \\
\hline & Betriebskosten & 7,6 & 9,1 & 4,1 \\
\hline & Gesamtkosten & 13,1 & 14,4 & 9,3 \\
\hline \multirow{3}{*}{ Kasko } & Provisionen & 10,4 & 9,9 & 9,8 \\
\hline & Betriebskosten & 13,2 & 12,2 & 8,8 \\
\hline & Gesamtkosten & 23,6 & 22,1 & 18,6 \\
\hline \multirow{3}{*}{$\begin{array}{c}\text { Feuer } \\
\text { Industrie }\end{array}$} & Provisionen & - & - & 12,4 \\
\hline & Betriebskosten & - & - & 13,1 \\
\hline & Gesamtkosten & - & - & 25,5 \\
\hline \multirow{3}{*}{$\begin{array}{l}\text { Allgemeine } \\
\text { Sturm }\end{array}$} & Provisionen & - & 12,4 & 12,0 \\
\hline & \begin{tabular}{|l} 
Betriebskosten \\
\end{tabular} & - & 21,9 & 22,0 \\
\hline & Gesamtkosten & - & 34,3 & 34,0 \\
\hline \multirow{3}{*}{$\begin{array}{l}\text { Verbundene } \\
\text { Wohngebăude }\end{array}$} & Provisionen & 12,0 & - & - \\
\hline & Betriebskosten & 13,3 & - & - \\
\hline & Gesamtkosten & 25,3 & - & - \\
\hline
\end{tabular}

Tabelle 5.10: Kostenquoten [\%]

\subsubsection{Rückversicherungsmodell}

\subsubsection{Vorbemerkungen}

Unter Rückversicherung soll im Verständnis dieser Arbeit die „Überwälzung eines Teils der von einem Erstversicherer gegenüber Versicherungsnehmern aufgrund von Versicherungsverträgen [...] übernommenen Gefahren oder Risiken“"726 verstanden werden. ${ }^{727}$ Im Sinne dieser Definition wird im Folgenden von modernen Formen der Rückversicherung, wie beispielsweise Alternativem Risikotransfer (ART) oder der Finanzrückversicherung abstrahiert. ${ }^{728}$ Wie bereits in Kapitel 5.3.1 diskutiert, ist die versicherungstechnische Bruttorisikoposition im Modellverlauf zunächst durch den Spartenmix und das Prämienniveau exogen vorgegeben. Die Rückversicherung stellt daher das einzige Instrument dar, eine angestrebte versicherungstechnische Nettorisikoposition zu realisieren. Es wird von der Prämisse ausgegangen, dass das Modellunternehmen nicht eigenständig als Rückversicherer am Markt auftritt und demzufolge nur die passive Rückversicherung betreibt. Vor diesem Hintergrund beschränkt sich die Funktion der Rückversicherung im Verlauf der weiteren Ausführungen auf die Teilung und Reduktion des versicherungstechnischen Risikos. Sie konkretisiert sich ins-

${ }^{226}$ GrossmanN (1977), S. 7. Vgl. zum Rückversicherungsbegriff außerdem PFEIFFER (1994), S. 11 und GERATHEWOHL (1976), S. 431.

727 In $\S 779$ Abs. 1 HGB (Seehandelsrecht) ist Rückversicherung als „die Versicherung der von dem Versicherer übernommenen Gefahr“ definiert. MACK versteht unter Rückversicherung die „Möglichkeit, einen Teil der übernommenen ungewissen Schadenkosten [...] durch fixe Kosten zu ersetzen.“MACK (2002), S. 325.

728 Vgl. hierzu ausführlich LIEBWEIN (2000), S. 303-432, SCHRAdIN (1998), S. 322-439, SwISS RE (2003) und ZIETSCH (2005), S. 351-373. 
besondere in dem Schutz vor extremen Schadenbelastungen aufgrund von Groß- und Kumulschäden. ${ }^{729}$ Durch eine zweckmäßige Gestaltung des Rückversicherungsprogramms, welches beispielsweise Einzelrisiken mit starker Exponierung bzw. hohen Versicherungssummen abdeckt, kann die Schaden- und Ergebnisentwicklung auf der Teil- und Gesamtkollektivebene homogenisiert und stabilisiert werden. ${ }^{730}$ Die geringere Volatilität des versicherungstechnischen Nettoergebnisses führt in der Regel zu einer Reduzierung des Risikokapitalsbedarfs bzw. zu einer Ausweitung der Zeichnungskapazität. ${ }^{731}$ Dieser Zusammenhang verdeutlicht die Funktion der Rückversicherung als Risikoträger und Risikokapitalsubstitut. Auf die Vielzahl von begleitenden Dienstleistungen, die Rückversicherer beispielsweise im Bereich des Kapitalanlagemanagements, der Schadenregulierung oder der aktuariellen Beratung anbieten, soll an dieser Stelle nicht eingegangen werden. ${ }^{732}$

Klassifiziert man die verschiedenen Rückversicherungsformen anhand ihrer vertragsrechtlichen Ausgestaltung, so kann zwischen fakultativen und obligatorischen Deckungen unterschieden werden. ${ }^{733}$ In der fakultativen Rückversicherung entscheiden Erst- und Rückversicherer von Fall zu Fall, welche Risiken zu welchen Konditionen rückversichert werden sollen. Für beide Vertragsparteien besteht grundsätzlich keine Verpflichtung zur Zession bzw. Übernahme von Risiken. Fakultative Verträge beziehen sich in der Regel auf Einzelrisiken, für die nach Ausschöpfung des Eigenbehalts entweder die obligatorische Kapazität nicht ausreicht oder die aufgrund ihrer Art und Größe von der obligatorischen Deckung ausgeschlossen sind. ${ }^{734}$ In der obligatorischen Rückversicherung besteht für beide Vertragsparteien Zessions- bzw. Annahmepflicht hinsichtlich aller im Vertrag spezifizierten Risiken. Obligatorische Verträge stellen die in der Praxis vorherrschende Rückversicherungsform dar und werden im Rahmen dieser Arbeit ausschließlich betrachtet.

729 In diesem Zusammenhang gewinnt der „Grundsatz der Schicksalsteilung“ besondere Bedeutung, da eine finanzielle Balance zwischen Erst- und Rückversicherer in einer Rechnungsperiode zumeist nicht erreicht werden kann. Vgl. hierzu vertiefend GERATHEWOHL (1988) und kritisch SCHINZLER (1988), S. $74 \mathrm{ff}$.

${ }^{730}$ Vgl. LiEBWEIN (2000), S. 43-44. Vgl. zu den Zielen der Rückversicherungsnahme auch SCHENK (1995).

731 Vgl. Mentzel (2004), S. 75-88, MaCK (2002), S. 325, Swiss Re (2002a), S. 12 und Straub (1988), S. 7.

${ }^{732}$ Vgl. hierzu Straub (1988), S. 10 und Liebwein (2000), S. 47-49.

733 Vgl. im Folgenden auch LIEBWEIN (2000), S. 51-57 und PFEIFFER (1994), S. 24-30.

734 Vgl. PfEIFFER (1994), S. 24-29, SWISS RE (2002a), S. 18 und LiEBWEIN (2000), S. 54. 
Darüber hinaus wird hinsichtlich der Art der Risikoteilung zwischen proportionalen und nicht-proportionalen Vertragsformen differenziert. ${ }^{735} \mathrm{Im}$ Rahmen der proportionalen Rückversicherung ${ }^{736}$ werden sowohl die Originalprämien als auch die auf einen bestimmten Vertrag entfallenden Schäden und Kosten zu einem festen Prozentsatz aufgeteilt. Mit dem Quotenvertrag und dem Summenexzedentenvertrag werden im folgenden Kapitel die Hauptformen proportionaler Verträge vorgestellt. In der nichtproportionalen Rückversicherung existiert keine prozentuale Bindung an das Originalrisiko. Es werden keine anteiligen Risiken zediert, sondern es findet eine Aufteilung der Schadenleistungen statt. Die Haftung des Rückversicherers tritt in Kraft, sobald eine festgelegte maximale Schadenhöhe (Priorität) überschritten wird. Als wichtigste Formen nicht-proportionaler Verträge werden der Schadenexzedentenvertrag und der Jahresüberschadenexzedentenvertrag (Stop Loss) behandelt.

Im weiteren Verlauf der Untersuchung wird hinsichtlich der Qualität des Rückversicherungsversprechens davon ausgegangen, dass sowohl die Bonität als auch die Liquidität der Rückversicherungspartner einwandfrei ist. ${ }^{737}$ Darüber hinaus ist die Rückversicherung nur in Bezug auf das simulierte Anfalljahr disponibel. ${ }^{738}$ Das im folgenden Kapitel zu erläuternde Rückversicherungsprogramm ${ }^{739}$ wurde im Interesse der Übersichtlichkeit einfach gehalten, kann jedoch hinsichtlich seiner Struktur durchaus als marktüblich bezeichnet werden.

Da für das Modellunternehmen und vermutlich auch für einen Großteil des Gesamtmarktes keine validen geschäftsfeldspezifischen Rückversicherungsdaten vorliegen, wird mit der Integration der Rückversicherung ebenfalls eine Eingrenzung auf die versicherungstechnische Spartensicht vorgenommen. Im nachfolgenden Kapitel werden die einzelnen Vertragsformen hinsichtlich ihrer Ausgestaltung und risikotheoretischen Wirkung vorgestellt. In Kapitel 5.4.5.3 wird schließlich ein möglicher Ansatz beschrieben, mit dessen Hilfe die jeweiligen Preiskomponenten der Verträge auf risikotheoretischer Grundlage quantifiziert werden können.

$735 \mathrm{Vgl}$. zur Differenzierung zwischen proportionaler und nicht-proportionaler Rückversicherung vertiefend FARNY (2000), S. 542-547, SWISS RE (1997a) und PFEIFFER (1994), S. 48-80.

736 Die proportionale Rückversicherung wird häufig auch als Summenversicherung bezeichnet, da sie im Gegensatz zur Gefahrenversicherung auf Original-Versicherungssummen bzw. wahrscheinlichen Höchstschäden (PML) basiert. Vgl. LiEBWEIN (2000), S. 61-62.

737 Vgl. zum Ausfallrisiko von Rückversicherungsforderungen auch MENTZEL (2004), S. 130-137.

$738 \mathrm{Vgl}$. zu einer übereinstimmenden Annahme SCHMEISER (2001), S. 144 und S. 154.

739 Eine detaillierte Übersicht des für das Modellunternehmen unterstellten Rückversicherungsprogramms und der zugrunde liegenden Vertragsparameter ist Tabelle $5.13 \mathrm{zu}$ entnehmen. 


\subsubsection{Modellierung einzelner Rückversicherungsverträge}

\subsection{Quotenvertrag}

Unter einem Quotenvertrag partizipiert der Rückversicherer an allen gezeichneten Risiken eines Teilbestands mit einem einheitlichen Prozentsatz $\mathrm{p}$ und erhält dafür einen entsprechenden Anteil der Originalprämie. ${ }^{740}$ Bezeichne die Zufallvariable S den Gesamtschaden eines Teilbestands im simulierten Anfalljahr, so ergibt sich die Schadenbelastung des Erstversicherers $\mathrm{S}_{\mathrm{EV}}$ unter einem Quotenvertrag als:

$$
\mathrm{S}_{\mathrm{EV}}=(1-\mathrm{p}) \cdot \mathrm{S} \quad \text { mit } 0<\mathrm{p}<1
$$

Der relative Schadenverlauf des Erstversicherers, beispielsweise gemessen an der Schadenquote, bleibt demzufolge unverändert. Es ergibt sich lediglich eine Reduzierung der absoluten Schadenbelastung. Der Quotenvertrag bietet somit keinen wirksamen Schutz vor einzelnen Großschäden und ermöglicht keine Homogenisierung des Schadenverlaufs eines Portfolios. Typische Einsatzgebiete sind daher weitgehend homogene Sparten mit schwankenden Grundwahrscheinlichkeiten oder Trends im Kleinund Mittelschadenbereich. ${ }^{741}$ Darüber hinaus werden Quotenverträge häufig zur Finanzierung einzelner Sparten in Aufbauphasen oder zur Erreichung einer ausgewogenen Bestandsstruktur eingesetzt. Sofern Erst- und Rückversicherer eine langjährige und umfangreiche Geschäftsbeziehung unterhalten, werden Quotenverträge bisweilen auch abgeschlossen, um im Interesse der Gesamtbeziehung eine angemessene Risikound Ergebnisteilung herbeizuführen. ${ }^{74}$

In der Regel weisen Erstversicherer aufgrund ihrer Akquisitions- und Bestandsverwaltungskosten ein deutlich höheres Kostenniveau als Rückversicherer auf. Die Kostenstruktur des Erstversicherers findet im Zuge der Tarifkalkulation ihren Niederschlag in den Originalprämien. Da der Rückversicherer im Rahmen des Quotenvertrags proportional an den Originalprämien partizipiert, erstattet er dem Erstversicherer einen Teil der eingerechneten Kosten in Form einer Rückversicherungsprovision. Die Rückversicherungsprovision stellt - abgesehen von ihrer ursprünglichen Funktion als Kostenerstattung - mittlerweile das zentrale Preisregulativ im Rahmen der proportionalen Rückversicherung dar. ${ }^{743}$ Über die Höhe der Provision kann beispielsweise die Qualität des zedierten Geschäfts in die Preisgestaltung einfließen. Sie wird entweder als fes-

\footnotetext{
740 Vgl. im Folgenden LIEBWEIN (2000), S. 62-66 und PFEIFFER (1994), S. 53-55.

741 Als Beispiele können die Sparten Allgemeine Haftpflicht, Kraftfahrt, Hagel, Sturm und Transport angeführt werden. Vgl. LIEBWEIN (2000), S. 62.

742 Vgl. auch GrosSMANN (1977), S. 92 und GeRATHEWOHL (1988).

743 Vgl. LIEBWEIN (2000), S. 86.
} 
ter Prozentsatz der zedierten Prämien oder als vom Schadenverlauf abhängige Staffelprovision vereinbart. ${ }^{744}$

\subsection{Summenexzedentenvertrag}

Im Rahmen eines Summenexzedentenvertrages wird der Rückversicherer nur noch proportional an Risiken beteiligt, deren Versicherungssumme bzw. wahrscheinlicher Höchstschaden (PML) einen zuvor festgelegten Selbstbehalt M (= Maximum) übersteigen. ${ }^{745}$ Im Gegensatz zum Quotenvertrag wird das Aufteilungsverhältnis $\mathrm{p}_{i}$ individuell für jede versicherungstechnische Einheit (= Einzelrisiko) i ermittelt als:

$$
\mathrm{p}_{\mathrm{i}}=\frac{\mathrm{PML}_{\mathrm{i}}-\mathrm{M}}{\mathrm{PML}_{\mathrm{i}}}=1-\frac{\mathrm{M}}{\mathrm{PML}_{\mathrm{i}}} \quad \text { mit } \mathrm{i} \in\{1, \ldots, \mathrm{n}\} \text { und } \mathrm{PML}_{\mathrm{i}}>\mathrm{M}
$$

Dieser Vorgang wird auch als „Maximierung“ bezeichnet. Somit gilt für die Aufteilung des Einzelschadens $S_{i}$ einer versicherungstechnischen Einheit i mit dem wahrscheinlichen Höchstschaden $\mathrm{PML}_{\mathrm{i}}$ im Rahmen eines unlimitierten Summenexzedentenvertrages:

$$
\mathrm{S}_{\mathrm{i}}=\underbrace{\mathrm{p}_{\mathrm{i}} \cdot \mathrm{S}_{\mathrm{i}}}_{\mathrm{S}_{\mathrm{RV}}}+\underbrace{\left(1-\mathrm{p}_{\mathrm{i}}\right) \cdot \mathrm{S}_{\mathrm{i}}}_{\mathrm{S}_{\mathrm{EV}}} \quad \text { mit } \mathrm{p}_{\mathrm{i}}=\max \left[1-\frac{\mathrm{M}}{\mathrm{PML}_{\mathrm{i}}} ; 0\right]
$$

In der Regel wird die Haftung des Rückversicherers unter einem Summenexzedentenvertrag durch einen Höchstbetrag begrenzt, der als Vielfaches u des Maximums $M$ angegeben wird. Die Entschädigungsleistung des Rückversicherers $\mathrm{S}_{\mathrm{RV}}$ für ein Portfolio von $n$ Einzelrisiken ergibt sich dann als

$$
\mathrm{S}_{\mathrm{RV}}=\sum_{\mathrm{i}=1}^{\mathrm{n}} \min \left[\mathrm{p}_{\mathrm{i}} ; \frac{\mathrm{u} \cdot \mathrm{M}}{\mathrm{PML}_{\mathrm{i}}}\right] \cdot \mathrm{S}_{\mathrm{i}}
$$

Diejenigen Schäden, welche die Haftstrecke $\mathrm{u} \cdot \mathrm{M}$ übersteigen, fallen wieder in den Selbstbehalt des Erstversicherers zurück, sofern sie nicht anderweitig, z.B. durch einen zweiten Summenexzedentenvertrag gedeckt sind. Der Summenexzedentenvertrag wird vorwiegend in Versicherungszweigen mit stark voneinander abweichenden Versicherungssummen bzw. PML (z.B. Feuerversicherung) eingesetzt und ermöglicht insge-

\footnotetext{
744 Vgl. StRaub (1988), S. 24. Im Rahmen dieser Arbeit werden nur Festprovisionen betrachtet. Vgl. zur Staffelprovision ausführlich PFEIFFER (1994), S. 57-58 und LIEBWEIN (2000), S. 87-88.

745 Vgl. im Folgenden MACK (2002), S. 325-329, LIEBWEIN (2000), S. 67-73 und PFEIFFER (1994), S. 48-53.
} 
samt eine Homogenisierung des Schadenverlaufs, da der Rückversicherer bei Risiken mit hohen Versicherungssummen bzw. PML überproportional beteiligt wird. Das weitgehend stabil verlaufende Basisgeschäft verbleibt im Selbstbehalt. Der Erstversicherer hat darüber hinaus die Möglichkeit, den Selbstbehalt eines Bestandes nach dem Gefährdungsgrad mittels einer Maximaltabelle zu staffeln und somit zwischen ,guten“ und „schlechten“ Risiken zu differenzieren. ${ }^{746}$ Vergleichbar dem Quotenvertrag wird die Kapazität des Erstversicherers durch den Summenexzedentenvertrag erweitert, insbesondere können vermehrt auch Risiken mit hohen Versicherungssummen bzw. PML gezeichnet werden. Neben dem erheblichen administrativen Aufwand weist der Summenexzedentenvertrag den wesentlichen Nachteil auf, dass er zur Deckung von Kumulschäden und für Sparten mit vielen Kleinschäden ungeeignet ist. Der Summenexzedentenvertrag kommt im vorliegenden Modell in den Sparten „Feuer Industrie“ und „VGV Feuer“ zur Anwendung.

Wie bereits in Kapitel 5.4.3.2.1 diskutiert, wird das versicherungstechnische Portfolio nicht auf der Einzelvertragsebene abgebildet. Um die homogenisierende Wirkung eines Summenexzedentenvertrages dennoch geeignet modellieren zu können, wird der Vertragsbestand der Sparten „Feuer Industrie“ und „VGV Feuer“ in so genannte PMLBänder aufgeteilt. Tabelle 5.11 verdeutlicht diesen Sachverhalt am Beispiel der Sparte „Feuer Industrie“. ${ }^{747}$

\begin{tabular}{|c|c|c|}
\hline Band-Nr. & PML Untergrenze & PML Obergrenze \\
\hline 1 & 0,0 & 1,5 \\
\hline 2 & 1,5 & 7,5 \\
\hline 3 & 7,5 & 13,5 \\
\hline
\end{tabular}

Tabelle 5.11: Bandstruktur der PML-Sparte „Feuer Industrie“ [Mio. GE]

Im Hinblick auf die Modellierung des Rückversicherungsprogramms ist zu beachten, dass bereits in der Bruttomodellierung die Bandstruktur der betreffenden PML-Sparten mit der Haftungsstruktur des Summenexzedentenvertrages (Höhe und Anzahl der Maxima) korrespondiert. Die Bandgrenzen sollten daher so gewählt werden, dass diese jeweils einem Vielfachen des Selbstbehalts (= Maximum) entsprechen. ${ }^{748}$

746 Bei „guten“ Risiken wird der Selbstbehalt relativ hoch ausfallen und vice versa. Es sei betont, dass diese Vorgehensweise eine bewusste Antiselektion zu Lasten Rückversicherers bewirkt. Vgl. vertiefend GrossmanN (1977), S. 98 und LiEBWEIN (2000), S. 67 und S. 70.

747 Im Basisszenario wird unterstellt, dass der Vertragsbestand der Sparte „Feuer Industrie“ keine Policen mit einem wahrscheinlichen Höchstschaden (PML) größer als 13,5 Mio. GE aufweist.

748 Im betrachteten Modellunternehmen beträgt der Selbstbehalt (= Maximum) des Summenexzedentenvertrages in der Sparte „Feuer Industrie“ 1,5 Mio. GE. Als Haftstrecke wurden 9 Maxima $(=13,5$ Mio. GE) festgesetzt. 
Hinsichtlich der Preisgestaltung des Summenexzedentenvertrages stellt die Rückversicherungsprovision - neben der proportionalen Beteiligung an der Originalprämie - eine wesentliche Preiskomponente dar. ${ }^{79}$

\subsection{Schadenexzedentenvertrag}

Der Schadenexzedentenvertrag ist der nicht-proportionalen Rückversicherung zuzuordnen. Er tritt sowohl in der Gestalt eines Einzelschaden- als auch eines Kumulschadenexzedentenvertrages auf. ${ }^{750}$ Der Einzelschadenexzedent sichert die versicherungstechnische Ergebnisentwicklung des Erstversicherers gegen einzelne Großschäden einer bestimmten Risikoeinheit (in der Regel einer Versicherungssparte) ab. Der Rückversicherer übernimmt die über der Priorität a (= Selbstbehalt) liegende Schadenlast maximal bis zur Höhe eines vereinbarten Haftungslimits $\mathrm{H}$. Bezeichne $\mathrm{X}_{\mathrm{i}}$ die Einzelschäden eines Teilbestands mit $i \in\{1, \ldots, n\}$ Risiken, so kann die Entschädigungsleistung des Rückversicherers $\mathrm{S}_{\mathrm{RV}}$ wie folgt ermittelt werden:

$$
\mathrm{S}_{\mathrm{RV}}=\sum_{\mathrm{i}=1}^{\mathrm{n}} \min \left(\max \left(\mathrm{X}_{\mathrm{i}}-\mathrm{a}, 0\right), \mathrm{H}\right) \quad \text { mit } \mathrm{a}>0 \text { und } \mathrm{H}>0
$$

Im Fall von Einzelschäden, die nach Abzug der Priorität das Haftungslimit überschreiten, fällt der überschießende Betrag wieder in den Selbstbehalt des Erstversicherers zurück, sofern kein anschließender Rückversicherungsschutz besteht. Die Entschädigungsleistung des Rückversicherers ist somit hinsichtlich jedes einzelnen Schadens auf das Haftungslimit begrenzt. Darüber hinaus kann die Haftung des Rückversicherers aber auch zeitlich limitiert werden, indem eine maximale Anzahl von Ausschöpfungen bzw. Wiederauffüllungen des Haftungslimits pro Jahr vereinbart wird. ${ }^{751}$ Die Wiederauffüllung des Haftungslimits erfolgt entweder entgeltfrei, oder es wird durch den Rückversicherer eine Prämie erhoben, die sich als Prozentsatz der originären Rückversicherungsprämie bemisst. ${ }^{752}$ In Versicherungszweigen, die auch Naturgefahren abdecken, wird in der Regel nur eine entgeltliche Wiederauffüllung gewährt. ${ }^{753}$

749 Weitere proportionale Preiskomponenten, wie z.B. Gewinnanteile oder Schaden- und Verlustselbstbeteiligungen, werden im Rahmen dieser Arbeit nicht betrachtet. Vgl. hierzu LIEBWEIN (2000), S. 93-100 und PFEIFFER (1994), S. 58-60.

750 Vgl. im Folgenden MACK (2002), S. 325-329, LIEBWEIN (2000), S. 151-166 und PFEIFFER (1994), S. 62-64.

751 Vgl. vertiefend LIEBWEIN (2000), S. 154.

752 Entgeltfreie Wiederauffüllungen des Haftungslimits treten in der Realität jedoch allenfalls pro forma auf, da der Rückversicherer die vereinbarte Anzahl der „freien“ Wiederauffüllungen vorab (,up-front") in die Kalkulation der Rückversicherungsprämie einbeziehen wird.

753 Vgl. PFEIFFER (1994), S. 74-75. 
Der Einzelschadenexzedentenvertrag schützt den Erstversicherer vor hohen Nettobelastungen durch einzelne Großschäden und ermöglicht auf diese Weise eine Homogenisierung der Schadenhöhen im Selbstbehalt. Sofern jedoch durch ein Schadenereignis (z.B. eine Naturkatastrophe) viele versicherungstechnische Einheiten gleichzeitig betroffen sind und die resultierenden Einzelschäden jeweils die Priorität unterschreiten, bietet der Einzelschadenexzedent keinen wirksamen Schutz vor diesem Frequenzbzw. Kumulrisiko. In Sparten mit entsprechend hoher Belastung durch Naturgefahren (z.B. Kasko) bietet sich daher der Abschluss eines Kumulschadenexzedentenvertrages an. Dieser greift, sofern die Summe aller aus dem Ereignis resultierenden Einzelschäden die Priorität übersteigt. ${ }^{754}$ Der Erstversicherer wird folglich durch das Naturereignis nur einmalig und maximal in Höhe der Priorität belastet. Der für einen Schadenexzedentenvertrag zu entrichtende Preis bemisst sich im Gegensatz zu proportionalen Deckungen an dem erwarteten Gesamtschadenaufwand des rückversicherten Portfolios. Es ist daher eine eigenständige Prämienkalkulation seitens des Rückversicherers (Quotierung) erforderlich. ${ }^{755}$ Die im Rahmen dieser Arbeit verfolgte Vorgehensweise einer modellimmanenten Preisermittlung für Rückversicherungsschutz wird in Kapitel 5.4.5.3 erläutert.

\subsection{Jahresüberschadenexzedentenvertrag (Stop Loss)}

Die logische Kette vom Einzelschaden- über den Kumulschadenexzedentenvertrag wird durch den Jahresüberschadenexzedentenvertrag (kurz: Stop Loss) vervollständigt, da dieser von der eigentlichen Schadenursache abstrahiert und eine Begrenzung des Jahresgesamtschadens vornimmt. ${ }^{756}$ In der Regel wird der Stop Loss mit anderen proportionalen oder nicht-proportionalen Rückversicherungsformen kombiniert, so dass für die Bemessung der Entschädigungsleistung der im Selbstbehalt verbleibende Jahresschaden des Erstversicherers herangezogen werden muss. Der Rückversicherer ist zur Leistung aus dem Stop Loss verpflichtet, sofern die Summe aller Selbstbehaltschäden des Erstversicherers innerhalb eines Jahres die gewählte Priorität a übersteigt. ${ }^{757}$ Die Priorität wird als Prozentsatz des auf den Selbstbehalt entfallenden Prämienvolumens $^{758}$ (z.B. als Schadenquote) angegeben und sollte so gewählt werden, dass sich der Erstversicherer bei Erreichung der Priorität bereits in der versicherungstechnischen

754 Dabei ist es unerheblich, ob der Kumulschaden durch eine starke Häufung von Kleinschäden oder durch einige Großschäden entsteht. Vgl. zur "Irrelevanz der Schadenstruktur“ auch WAGNER (2000), S. 358.

755 Vgl. zu Quotierungsverfahren ausführlich MACK (2002), S. 328 und S. 357-369, LIEBWEIN (2000), S. 183-210, SwISS RE (1997a), S. 30-32, PFEIFFER (1994), S. 66-76 und die Ausfuhrungen in Kapitel 5.4.5.3.

756 Vgl. hierzu und im Folgenden LIEBWEIN (2000), S. 167-172.

757 Vgl. MACK (2002), S. 328, PFEIFFER (1994), S. 65 und LiEBWEIN (2000), S. 167.

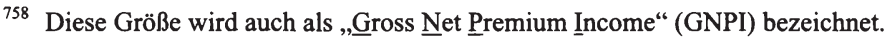


Verlustzone befindet. ${ }^{759}$ Analog zum Schadenexzedentenvertrag ist die Haftung des Rückversicherers in der Regel durch ein Haftungslimit $\mathrm{H}$ beschränkt. Bezeichne $\mathrm{X}_{\mathrm{T}}$ den nach Berücksichtigung sämtlicher anderer Rückversicherungsverträge verbleibenden Jahresschaden einer Sparte, so kann die Entschädigungsleistung des Rückversicherers $\mathrm{S}_{\mathrm{RV}}$ unter einem Stop-Loss-Vertrag wie folgt formalisiert werden: ${ }^{760}$

$$
\mathrm{S}_{\mathrm{RV}}=\min \left(\max \left(\mathrm{X}_{\mathrm{T}}-\mathrm{a}, 0\right), \mathrm{H}\right)
$$

mit $\mathrm{a}>0$ und $\mathrm{H}>0$

Stop-Loss-Verträge werden in der Regel in Sparten abgeschlossen, deren Jahresschaden aufgrund von Naturgefahren starken zufallsbedingten Schwankungen unterworfen ist (z.B. in der Sturmversicherung) ${ }^{761}$ Sie bewirken eine signifikante Glättung des versicherungstechnischen Ergebnisses im Zeitverlauf und bieten zudem einen umfassenden Bilanzschutz. ${ }^{762}$

\subsubsection{Ermittlung der Preiskomponenten (Pricing)}

Bei der Preisermittlung für Rückversicherungsschutz sollte grundsätzlich eine Differenzierung zwischen proportionalen und nicht-proportionalen Deckungen vorgenommen werden. ${ }^{763}$ Während der Rückversicherer im Rahmen proportionaler Verträge zu einem festen Prozentsatz an den Originalprämien des Erstversicherers partizipiert und im Gegenzug einen festen Anteil des Originalrisikos übernimmt, erstreckt sich der nicht-proportionale Rückversicherungsschutz ausschließlich auf Schadenzahlungen. Da eine Koppelung an die Originalprämie nicht mehr besteht, ist für die Ermittlung risikogerechter Marktpreise eine eigenständige Kalkulation (Quotierung) erforderlich. ${ }^{764}$ Die Bruttoprämie eines nicht-proportionalen Vertrages setzt sich aus folgenden Komponenten zusammen: ${ }^{765}$

759 Auf diese Weise soll verhindert werden, dass mit dem Stop Loss eine versicherungstechnische Gewinngarantie verbunden ist und der Erstversicherer das Interesse an der Durchsetzung risikogerechter Originalprämien verliert. Vgl. LiEBWEIN (2000), S. 169 und PFEIFFER (1994), S. 65.

760 Aus der Perspektive des Erstversicherers ist das Risikoprofil eines Stop Loss-Vertrags somit dem einer Call-Option vergleichbar. Vgl. hierzu vertiefend BROHM (2002), S. 63-64.

761 Vgl. MACK (2002), S. 328, PFEIFFER (1994), S. 65 und LIEBWEIN (2000), S. 167-168.

762 Vgl. übereinstimmend GROSSMANN (1977), S. 124 und LIEBWEIN (2000), S. 172.

763 Vgl. im Folgenden LIEBWEIN (2000), S. 183 und PFEIFFER (1994), S. 66-67.

764 Vgl. Flemming (1988), S. 102, GROSSMANN (1977), S. 133 und LiEBWEIN (2000), S. 183.

765 Die Bruttoprämie stellt eine kalkulatorische Preisuntergrenze dar, die jedoch in ,weichen“ Marktphasen, d.h. in Phasen mit einem Angebotsüberhang für Rückversicherungsschutz, noch unterschritten werden kann. 


\section{Risikoprämie \\ + Schwankungszuschlag \\ + Kosten- und Gewinnzuschlag \\ $=$ Bruttoprämie}

Die Risikoprämie entspricht dem Erwartungswert der Schäden aus dem jeweiligen Rückversicherungsvertrag. Zur Ermittlung der Risikoprämie kann sich der Rückversicherer verschiedener Quotierungsverfahren bedienen, die in Tabelle 5.12 im Überblick dargestellt sind. ${ }^{766}$

\begin{tabular}{|l|l|}
\hline Verfahren & Basis für die Quotierung \\
\hline Burning-Cost-Verfahren & Schadenerfahrung der Vergangenheit \\
\hline Exposure-Verfahren & Risikostruktur des geschützten Portfolios \\
\hline Pay-Back-Verfahren & $\begin{array}{l}\text { Schadenfrequenz und Wiederkehrperioden } \\
\text { bestimmter Schadenereignisse }\end{array}$ \\
\hline
\end{tabular}

Tabelle 5.12: Quotierungsverfahren

Die Notwendigkeit eines Schwankungszuschlags ergibt sich aus dem stochastischen Charakter des Schadenverlaufs. MACK weist darauf hin, „dass ein Versicherungsunternehmen deutlich mehr Kapital benötigt als den geschätzten Erwartungswert des Gesamtschadens, damit es auch einen diesen Schätzwert übersteigenden Gesamtschaden begleichen kann.“767 Darüber hinaus ermöglicht der Schwankungszuschlag bei Vertragsformen, die auf einen Ausgleich in der Zeit abstellen, die Bildung von Kumuloder Katastrophenreserven. ${ }^{768}$ Zur Abdeckung von Betriebs- und Retrozessionskosten des Rückversicherers sowie zur Entlohnung des Unternehmerrisikos sollte ferner ein Kosten- und Gewinnzuschlag erhoben werden. Die Summe aller kalkulierten Zuschläge wird als Loading bezeichnet. ${ }^{769}$

In der Praxis wird überwiegend die Zielsetzung verfolgt, ein bestehendes Rückversicherungsprogramm hinsichtlich seiner Haftungs- und Preisstruktur möglichst exakt in einem internen Risikomodell abzubilden. Der hier entwickelte Modellansatz bietet die Möglichkeit, die Preisstruktur der abgebildeten Verträge entweder exogen (z.B. als vertraglich fixierte Preise) vorzugeben oder diese auf risikotheoretischer Grundlage aus dem Modell heraus zu ermitteln. Ein wesentlicher Vorteil der letztgenannten „technischen“ Preisermittlung besteht darin, dass externe Einflüsse (z.B. ein hohes

\footnotetext{
766 Vgl. zur Darstellung und kritischen Beurteilung dieser Verfahren MACK (2002), S. 357-369, PFEIFFER (1994), S. 67-73, GrossmanN (1977), S. 133-135 und LiebWEIN (2000), S. 186-199.

767 MACK (2002), S. 28 [Hervorhebung im Original].

$768 \mathrm{Vgl}$. GROSSMANN (1977), S. 136.

769 Vgl. auch LiEBWEIN (2000), S. 183-200 und PFEIFFER (1994), S. 66-76.
} 
Prämienniveau aufgrund knapper Kapazitäten) ausgeblendet werden können und ein auf die spezifische Portfoliostruktur abgestimmter, risikoadäquater Preis ermittelt wird, der gegebenenfalls als Ausgangspunkt für Preisverhandlungen dienen kann. ${ }^{770}$ Soll hingegen das Pricing einer existierenden Rückversicherungsstruktur möglichst realitätsnah abgebildet werden, stellt das technische Pricing keine entscheidungsrelevante Vorgehensweise dar, da beispielsweise Marktzyklen oder die unzureichende Platzierbarkeit bestimmter Deckungsformen am Markt nicht abgebildet werden.

Im Folgenden wird ein Ansatz zur modellimmanenten Bestimmung der Bruttoprämie vorgestellt. Dieser greift zur Bemessung des Loadings auf den bereits in Kapitel 4.1.3.3 diskutierten CVaR-Ansatz zurück. Die Risikoprämie eines Vertrages ergibt sich unverändert als Schadenerwartung des Rückversicherers $E\left[S_{R V}\right]$. Es wird davon ausgegangen, dass der Rückversicherer zur Abdeckung des Schwankungsrisikos Risikokapital vorhält, dessen Höhe von dem gewählten Sicherheitsniveau $1-\varepsilon$ abhängt. Das erforderliche Risikokapital ergibt sich als: ${ }^{771}$

$$
\operatorname{CVaR}_{\varepsilon}\left(S_{\mathrm{RV}}\right)=\mathrm{E}\left[\mathrm{S}_{\mathrm{RV}} \mid \mathrm{S}_{\mathrm{RV}} \geq \operatorname{VaR}_{\varepsilon}\left(\mathrm{S}_{\mathrm{RV}}\right)\right]
$$

Aus der Sicht des Rückversicherers muss das für die jeweilige Deckungszusage vorzuhaltende Risikokapital angemessen verzinst werden. Die erwartete Einperiodenren-

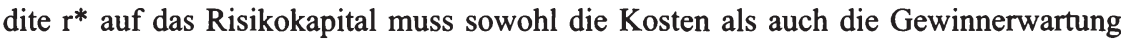
des Rückversicherers abdecken und kann daher als Loading interpretiert werden. Es wird unterstellt, dass der Rückversicherer das erforderliche Risikokapital bereits zum Zeitpunkt der Deckungszusage, d.h. am Anfang des betrachteten Jahres bereitstellt. Da sich das Rückversicherungsversprechen jedoch erst im Verlauf des Jahres in Form von Erstattungsleistungen konkretisiert, erscheint es sinnvoll, als Basisgröße für den Verzinsungsanspruch den Barwert des Risikokapitals heranzuziehen. Der risikoadäquate Preis $P_{R V, \varepsilon}$ eines Rückversicherungsvertrages setzt sich folglich aus der Risikoprämie $\mathrm{E}\left[\mathrm{S}_{\mathrm{RV}}\right]$ und dem Verzinsungsanspruch auf das barwertige Risikokapital (= Loading) zusammen.

$$
\mathrm{P}_{\mathrm{RV}, \varepsilon}=\mathrm{E}\left[\mathrm{S}_{\mathrm{RV}}\right]+\underbrace{\mathrm{CVaR}_{\varepsilon}\left(\mathrm{S}_{\mathrm{RV}}\right) \cdot \mathrm{r}^{*} \cdot\left(1+\mathrm{r}^{*}\right)^{-1}}_{\text {Loading }}
$$

Diese Vorgehensweise impliziert zunächst, dass der Rückversicherer hinsichtlich der Entschädigungsleistungen und des potenziellen Verlustes aus jedem Einzelvertrag über keine Diversifikationsmöglichkeiten verfügt. In der Realität können Rückversicherer

770 Vgl. zur Notwendigkeit eines ,technischen“ Pricing auch NIERHAUS (1990), S. 1158-1162.

771 Vgl. detailliert zur Ermittlung des Conditional Value-at-Risk Kapitel 4.1.3.3. 
jedoch in vielfältiger Hinsicht sowohl zeitliche und räumliche als auch kollektive Ausgleichseffekte erzielen. ${ }^{772}$ Kollektive Ausgleichseffekte ergeben sich in der Regel bereits zwischen den bei einem einzelnen Zedenten abgeschlossenen Verträgen. Darüber hinaus eröffnen sich für weltweit agierende und hinsichtlich ihrer Portfoliostruktur breit diversifizierte Rückversicherer erhebliche zusätzliche Diversifikationspotenziale. ${ }^{773}$ Der im betrachteten CVaR-Ansatz verwendete Parameter ${ }^{*}$ stellt vor diesem Hintergrund die Renditeerwartung für den Standalone-Fall dar und entspricht daher ausdrücklich nicht der Renditeanforderung, die ein Rückversicherer in Bezug auf sein diversifiziertes, d.h. auf der Unternehmensebene vorzuhaltendes Risikokapital stellt. Im Rahmen dieser Arbeit wird für den Parameter $r^{*}$ durchgängig ein Wert von 3,0\% angenommen. ${ }^{774}$ Das Sicherheitsniveau des Rückversicherers beträgt analog zum Modellunternehmen $1-\varepsilon=99,5 \%$. An dieser Stelle sei betont, dass das Sicherheitsniveau des Rückversicherers mindestens dem des Erstversicherers entsprechen sollte. In der Praxis ist sogar zu vermuten, dass die Rückversicherer auf der Basis konservativerer Sicherheitsniveaus steuern, um ein erstklassiges und somit für den Markt attraktives Rating zu erhalten. ${ }^{775}$

Im Rahmen proportionaler Verträge bemisst sich die Rückversicherungsprämie als fester Prozentsatz p der Originalprämie. Auf diese Rückversicherungsprämie vergütet der Rückversicherer dem Erstversicherer eine Rückversicherungsprovision, welche neben ihrer Funktion als Kostenerstattung eine zentrale Preiskomponente darstellt. ${ }^{776}$ Die Differenz zwischen erhaltener Prämie und erstatteter Provision kann als effektive Rückversicherungsprämie bzw. als risikoadäquater Preis für den proportionalen Rückversicherungsschutz interpretiert werden. Dies soll am Beispiel eines Quotenvertrages

772 Vgl. auch HERSBERgER (1996), S. 19-20.

773 Vgl. übereinstimmend MENTZEL (2004), S. 133-137 und HERSBERger (1996), S. 16-20. Als Beispiel sei an dieser Stelle die Hannover Rückversicherung AG genannt, die in ihrem Geschäftsbericht 2003 darauf hinweist, dass sie „die notwendige Eigenkapitalunterlegung in der SchadenRückversicherung durch einen optimalen Risikostrukturausgleich [...] in Summe um ca. 50\% reduzieren“ kann. Vgl. HANNOVER RÜCK (2003), S. 55.

774 Würde für das Pricing des hier unterstellten Rückversicherungsprogramms an Stelle des CVaRAnsatzes (mit $r^{*}=3,0 \%$ ) das sehr pauschale, jedoch in der Praxis vielfach anzutreffende Standardabweichungsprinzip herangezogen, müsste ein Loadingfaktor von $24 \%$ einer Standardabweichung angesetzt werden, um aus der Sicht des Rückversicherers ein äquivalentes Pricing sicherzustellen. Dieser Loadingfaktor kann im Marktdurchschnitt durchaus als realistisch eingestuft werden. Vgl. zum Standardabweichungsprinzip auch MACK (1983), S. 329-331.

775 Diese Einschätzung wird durch folgendes Zitat gestützt: „Die Schweizer Rück hat sich für die Risikokapitalermittlung die Vorgabe gesetzt, dass sie den größten Jahrhundertverlust (mit einer Wiederkehrwahrscheinlichkeit von höchstens einmal in hundert Jahren) nicht nur überleben, sondern nach diesem Verlustjahr noch genügend Kapital übrig haben will, um mit einem unverminderten Portefeuille im Markt zu bleiben." HERSBERGER (1996), S. 20. 
erläutert werden. ${ }^{777}$ Das Ergebnis des Rückversicherers aus dem Quotenvertrag setzt sich aus folgenden Komponenten zusammen:

$$
\begin{aligned}
& \text { Ergebnis }_{\mathrm{RV}}^{\text {Quote }}=\underbrace{\mathrm{P}^{\text {Orig }} \cdot \mathrm{p}}_{\mathrm{P}^{\text {Quote }}}-\text { Prov }-\underbrace{\mathrm{S}^{\text {Orig }} \cdot \mathrm{p}}_{\mathrm{S}^{\text {Quote }}} \\
& =\underbrace{\mathrm{P}^{\text {Quote }}-\mathrm{Prov}}_{\mathrm{P}_{\text {eff }}^{\text {Quote }}}-\mathrm{S}^{\text {Quote }}=\mathrm{P}_{\text {eff }}^{\text {Quote }}-\mathrm{S}^{\text {Quote }} \\
& \text { mit: } \quad \mathrm{P}^{\text {Orig }}=\text { Prämienvolumen des Originalgeschäfts } \\
& S^{\text {Orig }}=\text { Schadenaufwand des Originalgeschäfts } \\
& \mathrm{p} \quad=\text { Quotenabgabe [\%] } \\
& \text { Prov }=\text { Rückversicherungsprovision } \\
& \mathrm{P}^{\text {Quote }}=\text { zediertes Prämienvolumen } \\
& \mathrm{S}^{\text {Quote }}=\text { zedierter Schadenaufwand } \\
& \mathrm{P}_{\text {eff }}^{\text {Quote }}=\text { effektive Rückversicherungsprämie }
\end{aligned}
$$

Die Rückversicherungsprovision Prov wird in der Regel als Prozentsatz der zedierten Prämie $\mathrm{P}^{\text {Quote }}$ ausgedrückt. Bezeichne also $\mathrm{x}^{\text {Prov }}$ den Rückversicherungsprovisionssatz, so gelten folgende Beziehungen:

$$
\mathrm{P}_{\text {eff }}^{\text {Quote }}=\mathrm{P}^{\text {Quote }}-\text { Prov }=\mathrm{P}^{\text {Quote }}-\mathrm{x}^{\text {Prov }} \cdot \mathrm{P}^{\text {Quote }}=\mathrm{P}^{\text {Quote }}\left(1-\mathrm{x}^{\text {Prov }}\right)=\mathrm{P}^{\text {Orig }} \cdot \mathrm{p}\left(1-\mathrm{x}^{\text {Prov }}\right)
$$

und damit:

$$
\mathrm{x}^{\text {Prov }}=1-\frac{\mathrm{P}_{\text {eff }}^{\text {Quote }}}{\mathrm{P}^{\text {Quote }}}=1-\frac{\mathrm{P}_{\text {eff }}^{\text {Quote }}}{\mathrm{P}^{\text {Orig }} \cdot \mathrm{p}}
$$

Die vorstehenden Ausführungen verdeutlichen, dass der risikoadäquate Preis eines Quotenvertrages $P_{\text {eff }}^{\text {Quote }}$ in analoger Vorgehensweise zur nicht-proportionalen Rückversicherung mit Hilfe des CVaR-Ansatzes ermittelt werden kann. Unter Verwendung von Beziehung (5.42) bzw. (5.43) kann dieser risikoadäquate Preis wiederum in eine Prämien- und eine Provisionskomponente aufgeteilt werden. Dieses Vorgehen eröffnet die Möglichkeit, auch für proportionale Rückversicherungsverträge eine „technische“ Preisermittlung vorzunehmen. Den weiteren Ausführungen über das versicherungstechnische Nettogeschäft liegt der beschriebene risikotheoretische Preisermittlungsansatz zugrunde. Auf der Grundlage der simulierten Bruttoschäden eines Anfalljahres und der hieraus resultierenden Entschädigungsleistungen (Recoveries) des Rückversi-

777 Die beschriebene Vorgehensweise kann in analoger Form auch auf Summenexzedentenverträge übertragen werden. 
cherers können die Preisparameter der einzelnen Rückversicherungsverträge bestimmt werden. In Tabelle 5.13 sind für das im Basisszenario gültige Rückversicherungsprogramm sowohl die Haftungsstruktur als auch die durch „technisches“ Pricing ermittelten Preisparameter dargestellt.

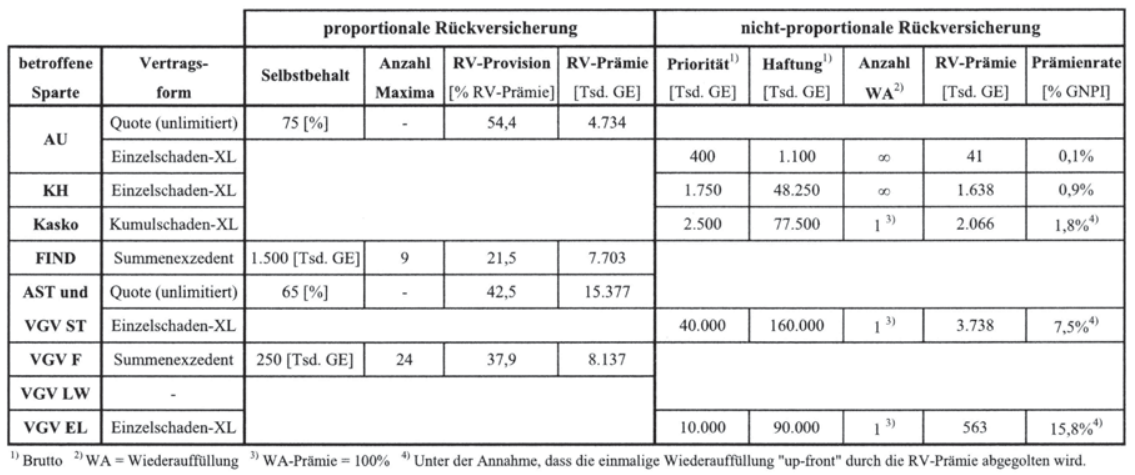

Tabelle 5.13: Haftungsstruktur und Parameter des Rückversicherungsprogramms im Basisszenario

Die Haftstrecke des Einzelschadenexzedentenvertrages für die Sparten „Allgemeine Sturm“ und „VGV Sturm“ ist auf das maximale Schadenpotenzial eines einzelnen Sturmereignisses (200 Mio. GE) abgestimmt. In sämtlichen Versicherungszweigen, die gegenüber Naturgefahren exponiert sind, ist zudem nur eine entgeltliche Wiederauffüllung des Haftungslimits möglich. Die gewählte XL-Haftstrecke in „KraftfahrtHaftpflicht“ (50 Mio. GE) trägt der Annahme Rechnung, dass der modellierte Bestand keine Policen mit unbegrenzter Deckung enthält. Die Feuersparten sind jeweils über einen Summenexzedentenvertrag gedeckt. Die Sparte „VGV Leitungswasser“ verfügt annahmegemäß über keinen Rückversicherungsschutz.

\subsubsection{Abwicklungsmodell}

5.4.6.1 Grundlagen zur Abwicklung von Vorjahres- und Geschäftsjahresschäden

In den vorangegangenen Ausführungen zur Schadenmodellierung wurde implizit auf diejenigen Versicherungsverträge Bezug genommen, deren Garantiezeitraum und Schadenanfall der modellierten Periode ( $=$ simuliertes Anfalljahr) direkt zuzurechnen ist. Diese Verträge werden auch als „laufendes Geschäft“ bezeichnet. ${ }^{778}$ Die Modellierung soll im Folgenden um so genannte „Altverträge“ erweitert werden, deren Versicherungsdauer zwar bereits abgelaufen ist, die jedoch weiterhin ein potenzielles Spät-

778 Vgl. BROHM (2002), S. 221-222 und KORYCIORZ (2004), S. 157-158. 
schadenrisiko tragen. Insbesondere in Versicherungszweigen mit langen Abwicklungsdauern, wie beispielsweise den Haftpflichtsparten oder auch in der Personenversicherung, sind die auf Altverträge ${ }^{779}$ entfallenden Schäden oft noch nicht vollständig reguliert bzw. noch gar nicht bekannt. ${ }^{780}$ Da der wirtschaftliche Anfall der Altschäden dem Modellbeginn vorausgeht, können diese der modellierten Periode nicht unmittelbar zugewiesen werden. Es bestehen grundsätzlich zwei Möglichkeiten, dieser Problematik zu begegnen. Die Annahme, dass zu Modellbeginn keine Verpflichtungen aus Altverträgen existieren, käme einer fiktiven Neugründung des Versicherungsunternehmens zu Beginn der Modellperiode bzw. einem fiktiven Verkauf der bestehenden Schadenrückstellungen an eine Abwicklungsgesellschaft gleich. In der Realität üben die Abwicklungsergebnisse vergangener Anfalljahre jedoch einen signifikanten Einfluss auf das versicherungstechnische Ergebnis des laufenden Geschäftsjahres aus, so dass eine Vernachlässigung der Altverträge grundsätzlich nicht in Betracht kommt. ${ }^{781}$ Die zweite Möglichkeit besteht darin, das Abwicklungsverhalten der Altschäden anhand empirisch geschätzter Abwicklungsmuster explizit in die Modellierung einzubeziehen. Dieser Vorgehensweise soll hier gefolgt werden.

In diesem Zusammenhang ist es zunächst erforderlich, den Schadenaufwand eines Geschäftsjahres in eine zahlungswirksame und eine nicht-zahlungswirksame Komponente aufzuteilen. Während die Zahlungen für Versicherungsfälle und Regulierungskosten den Cashflow des Unternehmens unmittelbar beeinflussen, wird die Höhe des versicherungstechnischen Ergebnisses darüber hinaus auch durch die Dotierung der Rückstellung für noch nicht abgewickelte Versicherungsfälle (synonym: „Schadenrückstellung" oder "Schadenreserve“) und die Veränderung der Schadenrückstellungen für vorangegangene Anfalljahre beeinflusst. Die Verpflichtung zur Bildung von Schadenrückstellungen ergibt sich im handelsrechtlichen Jahresabschluss aus $\S 341 \mathrm{~g} \mathrm{HGB}$ in Verbindung mit $\S 341$ e Abs. 1 Satz HGB. Demnach müssen zukünftige Auszahlungsverpflichtungen für Versicherungsfälle durch die Bildung einer Rückstellung antizipiert werden, sofern sie der jeweiligen Rechnungsperiode zuzuordnen sind. Die Schadenrückstellungen stellen in der Bilanz eines Schaden- und Unfallversicherers den weitaus größten versicherungstechnischen Passivposten dar. ${ }^{782}$

779 Die Begriffe „Altschäden“ und „Vorjahresschäden“ werden in dieser Arbeit synonym verwendet.

780 Vgl. hierzu und im Folgenden MACK (2002), S. 221-223 und KORYCIORZ (2004), S. 157-162.

781 Vgl. übereinstimmend GARSCHHAMMER/ZAGST (2005), S. 425-435, HOFFMANN/SANN (2002), S. 13 und BROHM (2002), S. 221.

782 Im Geschäftsjahr 2003 betrug der Anteil der versicherungstechnischen Nettorückstellungen an der Bilanzsumme aller durch die BAFIN beaufsichtigten deutschen Schaden- und Unfallversicherungsunternehmen 67,2\% (selbst abgeschlossenes Geschäft). Vgl. BAFIN (2005), Tab. 530. 
Hinsichtlich des Konkretisierungsgrads der zugrunde liegenden Verpflichtungen wird zwischen folgenden Teilrückstellungen differenziert. ${ }^{783}$

- Rückstellung für eingetretene, dem Versicherungsunternehmen am Bilanzstichtag bekannte, aber noch nicht abgewickelte Schadenfälle (bekannte Schäden)

- Rückstellung für eingetretene Schadenfälle, die zwischen Bilanzstichtag und hiervon abweichendem Schadenregisterschluss gemeldet werden (bekannte Spätschäden)

- Rückstellung für eingetretene, aber bis zum Schadenregisterschluss nicht gemeldete Schadenfälle (unbekannte Spätschäden)

- Rückstellung für Schadenregulierungskosten

Für die bekannten Schadenfälle (inklusive der bekannten Spätschäden) müssen durch das Versicherungsunternehmen so genannte Einzelfallreserven gebildet werden. ${ }^{784} \mathrm{Die}$ Dotierung sollte nach vernünftiger kaufmännischer Beurteilung erfolgen. ${ }^{785}$ Unbekannte Spätschäden entziehen sich per definitionem einer Einzelbewertung und werden daher im Rahmen der so genannten Spätschadenpauschale berücksichtigt. Die Teilrückstellung für Schadenregulierungskosten (SRK) sollte neben den internen Schadenbearbeitungskosten und externen Schadenermittlungskosten über einen pauschalen Ansatz auch diejenigen Regulierungskosten erfassen, die für unbekannte Spätschäden nach dem betrachteten Bilanzstichtag anfallen. Nach geltendem Handelsrecht dürfen die Schadenrückstellungen nicht abgezinst werden, sondern müssen in Höhe der erwarteten Gesamtverpflichtung gebildet werden. ${ }^{786}$ Das Steuerrecht schreibt hingegen die Abzinsung der Schadenreserven vor, sofern ihnen nicht verzinsliche Verpflichtungen mit einer Laufzeit von mehr als 12 Monaten zugrunde liegen. ${ }^{787}$

Wie bereits erläutert, wird durch den entwickelten Modellansatz der Endschadenbedarf (Ultimate) des Anfalljahres 2004 simuliert. Es wird zudem unterstellt, dass das Modellunternehmen seit einigen Jahren am Markt agiert und infolgedessen Schadenrück-

${ }^{783}$ Vgl. im Folgenden auch OECKING/SANNER (2004), S. 26-30 und ANGERER (1994), S. 36-37.

${ }^{784}$ Eine Gruppen- oder Pauschalbewertung bekannter Schäden ist nur in wenigen Ausnahmefällen zulässig. Vgl. hierzu OECKING/SANNER (2004), S. 27-28.

785 Vgl. § 253 Abs. 1 HGB.

786 Vgl. § 253 Abs. 1 Satz 1 HGB. Lediglich bei Verpflichtungen, die einen Zinsanteil enthalten, ist gemäß $§ 341$ g Abs. 5 HGB die Ermittlung eines Barwertes zulässig. Dieser Fall trifft in der Schaden- und Unfallversicherung lediglich auf Renten-Deckungsrückstellungen zu, die im weiteren Verlauf dieser Arbeit jedoch nicht betrachtet werden. Vgl. auch ANGERER (1994), S. 38 und OECKING/SANNER (2004), S. 30.

${ }^{787} \mathrm{Vgl}$. § 52 Abs. 16 Satz 10 EStG. Im Rahmen der realitätsnäheren Bewertung ist es darüber hinaus erforderlich, unter Rückgriff auf die Abwicklungsergebnisse der Vorjahre eine Neubewertung der Einzelschadenreserven vorzunehmen. Vgl. OECKING/SANNER (2004), S. 30 und DOTTERWEICH (2004), S. 216-218. 
stellungen für die Verpflichtungen aus vorangegangenen Anfalljahren gebildet hat, die nun sukzessive abgewickelt, d.h. zahlungswirksam werden. Der Zweck des Abwicklungsmodells besteht darin, mit Hilfe empirisch geschätzter Zahlungs- und Reservemuster („Abwicklungsmuster") den Gesamtschadenaufwand des simulierten Geschäftsjahres zu ermitteln. Dieser setzt sich aus den Schadenzahlungen sämtlicher Anfalljahre, der Dotierung der GJ-Schadenrückstellung und den Veränderungen der Schadenrückstellungen vorangegangener Anfalljahre zusammen. Der Modellierung des Schadenabwicklungsverhaltens liegt die intuitive Vorgehensweise zugrunde, die in Kapitel 5.4.3.2.2 zum Zwecke der Endschadenschätzung berechneten Entwicklungsfaktoren („Chain-Ladder-Faktoren“) nunmehr „in umgekehrter Richtung“" zu nutzen. ${ }^{788}$ Mit Hilfe der Entwicklungsfaktoren kann sowohl für den Endschaden des simulierten Anfalljahres 2004 als auch für die bestehenden Schadenrückstellungen zurückliegender Anfalljahre eine Zuordnung auf die einzelnen Abwicklungsjahre erfolgen. Das Abwicklungsmodell erfüllt somit grundsätzlich zwei Funktionen, die in Abbildung 5.14 zunächst schematisch dargestellt und im weiteren Verlauf der Ausführungen detailliert erläutert werden sollen:

1) Abwicklung des für das Anfalljahr 2004 simulierten Endschadens im GJ 2004

2) Abwicklung der Schadenrückstellungen zurückliegender Anfalljahre im GJ 2004

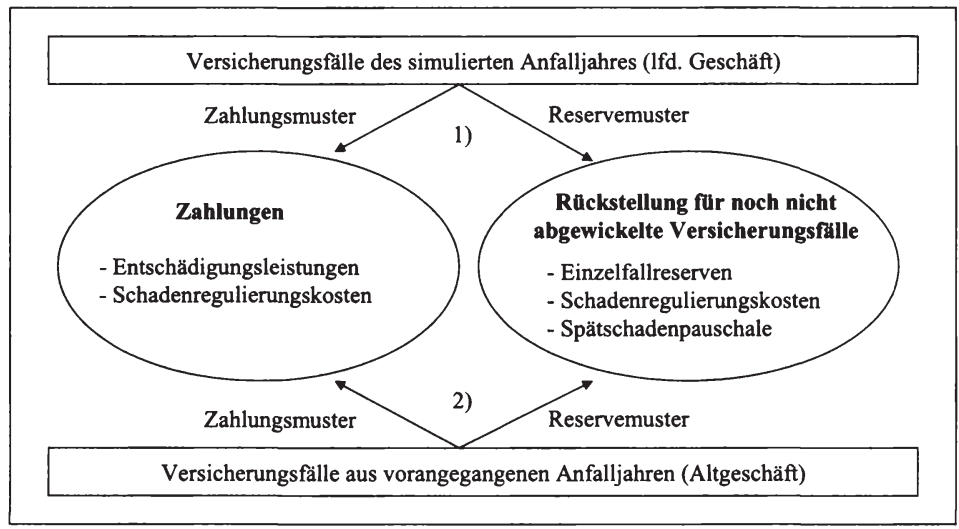

Abbildung 5.14: Systematik zur Abwicklung von Versicherungsfällen

Es ist $\mathrm{zu}$ berücksichtigen, dass sowohl das versicherungstechnische Gesamtportfolio als auch einzelne Teilbestände eines Schaden- und Unfallversicherungsunternehmens in Bezug auf ihr Abwicklungsverhalten häufig sehr heterogen sind. ${ }^{789}$ Um stabile Aus-

\footnotetext{
788 Vgl. im Folgenden auch DOTTERWEICH (2004), S. 150.

789 Vgl. im Folgenden MACK (2002), S. 227 und RADTKE (2004), S. 4-5.
} 
sagen über das Abwicklungsverhalten einzelner Teilbestände ableiten zu können, sollte der Modellierung eine angemessene Segmentierung und bestandsmäßige Abgrenzung vorausgehen. In der Praxis übliche Segmentierungskriterien sind z.B. Versicherungssparten, Untersparten und Geschäftsfelder oder auch die Unterscheidung zwischen groß- und kleinschadenbelasteten Segmenten. ${ }^{790}$ Im Rahmen dieser Arbeit erfolgt die Schadenabwicklung nur auf der Ebene der Versicherungssparten. Eine Ausweitung der Abwicklungsanalyse auf einzelne Sparte-Geschäftsfeld-Kombinationen (z.B. Kraftfahrt-Haftpflicht Privatkunden) ist jedoch möglich.

Ferner sei darauf hingewiesen, dass sämtliche Zahlungs- und Reservemuster als deterministisch angenommen werden. ${ }^{791}$ Diese vereinfachende Annahme dient hier dem Zweck einer übersichtlicheren Darstellung. Gleichwohl ist hiermit die Einschränkung verbunden, dass das Reserve- bzw. Abwicklungsrisiko des Modellunternehmens nicht hinreichend abgebildet wird. Wie bereits in Kapitel 2.2.3.1 erläutert, wird unter dem Reserverisiko das Risiko verstanden, die bekannten und unbekannten Schäden, die im simulierten Anfalljahr und in den vergangenen Anfalljahren eingetreten sind, nicht aus den hierfür gestellten Schadenrückstellungen begleichen zu können. Ebenfalls kann die Unsicherheit hinsichtlich der Auszahlungsgeschwindigkeit der Schäden dem Reserverisiko zugerechnet werden. Der hier vorgestellte Ansatz sollte daher um die Möglichkeit einer stochastischen Schadenabwicklung erweitert werden. In der einschlägigen Literatur werden in diesem Kontext mit der Bootstrap-Methode ${ }^{792}$, dem mittleren quadratischen Fehler der Gesamtreserve nach MACK $^{793}$ oder auch dem stochastischen Chain-Ladder-Verfahren unterschiedliche Schadenreservierungsmethoden diskutiert, welche die Modellierung stochastischer Abwicklungsmuster und eine Quantifizierung des Reserverisikos ermöglichen. ${ }^{794}$ Bei der Modellierung eines stochastischen $\mathrm{Ab}$ wicklungsverhaltens auf der Basis von Aufwandsdreiecken ist einschränkend zu berücksichtigen, dass die Reservierungspolitik in deutschen Schaden- und Unfallversicherungsunternehmen vielfach auch durch unternehmens- und fiskalpolitische Erwägungen motiviert ist. Die verzerrenden Effekte einer systematischen Überdotierung der Schadenrückstellungen können daher gegebenenfalls durch einen stochastischen Modellierungsansatz noch verstärkt werden.

\footnotetext{
790 Vgl. SANN/JUSTEN/OTTEN/FrOMME (2003), S. 7.

$791 \mathrm{Vgl}$. zu einer analogen Vorgehensweise beispielsweise SCHMEISER (2001), S. 144.

792 Vgl. hierzu EFron (1979), S. 1-26, VOSE (1998), S. 181-191 und ENGLAND/VERRALL (2002).

793 Vgl. MACK (2002), S. 248-257.

794 Einen Überblick über diese Verfahren findet man beispielsweise bei ROCKEL (2004), S. 116-119.
} 


\subsubsection{Abwicklung des Bruttogeschäfts}

Die Modellierung der Bruttoabwicklung soll am bekannten Beispiel der Sparte „Allgemeine Unfall“" erläutert werden. Der Schwerpunkt der Darstellung liegt auf der Abwicklung der Einzelfallreserven. Für die Spätschadenpauschale und die Teilrückstellung für Schadenregulierungskosten liegen in der Praxis vielfach keine validen $\mathrm{Ab}$ wicklungsmuster vor, so dass hier vereinfachend angenommen wird, dass sich diese Teilrückstellungen wie die Einzelfallreserven abwickeln. Als Grundlage der weiteren Ausführungen gilt es sich in Erinnerung zu rufen, dass der aus Tabelle 5.14 ersichtliche kumulierte Schadenaufwand sowohl die kumulierten Schadenzahlungen als auch die bestehenden Schadenrückstellungen (Einzelfallreserven) umfasst. Aus Kapitel 5.4.3.2.2 ist das nachfolgende vervollständigte Abwicklungsdreieck für den Schadenaufwand bereits bekannt. ${ }^{795}$

\begin{tabular}{|c|rrrrrrrr|r|}
\hline Anfalljahr & \multicolumn{7}{|c|}{ Abwicklungsjahr k } \\
$\mathbf{i}$ & $\mathbf{1}$ & $\mathbf{2}$ & $\mathbf{3}$ & $\mathbf{4}$ & $\mathbf{5}$ & $\mathbf{6}$ & Ultimate \\
\hline $\mathbf{1 9 9 7}(\mathrm{i}=1)$ & 13.043 & 10.940 & 9.806 & 9.618 & 9.606 & 9.546 & 9.526 \\
$\mathbf{1 9 9 8}(\mathrm{i}=2)$ & 12.708 & 10.385 & 9.413 & 9.088 & 9.107 & 8.811 & 8.792 \\
$\mathbf{1 9 9 9}(\mathrm{i}=3)$ & 14.390 & 12.307 & 11.225 & 10.936 & 10.984 & 10.775 & 10.752 \\
$\mathbf{2 0 0 0}(\mathrm{i}=4)$ & 16.275 & 13.347 & 12.095 & 10.859 & 10.879 & 10.672 & 10.649 \\
$\mathbf{2 0 0 1}(\mathrm{i}=5)$ & 19.652 & 15.854 & 12.039 & 11.462 & 11.484 & 11.265 & 11.241 \\
$\mathbf{2 0 0 2}(\mathrm{i}=6)$ & 16.219 & 13.002 & 11.293 & 10.752 & 10.772 & 10.567 & 10.545 \\
$\mathbf{2 0 0 3}(\mathrm{i}=7)$ & 18.394 & 15.115 & 13.129 & 12.500 & 12.523 & 12.285 & 12.259 \\
\hline \multicolumn{2}{|c|}{$\mathbf{C L}_{\mathbf{k}}^{\mathbf{A}}$} & 0,8217 & 0,8686 & 0,9521 & 1,0019 & 0,9810 & 0,9979 & 1,0000 \\
\hline $\mathbf{a}_{\mathbf{k}}$ & 1,5005 & 1,2330 & 1,0710 & 1,0196 & 1,0216 & 1,0021 & 1,0000 \\
\hline
\end{tabular}

Tabelle 5.14: Aufwandsdreieck mit Chain-Ladder-Faktoren und Aufwandsfaktoren

Zur Erläuterung von Tabelle 5.14 sei angemerkt, dass die Summe der schraffierten Elemente der Hauptdiagonalen den kumulierten Schadenaufwand der Anfalljahre 1997 bis 2003 im Geschäftsjahr 2003 darstellt. Der Schadenaufwand des Anfalljahres 2003 im Geschäftsjahr 2003 beträgt 18.394 Tsd. GE. Die Chain-Ladder-Faktoren $\mathrm{CL}_{\mathrm{k}}^{\mathrm{A}}$ dienten in Kapitel 5.4.3.2.2 dazu, den erwarteten Endschadenbedarf zu schätzen. Für die Rückrechnung dieses Endschadens auf die einzelnen Abwicklungsjahre bedarf es so genannter Abwicklungsfaktoren. ${ }^{796}$ Wie die Chain-Ladder-Faktoren können sich auch die Abwicklungsfaktoren entweder auf den Schadenaufwand oder die Schadenzahlungen beziehen. In einem ersten Schritt werden die Aufwandsfaktoren $\mathrm{a}_{\mathrm{k}}$ der Abwicklungsjahre $\mathrm{k} \in\{1, \ldots, \mathrm{n}\}$ betrachtet. Diese stellen das Verhältnis zwischen dem erwarteten Schadenaufwand $\mathrm{E}\left(\mathrm{C}_{\mathrm{i}, \mathrm{k}}\right)$ im jeweiligen Abwicklungsjahr $\mathrm{k}$ und dem erwarteten

795 Es wird unverändert unterstellt, dass das älteste Anfalljahr 1997 bereits vollständig abgewickelt ist. Da die Annahme einer festen Abwicklungsdauer in der Praxis häufig verletzt ist, wird das beobachtete Abwicklungsmuster vielfach um einen so genannten Nachlauf- bzw. Tailfaktor erweitert. Vgl. hierzu auch SCHMIDT (2004a), S. 20.

796 Vgl. im Folgenden auch SCHMIDT (2004a), S. 15-20 und SCHMIDT (2004b), S. 60. 
Endschaden $\mathrm{E}\left(\mathrm{C}_{\mathrm{i}, \mathrm{n}}\right)$ dar. Zwischen den Aufwandsfaktoren $\mathrm{a}_{\mathrm{k}}$ und den Chain-LadderFaktoren $\mathrm{CL}_{\mathrm{k}}^{\mathrm{A}}$ lässt sich folgende reziproke Beziehung herstellen.

$$
\mathrm{a}_{\mathrm{k}}=\prod_{\mathrm{i}=\mathrm{k}}^{\mathrm{n}} \frac{1}{\mathrm{CL}_{\mathrm{i}}^{\mathrm{A}}} \quad \text { mit } \mathrm{k} \in\{1, \ldots, \mathrm{n}\}
$$

Exemplarisch ergibt sich der Aufwandsfaktor des ersten Abwicklungsjahres $(k=1)$ als Produkt über alle reziproken Chain-Ladder-Faktoren $\mathrm{CL}_{\mathrm{k}}^{\mathrm{A}}$ als $\mathrm{a}_{1}=1,5005$. Von dem erwarteten Endschadenbedarf eines Anfalljahres werden somit ca. $150 \%$ im ersten Abwicklungsjahr als Schadenaufwand ergebniswirksam. Die Aufteilung des Endschadens auf die sonstigen Abwicklungsjahre kann formal durch Beziehung (5.45) beschrieben werden.

$$
E\left(C_{i, k}\right)=E\left(C_{i, n}\right) \cdot a_{k}
$$

Die Aufwandsfaktoren $a_{k}$ hängen offensichtlich nur vom Abwicklungsjahr $k$, nicht aber vom Anfalljahr i ab. Der gewählten Vorgehensweise liegt somit die Annahme zugrunde, dass die Abwicklung der Schäden eines Anfalljahres in den unterschiedlichen Abwicklungsjahren nach einem für alle Anfalljahre identischen Muster erfolgt. ${ }^{797}$ In Analogie zur obigen Vorgehensweise lassen sich aus dem kumulierten Zahlungs-

\begin{tabular}{|c|c|c|c|c|c|c|c|}
\hline \multirow{2}{*}{$\begin{array}{c}\text { Anfalljahr } \\
\text { i }\end{array}$} & \multicolumn{7}{|c|}{ Abwicklungsjahr k } \\
\hline & 1 & 2 & 3 & 4 & 5 & 6 & Ultimate \\
\hline $1997(\mathrm{i}=1)$ & 1.171 & 5.290 & 8.079 & 9.328 & 9.435 & 9.496 & 9.526 \\
\hline $1998(\mathrm{i}=2)$ & 1.297 & 4.914 & 7.694 & 8.638 & 8.724 & 8.764 & 8.792 \\
\hline $1999(\mathrm{i}=3)$ & 1.425 & 6.629 & 9.359 & 10.467 & 10.660 & 10.719 & 10.753 \\
\hline $2000(\mathrm{i}=4)$ & 1.384 & 6.134 & 9.176 & 10.416 & 10.557 & 10.616 & 10.649 \\
\hline $2001(i=5)$ & 1.127 & 6.508 & 9.709 & 10.994 & 11.144 & 11.205 & 11.241 \\
\hline $2002(i=6)$ & 1.261 & 6.099 & 9.108 & 10.314 & 10.454 & 10.512 & 10.545 \\
\hline $2003(\mathrm{i}=7)$ & 1.528 & 7.091 & 10.589 & 11.991 & 12.154 & 12.221 & 12.259 \\
\hline $\mathbf{C L}_{\mathrm{k}}^{\mathrm{Z}}$ & 4,6408 & 1,4934 & 1,1324 & 1,0136 & 1,0055 & 1,0031 & 1,0000 \\
\hline$z_{k}$ & 0,1246 & 0,5784 & 0,8638 & 0,9781 & 0,9914 & 0,9969 & 1,0000 \\
\hline
\end{tabular}
dreieck die Zahlungsfaktoren $\mathrm{z}_{\mathrm{k}}$ der Abwicklungsjahre $\mathrm{k} \in\{1, \ldots, \mathrm{n}\}$ bestimmen.

Tabelle 5.15: Zahlungsdreieck mit Chain-Ladder-Faktoren und Zahlungsfaktoren

Aus den auf diese Weise ermittelten Aufwands- und Zahlungsmustern lässt sich durch Differenzbildung das zugehörige Reservemuster berechnen. Der Reservefaktor $r_{k}$ ergibt sich als:

$$
r_{k}=a_{k}-z_{k} \quad \text { für } k \in\{1, \ldots, n\}
$$

797 Vgl. auch SCHMIDT (2004a), S. 15. 
Eine graphische Gegenüberstellung der Zahlungsfaktoren $z_{k}$ und Aufwandsfaktoren $a_{k}$ in Abbildung 5.15 zeigt in der Beispielsparte „Allgemeine Unfall“ den durchaus typischen Effekt einer starken Überreservierung in den ersten Abwicklungsjahren. Gegen Ende des Abwicklungszeitraums konvergieren die Abwicklungsfaktoren des Zahlungs- und Aufwandsmusters gegen 100\% (des Endschadens). Die Zahlungs- und Aufwandsstände nähern sich demnach mit fortschreitender Abwicklungszeit immer mehr dem erwarteten Endschaden (Ultimate) an. ${ }^{798}$ Die Differenz zwischen dem Zahlungs- und Aufwandsstand stellt die im jeweiligen Abwicklungsjahr bestehenden Einzelfallreserven dar.

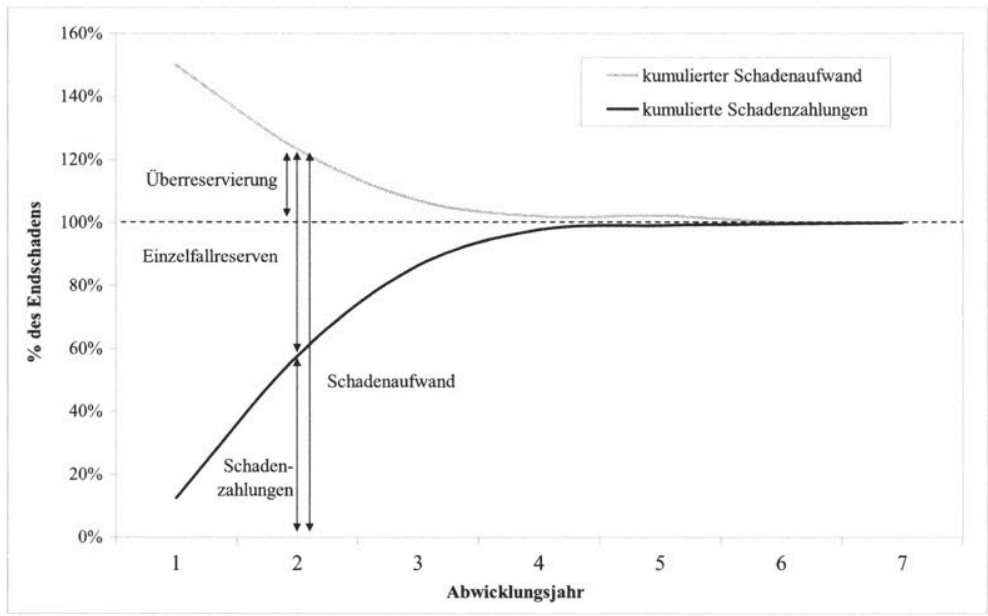

Abbildung 5.15: Abwicklung des erwarteten Endschadens in der Sparte „Allgemeine Unfall“

In einem ersten Schritt wird nachfolgend die Abwicklung des Endschadens des simulierten Anfalljahres 2004 im Geschäftsjahr 2004 beschrieben. ${ }^{799} \mathrm{Zu}$ diesem Zweck wird der erwartete Endschaden $E\left(C_{i, n}\right)$ unter Verwendung der Abwicklungsfaktoren $z_{1}$ bzw. $r_{1}$ in die erwarteten Schadenzahlungen und die im Geschäftsjahr 2004 zu stellenden Einzelfallreserven (Dotierung der GJ-Rückstellung) überführt. Diese Vorgehensweise wird in Abbildung 5.16 am Beispiel der Schadenzahlungen schematisch dargestellt.

798 Mit Blick auf Tabelle 5.14 und Tabelle 5.15 sei darauf hingewiesen, dass in der Beispielsparte „Allgemeine Unfall“ die zahlungs- und aufwandsbasierten Endschadenstände (Ultimates) übereinstimmen. Diese Tatsache ist darin begründet, dass das älteste Anfalljahr als vollständig abgewickelt angenommen wird.

799 Vgl. auch DOTTERWEICH (2004), S. 161-162. 


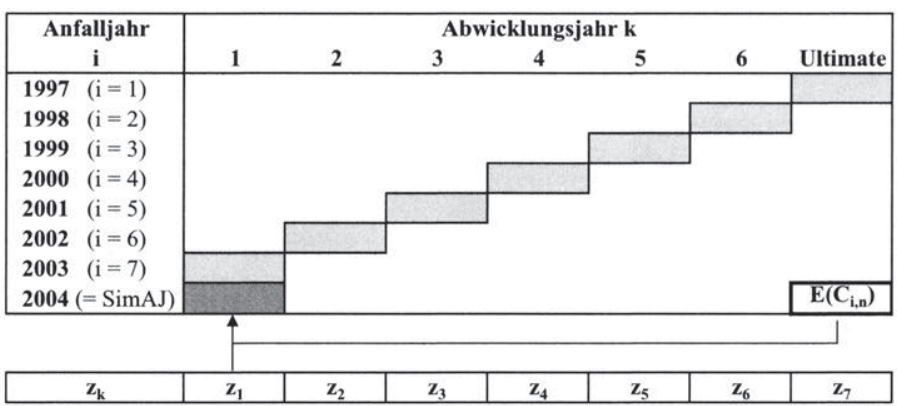

Abbildung 5.16: Abwicklung des simulierten Endschadens im Geschäftsjahr 2004

Die Ermittlung des Zuführungsbetrags zur GJ-Schadenrückstellung im Geschäftsjahr 2004 erfolgt in analoger Vorgehensweise unter Verwendung des Reservefaktors $r_{1}$. Von dem simulierten Endschaden $\mathrm{E}\left(\mathrm{C}_{\mathrm{i}, \mathrm{n}}\right)$ des Anfalljahres 2004 wird somit insgesamt der erwartete Schadenaufwand $\mathrm{E}\left(\mathrm{C}_{\mathrm{i}}\right)$ im Geschäftsjahr 2004 ergebniswirksam:

$$
\mathrm{E}\left(\mathrm{C}_{\mathrm{i}}\right)=\mathrm{E}\left(\mathrm{C}_{\mathrm{i}, \mathrm{n}}\right) \cdot \mathrm{a}_{1}=\mathrm{E}\left(\mathrm{C}_{\mathrm{i}, \mathrm{n}}\right) \cdot\left(\mathrm{z}_{1}+\mathrm{r}_{1}\right)
$$

In einem zweiten Schritt wird der Einfluss vorangegangener Anfalljahre modelliert. ${ }^{800}$ Es wird demnach ermittelt, wie sich die bestehenden Schadenrückstellungen zurückliegender Anfalljahre im Geschäftsjahr 2004 abwickeln. Anhand der Chain-LadderFaktoren kann für jedes vergangene Anfalljahr ermittelt werden, welcher Anteil der Schadenrückstellungen als Schadenzahlung in das versicherungstechnische Bruttoergebnis des Geschäftsjahres 2004 einfließt und welcher Anteil als Ausgangsreserve $\mathrm{AR}_{t}$ in der Bilanz verbleibt. Neben den bereits bekannten Chain-Ladder-Faktoren $\mathrm{CL}_{\mathrm{k}}^{\mathrm{Z}}$ und $\mathrm{CL}_{\mathrm{k}}^{\mathrm{A}}$ mit $\mathrm{k} \in\{1, \ldots, \mathrm{n}\}$ werden somit für jeden modellierten Versicherungszweig folgende Informationen benötigt:

- letzte Hauptdiagonale des kumulierten Zahlungsdreiecks (paid to date)

- letzte Hauptdiagonale des kumulierten Aufwandsdreiecks (incurred to date)

Da das Anfalljahr 1997 annahmegemäß bereits vollständig abgewickelt ist, wird das Geschäftsjahr 2004 folglich durch dieses Anfalljahr nicht mehr belastet. Ergebnisrelevant sind somit nur noch die Anfalljahre 1998 bis 2003. Die kumulierten Zahlungsund Aufwandsstände für diese Anfalljahre im Geschäftsjahr 2004 ergeben sich durch Multiplikation der bekannten Zahlungs- und Aufwandsstände aus dem vorangegangenen Geschäftsjahr 2003 mit den zugehörigen Chain-Ladder-Faktoren $\mathrm{CL}_{\mathrm{k}}^{z}$ bzw. $C L_{k}^{A}$.

${ }^{800}$ Vgl. ergänzend VerRALL (1994), S. 325-332 und DOTTERWEICH (2004), S. 161-162. 
Formal gilt:

$$
\begin{array}{ll}
\mathrm{E}\left(\mathrm{Z}_{\mathrm{i}, \mathrm{k}}\right)=\mathrm{E}\left(\mathrm{Z}_{\mathrm{i}, \mathrm{k}-1}\right) \cdot \mathrm{CL}_{\mathrm{k}}^{\mathrm{Z}} & \text { mit } \mathrm{k} \in\{2, \ldots, \mathrm{n}\} \text { und } \mathrm{i} \in\{1, \ldots, \mathrm{n}-\mathrm{k}+1\} \\
\mathrm{E}\left(\mathrm{C}_{\mathrm{i}, \mathrm{k}}\right)=\mathrm{E}\left(\mathrm{C}_{\mathrm{i}, \mathrm{k}-1}\right) \cdot \mathrm{CL}_{\mathrm{k}}^{\mathrm{A}} & \text { mit } \mathrm{k} \in\{2, \ldots, \mathrm{n}\} \text { und } \mathrm{i} \in\{1, \ldots, \mathrm{n}-\mathrm{k}+1\}
\end{array}
$$

Die somit beschriebene Vorgehensweise wird in Abbildung 5.17 schematisch dargestellt. Im Interesse einer übersichtlicheren Darstellung werden die Hauptdiagonalen der bekannten Zahlungs- und Aufwandsdreiecke jeweils als Säule abgebildet.

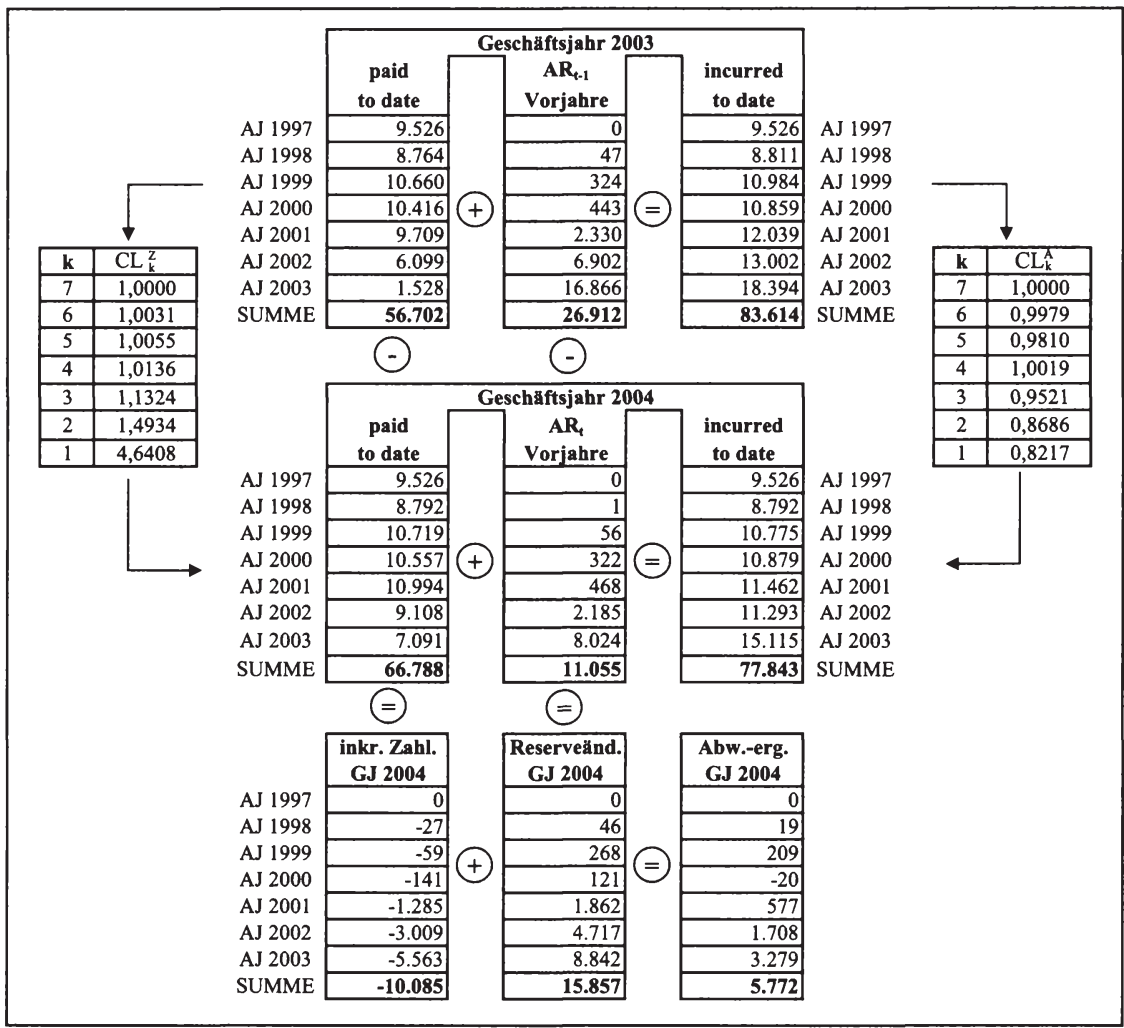

Abbildung 5.17: Abwicklung der vorangegangenen Anfalljahre im Geschäftsjahr 2004

Die Differenz zwischen den Zahlungsständen (paid to date) im Geschäftsjahr 2003 und 2004 entspricht den inkrementellen Schadenzahlungen im Geschäftsjahr 2004. Im Geschäftsjahr 2004 werden somit insgesamt 10.085 Tsd. GE für Schadenfälle gezahlt, die vergangenen Anfalljahren zuzuordnen sind. Die Differenz wiederum zwischen der 
Ausgangsreserve des vorangegangenen Geschäftsjahres $\mathrm{AR}_{\mathrm{t}-1}$ und der Ausgangsreserve des simulierten Geschäftsjahres $A R_{t}$ stellt die ertragswirksame Veränderung der Vorjahresreserven im Geschäftsjahr 2004 dar. Im betrachteten Fall werden demnach Schadenrückstellungen in Höhe von insgesamt 15.857 Tsd. GE ertragswirksam im Geschäftsjahr 2004 aufgelöst. Wie bereits in Kapitel 5.3.2 thematisiert, treten als Folge der Abweichung zwischen reservierter Schadenhöhe und letztendlich zu zahlender Entschädigungsleistung regelmäßig Abwicklungsergebnisse für frühere Anfalljahre auf, die kumuliert betrachtet das bilanzielle Abwicklungsergebnis des Geschäftsjahres darstellen. Formal lässt sich das Abwicklungsergebnis aus Vorjahresschäden im Geschäftsjahr $\mathrm{t}$ durch Beziehung (5.50) beschreiben:

$$
\begin{array}{ll}
\mathrm{AE}_{\mathrm{t}}=\mathrm{ER}_{\mathrm{t}}-\mathrm{Z}_{\mathrm{t}}-\mathrm{AR}_{\mathrm{t}}=\mathrm{AR} \mathrm{R}_{\mathrm{t}-1}-\mathrm{AR}_{\mathrm{t}}-\mathrm{Z}_{\mathrm{t}} \\
\text { mit: } \quad \mathrm{ER}_{\mathrm{t}}=\text { Eingangsreserve für Vorjahre im Geschäftsjahr } \mathrm{t} \\
& \mathrm{AR}_{\mathrm{t}}=\text { Ausgangsreserve für Vorjahre im Geschäftsjahr } \mathrm{t} \\
& \mathrm{Z}_{\mathrm{t}}=\text { Zahlungen für Vorjahresschäden im Geschäftsjahr } \mathrm{t}
\end{array}
$$

Für die Beispielsparte „Allgemeine Unfall“" ergibt sich demzufolge im Geschäftsjahr $\mathrm{t}=2004$ ein erwartetes Abwicklungsergebnis aus vorangegangenen Anfalljahren in Höhe von

$$
\begin{aligned}
\mathrm{AE}_{\mathrm{t}}^{\mathrm{AU}} & =\mathrm{AR}_{\mathrm{t}-1}^{\mathrm{AU}}-\mathrm{AR}_{\mathrm{t}}^{\mathrm{AU}}-\mathrm{Z}_{\mathrm{t}}^{\mathrm{AU}} \\
& =26.912-11.055-10.085=5.772[\text { Tsd. GE] }
\end{aligned}
$$

\subsubsection{Abwicklung des Nettogeschäfts}

Sowohl die Darstellung der handelsrechtlichen Gewinn- und Verlustrechnung als auch der zugehörige Bilanzausweis erfolgen bei Schaden- und Unfallversicherungsunternehmen im Allgemeinen auf Nettobasis, d.h. nach Berücksichtigung der Leistungen der Rückversicherer. ${ }^{801}$ Für eine realitätsnahe Abwicklung des simulierten NettoEndschadens und der Netto-Eingangsreserven für Vorjahresschäden benötigte man daher idealerweise die Netto-Abwicklungsmuster der zugrunde liegenden Einzelschäden, d.h. die Abwicklungsmuster unter Berücksichtigung der zugehörigen Rückversicherungsleistungen. In der Praxis liegen jedoch Netto-Abwicklungsmuster nur sehr selten in hinreichend valider Form vor. Aus diesem Grunde wird vereinzelt vorgeschlagen, als Abwicklungsmuster für die Netto-Schadenrückstellungen zurückliegender Anfalljahre das Netto-Abwicklungsmuster der Geschäftsjahresschäden heranzu-

${ }^{801}$ Der korrespondierende Bruttoausweis erfolgt vielfach durch Vorspaltenangaben. Vgl. zum Prinzip des Brutto- bzw. Nettoausweises FARNY (1992), S. 110-115 und KPMG (1994), S. 154. 
ziehen. ${ }^{802}$ Diese Vorgehensweise lässt sich jedoch „bei offensichtlichen Bilanzverwerfungen im Spiegel vergangenheitsbezogener Unter- und Überreservierungen nicht mehr verfolgen. ${ }^{،{ }^{\prime 803}}$ Im Rahmen dieser Arbeit wird vereinfachend unterstellt, dass sich der simulierte Netto-Endschaden und die Netto-Schadenrückstellungen für Vorjahresschäden entsprechend der Brutto-Abwicklungsmuster abwickeln. ${ }^{804}$ Als Ausgangspunkt für die Abwicklung dienen die Netto-Eingangsreserven, die sich aus der Bilanz eines Versicherungsunternehmens ablesen lassen. Auf eine Differenzierung zwischen Einzelfallreserven, Spätschadenpauschale und der Teilrückstellung für Schadenregulierungskosten wird im weiteren Verlauf im Interesse einer übersichtlicheren Darstellung verzichtet.

\subsubsection{Zentrale Ergebnisgrößen des versicherungstechnischen Geschäfts}

\subsubsection{Versicherungstechnisches Ergebnis auf Anfalljahresbasis}

Mit Blick auf die bereits in Kapitel 3.2.4 erläuterte Differenzierung zwischen Steuerungsebene und Bilanzebene ist es erforderlich, für beide Analysedimensionen eine Ergebnisgröße zu definieren, anhand derer die Entwicklung des versicherungstechnischen Portfolios beurteilt werden kann. Auf der Steuerungsebene wird als Indikator für das Zeichnungsrisiko des simulierten Anfalljahres (SimAJ) das versicherungstechnische Ergebnis auf Anfall- bzw. Zeichnungsjahresbasis herangezogen, welches für jede modellierte Sparte-Geschäftsfeld-Kombination ermittelt wird. ${ }^{805}$ Sowohl in der Bruttoals auch in der Nettobetrachtung kann nachfolgendes Berechnungsschema zugrunde gelegt werden:

Prämien ${ }^{\text {SimAJ }}$

- Schadenzahlungen ${ }^{\text {SimAJ }}$

- Bedarfsreserve ${ }^{\text {SimAJ }}\left(\mathrm{BR}^{\text {SimAJ }}\right)$

+ kalkulatorische Zinsen aus Anlage $\mathrm{BR}^{\text {SimAJ }}$

- Provisionen ${ }^{\text {SimAJ }}$

- Betriebskosten ${ }^{\text {SimAJ }}$

$=$ Anfalljahresergebnis ${ }^{\text {SimAJ }}$

\footnotetext{
$802 \mathrm{Vgl}$. HOFFMANN/SANN (2003a), S. 10.

${ }^{803}$ HofFMANN/SANN (2003a), S. 10.

${ }^{804}$ Diese Vorgehensweise lässt sich streng genommen nur unter der Annahme rechtfertigen, dass in sämtlichen vorangegangenen Anfalljahren lediglich proportionaler Rückversicherungsschutz bestanden hat. Vgl. übereinstimmend HOFFMANN/SANN (2003a), S. 10. 
In der Anfalljahressicht werden demnach ausschließlich Ergebniskomponenten berücksichtigt, die dem simulierten Anfalljahr direkt zuzurechnen sind. ${ }^{806}$ Hierzu zählt insbesondere der Endschadenbedarf (Ultimate), der mittels aktuarieller Schätzverfahren für das gesamte Anfalljahr prognostiziert wird. Der Endschadenbedarf setzt sich zusammen aus den bisher im simulierten Jahr geleisteten Schadenzahlungen und den in Folgejahren erwarteten Schadenzahlungen für dieses Anfalljahr $\left(\mathrm{BR}^{\mathrm{SimAJ}}\right)$. Da die Bedarfsreserve erst in den nachfolgenden Geschäftsjahren sukzessive zur Auszahlung gelangt, sollte diese - dem ökonomischen Postulat der Marktpreisbewertung und dem Opportunitätsgedanken folgend - auf diskontierter Basis in die Berechnung eingehen ${ }^{807}$ Die Ermittlung der diskontierten Bedarfsreserve bzw. der kalkulatorischen Zinsen aus der Anlage der Bedarfsreserve wird nachfolgend am Beispiel der Sparte „Allgemeine Unfall“" schematisch dargestellt: ${ }^{.08}$

\begin{tabular}{|c|c|c|c|c|c|c|c|}
\hline \multirow{2}{*}{ Erwartungswerte [Tsd. GE] } & \multicolumn{7}{|c|}{ Abwicklungsjahr k } \\
\hline & 1 & 2 & 3 & 4 & 5 & 6 & 7 \\
\hline Endschaden brutto $E\left(C_{i, n}\right)$ & 18.854 & & & & & & \\
\hline Zahlungsmuster $z_{k}$ & 0,1246 & 0,5784 & 0,8638 & 0,9781 & 0,9914 & 0,9969 & 1,0000 \\
\hline Schadenzahlungen ${ }^{\text {SimA }}$ & 2.350 & 8.555 & 5.380 & 2.156 & 250 & 104 & 59 \\
\hline Stand Bedarfsreserve $\mathrm{BR}_{\mathrm{k}}$ & 16.504 & 7.949 & 2.569 & 413 & 162 & 59 & 0 \\
\hline kalk. Zinsen aus Anlage $\mathrm{BR}_{\mathrm{k}}$ & & 303 & 130 & 37 & 7 & 3 & 1 \\
\hline & 296 & 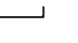 & & & 1 & & \\
\hline & 124 & & & & & & \\
\hline & 34 & & & & & & \\
\hline & 然 & & & & & & \\
\hline & & & & & & & \\
\hline kalk. Zinsen aus Anlage $\mathrm{BR}^{\mathrm{Sim} J}$ & 464 & & & & & & \\
\hline
\end{tabular}

Tabelle 5.16: Berechnungsschema der diskontierten Bedarfsreserve $(i=\mathbf{2}, \mathbf{4 8} \%)$

${ }^{806} \mathrm{Da}$ für die Steuerbemessung der handelsrechtliche Jahresüberschuss ausschlaggebend ist, erfolgt die Berücksichtigung von Unternehmenssteuern erst auf der Bilanzebene. Vgl. hierzu Kapitel 5.3.2.

807 ALBRECHT führt hierzu bereits 1987 übereinstimmend aus: „Ferner ist für uns nicht der bilanzielle Ansatz des versicherungstechnischen Fremdkapitals von Interesse, sondern es muß ein Ansatz gewählt werden, der den durchschnittlichen Zinseffekt, der aus den versicherungstechnischen Rückstellungen resultiert, korrekt wiedergibt. Insbesondere ist zu beachten, daß Teile der Schadenreserve in abnehmendem Umfang mehrere Jahre als Anlagekapital zur Verfügung stehen. Der damit verbundene Zinseffekt ist auf die Periode umzurechnen." ALBRECHT (1987a), S. 323.

$808 \mathrm{Vgl}$. in diesem Zusammenhang auch DOTTERwEICH (2004), S. 77-81. Für den Fall, dass die Prämien des Anfalljahres nach Abzug von Kosten und Schadenzahlungen des ersten Abwicklungsjahres $(k=1)$ nicht ausreichen, um die erforderliche Bedarfsreserve zu stellen, wird hier von den Annahme ausgegangen, dass das Modellunternehmen in Höhe der Unterdeckung einen Kredit zum risikolosen Geldmarktzins aufnimmt. 
Der für das Anfalljahr 2004 prognostizierte Brutto-Endschaden $\mathrm{E}\left(\mathrm{C}_{\mathrm{i}, \mathrm{n}}\right)$ für Basis- und Großschäden beträgt 18,9 Mio. GE. Durch Verwendung des Zahlungsmusters $z_{k}$ kann dieser Endschaden (Ultimate) in die zukünftig erwarteten Schadenzahlungen und den zukünftigen Stand der Bedarfsreserve $\mathrm{BR}_{\mathrm{k}}$ aufgespalten werden.

Unter Fortführung der Prämisse, dass die Schadenzahlungen jeweils zur Jahresmitte anfallen, errechnen sich die kalkulatorischen Zinsen aus der Anlage der Bedarfsreserve im simulierten Anfalljahr als: ${ }^{809}$

(5.53) kalk. Zinsen aus Anlage $\mathrm{BR}^{\operatorname{SimAJ}}=\sum_{\mathrm{k}=1}^{\mathrm{n}} \frac{\left(\mathrm{BR}_{\mathrm{k}}+\mathrm{BR}_{\mathrm{k}+1}\right) \cdot \mathrm{i}}{2 \cdot(1+\mathrm{i})^{\mathrm{k}}} \quad$ mit $\mathrm{BR}_{\mathrm{n}+1}:=0$

Als Diskontierungssatz i wird die Renditeerwartung (quasi) risikoloser Geldmarktpapiere herangezogen. ${ }^{810}$ Diese Rendite wird vereinfachend für die gesamte Abwicklungsdauer als konstant angenommen (flache Zinsstruktur). Eine Weiterentwicklung des hier betrachteten Modellansatzes könnte jedoch vorsehen, die Bedarfsreserve auf der Grundlage einer normalen Zinsstrukturkurve zu diskontieren. Der Verwendung des risikolosen Zinses liegt die konservative Überlegung zugrunde, dass die Bedarfsreserve eine zukünftige Auszahlungsverpflichtung gegenüber den Versicherungsnehmern darstellt. Um die sichere Erfüllbarkeit dieser Auszahlungsverpflichtung zu gewährleisten, sollte das Versicherungsunternehmen die Bedarfsreserve im Rahmen ihrer strategischen Asset Allocation daher nicht unter Risiko stellen. ${ }^{811}$

Die diskontierte Bedarfsreserve der Sparte „Allgemeine Unfall“ im simulierten Anfalljahr ergibt sich abschließend als:

$$
\begin{aligned}
\mathrm{BR}_{\text {disk. }}^{\text {SimAJ }} & =\mathrm{BR}^{\mathrm{SimAJ}}-\text { kalk. Zinsen aus Anlage } \mathrm{BR}^{\mathrm{SimAJ}} \\
& =16.504-464=16.040[\text { Tsd. } \mathrm{GE}]
\end{aligned}
$$

${ }^{809}$ Abweichend von dem hier gewählten jährlichen Zahlungsmuster ist in der Praxis die Verwendung monatlicher Zahlungsmuster für Prämien, Schäden (insbesondere Großschäden) und Kosten anzuraten.

810 Vgl. übereinstimmend KROMSCHRÖDER (1994), S. 314 und BACH (2003), S. 644. Die Existenz eines (quasi) risikolosen Zinses wird in der betrachteten Modellwelt vorausgesetzt. Vgl. hierzu vertiefend LISTER (1997), S. 204-212.

811 In der versicherungswirtschaftlichen Praxis wird bisweilen zur Diskontierung der Bedarfsreserve diejenige Renditeerwartung herangezogen, die als Benchmark bzw. Mindestrendite an das Kapitalanlage-Management formuliert wird. Dieser Vorgehensweise ist unter Steuerungsaspekten jedoch nur zuzustimmen, sofern diese Benchmark durch entsprechende Hedgingmaßnahmen abgesichert ist. 


\title{
5.4.7.2 Versicherungstechnisches Ergebnis auf Geschäftsjahresbasis
}

Auf der Bilanzebene wird zur Beurteilung des versicherungstechnischen Geschäfts das Ergebnis auf Geschäftsjahresbasis herangezogen. ${ }^{812}$ Im Rahmen des versicherungstechnischen Geschäftsjahresergebnisses wird die Schadenentwicklung des simulierten Anfalljahres und sämtlicher vorangegangener Anfalljahre in einer Ergebnisgröße verdichtet. ${ }^{813}$ Diese Verdichtung geht mit einem signifikanten Informations- und Transparenzverlust einher. So kann beispielsweise eine hohe Ergebnisbelastung aus neu gezeichneten Risiken durch die Realisierung von Abwicklungsgewinnen aus Vorjahren ausgeglichen oder sogar überkompensiert werden. Der mit diesem Vorgang korrespondierende Abbau stiller Reserven in den Schadenrückstellungen kann aus der bilanziellen Darstellungsweise jedoch nicht mehr abgelesen werden. Darüber hinaus werden in der Geschäftsjahressicht - dem handelsrechtlichen Erfolgsprinzip ${ }^{814}$ folgend erfolgswirksame Erträge und Aufwendungen ausgewiesen. Die in diesem Zusammenhang erforderliche Abgrenzung bzw. Periodisierung von Zahlungsströmen erfolgt in der Regel jedoch nicht willkürfrei, sondern ist durch die Ausübung bilanzieller Wahlrechte unternehmensindividuell gestaltbar. Die Zusammensetzung des versicherungstechnischen Ergebnisses des simulierten Geschäftsjahres (SimGJ) wird durch folgendes Berechnungsschema verdeutlicht. ${ }^{815}$

\author{
Bruttoprämien ${ }^{\text {SimGJ }}$ \\ - Bruttoaufwendungen für GJ-Versicherungsfälle ${ }^{\text {SimGJ }}$ \\ davon: Zahlungen für GJ-Versicherungsfalle \\ Dotierung der Rückstellung für GJ-Versicherungsfälle \\ +/- Bruttoabwicklungsergebnis ${ }^{\text {SimGJ }}$ \\ davon: Zahlungen für VJ-Versicherungsfälle \\ Veränderung der Rückstellung für VJ-Versicherungsfälle \\ $+\quad$ Bruttoaufwendungen für den Versicherungsbetrieb ${ }^{\text {SimGJ }}$ \\ $=\quad$ versicherungstechnisches Bruttoergebnis ${ }^{\text {SimGJ }}$ \\ $+/$ - Rückversicherungsergebnis ${ }^{\text {SimGJ }}$ \\ $=$ versicherungstechnisches Nettoergebnis ${ }^{\text {SimGJ }}$
}

\footnotetext{
${ }^{812} \mathrm{Vgl}$. in diesem Zusammenhang auch Kapitel 5.4.1.2.

813 Vgl. im Folgenden auch ALFERMANN/RADTKE/REICH (2004), S. 67-70.

${ }^{814}$ Vgl. zur versicherungsspezifischen Unterscheidung von zahlungswirtschaftlichem und erfolgswirtschaftlichem Prinzip FARNY (1992), S. 107-108.

815 Aufgrund der Tatsache, dass keine hinreichend validen Informationen über die Verteilung der Rückversicherungsprämien, Rückversicherungsprovisionen und Recoveries auf die einzelnen $\mathrm{Ge}-$ schäftsfelder vorliegen, wird das versicherungstechnische Ergebnis auf Geschäftsjahresbasis nur in Form einer Spartenerfolgsrechnung ermittelt. Vgl. zum Begriff der Spartenerfolgsrechnung KPMG (1994), S. 160-166 sowie zur Unterscheidung von Gesamtbestands- und Spartenerfolgsrechnungsprinzip FARNY (1992), S. 109-110.
} 
Unter Fortführung der Prämisse, dass sämtliche Versicherungspolicen des Modellunternehmens eine Vertragslaufzeit von einem Jahr aufweisen und genau zu dessen Beginn gezeichnet werden, sind die vereinnahmten Bruttoprämien auf Anfalljahres- und Geschäftsjahresebene ihrer Höhe nach identisch. Die Zahlungen für GJ-Versicherungsfälle korrespondieren in der Anfalljahressicht mit der Position Schadenzahlungen $^{\text {SimAJ }}$. Hinsichtlich des Ausweises der internen Schadenregulierungskosten ist zu berücksichtigen, dass diese in der bilanziellen Darstellung - wie in der Praxis üblich als Aufwendungen für Versicherungsfälle in die Berechnung eingehen ${ }^{816}$ In der Anfalljahressicht hingegen werden die internen Schadenregulierungskosten ihrem ökonomischen Charakter entsprechend als Betriebskosten ausgewiesen.

Der wesentliche Unterschied zwischen dem versicherungstechnischen Ergebnis auf Geschäftsjahresbasis und dem Anfalljahresergebnis besteht einerseits in dem Differenzbetrag zwischen der Rückstellung für GJ-Versicherungsfälle und der Bedarfsreserve und andererseits in der Einbeziehung der Abwicklungsergebnisse vorangegangener Anfalljahre.

Die Differenz entspricht demnach dem Saldo aus neu gelegten stillen Reserven und im Geschäftsjahr realisierten stillen Reserven in den VJ-Schadenrückstellungen. Die im simulierten Geschäftsjahr neu gelegten stillen Reserven entsprechen der positiven Differenz zwischen dem Stand der Rückstellung für GJ-Versicherungsfälle und der Bedarfsreserve des korrespondierenden Anfalljahres. Das Abwicklungsergebnis des simulierten Geschäftsjahres stellt die Summe sämtlicher Abwicklungsergebnisse vorangegangener Anfalljahre dar. Es entspricht demzufolge der Summe an stillen Reserven in den VJ-Schadenrückstellungen, die im simulierten Geschäftsjahr realisiert werden.

Vor dem Hintergrund der obigen Erläuterungen kann das versicherungstechnische Bruttoergebnis durch nachstehende Überleitungsrechnung aus dem Brutto-Anfalljahresergebnis hergeleitet werden. ${ }^{817}$

${ }^{816}$ Auf eine Umgliederung der primären Aufwandsarten nach Funktionsbereichen („Kostenverteilung") wird im Interesse einer transparenteren und willkürfreien Darstellung verzichtet. Vgl. zur Unterscheidung und kritischen Würdigung von Primär- und Sekundärprinzip FARNY (1992), S. 107 und KPMG (1994), S. 166-176.

817 Vgl. auch ALFERMANN/RADTKE/REICH, die bemerken, dass eine „Überführung der unter Underwriting-Gesichtspunkten entscheidungsrelevanten Anfalljahrergebnisse in die zugehörige bilanzwirksame Kalenderjahrperspektive [...] nach einer Schätzung der kompletten Abwicklungsstruktur leicht möglich“ ist. ALFERMANN/RADTKE/REICH (2004), S. 68. Die Überleitungsrechnung kann nach Berücksichtigung der Rückversicherung ebenfalls auf Nettobasis erfolgen. 
Anfalljahresergebnis ${ }^{\text {SimAJ }}$ brutto

- Dotierung d. Rückst. für GJ-Versicherungsfälle brutto

+ Bedarfsreserve ${ }^{\text {SimAJ }}$ brutto

+ Bedarfsreserve ${ }^{\text {SimAJ }}$ SRK brutto

- kalkulat. Zinsen aus der Anlage der Bedarfsreserve ${ }^{\text {SimAJ }}$

+ - Bruttoabwicklungsergebnis ${ }^{\text {SimGJ }}$

$=$ versicherungstechnisches Bruttoergebnis ${ }^{\text {SimGJ }}$

Nach Berücksichtigung des Rückversicherungsergebnisses, welches den Saldo aus RV-Prämien, RV-Provisionen, Recoveries und dem RV-Abwicklungsergebnis darstellt, verbleibt schließlich das versicherungstechnische Nettoergebnis des simulierten Geschäftsjahres. ${ }^{818}$

Die Darstellung der versicherungstechnischen Modellierung ist nunmehr abgeschlossen. Mit Prämien, Schäden und Kosten wurden die wesentlichen Komponenten des versicherungstechnischen Bruttoergebnisses schrittweise dargestellt und in einzelnen Teilmodellen abgebildet. Während die Prämien und Kosten deterministisch modelliert wurden, bildete die stochastische Schadensimulation eines Anfalljahres zweifellos den Schwerpunkt der bisherigen Modellbeschreibung. Für jedes einzelne Feld der SparteGeschäftsfeld-Matrix erfolgte eine separate Modellierung der Schadensumme, welche sich wiederum aus Basisschäden, Großschäden und ggf. Schäden aus Naturgefahrereignissen zusammensetzte. Im Rahmen des Rückversicherungsmodells wurde die zweidimensionale Segmentierung des versicherungstechnischen Bruttoportfolios auf die eindimensionale Spartensicht reduziert. Auf der Grundlage einer vorgegebenen Rückversicherungsstruktur wurden die simulierten (Einzel-) Schäden szenarioweise abgerechnet und in eine Nettobetrachtung überführt. Am Ende dieses Kapitels steht somit das versicherungstechnische Nettoergebnis, welches nachfolgend um den Ergebnisbeitrag der Kapitalanlagetätigkeit ergänzt wird.

${ }^{818}$ Da sonstige versicherungstechnische Ergebnisbeiträge nicht betrachtet werden, entspricht das versicherungstechnische Nettoergebnis des Geschäftsjahres der Zwischensumme I.9 gemäß Formblatt 2 der RechVersV. 


\subsection{Modellierung der Kapitalanlagetätigkeit}

\subsubsection{Vorüberlegungen}

Wie bereits in Kapitel 5.3.2 skizziert, kann das Gesamtergebnis eines Schaden- und Unfallversicherungsunternehmens in die Kernkomponenten „Versicherungstechnisches Ergebnis“ und „Kapitalanlageergebnis“ aufgespalten werden. ${ }^{819}$ Wenngleich im Rahmen dieser Arbeit unter Steuerungsaspekten primär das versicherungstechnische Portfolio im Mittelpunkt steht, ist für eine gesamthafte Erfassung des Unternehmensrisikos eine Auseinandersetzung mit dem Kapitalanlagerisiko unverzichtbar. Nachdem im vorangegangenen Abschnitt ausführlich auf die Modellierung des versicherungstechnischen Portfolios eingegangen wurde, wendet sich Kapitel 5.5 dem Kapitalanlageportfolio zu. Hinsichtlich der Modellierung von Kapitalmarktentwicklungen kann grundlegend zwischen deterministischen und stochastischen Investmentmodellen unterschieden werden. Deterministische Projektionen werden vielfach im Rahmen singulärer Szenarioanalysen oder aufsichtsrechtlich motivierter Stresstests durchgeführt und lassen lediglich kurzfristige Tendenzaussagen über die Risikopotenziale des Anlagebestandes zu. ${ }^{820}$ Darüber hinaus lassen sich den einzelnen Szenarien keine Eintrittswahrscheinlichkeiten zuordnen. Im Interesse einer differenzierten und objektivierbaren Risikoanalyse, die auf die Evaluation von Rendite-Risiko-Profilen abstellt, sollte daher ein stochastischer Ansatz gewählt werden.

Im Kontext stochastischer Investmentmodelle ${ }^{821}$ wird traditionell zwischen theoretischen (ökonometrischen) und atheoretischen (empirischen) Modellalternativen unterschieden. ${ }^{822}$ Ökonometrische Modelle fußen auf einer ökonomischen Theorie und stellen einen Erklärungsversuch empirisch beobachteter Zusammenhänge dar. ${ }^{823} \mathrm{Die} \mathrm{Pa-}$ rameterschätzungen basieren vielfach auf einem System interdependenter stochastischer Prozesse und rekursiven Mehrgleichungssystemen, die nur noch schwer zu handhaben sind. ${ }^{824}$

${ }^{819}$ Vgl. hierzu auch ALBRECHT (1995), S. 24 ff. Von der Existenz sonstiger Ergebnisbeiträge wird an dieser Stelle weiterhin abstrahiert. Vgl. BUSSON/RUB/ZWIESLER (2000), S. 104 und AlbRECHT (2001), S. 12-13.

${ }^{821} \mathrm{Vgl}$. zu prominenten stochastischen Investmentmodellen insbesondere WILKIE (1995), SMITH (1997), MULVEY/THORLACIUS (1998). Die Basisgrößen dieser Modelle werden zumeist kaskadenartig modelliert.

${ }^{822}$ Vgl. im Folgenden ausführlich EBERTS (2002), S. $47 \mathrm{ff}$.

${ }^{823}$ Von der Gültigkeit ökonometrischer Modelle wird so lange ausgegangen, bis sie aufgrund empirischer Beobachtungen falsifiziert werden. Vgl. zur erkenntnistheoretischen Philosophie des kritischen Rationalismus insbesondere POPPER (1963).

${ }^{824} \mathrm{Vgl}$. außerdem zu grundlegender Kritik an ökonometrischen Modellen LuCAS (1976). 
Demgegenüber versuchen empirische Modelle, auf der Grundlage explorativer Datenanalysen die zukünftige Kapitalmarktentwicklung möglichst gut zu approximieren. Die Zielsetzung besteht in einer plausiblen Fortschreibung der bisher beobachteten Renditeentwicklungen von Hauptanlageklassen sowie deren Interdependenzen. EBERTS weist darauf hin, dass ,sparsam parametrisierte Zeitreihenmodelle [...] oft bessere und robustere längerfristige Prognosen produzieren, insbesondere in der $\mathrm{zu}$ Prognosezwecken interessierenden Out-of-sample-Periode. ${ }^{\text {“825 }}$

Vor diesem Hintergrund und im Interesse einer vereinfachten Darstellung wird in dieser Arbeit zur Modellierung des Kapitalmarktes eine empirische Herangehensweise gewählt. Die erwarteten Renditen der betrachteten Hauptanlageklassen (Assetklassen) sowie deren Risikopotenzial ${ }^{826}$ werden über einen Mean-Variance-Ansatz abgebildet, der sich an der zweidimensionalen Rendite-Risikobetrachtung nach MARKOWITZ orientiert. ${ }^{827}$ Die zwischen den Assetklassen bestehende Abhängigkeitsstruktur wird über eine Korrelationsmatrix berücksichtigt.

An dieser Stelle sei darauf hingewiesen, dass das nachfolgend beschriebene Investmentmodell lediglich ein Beschreibungsmodell darstellt, welches nicht unreflektiert zu Optimierungszwecken im Sinne einer effizienten Portfolioselektion nach MARKOWITZ herangezogen werden kann. Die Betrachtung beschränkt sich zudem auf das Zinsänderungs- und Aktienkursrisiko in den Kapitalanlagen. Ausfall-, Währungs- und Liquiditätsrisiken werden vernachlässigt. ${ }^{828}$ Die laufenden Kosten für die Verwaltung der Kapitalanlagen werden in Prozent des durchschnittlichen Marktwertes einer jeden Assetklasse berücksichtigt. ${ }^{829}$ Die Vernachlässigung von Transaktionskosten erscheint unter der Annahme einer Buy-and-hold-Strategie, d.h. dass unterjährig keine spekulativen Umschichtungen im Kapitalanlagebestand erfolgen, vertretbar. ${ }^{830}$

${ }^{825}$ EBERTS (2002), S. 49. Vgl. auch die dort in Fußnote 129 angegebene Literatur.

${ }^{826}$ Der gängigen Praxis folgend wird als Risikomaß durchweg auf die Kennzahl der Volatilität abgestellt, ausgedrückt als geschätzte jährliche Standardabweichung der jeweils betrachteten Renditegtöße. Vgl. auch ALBRECHT/MAURER (2002), S. 106-108 und STEINER/BRUNS (2002), S. 58-624.

827 Vgl. MARKowitz (1952), EBERTS (2002), S. 21-25, SCHERER (2002), S. 93-126 und STEINER/ BRUNS (2002), S. 7-16. Vgl. zu einer ähnlichen Vorgehensweise auch WENGERT (2000), S. $121 \mathrm{ff}$.

${ }^{828}$ Vgl. hierzu vertiefend SPELLMANN (2002), S. 19-23, BROHM (2002), S 214-215 und BÄHRLE (1997), S. 21-24.

${ }^{829} \mathrm{Vgl}$. zu einer analogen Vorgehensweise HEINKE (2002a), S. 635. Aus Vereinfachungsgründen wird für alle Assetklassen ein einheitlicher Kostensatz von $0,3 \%$ p.a. gewählt. Die Kosten für die Verwaltung der Kapitalanlagen fallen annahmegemäß am Jahresende an.

${ }^{830}$ Vgl. übereinstimmend EBERTS (2002), S. 11. 


\subsubsection{Einperiodige vs. mehrperiodige Investmentmodelle unter Unsicherheit}

Das Risiko eines Finanzinvestments liegt in der ex-ante bestehenden Ungewissheit über dessen künftige Wertentwicklung begründet. ${ }^{831}$ Das bereits im Rahmen der versicherungstechnischen Modellierung verwendete Instrumentarium der Wahrscheinlichkeitstheorie kann ebenfalls zur Charakterisierung unsicherer Finanzinvestments herangezogen werden. In diesem Kontext ist grundlegend zwischen Einperioden- und Mehrperiodenmodellen $\mathrm{zu}$ unterscheiden. In einperiodigen Investmentmodellen sind Finanzinvestitionen durch einen Anlagehorizont von einer Periode charakterisiert. Während der Vermögenswert $V_{0}$ zu Beginn des Anlagehorizontes $t=0$ bekannt ist, stellt der Vermögensendwert $V_{1}$ am Ende des Anlagehorizontes $t=1$ eine Zufallsgröße dar. Die Einperiodenrendite R ergibt sich im zeitdiskreten Fall als:

$$
\begin{aligned}
& \mathrm{R}^{\text {disket }}=\frac{\mathrm{V}_{1}-\mathrm{V}_{0}}{\mathrm{~V}_{0}}=\frac{\mathrm{V}_{1}}{\mathrm{~V}_{0}}-1 \quad \text { bzw. als } \\
& \frac{\mathrm{V}_{1}}{\mathrm{~V}_{0}}=\left(1+\frac{\mathrm{R}^{\text {setetig }}}{\mathrm{n}}\right)^{\mathrm{n}} \stackrel{\mathrm{n} \rightarrow \infty}{\longrightarrow} \mathrm{e}^{\mathrm{R}^{\text {veiig }}} \Rightarrow \ln \left(\frac{\mathrm{V}_{1}}{\mathrm{~V}_{0}}\right)=\mathrm{R}^{\text {stetig }} \\
& \text { im zeitstetigen Fall. }
\end{aligned}
$$

Aufgrund der Unsicherheit bezüglich des Vermögensendwertes $V_{1}$ stellt auch $R$ eine zufallsabhängige Größe dar, deren Zufallsgesetzmäßigkeit im einperiodigen Kontext durch Verteilungsannahmen spezifiziert werden kann. Aufgrund ihrer einfachen analytischen Handhabbarkeit wird zur Darstellung zufallsabhängiger Renditen vielfach die Normalverteilung herangezogen. ${ }^{833}$ Der Wertebereich einer normalverteilten Zufallsgröße ist durch $(-\infty,+\infty)$ gegeben. Da Anlagerenditen jedoch über einen Wertebereich der Form $[-1, \infty)$ verfügen, kann der Ansatz einer Normalverteilung lediglich eine Approximation empirischer Renditen darstellen. ${ }^{834}$ Darüber hinaus sind Verteilungen empirischer Renditen häufig rechtsschief, so dass an Stelle der symmetrischen Normalverteilung vielfach auf die logarithmische Normalverteilung (Lognormalverteilung) zurückgegriffen wird. ${ }^{835}$

831 Vgl. im Folgenden ALBrechT/MaURER (2002), S. 89.

${ }^{832} \mathrm{Vgl}$. grundlegend zur Differenzierung zwischen diskreten und stetigen Renditen EBERTS (2002), S. 11-15 und ALBRECHT/MAURER (2002), S. 54-55.

833 Vgl. im Folgenden ALBRECHT/MAURER (2002), S. 95.

834 Von dem Spezialfall einer kreditfinanzierten Finanzinvestition wird an dieser Stelle abstrahiert.

${ }^{835}$ ALBRECHT/MAURER weisen darauf hin, dass die Normalverteilung insbesondere für kurze Zeitintervalle von ein bis drei Monaten als „akzeptable Approximation“ angesehen werden kann. Vgl. ALBRECHT/MAURER (2002), S. 95-97. 
In mehrperiodigen Investmentmodellen wird die Unsicherheit zukünftiger Vermögenswerte und daraus abgeleiteter Renditen durch stochastische Prozesse abgebildet. ${ }^{836}$ Bei diskreter Modellierung der Zeit stehen häufig die Random Walk-Hypothese oder Binomialgitterprozesse im Mittelpunkt. ${ }^{837}$ Die Wertentwicklung eines Finanztitels kann durch einen so genannten Random Walk mit Drift beschrieben werden, sofern folgende Eigenschaften erfüllt sind:

$$
\begin{aligned}
& V_{t}-V_{t-1}=d+\hat{Z}_{t} \\
& \operatorname{Cov}\left(\hat{Z}_{t}, \hat{Z}_{t-1}\right)=0 \\
& E\left(\hat{Z}_{t}\right)=0 \\
& \operatorname{Var}\left(\hat{Z}_{t}\right)=\sigma^{2}
\end{aligned}
$$

mit $d \in \Re$ und $t \geq 1$.

Die absolute Wertveränderung eines bestimmten Finanztitels kann dann durch eine zeitlich konstante Komponente (Driftparameter d) beschrieben werden, die durch einen Zufallsprozess $\left\{\hat{Z}_{t}: t \in \Re_{0}^{+}\right\}$überlagert wird. Die normalverteilten Zufallsgrößen $\hat{Z}_{\mathrm{t}}$ werden als zentriert, unkorreliert und identisch verteilt angenommen. Es wird zudem eine konstante Varianz unterstellt. ${ }^{838}$ Ausgehend von einem Startwert $v_{0}$ ergibt sich der zukünftige Wert einer Vermögensposition $V_{t}$ als:

$$
\mathrm{V}_{\mathrm{t}}=\mathrm{v}_{0}+\mathrm{dt}+\hat{\mathrm{Z}}_{1}+\ldots+\hat{\mathrm{Z}}_{\mathrm{t}}
$$

Unter Rückgriff auf die Beziehungen (5.61) und (5.62) folgt außerdem:

$$
\mathrm{E}\left(\mathrm{V}_{\mathrm{t}}\right)=\mathrm{v}_{0}+\mathrm{dt} \quad \text { und } \quad \operatorname{Var}\left(\mathrm{V}_{\mathrm{t}}\right)=\sigma^{2} \mathrm{t}
$$

Bei stetiger Zeitmodellierung wird häufig auf den verallgemeinerten Wiener Prozess zurückgegriffen, der auch als „Brownsche Bewegung“ bekannt ist. ${ }^{839}$ Der Wiener Prozess ist ein spezieller Markov-Prozess und wird durch die Parameter $\hat{\mu}$ (Driftkoeffizient) und $\hat{\sigma}^{2}$ (Diffusionskoeffizient) wie folgt charakterisiert:

836 Vgl. vertiefend WENGERT (2000), S. 122-123 und HULL (2003), S. 216-229.

837 Vgl. im Folgenden auch Albrecht/MAURER (2002), S. 137-143 und STEINER/BRUNS (2002), S. 227-228.

${ }^{838}$ Zufallsprozesse mit Zufallsgrößen $\hat{Z}_{t}(t \geq 1)$, die über die Eigenschaften (5.59) bis (5.62) verfügen, werden auch als White-Noise-Prozesse ("Weißes Rauschen") bezeichnet. Vgl. ALBRECHT/MAURER (2002), S. 140.

${ }^{839}$ Vgl. im Folgenden auch Hull (2003), S. 218-222, ALBRECHT/MAURER (2002), S. 144-152 und KÖNIG (2002a), S. 56-64. 


$$
W P_{t}=w_{0}+\hat{\mu} \cdot t+\hat{\sigma} \cdot \sqrt{t} \cdot Z
$$

Die Variable Z stellt in diesem Kontext eine standardnormalverteilte Zufallsgröße dar. Der verallgemeinerte Wiener Prozess verfügt über die so genannte MarkovEigenschaft. Demnach ist für die künftige (zufällige) Entwicklung einer Vermögensposition lediglich ihr aktueller Wert ausschlaggebend. ${ }^{840}$

Im weiteren Verlauf dieser Arbeit werden die Renditen der modellierten Hauptanlageklassen durchgängig über einen einperiodigen Verteilungsansatz abgebildet und verstehen sich demzufolge als diskrete Einjahresrenditen. Diese Vorgehensweise ist konsistent zum gewählten Modellhorizont eines (Anfall-) Jahres und bietet neben einer vereinfachten Darstellung den Vorteil, dass unterjährige Schwankungen von der Betrachtung ausgeschlossen werden.

\subsubsection{Buchwert- vs. Marktwertmodellierung}

In Abhängigkeit der eingenommenen Analyseperspektive können für den Modelladressaten entweder Marktwerte oder Buchwerte von Interesse sein. ${ }^{841}$ Eine praxistaugliche Modellierung sollte daher grundsätzlich die Abbildung beider Bewertungsmaßstäbe für Aktiva und Passiva unterstützen. Während sich Marktwerte im freihändigen Handel an Börsen- bzw. Marktplätzen und häufig unter Anwendung eines Barwertkalküls bilden, werden Buchwerte unter Berücksichtigung handels-, steuer- oder aufsichtsrechtlicher Vorschriften aus den zugehörigen Marktwerten abgeleitet. Es kommt daher vielfach zu Bewertungsdifferenzen, welche als stille Reserven bzw. stille Lasten bezeichnet werden. Aufgrund fehlender institutionalisierter Märkte ist die Ermittlung von Marktwerten für versicherungstechnische Verbindlichkeiten nur sehr eingeschränkt möglich. ${ }^{842}$ Nach geltendem Handelsrecht dürfen Schadenrückstellungen nicht auf ihren jeweiligen Zeit- bzw. Marktwert abgezinst werden, sondern müssen in Höhe der erwarteten Gesamtverpflichtung dotiert werden. ${ }^{843}$ Aufgrund des handelsrechtlichen Imparitätsprinzips und der fehlenden marktnahen Bewertung übersteigen die Buchwerte der Passiva eines Schaden- und Unfallversicherungsunternehmens daher in der Regel die zugehörigen Marktwerte bzw. Best-Estimate-Werte in erhebli-

\footnotetext{
${ }^{840} \mathrm{Vgl}$. AlBrecht/Maurer (2002), S. 146. Man spricht in diesem Zusammenhang auch von der "Gedächtnislosigkeit“" von Markov-Prozessen. Vgl. hierzu auch KÖNIG (2002a), S. 57.

841 Vgl. im Folgenden auch BROHM (2002), S. 215-219.

${ }^{842}$ Vgl. hierzu BROHM (2002), S. 217 und Rockel (2004), S. 63. Auf der Steuerungsebene wird durch die Diskontierung der Bedarfsreserve der Zeitwert der versicherungstechnischen Verbindlichkeiten des simulierten Anfalljahres näherungsweise ermittelt. Die Diskontierung lässt sich auf die versicherungstechnischen Verpflichtungen aus vorangegangenen Anfalljahren ausweiten, sofern hinreichend valide Abwicklungsmuster vorliegen.

${ }^{843}$ Vgl. § 253 Abs. 1 Satz 1 HGB und ergänzend Fußnote 786.
} 
chem Maße ${ }^{844}$ Insbesondere in Sparten mit langer Abwicklungsdauer können die stillen Reserven in den Schadenrückstellungen ein beträchtliches Ausmaß annehmen. ${ }^{845}$

Während auf der Kapitalanlageseite notwendigerweise Marktwertentwicklungen modelliert werden müssen, stellen im Rahmen der versicherungstechnischen Modellierung Buchwerte den Ausgangspunkt der Bewertung dar. Hieraus erwächst die generelle Anforderung, sowohl Markt- als auch Buchwerte durch das Modell bereitzustellen. ${ }^{846}$ EBERTS weist darauf hin, dass Marktwerte ,in einem stochastischen Investmentmodell unter strategischem Blickwinkel - gerade bei Gestaltungsfreiheiten auch betreffend der Passivseite - die betriebswirtschaftlich richtigen langfristigen ZielgröBen“ darstellen. ${ }^{847}$ Im Rahmen dieser Arbeit wird davon ausgegangen, dass nicht von dem in $\S 341 \mathrm{~b}$ HGB formulierten Wahlrecht Gebrauch gemacht wird und insofern das strenge Niederstwertprinzip gemäß $\S 253$ Abs. 3 HGB Anwendung findet. Der Buchwert am Ende der simulierten Bilanzperiode (= Geschäftsjahr) ergibt sich daher als Minimum aus dem Buchwert der vorangegangenen Periode und dem simulierten Marktwert. Hiervon unberührt bleiben so genannte „held-to-maturity-Papiere“ wie beispielsweise Namensschuldverschreibungen. Diese werden gemäß § 341c HGB zum Nennwert bilanziert. Dies bedeutet, dass für diese Wertpapiergattung ein Abschreibungsrisiko aufgrund gestiegener Marktzinsen nicht existent ist. ${ }^{848}$

\subsubsection{Ermittlung des Kapitalanlagevolumens und}

Integration des versicherungstechnischen Netto Cashflows

Das Kapitalanlagevolumen wird im Wesentlichen durch die Versicherungstechnik, d.h. die Art und Weise der Produktion von Versicherungsschutz induziert. Es kann daher auch als derivatives Portfolio bezeichnet werden ${ }^{849}$ Wie bereits in Kapitel 5.3.2 thematisiert, setzt sich der für Anlagezwecke nutzbare Zinsträger in der Bilanzperspektive aus Eigenkapital, den versicherungstechnischen Netto-Rückstellungen und den vorschüssig vereinnahmten Prämien zusammen. In der versicherungswirtschaftlichen Praxis weist somit der überwiegende Anteil des Anlagevolumens eine versicherungstechnische Herkunft auf. Der Kapitalanlagetätigkeit kommt einerseits die grundlegende Aufgabe zu, einen Beitrag zur sicheren Erfüllbarkeit der Verpflichtungen aus Versicherungsverträgen zu leisten. Andererseits sollte die Kapitalanlage innerhalb ei-

\footnotetext{
844 Vgl. BROHM (2002), S. 216.

${ }^{845} \mathrm{Vgl}$. auch die Ausführungen in Kapitel 6.3.2.

846 Vgl. Brohm (2002), S. 217, Albrecht (2001), S. 18 und JUNKER/SCHWARZ (2000), S. 1490.

847 EBERTS (2002), S. 10.

848 Vgl. vertiefend STEPHAN (1995), S. 60-61 und S. 183-194.

849 „Ohne das primäre Portefeuille des Bestandes an Versicherungsverträgen würde [...] das derivative Portefeuille des Kapitalanlagebestandes nicht bestehen." ALBRECHT (1995), S. 35.
} 
nes vorgegebenen Risikorahmens ein möglichst hohes Ergebnis erwirtschaften (Wettbewerbsaspekt) ${ }^{850}$ In der Literatur wird bisweilen argumentiert, dass zwischen der Entwicklung kapitalmarktspezifischer Einflussfaktoren (z.B. der Inflationsrate) und der Schadenentwicklung signifikante Abhängigkeiten bestehen. ${ }^{851}$ In Versicherungsunternehmen, die aufgrund ihrer Portfoliogröße und -struktur als hinreichend diversifiziert bezeichnet werden können, sind jedoch zumindest Zweifel angebracht, ob diese Abhängigkeiten ein signifikantes Ausmaß annehmen. ${ }^{852}$ Stärkere Bedeutung ist hingegen der Tatsache beizumessen, dass die Höhe des für Kapitalanlagezwecke verfügbaren Zinsträgers neben den versicherungstechnischen Netto-Rückstellungen auch wesentlich durch die Stochastizität der Schadenzahlungen beeinflusst wird. ${ }^{853} \mathrm{Im}$ Rahmen dieser Arbeit wird daher die Interaktion zwischen dem versicherungstechnischen Portfolio und dem Kapitalanlageportfolio ausschließlich über den versicherungstechnischen Netto Cashflow berücksichtigt. Dieser kann in Abhängigkeit des Schadenverlaufs positiv oder (z.B. im Fall von Naturkatastrophen) auch negativ ausfallen. Das in Kapitel 5.4.6 beschriebene Abwicklungsmodell dient in diesem Zusammenhang dem Zweck, den simulierten Schadenaufwand in eine zahlungswirksame Komponente (Schadenzahlungen) und eine nicht-zahlungswirksame Komponente (Schadenrückstellungen) zu überführen. Der versicherungstechnische Netto Cashflow lässt sich wie folgt ermitteln:

\begin{tabular}{ll} 
& Nettoprämien \\
- & Nettoschadenzahlungen für das SimAJ im SimGJ \\
- & Nettoschadenzahlungen für Vorjahre im SimGJ \\
\hline & Nettokosten $^{\text {SimGJ }}$ \\
\hline & versicherungstechnischer Netto Cashflow
\end{tabular}

Bezüglich der Zahlungszeitpunkte der einzelnen Cashflow-Komponenten müssen vereinfachende Annahmen getroffen werden, um die Interaktion zwischen Versicherungstechnik und Kapitalanlagetätigkeit im Modell handhabbar zu machen. ${ }^{854}$ Es wird unterstellt, dass die Nettoprämien in voller Höhe zu Beginn des betrachteten Geschäftsjahres zahlungswirksam vereinnahmt werden. Die Schadenzahlungen und Betriebskos-

$850 \mathrm{Vgl}$. ALBRECHT (1995), S. 36-37.

851 Vgl. stellvertretend ALBRECHT (1995), S. 40.

${ }^{852}$ Vgl. übereinstimmend SCHMEISER (1999), S. 91 und SANN (2001), S. 12. MAURER kommt in seiner empirischen Untersuchung für den deutschen Versicherungsmarkt sogar zu dem Ergebnis, „daß zwischen den Prämienrenditen [...] und den Renditen der angeführten Finanzmarktindizes kein Zusammenhang besteht und beobachtbare Korrelationen rein zufällig sind." MAURER (2000), S. 252.

${ }^{853}$ Vgl. für eine formale Darstellung dieses Zusammenhangs SCHMEISER (1999), S. 91. Vgl. hierzu außerdem MENTZEL (2004), S. 20 und HEINKE (2000), S. 630.

${ }^{854}$ Einen Überblick über die zeitliche Struktur des Cashflows vermittelt außerdem Abbildung 6.18. 
ten ${ }^{855}$ fallen annahmegemäß in der Mitte des Geschäftsjahres an. ${ }^{856}$ Die Nettoprämien stehen somit für 6 Monate als Zinsträger zur Verfügung. In Abhängigkeit des Schadenverlaufs kann der in der Mitte des Jahres fällige versicherungstechnische Netto Cashflow den Kapitalanlagebestand entweder erhöhen oder auch reduzieren. Es wird davon ausgegangen, dass ein positiver Cashflow bis zum Jahresende, d.h. für insgesamt 6 Monate in Geldmarkttitel oder wahlweise auch in andere Assetklassen investiert wird. Ein negativer Cashflow kann bis zum Jahresende über kurzfristige Fremdmittel (z.B. von Kreditinstituten) ausgeglichen werden. Es wird unterstellt, dass dieser kurzfristige Kredit ebenfalls zum Geldmarktzins aufgenommen werden kann. Die laufenden Erträge ${ }^{857}$ und Kosten der Kapitalanlagetätigkeit fließen in die so genannte Liquiditätskasse ein. ${ }^{858}$

Sollte am Ende des simulierten Geschäftsjahres nach Abzug von Steuern und Gewinnausschüttungen eine positive Liquiditätskasse für Anlagezwecke zur Verfügung stehen, wäre im mehrperiodigen Kontext eine Wiederanlageregel zu definieren. Vielfach wird im Sinne einer vereinfachten Managementregel davon ausgegangen, dass die Wiederanlage entweder die Ausgangsallokation repliziert oder im Sinne einer vorgegebenen Zielallokation umschichtet. ${ }^{859}$ Weist die Liquiditätskasse hingegen einen negativen Stand auf, ist durch das Management eine sinnvolle Liquidationsrangfolge festzulegen, die sich in der Regel an der Fristigkeit der einzelnen Assetklassen oder auch an der Höhe der stillen Reserven bzw. stillen Lasten orientiert. ${ }^{860}$ In einem ersten Schritt sollten dann Geldmarkttitel oder Anleihen mit kurzer Restlaufzeit herangezogen werden. Sollten diese Beträge nicht ausreichen, um den Liquiditätsbedarf zu decken, müssen in einem zweiten Schritt mittel- und langfristige Anleihen oder auch Aktien - gegebenenfalls unter Realisierung von Kursverlusten - liquidiert werden.

Im vorliegenden Modell setzt sich der Zinsträger zu Beginn des simulierten Geschäftsjahres aus dem bilanziellen Eigenkapital, den versicherungstechnischen Nettorückstel-

${ }^{855}$ Es wird hier unterstellt, dass sämtliche Betriebskosten in voller Höhe im Geschäftsjahr zahlungswirksam werden. Der hierzu korrespondierende bilanzielle „Aufwand für Versicherungsbetrieb“ enthält jedoch in der Regel auch nicht zahlungswirksame Komponenten (z.B. Aufwendungen für Altersversorgung). Es wäre daher auch denkbar, lediglich einen prozentualen Anteil der Betriebskosten als zahlungswirksam anzusetzen.

${ }^{856}$ Eine differenziertere zeitliche Erfassung der Zahlungsmuster, z.B. auf Monatsbasis, ist aus konzeptioneller Sicht wünschenswert. Unter Liquiditätsaspekten betrifft dies insbesondere auch zeitliche Eintrittsmuster für Großschäden und Naturkatastrophen.

${ }^{857}$ Im vorliegenden Fall setzen sich die laufenden Kapitalanlageerträge aus den zur Jahresmitte anfallenden Dividenden- und Kuponzahlungen und den Geldmarktzinsen am Jahresende zusammen.

858 Vgl. zu einer analogen Vorgehensweise HEINKE (2002b), S. 722.

859 Vgl. Heinke (2002a), S. 636, HeINKE (2002b), S. 722 und HofFMANN/SANN (2003b), S. 7. Vgl. zur Konzeption des „Rebalancing“ auch RAUSCHER/TEMPLER (2004).

${ }^{860}$ Vgl. auch HEINKE (2002a), S. 636. 
lungen des Vorjahres sowie den Prämien des simulierten Geschäftsjahres zusammen. Dieser Zinsträger wird in der Regel in eine Vielzahl von Einzeltiteln investiert. Im Interesse einer systematischen und risikotheoretisch motivierten Betrachtung sollte sich die Modellierung jedoch auf die Ebene der strategischen Asset Allocation beschränken. ${ }^{861}$ Die Einzeltitel werden daher zu so genannten Hauptanlageklassen bzw. Assetklassen verdichtet. Bei der Verdichtung ist zu berücksichtigen, dass die Einzeltitel innerhalb einer Assetklasse hinsichtlich ihres Rendite-Risiko-Profils, ihrer Fungibilität und Cashflow-Muster sowie ihrer bilanziellen Behandlung weitgehend homogen sind. Darüber hinaus sollten die Einzeltitel einer Assetklasse untereinander möglichst hoch korreliert sein und gegenüber anderen Assetklassen zumindest ähnliche Diversifikationseigenschaften besitzen. ${ }^{862}$ Im Rahmen dieser Arbeit wird in grober Einteilung zwischen den Hauptanlageklassen Aktien, Anleihen und Geldmarkt unterschieden. ${ }^{863}$ Diese Assetklassen zeichnen sich dadurch aus, dass sie standardisiert handelbar sind und demzufolge eine hinreichende Datenbasis zur Abschätzung der Renditeparameter zur Verfügung steht. Da der betrachtete Modellansatz unter der Zielsetzung entwickelt wird, die strategische Steuerung der Rendite-Risiko-Position zu unterstützen, erscheint eine Beschränkung auf drei Hauptanlageklassen zunächst vertretbar. ${ }^{864}$

\subsubsection{Modellierung der Hauptanlageklassen}

\subsubsection{Aktien}

Unter Aktien werden Teilhaberpapiere verstanden, die ein wirtschaftliches und rechtliches Eigentum an der emittierenden Aktiengesellschaft verbriefen. ${ }^{865}$ Im Gegensatz zu festverzinslichen Wertpapieren werden somit keine Forderungen gegenüber Emittenten, sondern ein Anteil am Gesellschaftsvermögen verbrieft. Bei der Investition in Aktien steht zumeist die Erzielung von Kursgewinnen im Vordergrund, um die im Vergleich zu festverzinslichen Wertpapieren zumeist niedrigeren (Dividenden-) Renditen überzukompensieren.

Der in dieser Arbeit verfolgte (portfolioorientierte) Ansatz geht davon aus, dass zwischen den Renditen einzelner Aktien eines Portfolios Interdependenzen bestehen. ${ }^{866}$

861 Vgl. zum Begriff der strategischen Asset Allocation und zur Einordnung in den Investmentprozess STEPHAN (1995), S. 3-14, EBERTS (2002), S. 7 und STEINER/BRUNS (2002), S. 90.

${ }^{862} \mathrm{Vgl}$. EBerts (2002), S. 8.

${ }^{863}$ Von der Investition in Immobilienwerte wird in dieser Arbeit abstrahiert. Vgl. hierzu vertiefend EBERTS (2002), S. 171-176 und HOPP (2001).

${ }^{864} \mathrm{Vgl}$. zur einer ähnlichen Vorgehensweise OSETROVA/SCHMEISER (2005), S. 20.

${ }^{865}$ Vgl. im Folgenden auch STEINER/Bruns (2002), S. 205 ff. und ALBRECHT/MAURER (2002), S. 19-21.

${ }^{866} \mathrm{Vgl}$. zur Differenzierung zwischen einzelwertorientierter und portfolioorientierter Aktienanalyse ausführlich STEINER/BRUNS (2002), S. 227-312. 
Die Erfassung sämtlicher Renditekorrelationen würde ein erhebliches Datenbeschaffungsproblem und eine nur schwer beherrschbare Komplexität mit sich bringen. Es bietet sich daher an, das Aktienportfolio durch einen möglichst marktbreiten Index zu approximieren und auf diese Weise die Aktienmarktentwicklung in nur einer einzigen Größe zu verdichten. Hiermit verbindet sich die grundlegende Annahme, ,dass Indexportfolios effizient sind, d.h. durch kein anderes Portefeuille von Titeln der gleichen Anlageklasse unter Renditeerwartung/Renditerisiko-Aspekten dominiert werden und sich gleichläufig zur Gesamtentwicklung der Asset-Klasse verhalten. ${ }^{\text {“867 }}$ In dieser Arbeit wird zur Modellierung des Aktienportfolios auf den Dow Jones EURO STOXX $50^{\mathrm{SM}}$ (kurz: Euro Stoxx) als Benchmark zurückgegriffen. Dieser Blue-Chip-Index enthält 50 führende europäische Börsenunternehmen und weist in Bezug auf die abgebildete Branchenstruktur einen hohen Diversifikationsgrad auf. ${ }^{868}$

Aktiengesellschaften können einen Teil der erwirtschafteten Gewinne in Form einer Dividende an ihre Aktionäre ausschütten. Demzufolge setzt sich die jährliche Gesamtrendite eines Aktieninvestments aus der Dividendenzahlung und der Kursveränderung im Jahresverlauf zusammen. Die Modellierung der Gesamtrendite kann z.B. unter Rückgriff auf einen Performanceindex bzw. Total-Return-Index erfolgen, welcher eine fiktive Reinvestition der zugeflossenen Dividenden mittels einer „Operation Blanche“ vornimmt. ${ }^{869}$ Für bestimmte Analysezwecke bietet es sich jedoch an, zwischen der eigentlichen Wertentwicklung und dem laufenden Einkommensrückfluss (= Dividende) zu differenzieren. ${ }^{870}$ Dieser Vorgehensweise wird auch in dieser Arbeit gefolgt. Im Hinblick auf die separate Modellierung von Kursveränderung und Dividendenstrom ist es nützlich, dass der Euro Stoxx börsentäglich sowohl als Performanceindex als auch als Kursindex ermittelt wird. Die Differenz zwischen beiden Indizes kann als Dividendenstrom interpretiert werden. ${ }^{871}$ Der Beobachtungszeitraum für die Abschätzung der erforderlichen Verteilungsparameter umfasst die Jahre 1991 bis $2003 .{ }^{872}$

${ }^{867}$ EBERTS (2002), S. 9. Vgl. außerdem übereinstimmend SCHERER (2002), S. 95

${ }^{868}$ Vgl. hierzu O.V. (2004), S. 11.

${ }^{869} \mathrm{Vgl}$. zu Konstruktionsprinzipien von Investmentindizes vertiefend ALBRECHT/MAURER (2002), S. 83-86. Im Rahmen einer „Operation Blanche“ wird der Barzufluss aus Dividenden oder Bezugsrechtsverkäufen unmittelbar zum Erwerb desselben Finanztitels verwendet. Vgl. auch ALBRECHT/MAURER (2002), S. 85.

${ }^{870}$ Vgl. JOHN (2002), S. 185. Dividendenzahlungen sind insbesondere für Lebensversicherungsunternehmen bedeutsam, da diese aufgrund des Gewinnversprechens gegenüber den Versicherungsnehmern auf hinreichend hohe laufende Erträge angewiesen sind. Vgl. WEIGEL (1991), S. 200. Vgl. auch STEPHAN (1995), S. 220-222.

${ }^{872}$ Der Zeitraum von 1991 bis 2003 wurde ausgewählt, da der Performanceindex des Dow Jones EURO STOXX $50^{\mathrm{SM}}$ erstmalig zum 31.12.1991 ermittelt wurde. 
Die Modellierung der einperiodigen Kursveränderung von Aktien erfolgt unter Rückgriff auf die Lognormalverteilung. Hiermit wird der bereits in Kapitel 5.5.2 beschriebenen Tatsache Rechnung getragen, dass empirische Renditeverteilungen tendenziell rechtsschief sind. ${ }^{873}$ Eine Zufallsvariable $\mathrm{X}>0$ ist lognormalverteilt, wenn ihr logarithmierter Wert einer Normalverteilung genügt, formal ausgedrückt: ${ }^{874}$

$$
\mathrm{X} \sim \mathrm{LN}\left(\mathrm{m}, v^{2}\right) \Leftrightarrow \ln \mathrm{X} \sim \mathrm{N}\left(\mathrm{m}, v^{2}\right)
$$

Die Dichtefunktion einer lognormalverteilten Zufallsvariable ist gegeben durch

$$
f(x)=\frac{1}{\sqrt{2 \pi} \cdot x \cdot v} \cdot e^{-\frac{(\ln x-m)^{2}}{2 v^{2}}} \quad \text { für } x>0
$$

Während unter einer Normalverteilungsannahme der Erwartungswert mit dem Median übereinstimmt, liegt der Erwartungswert der Lognormalverteilung aufgrund der Rechtsschiefe der Verteilung immer oberhalb des Medians. Die Schiefe einer Lognormalverteilung ist zudem immer größer als Null und nimmt mit steigendem $v^{2}$ zu (et vice versa). Die Parameter der Lognormalverteilung können durch die vorgegebenen bzw. empirisch ermittelten Werte von Erwartungswert und Varianz bestimmt werden als:

$$
\begin{aligned}
& v^{2}=\ln \left[1+\frac{\operatorname{Var}(X)}{E(X)^{2}}\right] \\
& \mathrm{m}=\ln \left[\frac{\mathrm{E}(\mathrm{X})^{2}}{\sqrt{\mathrm{E}(\mathrm{X})^{2}+\operatorname{Var}(X)}}\right]=\ln \mathrm{E}(\mathrm{X})-\frac{1}{2} v^{2}
\end{aligned}
$$

Unter der Annahme, dass der Aktienkurs $\mathrm{K}_{1}$ am Ende der Modellperiode lognormalverteilt ist, gilt dies auch für den zugehörigen Aufzinsungsfaktor $1+\mathrm{R}^{\text {Aktie }}: 875$

$$
1+\mathrm{R}^{\mathrm{Aktic}}=\frac{\mathrm{K}_{1}}{\mathrm{~K}_{0}} \sim \mathrm{LN}\left(\mathrm{m}_{1}, v^{2}\right)
$$

\footnotetext{
${ }^{873} \mathrm{Vgl}$. in diesem Zusammenhang vertiefend ALBRECHT/MAURER/RUCKPAUL (2001).

${ }^{874}$ Die folgenden Ausführungen lehnen sich an die Darstellungsweise in ALBRECHT/MAURER (2002), S. 95-96 an.

${ }^{875} \mathrm{Vgl}$. zur Herleitung dieser Aussage ALBRECHT/MAURER (2002), S. 95-96.
} 
Die Kursrendite $\mathrm{R}^{\text {Aktie }}$ genügt folglich einer um eine Einheit nach links verschobenen Lognormalverteilung. Auf diese Weise ist sichergestellt, dass der Wertebereich der modellierten Kursrenditen $(-1, \infty)$ beträgt. Sollen in umgekehrter Vorgehensweise die Parameter der Lognormalverteilung (5.71) von $1+\mathrm{R}^{\text {Aktie }}$ so festgelegt werden, dass Erwartungswert und Varianz von $\mathrm{R}^{\text {Aktie }}$ mit den entsprechenden Parametern der Normalverteilung identisch sind, d.h. $E\left(R^{\text {Aktie }}\right)=\mu$ und $\operatorname{Var}\left(R^{\text {Aktie }}\right)=\sigma^{2}$, so gelten folgende Beziehungen:

$$
\begin{aligned}
& v^{2}=\ln \left[1+\left(\frac{\sigma}{1+\mu}\right)^{2}\right] \\
& \mathrm{m}_{1}=\ln (1+\mu)-\frac{1}{2} v^{2}
\end{aligned}
$$

Die erforderlichen Werte für $\mu$ und $\sigma$ werden aus den Jahresultimoständen des Euro Stoxx Kursindex über den Zeitraum von 1991 bis 2003 geschätzt und betragen $\mu=0,07831$ bzw. $\sigma=0,15631$. Um den volatilitätsreduzierenden Einfluss von Sicherungsgeschäften zu berücksichtigen, wird an dieser Stelle unterstellt, dass das Modellunternehmen den Aktienbestand über Optionskontrakte gesichert hat. Stark vereinfachend wird angenommen, dass diese Optionskontrakte eine Reduzierung der Volatilität $\sigma$ um 10\%-Punkte bewirken und Sicherungskosten verursachen, die einer Renditeeinbuße von einem Prozentpunkt entsprechen. ${ }^{876}$ Unter Ausnutzung der Beziehungen (5.72) und (5.73) ergeben sich die Parameter der Lognormalverteilung zu $v^{2}=0,02079$ bzw. $\mathrm{m}_{1}=0,06499$. Um eine Vergleichbarkeit mit den normalverteilten Renditen herzustellen, ist die Dichtefunktion der Lognormalverteilung noch um -1 zu verschieben.

In Abbildung 5.18 sind die Dichtefunktionen der normalverteilten und lognormalverteilten Kursrenditen jeweils für $\mu=0,07831$ bzw. $\sigma=0,15631$ dargestellt. Aus der Gegenüberstellung der Dichtefunktionen wird ersichtlich, dass im Falle der Lognormalverteilung aufgrund ihrer Rechtsschiefe mehr als die Hälfte der Realisationen unter dem Erwartungswert in Höhe von $\mu=0,07831$ liegen. Die erwartete Kursrendite wird also mit einer Wahrscheinlichkeit von mehr als $50 \%$ nicht erreicht.

\footnotetext{
${ }^{876}$ Die unterstellte Sicherungsstrategie ist somit weitgehend einer so genannten Collar-Position vergleichbar. Zum Zwecke einer Verbilligung der Sicherungskosten besteht sowohl eine effektive Verlustbegrenzung (Long Put) als auch eine Gewinnbegrenzung (Short Call). Vgl. ALBRECHT/ MAURER (2002), S. 544-546 und ElLER/DEUTSCH (1998), S. 161-162.
} 


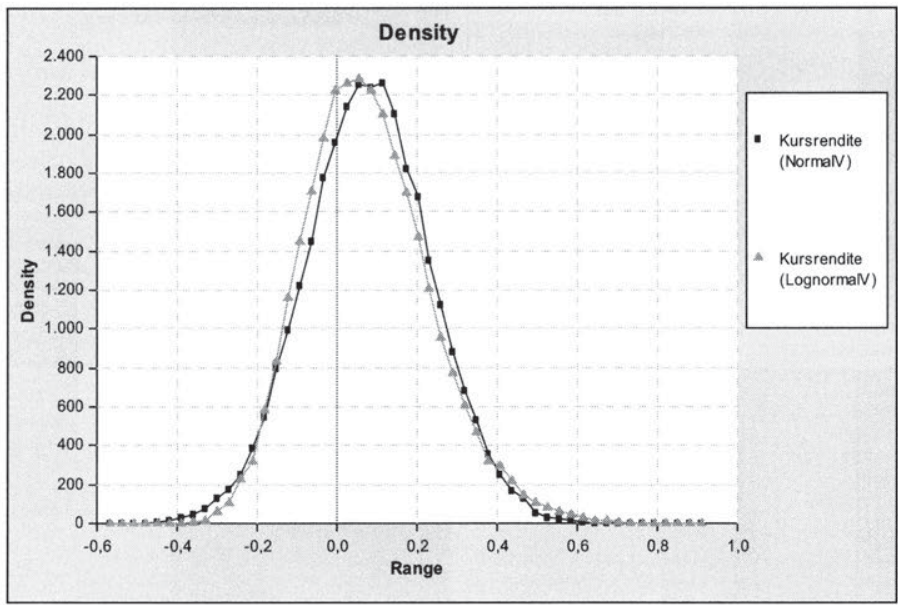

Abbildung 5.18: Dichtefunktionen normalverteilter und lognormalverteilter Aktienkursrenditen

Die Modellierung der Dividendenrendite erfolgt über einen Normalverteilungsansatz. Für jedes Beobachtungsjahr im Zeitraum von 1991 bis 2003 wird die Dividendenrendite $\mathrm{R}^{\text {Div }}$ näherungsweise als Differenz zwischen der Rendite des Performance- und Kursindexes berechnet. Aus dieser Zeitreihe wiederum können die Verteilungsparameter $\mu$ und $\sigma$ geschätzt werden. Die Dividendenzahlung ergibt sich schließlich durch Multiplikation der Dividendenrendite $\mathrm{R}^{\text {Div }}$ mit dem durchschnittlichen Marktwert des Aktienbestandes als:

$$
\text { Dividende }=\mathrm{R}^{\mathrm{Div}} \cdot \frac{\mathrm{MW}_{0}^{\text {Aktien }}+\mathrm{MW}_{1}^{\text {Aktien }}}{2}
$$

\subsubsection{Anleihen}

Unter Anleihen sollen im weiteren Verlauf festverzinsliche Wertpapiere verstanden werden. Diese lassen sich als Folge von vereinbarten Zins- und Tilgungszahlungen zu bestimmten Zeitpunkten charakterisieren. ${ }^{877}$ Im Gegensatz zu einem Aktieninvestment, im Rahmen dessen der Aktionär unsichere Rückflüsse in Form von Dividenden und Veräußerungserlösen erzielt, verfügt der Inhaber einer Anleihe über vertraglich fixierte, demnach auch der Höhe nach begrenzte Nominalansprüche gegenüber dem Emittenten. In den Kapitalanlageportfolios der deutschen Versicherungswirtschaft und im gesamtwirtschaftlichen Finanzierungsprozess nimmt die Gattung der festverzinsli-

877 Vgl. im Folgenden auch ALBRECHT/MAURER (2002), S. 337-434 und STEINER/BRUNS (2002), S. 135-204. 
chen Wertpapiere unverändert eine herausragende Stellung ein. ${ }^{878}$ Während im Segment lang laufender Anleihen der Rentenmarkt direkt mit dem Aktienmarkt konkurriert, ist im Bereich kurzer Restlaufzeiten der Übergang zwischen Rentenmarkt und Geldmarkt fließend. ${ }^{879}$ Zum Zwecke der Modellierung erscheint es sinnvoll, den Anleihebestand in verschiedene Restlaufzeitklassen aufzuteilen. Auf diese Weise kann sowohl der Zahlungsstrom aus Kupons und Tilgungen als auch der Einfluss des Zinsänderungsrisikos differenziert abgebildet werden. Im Rahmen dieser Arbeit wird der Anleihebestand in vier Restlaufzeitklassen aufgeteilt:

- Restlaufzeit $<1 \mathrm{Jahr}$

- Restlaufzeit 1 bis 3 Jahre

- Restlaufzeit 4 bis 8 Jahre

- $\quad$ Restlaufzeit $>8$ Jahre

In Abgrenzung hierzu ist die Assetklasse der Geldmarkttitel dadurch gekennzeichnet, dass die enthaltenen Papiere eine Restlaufzeit von durchschnittlich 3 Monaten aufweisen. ${ }^{880}$ Es wird ausschließlich der Währungsraum des Euro betrachtet und zudem unterstellt, dass die Anleihen keinerlei Ausfallrisiko unterliegen. ${ }^{881}$ Im Zuge einer Steigerung des Marktzinsniveaus sinkt grundsätzlich der Zeitwert (= Barwert) festverzinslicher Portfolios, da zukünftige Zahlungsströme wie Kupons oder Tilgungen mit einem höheren Marktzins diskontiert werden. Dieses Risiko wird auch als Zinsänderungsrisi$k o$ bezeichnet. ${ }^{882} \mathrm{Im}$ Vergleich zu Aktien fallen die Kursänderungen bei Anleihen zwar nicht so hoch aus, sie betreffen jedoch in der Regel alle im Bestand befindlichen Titel. Eine Diversifizierung des Zinsänderungsrisikos ist daher nicht möglich. ${ }^{883}$

Aufgrund des unterschiedlichen Bilanzausweises und der hohen praktischen Relevanz erscheint eine Differenzierung in Inhaber- und Namenspapiere zweckmäßig. ${ }^{884}$ Inhaberpapiere des Umlaufvermögens müssen nach dem strengen Niederstwertprinzip auf

${ }^{878}$ Vgl. EBERTS (2002), S. 166.

879 Vgl. übereinstimmend EBERTS (2002), S. 167-168 und STEINER/BRUNS (2002), S. 1.

${ }^{880}$ Vgl. im Einzelnen Kapitel 5.5.5.3.

${ }^{881}$ Diese Prämissen treffen näherungsweise auf Anleihen der öffentlichen Hand zu. Vgl. hierzu ausführlich WEIGEL (1991), S. 165-173. Vgl. zur Einbeziehung von Währungsrisiken STEPHAN (1995), S. 148-153.

${ }^{882} \mathrm{Vgl}$. STEINER/BRUNS (2002), S. 58. Gleichzeitig verbessern sich jedoch die Wiederanlagebedingungen für zukünftige Zinszahlungen. Vgl. zur Problematik der Wiederanlageprämisse im Rahmen von renditebasierten Selektionsentscheidungen ALBRECHT/MAURER (2002), S. 346-347.

${ }^{883}$ Vgl. WENGERT (2000), S. 33.

${ }^{884}$ Im Geschäftsjahr 2003 betrug der Buchwertanteil von Namenspapieren (Namensschuldverschreibungen, Schuldscheinforderungen und Darlehen) an den gesamten Kapitalanlagen der von der BAFIN beaufsichtigten deutschen Schaden- und Unfallversicherungsunternehmen $29,7 \%$. Vgl. BAFIN (2005), Tab. 510. 
ihren jeweiligen Marktwert abgeschrieben werden, falls dieser den Anschaffungswert unterschreitet. ${ }^{885}$ Namenspapiere hingegen werden gemäß $\S 341 \mathrm{c}$ HGB unabhängig von ihrer Zughörigkeit zum Anlage- oder Umlaufvermögen zum Nennwert bilanziert und unterliegen demzufolge keinem Abschreibungsrisiko. Dieser ausweistechnische Vorteil muss in der Regel durch einen leichten Renditeabschlag, vor allem jedoch durch eine weitgehende Illiquidität der Namenspapiere erkauft werden. ${ }^{886}$ Die Differenz zwischen dem finanzmathematisch ermittelten Zeitwert und dem Buchwert muss nicht im Anhang des Jahresabschlusses angegeben werden.

Im Zuge der Modellierung des Anleihebestands wird durchgängig von einer Buy-andHold-Strategie ausgegangen, d.h. sämtliche Anleihen werden bis zu ihrer Endfälligkeit im Portfolio gehalten. Darüber hinaus wird vereinfachend unterstellt, dass alle festverzinslichen Titel zu pari, also zu einem Kurswert von 100\% erworben wurden und auch Zukäufe zu pari erfolgen. ${ }^{887}$ Folglich stimmen die historischen Anschaffungskosten der Anleihen mit der Summe ihrer Nennwerte überein. Die Kuponzahlungen sind annahmegemäß zur Jahresmitte fällig und fließen in die bereits angesprochene Liquiditätskasse ein, die bis zum Jahresende in Geldmarkttitel investiert wird. Für die Modellierung der Kurs- bzw. Marktwerte sind Informationen über die aktuelle Renditestruktur und die durchschnittlichen Kupons der vier Restlaufzeitklassen erforderlich.

Der Marktwert MW ${ }^{\text {Anl }}$ einer Standardanleihe ${ }^{88}$ mit der Restlaufzeit $t \in\{1, \ldots, T\}$ ergibt sich unter Rückgriff auf das Present-Value-Konzept als Barwert der künftigen (konstanten) Kuponzahlungen $\hat{\mathrm{K}}$ und der Tilgungszahlung $\mathrm{Q} .{ }^{889}$

$$
\mathrm{MW}^{\mathrm{Anl}}=\hat{\mathrm{K}} \cdot \sum_{\mathrm{j}=1}^{\mathrm{t}} \mathrm{q}^{-\mathrm{j}}+\mathrm{Q} \cdot \mathrm{q}^{-\mathrm{t}} \quad \text { mit } \mathrm{q}=1+\mathrm{r}_{\mathrm{I}}
$$

${ }^{885}$ Inhaberpapiere des Anlagevermögens werden im Rahmen dieser Arbeit nicht modelliert, da sie hinsichtlich ihrer bilanziellen Behandlung den Namenspapieren vergleichbar sind. Bei Inhaberpapieren des Anlagevermögens kann das Versicherungsunternehmen gemäß $\S 341 \mathrm{~b}$ HGB auf eine Abschreibung verzichten, sofern die Wertminderung als vorübergehend einzuschätzen ist. Im Gegensatz zu Aktien ist diese Bedingung bei festverzinslichen Wertpapieren regelmäßig erfüllt, weil die endfällige Rückzahlung der Titel nicht durch eine Steigerung des Marktzinsniveaus gefährdet wird. Die unterlassenen Abschreibungen müssen im Anhang des Jahresabschlusses als stille Lasten angegeben werden. Vgl. HEISTERMANN (2004), S. 128.

${ }^{886} \mathrm{Vgl}$. im Folgenden HEISTERMANN (2004), S. 128.

${ }^{887} \mathrm{Vgl}$. zu einer analogen Vorgehensweise HEINKE (2002a), S. 635.

${ }^{888}$ Eine Standardanleihe ist gekennzeichnet durch periodische Zinszahlungen in konstanter Höhe und eine endfällige Tilgung. Vgl. AlBrecht/MAURER (2002), S. 338.

889 Vgl. Albrecht/Maurer (2002), S. 342-345 und Steiner/Bruns (2002), S. 140-143. 
Der Renditeparameter $r_{I}$ entspricht dabei der so genannten internen Rendite oder Yield-to-Maturity (YTM) ${ }^{890}$ Der funktionale Zusammenhang zwischen internen Renditen und der zugehörigen Restlaufzeit wird als Renditestrukturkurve bezeichnet. Diese wird im betrachteten Einperiodenmodell als statisch unterstellt und genügt nicht dem Kriterium der Arbitragefreiheit. ${ }^{891}$ Die Modellierung der Renditestrukturkurve erfolgt im Rahmen dieser Arbeit vereinfachend über einen Normalverteilungsansatz. Für jede der vier Anleiheklassen werden die mittlere Restlaufzeit und die durchschnittliche Kuponhöhe der enthaltenen Titel näherungsweise bestimmt. ${ }^{892}$ Als zugehöriger Renditeerwartungswert $\mu$ wird für jede Klasse die am Kapitalmarkt aktuell erzielbare Rendite für Staatsanleihen mit vergleichbarer Restlaufzeit herangezogen. Die Standardabweichung $\sigma$ der Marktrendite kann für jede Restlaufzeitklasse aus monatlichen Zeitreihen für Staatsanleiherenditen geschätzt werden. Für das Modellunternehmen wird eine normale Renditestrukturkurve zugrunde gelegt, welche durch nachfolgende Parameterkonstellationen charakterisiert ist.

\begin{tabular}{|c|c|c|c|c|c|c|c|}
\hline & $\begin{array}{c}\text { ( RLZ }{ }^{1)} \\
\text { [Jahre] }\end{array}$ & $\begin{array}{c}\text { Ø Kupon } \\
{[\%]}\end{array}$ & $\begin{array}{c}\mu \\
{[\%]}\end{array}$ & $\begin{array}{c}\sigma \\
{[\%]}\end{array}$ & $\begin{array}{c}\mathbf{R B F}{ }^{2 \text { ) }} \\
\text { (nachschüssig) }\end{array}$ & $\begin{array}{r}\text { Renditeko } \\
\text { Untergrenze }\end{array}$ & $\begin{array}{l}\text { rridor [\%] } \\
\text { Obergrenze }\end{array}$ \\
\hline $\mathrm{RLZ}<1 \mathrm{Jahr}$ & 1 & 2,50 & 2,66 & 0,78 & 1,93 & 0,5 & 15,0 \\
\hline RLZ 1-3 Jahre & 2 & 3,00 & 2,90 & 0,77 & 2,71 & 0,5 & 15,0 \\
\hline RLZ 4-8 Jahre & 6 & 4,00 & 3,85 & 0,73 & 3,85 & 0,5 & 15,0 \\
\hline RLZ > 8 Jahre & 10 & 4,90 & 4,79 & 0,69 & 4,79 & 0,5 & 15,0 \\
\hline
\end{tabular}

${ }^{1)}$ Restlaufzeit ${ }^{2)}$ Rentenbarwertfaktor

Tabelle 5.17: Parameter der Anleihemodellierung

Um zu gewährleisten, dass keine unrealistisch hohen Renditen simuliert und außerdem durch den Normalverteilungsansatz keine negativen Zinsszenarien erzeugt werden, wird ein zulässiger Renditekorridor definiert. ${ }^{893}$ Die Abweichungen zwischen dem mittleren Kuponzins und der erwarteten Marktrendite $\mu$ erklären sich dadurch, dass analog zur Versicherungstechnik ein ,historisch gewachsener Bestand“ betrachtet wird. In jeder Restlaufzeitklasse sind daher Anleihen mit unterschiedlicher Ursprungslaufzeit und unterschiedlichen Kuponhöhen enthalten. In jedem Jahr wechselt ein bestimmter Anteil der Anleihen in die angrenzende Restlaufzeitklasse. In einem mehrperiodigen Modell wäre daher zu definieren, welcher Anteil der Anleihen einer bestimm-

${ }^{890}$ Vgl. im Folgenden Albrecht/Maurer (2002), S. 342.

891 Vgl. vertiefend zur Annahme arbitragefreier Kapitalmärkte ALBRECHT/MAURER (2002), S. 175 ff. Im mehrperiodigen Kontext kann die Renditestruktur über arbitragefreie Einfaktormodelle erzeugt werden. Stellvertretend seien hier die Modelle von VASICEK und COX/INGERSOLL/ROsS genannt. Vgl. hierzu VASICEK (1977), COX/INGERSOLL/ROSS (1985a), COX/INGERSOLL/ROSS (1985b), WENGERT (2000), S. 127-130 und SCHÜRLE (1998), S. 117-124.

892 In diesem Kontext sind in der Praxis detaillierte Auswertungen des Anleiheportfolios erforderlich.

893 Diejenigen Renditerealisationen, welche nicht in den definierten Korridor fallen, werden verworfen und erneut simuliert (sog. „Accept-Bedingung“). 
ten Restlaufzeitklasse am Ende eines jeden Jahres in die angrenzende Klasse wechselt bzw. im Falle von Restlaufzeiten < 1 Jahr getilgt wird ${ }^{894}$ Im hier betrachteten Einperiodenmodell werden sämtliche Anleihen mit einer Restlaufzeit bis zu einem Jahr am Ende der Modellperiode fällig. Die Tilgungsbeträge fließen in die Liquiditätskasse ein.

\subsubsection{Geldmarkttitel}

In Abgrenzung zu mittel- und langfristigen Kapitalmarkttiteln stellen Geldmarktpapiere kurzfristige Finanztitel mit einer Laufzeit bis zu einem Jahr dar. ${ }^{895}$ Man unterscheidet in diesem Zusammenhang den Geldmarkt im engeren Sinne, an dem Kreditinstitute und die Zentralinstitute (z.B. Europäische Zentralbank, Deutsche Bundesbank) teilnehmen, und den Geldmarkt im erweiterten Sinne, an dem auch institutionelle Anleger, wie z.B. Versicherungsunternehmen partizipieren. Geldmarktgeschäfte dienen in Versicherungsunternehmen vorrangig dem innerbetrieblichen Liquiditätsmanagement und der gezielten Ertragsoptimierung der Zahlungsströme.

Durch die Einführung des Euro wurde sowohl die Markttiefe als auch die Breite des Geldmarktes signifikant erhöht. ${ }^{896}$ Neben den klassischen Geldmarkttiteln wie Tagesund Festgeldern stehen den Marktteilnehmern nunmehr mit verbrieften Anlagen wie Commercial Papers, Certificates of Deposit oder Geldmarktfonds erweiterte Anlagemöglichkeiten zur Verfügung. Ein Großteil der durch Versicherungsunternehmen gehaltenen Liquidität wird jedoch nach wie vor in Einlagen bei Eurobanken angelegt. Die aufsichtsrechtliche Zulässigkeit dieser Geschäfte ergibt sich aus § 54a Abs. 2 Nr. 9c VAG. ${ }^{897}$

Im Rahmen dieser Arbeit wird unterstellt, dass die durchschnittliche Laufzeit der Geldmarkttitel 3 Monate beträgt. Die Modellierung erfolgt erneut über einen Normalverteilungsansatz. Als Erwartungswert $\mu$ wurde die zum Zeitpunkt der Modellierung gültige Rendite des 3-Monats-EURIBOR herangezogen. Die Standardabweichung $\sigma$ wurde aus den Monatsultimoständen des 3-Monats-EURIBOR über einen Zeitraum von 2001 bis 2004 geschätzt. Analog zur Modellierung der Anleiherenditen wird auch für die Simulation der Geldmarktrenditen ein entsprechender (positiver) Renditekorridor vorgegeben (vgl. Tabelle 5.19).

\footnotetext{
${ }^{894}$ Vgl. hierzu HeINKE (2002a), S. 635.

895 Vgl. im Folgenden PERridon/STEINER (2004), S. 169-170 und AlbreCHT/MAURER (2002), S. 27-28.

896 Vgl. auch KRÜGER/WEHLING (2001), S. 194-201.

897 Vgl. hierzu vertiefend KRÜGER/WEHLING (2001), S. 192-194.
} 


\subsubsection{Abbildung der Korrelationsstruktur zwischen den Hauptanlageklassen}

Für das Risiko eines Gesamtportfolios ist neben der Aufteilung des Kapitalanlagevolumens auf die einzelnen Assetklassen insbesondere der Grad der Gleich- bzw. Gegenläufigkeit der einzelnen Assetklassenrenditen von zentraler Bedeutung. ${ }^{898}$ Die von MARKOWITZ für einen einperiodigen Modellkontext formulierte Portfoliotheorie gelangt zu der Erkenntnis, dass das Risiko einer Gesamtposition nicht der Summe der Risiken der Einzelpositionen entspricht. ${ }^{899}$ Der Diversifikationseffekt fällt umso größer aus, je weniger die Renditen der einzelnen Assetklassen miteinander korreliert sind. Grundsätzlich beschränkt sich das Diversifikationspotenzial auf den unsystematischen Teil des Gesamtrisikos bzw. Marktrisikos. ${ }^{900}$ So genannte systematische, d.h. marktinhärente Risiken sind hingegen nicht diversifizierbar. Als Messgröße für den Grad der linearen Koppelung der Renditen wird in der Regel der Korrelationskoeffizient $\rho$ herangezogen. ${ }^{901}$ Bezeichnen $\mathrm{X}$ und $\mathrm{Y}$ die zufallsabhängigen Renditen zweier Assetklassen, so ist $\rho$ definiert als:

$$
\rho(\mathrm{X}, \mathrm{Y})=\frac{\operatorname{Cov}(\mathrm{X}, \mathrm{Y})}{\sigma(\mathrm{X}) \sigma(\mathrm{Y})}=\frac{\mathrm{E}[(\mathrm{X}-\mathrm{E}(\mathrm{X}))(\mathrm{Y}-\mathrm{E}(\mathrm{Y}))]}{\sigma(\mathrm{X}) \sigma(\mathrm{Y})} \quad \rho \in[-1,1]
$$

Soll der lineare Zusammenhang zweier zufallsabhängiger Renditegrößen X und Y zum Zweck der Parameterschätzung aus einer Stichprobe unabhängiger Beobachtungen $\mathrm{x}_{1}, \ldots, \mathrm{x}_{\mathrm{n}}$ und $\mathrm{y}_{1}, \ldots, \mathrm{y}_{\mathrm{n}}$ ermittelt werden, kann der Korrelationskoeffizient $\rho^{\mathrm{P}}(\mathrm{x}, \mathrm{y})$ nach PEARSON als verteilungsunabhängiger Schätzer verwendet werden. Dieser ist definiert als: ${ }^{902}$

$$
\begin{aligned}
& \rho^{p}(x, y)=\frac{\sum_{i=1}^{n}\left(x_{i}-\bar{x}\right)\left(y_{i}-\bar{y}\right)}{\sqrt{\sum_{i=1}^{n}\left(x_{i}-\bar{x}\right)^{2} \sum_{i=1}^{n}\left(y_{i}-\bar{y}\right)^{2}}} \\
& \text { mit } \quad \bar{x}=\frac{1}{n} \sum_{i=1}^{n} x_{i} \text { und } \bar{y}=\frac{1}{n} \sum_{i=1}^{n} y_{i}
\end{aligned}
$$

898 Vgl. im Folgenden ALBRECHT/MAURER (2002), S. 71.

899 Vgl. MARKOWITZ (1952). Die Portfoliotheorie bezieht sich ursprünglich auf Einzelinvestments. Eine Übertragung auf aggregierte Assetklassen ist jedoch möglich und angesichts des erheblichen Umfangs der erforderlichen Inputdaten sogar zu empfehlen. Vgl. ALBRECHT (1995), S. 70 und EBERTS (2002), S. 23.

900 Vgl. im Folgenden auch SteINER/BRUNS (2002), S. 56-57 und PERRIDON/STEINER (2004), S. 281.

901 Vgl. im Folgenden AlBrECHT/MAURER (2002), S. 97-102 und STEINER/BRUNS (2002), S. 70-72.

902 Eine einfache, auf Plausibilität gestützte Herleitung des Korrelationskoeffizienten liefert NEUBAUER (1994), S. 251-253. 
In dieser Arbeit wird die Korrelationsstruktur der einzelnen Assetklassenrenditen unter Verwendung des Korrelationskoeffizienten nach PEARSON aus historischen Zeitreihen ermittelt bzw. öffentlich verfügbaren Datenquellen wie RiskMetrics ${ }^{\mathrm{TM}}$ abgeleitet. $^{903}$ Im Rahmen der stochastischen Modellierung der Kapitalanlageentwicklung wird unterstellt, dass die ermittelte Korrelationsmatrix der Assetklassenrenditen für alle Simulationen konstant bleibt (vgl. Tabelle 5.18). ${ }^{904}$

\begin{tabular}{|l|c|c|c|c|c|c|c|}
\hline & $\begin{array}{c}\text { Kursrendite } \\
\text { Aktien }\end{array}$ & $\begin{array}{c}\text { Dividenden- } \\
\text { rendite }\end{array}$ & $\begin{array}{c}\text { Anleihen } \\
\text { RLZ < 1 J. }\end{array}$ & $\begin{array}{c}\text { Anleihen } \\
\text { RLZ 1-3 J. }\end{array}$ & $\begin{array}{c}\text { Anleihen } \\
\text { RLZ 4-8 J. }\end{array}$ & $\begin{array}{c}\text { Anleihen } \\
\text { RLZ }>8 \text { J. }\end{array}$ & $\begin{array}{c}\text { Geldmarkt } \\
\text { RLZ Ø 3 M. }\end{array}$ \\
\hline Kursrendite Aktien & 1,00 & & & & & & \\
\hline Dividendenrendite & 0,56 & 1,00 & & & & & \\
\hline Anleihen RLZ < 1 J. & 0,47 & 0,11 & 1,00 & & & & \\
\hline Anleihen RLZ 1-3 J. & 0,45 & 0,25 & 0,95 & 1,00 & & & \\
\hline Anleihen RLZ 4-8 J. & 0,38 & 0,28 & 0,75 & 0,72 & 1,00 & & \\
\hline Anleihen RLZ > 8 J. & 0,31 & 0,31 & 0,54 & 0,64 & 0,79 & 1,00 & \\
\hline Geldmarkt RLZ Ø 3 M. & 0,49 & 0,10 & 0,94 & 0,72 & 0,75 & 0,52 & 1,00 \\
\hline
\end{tabular}

Tabelle 5.18: Korrelationsmatrix der modellierten Assetklassen

Exemplarisch ergibt sich zwischen der Aktienkursrendite und der Dividendenrendite ein Korrelationskoeffizient $\rho^{P}$ von $0,56 .{ }^{905}$ Die Renditen der betrachteten Assetklassen werden durchgängig als positiv korreliert unterstellt. Da jedoch keine vollständigen Korrelationen $(\rho=1)$ vorliegen, können durch alternative Mischungsverhältnisse der Assetklassen unterschiedliche Rendite-Risiko-Positionen realisiert bzw. Diversifikationspotenziale erschlossen werden. ${ }^{906}$

Für einen $\mu$ - $\sigma$-rationalen Investor kommen nur solche Portfoliozusammensetzungen in Frage, die im Sinne der Portfoliotheorie effizient sind, d.h. die bezüglich ihrer RenditeRisiko-Charakteristik nicht von anderen Portfolios dominiert werden. ${ }^{907}$ Bei Umschichtungen im Kapitalanlagebestand ist grundsätzlich zu beachten, dass sowohl aufsichtsrechtliche als auch interne Restriktionen minimale und maximale ExposureGrenzen vorgeben.

903 Vgl. zu Risk Metrics ${ }^{\text {TM }}$ vertiefend KÖNIG (2002b), S. 243-254, MINA/YI XIAO (2001) und JPMORGAN/REUTERS (1996).

904 Vgl. zu einer übereinstimmenden Vorgehensweise SCHMEISER (2004a), S. 46, HEINKE (2002a), S. 636, WENGERT (2000), S. 163-165 und CORELL (1998b), S. 68.

${ }_{905}$ Der Berechnung liegt erneut der Beobachtungszeitraum von 1991 bis 2003 zugrunde.

906 Vgl. vertiefend ALBRECHT (1995), S. 70-96.

${ }^{907}$ In der Versicherungspraxis wird die Realisierung effizienter Portfolios vielfach durch aufsichtsrechtliche Kapitalanlagevorschriften und Restriktionen erschwert. Vgl. hierzu auf die Ausführungen in Kapitel 2.2.3.2. Dieser normative Rahmen führt dazu, dass ,nur noch eine Teilmenge der prinzipiell erreichbaren Portefeuilles realisiert werden darf [...].“ ALBRECHT (1995), S. 88. 
Die für das Modellunternehmen gewählte Ausgangsallokation der Kapitalanlagen sowie die erwarteten Rendite- und Kostenparameter der einzelnen Assetklassen können abschließend Tabelle 5.19 entnommen werden. ${ }^{908}$

\begin{tabular}{|c|c|c|c|c|c|c|c|}
\hline & \multicolumn{2}{|c|}{ Allokation (Buchwerte) } & \multicolumn{2}{|c|}{ Renditeparameter } & \multicolumn{2}{|c|}{ Renditekorridor } & \multirow{2}{*}{$\begin{array}{c}\text { Kostensatz } \\
{[\%]} \\
\end{array}$} \\
\hline & [Mio. GE] & {$[\%]$} & $\mu$ & $\sigma$ & Untergrenze & Obergrenze & \\
\hline Kursrendite Aktien & \multirow{2}{*}{63,9} & \multirow{2}{*}{$20 \%$} & $7,83 \%$ & $15,63 \%$ & - & - & \multirow{2}{*}{$0,3 \%$} \\
\hline Dividendenrendite & & & $2,47 \%$ & $1,26 \%$ & - & - & \\
\hline Anleihen RLZ < $1 \mathrm{~J}$. & 63,9 & $20 \%$ & $2,66 \%$ & $0,78 \%$ & $0,5 \%$ & $15,0 \%$ & $0,3 \%$ \\
\hline Anleihen RLZ 1-3 J. & 63,9 & $20 \%$ & $2,90 \%$ & $0,77 \%$ & $0,5 \%$ & $15,0 \%$ & $0,3 \%$ \\
\hline Anleihen RLZ 4-8 J. & 63,9 & $20 \%$ & $3,85 \%$ & $0,73 \%$ & $0,5 \%$ & $15,0 \%$ & $0,3 \%$ \\
\hline Anleihen RLZ > $8 \mathrm{~J}$. & 47,9 & $15 \%$ & $4,79 \%$ & $0,69 \%$ & $0,5 \%$ & $15,0 \%$ & $0,3 \%$ \\
\hline Geldmarkt RLZ Ø $3 \mathrm{M}$. & 15,9 & $5 \%$ & $2,48 \%$ & $0,79 \%$ & $0,5 \%$ & $15,0 \%$ & $0,3 \%$ \\
\hline Summe & 319,4 & $100 \%$ & & & & & \\
\hline
\end{tabular}

Tabelle 5.19: Ausgangsallokation der Kapitalanlagen, Rendite- und Kostenparameter

Aufgrund der Verdichtung auf lediglich drei Hauptanlageklassen entspricht die aus Tabelle 5.19 ersichtliche Ausgangsallokation naturgemäß nicht mehr exakt der Kapitalanlagestruktur des zugrunde liegenden Originalunternehmens. Die Aufteilung des Kapitalanlagebestandes auf Aktien, festverzinsliche Wertpapiere und Geldmarkttitel kann jedoch näherungsweise als marktüblich bezeichnet werden, so dass auch an dieser Stelle zumindest eine strukturelle Repräsentativität für den deutschen Schaden- und Unfallversicherungsmarkt gewahrt bleibt.

\subsubsection{Zentrale Ergebnisgrößen der Kapitalanlagetätigkeit}

In Analogie zum versicherungstechnischen Portfolio muss auch für die Beurteilung der Kapitalanlagetätigkeit eine geeignete Ergebnisgröße definiert werden. Hierzu können in Abhängigkeit von der Zielsetzung grundsätzlich unterschiedliche Kennziffern in Betracht gezogen werden. ${ }^{909}$ Im Rahmen dieser Arbeit wird das Kapitalanlagerisiko definiert als die Unsicherheit in Bezug auf den im simulierten Jahr erzielbaren Total Return aus Kapitalanlagetätigkeit, mithin als die Gefahr einer negativen Marktwertveränderung des Kapitalanlagebestandes. ${ }^{910}$

908 Die Kosten der Kapitalanlageverwaltung sind unter Steuerungsaspekten aufgrund ihres hohen Fixkostenanteils und ihrer limitierten Beeinflussbarkeit durch das Management nur von untergeordneter Bedeutung. Sie werden für jede Hauptanlageklasse vereinfachend als Prozentsatz des Buchwertes angegeben.

909 Vgl. ausführlich zur versicherungsspezifischen Unterscheidung von bilanzorientierten und marktwertorientierten Zielsetzungen im Rahmen des Kapitalanlage-Controllings GRITZMANN (1998), S. 42-61.

910 Eine ebenfalls in der Praxis diskutierte Bezugsgröße ist die Differenz zwischen bilanziellem Kapitalanlageergebnis bzw. Total Return und der risikolosen Anlage des gesamten Zinsträgers. Diese Vorgehensweise trägt dem Opportunitätsgedanken Rechnung. 
Das bilanzielle Ergebnis aus Kapitalanlagen ist aufgrund der durch das Handelsrecht zur Verfügung gestellten Ansatz- und Bewertungswahlrechte zur Beurteilung des Erfolgs der Kapitalanlagetätigkeit und zu Steuerungszwecken ungeeignet. ${ }^{911}$ Der Total Return hingegen misst die Veränderung des Marktwertes des gesamten Kapitalanlageportfolios und stellt somit die ökonomisch relevante Bezugsgröße dar. Aufgrund des stochastischen Charakters der einzelnen Assetklassenrenditen ist der Total Return ebenfalls eine Zufallsvariable, welche im vorliegenden Fall wie folgt ermittelt werden kann:

\begin{tabular}{ll} 
& Dividendenerträge \\
+ & Kuponerträge aus Anleihen \\
+ & Zinserträge aus Geldmarkttiteln (inkl. Liquiditätskasse) \\
$=$ & lfd. Erträge aus Kapitalanlagen \\
$=$ & lfd. Aufwendungen für die Kapitalanlageverwaltung \\
\hline$=$ & lfd. Ergebnis aus Kapitalanlagen \\
+ & Zuschreibungen \\
- & Abschreibungen \\
\hline$=$ & bilanzielles Ergebnis aus Kapitalanlagen \\
$+/-$ & Veränderung der Bewertungsreserven \\
$=$ & Total Return aus Kapitalanlagen
\end{tabular}

Für Kapitalgesellschaften gilt nach $\S 280$ Abs. 1 HGB ein Wertaufholungsgebot für alle außerplanmäßigen Abschreibungen bei Wegfall der Gründe. ${ }^{912}$ Die fortgeführten Anschaffungs- und Herstellungskosten bilden dabei die Obergrenze für die Zuschreibungen. Da im Rahmen dieser Arbeit vereinfachend davon ausgegangen wird, dass die Buchwerte der einzelnen Assetklassen ihren historischen Anschaffungskosten entsprechen, können Zuschreibungen per definitionem nicht auftreten. Aufgrund des postulierten strengen Niederstwertprinzips konkretisieren sich Kursrückgänge nicht in einem Aufbau von stillen Lasten, sondern werden als Abschreibungen unmittelbar erfolgswirksam. Kurssteigerungen führen hingegen zu einem Aufbau von Bewertungsreserven. Dies hat insbesondere in Bezug auf den Aktienbestand zur Folge, dass in das laufende Ergebnis aus Kapitalanlagen lediglich die Dividenden einfließen und außerordentliche Ergebnisbeiträge in Form realisierter Kursgewinne nicht auftreten.

911 Vgl. übereinstimmend CORELL (1999), S. 1152, FORST, VON DER (1998), S. 91-93 und GRITZMANN (1998), S. 46-52. Insbesondere ergeben sich bilanzielle Gestaltungsspielräume durch die gezielte Steuerung von Ausschüttungen aus Fondsvermögen. Vgl. in diesem Zusammenhang detailliert HEINKE (2000), S. 633-636.

912 In Verbindung mit $\S 6$ Abs. $1 \mathrm{Nr} .1$ Satz 4 und $\S 6$ Abs. 1 Nr. 2 Satz 3 EStG wird aus dem Zuschreibungswahlrecht ein striktes Wertaufholungsgebot, sofern der Steuerpflichtige nicht nachweisen kann, dass eine voraussichtlich dauernde Wertminderung vorliegt. Vgl. auch BAETGE/KIRSCH/ THIELE (2003), S. 236-238. 
Unter der Annahme, dass unterjährig keine Umschichtungen in den Kapitalanlagen vorgenommen werden, setzt sich das bilanzielle Kapitalanlageergebnis daher im hier betrachteten Modellkontext aus dem laufenden Kapitalanlageergebnis abzüglich Abschreibungen zusammen.

Um den ökonomischen Erfolg der Kapitalanlagetätigkeit anhand ihres Total Returns bewerten zu können, muss das bilanzielle Kapitalanlageergebnis noch um die Veränderung der Bewertungsreserven korrigiert werden. Auf diese Weise wird sichergestellt, dass die Ergebnisgröße des Total Return exakt der Marktwertveränderung des Kapitalanlageportfolios entspricht. Der Quotient aus Total Return und dem korrespondierenden durchschnittlichen Kapitalanlagevolumen zu Marktwerten wird auch als Kurswert- bzw. Marktwertrendite bezeichnet.

\subsection{Integration der Teilmodelle in ein Gesamtmodell}

In den vorangegangenen Kapiteln 5.4 und 5.5 wurde die Abbildung der einzelnen Ergebniskomponenten eines Schaden- und Unfallversicherungsunternehmens in separaten Teilmodellen vorgestellt. Es wurde deutlich, dass sich das Gesamtergebnis in das versicherungstechnische Ergebnis und das Ergebnis der Kapitalanlagetätigkeit aufspalten lässt. Hinsichtlich des modellierten Zeithorizonts kann ferner zwischen der risikoorientierten Anfalljahressicht und der handelsrechtlich motivierten Geschäftsjahressicht unterschieden werden. Zum Abschluss der Modellbeschreibung werden die bisher isoliert beschriebenen Teilmodelle zu einem Gesamtmodell zusammengeführt und der Simulationsablauf im Überblick beschrieben.

In Abbildung 5.19 wird zunächst die Ermittlung des Gesamtergebnisses eines Anfalljahres schematisch dargestellt. Ausgehend von einer geeigneten Segmentierung des versicherungstechnischen Gesamtportfolios in weitgehend homogene Teilkollektive erfolgt im Bruttomodell für jedes Feld der Sparte-Geschäftsfeld-Matrix eine separate Modellierung des Brutto-Anfalljahrergebnisses. Während die Ergebniskomponenten Prämien, Betriebskosten und Provisionen als deterministische Größen in die Modellierung einfließen, wird der Schadenaufwand mittels stochastischer Simulation erzeugt. Auf der Grundlage von Bestandsinformationen und empirisch geschätzten Schadenparametern werden für jedes Teilkollektiv sowohl die Summe der Basisschäden (Basisschadenlast) als auch einzelne Großschäden generiert. Die Modellierung von Naturgefahrereignissen erfolgt in einem separaten Teilmodell. Um zu gewährleisten, dass ein Naturereignis simultan, d.h. innerhalb desselben Simulationslaufs, auf die betroffenen Sparte-Geschäftsfeld-Kombinationen wirkt, werden die erzeugten Sturm-, Hagel- und Überschwemmungsschäden je Simulationslauf auf die Teilkollektive verteilt. 
Aus der Aggregation von Bruttoprämien, Bruttobetriebskosten, Provisionen und Bruttoschadenaufwand resultiert schließlich ein Set von $n=30.000$ Realisationen des Brutto-Anfalljahrergebnisses.

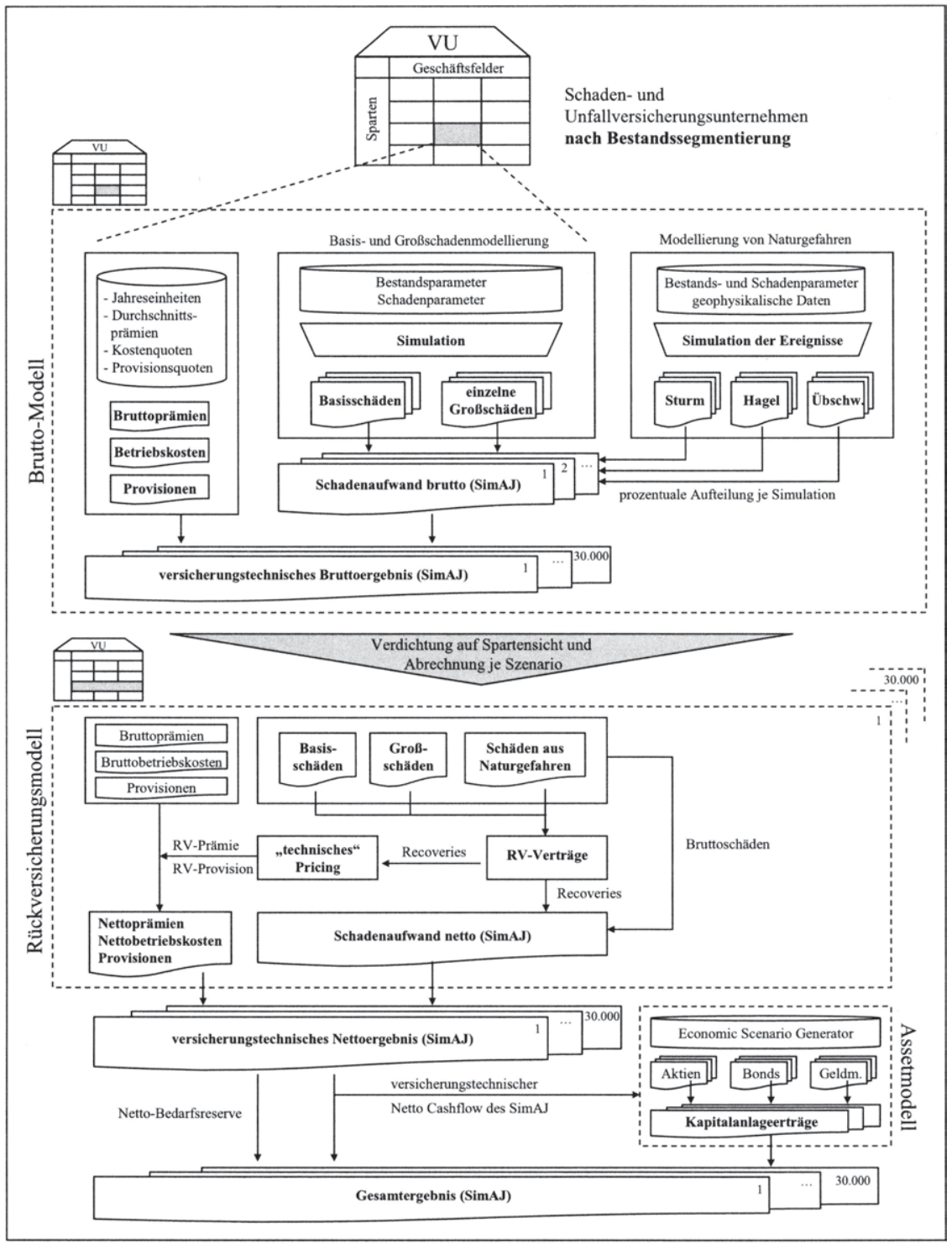

Abbildung 5.19: Modellaufbau und Simulationsablauf in der Anfalljahressicht 
Da der Ermittlung des Netto-Anfalljahrergebnisses eine spartenspezifische Rückversicherungsstruktur zugrunde liegt, ist es zunächst erforderlich, die je Sparte-Geschäftsfeld-Kombination erzeugten Bruttosimulationen auf die einzelnen Versicherungssparten zu verdichten. Die bisher betrachtete zweidimensionale Portfoliostruktur wird demzufolge aufgegeben. Im Rückversicherungsmodell wird der simulierte Bruttoschadenaufwand simulationsweise durch die betroffenen Rückversicherungsverträge geführt und vertragsspezifisch abgerechnet. Der Bruttoschadenaufwand wird somit in den beim Erstversicherer verbleibenden Anteil (Selbstbehalt) und die Entschädigungsleistungen des Rückversicherers (Recoveries) aufgeteilt. Die Recoveries der Rückversicherer dienen wiederum als Grundlage für die „technische“ Ermittlung des Rückversicherungsprämie und der Rückversicherungsprovision. Der Saldo aus Nettoprämien, Nettobetriebskosten, Provisionen und Nettoschadenaufwand stellt das versicherungstechnische Nettoergebnis des simulierten Anfalljahres dar. Dieses kann wiederum in zahlungswirksame Komponenten (Nettoprämien, Nettoschadenzahlungen, Nettobetriebskosten, Provisionen) und nicht-zahlungswirksame Komponenten (Netto-Bedarfsreserve) aufgespalten werden. Der für Kapitalanlagezwecke nutzbare Zinsträger setzt sich zu Beginn des Anfalljahres aus den vorschüssig vereinnahmten Nettoprämien zusammen und wird annahmegemäß in der Periodenmitte durch abfließende Nettoschadenzahlungen, Nettobetriebskosten und Provisionen reduziert. ${ }^{913}$ Der versicherungstechnische Netto Cashflow wird in die Hauptanlageklassen Aktien, Anleihen und Geldmarktpapiere investiert. Die erforderlichen Renditeverteilungen dieser Anlageklassen werden durch einen vereinfachten „Economic Scenario Generator" erzeugt. Aus der Aggregation des Netto-Anfalljahrergebnisses mit den Anlageerträgen des versicherungstechnischen Netto Cashflows resultiert ein Set von Realisationen für das Gesamtergebnis des simulierten Anfalljahres.

In der bilanziellen Geschäftsjahressicht wird der Einfluss des Schadengeschehens vorangegangener Anfalljahre explizit in die Betrachtung integriert. Neben die bereits bekannten Ergebniskomponenten des simulierten Anfalljahres tritt die Abwicklung der bestehenden Netto-Schadenrückstellungen im simulierten Geschäftsjahr. Im Abwicklungsmodell wird der simulierte Netto-Endschaden jeder Versicherungssparte in eine zahlungswirksame Komponente (GJ-Zahlungen) und eine nicht-zahlungswirksame Komponente (Dotierung GJ-Reserve) überführt. Darüber hinaus wird ermittelt, wie sich die bestehenden Netto-Schadenrückstellungen der früheren Anfalljahre im simulierten Geschäftsjahr verändern bzw. welcher Anteil in Form von VJ-Schadenzahlungen abfließt. Die Abwicklung der GJ- und VJ-Schäden erfolgt je Versicherungszweig und greift auf empirisch geschätzte und als deterministisch unterstellte Zah-

913 Vgl. zum zeitlichen Anfall des versicherungstechnischen Cashflows und zur zeitlichen Struktur des verfügbaren Zinsträgers auch Abbildung 6.18. 
lungs- und Reservemuster zurück. Aus der Saldierung von Nettoprämien, Nettobetriebskosten, Provisionen sowie GJ- und VJ-Schadenaufwand resultiert ein Set von $\mathrm{n}=30.000$ Realisationen für das versicherungstechnische Nettoergebnis des simulierten Geschäftsjahres.

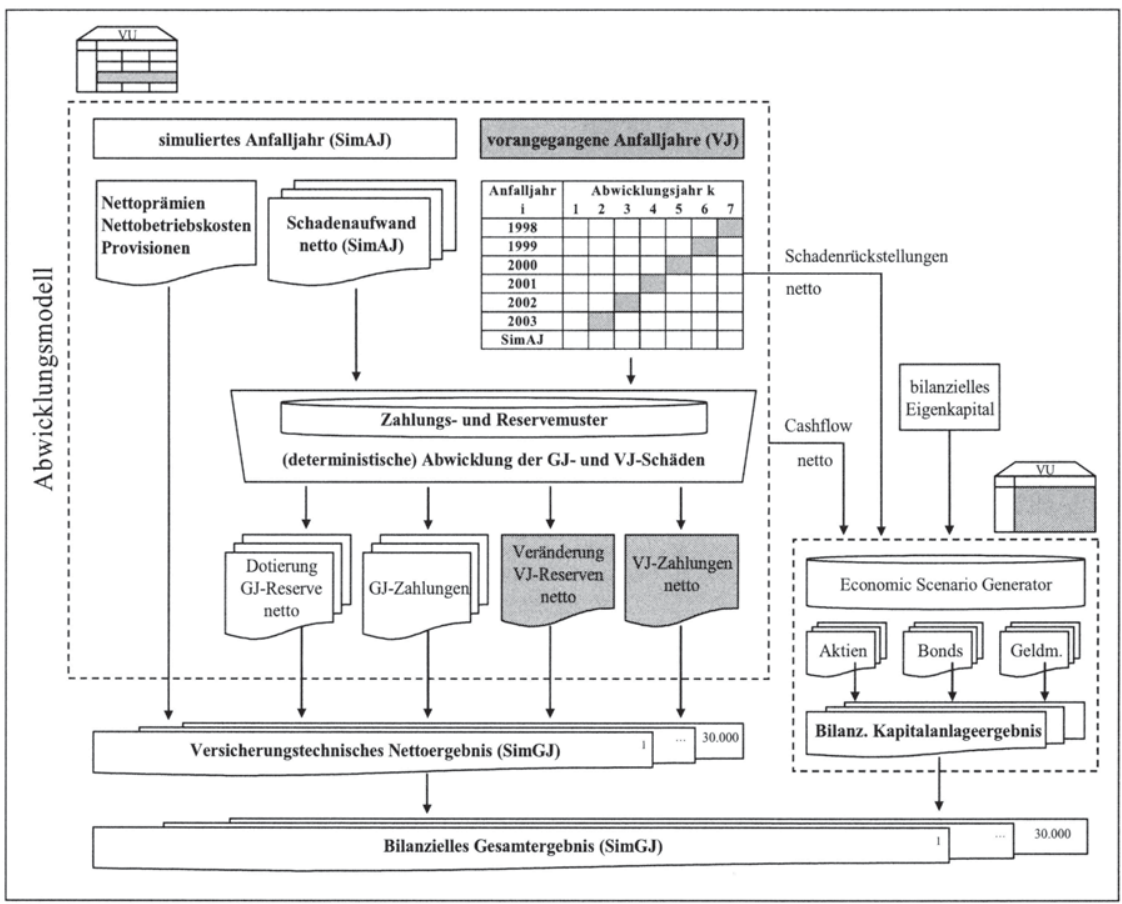

Abbildung 5.20: Modellaufbau und Simulationsablauf in der Geschäftsjahressicht

In der Geschäftsjahressicht setzt sich das investierte Kapitalanlagevolumen zu Periodenbeginn aus den vorschüssig erhaltenen Nettoprämien, den Netto-Schadenrückstellungen und dem bilanziellen Eigenkapital zusammen. In der Periodenmitte wird dieser Zinsträger durch die Schadenzahlungen sämtlicher Anfalljahre sowie durch Betriebskosten und Provisionen reduziert. Das durch die Investition in Aktien, Anleihen und Geldmarktpapiere generierte bilanzielle Kapitalanlageergebnis wird schließlich je Simulationslauf mit dem versicherungstechnischen Nettoergebnis zum bilanziellen Gesamtergebnis aggregiert. 
Mirko Tillmann - 978-3-631-75327-9

Downloaded from PubFactory at 01/11/2019 06:10:15AM

via free access 


\section{Umsetzung des Modellansatzes am Beispiel eines deutschen Schaden- und Unfallversicherungsunternehmens}

\subsection{Strukturierung und Eingrenzung des Analyserahmens}

Der im vorangegangenen Hauptkapitel beschriebene Modellansatz ist unter der Zielsetzung entwickelt worden, die Auswirkungen ausgewählter Unternehmensstrategien und Parametervariationen auf die Rendite-Risikoposition eines Schaden- und Unfallversicherungsunternehmens zu analysieren und hieraus Implikationen für die strategische Steuerung abzuleiten. Die Vorteilhaftigkeit der strategischen Alternativen soll stets unter der Fragestellung untersucht werden, inwiefern diese dazu geeignet sind, auf der Gesamtunternehmensebene die Relation von Rentabilität und Risiko zu verbessern. ${ }^{914}$ Der inhaltliche Schwerpunkt dieser Arbeit ist daher im Bereich der zentralen Struktursteuerung angesiedelt. In Kapitel 5.4.1.2 wurde bereits erläutert, dass die so genannte Anfalljahressicht hinsichtlich des versicherungstechnischen Portfolios die steuerungsrelevante Perspektive darstellt. Während der Einfluss vergangener Anfalljahre auf die Rendite-Risikoposition bereits weitgehend determiniert ist, ergeben sich im Hinblick auf die Zeichnungs- und Rückversicherungspolitik für das simulierte (zukünftige) Anfalljahr noch vielfältige Steuerungsmöglichkeiten. ${ }^{915}$

Auf der Steuerungsebene konzentriert sich die weitere Analyse daher auf das Zeichnungsrisiko des simulierten Anfalljahres sowie die Auswirkungen unterschiedlicher Rückversicherungsstrategien auf die Rendite-Risiko-Position des Modellunternehmens. Das Reserverisiko, verstanden als Unsicherheit in Bezug auf die Auszahlungsgeschwindigkeit und Auskömmlichkeit von Schadenrückstellungen zurückliegender Anfalljahre, wird nicht betrachtet. Eine Integration der Kapitalanlagetätigkeit ist auf der Steuerungsebene nur insofern möglich, als dass die verzinsliche Anlage der vorschüssig vereinnahmten Prämien und des versicherungstechnischen Netto Cashflows des simulierten Anfalljahres in die Analyse einfließen. Im Interesse einer zeitlich konsistenten Betrachtungsweise bleiben jedoch auf der Steuerungsebene sowohl der Einfluss vorangegangener Anfalljahre auf den versicherungstechnischen Netto Cashflow als auch die Möglichkeit zur Anlage der Schadenrückstellungen „alter“ Anfalljahre außer Betracht. Im Mittelpunkt der nachfolgenden Ausführungen steht die Steuerung des versicherungstechnischen Portfolios unter expliziter Betrachtung der Zeichnungsund Rückversicherungspolitik.

914 Vgl. übereinstimmend zur Steuerung in Kreditinstituten WöHLE (2003), S. 82. An dieser Stelle wird bewusst auf den Begriff „Optimierung der Rendite-Risiko-Relation“ verzichtet, da der entwickelte Modellansatz über keinen simultanen Optimierungsalgorithmus verfügt.

915 Neben einer restriktiveren Schadenregulierungspolitik besteht hinsichtlich vergangener Anfalljahre lediglich die Möglichkeit eines „Loss Portfolio Transfers“, d.h. des Verkaufs eines Teilbestandes einschließlich der korrespondierenden Spätschadenlast. Vgl. hierzu und zu weiteren Formen der Finite Risk-Rückversicherung SWISS RE (1997b), S. 13-15. 
Auf der Bilanzebene wird der Einfluss vergangener Zeichnungsjahre in die Analyse einbezogen und modelltechnisch über das Abwicklungsmodul berücksichtigt. Das bilanzielle Kapitalanlageergebnis ist in Bezug auf den Zeithorizont (= simuliertes Geschäftsjahr) und den zugrunde liegenden Zinsträger mit der versicherungstechnischen Ergebnisgröße konsistent, so dass beide Größen zum bilanziellen Gesamtergebnis aggregiert werden können. Auf der Bilanzebene lassen sich somit in vereinfachter Form sowohl eine Gewinn- und Verlustrechnung als auch eine Bilanz aufstellen. Der skizzierte zweidimensionale Untersuchungsaufbau wird in Abbildung 6.1 im Überblick dargestellt. Er gibt gleichzeitig die Gliederung des nachfolgenden Hauptkapitels vor.

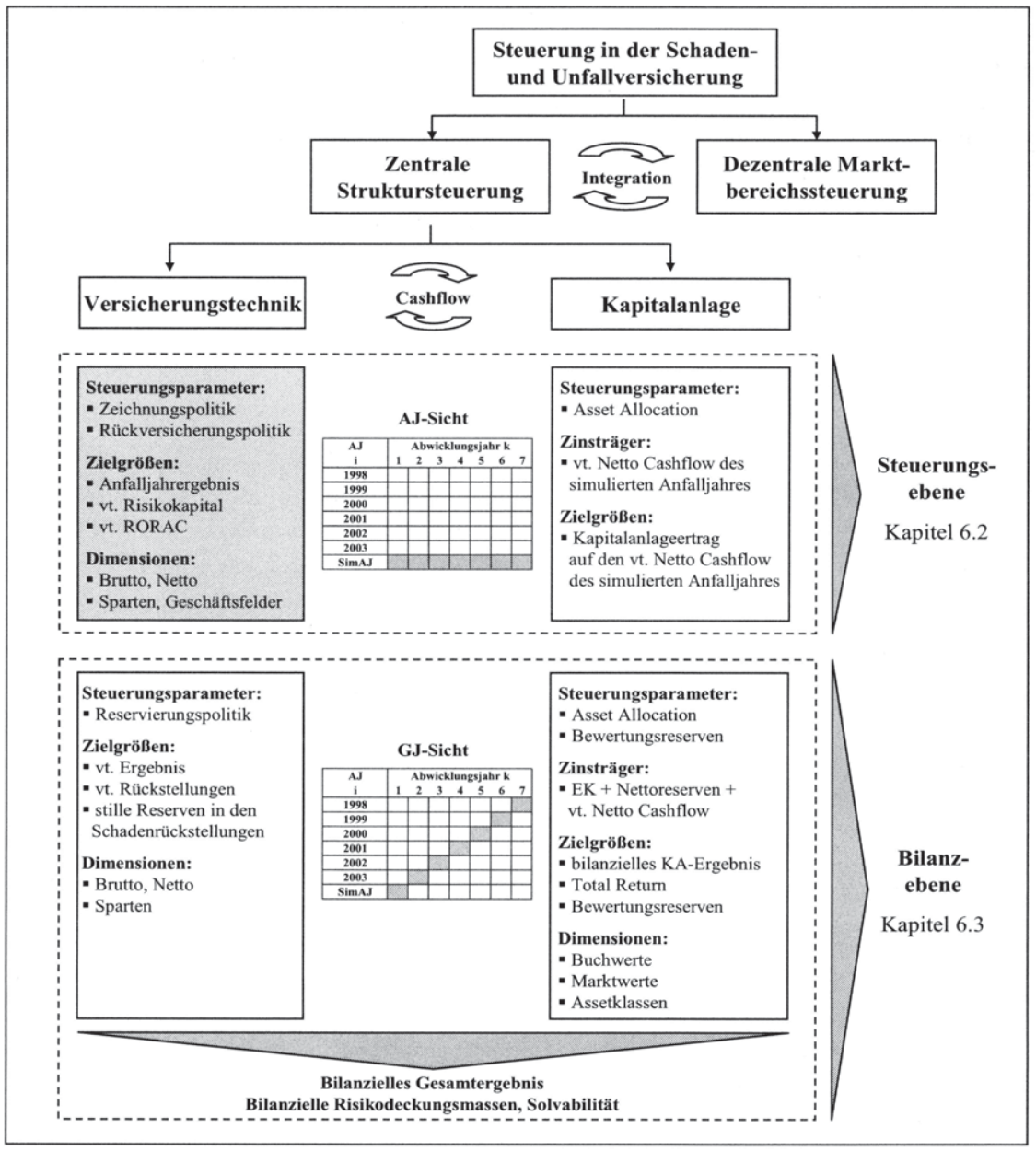

Abbildung 6.1: Zweidimensionaler Untersuchungsaufbau 
Die Steuerungsebene ist Gegenstand von Kapitel 6.2. Es wird zunächst die Entwicklung des versicherungstechnischen Portfolios im simulierten Anfalljahr dargestellt und sowohl sparten- als auch geschäftsfeldspezifisch analysiert. In einem zweiten Schritt wird die Anfalljahresbetrachtung um Kapitalerträge aus der Anlage des versicherungstechnischen Netto Cashflows ergänzt. Schließlich wird im Rahmen von parametrischen Variationsanalysen ${ }^{916}$ untersucht, ob und in welchem Ausmaß die Veränderungen zentraler Risikoparameter Einfluss auf die Rendite-Risikoposition des Modellunternehmens nehmen. In diesem Kontext werden insbesondere der Einfluss stochastischer Abhängigkeitsstrukturen zwischen einzelnen Risikokollektiven untersucht (Kapitel 6.2.2) und exemplarisch die Auswirkungen unterschiedlicher Rückversicherungsund Zeichnungsstrategien aufgezeigt (Kapitel 6.2.3 und 6.2.4).

In Kapitel 6.3 wird die Analyse um den Einfluss vorangegangener Anfalljahre erweitert und untersucht, zu welchen strukturellen Effekten die o.g. Strategien auf der Bilanzebene führen. Neben der Interpretation des bilanziellen Gesamtergebnisses kommt hier auch der Entwicklung der bilanziellen und außerbilanziellen Risikodeckungsmassen besondere Bedeutung zu. Darüber hinaus wird überprüft, ob die strategischen Handlungsoptionen auch aus aufsichtsrechtlicher Perspektive zulässig sind, mithin die Einhaltung der momentan gültigen Solvabilitätsanforderungen (Solvency I) gewährleistet ist.

\subsection{Steuerungsebene}

6.2.1 Darstellung und Analyse der Modellergebnisse im Basisszenario

Bevor die Modellergebnisse im Einzelnen vorgestellt und analysiert werden, soll die Ausgangssituation des Modellunternehmens grob skizziert werden. ${ }^{917}$ Es handelt sich um ein Schaden- und Unfallversicherungsunternehmen in der Rechtsform einer Aktiengesellschaft. Das versicherungstechnische Portfolio kann in weitgehend homogene Teilkollektive zergliedert und in einer Matrixform dargestellt werden. Neben der bilanziell geprägten Spartensicht hat das Unternehmen den Versicherungsbestand anhand kundenspezifischer Merkmale in die Geschäftsfelder Privatkunden, Gewerbekunden und Industriekunden segmentiert. ${ }^{918}$

Tabelle 6.1 stellt die Prämien- und Geschäftsstruktur des Modellunternehmens im Überblick dar.

\footnotetext{
916 Zur Abgrenzung der Begriffe Sensitivitätsanalyse und parametrische Variationsanalyse vgl. SCHIERENBECK (2003b), S. 569-574.

917 Vgl. ergänzend auch die Ausführungen zur Datenbasis in Kapitel 5.3.2.

918 Vgl. auch Kapitel 5.4.1.1.
} 


\begin{tabular}{|c|c|c|c|c|c|}
\hline \begin{tabular}{|ll} 
Sparte & Geschätsfeld
\end{tabular} & Privatkunden & Gewerbekunden & Industriekunden & \multicolumn{2}{|c|}{ GESAMT } \\
\hline Allgemeine Unfall & 36,8 & 3,9 & 0,7 & 41,5 & $8 \%$ \\
\hline Kraftfahrt-Haftpflicht & 125,6 & 41,1 & 10,0 & 176,6 & $33 \%$ \\
\hline Kasko & 80,3 & 28,3 & 4,6 & 113,2 & $21 \%$ \\
\hline HUK GESAMT & 242,7 & 73,3 & 15,3 & 331,3 & $62 \%$ \\
\hline Feuer Industrie & & & 12,2 & 12,2 & $2 \%$ \\
\hline Allgemeine Sturm & & 13,4 & 0,3 & 13,7 & $3 \%$ \\
\hline VGV Feuer & 45,1 & & & 45,1 & $8 \%$ \\
\hline VGV Leitungswasser & 66,2 & & & 66,2 & $12 \%$ \\
\hline \begin{tabular}{|l} 
VGV Sturm \\
\end{tabular} & 62,7 & & & 62,7 & $12 \%$ \\
\hline VGV Elementar & 3,6 & & & 3,6 & $1 \%$ \\
\hline SACH GESAMT & 177,6 & 13,4 & 12,6 & 203,5 & $38 \%$ \\
\hline \multirow{2}{*}{ GESAMT } & 420,3 & 86,6 & 27,8 & \multirow{2}{*}{534,7} & \multirow{2}{*}{$100 \%$} \\
\hline & $79 \%$ & $16 \%$ & $5 \%$ & & \\
\hline
\end{tabular}

Tabelle 6.1: Prämien- und Geschäftsstruktur im Basisszenario [Brutto in Mio. GE]

Es ist zu berücksichtigen, dass in dieser Arbeit im Interesse einer übersichtlicheren Darstellung lediglich ausgewählte Versicherungszweige betrachtet werden. ${ }^{919}$ Das Modellunternehmen agiert primär im Privatkundensegment. Gleichwohl entfällt ca. ein Fünftel des Bruttoprämienvolumens auf das Geschäft mit Gewerbe- und Industriekunden. Das Bruttoprämienvolumen teilt sich zu $62 \%$ auf die HUK-Sparten und zu $38 \%$ auf die Sachsparten auf. Dieses Aufteilungsverhältnis entspricht näherungsweise dem Durchschnitt der deutschen Schaden- und Unfallversicherungsunternehmen im Geschäftsjahr 2003. ${ }^{920}$

\subsubsection{Versicherungstechnik}

\subsection{Darstellung und Analyse des Bruttogeschäfts}

Nachfolgend wird die Struktur des versicherungstechnischen Bruttoergebnisses im simulierten Anfalljahr dargestellt und sowohl sparten- als auch geschäftsfeldspezifisch analysiert. In diesem Kontext gilt es sich zunächst in Erinnerung zu rufen, dass die Ergebniskomponenten Prämien, Provisionen und Betriebskosten über deterministische Parameter in das Modell eingehen. Demzufolge resultieren die Schwankungen des Bruttoergebnisses ausschließlich aus dem stochastischen Charakter der Schadenmodellierung. Den Ausgangspunkt der Untersuchung bildet eine Aufspaltung des Gesamtergebnisses in seine wesentlichen Komponenten, wobei in einem ersten Schritt ausschließlich Erwartungswerte betrachtet werden. Im weiteren Verlauf wird die Analyse

919 Insbesondere werden die Versicherungszweige Allgemeine Haftpflicht, Verbundene Hausrat, Rechtschutz und Transport nicht abgebildet. Das Bruttoprämienvolumen dieser Sparten hatte im Geschäftsjahr 2003 einen Anteil von $24,1 \%$ am gesamten Prämienaufkommen des deutschen Schaden- und Unfallversicherungsmarktes. Vgl. BAFIN (2005), Tab. 541.

Vgl. BAFin (2005), Tab. 541. 
durch die Betrachtung von Verteilungen bzw. lagetypischen Streuungsmaßen (Perzentile) erweitert. Die Untersuchung gewinnt demzufolge schrittweise an Komplexität.

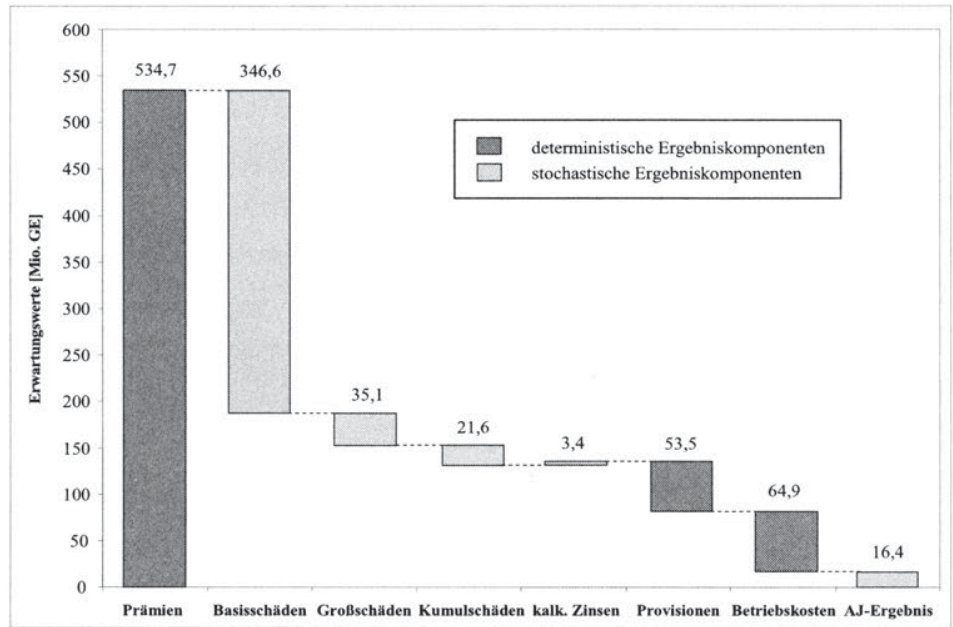

Abbildung 6.2: Entstehung des Brutto-Anfalljahrergebnisses

Abbildung 6.2 verdeutlicht den dominierenden Einfluss der Schadenentwicklung auf das versicherungstechnische Bruttoergebnis. Im Erwartungswert beträgt die Grundschadenlast, d.h. die Summe der Basisschäden 64,8\% der gesamten Prämieneinnahmen. Insgesamt müssen im Erwartungswert 75,4\% der Prämien für die Regulierung von Basis-, Groß- und Kumulschäden aufgewendet werden. Unter Berücksichtigung der kalkulatorischen Zinsen aus der Anlage der Bedarfsreserve reduziert sich die Bruttoschadenquote leicht auf 74,8\%. Darüber hinaus entfallen insgesamt $10,0 \%$ der Prämien auf die Vergütung der Vertriebsorgane (Provisionen) und 12,1\% auf Betriebskosten. Das erwartete Brutto-Anfalljahrergebnis beträgt 16,4 Mio. GE bzw. 3,1\% des Prämienvolumens (Return on Sales).

Aufgrund der zweidimensionalen Modellstruktur kann das Brutto-Anfalljahrergebnis des Gesamtunternehmens linear auf sämtliche Felder der Sparte-Geschäftsfeld-Matrix heruntergebrochen werden. ${ }^{921}$ Dies ermöglicht eine detaillierte, jedoch lediglich erwartungswertbasierte Analyse der Ergebnisstruktur und die Identifikation wesentlicher Gewinn- und Verlustquellen. Tabelle 6.2 stellt die versicherungstechnische Ergebnisstruktur des Modellunternehmens im Überblick dar.

${ }^{921}$ Die Möglichkeit zur linearen Zerlegung des Gesamtergebnisses in einzelne Segmentergebnisse beruht auf der Additivitätseigenschaft des Erwartungswertes. Vgl. vertiefend BOHLEY (2000), S. $122-131$. 


\begin{tabular}{|c|c|c|c|c|c|c|c|c|c|c|}
\hline \multirow{2}{*}{$\begin{array}{l}\text { Erwartungswerte } \\
\text { [Tsd. GE] }\end{array}$} & \multicolumn{10}{|c|}{ Privatkunden } \\
\hline & $\mathbf{A U}$ & KH & Kasko & FIND & AST & VGVF & VGV LW & \begin{tabular}{|l|} 
VGV ST \\
\end{tabular} & \begin{tabular}{|l|} 
VGVEL \\
\end{tabular} & SUMME \\
\hline Bruttoprämie & 36.849 & 125.569 & 80.284 & & & 45.089 & 66.176 & 62.730 & 3.567 & 420.265 \\
\hline - Endschaden brutto & 16.396 & 104.530 & 62.520 & & & 34.052 & 61.516 & 31.538 & 2.975 & 313.528 \\
\hline + kalk. Zinsen & 403 & 1.511 & 109 & & & 155 & 167 & 129 & 9 & 2.484 \\
\hline - Provisionen & 6.968 & 6.906 & 8.325 & & & 5.420 & 7.954 & 7.540 & 429 & 43.543 \\
\hline - Betriebskosten & 7.819 & 9.593 & 10.630 & & & 6.015 & 8.828 & 8.368 & 476 & 51.729 \\
\hline$=$ AJ-Ergebnis brutto & 6.070 & 6.049 & -1.082 & & & -243 & -11.955 & 15.413 & -303 & 13.949 \\
\hline \multirow{2}{*}{$\begin{array}{c}\text { Erwartungswerte } \\
\text { [Tsd. GE] }\end{array}$} & \multicolumn{10}{|c|}{ Gewerbekunden } \\
\hline & $\mathbf{A U}$ & KH & Kasko & FIND & AST & VGV F & VGV LW & VGV ST & VGV EL & SUMME \\
\hline Bruttoprămie & 3.948 & 41.080 & 28.271 & & 13.351 & & & & & 86.649 \\
\hline - Endschaden brutto & 2.267 & 33.232 & 22.170 & & 7.162 & & & & & 64.830 \\
\hline + kalk. Zinsen & 56 & 480 & 39 & & 18 & & & & & 592 \\
\hline - Provisionen & 702 & 2.177 & 2.785 & & 1.650 & & & & & 7.314 \\
\hline - Betriebskosten & 544 & 3.718 & 3.460 & & 2.921 & & & & & 10.644 \\
\hline$=$ AJ-Ergebnis brutto & 491 & 2.433 & -105 & & 1.635 & & & & & 4.454 \\
\hline \multirow{2}{*}{$\begin{array}{c}\text { Erwartungswerte } \\
\text { [Tsd. GE] }\end{array}$} & \multicolumn{10}{|c|}{ Industriekunden } \\
\hline & $\mathbf{A U}$ & KH & Kasko & FIND & AST & VGV F & VGV LW & VGV ST & \begin{tabular}{|l|} 
VGV EL \\
\end{tabular} & SUMME \\
\hline Bruttoprämie & 688 & 9.956 & 4.629 & 12.219 & 344 & & & & & 27.836 \\
\hline - Endschaden brutto & 199 & 9.512 & 3.885 & 10.912 & 437 & & & & & 24.946 \\
\hline + kalk. Zinsen & 5 & 137 & 7 & 131 & 1 & & & & & 282 \\
\hline - Provisionen & 121 & 518 & 455 & 1.519 & 41 & & & & & 2.653 \\
\hline - Betriebskosten & 56 & 412 & 406 & 1.603 & 76 & & & & & 2.552 \\
\hline$=\mathrm{AJ}$-Ergebnis brutto & 316 & -349 & -108 & -1.683 & -210 & & & & & -2.034 \\
\hline \multirow{2}{*}{$\begin{array}{c}\text { Erwartungswerte } \\
\text { [Tsd. GE] }\end{array}$} & \multicolumn{10}{|c|}{ GESAMT } \\
\hline & $\mathbf{A U}$ & KH & Kasko & FIND & AST & VGV F & VGV LW & VGV ST & \begin{tabular}{|l|} 
VGV EL \\
\end{tabular} & SUMME \\
\hline Bruttoprämie & 41.485 & 176.604 & 113.184 & 12.219 & 13.695 & 45.089 & 66.176 & 62.730 & 3.567 & 534.750 \\
\hline - Endschaden brutto & 18.862 & 147.275 & 88.574 & 10.912 & 7.599 & 34.052 & 61.516 & 31.538 & 2.975 & 403.304 \\
\hline + kalk. Zinsen & 464 & 2.129 & 155 & 131 & 19 & 155 & 167 & 129 & 9 & 3.358 \\
\hline - Provisionen & 7.791 & 9.601 & 11.565 & 1.519 & 1.692 & 5.420 & 7.954 & 7.540 & 429 & 53.510 \\
\hline - Betriebskosten & 8.419 & 13.723 & 14.495 & 1.603 & 2.997 & 6.015 & 8.828 & 8.368 & 476 & 64.924 \\
\hline$=$ AJ-Ergebnis brutto & 6.877 & 8.133 & -1.296 & -1.683 & 1.426 & -243 & -11.955 & 15.413 & -303 & 16.369 \\
\hline Schadenquote (disk.) & $44,3 \%$ & $82,2 \%$ & $78,1 \%$ & $88,2 \%$ & $55,4 \%$ & $75,2 \%$ & $92,7 \%$ & $50,1 \%$ & $83,1 \%$ & $74,8 \%$ \\
\hline Kostenquote & $39,1 \%$ & $13,2 \%$ & $23,0 \%$ & $25,5 \%$ & $34,2 \%$ & $25,4 \%$ & $25,4 \%$ & $25,4 \%$ & $25,4 \%$ & $22,1 \%$ \\
\hline Combined Ratio & $83,4 \%$ & $95,4 \%$ & $101,1 \%$ & $113,8 \%$ & $89,6 \%$ & $100,5 \%$ & $118,1 \%$ & $75,4 \%$ & $108,5 \%$ & $96,9 \%$ \\
\hline Return on Sales & $16,6 \%$ & $4,6 \%$ & $-1,1 \%$ & $-13,8 \%$ & $10,4 \%$ & $-0,5 \%$ & $-18,1 \%$ & $24,6 \%$ & $-8,5 \%$ & $3,1 \%$ \\
\hline
\end{tabular}

Tabelle 6.2: Struktur und Kennziffern des Brutto-Anfalljahrergebnisses

Das Privatkundengeschäft nimmt bezüglich seines absoluten Ergebnisbeitrages eine dominante Stellung ein. Es erwirtschaftet im Erwartungswert ein Brutto-Anfalljahrergebnis von 13,9 Mio. GE. Dies entspricht einem Return on Sales (ROS) von 3,3\% der Bruttoprämien. Hauptergebnisträger sind die Versicherungszweige „Allgemeine Unfall“" und „Kraftfahrt-Haftpflicht“. Der Ergebnisverlauf der durch die „Verbundene Wohngebäudeversicherung" (VGV) abgedeckten Gefahren ist hingegen sehr heterogen. Während die Subsparte „VGV Sturm“ im Erwartungswert sehr profitabel verläuft (24,6\% ROS), ist das Leitungswasserrisiko angesichts einer durchschnittlichen Combined Ratio ${ }^{922}$ von $118,1 \%$ signifikant untertarifiert. Die Hauptsparte „Verbundene Wohngebäudeversicherung" erwirtschaftet insgesamt nur ein Bruttoergebnis von 2,9 Mio. GE (1,6\% ROS). In Relation zur Bruttoprämie stellt das Gewerbekundengeschäft das ertragsstärkste Geschäftsfeld dar. Es erwirtschaftet durchschnittlich einen ROS von 5,1\%. Mit Ausnahme der Sparte „Kasko“ verzeichnen alle betriebenen Versiche-

${ }^{922}$ Die Combined Ratio entspricht hier der Summe aus Endschadenquote (inkl. kalkulatorischer Zinsen aus der Anlage der Bedarfsreserve) und Kostenquote. 
rungszweige positive Anfalljahresergebnisse. Das kleinste Geschäftsfeld Industriekunden verläuft im Erwartungswert defizitär und weist einen negativen ROS von $-7,3 \%$ auf. Wesentlicher Verlusttreiber ist hier das industrielle Feuergeschäft. Trotz einer vergleichsweise günstigen Kostenquote von 18,7\% bedingt die hohe Schadenerwartung dieses Geschäftsfelds eine Combined Ratio von 107,3\%. Abbildung 6.3 verdeutlicht in Form eines Rankings, in welchem Umfang die einzelnen Versicherungszweige im Erwartungswert zum Gesamtergebnis des simulierten Anfalljahres beitragen.

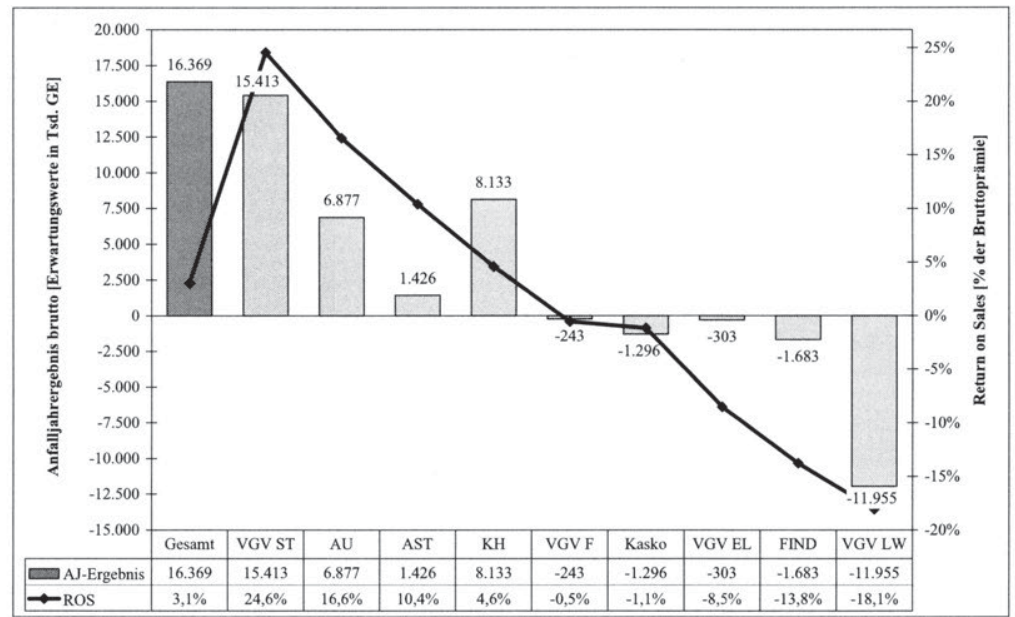

Abbildung 6.3: Brutto-Anfalljahrergebnisse und Return on Sales (ROS) nach Versicherungszweigen

Insgesamt erzielen die HUK-Sparten im Erwartungswert ein Ergebnis in Höhe von 13,7 Mio. GE. Die Sachversicherungssparten weisen insgesamt nur ein Ergebnis von 2,7 Mio. GE aus. In Relation zu den Bruttoprämien verläuft das HUK-Geschäft angesichts eines ROS von 4,1\% deutlich profitabler als das Sachgeschäft mit 1,3\%. Dieser Unterschied liegt jedoch ausschließlich in der geringeren Kostenbelastung der HUKSparten (Kostenquote 19,8\%) gegenüber den Sachversicherungssparten (Kostenquote $26,0 \%$ ) begründet. Im Erwartungswert weist das HUK-Geschäft mit 76,1\% eine höhere Endschadenquote auf als das Sachversicherungsgeschäft mit 72,7\%.

Die bisherige Analyse des Bruttogeschäfts erfolgte ausschließlich auf der Basis von Erwartungswerten. Diese Darstellungsweise besitzt den analytischen Vorzug, dass sämtliche Ergebniskomponenten der Sparten-Geschäftsfeld-Matrix linear zum Gesamtergebnis aggregiert werden können. Im Anwendungskontext der Schaden- und Unfallversicherung, der typischerweise durch asymmetrische und vielfach hochvolatile 
Schaden- bzw. Ergebnisverteilungen charakterisiert ist, lässt diese Vorgehensweise jedoch keine Aussagen über den Risikogehalt und damit die "Gefährlichkeit" des zugrunde liegenden Geschäfts $\mathrm{zu}^{923} \mathrm{Um}$ Informationen über das Schwankungsverhalten zu gewinnen, wird die Analyse nachfolgend um die Betrachtung vollständiger Verteilungen bzw. lagetypischer Streuungsmaße (Perzentile) erweitert. Dies soll zunächst am Beispiel der drei modellierten Schadenkategorien verdeutlicht werden. In Abbildung 6.4 wird das Streuungsverhalten von Basis-, Groß- und Kumulschäden anhand einer Boxplot-Graphik untersucht. Die obere bzw. untere Kante der Schachtel entsprechen jeweils dem 99\% bzw. 1\%-Perzentil der betrachteten Schadenverteilung. Ferner sind das Maximum und Minimum der Verteilung sowie deren Erwartungswert $(\mu)$ und Median $(\mathrm{Me})$ angegeben.

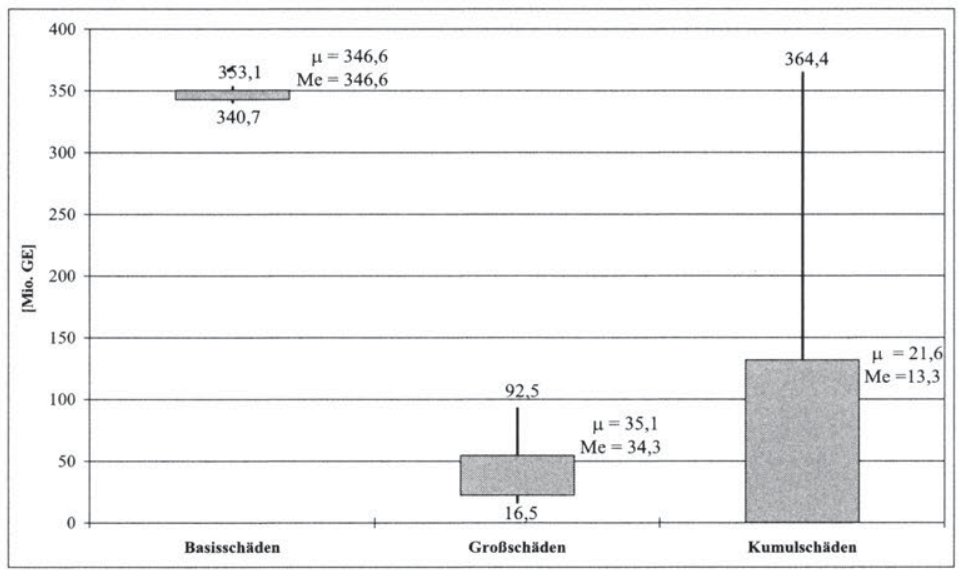

Abbildung 6.4: Streuungsverhalten der Schadenkategorien

Die Basisschäden erreichen im Erwartungswert das höchste Niveau, weisen jedoch eine sehr geringe Schwankungsbreite auf. Erwartungswert und Median stimmen annähernd überein, was auf eine symmetrische Verteilung der Basisschadenlast schließen lässt. An dieser Stelle gewinnt der Zentrale Grenzwertsatz an Bedeutung, welcher besagt, dass die standardisierte Summe einer großen Zahl von unabhängigen, reellwertigen (nicht notwendigerweise identisch verteilten) Zufallsvariablen annähernd normal-

${ }^{923}$ Vgl. übereinstimmend SCHRADIN (1998), S. 203-206. Darüber hinaus ist die Aussagekraft des empirischen Erwartungswertes aufgrund seiner Anfälligkeit gegen so genannte „Ausreißer“, d.h. weit vom Zentrum der Verteilung gelegene Simulationswerte, gerade im versicherungsspezifischen Kontext sehr eingeschränkt. Vgl. BLEYMÜLLER/GEHLERT/GÜLICHER (2002), S. 13-15 sowie BOHLEY (2000), S. 128-131. 
verteilt ist. ${ }^{924}$ Die Basisschäden der einzelnen Teilkollektive werden zwar jeweils über eine (rechtsschiefe) Lognormalverteilung modelliert, angesichts der großen Anzahl von Einzelrisiken genügt die Summe der Basisschäden über alle Teilkollektive jedoch approximativ einer Normalverteilung. Demgegenüber zeichnen sich die Groß- und insbesondere die Schäden aus Naturgefahrereignissen durch eine deutlich größere Schwankungsbreite und rechtsschiefe Verteilungen aus. Das erhebliche Risikopotenzial dieser Schadenkategorien wird z.B. daran ersichtlich, dass der maximale Kumulschaden die maximale Basisschadenlast übersteigt. Es kann daher bereits an dieser Stelle vermutet werden, dass der Risikokapitalbedarf des Modellunternehmens maßgeblich durch die Groß- und Kumulschäden und weniger durch die Basisschadenlast determiniert wird. Abbildung 6.5 visualisiert das asymmetrische Streuungsverhalten des Brutto-Gesamtschadens in Form einer empirischen Dichtefunktion ${ }^{925}$ (= Häufigkeitspolygon). $\mathrm{Zu}$ diesem Zweck werden die Simulationswerte zunächst auf der Abszisse klassifiziert (Range). Die zugehörigen absoluten Klassenhäufigkeiten (Density) sind auf der Ordinate abgetragen.

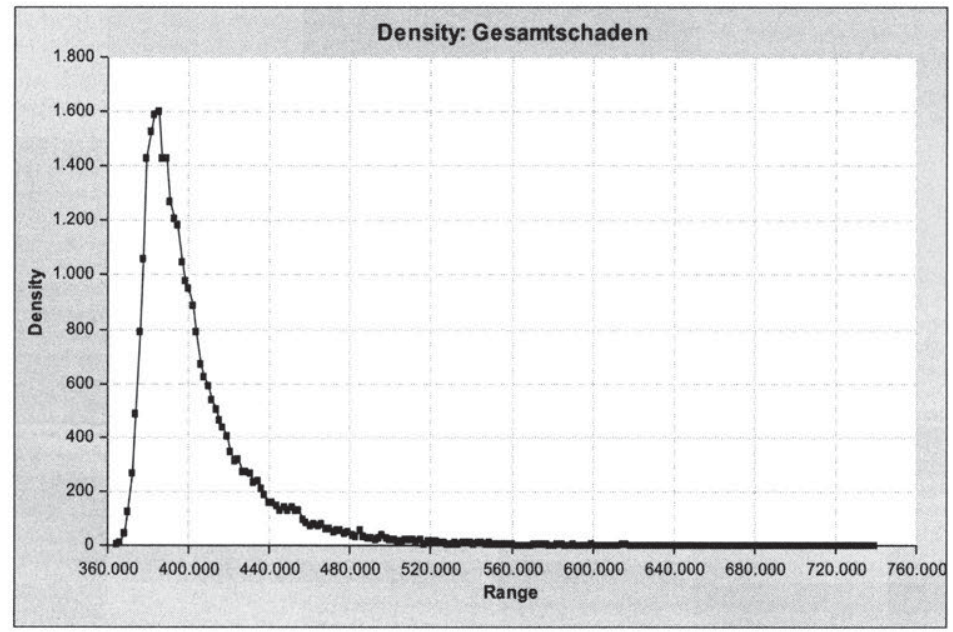

Abbildung 6.5: Empirische Dichtefunktion des Brutto-Gesamtschadens

924 Vgl. vertiefend KERSTING (2004), S. 167-174 sowie im versicherungsspezifischen Kontext KORYCIORZ (2004), S. 279 und ALBRECHT (1982).

${ }^{925}$ Es bezeichne $y_{i}$ mit $i \in\{1, \ldots, n\}$ die Realisation einer Zufallsvariablen auf dem Wertebereich $[a, b]$. Weiterhin sei angenommen, dass $Z_{k}$ mit $k \in\{1, \ldots, r\}$ eine Unterteilung des Wertebereichs mit dem Repräsentanten $z_{k}$ (z.B. für die Klassenmitte) darstelle. Dann sei die empirische Dichtefunktion in dieser Arbeit definiert als $f\left(z_{k}\right)=\left|\left\{y_{i} ; y_{i} \in Z_{k}\right\}\right|$. In Abbildung 6.5 wurden die Simulationswerte linear interpoliert. 
Die nunmehr für das Gesamtunternehmen gewonnenen Erkenntnisse über die Stochastizität der einzelnen Schadenkategorien werden nachfolgend auf die Analyse der gesamten Sparte-Geschäftsfeld-Matrix übertragen. Abbildung 6.6 stellt zunächst die Ergebnisstreuung der modellierten Versicherungszweige in der Form eines Perzentilplots dar. Im Gegensatz zur Boxplot-Graphik visualisiert der Perzentilplot lediglich den Wertebereich zwischen dem 1\%- und 99\%-Perzentil. Die Extremwerte der Ergebnisverteilungen fließen nicht in die graphische Darstellung ein, da diese die Skalierung der Ordinate übersteigen würden. Die jeweils korrespondierenden Maxima und Minima sind jedoch Tabelle $6.3 \mathrm{zu}$ entnehmen. ${ }^{926}$

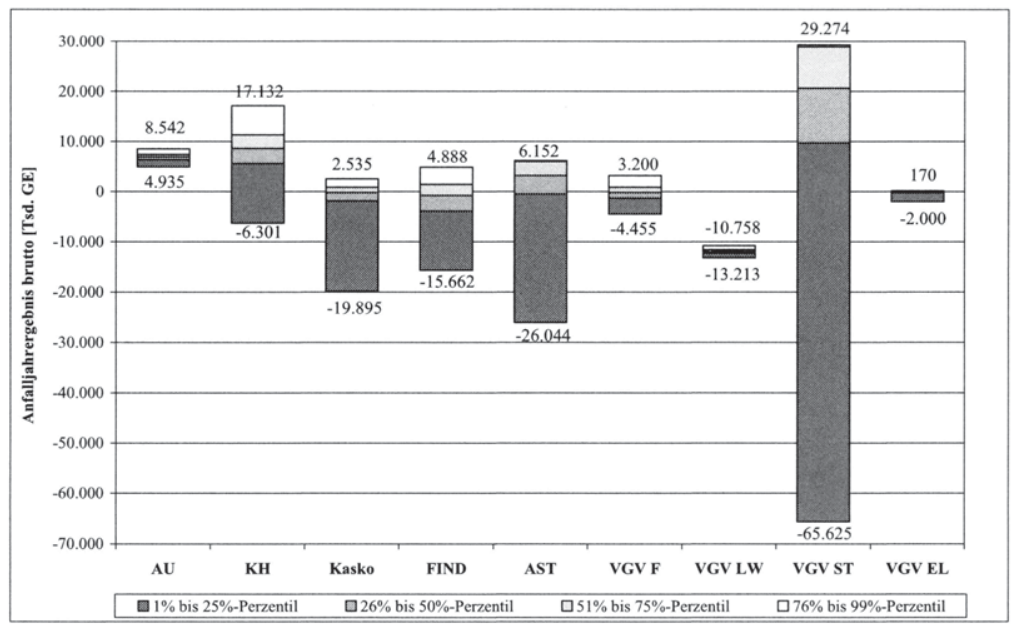

Abbildung 6.6: Streuungsverhalten der Brutto-Anfalljahresergebnisse nach Versicherungszweigen

\begin{tabular}{|l|r|r|r|r|r|r|r|r|r|r|}
\hline \multicolumn{1}{|c|}{ [Tsd. GE] } & \multicolumn{1}{c|}{ AU } & \multicolumn{1}{c|}{ KH } & \multicolumn{1}{c|}{ Kasko } & \multicolumn{1}{c|}{ FIND } & \multicolumn{1}{c|}{ AST } & VGV F & VGV LW & VGV ST & VGV EL & GESAMT \\
\hline Erwartungswert & 6.877 & 8.133 & -1.296 & -1.683 & 1.426 & -243 & -11.955 & 15.413 & -303 & $\mathbf{1 6 . 3 6 9}$ \\
\hline Standardabweichung & 779 & 4.863 & 4.924 & 4.387 & 6.894 & 1.639 & 530 & 20.442 & 2.419 & $\mathbf{2 8 . 8 2 3}$ \\
\hline Variationskoeffizient [\%] & $11 \%$ & $60 \%$ & $380 \%$ & $261 \%$ & $484 \%$ & $675 \%$ & $4 \%$ & $133 \%$ & $798 \%$ & $\mathbf{1 7 6 \%}$ \\
\hline Median & 6.912 & 8.637 & -199 & -781 & 3.207 & -164 & -11.955 & 20.604 & -147 & $\mathbf{2 4 . 8 9 4}$ \\
\hline Maximum & 9.476 & 22.772 & 3.724 & 6.562 & 6.321 & 5.262 & -9.897 & 29.596 & 355 & $\mathbf{5 5 . 8 0 7}$ \\
\hline Minimum & 2.617 & -51.324 & -156.730 & -31.782 & -84.357 & -8.002 & -13.958 & -241.216 & -107.871 & $\mathbf{- 3 1 8 . 6 5 6}$ \\
\hline 1\%-Perzentil & 4.935 & -6.301 & -19.895 & -15.662 & -26.044 & -4.455 & -13.213 & -65.625 & -2.000 & $\mathbf{- 9 4 . 1 8 0}$ \\
\hline Verlustwahrscheinlichkeit [\%] & $0,0 \%$ & $5,0 \%$ & $54,4 \%$ & $58,5 \%$ & $27,1 \%$ & $53,9 \%$ & $100,0 \%$ & $15,3 \%$ & $85,3 \%$ & $\mathbf{1 8 , 9 \%}$ \\
\hline
\end{tabular}

Tabelle 6.3: Kennziffern des Brutto-Anfalljahresergebnisses nach Versicherungszweigen

926 Bei der Interpretation von Tabelle 6.3 und im weiteren Verlauf gilt es zu beachten, dass die Spalte „Gesamt“ jeweils die Ergebniskennziffern des diversifizierten Gesamtunternehmens enthält. Diese entsprechen nur im Falle linearer Maßzahlen (z.B. Erwartungswert) der Summe sämtlicher Teilkollektive. 
Die Sparte „Allgemeine Unfall“ weist bei einer Ergebniserwartung von 6,9 Mio. GE nur eine sehr geringe Schwankungsbreite auf. In keiner der erzeugten Simulationen tritt ein negatives Brutto-Anfalljahrergebnis ein. ${ }^{927}$ Demgegenüber offenbart die Sparte „Kraftfahrt-Haftpflicht“ bei einer nur leicht erhöhten Ergebniserwartung eine deutlich stärkere Volatilität. Das Bruttoergebnis schwankt in einem Intervall von 22,8 Mio. GE bis -51,3 Mio. GE und wird in 5\% aller Fälle negativ. Der Variationskoeffizient als relatives Maß für das Streuungsverhalten nimmt in der Sparte „Kraftfahrt-Haftpflicht“ mit $60 \%$ einen höheren Wert an als in der Sparte „Allgemeine Unfall“" mit lediglich $11 \% .{ }^{928}$ Die Kaskoversicherung zeigt eine noch volatilere Ergebnischarakteristik. Zwar liegen 99\% aller Ergebnissimulationen im Wertebereich zwischen 3,7 Mio. GE und -19,9 Mio. GE, allerdings birgt der Tail der Ergebnisverteilung ein erhebliches Verlustpotenzial. Im Extremfall tritt ein Bruttoverlust in Höhe von -156,7 Mio. GE auf. Dieses Phänomen liegt in der starken Exponierung der Sparte „Kasko“ gegenüber Hagelschäden begründet, die durch sehr geringe lokale Eintrittswahrscheinlichkeiten bei gleichzeitig hoher Schadendichte gekennzeichnet sind. ${ }^{929}$ Die Sparte „VGV Elementar" weist in Bezug auf Überschwemmungsschäden ein vergleichbar riskantes Schaden- und Ergebnisprofil auf. Die hohe Verlustwahrscheinlichkeit von 85,3\% liegt darüber hinaus in einer deutlichen Untertarifierung begründet. Die sturmexponierten Sparten „Allgemeine Sturm“ und „VGV Sturm“ zeichnen sich demgegenüber durch ein höheres Prämienniveau, deutlich positive Ergebniserwartungen und geringere Verlustwahrscheinlichkeiten aus. Gleichzeitig bergen jedoch die Ränder der Verteilungen erhebliche Risiken. Insbesondere in der Sparte „VGV Sturm“ ist das Verlustpotenzial aufgrund des hohen Marktanteils und begrenzter regionaler Diversifikationsmöglichkeiten sehr hoch. ${ }^{930}$ Die Sparte „VGV Leitungswasser“ weist nur eine sehr geringe Schwankungsbreite der Bruttoergebnisse auf. Angesichts einer Verlustwahrscheinlichkeit von $100 \%$ ist für diesen Versicherungszweig eine systematische Untertarifierung festzustellen. Die defizitäre Ergebnissituation der Sparte „VGV Leitungswasser“ ist im

927 In diesem Kontext ist einschränkend zu berücksichtigen, dass für die Sparte „Allgemeine Unfall“ lediglich Basis- und Großschäden (> 50 Tsd. GE) modelliert werden. Mögliche Kumulrisiken, wie beispielsweise die Gruppenversicherung eines Betriebsausfluges eines Großunternehmens, bleiben demnach unberücksichtigt. Vgl. hierzu auch BoETIUS (1996), S. 264.

928

Eine Interpretation des Schwankungsverhaltens zweier Zufallsvariablen anhand eines absoluten Streuungsmaßes (z.B. der Standardabweichung oder Varianz) kann zu falschen Ergebnissen führen, sofern die Zufallsvariablen signifikant voneinander abweichende Erwartungswerte aufweisen. In derartigen Fällen ist es empfehlenswert, auf ein relatives (bzw. normiertes) Streuungsmaß wie den Variationskoeffizienten zurückzugreifen. Der Variationskoeffizient ermittelt sich als Quotient aus Standardabweichung und Erwartungswert. Er wird in der Regel als Prozentwert angegeben. Vgl. vertiefend BLEYMÜLLER/GEHLERT/GÜLICHER (2002), S. 22 sowie SACHS (2002), S. 139140. Vgl. kritisch zur Verwendung des Variationskoeffizienten als Risikomaß ALBRECHT (1982), S. 515-519.

929 Vgl. auch die Ausführungen in Kapitel 5.4.3.4.3.

930 Vgl. auch die Ausführungen zur Datenbasis des Originalunternehmens in Kapitel 5.3.2. 
deutschen Schaden- und Unfallversicherungsmarkt durchaus als realitätsnah einzuschätzen. ${ }^{931}$ Die Wahrscheinlichkeit für das Gesamtunternehmen, im simulierten Anfalljahr einen Verlust vor Rückversicherung zu erzielen, beträgt 18,9\%. Angesichts einer Spannweite von 55,8 Mio. GE bis -318,7 Mio. GE und eines Erwartungswertes von 16,4 Mio. GE weist das versicherungstechnische Bruttogeschäft eine stark linksschiefe Ergebnisverteilung auf (vgl. auch Abbildung 6.8).

Darüber hinaus kann bereits auf der Spartenebene ein signifikanter Diversifikationseffekt konstatiert werden. ${ }^{932}$ Der Diversifikationseffekt kann grundsätzlich auf unterschiedliche Steuerungsgrößen (z.B. Anfalljahrergebnis, Risikokapital) bezogen werden. Als Bewertungsmaß können darüber hinaus verschiedene Risikomaße R (z.B. Standardabweichung, Perzentile, VaR, CVaR) Verwendung finden. Die formale Darstellung des in Kapitel 4.2.1 bereits in Abhängigkeit einer Verlustvariablen $\mathrm{V}$ definierten Diversifikationsmaßes $D_{R}$ wird nunmehr für einen simulationsgestützten Kontext erweitert. Bezeichne $\mathrm{x}_{\mathrm{ij}}$ die Simulationsergebnisse für das $\mathrm{i}$-te Teilkollektiv in der $\mathrm{j}$-ten Simulation, so sei der Diversifikationseffekt im Folgenden definiert als: ${ }^{933}$

$$
\begin{aligned}
& \mathrm{D}(\mathrm{R}):=\sum_{\mathrm{i}=1}^{\mathrm{m}} \mathrm{R}\left(\mathrm{x}_{\mathrm{i} 1}, \ldots, \mathrm{x}_{1 \mathrm{n}}\right)-\mathrm{R}\left(\sum_{\mathrm{i}=1}^{\mathrm{m}} \mathrm{x}_{\mathrm{i} 1}, \ldots, \sum_{\mathrm{i}=1}^{\mathrm{m}} \mathrm{x}_{\mathrm{in}}\right) \quad \mathrm{i} \in\{1, \ldots, \mathrm{m}\} \text { und } \mathrm{j} \in\{1, \ldots, \mathrm{n}\} \\
& \text { mit: } \mathrm{R}(\cdot)= \text { Risikomaß } \\
& \mathrm{m}=\text { Anzahl der betrachteten Teilkollektive } \\
& \mathrm{n}=\text { Anzahl der durchgeführten Simulationen }
\end{aligned}
$$

Der somit formal beschriebene Diversifikationseffekt soll nunmehr exemplarisch für den Fall ermittelt werden, dass als Risikomaß R das 1\%-Perzentil des Brutto-Anfalljahrergebnisses herangezogen wird. Tabelle 6.3 ist zu entnehmen, dass die Summe der das 1\%-Perzentil repräsentierenden Gesamtergebnisse der $m=9$ Versicherungszweige $-148,3$ Mio. GE beträgt. Auf der Unternehmensebene hingegen tritt mit einprozentiger Wahrscheinlichkeit lediglich ein Gesamtsergebnis ein, das kleiner als -94,2 Mio. GE ist. Der Diversifikationseffekt kann demnach auf 54,1 Mio. GE beziffert werden. Hinsichtlich der Interpretation des Diversifikationseffektes sei daran erinnert, dass zum jetzigen Zeitpunkt der Untersuchung die Schadenentwicklung zwischen den einzelnen Versicherungszweigen noch als stochastisch unabhängig angenommen wird.

\footnotetext{
931 Die versicherungstechnische Bruttoergebnisquote (versicherungstechnisches Bruttoergebnis in \% der verdienten Bruttoprämie) der Einzelsparte „Leitungswasser“ betrug 2003 im deutschen Schaden- und Unfallversicherungsmarkt -22,3\%. Vgl. BAFIN (2005), Tab. 541.

$932 \mathrm{Vgl}$. zur formalen Definition des Diversifikationseffektes auch Kapitel 4.2.1.

933 Vgl. auch KORYCIORZ (2004), S. 185-189 und die Ausführungen in Kapitel 4.2.1.
} 
Da auf der Ebene der Versicherungszweige andere Risikoausgleichseffekte wirken als beispielsweise zwischen den drei betrachteten Geschäftsfeldern, wird der Diversifikationseffekt auf der Geschäftsfeldebene ein anderes Ausmaß annehmen. Bevor dieser Effekt jedoch quantifiziert wird, soll das Streuungsverhalten der Bruttoergebnisse der Geschäftsfelder im Einzelnen analysiert werden. In Abbildung 6.7 wird erneut auf die Darstellungsweise eines Perzentilplots zurückgegriffen. Dieser wird in Tabelle 6.4 durch zusätzliche Ergebniskennziffern präzisiert.

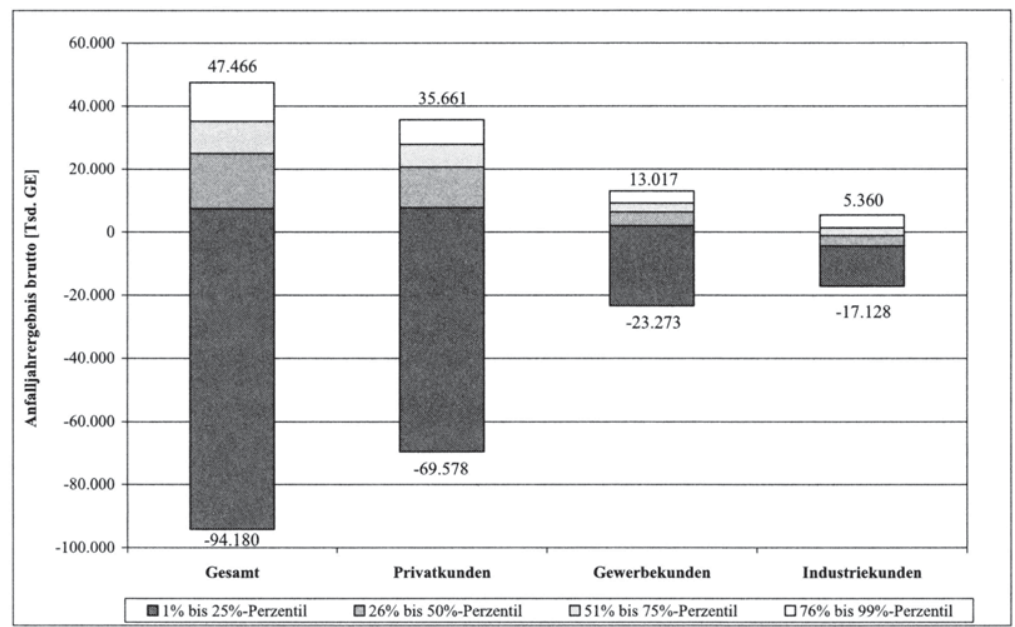

Abbildung 6.7: Streuungsverhalten der Brutto-Anfalljahrergebnisse nach Geschäftsfeldern

\begin{tabular}{|l|r|r|r|r|}
\hline \multicolumn{1}{|c|}{ [Tsd. GE] } & \multicolumn{1}{c|}{ PRI } & \multicolumn{1}{c|}{ GEW } & \multicolumn{1}{c|}{ IND } & GESAMT \\
\hline Erwartungswert & 13.949 & 4.454 & -2.034 & $\mathbf{1 6 . 3 6 9}$ \\
\hline Standardabweichung & 21.336 & 7.252 & 4.755 & $\mathbf{2 8 . 8 2 3}$ \\
\hline Variationskoeffizient [\%] & $153 \%$ & $163 \%$ & $234 \%$ & $\mathbf{1 7 6 \%}$ \\
\hline Median & 20.623 & 6.390 & -1.120 & $\mathbf{2 4 . 8 9 4}$ \\
\hline Maximum & 40.580 & 15.795 & 7.800 & $\mathbf{5 5 . 8 0 7}$ \\
\hline Minimum & -243.082 & -70.894 & -43.258 & $\mathbf{- 3 1 8 . 6 5 6}$ \\
\hline 1\%-Perzentil & -69.578 & -23.273 & -17.128 & $\mathbf{- 9 4 . 1 8 0}$ \\
\hline Verlustwahrscheinlichkeit [\%] & $17,3 \%$ & $18,6 \%$ & $61,4 \%$ & $\mathbf{1 8 , 9 \%}$ \\
\hline
\end{tabular}

Tabelle 6.4: Kennziffern des Brutto-Anfalljahresergebnisses nach Geschäftsfeldern

Von den drei Geschäftsfeldern weist das Privatkundengeschäft die größte absolute Ergebnisschwankung auf. Die starke Exponierung gegenüber Naturereignisschäden manifestiert sich in einem Maximalverlust von -243,1 Mio. GE. Ein Vergleich der Variationskoeffizienten verdeutlicht jedoch, dass die relative Schwankung des Privatkundenergebnisses am geringsten ausfällt. Auch die Verlustwahrscheinlichkeit nimmt mit 17,3\% den geringsten Wert an. Das Gewerbekundengeschäft ist im Hinblick auf seine Verlustwahrscheinlichkeit und relative Ergebnisschwankung mit dem Privatkundenge- 
schäft vergleichbar, bewegt sich jedoch insgesamt auf deutlich geringerem Niveau. Der mögliche Maximalverlust von 70,9 Mio. GE resultiert vornehmlich aus den kumulexponierten Sparten „Kasko“ und „Allgemeine Sturm“. Das Industriekundengeschäft verläuft in mehr als der Hälfte aller Simulationsläufe defizitär und weist die größte relative Ergebnisschwankung auf. Der erneut auf das 1\%-Perzentil des BruttoAnfalljahrergebnisses bezogene Diversifikationseffekt beträgt 15,8 Mio. GE und fällt somit auf der Geschäftsfeldebene deutlich geringer aus als auf der Spartenebene. ${ }^{934}$

Ausgehend von der in Kapitel 2.3 gewählten Definition wird nachfolgend der versicherungstechnische Risikokapitalbedarf des Modellunternehmens ermittelt. Das Risikokapital dient der Absicherung versicherungstechnischer Bruttoverluste, die sich aus dem Zeichnungs- bzw. Tarifierungsrisiko des simulierten Anfalljahres ergeben. Die in Abbildung 6.8 dargestellte empirische Dichtefunktion vermittelt zunächst einen Eindruck über das asymmetrische Streuungsverhalten des Bruttoergebnisses.

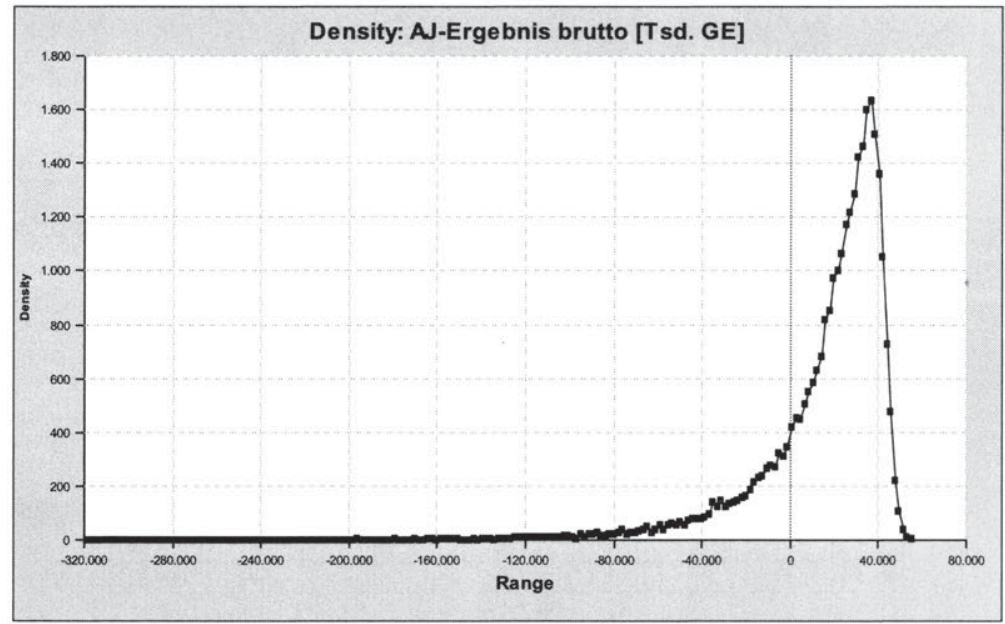

Abbildung 6.8: Empirische Dichtefunktion des Brutto-Anfalljahrergebnisses

Das erhebliche Verlustpotenzial, welches der Tail der linksschiefen Ergebnisverteilung birgt, tritt in dieser Darstellungsform noch deutlicher zu Tage. Darüber hinaus wird ersichtlich, dass die Verteilung des Brutto-Anfalljahrergebnisses lediglich einer linearen Transformation der Zufallsgesetzmäßigkeit des Brutto-Gesamtschadens entspricht, da sonstige Ergebniskomponenten wie Prämien und Kosten als deterministisch ange-

934 Es sei daran erinnert, dass die Schadenentwicklung zwischen den Geschäftsfeldern als stochastisch unabhängig unterstellt wird. Der Diversifikationseffekt fällt daher höher aus als im Falle positiver Abhängigkeit. 
nommen werden. ${ }^{935}$ Der korrespondierende Risikokapitalbedarf lässt sich jedoch anschaulicher aus der Verteilungsfunktion bzw. dem Perzentilgraphen ableiten.

Wie bereits in Kapitel 4.1.3 begründet, wird im Rahmen dieser Arbeit als Risikomaß der Conditional Value-at-Risk (CVaR) herangezogen und ein Sicherheitsniveau 1- $\varepsilon$ von $99,5 \%$ zugrunde gelegt. Angewendet auf das modellierte Risikokollektiv entspricht der als CVaR ermittelte Risikokapitalbedarf näherungsweise dem Kapitalbedarf auf der Basis des Value-at-Risk $(\mathrm{VaR})^{936}$ mit einem Sicherheitsniveau von 99,78\%. Dieses entspricht wiederum der einjährigen Ausfallwahrscheinlichkeit eines BBB-Rating nach STANDARD \& POOR's. ${ }^{937}$ In Abbildung 6.9 wird dieser Zusammenhang anhand des Perzentilgraphen des Brutto-Anfalljahrergebnisses visualisiert.

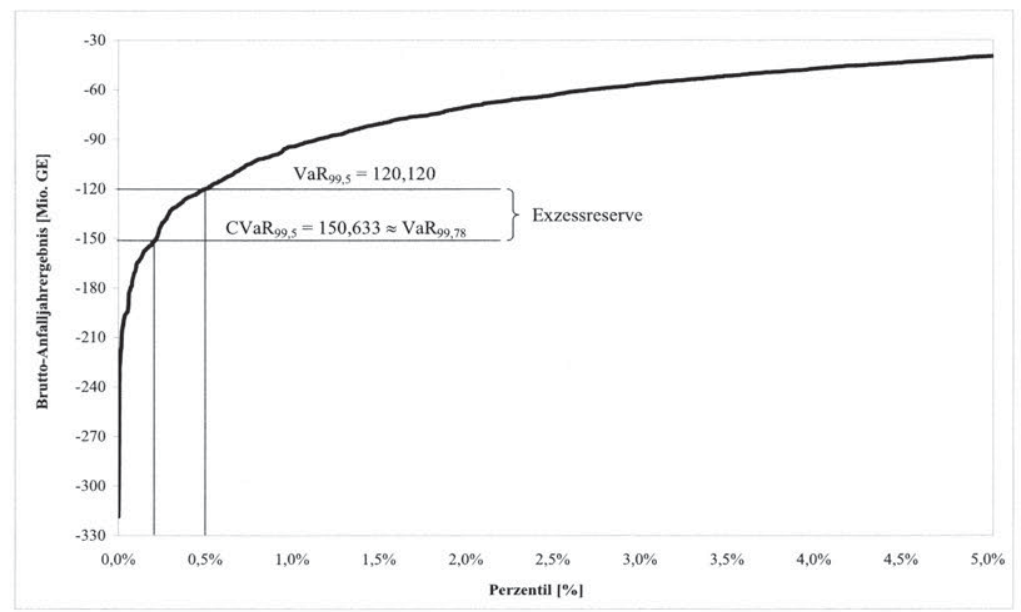

935 Die empirische Dichtefunktion der Ergebnisgröße kann daher als spiegelbildliche Darstellung der aus Abbildung 6.5 bekannten Gesamtschadenverteilung interpretiert werden, welche lediglich um den deterministischen Saldo aus Prämien und Kosten verschoben ist. Vgl. auch KORYCIORZ (2004), S. 74.

${ }^{936}$ Diese Relation stellt ausdrücklich keine allgemeingültige Transformationsbeziehung dar, sondern trifft lediglich approximativ auf die Risikostruktur des hier betrachteten Modellunternehmens zu. Es gilt ferner zu beachten, dass der Steuerungsparameter $\varepsilon$ im Gegensatz zum VaR-Konzept im Anwendungskontext des CVaR ausdrücklich nicht mehr als Ruin- bzw. Ausfallwahrscheinlichkeit interpretiert werden kann. Vgl. hierzu auch Kapitel 4.1.3.3 und KORYCIORZ (2004), S. 79.

937 Durch die Wahl eines Sicherheitsniveaus von 99,5\% für den CVaR stellt das Modellunternehmen für das hier betrachtete Portfolio sicher, dass die einperiodige Ausfallwahrscheinlichkeit auf der Basis des VaR mindestens einem ,investment-grade-Rating“ entspricht. Im Rahmen der Neuausrichtung des europäischen Solvabilitätssystems (Solvency II) wird erwartet, dass sich die Kapitalanforderungen an dieser Ratingstufe orientieren werden. Vgl. übereinstimmend GDV (2002b), S. 25 und zum versicherungsspezifischen Ratingprozess STANDARD \& POOR's (2000). 
Das für das Zeichnungsrisiko des simulierten Anfalljahres vorzuhaltende Risikokapital beträgt demnach auf der Gesamtunternehmensebene 150,6 Mio. GE. Würde hingegen der VaR als Risikomaß herangezogen und unverändert ein Sicherheitsniveau von $99,5 \%$ unterstellt, betrüge der Risikokapitalbedarf lediglich 120,1 Mio. GE. ${ }^{938}$ Dies verdeutlicht, dass durch den $\mathrm{CVaR}$ das Gefährdungspotenzial von Extremereignissen angemessener berücksichtigt wird, da über den VaR hinaus auch der Erwartungswert sämtlicher Ergebnisrealisationen jenseits des VaR (die sog. Exzessreserve) in die Betrachtung einfließt.

KORYCIORZ zeigt für normal- und lognormalverteilte Schadenvariablen, dass das Vorhalten einer Exzessreserve - unabhängig vom gewählten Sicherheitsniveau - eine signifikante Reduzierung der Ruinwahrscheinlichkeit induziert, und dass ferner mit zunehmendem Sicherheitsniveau 1- $\varepsilon$ der „relative Vorteil“ des CVaR gegenüber dem VaR abnimmt. ${ }^{939}$ Allerdings kann ebenfalls gezeigt werden, dass mit zunehmender Rechtsschiefe der jeweils zugrunde liegenden Schadenverteilung die Exzessreserve sogar absolut zunimmt. ${ }^{940}$ Dies verdeutlicht einerseits, dass das Verlustpotenzial von Katastrophenszenarien, wie beispielsweise großen Sturmereignissen, auch bei hohen Sicherheitsniveaus weiterhin steuerungsrelevant ist. ${ }^{941}$ Andererseits gewinnt die Exzessreserve mit zunehmender Rechtsschiefe der zugrunde liegenden Schadenverteilung an Bedeutung, was im spezifischen Kontext der Schaden- und Unfallversicherung die Vorteilhaftigkeit des $\mathrm{CVaR}$ gegenüber dem VaR als Risikomaß untermauert.

In Kapitel 3.2.2 wurde die Notwendigkeit einer integrierten Rendite-Risikosteuerung bereits aufgezeigt und der RORAC als geeignetes risikoadjustiertes Performancemaß identifiziert. Auf der Gesellschaftsebene ergibt sich der RORAC des versicherungstechnischen Bruttogeschäfts als Quotient aus erwartetem Brutto-Anfalljahrergebnis und Risikokapital zu:

$$
\operatorname{RORAC}_{\text {Bruto }}^{\mathrm{VT}}=\frac{\mathrm{E}\left(\mathrm{G}_{\text {Bruto }}^{\mathrm{VT}}\right)}{\mathrm{CVaR}_{\text {Bruto }}^{\mathrm{VT}}}=\frac{16.369}{150.633}=10,87 \%
$$

938 Die (definitionsgemäß) positive Differenz zwischen CVaR und VaR wird auch als Mean-ExcessLoss bzw. Exzessreserve bezeichnet. Vgl. KORYCIORZ (2004), S. 75-79 und die Ausführungen in Kapitel 4.1.3.3.

939 Vgl. KORYCIORZ (2004), S. 74-86.

940 Vgl. KORYCIORZ (2004), S. 86.

941 Dieses Phänomen wird gelegentlich auch als „Persistenz des Katastrophenrisikos“ bezeichnet. Vgl. KORYCIORZ (2004), S. 79 und zur originären Interpretation des Begriffs „Persistenz des Risikos“ ALBRECHT/MAURER/RUCKPAUL (2001), S. 486. 
Auf der Grundlage dieses Ergebnisses kann noch nicht abschließend beurteilt werden, ob das versicherungstechnische Bruttogeschäft eine Rendite erwirtschaftet, die den Anforderungen der Kapitalgeber genügt. Auf die Vorgabe einer expliziten ,hurdle rate" für das Bruttogeschäft soll hier jedoch zunächst verzichtet werden. Da Versicherungsunternehmen ihren Bruttokapitalbedarf noch substanziell durch Rückversicherungsnahme reduzieren können, bezieht sich die Renditeforderung der Kapitalgeber in der Regel auf den Nettokapitalbedarf. ${ }^{942}$

Die Anwendung der in Kapitel 5.3.3.4 vorgestellten Allokationsmethodik nach dem CVaR-Konzept führt zu nachstehender Verteilung des Risikokapitalbedarfs auf die modellierten Sparte-Geschäftsfeld-Kombinationen. ${ }^{943}$ Die Allokationsergebnisse sollen nachfolgend zunächst strukturell erläutert und anschließend einer kritischen Würdigung unterzogen werden.

\begin{tabular}{|l|r|r|r|r|}
\hline \multicolumn{1}{|c|}{ [Tsd. GE] } & \multicolumn{1}{c|}{ PRI } & \multicolumn{1}{c|}{ GEW } & \multicolumn{1}{c|}{ IND } & \multicolumn{1}{c|}{ SUMME } \\
\hline Allgemeine Unfall & -6.061 & -488 & -323 & $\mathbf{- 6 . 8 7 2}$ \\
\hline Kraftfahrt-Haftpflicht & -5.851 & -2.118 & 439 & $\mathbf{- 7 . 5 3 0}$ \\
\hline Kasko & 3.355 & 565 & 246 & $\mathbf{4 . 1 6 5}$ \\
\hline Feuer Industrie & & & 2.037 & $\mathbf{2 . 0 3 7}$ \\
\hline Allgemeine Sturm & & 36.061 & 3.488 & $\mathbf{3 9 . 5 4 9}$ \\
\hline VGV Feuer & 208 & & & $\mathbf{2 0 8}$ \\
\hline VGV Leitungswasser & 11.991 & & & $\mathbf{1 1 . 9 9 1}$ \\
\hline VGV Sturm & 104.994 & & & $\mathbf{1 0 4 . 9 9 4}$ \\
\hline VGV Elementar & 2.090 & & & $\mathbf{2 . 0 9 0}$ \\
\hline SUMME & $\mathbf{1 1 0 . 7 2 6}$ & $\mathbf{3 4 . 0 2 0}$ & $\mathbf{5 . 8 8 7}$ & $\mathbf{1 5 0 . 6 3 3}$ \\
\hline
\end{tabular}

Tabelle 6.5: Allokation des Risikokapitalbedarfs (Brutto)

Das Geschäftsfeld Privatkunden verfügt aufgrund seiner dominanten Bestandsgröße auch über den höchsten absoluten Risikokapitalbedarf. Die Geschäftsfelder Gewerbeund Industriekunden weisen hingegen - gemessen am absoluten Kapitalbedarf - eine deutlich geringere Risikoexponierung auf. Insgesamt wird der Risikokapitalbedarf des Modellunternehmens maßgeblich durch die „Verbundene Wohngebäudeversicherung“ und die Sparte „Allgemeine Sturm“ dominiert. Der für die Risikokapitalbestimmung relevante Randbereich der Ergebnisverteilung wird offenbar stark durch Simulationen repräsentiert, die eine hohe Schadenbelastung durch Sturmereignisse aufweisen. ${ }^{944}$

${ }_{942}$ Hierzu wird auf die Ausführungen in Kapitel 6.2.1.1.2 verwiesen.

943 Aufgrund der Linearitätseigenschaft bedingter Erwartungswerte kann der CVaR additiv auf die betrachteten Teilkollektive aufgeteilt werden. Vgl. hierzu auch Kapitel 4.2.4.

944 Der besonderen Bedeutung der Naturereignisschäden für das Tailverhalten bzw. den Risikokapitalbedarf Rechnung tragend, wird deren Einfluss in Kapitel 5.4.3.4 vertiefend analysiert. Darüber hinaus wird die Struktur des Randbereichs der Bruttoergebnisverteilung in Anhang E für ausgewählte Versicherungszweige graphisch dargestellt. 
Hingegen wird den Sparten „Allgemeine Unfall“ und „Kraftfahrt-Haftpflicht“ negatives Risikokapital zugewiesen. In der Unfallsparte ist dieses Allokationsergebnis intuitiv interpretierbar, da sich deren Ergebnisverteilung ausschließlich im positiven Wertebereich bewegt. In der Sparte „Kraftfahrt-Haftpflicht“ treten jedoch in einer isolierten Betrachtung durchaus negative Ergebnisrealisationen auf, die einen positiven Risikokapitalbedarf implizieren würden. Der Versicherungszweig „Kraftfahrt-Haftpflicht“ profitiert jedoch von kollektiven Risikoausgleichseffekten dergestalt, dass singuläre Spartenverluste durch positive Ergebnisse in anderen Bereichen überkompensiert werden und diese Szenarien letztlich nicht mehr in den Randbereich der Gesamtverteilung fallen. In den für die Risikokapitalbestimmung relevanten 150 schlechtesten Ergebnisszenarien wird in der Sparte „Kraftfahrt-Haftpflicht“ im Erwartungswert sogar ein Gewinn von 7.530 Tsd. GE erzielt. Dieser entspricht betragsmäßig genau dem aus Tabelle 6.5 ersichtlichen (negativen) Risikokapitalbedarf dieses Segments.

Das Beispiel verdeutlicht, dass eine Allokation nach dem CVaR-Konzept eine intuitiv nachvollziehbare Interpretation der Zuweisungsbeträge ermöglicht. Jeder Geschäftseinheit wird gerade so viel Risikokapital zugewiesen, wie sie im Erwartungswert zur Entstehung der außergewöhnlichen Verluste jenseits des Value-at-Risk beiträgt. ${ }^{945}$ Sofern ein Geschäftsbereich in den Randszenarien im Erwartungswert einen Gewinn erwirtschaftet, resultiert hieraus ein negativer Zuweisungsbetrag.

In Abhängigkeit von der Art und Weise, wie die Ergebnisverteilungen einzelner Teilkollektive zu übergeordneten Kollektiven zusammengefasst werden, treten unterschiedliche Risikoausgleichseffekte zu Tage. ${ }^{946}$ In Abbildung 6.10 wird exemplarisch der Risikokapitalbedarf dargestellt, der sich insgesamt für unterschiedliche Aggregationsstufen („Schnitte durch das Gesamtkollektiv“) ergeben würde. ${ }^{947}$ Aufgrund der Linearität des Erwartungswertes ist das erwartete Brutto-Anfalljahrergebnis für alle betrachteten Aggregationsstufen identisch. Der RORAC wird daher ausschließlich durch die Höhe des Risikokapitalbedarfs beeinflusst.

\footnotetext{
945 Vgl. auch KORYCIORZ (2004), S. 250.

946 Vgl. hierzu vertiefend Kapitel 4.2.1 sowie LEYHERR/SCULLY/SOMMERFELD (2003), S. 1796.

${ }^{947}$ Die hiermit korrespondierenden Allokationsergebnisse sind dem Anhang B zu entnehmen.
} 


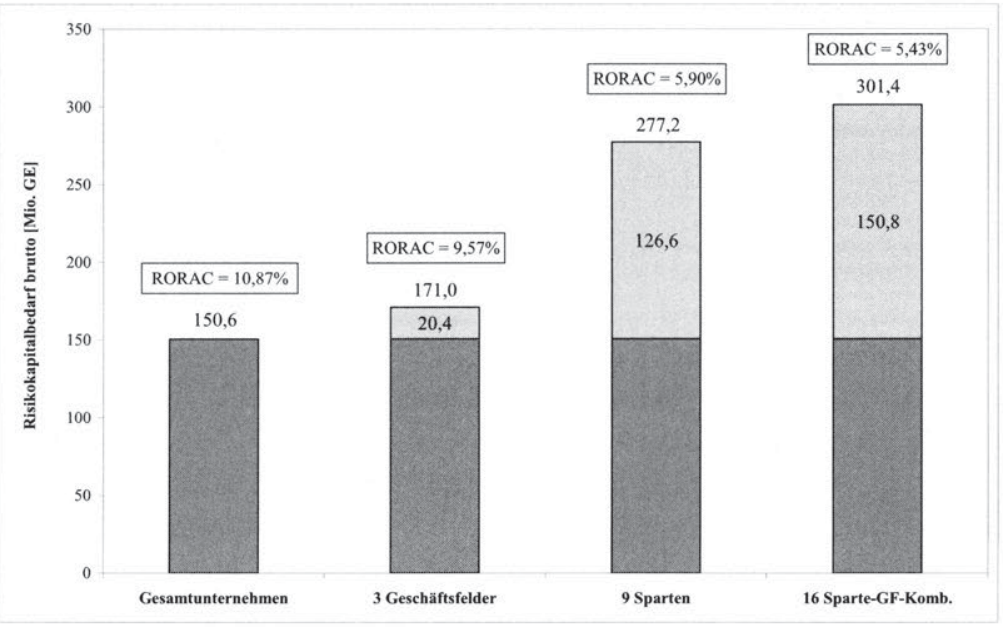

Abbildung 6.10: Diversifikationseffekte unterschiedlicher Portfolio-Aggregationen

Werden die drei Geschäftsfelder isoliert voneinander (als fiktive Teilunternehmen) betrachtet, so können Risikoausgleichseffekte innerhalb eines Geschäftsfelds nur noch zwischen den jeweils betriebenen Versicherungszweigen auftreten. Gegenüber der Betrachtung des Gesamtportfolios ergibt sich für diesen Fall ein zusätzlicher Risikokapitalbedarf in Höhe von 20,4 Mio. GE, welcher mit 17,6 Mio. GE weitgehend auf das Geschäftsfeld Industriekunden entfällt. Der Kapitalbedarf der anderen Geschäftsfelder erhöht sich nur geringfügig. Dieser Effekt liegt darin begründet, dass der Industriekundenbestand des Modellunternehmens nur sehr schwach gegenüber Sturmrisiken exponiert ist. Das Risikopotenzial, welches beispielsweise aus dem hochvolatilen industriellen Feuergeschäft resultiert, wird im Gesamtkollektiv durch das Sturmrisiko der anderen Geschäftsfelder überlagert. In der isolierten Betrachtung der Geschäftsfelder wird jedoch gerade das Verlustpotenzial der hochvolatilen Sparte „Feuer Industrie“ relevant und induziert einen zusätzlichen Kapitalbedarf.

Die Tatsache, dass die übrigen Geschäftsfelder in der Standalone-Betrachtung nur geringfügig mehr Risikokapital erfordern als im diversifizierten Gesamtportfolio, darf jedoch nicht zur der Schlussfolgerung verleiten, dass diese aufgrund ihres größeren Bestandes bereits hinreichend diversifiziert seien. Vielmehr lässt die starke Exponierung dieser Geschäftsfelder gegenüber Naturereignisschäden eine weitere Diversifizierung gar nicht erst zu, da der Randbereich der Ergebnisverteilung durch die Katastrophenszenarien dominiert wird. ${ }^{948}$

948 Vgl. hierzu auch die Ausführungen in Anhang E. 
In der isolierten Spartenbetrachtung können Diversifikationseffekte nur noch zwischen den Geschäftsfeldern wirken. Dies bedeutet insbesondere, dass sich günstige und ungünstige Ergebnisverläufe in kumulbelasteten Sparten nicht mehr ausgleichen können und einen erheblichen Mehrbedarf an Risikokapital hervorrufen. Außerdem wird das bisher überlagerte Ergebnisrisiko nicht kumulbelasteter Sparten (z.B. „Kraftfahrt-Haftpflicht") relevant. Insgesamt ergibt sich für den fiktiven Fall, dass die einzelnen Versicherungszweige als selbstständige Teilunternehmen geführt würden, ein zusätzlicher Kapitalbedarf in Höhe von 126,6 Mio. GE.

In der Standalone-Betrachtung schließlich verdoppelt sich der Kapitalbedarf nahezu. Dieser Fall stellt gewissermaßen den „worst case“ dar, da zwischen den einzelnen Teilkollektiven überhaupt keine Ausgleichseffekte mehr wirksam werden können.

Es wird deutlich, dass durch die künstliche Aufteilung des Gesamtkollektivs in einzelne Steuerungseinheiten (fiktive Teilunternehmen) ein segmentübergreifender Risikoausgleich unterbunden würde. Für den realistischen Fall, dass die Steuerungseinheiten nicht vollständig positiv miteinander korreliert sind, wird die Summe der Risikokapitalbeträge sämtlicher Teilunternehmen den Kapitalbedarf des Gesamtkollektivs übersteigen. Fehlsteuerungsimpulse wären in der Folge nicht auszuschließen. Auf eine weitere Problematik segmentspezifischer RORAC-Kennziffern sei hier ebenfalls hingewiesen. Die aus Tabelle 6.5 ersichtlichen negativen Risikokapitalbeträge bedingen für einzelne Teilkollektive konsequenterweise auch negative Ausprägungen des RORAC, obwohl (bzw. gerade weil) diese Segmente positive Anfalljahrergebnisse erwirtschaften. Für den Fall, dass diesen Geschäftsbereichen vereinfachend ein Risikokapital von Null zugewiesen würde („Nullallokation“), ergäbe sich eine unendliche Bereichsperformance. Die Ableitung steuerungsrelevanter Informationen zur Verbesserung der Rendite-Risikoposition erscheint daher allenfalls zufällig möglich. ${ }^{949}$

Vor dem Hintergrund dieser Erkenntnisse und angesichts der bereits in Kapitel 4.2.2 formulierten Kritik an einer dezentralen Marktbereichssteuerung über risikoadjustierte Performancemaße wird im weiteren Verlauf der Untersuchung zur Bewertung einzelner Strategien immer der versicherungstechnische RORAC auf der Gesamtunternehmensebene, d.h. unter Berücksichtigung sämtlicher Risikoausgleichseffekte herangezogen. ${ }^{950}$ Nachdem bisher ausschließlich das versicherungstechnische Bruttogeschäft

949 Vgl. übereinstimmend DRESEL (2003), S. 75.

950 „The proper and straightforward way to make capital budgeting decisions for lines of business is to directly evaluate whether and to what extent expanding or contracting a line of business will lead to higher or lower profitability of the firm as a whole." GRÜNDL/SCHMEISER (2004c), S. 13 [Hervorhebung nicht im Original]. 
betrachtet wurde, wird die Analyse der Rendite-Risiko-Position nachfolgend um den Einfluss der Rückversicherung erweitert.

\subsection{Darstellung und Analyse des Nettogeschäfts}

Hinsichtlich der Steuerung des versicherungstechnischen Portfolios stellt die Rückversicherung ein zentrales Gestaltungsmittel dar. Ein portfoliospezifisch abgestimmtes Rückversicherungsprogramm, welches die finanziellen Auswirkungen einzelner Großschäden auf ein vorgegebenes Ausmaß beschränkt und außerdem in kumulexponierten Versicherungszweigen Haftung übernimmt, kann zu einer signifikanten Reduzierung der Ergebnisvolatilität und folgerichtig auch des Risikokapitalbedarfs beitragen. ${ }^{951}$ Neben ihrer Funktion als Kapitalsubstitut verursacht die Inanspruchnahme von Rückversicherungsschutz jedoch auch Kosten, welche sowohl das versicherungstechnische Nettoergebnis als auch den korrespondierenden Cashflow tangieren. Angesichts dieser gegenläufigen Effekte ist es notwendig, ein Kriterium zu formulieren, welches die Rendite-Risiko-Effizienz alternativer Handlungsalternativen quantifiziert. In diesem Kontext gilt es zunächst zu berücksichtigen, dass ein Rückversicherungsprogramm in der Regel durch den kombinierten Einsatz unterschiedlichster Vertragsformen und Deckungsvereinbarungen charakterisiert ist. ${ }^{952}$ Sofern Erst- und Rückversicherer eine langjährige und umfangreiche Geschäftsbeziehung unterhalten, kann bisweilen auch eine „Quersubventionierung“ zwischen einzelnen Vertragsformen bzw. Sparten beobachtet werden, die im Interesse der Gesamtverbindung eine angemessene Risiko- und Ergebnisteilung herbeiführen sollen. ${ }^{953}$ Als Beispiel für derartige Konstellationen kann im betrachteten Modellunternehmen der Quotenrückversicherungsvertrag in der Sparte „Allgemeine Unfall“ interpretiert werden. Bei isolierter Betrachtung wäre es für das Modellunternehmen vermutlich nicht sinnvoll, $25 \%$ des profitablen Unfallgeschäfts an einen Rückversicherer zu zedieren und in der Folge auf einen ,sicheren Ertrag“ zu verzichten. ${ }^{954}$ Der Quotenvertrag in der Sparte „Allgemeine Unfall“ ist jedoch in Kombination mit dem (unlimitierten) Quotenrückversicherungsvertrag in den Sturmsparten zu beurteilen, welcher angesichts des erheblichen regionalen Sturmkumuls bei isolierter Betrachtung vermutlich nur schwer am Markt zu platzieren wäre.

951 Vgl. übereinstimmend HERSBERGER (1996), S. 16, MACK (2002), S. 325 und insbesondere KORYCIORZ (2004), S. 140-157.

952 Vgl. LIEBWEIN (2000), S. 51 ff. und die Ausführungen in Kapitel 5.4.5.

953 „So sind gerade in der Rückversicherung der konkrete Preis und damit die darin enthaltenen Kosten ausschließlich das individuelle Ergebnis der abwägenden Überlegungen der Beteiligten. Allein diesen beiden ist es daher überlassen, ob sie einen ihrer individuellen Beziehung entsprechenden optimalen Gleichgewichtspunkt finden können.“ MACK (1983), S. 336. Vgl. auch LIEBWEIN (2000), S. 74 und S. 168.

954 An dieser Stelle wird davon ausgegangen, dass der Quotenvertrag nicht zum Zwecke der Aufbaufinanzierung der Sparte „Allgemeine Unfall“ abgeschlossen wurde (sog. Aufbauquote). Vgl. zu diesem Sachverhalt vertiefend ARNOLDUSSEN (1991), S. 177-181. 
Im beschriebenen Fall wird demnach das Nettoergebnis der Sparte „Allgemeine Unfall“" im Interesse der Risikoexponierung des Gesamtunternehmens belastet. Die Rückversicherungsverträge der Sturmsparten werden durch das Unfallgeschäft subventioniert. Dieses Beispiel verdeutlicht bereits, dass eine direkte Zuordnung der Rückversicherungskosten auf einzelne Teilkollektive (z.B. Versicherungszweige) vielfach nicht möglich und unter Steuerungsaspekten nicht sachgerecht ist. Abgesehen von dieser in der Praxis häufig anzutreffenden Zuordnungsproblematik wird hier die Ansicht vertreten, dass ein Rückversicherungsprogramm immer unter der Zielsetzung beurteilt werden sollte, eine dem Risikotragfähigkeitspotenzial des Gesamtunternehmens angemessene Exponierung zu erreichen. ${ }^{955}$ Ein derartig formuliertes Verständnis schließt jedoch isolierte Optimierungskalküle für einzelne Teilkollektive ausdrücklich aus.

Im betrachteten Modellansatz wird durch die „technische“ Ermittlung des Rückversicherungspreises eine risikoadäquate Belastung der einzelnen Versicherungszweige gewährleistet und die Grundlage für eine Spartenerfolgsrechnung nach Rückversicherung geschaffen. Aufgrund der beschriebenen, vielfach unternehmenspolitisch motivierten Subventionierungseffekte sollten jedoch Nettoergebnisse einzelner Sparten oder hierauf basierende RORAC-Kennziffern nicht unreflektiert zu Steuerungszwecken herangezogen werden. ${ }^{956}$ Vielmehr sollte die Effizienz eines Rückversicherungsprogramms danach beurteilt werden, ob und in welchem Ausmaß dieses zu einer Verbesserung der versicherungstechnischen Rendite-Risikoposition des Gesamtunternehmens beiträgt. Die Rückversicherung kann demzufolge als effizient bezeichnet werden, sofern auf der Unternehmensebene gilt:

$$
\begin{aligned}
& \mathrm{RORAC}_{\text {Netto }}^{\mathrm{VT}}>\mathrm{RORAC}_{\text {Brutto }}^{\mathrm{VT}} \Leftrightarrow \frac{\mathrm{E}\left(\mathrm{G}_{\text {Netro }}^{\mathrm{VT}}\right)}{\mathrm{RK}_{\text {Netto }}^{\mathrm{VT}}}>\frac{\mathrm{E}\left(\mathrm{G}_{\mathrm{BButt}}^{\mathrm{VT}}\right)}{\mathrm{RK}_{\mathrm{Brutto}}^{\mathrm{VT}}} \\
& \text { mit: } \quad \mathrm{G}^{\mathrm{VT}}=\text { versicherungstechnisches Ergebnis } \\
& \mathrm{RK}^{\mathrm{VT}}=\text { versicherungstechnisches Risikokapital }
\end{aligned}
$$

Dies ist der Fall, sofern die relative Risikokapitalersparnis durch die Rückversicherungsnahme größer ist als die relative Reduzierung des versicherungstechnischen Ergebnisses durch die Kosten der Rückversicherung. Formal ergibt sich nach Umformung von $(6.3):^{957}$

\footnotetext{
955 Vgl. übereinstimmend MENTZEL (2004), S. 263. Vgl. zu weiteren Zielen der Rückversicherungsnahme ausführlich GRAUMANN (1997), S. 367-370.

956 Vgl. zur grundlegenden Kritik an der Verwendung risikoadjustierter Performancemaße im Rahmen dezentraler Marktbereichssteuerung auch Kapitel 4.2.2.

957 Vgl. MENTZel (2004), S. 85-86, HeRSBERGER (1996), S. 16-17 und SCHRADIN (1994), S. 319.
} 


$$
\begin{aligned}
\frac{\mathrm{RK}_{\mathrm{Brutto}}^{\mathrm{VT}}-\mathrm{RK}_{\text {Netto }}^{\mathrm{VT}}}{\mathrm{RK}_{\text {Netto }}^{\mathrm{VT}}}>\frac{\mathrm{P}^{\mathrm{RV}}-\mathrm{Prov}^{\mathrm{RV}}}{\mathrm{E}\left(\mathrm{G}_{\text {Netto }}^{\mathrm{VT}}\right)} \Leftrightarrow \frac{\Delta \mathrm{RK}^{\mathrm{VT}}}{\mathrm{RK}_{\text {Netto }}^{\mathrm{VT}}}>\frac{\mathrm{K}^{\mathrm{RV}}}{\mathrm{E}\left(\mathrm{G}_{\text {Netto }}^{\mathrm{VT}}\right)} \\
\text { mit: } \quad \mathrm{P}^{\mathrm{RV}}=\text { Rückversicherungsprämie } \\
\mathrm{Prov}^{\mathrm{RV}}=\text { Rückversicherungsprovision } \\
\mathrm{K}^{\mathrm{RV}}=\text { Kosten des Rückversicherungsschutzes } \\
\Delta \mathrm{RK}^{\mathrm{VT}}=\text { Risikokapitalersparnis }
\end{aligned}
$$

Im weiteren Verlauf wird die Struktur des in Kapitel 5.4.5 definierten Rückversicherungsprogramms hinsichtlich seiner Rendite-Risiko-Effizienz analysiert. Auf eine risikotheoretische Wirkungsanalyse der verschiedenen Rückversicherungsformen anhand der zugrunde liegenden Schadenkollektive einzelner Sparten wird jedoch verzichtet. ${ }^{958}$ In einem ersten Schritt wird die Wirkung des Rückversicherungsprogramms anhand einer Gegenüberstellung der Gesamtschadenverteilungen illustriert.

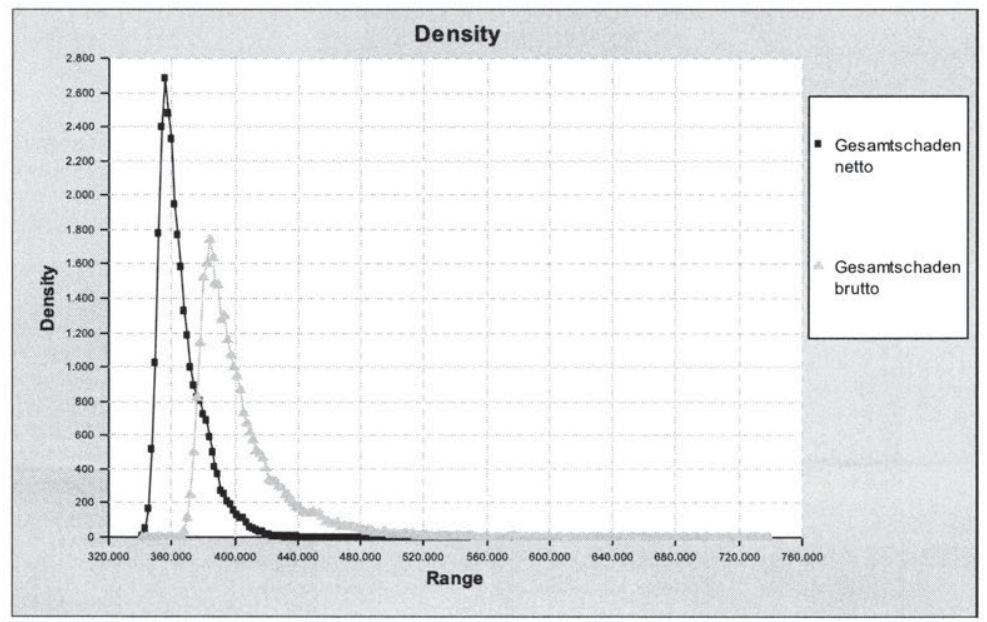

Abbildung 6.11: Empirische Dichtefunktionen des Gesamtschadens

Bei kombinierter Betrachtung von Abbildung 6.11 und Tabelle 6.6 werden bereits wesentliche Effekte deutlich, die sich durch die Rückversicherungsnahme einstellen. 


\begin{tabular}{|l|r|r|}
\hline \multicolumn{1}{|c|}{ [Tsd. GE] } & \multicolumn{1}{c|}{ Brutto } & \multicolumn{1}{c|}{ Netto } \\
\hline Erwartungswert & 403.304 & 366.106 \\
\hline Standardabweichung & 28.943 & 13.950 \\
\hline Variationskoeffizient [\%] & $7,2 \%$ & $3,8 \%$ \\
\hline Schiefekoeffizient $\gamma$ & 2,38 & 1,33 \\
\hline Median & 394.760 & 362.339 \\
\hline Minimum & 363.550 & 340.800 \\
\hline Maximum & 739.515 & 461.837 \\
\hline 99\%-Perzentil & 514.311 & 410.200 \\
\hline
\end{tabular}

Tabelle 6.6: Statistische Kenngrößen der Gesamtschadenverteilungen

Zunächst fällt auf, dass die Streuung des Gesamtschadens, zunächst gemessen an der Standardabweichung, um mehr als die Hälfte reduziert wird. Eine Aussage über die Wirksamkeit des Rückversicherungsprogramms ist jedoch auf der Basis des symmetrischen Streuungsmaßes der Standardabweichung nicht möglich, zumal die Bruttoschadenverteilung aufgrund der hohen Belastung durch Naturgefahrereignisse einen „fat tail“" aufweist. Durch die Rückversicherung erfährt die Gesamtverteilung der Originalschäden jedoch eine signifikante Stutzung am rechten Rand. Dieser Effekt ist auf den Einfluss der nicht-proportionalen Rückversicherungsverträge zurückzuführen, da Wahrscheinlichkeitsmasse am rechten Rand der Schadenverteilung abgeschnitten und in der Priorität konzentriert wird. ${ }^{959}$ Nicht-proportionale Verträge bewirken somit einen signifikanten Entlastungseffekt in Bezug auf den ökonomisch relevanten rechten Rand der Gesamtschadenverteilung. In der Praxis wird der Nettorisikokapitalbedarf zuweilen ermittelt, indem das Bruttorisikokapital mit der Selbstbehaltsquote des jeweiligen Unternehmens multipliziert wird. Diese Vorgehensweise bildet jedoch gerade den asymmetrischen Entlastungseffekt nicht-proportionaler Rückversicherungsverträge nicht ab und ist demzufolge abzulehnen. ${ }^{960}$

Die Effekte der Quotenrückversicherung konkretisieren sich lediglich in einer Reduzierung des Skalenparameters (z.B. Erwartungswert) der Schadenverteilung, während der Formparameter unverändert bleibt. ${ }^{961}$ Die proportionale Risikoteilung eines Quotenvertrages nimmt demnach keinen Einfluss auf die relativen Gefährlichkeitsmaße der Schadenverteilung, wie z.B. den Variations- bzw. Schiefekoeffizienten. ${ }^{962} \mathrm{Da}$ im Rahmen eines Quotenvertrags jedes Originalrisiko gleichermaßen zwischen Erst- und Rückversicherer aufgeteilt wird, mithin die Schadenanzahlverteilung durch die Rück-

\footnotetext{
959 Vgl. KORYCIORZ (2004), S. 152-157 und MACK (2002), S. 332-342.

960 Vgl. übereinstimmend LEYHERR/SCULLY/SOMMERFELD (2003), S. 1797.

${ }^{961}$ Die Gestalt der Verteilungsdichte einer Schadenvariablen bleibt somit nach Abschluss eines Quotenrückversicherungsvertrags sowohl beim Erst- als auch beim Rückversicherer dieselbe. Lediglich deren Größe ändert sich. Vgl. übereinstimmend MACK (2002), S. 329-332 und MENTZEL (2004), S. 95. Vgl. außerdem zur Unterscheidung von Skalen- und Formparameter in empirisch relevanten Verteilungsmodellen MACK (2002), S. 86-100.

962 Vgl. MACK (2002), S. 330.
} 
versicherung unberührt bleibt, ergeben sich auch auf der Kollektivebene keine strukturellen Änderungen des versicherten Risikos. Bei einem Summenexzedentenvertrag hingegen bewirkt das vertragsindividuelle Aufteilungsverhältnis in Bezug auf die relativen Gefährlichkeitsmaße in der Regel eine Reduzierung im Portfolioanteil des Erstversicherers und spiegelbildlich eine Erhöhung im Portfolioanteil des Rückversicherers. ${ }^{963}$

Die Wirkung des vorgegebenen Rückversicherungsprogramms wird nachfolgend anhand der versicherungstechnischen Nettoergebnisse der einzelnen Versicherungszweige analysiert. ${ }^{964}$ Die Skalierung der Ordinate in Abbildung 6.12 wurde identisch zur Skalierung der Bruttoergebnisse in Abbildung 6.6 gewählt, um auch optisch einen Brutto-Netto-Vergleich zu ermöglichen. Die Darstellung wird in Tabelle 6.7 um zusätzliche Ergebniskennziffern ergänzt.

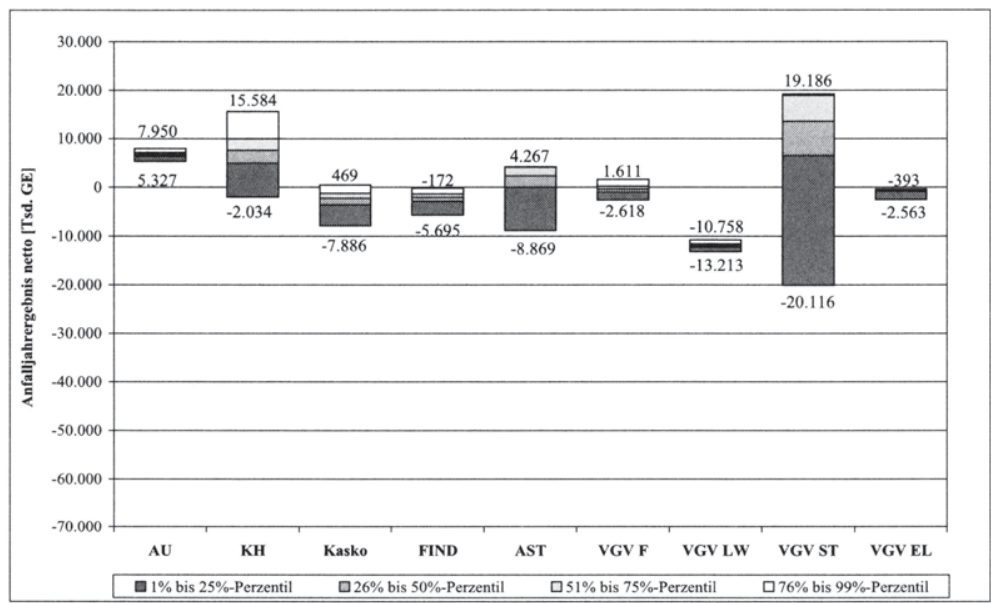

Abbildung 6.12: Streuungsverhalten der Netto-Anfalljahrergebnisse nach Versicherungszweigen

\begin{tabular}{|c|c|c|c|c|c|c|c|c|c|c|}
\hline [Tsd. GE] & $\mathbf{A U}$ & KH & Kasko & FIND & AST & VGV F & VGV LW & VGV ST & VGV EL & GESAMT \\
\hline Erwartungswert & 6.726 & 7.426 & -2.554 & -2.232 & 1.639 & -383 & -11.955 & 11.374 & -783 & 9.257 \\
\hline Standardabweichung & 567 & 3.774 & 1.843 & 1.202 & 3.281 & 907 & 530 & 9.826 & 728 & 13.886 \\
\hline Variationskoeffizient [\%] & $8 \%$ & $51 \%$ & $72 \%$ & $54 \%$ & $200 \%$ & $237 \%$ & $4 \%$ & $86 \%$ & $93 \%$ & $150 \%$ \\
\hline Median & 6.747 & 7.578 & -2.253 & -2.068 & 2.362 & -354 & -11.955 & 13.550 & -709 & 13.031 \\
\hline Maximum & 8.651 & 21.134 & 1.658 & 377 & 4.376 & 2.989 & -9.897 & 19.395 & -207 & 34.402 \\
\hline Minimum & 3.543 & -8.885 & -25.267 & -9.392 & -22.360 & -4.427 & -13.958 & -60.969 & -18.712 & -86.101 \\
\hline $1 \%$-Perzentil & 5.327 & -2.034 & -7.886 & -5.695 & -8.869 & -2.618 & -13.213 & -20.116 & -2.563 & -34.625 \\
\hline
\end{tabular}

Tabelle 6.7: Kennziffern des Netto-Anfalljahrergebnisses nach Versicherungszweigen

963 Vgl. MACK (2002), S. 330.

964 Wie bereits in Kapitel 5.4.5.1 angesprochen, erfolgt in der versicherungstechnischen Nettobetrachtung eine Eingrenzung auf die Spartensicht. In der Praxis liegen valide Rückversicherungsdaten in der Regel nur auf der Ebene der Versicherungszweige und nicht für einzelne Geschäftsfelder vor. 
Der volatilitätsreduzierende Einfluss der Rückversicherung wird durch eine signifikante Stauchung der einzelnen Perzentilplots dokumentiert. ${ }^{965}$ Diese decken jedoch nur den Wertebereich zwischen dem 1\%-Perzentil und dem 99\%-Perzentil ab. Eine abschließende Aussage über das Tailverhalten, d.h. die „Gefährlichkeit“ der einzelnen Ergebnisverteilungen, lässt sich daher anhand der Perzentilplots noch nicht treffen. Es fallt jedoch auf, dass sich die Variationsbreite der Ergebnisse, definiert als Spannweite zwischen maximaler und minimaler Ergebnisausprägung, über alle Versicherungszweige hinweg stark vermindert hat. ${ }^{966}$ Insbesondere in den kumulbelasteten Sparten und in „Kraftfahrt-Haftpflicht“ bietet die Rückversicherung offenbar einen wirksamen Schutz gegen die finanziellen Auswirkungen von Naturkatastrophenszenarien bzw. hochsummigen Personenschäden. Die Kosten der Rückversicherung manifestieren sich zudem in einer deutlichen Reduzierung der erwarteten Nettoergebnisse je Sparte.

Eine isolierte Beurteilung der Rückversicherung auf der Basis des erwarteten Gewinns erscheint jedoch nicht zweckmäßig. ${ }^{967}$ Vielmehr sollte die Risikokomponente in die Beurteilung einbezogen werden. Mit Blick auf das Nettoergebnis des Gesamtunternehmens ist es daher wichtig festzuhalten, dass sich dessen Streuung gegenüber der Bruttobetrachtung stark reduziert. Das 1\%-Perzentil des Gesamtergebnisses hat sich von brutto -94,2 Mio. GE auf netto -34,6 Mio. GE verbessert. Aufgrund der Rückversicherungsnahme wird ein Verlust in Höhe von -34,6 Mio. GE nur noch mit einprozentiger Wahrscheinlichkeit überschritten. Es wurde bereits darauf hingewiesen, dass die Spannweite als Streuungsmaß eine starke Anfälligkeit gegenüber Extremwerten zeigt. Die Verteilung dieser „Ausreißer“ hängt stark von dem Umfang der zugrunde liegenden Stichprobe ab. Je größer die Stichprobe, desto höher ist die Zufallsschwankung der Extremwerte. ${ }^{968}$ Zum Zweck eines Brutto-Netto-Vergleichs einzelner Versicherungszweige wird daher erneut auf das relative Streuungsmaß des Variationskoeffizienten zurückgegriffen.

Die Gegenüberstellung der Variationskoeffizienten in Abbildung 6.13 verdeutlicht die homogenisierende Wirkung des Rückversicherungsprogramms im Basisszenario. In allen rückversicherten Sparten ist eine signifikante Reduktion des relativen Streuungsverhaltens zu beobachten.

\footnotetext{
965 Keine Veränderungen ergeben sich hingegen in der Sparte „VGV Leitungswasser“, da diese über keinen Rückversicherungsschutz verfügt.

966 Kritisch ist an dieser Stelle anzumerken, dass die Variationsbreite als absolutes Streuungsmaß stark durch untypische Extremwerte (,Ausreißer") beeinflusst wird und daher lediglich ein schwaches Indiz für die Vorteilhaftigkeit der Rückversicherung liefert. Vgl. übereinstimmend NEUBAUER (1994), S. 81.

967 Vgl. übereinstimmend MENTZEL (2004), S. 50.

968 Vgl. hierzu vertiefend SACHS (2002), S. 163-165.
} 


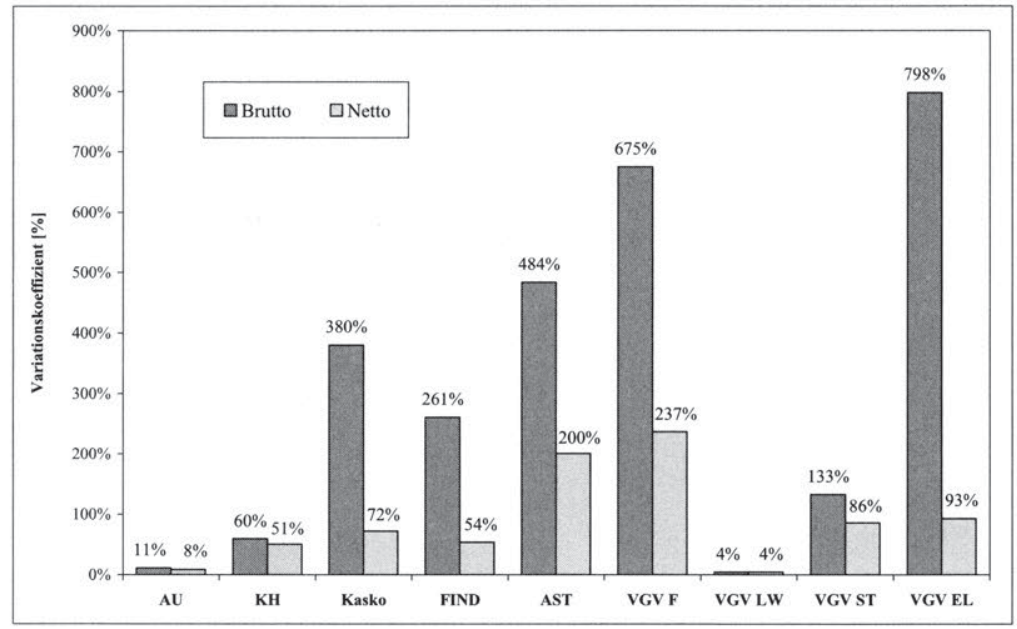

Abbildung 6.13: Relatives Streuungsverhalten der Spartenergebnisse

Nachfolgend soll untersucht werden, in welcher Form sich die Rückversicherungsnahme auf die Verteilung des versicherungstechnischen Gesamtergebnisses auswirkt. Hinsichtlich der hier und im weiteren Verlauf vielfach gewählten Darstellungsformen des Perzentilgraphen ist folgender grundlegender Hinweis erforderlich: Aufgrund der Tatsache, dass die betrachteten Realisationen von Zufallsvariablen im Perzentilgraphen jeweils unabhängig voneinander sortiert sind, stammen nach der Sortierung die auf ein bestimmtes Perzentil entfallenden Realisationen nicht mehr notwendigerweise (sondern allenfalls zufällig) aus demselben Simulationslauf. In der Betrachtung des versicherungstechnischen Geschäfts fällt der hiermit verbundene „Fehler“ aufgrund des weitgehenden Gleichlaufs der Brutto- und Nettoergebnisse jedoch vernachlässigbar klein aus. ${ }^{969}$

Aus Abbildung 6.14 ist ersichtlich, dass das Nettoergebnis lediglich in ca. 17\% aller Szenarien das Bruttoergebnis übersteigt. ${ }^{970}$ Unter dem Aspekt der Risikokapitalermittlung ist jedoch von maßgeblichem Interesse, in welchem Ausmaß der Randbereich der Verteilung durch die Rückversicherung entlastet wird. Offensichtlich bietet das Rückversicherungsprogramm bereits im Basisszenario einen wirksamen Schutz vor Kata-

969 Unter der Annahme, dass ausschließlich proportionaler Rückversicherungsschutz besteht, entfiele die Problematik gänzlich.

$970 \mathrm{Zu}$ Ergebnissen vergleichbarer Größenordnung kommt eine Untersuchung von LEYHERR/SCULLY/ SOMMERFELD, die hierzu auf Originaldaten der Allianz-Gruppe zurückgreifen. Vgl. LEYHERR/ SCULLY/SOMMERFELD (2003), S. 1795-1797. 
strophen- und Großschadenszenarien. Tabelle 6.8 zeigt die in Erwartungswerten formulierte Struktur des Brutto- und Nettoergebnisses im simulierten Anfalljahr.

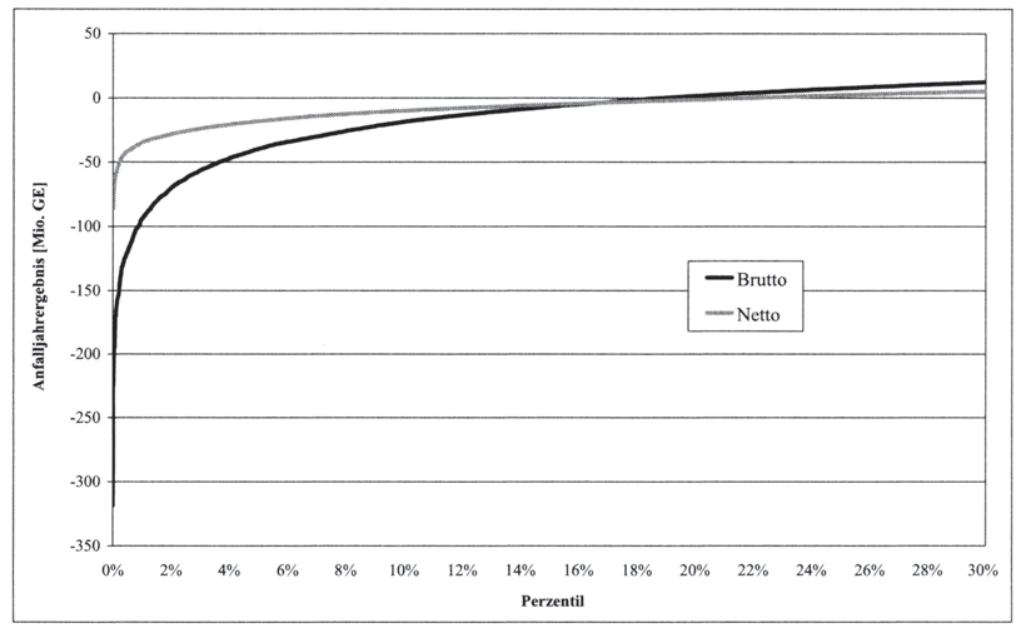

Abbildung 6.14: Perzentilgraph der Anfalljahrergebnisse zwischen $0 \%$ und $30 \%$

\begin{tabular}{|l|c|c|}
\hline \multicolumn{1}{|c|}{ Erwartungswerte [Tsd. GE] } & Brutto & Netto \\
\hline Prämien & 534.750 & 466.678 \\
\hline - Endschaden & 403.304 & 366.106 \\
\hline - Kosten (Prov., Betriebskosten) & 118.434 & 94.359 \\
\hline = AJ-Ergebnis 1 & 13.012 & 6.213 \\
\hline + kalk. Zinsen aus Anlage der BR & 3.358 & 3.044 \\
\hline = AJ-Ergebnis 2 & $\mathbf{1 6 . 3 6 9}$ & $\mathbf{9 . 2 5 7}$ \\
\hline CVaR $_{99,5}^{\mathrm{VT}}$ & $\mathbf{1 5 0 . 6 3 3}$ & $\mathbf{5 1 . 5 5 8}$ \\
\hline RORAC $^{\mathrm{VT}}$ & $\mathbf{1 0 , 8 7 \%}$ & $\mathbf{1 7 , 9 6 \%}$ \\
\hline
\end{tabular}

Tabelle 6.8: Ergebnisstruktur, Risikokapitalbedarf und RORAC

Eine ausschließlich auf Erwartungswerten basierende Analyse käme somit zu der Erkenntnis, dass das bestehende Rückversicherungsprogramm nicht vorteilhaft ist, da das erwartete Nettoergebnis das Bruttoergebnis unterschreitet. Diese Schlussweise ignoriert jedoch gänzlich den aus der nicht-proportionalen Rückversicherung resultierenden Risikotransfer, der eine substanzielle Entlastung des Tails bewirkt. Insgesamt wird der Risikokapitalbedarf durch die Rückversicherung um ca. zwei Drittel auf 51,6 Mio. GE reduziert. ${ }^{971}$

971 Der Nettorisikokapitalbedarf auf der Basis des Value-at-Risk $\left(\mathrm{VaR}_{99,5}\right)$ beträgt 50,2 Mio. GE. Die nur geringfügige Abweichung zum $\mathrm{CVaR}_{99,5}$ ist darauf zurückzuführen, dass extreme Schadenszenarien bereits durch die bestehende Rückversicherung abgefangen werden und somit keinen nennenswerten Einfluss mehr auf die Höhe der Exzessreserve nehmen. 
Eine Aussage über die Effizienz des Rückversicherungsprogramms muss daher neben der erwarteten Ergebniswirkung auch das mit diesem Ergebnis verbundene Risiko berücksichtigen. Ein Vergleich der RORAC-Kennziffern in Tabelle 6.8 verdeutlicht, dass die risikomindernde Wirkung der Rückversicherung die erwartete Ergebniseinbuße deutlich überkompensiert. Der RORAC verbessert sich von 10,87\% auf 17,96\%. Das Rückversicherungsprogramm kann daher im Sinne des in Beziehung (6.3) formulierten Kriteriums als effizient bezeichnet werden. Es sei an dieser Stelle jedoch daran erinnert, dass ein Vergleich der RORAC-Kennziffern keine Aussage darüber erlaubt, ob die bestehende Rückversicherungsstruktur im Sinne eines Unternehmenswertkalküls „optimal“ ist.

Unter Vernachlässigung kalkulatorischer Zinsen ist mit dem Rückversicherungsprogramm im Erwartungswert eine Ergebniseinbuße von 6,8 Mio. GE verbunden. Dieser steht ein um 99,1 Mio. GE reduzierter Risikokapitalbedarf gegenüber. Eine ausschließlich kapitalmarktorientierte Betrachtungsweise käme somit zu dem Ergebnis, dass das Modellunternehmen den rückversicherungsbedingten „Kapitalersatz“ mit $6,9 \%$ verzinst. Allerdings darf an dieser Stelle nicht der Schluss gezogen werden, dass das Unternehmen seine Rendite-Risiko-Position verbessern würde, sofern es den Betrag von 99,1 Mio. GE am Kapitalmarkt zu einem geringeren Zinssatz beziehen könnte. Diese Überlegung ignorierte nämlich die Garantie- und Liquiditätssicherungsfunktion der Rückversicherung und mithin den Sachverhalt, dass das mittels CVaR-Konzept ermittelte Risikokapital lediglich mit einer vorgegebenen Wahrscheinlichkeit 1- $\varepsilon$ zur Deckung von Extremverlusten ausreicht. In denjenigen Tailszenarien, deren Verluste den ermittelten Brutto-Risikokapitalbedarf von 150,6 Mio. GE übersteigen, erhält das Modellunternehmen Entschädigungsleistungen vom Rückversicherer, welche alternativ durch einen Kapitalmarktinvestor nicht erbracht würden. ${ }^{972}$ Dies unterstreicht, dass die Rückversicherungsnahme und der Kapitalmarkt hinsichtlich der erforderlichen Garantie- und Liquiditätssicherungsfunktion nicht als gleichwertig einzustufen sind.

Das für das Gesamtunternehmen ermittelte Netto-Risikokapital kann erneut unter Rückgriff auf das CVaR-Konzept auf die einzelnen Versicherungszweige alloziert werden. Abbildung 6.15 stellt die Ergebnisse der Nettoallokation denen der Bruttoallokation gegenüber.

972 Über die angesprochene Garantiefunktion hinaus erbringt der Rückversicherer auch weitere nichtmonetäre Serviceleistungen wie beispielsweise Unterstützung bei der Schadenregulierung oder aktuariellen Knowhow-Transfer. Vgl. vertiefend LIEBWEIN (2000), S. 47-49. 


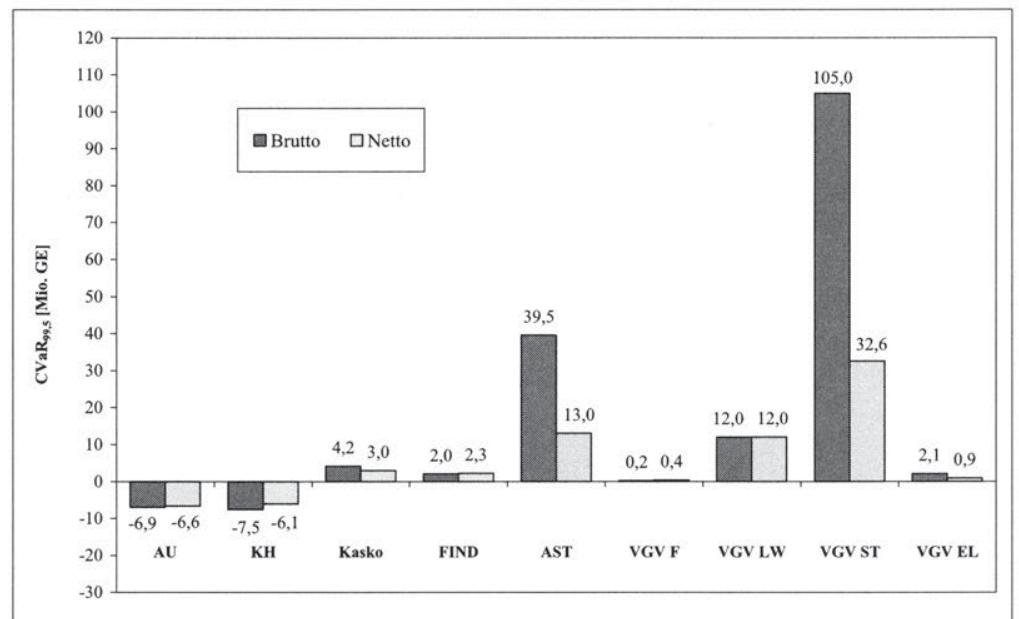

Abbildung 6.15: Allokation des Risikokapitalbedarfs auf Versicherungszweige

Der Risikokapitalbedarf kann in den sturmexponierten Sparten „Allgemeine Sturm“ und „VGV Sturm“ jeweils auf ein Drittel reduziert werden. Allerdings üben diese Versicherungszweige offenbar auch in der Nettobetrachtung einen so dominanten Einfluss auf den Kapitalbedarf aus, dass Hagel- und Überschwemmungsschäden bzw. hochsummige Feuer- und Haftpflichtschäden noch keinen nennenswerten Einfluss auf den Randbereich der Ergebnisverteilung nehmen. Es sei zudem betont, dass aus den obigen Allokationsergebnissen keine isolierten Aussagen über die Effizienz der Rückversicherung in einzelnen Versicherungszweigen abgeleitet werden sollten. Bei der Interpretation der Ergebnisse aus Abbildung 6.15 ist zu berücksichtigen, dass die der Bruttoallokation zugrunde liegenden 150 Tailszenarien nicht notwendigerweise mit denen der Nettoallokation übereinstimmen müssen. Einige Simulationsläufe fallen gegebenenfalls erst in der Nettosicht in den Randbereich der Ergebnisverteilung, da ihr Schadenpotenzial in der Bruttobetrachtung noch durch Naturereignisschäden überlagert wurde. Der Tail der Nettoergebnisverteilung weist daher eine veränderte Struktur auf.

Angesicht des weiterhin dominanten Einflusses der Sturmschäden auf den Nettorisikokapitalbedarf erscheint es angezeigt, die Wirkung der sturmbezogenen Rückversicherungsverträge eingehender zu untersuchen. Zu diesem Zweck werden in Abbildung 6.16 für jeden einzelnen der 30.000 Simulationsläufe die Brutto- und Nettoschäden aus Sturmereignissen in der Form eines Streudiagramms („Scatterplot") gegenübergestellt. Dabei sind die Nettoschäden auf der Ordinate, die zugehörigen Bruttoschäden auf der Abszisse abgetragen. Jeder Punkt des Streudiagramms repräsentiert die Schadensumme aus Sturmereignissen in der Sparte „VGV Sturm“ je Simulationslauf. 
Die Schadensumme setzt sich daher gegebenenfalls aus mehreren einzelnen Sturmereignissen zusammen.

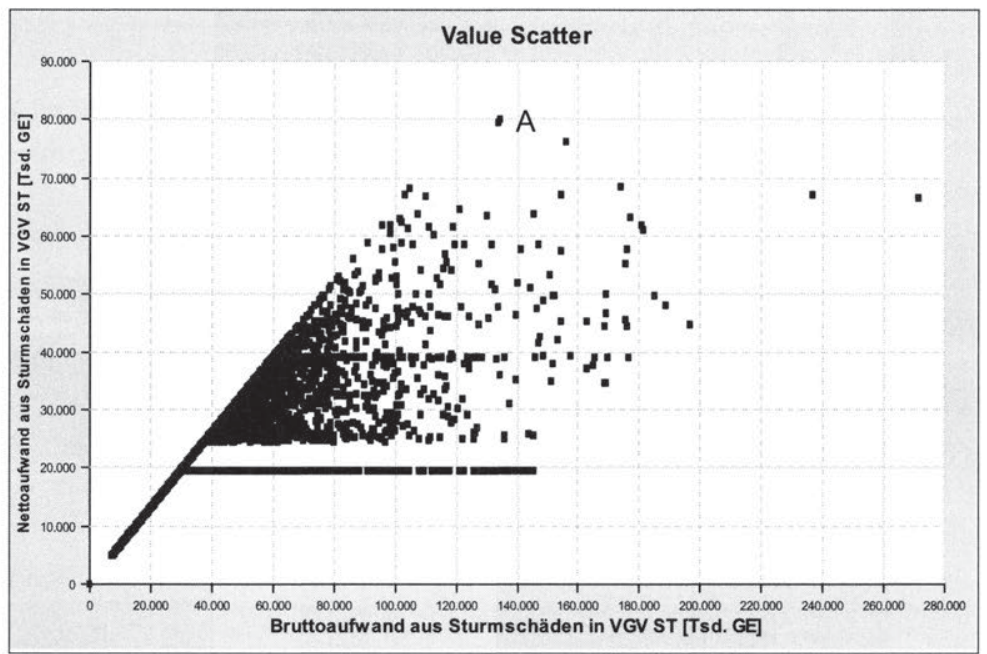

Abbildung 6.16: Streudiagramm des Schadenaufwands aus Sturmschäden in VGV ST (Basisszenario)

Für die Interpretation von Abbildung 6.16 erscheint es zunächst zweckmäßig, sich die wesentlichen Parameter des sturmbezogenen Rückversicherungsprogramms (kurz: Sturmprogramm) in Erinnerung zu rufen. Die Sparten „VGV Sturm“ und „Allgemeine Sturm" werden gemeinsam über einen unlimitierten Quotenrückversicherungsvertrag mit einer Abgabe in Höhe von 35\% gedeckt. Der verbleibende Quotenselbstbehalt ist wiederum über einen Einzelschadenexzedentenvertrag (XL) abgesichert, der eine Bruttopriorität von 40 Mio. GE sowie eine Bruttohaftung von 160 Mio. GE aufweist. Mit dem Rückversicherer ist lediglich eine kostenpflichtige Wiederauffüllung vereinbart. Darüber hinaus gilt es sich in Erinnerung zu rufen, dass der simulierte Bruttoschadenaufwand je Sturmereignis eine Höhe von mindestens 10 Mio. GE und höchstens 200 Mio. GE aufweist. ${ }^{973}$ Annahmegemäß wird jeder Einzelschaden im Verhältnis 75:25 auf die Versicherungszweige „VGV Sturm“ und „Allgemeine Sturm“ aufgeteilt. Mit diesen Informationen können nun Wirkungsweise und Effizienz des Sturmprogramms analysiert werden.

Auf der unteren Waagerechten des Streudiagramms liegen sämtliche Simulationsläufe, in denen genau ein Sturmeinzelschaden eintritt, welcher gleichzeitig auch die Netto-

973 Vgl. zur Modellierung der Sturmschäden detailliert Kapitel 5.4.3.4.2. 
priorität des XL überschreitet. Für beide Sturmsparten beträgt diese Nettopriorität 26,0 Mio. GE. ${ }^{974} \mathrm{Da}$ annahmegemäß von jedem Sturmeinzelschaden $75 \%$ auf die Sparte „VGV Sturm“ entfallen, verläuft die untere Waagerechte auf einem Nettoniveau von 19,5 Mio. GE. Darüber hinaus kann in Höhe eines Nettoschadens von 39,0 Mio. GE eine obere Waagerechte identifiziert werden. Auf dieser Linie liegen Simulationsläufe, in denen genau zwei Sturmschäden auftreten, welche beide die Nettopriorität des XL überschreiten. In diesen Simulationen macht das Modellunternehmen demnach von seinem Recht zur Wiederauffüllung des Haftungslimits Gebrauch. Auf der Diagonalen befinden sich sämtliche Simulationsläufe, in denen ein oder mehrere Sturmschäden auftreten. Jeder einzelne dieser Schäden unterschreitet jedoch die Nettopriorität des XL, so dass diese Schadenszenarien lediglich durch den Quotenrückversicherungsvertrag gedeckt sind. Folglich weist die Diagonale eine Steigung in Höhe des Quotenselbstbehalts (65\%) auf. Es fällt auf, dass eine signifikante Anzahl von Szenarien verbleibt, die nicht unmittelbar den erläuterten „Hauptachsen“ des Streuungsdiagramms zugeordnet werden können. Diese Punktwolke muss folglich Szenarien enthalten, in denen mehrere Stürme auftreten, von denen mindestens einer die Nettopriorität des XL überschreitet. Diese Konstellation wird nachfolgend am Beispiel des mit „A“ bezeichneten Simulationslaufs verdeutlicht, welcher insgesamt einen Bruttosturmschaden in Höhe von 133,7 Mio. GE aufweist. Dieser Bruttoschaden setzt sich aus fünf einzelnen Sturmereignissen zusammen.

\begin{tabular}{|l|c|c|c|c|c|c|}
\hline \multicolumn{1}{|c|}{ [Tsd. GE] } & Sturm 1 & Sturm 2 & Sturm 3 & Sturm 4 & Sturm 5 & GESAMT \\
\hline Einzelschaden brutto & 41.177 & 28.226 & 21.842 & 13.614 & 28.885 & $\mathbf{1 3 3 . 7 4 4}$ \\
\hline Netto nach Quote (SB = 65\%) & 26.765 & 18.347 & 14.197 & $\mathbf{8 . 8 4 9}$ & 18.775 & $\mathbf{8 6 . 9 3 4}$ \\
\hline Netto nach XL & 19.500 & 18.347 & 14.197 & $\mathbf{8 . 8 4 9}$ & 18.775 & $\mathbf{7 9 . 6 6 8}$ \\
\hline
\end{tabular}

Tabelle 6.9: Frequenzrisiko der Naturgefahr Sturm im Basisszenario (Beispiel)

Es wird transparent, dass lediglich Sturm 1 die Bruttopriorität des Einzelschadenexzedentenvertrags übersteigt. Die anderen vier Sturmereignisse werden somit nicht durch den XL erfasst und erfahren lediglich eine proportionale Deckung in Höhe der Quotenabgabe. Diese vier Einzelereignisse weisen jedoch in ihrer Summe durchaus ein signifikantes Volumen von 60,2 Mio. GE auf, welches in Höhe des Quotenselbstbehalts netto getragen werden muss. Dieses Beispiel verdeutlicht bereits, dass das Modellunternehmen mit dem im Basisszenario bestehenden Sturmprogramm einem erheblichem Frequenzrisiko ausgesetzt ist. Die Tatsache, dass der Risikokapitalbedarf des Gesamtunternehmens durch die sturmexponierten Sparten dominiert wird, kann maßgeblich auf dieses Frequenzrisiko zurückgeführt werden.

974 Die Nettopriorität errechnet sich als Produkt aus der Bruttopriorität des XL (40 Mio. GE) und dem Quotenselbstbehalt (65\%). 
Zum Abschluss dieses Kapitels soll das gesamte Rückversicherungsprogramm in Form eines Exkurses aus der Perspektive des Rückversicherers betrachtet werden. Die nachfolgenden Ausführungen können sich naturgemäß lediglich auf diejenigen Verträge beziehen, die aus der Geschäftsbeziehung mit dem Modellunternehmen erwachsen. Aussagen über den Wertschöpfungsbeitrag, den der Rückversicherer gegebenenfalls aufgrund seines weltweit diversifizierten Mehrbranchenportfolios und daraus resultierender Risikoausgleichseffekte erbringt, können jedoch nicht getroffen werden. ${ }^{975}$ Für den fiktiven Fall, dass der Rückversicherer keine Vertragsbeziehungen zu anderen Zedenten unterhält, kann sein (isolierter) Risikokapitalbedarf mit Hilfe des CVaRAnsatzes quantifiziert und anschließend auf die rückversicherten Versicherungszweige alloziert werden. Abbildung 6.17 stellt die Allokationsergebnisse im Überblick dar.

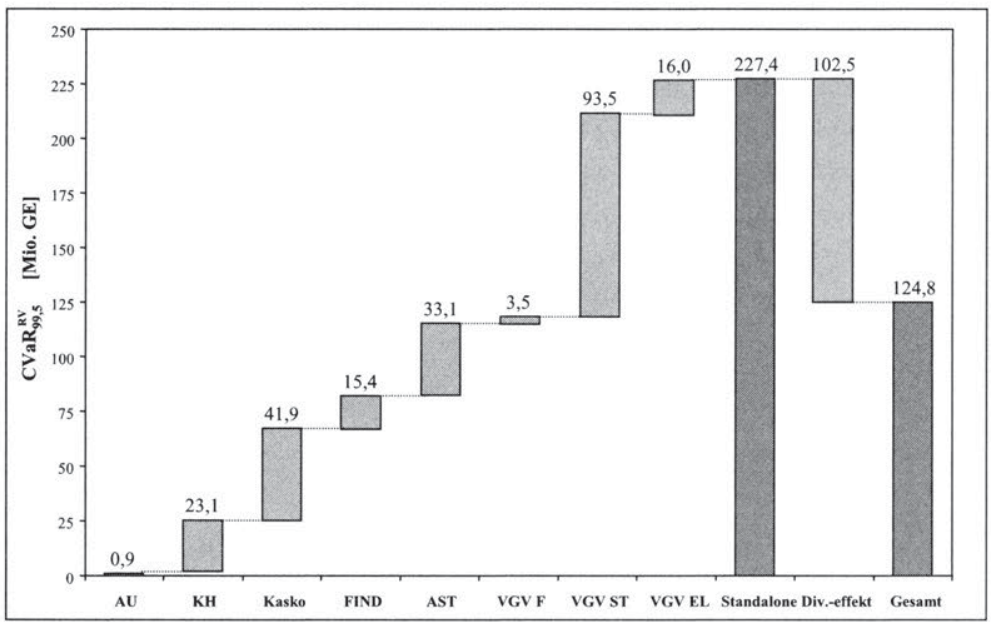

Abbildung 6.17: Risikokapitalbedarf des Rückversicherers in der isolierten Betrachtung

In einer Standalone-Betrachtung müsste der Rückversicherer demnach für die bestehenden Verträge mit dem Modellunternehmen ein Risikokapital in Höhe von 227,4 Mio. GE vorhalten. Aufgrund der Risikoausgleichseffekte, die sich bereits innerhalb des versicherungstechnischen Portfolios des Modellunternehmens einstellen, reduziert sich der Kapitalbedarf jedoch bereis auf 124,8 Mio. GE. Die Einbettung in ein breit diversifiziertes Rückversicherungsportfolio würde darüber hinaus vermutlich zu einer

975 Der volkswirtschaftliche Wertschöpfungsbeitrag der Rückversicherung entspricht der Differenz zwischen dem beim Erstversicherer eingesparten und dem beim Rückversicherer zusätzlich benötigten Risikokapital. Vgl. übereinstimmend HERSBERGER (1996), S. 16. Ein fiktives Beispiel für diesen „Produktionskostenvorteil des Rückversicherers“ findet sich bei HERSBERGER (1996), S. 17. Vgl. außerdem in diesem Kontext MENTZEL (2004), S. 133-137 und SCHRADIN (1998), S. 191-198. 
weitaus stärkeren Reduktion des Kapitalbedarfs führen. Das Ergebnis des Rückversicherers ${ }^{976}$ beträgt im Erwartungswert 6,8 Mio. GE, so dass sich bereits in der isolierten Betrachtung ein RORAC in Höhe von 5,45\% aus Sicht des Rückversicherers ergibt. Im Rahmen der „technischen“ Preisermittlung hatte der Rückversicherer jedem Einzelvertrag lediglich eine Renditeerwartung von 3\% zugrunde gelegt. ${ }^{977}$ Dies verdeutlicht, dass der Rückversicherer bereits im Rahmen seiner Geschäftsbeziehung mit dem Modellunternehmen signifikante Risikoausgleichseffekte erzielt.

\subsubsection{Integration von Kapitalanlageerträgen}

Im Rahmen der bisherigen Ausführungen stand unter dem Steuerungsaspekt lediglich das versicherungstechnische Portfolio im Mittelpunkt. Bei der Ermittlung des versicherungstechnischen Anfalljahrergebnisses wurde jedoch bereits dem Umstand Rechnung getragen, dass die Bedarfsreserve erst in den zukünftigen Geschäftsjahren sukzessive zur Auszahlung gelangt und daher als Zinsträger zu Verfügung steht. Durch die Einbeziehung kalkulatorischer Zinserträge aus der Anlage der Bedarfsreserve wurde der Schadenbedarf des simulierten Anfalljahres - insbesondere in Versicherungszweigen mit langer Abwicklungsdauer - bereits erheblich realitätsnäher bewertet. Allerdings ist bis zum jetzigen Zeitpunkt unberücksichtigt geblieben, dass die vorschüssig geleisteten Prämienzahlungen ebenfalls Anlagekapital generieren. Dieses Kapital steht dem Versicherungsunternehmen einerseits als Zinsträger für Kapitalmarktaktivitäten, andererseits für die Regulierung von Schäden bzw. Deckung von Betriebskosten und Provisionen zur Verfügung. Die Stochastizität der Schadenzahlungen beeinflusst das Anlagekapital sowohl in zeitlicher Hinsicht (Timing Risk) als auch in seiner Höhe (Underwriting Risk). ${ }^{978}$ Das zeitliche Auseinanderfallen von Prämieneinzahlungen und Schadenauszahlungen begründet für Versicherungsunternehmen die ökonomische Notwendigkeit, Kapitalanlagegeschäfte zu betreiben. ${ }^{979}$ Nachfolgend wird daher die verzinsliche Anlage des versicherungstechnischen Netto Cashflows in die Betrachtung integriert. Dabei gilt es zu beachten, dass das simulierte Anfalljahr weiterhin den relevanten Analysehorizont darstellt. Somit werden lediglich diejenigen CashflowKomponenten berücksichtigt, die das simulierte Anfalljahr betreffen. Die Einbezie-

976 Das Ergebnis des Rückversicherers setzt sich zusammen aus den erwarteten RV-Prämien abzüglich erwarteter Entschädigungsleistungen (Recoveries) und RV-Provisionen. Es entspricht folglich im Erwartungswert der Differenz aus Brutto- und Nettoergebnis des Erstversicherers (vor kalkulatorischen Zinsen aus der Anlage der Bedarfsreserve). Vgl. zur Berechnung auch Tabelle 6.8.

977 Vgl. hierzu detailliert Kapitel 5.4.5.3.

978 Vgl. auch MENTZEL (2004), S. 246.

979 In der einschlägigen Literatur wird dieses Phänomen vielfach als wirtschaftliche Verbundproduktion bzw. Kuppelproduktion bezeichnet. Vgl. vertiefend ALBRECHT (1987a), FARNY (2000), S. 593-601. Vgl. kritisch zum Begriff der „Verbundproduktion“ im versicherungsspezifischen Kontext SCHRADIN (1994), S. 196-204. 
hung von Zahlungsströmen, deren wirtschaftliche Entstehung vorangegangenen Anfalljahren zuzuordnen ist, erfolgt erst auf der Bilanzebene. ${ }^{980}$

Zunächst werden vereinfachende Annahmen über den zeitlichen Anfall der CashflowKomponenten getroffen. Es wird unverändert davon ausgegangen, dass zu Beginn des Anfalljahres die Bruttoprämien in voller Höhe vereinnahmt und die Rückversicherungsprämien auszahlungswirksam werden. Zur Jahresmitte fallen die Schadenzahlungen, sämtliche Provisionen und Betriebskosten sowie die Entschädigungsleistungen (Recoveries) und Provisionen des Rückversicherers an. ${ }^{981}$ Der resultierende Netto Cashflow kann daher grundsätzlich sowohl positive als auch negative Werte annehmen. Positive Cashflows werden am Kapitalmarkt investiert, negative Cashflows können annahmegemäß bis zum Ende des Geschäftsjahres zum risikolosen Zinssatz fremdfinanziert werden. Abbildung 6.18 stellt den zeitlichen Anfall des Cashflows im Überblick dar und präzisiert gleichzeitig die Struktur des für Kapitalanlagezwecke nutzbaren Zinsträgers.

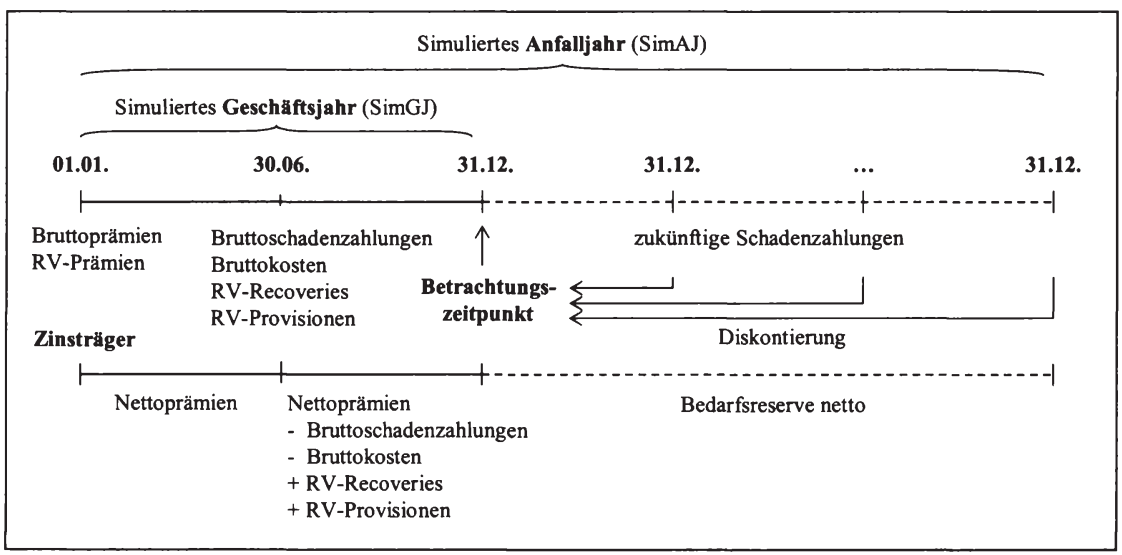

Abbildung 6.18: Berücksichtung von Kapitalanlageerträgen im simulierten Anfalljahr

Die hier gewählte Vorgehensweise orientiert sich an dem in der einschlägigen Literatur als Zinsträgermodell beschriebenen Ansatz, welcher das Versicherungsgeschäft als unabhängige Variable und das Kapitalanlagegeschäft als abhängige Variable postuliert. ${ }^{982}$

\footnotetext{
980 Vgl. hierzu Kapitel 6.3.

981 Die Prämisse eines Schadenanfalls in der Periodenmitte entspricht approximativ der Annahme eines kontinuierlichen Schadenanfalls. Vgl. übereinstimmend FARNY (1983a), S. 400.

982 Vgl. hierzu vertiefend FARNY (2000), S. 64-67 und S. 601 sowie die dort angegebene Literatur.
} 
In finanzierungstheoretischen Ansätzen ${ }^{983}$ hingegen wird diese Kausalität weitgehend aufgehoben und das Versicherungsunternehmen als ,einheitlicher Kapitalfonds gesehen, der von außen durch Versicherungsgeschäfte und andere Finanzgeschäfte gespeist und durch Investitionen in Kapitalanlagen verwendet wird." ${ }^{\text {“984 }}$ Aufgrund des restriktiven Prämissenrahmens, welcher derartigen Erklärungsansätzen insbesondere im Hinblick auf den zugrunde liegenden Markt und das Verhalten der Investoren zugrunde liegt, sind diese Ansätze jedoch primär dazu geeignet, die Kapitalanlagetätigkeit in Holdingsgesellschaften großer Finanzkonglomerate als Teil aller Finanzierungsvorgänge zu fundieren. ${ }^{985}$ Für einzelne operative Versicherungsunternehmen hingegen erscheint die Verwendung kapitalmarkttheoretischer Ansätze nicht angemessen. ${ }^{986}$

Das Gesamtnettoergebnis des simulierten Anfalljahres ermittelt sich nach Integration der Kapitalanlageerträge nunmehr als:

Anfalljahrergebnis netto vor kalkulat. Zinserträgen

+ kalkulatorische Zinserträge aus der Anlage der BR ${ }^{\text {SimAJ }}$

$=$ Anfalljahrergebnis netto

(AJ-Ergebnis 1)

+ Erträge aus der Anlage des vt. Netto Cashflows

$=$ Gesamtergebnis des Anfalljahres netto

(AJ-Ergebnis 2)

(AJ-Ergebnis 3)

Es soll zunächst der Fall betrachtet werden, dass der durch das Versicherungsgeschäft generierte Cashflow risikolos am Geldmarkt investiert wird. Dieser Vorgehensweise liegt die Überlegung zugrunde, dass das Versicherungsunternehmen risikoavers am Kapitalmarkt agiert und die jederzeitige Erfüllbarkeit zukünftiger Auszahlungsverpflichtungen durch eine Anlage in risikolos veräußerbare Assets sicherstellen möchte. Die aus einer derartig formulierten Anlagestrategie resultierenden Ergebnisverteilungen sind in Abbildung 6.19 als Perzentilgraph dargestellt.

983 An dieser Stelle sind insbesondere das Capital Asset Pricing Model (CAPM) oder auch die Optionspreistheorie zu nennen. Vgl. hierzu exemplarisch ROCKEL (2004), S. 67-84 und S. 95-109, ALBRECHT (1991), BREUER (1992), GROFFMANN (1995), S. 124-180, ALBRECHT (1995), S. 129155 und GRÜNDL/SCHMEISER (1999).

${ }^{984}$ FARNY (2000), S. 601.

985 Eine Übersicht der zentralen Annahmen des (versicherungsspezifisch eingesetzten) CAPM findet man z.B. bei ALBRECHT (1991), S. 503-515, BREUER (1992), S. 618-619 und GROFFMANN (1995), S. 135-136.

986 Vgl. übereinstimmend FARNY (2000), S. 601 und ALBRECHT (1991), S. 499-515. 


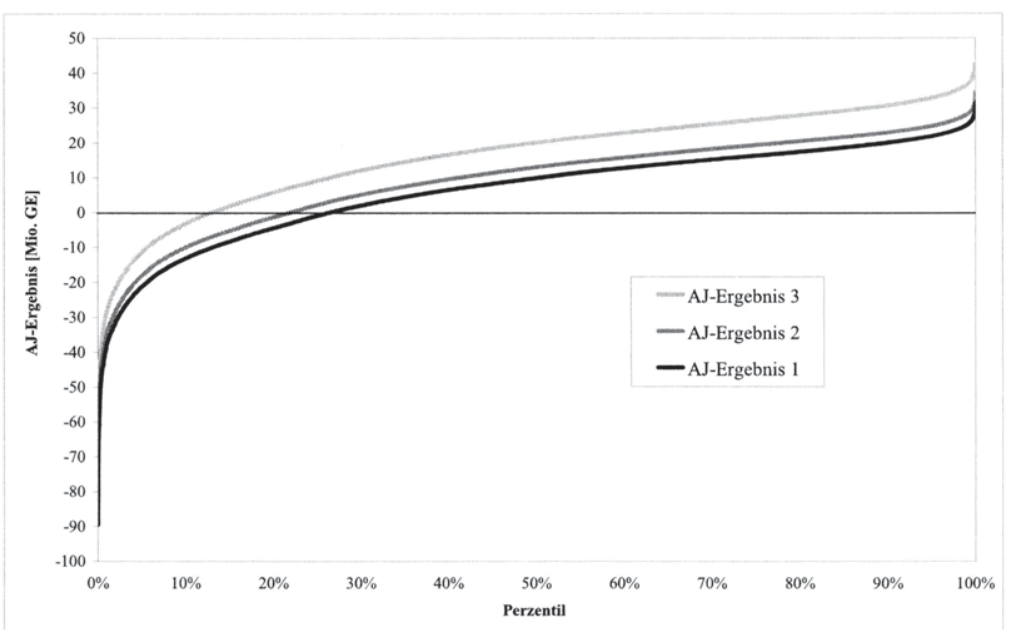

Abbildung 6.19: Netto-Anfalljahrergebnis unter Berücksichtigung von Kapitalanlageerträgen

Die Einbeziehung der erwarteten Anlageerfolge am Geldmarkt bewirkt eine Verschiebung der Ergebnisverteilung nach oben. Dieser Effekt ist darauf zurückzuführen, dass der versicherungstechnische Netto Cashflow aufgrund des bestehenden Rückversicherungsprogramms nur in sehr wenigen Szenarien negative Werte annimmt. Demzufolge generiert die risikolose Anlage der Cashflows fast ausschließlich positive Erfolgsbeiträge. Es verwundert daher nicht, dass sich der Risikokapitalbedarf des Unternehmens infolge der (quasi) sicheren Ergebnisverbesserung reduziert. Die beschriebenen Effekte werden in Tabelle 6.10 zusammengefasst.

\begin{tabular}{|c|c|c|c|}
\hline [Tsd. GE] & Erwartungswert & $\mathbf{C V a R}_{\mathbf{9 9}, \mathbf{5}}$ & RORAC \\
\hline AJ-Ergebnis 1 & 6.213 & 54.843 & $11,33 \%$ \\
\hline AJ-Ergebnis 2 & 9.257 & 51.558 & $17,96 \%$ \\
\hline AJ-Ergebnis 3 & 16.429 & 45.253 & $36,30 \%$ \\
\hline
\end{tabular}

Tabelle 6.10: Rendite-Risiko-Relationen unterschiedlicher Ergebnisstufen

Während die Differenz zwischen den AJ-Ergebnissen 2 und 3 im Erwartungswert genau dem erwarteten Ertrag aus der Anlage des versicherungstechnischen Cashflows entspricht, reduziert sich der korrespondierende CVaR nur unterproportional. Dieser Effekt kann auf die bereits angesprochenen Verbundwirkungen zwischen Versicherungsgeschäft und Kapitalanlage zurückgeführt werden, die hier in Form des versicherungstechnischen Cashflows modelliert sind. In denjenigen Simulationsläufen, die durch eine hohe Schadenbelastung gekennzeichnet sind, steht nur ein reduzierter Zinsträger in Form von Prämien bzw. Bedarfsreserven zur Verfügung. Werden neben der risikolosen Geldmarktanlage auch andere Anlageformen in die Betrachtung integriert, 
ist es natürlich denkbar, dass ein schlechtes Schadenjahr, mithin ein verminderter Zinsträger mit einer besonders positiven Kapitalmarktentwicklung zusammenfällt. Der Einfluss der Stochastizität des Anlagegeschäfts auf den erwarteten Gesamterfolg und den korrespondierenden Risikokapitalbedarf ist somit ex-ante nicht eindeutig zu bestimmen. ${ }^{987}$

In einem weiteren Schritt wird nun die Prämisse einer ausschließlich risikolosen Anlage aufgehoben. Neben der Anlage am Geldmarkt besteht zusätzlich die Möglichkeit, einen positiven versicherungstechnischen Cashflow teilweise in risikobehaftete Anlageklassen $\mathrm{zu}$ investieren. ${ }^{988}$ Beispielhaft wird dies nachfolgend für den Fall einer partiellen Anlage in Aktien untersucht. Ein rationaler Investor (z.B. ein Versicherungsunternehmen) wird im Sinne der klassischen Portfoliotheorie nach MARKOWITZ nur diejenigen Portfolios in seine Auswahlentscheidungen einbeziehen, die als effizient bezeichnet werden können, d.h. welche hinsichtlich ihrer Rendite-Risiko-Relation nicht von anderen Portfolios dominiert werden (Prinzip der „Mean-Variance-Diversification"). ${ }^{989}$ Die Menge aller zulässigen Portfolios bildet in der $\mu-\sigma$-Darstellung einen effizienten Rand, der auch als Effizienzlinie bezeichnet wird. Um entscheiden zu können, welches auf der Effizienzlinie gelegene Portfolio der Investor wählen soll, muss seine individuelle Risikoneigung bekannt und in Form einer Nutzenfunktion quantifizierbar sein. Graphisch veranschaulicht, befindet sich das optimale Portfolio eines Anlegers genau im Tangentialpunkt der Effizienzlinie mit seiner individuellen Isonutzenkurve. Die optimale Aufteilung des Budgets ist somit an der Stelle erreicht, wo das Portfolio risikoeffizient ist und gleichzeitig der Risikoneigung des Investors entspricht. Im hier betrachteten Fall kann das Modellunternehmen in kombinierte Portfolios aus Versicherungsverträgen und Kapitalanlagen investieren, wobei sich das Kapitalanlagevolumen aus dem Versicherungsgeschäft ableitet (derivatives Portfolio). ${ }^{990}$ Die Risikoneigung des Investors konkretisiert sich in Form des gewählten Sicherheitsniveaus 1-E. Im Rahmen der Quantifizierung des Risikokapitalbedarfs wird somit die Risikopräferenz des Anlegers bereits explizit berücksichtigt.

987 KORYCIORZ formuliert zutreffend: „Sind Versicherungstechnik und Anlagegeschäft negativ korreliert - besteht also eine Tendenz dafür, dass steigende Entschädigungsleistungen mit fallenden Investmenterträgen einhergehen -, so fällt der Schwankungszuschlag eindeutig positiv aus, während für den Fall einer positiven Korrelation keine generellen Aussagen möglich sind.“ KORYCIORZ (2004), S. 171-172.

988 Negative Cashflows werden weiterhin über kurzfristige Fremdmittel ausgeglichen. Dem Vorsichtsgedanken Rechnung tragend, wird bei der Ermittlung kalkulatorischer Zinserträge aus der Anlage der Bedarfsreserve unverändert eine risikolose Verzinsung auf Geldmarktniveau unterstellt.

989 Vgl. zur Portfoliotheorie im Folgenden STEINER/BRUNS (2002), S. 7-16 sowie im versicherungsspezifischen Kontext SCHERER (2002), S. 93-126, HARTUNG (2000), S. 206-224 und GROFFMANN (1995), S. 132-135.

990 Vgl. auch ALBRECHT (1995), S. 35. 
Im Folgenden soll nun untersucht werden, welchen Einfluss unterschiedlich stark ausgeprägte Aktienengagements auf die Risiko-Renditeposition des Modellunternehmens nehmen. ${ }^{991}$ Betrachtet werden Portfolios mit variierenden Aktienquoten, die in Schritten von fünf Prozentpunkten sukzessive von $0 \%$ bis $100 \%$ ansteigen. Abbildung 6.20 zeigt die Einordnung der sich ergebenden Konstellationen in das ErwartungswertRisikokapital-Diagramm.

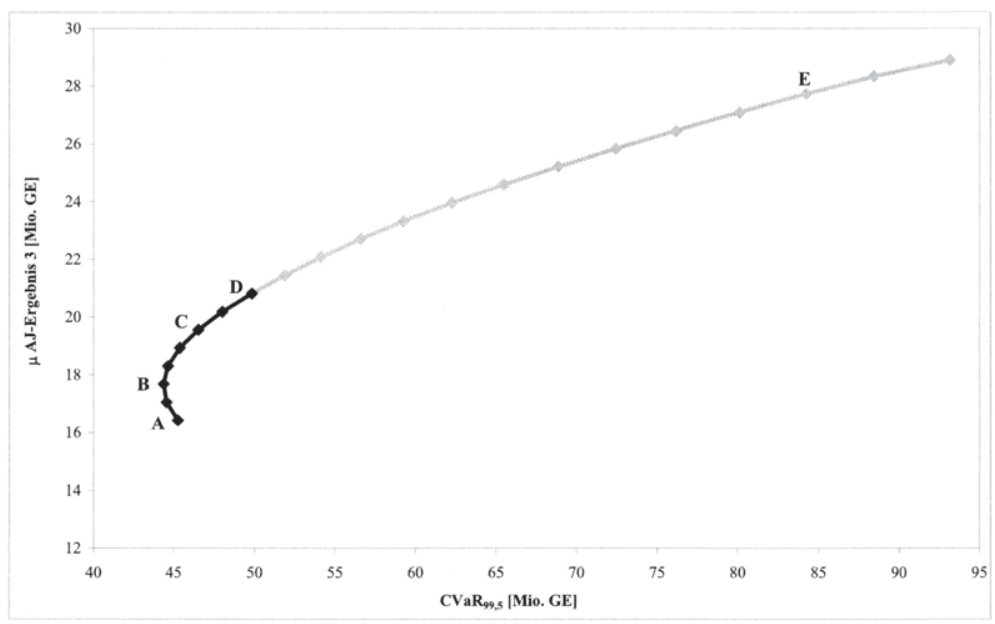

Abbildung 6.20: „Effizienzlinie“ kombinierter Portfolios aus Versicherungsverträgen und Kapitalanlagen

\begin{tabular}{|c|c|c|c|c|c|}
\hline [Mio. GE] & Aktienquote & $\mu$ AJ-Ergebnis 3 & $\boldsymbol{\sigma}$ AJ-Ergebnis 3 & CVaR $_{\mathbf{9 9}, \mathbf{5}}$ & RORAC \\
\hline A & $0 \%$ & 16,43 & 14,17 & 45,25 & $36,3 \%$ \\
\hline B & $10 \%$ & 17,69 & 14,80 & 44,38 & $39,9 \%$ \\
\hline C & $27 \%$ & 19,82 & 18,12 & 47,09 & $42,1 \%$ \\
\hline D & $35 \%$ & 20,82 & 20,37 & 49,84 & $41,8 \%$ \\
\hline E & $90 \%$ & 27,71 & 40,18 & 84,18 & $32,9 \%$ \\
\hline
\end{tabular}

Tabelle 6.11: Risiko-Rendite-Relationen unterschiedlicher Aktien-Geldmarkt-Portfolios

Ein vollständig risikoaverser Anleger müsste demnach Portfolio A realisieren, da dieses ausschließlich in risikolose Geldmarktpapiere investiert und infolgedessen die geringste Standardabweichung aufweist. Das Modellunternehmen hingegen akzeptiert über das gewählte Sicherheitsniveau von $99,5 \%$ mit einer gewissen Wahrscheinlichkeit auch das Auftreten negativer Portfolioergebnisse. Es fällt auf, dass Portfolio B trotz einer Aktienquote von $10 \%$ einen geringeren Risikokapitalbedarf induziert als

991 Vgl. in diesem Zusammenhang auch CORELL (1999), S. 1154-1156. 
Strategie A. ${ }^{992}$ Dies ist darauf zurückzuführen, dass im Rahmen der Modellierung davon ausgegangen wird, dass sich die Kapitalmarktrenditen unabhängig von der Rendite des versicherungstechnischen Portfolios entwickeln. ${ }^{993}$ Somit treten nicht nur innerhalb des versicherungstechnischen Portfolios, sondern auch zwischen Versicherungsgeschäft und Kapitalanlagetätigkeit Risikoausgleichseffekte auf. ${ }^{994}$ Unter der Zielsetzung einer RORAC-Maximierung stellt Portfolio C mit einer Aktienquote von 27\% die optimale Budgetaufteilung dar. Für Aktienquoten oberhalb von $27 \%$ ist der RORAC wiederum rückläufig, da die Ergebniserwartung nur noch unterproportional mit dem Risikokapitalbedarf ansteigt. Punkt D repräsentiert mit einer Aktienquote von $35 \%$ schließlich dasjenige Portfolio, welches aufsichtsrechtlich gerade noch zulässig wäre. ${ }^{995}$ Eine maximale Ausschöpfung der aufsichtsrechtlich eingeräumten Risikoquote führt offenbar in der hier vorliegenden Datenkonstellation nicht zu einer optimalen Risiko-Renditerelation des Gesamtportfolios. Portfolio E illustriert letztlich den Effekt, dass extrem hohe Aktienquoten zu einem überproportionalen Anstieg des Risikokapitalbedarfs führen, da die Volatilität des Kapitalmarktes einen zu dominanten Einfluss auf den Randbereich der Ergebnisverteilung nimmt und demzufolge der oben beschriebene „natürliche Hedgingeffekt“ zwischen Versicherungsgeschäft und Kapitalanlage nicht mehr greift.

Abschließend stellt sich nun die Frage, welche Ergebnisgröße letztlich für die Steuerung des Gesamtportfolios herangezogen werden sollte. Eine gänzliche Vernachlässigung des Sachverhalts, dass das Versicherungsgeschäft anlagefähiges Kapital generiert, erscheint aus betriebswirtschaftlicher Perspektive nicht angemessen. Allerdings hat sich insbesondere in den zurückliegenden Jahren die Einbeziehung erwarteter Kapitalanlageerlöse in die Prämienkalkulation vielfach als trügerisch erwiesen. Auf die-

992 ZIELKE weist in einer aktuellen Studie für den deutschen Lebensversicherungsmarkt darauf hin, dass ,eine 0 Prozent-Aktienstrategie langfristig die Ruinwahrscheinlichkeit der Lebensversicherer sogar erhöht" und der Verzicht auf Aktien „langfristig riskanter ist als eine Beimischung von derzeit 12 bis 15 Prozent Aktien." ZIELKE (2004), S. 1721-1722. Es ist jedoch ausdrücklich herauszustellen, dass dieses Ergebnis in der Lebensversicherung auch darauf zurückzufuihren ist, dass ein Verzicht auf Aktien langfristig die Renditeerwartung des Produktes „Lebensversicherung“ vermindert und somit im Asset-Liability-Kontext ein rückläufiges Neugeschäft und höhere Stornoquoten impliziert. Eine unreflektierte Übertragung dieses Zusammenhangs auf die Schaden- und Unfallversicherung ist daher nicht zulässig.

993 Die Interdependenz zwischen versicherungstechnischem Geschäft und Kapitalanlage wird ausschließlich über den Zinsträger (= versicherungstechnischer Netto Cashflow) hergestellt. Vgl. hierzu auch die Ausführungen in Kapitel 5.5.4.

994 Vgl. zu den Auswirkungen negativer Autokorrelationen zwischen Schadenentwicklung und Kapitalmarktrenditen SCHMEISER (2004b), S. 13-14.

995 An dieser Stelle muss betont werden, dass sich die in $\S 2$ Abs. 3 AnlV kodifizierte allgemeine Risikoquote von $35 \%$ ausdrücklich auf das gesamte Sicherungsvermögen und sonstige gebundene Vermögen eines Versicherungsunternehmens bezieht und nicht nur (wie hier) auf den versicherungstechnischen Cashflow. 
ses in der Literatur kontrovers diskutierte Phänomen des „Cashflow Underwriting“ soll an dieser Stelle nicht detailliert eingegangen werden. ${ }^{996}$ Die Kapitalmarktkrise der Jahre 2000 bis 2002 hat eindrucksvoll belegt, dass ein risikoadäquates Prämienniveau sowie eine selektive Zeichnungspolitik für einen nachhaltigen Geschäftserfolg unverzichtbar sind ${ }^{997}$ Es kommt daher darauf an, einen „,betriebswirtschaftlich sachgerechten, kontrollierten und vorsichtigen Ansatz von Zinserträgen““998 in das Steuerungskalkül zu integrieren.

Die Einbeziehung kalkulatorischer Zinserträge aus der Anlage der Bedarfsreserve erscheint betriebswirtschaftlich sinnvoll, da einzelne Versicherungszweige in unterschiedlichem Ausmaß und für eine unterschiedliche Dauer anlagefähiges Kapital generieren. Dem bereits zitierten Vorsichtsgedanken Rechnung tragend, erfolgt die Diskontierung zukünftiger Auszahlungsverpflichtungen mit dem (quasi) risikolosen Geldmarktzins. Auf diese Weise wird einerseits eine marktwertorientierte Betrachtungsweise des Versicherungsgeschäfts in den Steuerungsprozess integriert und andererseits sichergestellt, dass die kalkulatorischen Zinserträge „wie Sicherheitszuschläge interpretiert und bei Nichtinanspruchnahme dem versicherungstechnischen Bereich ,gutgeschrieben'“999 werden können. Der Umstand, dass bestimmte Versicherungszweige bzw. Geschäftsfelder durch ihre Geschäftstätigkeit dem Unternehmen erst den Aufbau eines längerfristigen Zinsträgers ermöglichen, wird auf diese Weise angemessen berücksichtigt.

Der versicherungstechnische Netto Cashflow trägt hingegen keinen kalkulatorischen Charakter, sondern wird unmittelbar im Geschäftsjahr zahlungswirksam. Er steht daher für zur Anlage am Kapitalmarkt zur Verfügung. Sofern das Versicherungsunternehmen die Zielsetzung verfolgt, seine zu Beginn des Geschäftsjahres bestehende Anlagestruktur im Jahresverlauf unverändert fortzuschreiben, sollte die Investitionsstrategie für den versicherungstechnischen Netto Cashflow genau die anfängliche Asset Allocation replizieren.

Im weiteren Verlauf dieser Arbeit wird davon ausgegangen, dass der versicherungstechnische Cashflow ausschließlich (quasi) risikolos in Geldmarktpapiere investiert wird. Neben einer Betonung des Vorsichtsgedankens dient diese Vorgehensweise ins-

996 Vgl. kritisch zum Phänomen des „Cashflow Underwriting“ FARNY (1983a), FARNY (1983b) mit der dort auf S. 403 angegebenen Literatur sowie ALBRECHT (1987a).

997 Vgl. übereinstimmend GROFFMANN (1995), S. 32-35 und insbesondere SwISS RE (2001).

998 FARNY (1983b), S. 485.

999 FARNY (1983b), S. 482. Vgl. außerdem zur Beurteilung einer ex-ante- oder ex-post-Zurechnung von Kapitalanlageerträgen im Kontext einer dem Äquivalenzprinzip folgenden Prämienkalkulation FARNY (1983a), S. 482-484. 
besondere einer transparenteren Darstellung, da sichergestellt wird, dass die Auswirkungen der versicherungstechnischen Strategien auf den Risikokapitalbedarf isolierbar bleiben und nicht durch Kapitalanlageeffekte überlagert werden. Als Entscheidungskriterium für die im nachfolgenden Abschnitt zu bewertenden Zeichnungs- bzw. Rückversicherungsstrategien wird daher unverändert der RORAC auf der Basis des AJ-Ergebnisses 2 herangezogen.

Die Darstellung und Analyse der in den zurückliegenden Abschnitten als „Basisszenario“ bezeichneten Parameter- bzw. Risikostruktur ist hiermit abgeschlossen. In den nachfolgenden drei Kapiteln wird mit Hilfe parametrischer Variationsanalysen untersucht, in welchem Ausmaß Veränderungen zentraler Risikoparameter Einfluss auf die Rendite-Risiko-Position des Modellunternehmens nehmen. In diesem Zusammenhang wird in Kapitel 6.2.2 zunächst der Einfluss stochastischer Abhängigkeitsstrukturen analysiert. In Kapitel 6.2.3 und 6.2.4 werden exemplarisch die Auswirkungen unterschiedlicher Rückversicherungs- bzw. Zeichnungsstrategien aufgezeigt. Das bekannte „Basisszenario“ dient in diesem Zusammenhang jeweils als Referenzszenario fur die untersuchten Parametervariationen und Handlungsstrategien.

\subsubsection{Einfluss stochastischer Abhängigkeitsstrukturen}

\subsubsection{Lineare Abhängigkeiten}

Das Auftreten kollektiver Risikoausgleichseffekte wird in Portfolios, deren Einzelrisiken gegenseitige Abhängigkeiten aufweisen, erheblich erschwert. ${ }^{1000}$ Im Verlauf der bisherigen Ausführungen wurde durchgängig unterstellt, dass sowohl die Basisschadenlast als auch die Großschäden in den einzelnen Teilkollektiven stochastisch unabhängig voneinander eintreten. ${ }^{1001}$ Demgegenüber wurden stochastische Abhängigkeitsstrukturen zwischen Versicherungszweigen, die durch eine starke Exponierung gegenüber Naturgefahren gekennzeichnet sind, bereits durch eine separate Modellierung dieser Kumulschäden berücksichtigt. Der simulierte Gesamtschaden eines Sturm-, Hagel- oder Überschwemmungsereignisses wurde anhand von Erfahrungswerten prozentual auf die betroffenen Sparte-Geschäftsfeld-Kombinationen verteilt. Auf diese Weise wurde sichergestellt, dass ein Ereignis simultan, d.h. modelltechnisch innerhalb des-

${ }^{1000}$ Vgl. auch Swiss RE (1999), S. 16-18, SCHRADIN (1998), S. 121-134 und KORYCIORZ (2004), S. 114-115.

${ }^{1001}$ In der einschlägigen Literatur ist die Annahme unkorrelierter Schadenkollektive häufig anzutreffen. Vgl. in diesem Zusammenhang exemplarisch MACK (2002), S. 30. EMBRECHTS/HÖING/JURI führen hierzu kritisch aus: „Very often, all or part of the constituent random variables are taken to be independent. This is due more to the tractibility of such models than to the nature of the phenonemon being modelled.“ EMBRECHTS/HÖING/JURI (2003), S. 147. 
selben Simulationslaufs, auf mehrere Teilkollektive wirkt. ${ }^{1002}$ Wenngleich zu vermuten ist, dass durch Kumulschäden bereits per definitionem ein nennenswerter Teil stochastischer Abhängigkeitsstrukturen erklärt werden kann, soll nachfolgend die Unabhängigkeitsannahme auch für Basis- und Großschäden aufgehoben werden.

In einem ersten Schritt werden lineare Abhängigkeitsstrukturen explizit in die Analyse einbezogen. Da die Auswirkungen auf das originäre Schadengeschehen untersucht werden sollen, erfolgt die Analyse ausschließlich auf Bruttobasis, d.h. ohne Berücksichtigung der Rückversicherung. Als Maß für die Stärke und Art der linearen Abhängigkeit findet nachfolgend der Rangkorrelationskoeffizient $\rho^{S}$ nach SPEARMAN Verwendung. ${ }^{1003}$ Dieser ergibt sich anschaulich, wenn in dem aus Kapitel 5.5.5.4 bereits geläufigen Korrelationskoeffizienten nach PEARSON die Werte $x_{i}$ und $y_{i}$ durch die sortierten Größen $\mathrm{x}_{[\mathrm{i}]}$ und $\mathrm{y}_{[\mathrm{i}]}$ ersetzt werden.

$$
\begin{aligned}
& \rho^{s}(x, y)=\frac{\sum_{i=1}^{n}\left(x_{[i]}-\bar{x}\right)\left(y_{[i]}-\bar{y}\right)}{\sqrt{\sum_{i=1}^{n}\left(x_{[i]}-\bar{x}\right)^{2} \sum_{i=1}^{n}\left(y_{[i]}-\bar{y}\right)^{2}}} \\
& \text { mit: } \quad \bar{x}=\frac{1}{n} \sum_{i=1}^{n} x_{i} \text { und } \bar{y}=\frac{1}{n} \sum_{i=1}^{n} y_{i}
\end{aligned}
$$

Dabei bezeichnen die zufallsabhängigen Größen $\mathrm{X}$ und $\mathrm{Y}$ jeweils die teilkollektive Summe aus Basis- und Großschäden in der Stichprobe. Der Rangkorrelationskoeffizient nach SPEARMAN misst somit die lineare Abhängigkeit der Verteilungsfunktionen zweier Zufallsvariablen und kann als Monotoniemaß interpretiert werden. ${ }^{1004}$ Lineare Transformationen der Zufallsvariablen $X$ und $Y$ lassen $\rho^{S}$ unverändert. Unter der hypothetischen Annahme einer perfekt positiven Korrelation $\left(\rho^{S}=1\right)$ sämtlicher Einzelrisiken kann ein Versicherungsunternehmen keine Ausgleichseffekte erzielen, da

\footnotetext{
1002 „Statistisch nachweisbare Abhängigkeiten entstehen im Versicherungsbereich auf natürliche Weise etwa zwischen ähnlichen Sparten wie in der Hausrat- und Gebäudeversicherung, auf Grund räumlicher Benachbartheit [sic] wie bei Hochwasser- und Sturmschäden oder durch gemeinsame auslösenden Faktoren [...].“ PFEIFER (2004), S. 476. MACK weist darauf hin, dass korrelierte Risiken ,außer in der konjunkturabhängigen Kreditversicherung insbesondere bei der Versicherung gegen Naturgefahren (Sturm, Hagel, Erdbeben, Überschwemmung)“ auftreten, da „geografisch benachbarte Risiken stark miteinander korrelieren." MACK (2002), S. 31. Vgl. außerdem zum Phänomen der „Extreme Correlation“ WANG (2002), S. 69.

${ }^{1003} \mathrm{Vgl}$. im Folgenden auch KORYCIORZ (2004), S. 287-288 und NEUBAUER (1994), S. 274-277.

${ }^{1004}$ Vgl. KorycioRZ (2004), S. 287 und EMBrechtS/MCNEIL/STraumanN (2002), S. 195 f.
} 
die Standardabweichung des Gesamtschadens ihr Maximum annimmt. ${ }^{1005}$ Diese Konstellation korrespondiert folglich mit dem bereits diskutierten Standalone-Fall, in dem kollektive Ausgleichseffekte zwischen den Teilkollektiven gar nicht mehr zu Stande kommen und demzufolge der Hauptnutzen der Kollektivbildung aufgehoben ist. ${ }^{1006}$

Demgegenüber bleiben negative Korrelationsbeziehungen im weiteren Verlauf der Untersuchung außer Betracht, da diese in der Praxis allenfalls durch gemeinsame externe Einflüsse, wie z.B. eine negative Konjunkturentwicklung, ausgelöst werden und in der Regel nur schwer zu quantifizieren sind. ${ }^{1007}$ In Form einer parametrischen Variationsanalyse wird nachfolgend untersucht, welchen Einfluss zunehmende lineare Abhängigkeiten auf die Rendite-Risikoposition des Modellunternehmens nehmen. Betrachtet werden Abhängigkeitsstrukturen zwischen den Versicherungszweigen in Bezug auf die Summe aus Basis- und Großschäden. Auf die Modellierung von Abhängigkeiten zwischen den Geschäftsfeldern wird verzichtet. Im Interesse einer transparenten Darstellung wird vereinfachend davon ausgegangen, dass die jeweils untersuchte Abhängigkeitsstruktur für sämtliche Versicherungszweige identisch ist. Tabelle 6.12 stellt die Simulationsergebnisse im Überblick dar.

\begin{tabular}{|c|c|c|c|c|c|c|}
\hline [Tsd. GE] & $\boldsymbol{\rho}^{\mathbf{s}}=\mathbf{0}$ & $\boldsymbol{\rho}^{\mathbf{s}}=\mathbf{0 , 2}$ & $\boldsymbol{\rho}^{\mathbf{s}}=\mathbf{0 , 4}$ & $\boldsymbol{\rho}^{\mathbf{s}}=\mathbf{0 , 6}$ & $\boldsymbol{\rho}^{\mathbf{s}}=\mathbf{0 , 8}$ & $\boldsymbol{\rho}^{\mathbf{s}}=1$ \\
\hline$\mu$ AJ-Ergebnis brutto & 16.369 & 16.369 & 16.369 & 16.369 & 16.369 & 16.369 \\
\hline $\mathrm{CVaR}_{99,5}$ brutto & 150.633 & 151.497 & 151.978 & 152.481 & 152.992 & 153.706 \\
\hline RORAC brutto & $\mathbf{1 0 , 8 7 \%}$ & $\mathbf{1 0 , 8 0} \%$ & $\mathbf{1 0 , 7 7 \%}$ & $\mathbf{1 0 , 7 4 \%}$ & $\mathbf{1 0 , 7 0 \%}$ & $\mathbf{1 0 , 6 5 \%}$ \\
\hline
\end{tabular}

Tabelle 6.12: Lineare Abhängigkeiten im Basisszenario

Offensichtlich üben lineare Korrelationen in Bezug auf Basis- und Großschäden nur noch einen marginalen Einfluss auf den Risikokapitalbedarf des Modellunternehmens aus. Dies bestätigt die zu Beginn des Abschnitts formulierte Hypothese, dass in dem hier betrachteten Schadenportfolio bereits ein Großteil der existierenden Abhängigkeitsstrukturen durch Kumul- bzw. Naturereignisschäden erklärt werden kann. Der relevante Randbereich der Ergebnisverteilung wird derartig stark durch Naturkatastrophenschäden dominiert, dass sogar perfekt positiv korrelierte Basis- bzw. Großschäden $\left(\rho^{S}=1\right)$ nur noch zu einem geringfügigen Anstieg des Risikokapitalbedarfs führen. Gleichwohl darf angesichts der in Tabelle 6.12 dokumentierten Ergebnisse nicht pauschalierend geschlossen werden, dass korrelierte Basis- und Großschäden sich grundsätzlich nicht auf den Risikokapitalbedarf eines Versicherungsunternehmens auswirken. Um dies zu verdeutlichen, wird das bisher betrachtete Basisszenario dahingehend

\footnotetext{
${ }^{1005} \mathrm{Vgl}$. zur formalen Darstellung dieses Zusammenhangs im Fall normalverteilter Zufallsvariablen KORYCIORZ (2004), S. 114. Vgl. zur Konzeption der Komonotonie KORYCIORZ (2004), S. 275.

${ }^{1006} \mathrm{Vgl}$. auch KORYCIORZ (2004), S. 114-115.

${ }^{1007}$ Vgl. übereinstimmend MACK (2002), S. 32 und MCNEIL (2000), S. 16.
} 
modifiziert, dass nur noch diejenigen Versicherungszweige in die Analyse einbezogen werden, die nicht durch Naturereignisschäden belastet werden. In dieser As-IfBetrachtung reduziert sich das versicherungstechnische Portfolio folglich auf die Sparten „Allgemeine Unfall“, „Kraftfahrt-Haftpflicht“, „Feuer Industrie“, „VGV Feuer“ und „VGV Leitungswasser". ${ }^{1008}$ Abbildung 6.21 stellt den Risikokapitalbedarf des modifizierten Portfolios exemplarisch für ansteigende lineare Korrelationsniveaus dar.

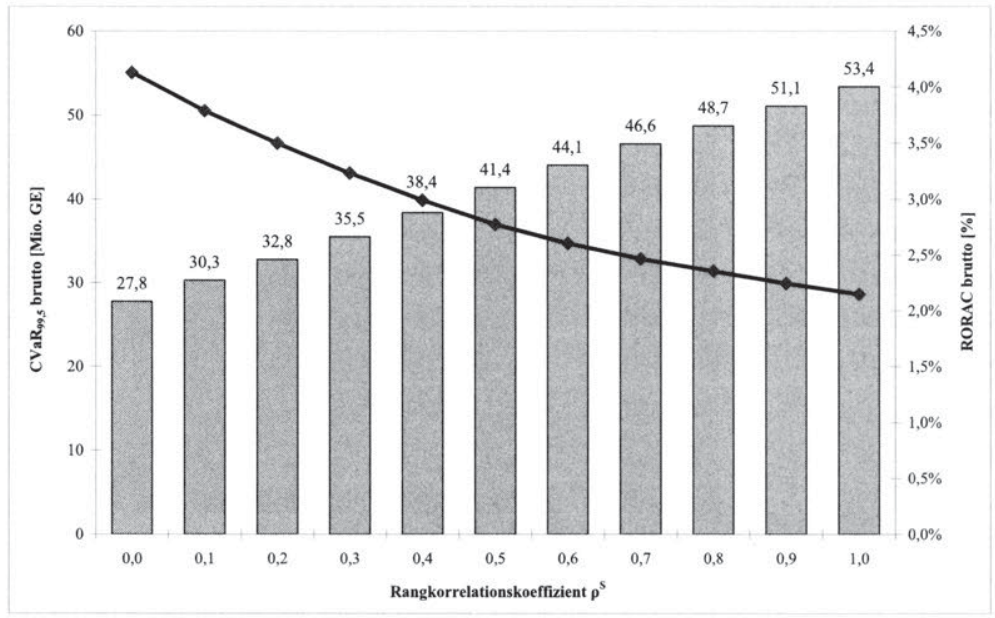

Abbildung 6.21: Lineare Abhängigkeiten im modifizierten Basisszenario

Es wird deutlich, dass der Risikokapitalbedarf des Modellunternehmens nahezu linear mit dem zugrunde gelegten Abhängigkeitsgrad ansteigt. ${ }^{1009}$ Die Darstellung illustriert somit eine der Kerngefahren des Versicherungsgeschäfts. Geht ein Versicherungsunternehmen, z.B. im Rahmen seiner Tarifgestaltung, fälschlicherweise von unkorrelierten oder nur schwach korrelierten Risiken aus, so läuft es Gefahr, dass die vorhandenen Risikodeckungsmassen nicht ausreichen, um das angestrebte Sicherheitsniveau 1- $\varepsilon$ zu gewährleisten. ${ }^{1010}$ In der Versicherungspraxis wird häufig noch unterstellt, dass das lineare Korrelationsmaß $\rho$ dazu geeignet ist, bestehende Abhängigkeitsstrukturen in ihrer Gesamtheit abzubilden. ${ }^{1011}$ Ferner wird aus dem Umstand, dass unabhängige $\mathrm{Zu}-$

\footnotetext{
${ }^{1008}$ Vereinfachend wird unterstellt, dass sämtliche Kostenpositionen der kumulexponierten Sparten variablen Charakter tragen und daher im modifizierten Basisszenario nicht berücksichtigt werden müssen.

${ }^{1009}$ In einem mehrperiodigen Modellkontext fiele die Erhöhung des Risikokapitalbedarfs vermutlich noch massiver aus. Vgl. hierzu exemplarisch SCHMEISER (2004b), S. 12-13.

${ }^{1010} \mathrm{Vgl}$. zur formalen Darstellung dieses Zusammenhangs am Beispiel der Risikomaße VaR und CVaR auch KORYCIORZ (2004), S. 112-115.

${ }^{1011}$ Vgl. hierzu und im Folgenden KORYCIORZ (2004), S. 112-120.
} 
fallsvariablen stets auch unkorreliert sind, im Umkehrschluss gefolgert, dass ein Korrelationskoeffizient von Null ebenfalls Unabhängigkeit impliziere. Diese Interpretation kann zu substanziellen Fehleinschätzungen der tatsächlichen Risikoposition führen, da die Existenz nicht-linearer Abhängigkeiten gänzlich außer Acht gelassen wird. ${ }^{1012}$ Die Verwendung linearer Korrelationsmaße ist zudem lediglich innerhalb der Klasse elliptischer Verteilungen unproblematisch. ${ }^{1013}$ Diese Verteilungen zeichnen sich durch die Eigenschaft aus, dass die Randverteilungen der betrachteten Zufallsvariablen zusammen mit ihrer Korrelationsmatrix die gemeinsame Verteilung eindeutig festlegen..$^{1014}$ Dies ist beispielsweise dann gewährleistet, wenn die gemeinsame (multivariate) Verteilung der Zufallsvariablen der Normalverteilungsannahme genügt. ${ }^{1015}$ Die Annahme elliptischer Verteilungen ist jedoch gerade im versicherungsspezifischen Kontext vielfach nicht angemessen und als sehr restriktive Anforderung an die ZufallsgesetzmäBigkeit des Schadengeschehens zu werten. ${ }^{1016}$ Daher wird im folgenden Abschnitt mit dem Copula-Konzept ein Ansatz vorgestellt, der die Berücksichtigung nicht-linearer Abhängigkeitsstrukturen erlaubt und daher zur Beschreibung von Tail-Abhängigkeiten besonders geeignet erscheint.

${ }^{1012}$ Vgl. vertiefend zur Kritik an linearen Abhängigkeitsmaßen EMBRECHTS/MCNEIL/STRAUMANN (1999), EMBRECHTS/MCNEIL/STRAUMANN (2002) sowie KORYCIORZ (2004), S. 115-120.

${ }^{1013} \mathrm{Vgl}$. auch KORYCIORZ (2004), S. 209.

${ }^{1014}$ EMBRECHTS/MCNEIL/STRAUMANN führen diesbezüglich aus: „As we have indicated, if the risks $\mathrm{X}_{1}, \ldots, \mathrm{X}_{\mathrm{n}}$ have a multivariate normal distribution, then everything is fine. However, it is not enough that each of the risks has a normal distribution, you must be convinced that they have jointly a multivariate normal distribution." EMBRECHTS/MCNEIL/STRAUMANN (1999), S. 2 [Hervorhebungen im Original].

1015 Vgl. EMBRECHTS/MCNEIL/STRAUMANN (2002), S. 8-10.

${ }^{1016}$ Vgl. hierzu übereinstimmend EMBRECHTS/MCNEIL/STRAUMANN (1999), S. 2 und KORYCIORZ (2004), S. 117. 


\subsubsection{Nicht-lineare Abhängigkeiten}

Im Falle von Tail-Abhängigkeiten besteht eine erhöhte Wahrscheinlichkeit, dass extreme Schadenrealisationen innerhalb eines Versicherungszweiges mit hohen Schadenrealisationen in anderen Versicherungszweigen zusammenfallen. ${ }^{1017}$ Mit Blick auf die Bestimmung des Risikokapitalbedarfs sind diese Fälle von übergeordnetem Interesse, da die zumeist verwendeten Shortfallmaße gerade den Tail der Ergebnisverteilung zur Risikomessung heranziehen. Das Copula-Konzept basiert auf der Grundidee, dass die Abhängigkeit zwischen Zufallsvariablen vollständig durch die Randverteilungen und ihre gemeinsame Verteilungsfunktion beschrieben werden kann. ${ }^{1018}$ In allgemeiner Form ist eine Copula daher definiert als multivariate Verteilungsfunktion $\mathrm{F}$ mit auf dem Intervall $[0,1]$ gleichverteilten Randverteilungen.

$$
\mathrm{F}\left(\mathrm{x}_{1}, \ldots, \mathrm{x}_{\mathrm{n}}\right)=\mathrm{P}\left(\mathrm{X}_{1} \leq \mathrm{x}_{1}, \ldots, \mathrm{X}_{\mathrm{n}} \leq \mathrm{x}_{\mathrm{n}}\right) \quad \text { mit } \mathrm{X}_{\mathrm{i}} \sim \mathrm{U}(0,1) \text { und } \mathrm{i}=1, \ldots, \mathrm{n}
$$

Dabei bezeichne $\mathrm{F}_{\mathrm{i}}(\mathrm{x})=\mathrm{P}\left(\mathrm{X}_{\mathrm{i}} \leq \mathrm{x}\right)$ die Wahrscheinlichkeit, dass die Zufallsvariable $\mathrm{X}_{\mathrm{i}}$ eine Realisierung unterhalb des Wertes $\mathrm{x}$ annimmt. Unter Bezugnahme auf das Theorem von $S K L A R^{1019}$ existiert eine Copula C, so dass für alle $\mathrm{x}=\left(\mathrm{x}_{1}, \ldots, \mathrm{x}_{\mathrm{n}}\right) \in \mathfrak{R}^{\mathrm{n}}$ gilt:

$$
\begin{aligned}
F\left(x_{1}, \ldots, x_{n}\right) & =P\left(F_{1}\left(X_{1}\right) \leq F_{1}\left(x_{1}\right), \ldots, F_{n}\left(X_{n}\right) \leq F_{n}\left(x_{n}\right)\right) \\
& =C\left(F_{1}\left(x_{1}\right), \ldots, F_{n}\left(x_{n}\right)\right)
\end{aligned}
$$

Sofern die Verteilungsfunktionen $\mathrm{F}_{1}, \ldots, \mathrm{F}_{\mathrm{n}}$ stetig sind, ist $\mathrm{C}$ eindeutig definiert. ${ }^{1020}$ Die Funktion $\mathrm{C}$ wird als Copula bezeichnet, da sie eine Verbindung zwischen der multivariaten Verteilungsfunktion $\mathrm{F}$ und ihren univariaten Randverteilungen $F_{1}, \ldots, F_{n}$ herstellt. ${ }^{1021}$ Mit Hilfe des Copula-Konzepts lassen sich paarweise Realisationen stochastisch abhängiger Zufallsvariablen erzeugen, die nicht-linearen Abhängigkeitsstrukturen unterliegen. ${ }^{1022}$

\footnotetext{
${ }^{1017}$ Vgl. ausführlich zum „Konzept asymptotischer Tail-Dependence“ KORYCIORZ (2004), S. 129-140 sowie JOE (1997), S. 33-35 und ROMANO (2002), S. 4-5.

${ }^{1018}$ Vgl. im Folgenden auch NELSEN (1999), EMBRECHTS/HÖING/JURI (2003), EMBRECHTS/LINDSKOG/MCNEIL (2001), PFeIFER/NESLEHOVÁ (2003a), PFEIFER/NESLEHOVÁ (2003b), FABIEN (2003), CHARPENTIER (2003) und NESLEHOVÁ (2004).

${ }^{1019}$ Vgl. zum Theorem von SKLAR vertiefend SCHWEIZER/SKLAR (1983), NELSEN (1999), S. 14-20 SOWie EMBRECHTS/LINDSKOG/MCNEIL (2001), S. 4.

${ }^{1020} \mathrm{Vgl}$. EMBRECHTS/LINDSKOG/MCNEIL (2001), S. 4.

${ }^{1021}$ Vgl. hierzu vertiefend EMBRECHTS/MCNEIL/STRAUMANN (2002), S. 4-5, EMBRECHTS/LINDSKOG/ MCNEIL (2001), S. 2-4, HÜRLIMANN (2002b), S. 3 und KORYCIORZ (2004), S. 120-123.

${ }^{1022}$ Einen verallgemeinerten Simulationsalgorithmus beschreibt KORYCIORZ (2004), S. 290-291.
} 
Im Kontext einer quantitativen Modellbildung verfügt das Copula-Konzept über wünschenswerte mathematische Eigenschaften, die hier stichpunktartig genannt seien: ${ }^{1023}$

- Die Copula ist von den Randverteilungen der Einzelrisiken unabhängig.

- Die gemeinsame Abhängigkeitsstruktur von Einzelrisiken wird bei Stetigkeit der Randverteilungen durch die Copula eindeutig beschrieben.

- Die Copula ist invariant gegenüber linearen und nicht-linearen Transformationen der Einzelrisiken.

Aus der Vielzahl der in der Literatur diskutierten Copula-Formen ${ }^{1024}$ soll nachfolgend die Gumbel-Copula zum Einsatz kommen, da diese zur Beschreibung von TailAbhängigkeiten besonders geeignet ist. ${ }^{1025}$ Die Gumbel-Copula ist für den bivariaten Fall definiert als:

$$
C_{\beta}^{G}(x, y)=\exp \left[-\left((-\ln x)^{1 / \beta}+(-\ln y)^{1 / \beta}\right)^{\beta}\right] \quad \text { mit } 0<\beta \leq 1
$$

Für den Spezialfall stochastischer Unabhängigkeit nimmt der Parameter $\beta$ den Wert 1 an. Sinkende $\beta$-Werte bedingen hingegen ein steigendes Abhängigkeitsniveau, wobei der definitionsgemäß ausgeschlossene Fall $\beta=0$ die stärkste Form positiver Abhängigkeiten (Komonotonie) implizieren würde. Negative Abhängigkeitsstrukturen können durch die Gumbel-Copula nicht beschrieben werden, was jedoch im versicherungsspezifischen Anwendungskontext als unproblematisch zu werten ist. ${ }^{1026}$ Für den Verlauf der weiteren Untersuchung ist es hilfreich, dass der Rangkorrelationskoeffizient $\rho^{\mathrm{S}}$ ein nicht-parametrisches Abhängigkeitsmaß darstellt, welches als spezifisches Merkmal zur Charakterisierung einer Copula interpretiert werden kann. ${ }^{1027}$ Dies eröffnet die Möglichkeit, in der nachfolgenden Simulationsstudie lineare und nicht-lineare Abhängigkeitsstrukturen näherungsweise miteinander zu vergleichen.

\footnotetext{
${ }^{1023}$ Vgl. im Folgenden auch PFEIFER (2003), S. 678-679.

${ }^{1024}$ Vgl. hierzu NELSEN (1999), EMBRECHTS/LINDSKOG/MCNEIL (2001), S. 17-39 und JOE (1997), S. $139-168$.

${ }^{1025}$ Die in der nordamerikanischen Literatur häufig anzutreffende Normal-Copula, welche die Abhängigkeitsstruktur einer multivariaten Normalverteilung ausdrückt, ist hingegen im versicherungsspezifischen Kontext zur Modellierung von Katastrophenszenarien nicht angemessen. Vgl. übereinstimmend KORYCIORZ (2004), S. 132 und die dort in Fußnote 380 angegebene Literatur.

${ }^{1026}$ Vgl. übereinstimmend MACK (2002), S. 32 und MCNEIL (2000), S. 16.

${ }^{1027}$ Vgl. zur formalen Herleitung dieses Sachverhalts KORYCIORZ (2004), S. 287-288 sowie EMBRECHTS/MCNEIL/STRAUMANN (2002), S. 16.
} 
Analog zur Vorgehensweise im vorangegangenen Kapitel wird zunächst der Einfluss nicht-linearer Abhängigkeiten auf das Basisszenario, d.h. unter Einbeziehung der kumulexponierten Versicherungszweige untersucht. Der Gumbel-Parameter $\beta$ wurde jeweils so gewählt, dass die resultierenden empirischen Rangkorrelationen $\rho^{\mathrm{S}}$ näherungsweise mit den Rangkorrelationen aus Tabelle 6.12 übereinstimmen. ${ }^{1028}$

\begin{tabular}{|c|c|c|c|c|c|c|}
\hline \multirow{2}{*}{ Tsd. GE] } & $\boldsymbol{\beta}=\mathbf{1}$ & $\boldsymbol{\beta}=\mathbf{0 , 8 7 0}$ & $\boldsymbol{\beta}=\mathbf{0 , 7 2 5}$ & $\boldsymbol{\beta}=\mathbf{0 , 5 7 1}$ & $\boldsymbol{\beta}=\mathbf{0 , 3 9 1}$ & $\boldsymbol{\beta}=\mathbf{0 , 0 1 6}$ \\
\cline { 2 - 7 } & $\rho^{\mathrm{s}}=0$ & $\rho^{\mathrm{s}} \approx 0,2$ & $\rho^{\mathrm{s}} \approx 0,4$ & $\rho^{s} \approx 0,6$ & $\rho^{s} \approx 0,8$ & $\rho^{s} \approx 1$ \\
\hline$\mu$ AJ-Ergebnis brutto & 16.369 & 16.369 & 16.369 & 16.369 & 16.369 & 16.369 \\
\hline CVaR $_{99,5}$ brutto & 150.633 & 151.966 & 152.204 & 152.777 & 153.541 & 153.742 \\
\hline RORAC brutto & $\mathbf{1 0 , 8 7 \%}$ & $\mathbf{1 0 , 7 7 \%}$ & $\mathbf{1 0 , 7 5 \%}$ & $\mathbf{1 0 , 7 1 \%}$ & $\mathbf{1 0 , 6 6 \%}$ & $\mathbf{1 0 , 6 5 \%}$ \\
\hline
\end{tabular}

Tabelle 6.13: Nicht-lineare Abhängigkeiten im Basisszenario (Gumbel-Copula)

Es ist zu konstatieren, dass im Basisszenario selbst starke nicht-lineare Abhängigkeiten von Basis- und Großschäden keinen nennenswerten Einfluss auf den Risikokapitalbedarf des Modellunternehmens ausüben. Dieses Phänomen liegt erneut in der Dominanz von Naturereignisschäden begründet. ${ }^{1029}$ Es fällt jedoch auf, dass der erforderliche Risikokapitalbetrag in den hier betrachteten „nicht-linearen Fällen“ zwar nur geringfügig, jedoch ausnahmslos oberhalb des Bedarfs des jeweils korrespondierenden „linearen Falls“ liegt. Dies belegt, dass die durch den Copula-Ansatz modellierten Tail-Abhängigkeiten zu höheren Kapitalanforderungen führen.

Diese Hypothese soll nachfolgend wiederum für den fiktiven Fall untersucht werden, dass keine kumulbelasteten Versicherungszweige durch das Modellunternehmen betrieben werden (modifiziertes Basisszenario). Hierzu wird der Risikokapitalbedarf für sukzessiv fallende $\beta$-Werte, d.h. für steigende nicht-lineare Abhängigkeitsniveaus von Basis- und Großschäden ermittelt. Durch die gewählte Höhe des Copula-Parameters $\beta$ werden Datenkonstellationen erzeugt, die jeweils approximativ den aus Abbildung 6.22 bekannten Rangkorrelationen $\rho^{\mathrm{S}}$ entsprechen. ${ }^{1030}$

\footnotetext{
${ }^{1028}$ Es sei betont, dass die aus Tabelle 6.13 ersichtlichen Relationen zwischen Gumbel-Parameter und Rangkorrelationskoeffizient lediglich für das betrachtete Modellportfolio näherungsweise Gültigkeit besitzen.

${ }^{1029}$ Vgl. hierzu auch Anhang E.

${ }^{1030}$ Modelltechnisch werden die Abhängigkeitsstrukturen durch einfaches Umsortieren der realisierten Zufallsgrößen erzeugt. Da bei diesem Vorgang keine neuen Realisationen erzeugt werden, wird die vorgegebene Korrelationsstruktur in Abhängigkeit von der Simulationsanzahl nur approximativ erreicht.
} 


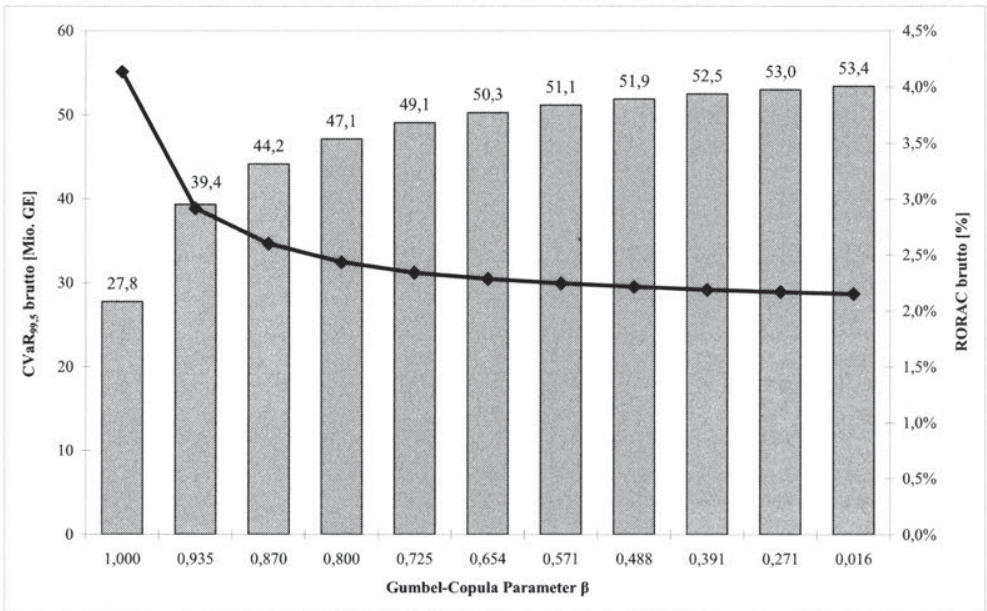

Abbildung 6.22: Nicht-lineare Abhängigkeiten im modifizierten Basisszenario (Gumbel-Copula)

Für die Extremfälle vollständiger Unabhängigkeit $(\beta=1)$ bzw. perfekt positiver Abhängigkeit $(\beta \rightarrow 0)$ entspricht der Risikokapitalbedarf respektive der RORAC exakt dem „linearen Fall““. Ein Vergleich mit Abbildung 6.21 legt jedoch offen, dass der Risikokapitalbedarf bereits für schwache nicht-lineare Abhängigkeitsstrukturen $(\beta=0,935)$ signifikant oberhalb des Kapitalbedarfs liegt, welcher sich - bei näherungsweise identischer Rangkorrelation - im Falle linearer Korrelation ergeben würde. Offenbar wirkt sich die Gefahr eines gleichzeitigen Auftretens von Großschäden in mehreren Sparten in beträchtlichem Maße auf den Randbereich der Ergebnisverteilung aus. Darüber hinaus zeigt sich, dass der Risikokapitalbedarf mit zunehmender TailAbhängigkeit $(\beta \rightarrow 0)$ ansteigt und asymptotisch gegen das im Standalone-Fall vorzuhaltende Kapital konvergiert.

Als Ergebnis ist somit festzuhalten: Vernachlässigt ein Versicherungsunternehmen bei der Quantifizierung und Steuerung seiner versicherungstechnischen Risikoposition (z.B. bei der Tarifierung) die Existenz von Abhängigkeitsstrukturen, kann dies zu einer substanziellen Unterschätzung des Risikokapitalbedarfs führen. ${ }^{1031}$ Sofern lineare Abhängigkeitsmaße, wie z.B. der Korrelationskoeffizient, in die Risikoanalyse einbezogen werden, gilt es zu berücksichtigen, dass deren „Einsatzvoraussetzungen“ gerade im versicherungsspezifischen Kontext nur selten anzutreffen sind. Darüber hinaus darf aus dem intuitiven Zusammenhang, dass unabhängige Zufallsvariablen stets auch unkorreliert sind, nicht im Umkehrschluss geschlossen werden, dass ein Korrelationsko-

${ }^{1031}$ Vgl. auch KORYCIORZ (2004), S. 113. 
effizient von Null gleichzeitig auch eine stochastische Unabhängigkeit signalisiert. Diese Schlussweise ignorierte gänzlich die Existenz nicht-linearer Abhängigkeiten, welche ein erhebliches Gefährdungspotenzial bergen und demzufolge ein zusätzliches Kapitalerfordernis induzieren. Mit Blick auf die Versicherungspraxis muss jedoch ausdrücklich herausgestellt werden, dass die Ermittlung der notwendigen Parameter, mithin eine exakte Aufdeckung der Abhängigkeitsstrukturen komplexer Versicherungsbestände, wenn überhaupt nur in grober Näherung möglich ist. Dies trifft insbesondere auf die zuletzt untersuchten nicht-linearen Abhängigkeiten zu. ${ }^{1032}$ Die Einbeziehung stochastischer Abhängigkeiten sollte daher primär als Sensitivitätsanalyse oder "Stresstest" verstanden werden und somit die realistische Zielsetzung verfolgen, ein Gefühl für relevante Größenordnungen und existenzgefährdende Schadenpotenziale zu entwickeln.

Angesichts der hier nur in knapper Form angesprochenen Problematik einer realitätsnahen Parametrisierung von Copula-Ansätzen fußen die nachfolgenden Untersuchungen zur Rückversicherungs- und Zeichnungspolitik wieder auf der Annahme, dass die Basis- und Großschäden der modellierten Teilkollektive unabhängig voneinander auftreten. Diese Vorgehensweise erscheint zudem zweckmäßig, um die nachfolgenden Analyseergebnisse unmittelbar mit dem in Kapitel 6.2.1 beschriebenen „Basisszenario" vergleichen zu können.

\subsubsection{Einfluss der Rückversicherungspolitik}

\subsubsection{Stop-Loss-Deckung für die sturmexponierten Sparten}

Die versicherungstechnische Nettoanalyse des Basisszenarios in Kapitel 6.2.1.1.2 hat bereits gezeigt, dass die Versicherungszweige „VGV Sturm“ und „Allgemeine Sturm“ einen starken Einfluss auf den Risikokapitalbedarf des Modellunternehmens ausüben. Wenngleich der Kapitalbedarf durch das im Basisszenario unterstellte Rückversicherungsprogramm bereits substanziell reduziert werden konnte, entfallen noch ca. $88 \%$ des Kapitalbedarfs auf die sturmexponierten Sparten. Als Ursache für dieses Phänomen wurde das so genannte Frequenzrisiko identifiziert. Der Jahresüberschadenexzedentenvertrag bzw. Stop Loss (SL) stellt das klassische Instrument zur Begrenzung des Frequenzrisikos dar. Er wird daher vielfach auch zum Zweck des „Bilanzschutzes“ abgeschlossen. ${ }^{1033}$ Wie im vorliegenden Fall gehen einem SL in der Regel andere

\footnotetext{
${ }^{1032}$ EMBRECHTS/LINDSKOG/MCNEIL führen in diesem Zusammenhang sehr zutreffend aus: „However there is much uncertainty in choosing a suitable copula family representing the dependence between potential losses for the 1 lines of business. The data may give indications of properties such as tail dependence but it should be combined with careful considerations of the nature of the underlying loss causing mechanisms.“ EMBRECHTS/LINDSKOG/MCNEIL (2001), S. 40.

${ }^{1033}$ Vgl. MACK (2002), S. 328.
} 
Rückversicherungsdeckungen voraus, so dass für die Bemessung der Entschädigungsleistungen der im Selbstbehalt verbleibende Jahresschaden der rückversicherten Sparten maßgeblich ist. Um das moralische Risiko ${ }^{1034}$ auf Seiten des Erstversicherers einzugrenzen, wird diese Vertragsart vornehmlich in Sparten abgeschlossen, die nahezu ausschließlich zufallsbedingten Schwankungen unterliegen. ${ }^{1035}$ Darüber hinaus sollte die Höhe der Priorität so bemessen sein, dass sich der Erstversicherer bereits versicherungstechnisch in der Verlustzone befindet, bevor die SL-Deckung einsetzt. Sowohl die Priorität als auch die Haftung werden in der Regel in Relation zum rückgedeckten Prämienvolumen, d.h. als Schadenquote angegeben. Die Verwendung einer relativen Maßzahl birgt für den Rückversicherer jedoch die grundlegende Gefahr, dass die vereinbarte Priorität auch bei konstantem Schadenniveau überschritten werden kann, wenn beispielsweise das Niveau der Originalprämien in ,weichen" Marktphasen absinkt. ${ }^{1036}$ Der Abschluss eines SL setzt daher eine langjährige und vertrauensvolle Geschäftsbeziehung zwischen Erst- und Rückversicherer voraus.

Nachfolgend soll untersucht werden, welchen Einfluss der Abschluss eines SLVertrages in den sturmexponierten Sparten „VGV Sturm“ und „Allgemeine Sturm“ auf die Rendite-Risiko-Position des Modellunternehmens ausübt. Beide Sparten sind bereits über einen Einzelschadenexzedentenvertrag mit Vorwegquote gedeckt, so dass sich die Haftung des abzuschließenden SL lediglich auf den verbleibenden Selbstbehalt beschränkt. Während die Höhe der Priorität sowohl durch die unternehmensseitig maximal tolerierte Verlusthöhe als auch die Platzierbarkeit am Markt determiniert wird, sind für die Festlegung einer hinreichenden Haftstrecke (= Priorität + Haftung) spezifische Kenntnisse über das Schadenpotenzial des zugrunde liegenden Portfolios notwendig. In diesem Zusammenhang können quantitative Risikomodelle eine wertvolle Entscheidungshilfe leisten, da sie geophysikalische Daten mit portfoliospezifischen Informationen verknüpfen. ${ }^{1037}$ Im Modellkontext wird die Haftstrecke des SL auf $270 \%$ festgelegt. Bis zu einer Schadenquote von $150 \%$ (= Priorität) trägt das Modellunternehmen sämtliche Schäden im Selbstbehalt. Darüber hinaus übernimmt der Rückversicherer Schäden bis zu einer Schadenquote von insgesamt 270\%. Die Haftung des Rückversicherers beträgt demzufolge $120 \%$ (,120\% xs 150\%“).

\footnotetext{
${ }^{1034} \mathrm{Vgl}$. zum moralischen Risiko und seinen versicherungsspezifischen Erscheinungsformen vertiefend NELL (1998), S. 7-33 sowie ZWEIFEL/EISEN (2003), S. 293-342.

${ }^{1035}$ Vgl. im Folgenden LieBwEIN (2000), S. 169 und GROSSMANN (1977), S. 124.

${ }^{1036}$ Vgl. vertiefend LIEBWEIN (2000), S. 168-171.

${ }^{1037}$ Vgl. Pfeifer (2003), S. 682-692 und PFEIFER (2004), S. 468-476.
} 
Abbildung 6.23 stellt die Wirkungsweise des abgeschlossenen SL-Vertrags anschaulich in Form eines Streudiagramms dar. Um einen konsistenten Vergleich mit Abbildung 6.16 zu gewährleisten, wird erneut nur der Schadenaufwand aus Sturmschäden der Sparte „VGV Sturm“ betrachtet. $^{1038}$

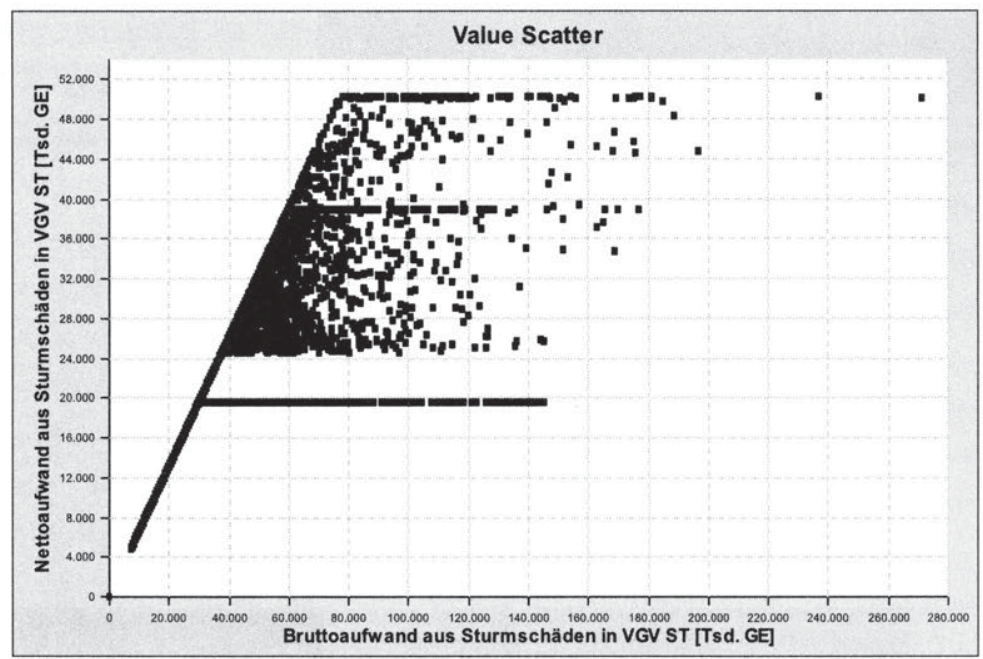

Abbildung 6.23: Streudiagramm des Schadenaufwands aus Sturmschäden in VGV ST mit Stop-Loss-Deckung

In denjenigen Simulationsläufen, welche durch mehrere - jeweils die XL-Priorität unterschreitende - Sturmschäden betroffen sind, wird die Gesamtschadenlast des Erstversicherers in der Sparte „VGV Sturm“ auf die Priorität beschränkt. Es wird außerdem deutlich, dass die gewählte Haftstrecke von $270 \%$ in den betrachteten 30.000 Simulationsläufen offenbar ausreicht, um das Sturmrisiko in „VGV Sturm“ auf einen maximalen Nettoschaden von ca. 50 Mio. GE zu beschränken. Auf der Unternehmensebene manifestiert sich die Begrenzung des Frequenzrisikos aus Sturmereignissen in einem deutlichen Rückgang des Risikokapitalsbedarfs. Der Nettokapitalbedarf des Modellunternehmens reduziert sich von 51,6 Mio. GE auf 40,4 Mio. GE. Das erwartete Nettoergebnis vermindert sich durch die zusätzliche Rückversicherungsprämie nur unterproportional, so dass sich letztlich der Netto-RORAC von $18,0 \%$ auf $21,8 \%$ verbessert. Eine CVaR-basierte Allokation des Nettorisikokapitals auf die Versicherungszweige liefert folgende Ergebnisse.

${ }^{1038}$ Die Beschränkung auf Sturmschäden in Abbildung 6.23 erfolgt ausschließlich im Interesse einer transparenteren Darstellung. Der Natur des Stop-Loss-Vertrags entsprechend, deckt dieser im vorliegenden Modell neben Sturmschäden die Gesamtschadenlast, d.h. auch Basis- und Überschwemmungsschäden, welche die Sparten „VGV Sturm“ und „Allgemeine Sturm“ betreffen. 


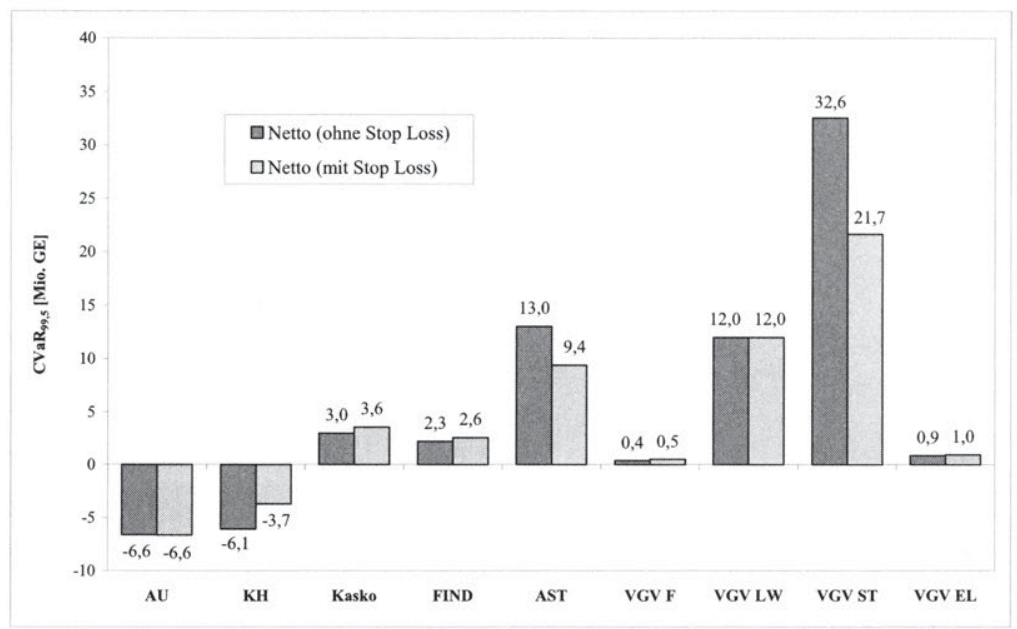

Abbildung 6.24: Einfluss der Stop-Loss-Deckung auf den Netto-Risikokapitalbedarf

Im Vergleich zu der im Basisszenario ermittelten Nettoallokation sinkt der Risikokapitalbedarf in den sturmexponierten Sparten „VGV Sturm“ und „Allgemeine Sturm“ durch den Abschluss des Stop-Loss-Vertrages ca. um ein Drittel. Dieser Entlastungseffekt wird jedoch nicht in voller Höhe im Gesamtportfolio wirksam, da der Kapitalbedarf in anderen Sparten wie beispielsweise „Kasko“ und „Feuer Industrie“ leicht ansteigt. Außerdem fällt der durch die Sparte „Kraftfahrt-Haftpflicht“ hervorgerufene Entlastungseffekt geringer aus. Dieses Resultat ist darauf zurückzuführen, dass der Randbereich der Gesamtergebnisverteilung nach Abschluss der Stop-Loss-Deckung eine neue Struktur aufweist und zumindest teilweise durch andere Ergebnisszenarien bestimmt wird. In diesen Szenarien sind einige Versicherungszweige (z.B. „KraftfahrtHaftpflicht“" und „Feuer Industrie“) vermehrt durch Großschäden bzw. durch Hagelschäden (z.B. „Kasko“) betroffen, so dass diese Sparten in stärkerem Maße zur Entstehung außergewöhnlicher Nettoverluste des Gesamtunternehmens beitragen. Wenngleich die Risikostruktur dieser Sparten insgesamt unverändert geblieben ist, wird ihnen im Rahmen der Allokation ein erhöhter Kapitalbedarf zugewiesen.

Im Hinblick auf die Ausgestaltung eines Steuerungsansatzes vermittelt dieses Phänomen eine wichtige Erkenntnis. Angesichts des erheblichen Risikopotenzials, dem das Modellunternehmen aufgrund seiner Exponierung gegenüber Naturgefahren ausgesetzt ist, kann der Abschluss des Stop-Loss-Vertrages als Entscheidung klassifiziert werden, die dem Schutz des Gesamtunternehmens dient. Ebenso dient das vorzuhaltende Risikokapital der Sicherheit des Unternehmens als Ganzes und nicht der Absicherung ein- 
zelner Versicherungszweige. ${ }^{1039}$ Obwohl die Ergebnis- und Risikostruktur der Teilkollektive „Kraftfahrt-Haftpflicht“, „Kasko“ und „Feuer Industrie“ unverändert geblieben ist, wird diesen Sparten nach Abschluss des Stop-Loss-Vertrages ein erhöhter Kapitalbedarf zugeordnet. Der RORAC dieser Teilkollektive reduziert sich demzufolge bei unveränderter Ergebniserwartung. Würde das Modellunternehmen seine Entscheidungen über den Auf- und Abbau strategischer Investitionsfelder im Sinne eines „Profit Rankings" anhand von risikoadjustierten Spartenkennziffern vornehmen, so könnten Fehlsteuerungsimpulse dergestalt auftreten, dass der Ausbau bzw. die Aufrechterhaltung eines Versicherungszweiges nicht mehr vorteilhaft erscheint, nachdem beispielsweise der Rückversicherungsschutz in einer anderen Sparte verändert wurde. ${ }^{1040}$ Aus einer ökonomisch motivierten Steuerungsperspektive erscheint dieser Effekt unerwünscht, da die Profitabilität eines einzelnen Geschäftsbereichs durch zentrale Unternehmensentscheidungen oder auch dezentrale Entscheidungen anderer Geschäftsbereiche beeinflusst werden kann. Vor diesem Hintergrund ist eine direkte Alternativenbeurteilung auf der Grundlage des RORAC auf der Unternehmensebene einer dezentralen Geschäftsbereichssteuerung anhand von „Profit Rankings“ vorzuziehen. ${ }^{1041}$

\subsubsection{XL-Deckung für die Sparte „Kraftfahrt-Haftpflicht“}

Die nachfolgende Untersuchung geht der Frage nach, ob die im Basisszenario unterstellte Rückversicherungsstruktur des Versicherungszweiges „Kraftfahrt-Haftpflicht“ (KH) dem zugrunde liegenden Schadenpotenzial angemessen ist oder gegebenenfalls hinsichtlich der Rendite-Risiko-Position des Gesamtportfolios verbessert werden könnte. Die Großschäden der Sparte KH sind im Basisszenario durch einen Einzelschadenexzedentenvertrag gedeckt, der folgende Haftungsstruktur aufweist: Priorität 1,75 Mio. GE, Haftung 48,25 Mio. GE („48,25 xs 1,75“). Die gewählte Haftungsstrecke von 50 Mio. GE trägt der Tatsache Rechnung, dass der KH-Bestand des Modellunternehmens annahmegemäß keine Verträge mit unbegrenzter Haftung (sog. „Illimité-Deckungen“) enthält. ${ }^{1042}$ Die Haftung für Personen- und Sachschäden sei vertrag-

\footnotetext{
${ }^{1039}$ Vgl. auch GRÜNDL/SCHMEISER (2004b), S. 6-7 und GRÜNDL/SCHMEISER (2004c), S. 12.

${ }^{1040} \mathrm{Vgl}$. ebenfalls kritisch zur Verwendung risikoadjustierter Performancemaße zum Zweck der Kapitalbudgetierung und dezentralen Steuerung einzelner Geschäftsbereiche VENTER (2002), VENTER (2003), GRÜNDL/SCHMEISER (2004b) und GRÜNDL/SCHMEISER (2004c).

${ }^{1041} \mathrm{Vgl}$. hierzu auch GRÜNDL/SCHMEISER (2002), S. 816, die eine übereinstimmende Aussage im Kontext des Kapitalwertkalküls treffen.

${ }^{1042}$ Als Reaktion auf diverse Katastrophenschäden in der Autoversicherung wurde die bisher in Deutschland gewährte Illimité-Deckung durch viele Rückversicherungsunternehmen (für Neuverträge) zum 01.01.2003 abgeschafft und die Haftung pro Schadenereignis auf 50 Mio. $€$ begrenzt. Bisher wurde jedoch nur ca. die Hälfte aller Verträge auf die neue Regelung umgestellt, so dass die „Altverträge“ in der Praxis noch ein erhebliches Gefährdungspotenzial bergen. Vgl. auch BERKENKOPF/FROMME (2004).
} 
lich auf 50 Mio. GE je Ereignis beschränkt. ${ }^{1043}$ Auf dem Sachverhalt aufbauend, dass der Kapitalbedarf des Modellunternehmens in erheblichem Maße durch Naturschadenereignisse beeinflusst wird, soll nachfolgend analysiert werden, inwiefern der Einzelschadenexzedent in der Sparte $\mathrm{KH}$ überhaupt zur Entlastung der Risikoposition des Gesamtunternehmens beiträgt. Hierzu wird in Abbildung 6.25 zunächst die Wirkung des XL-Vertrages in der Form eines Streudiagramms illustriert. Es gilt zu beachten, dass jeder Punkt des Streudiagramms die Summe über sämtliche KH-Großschäden je Simulationslauf abbildet.

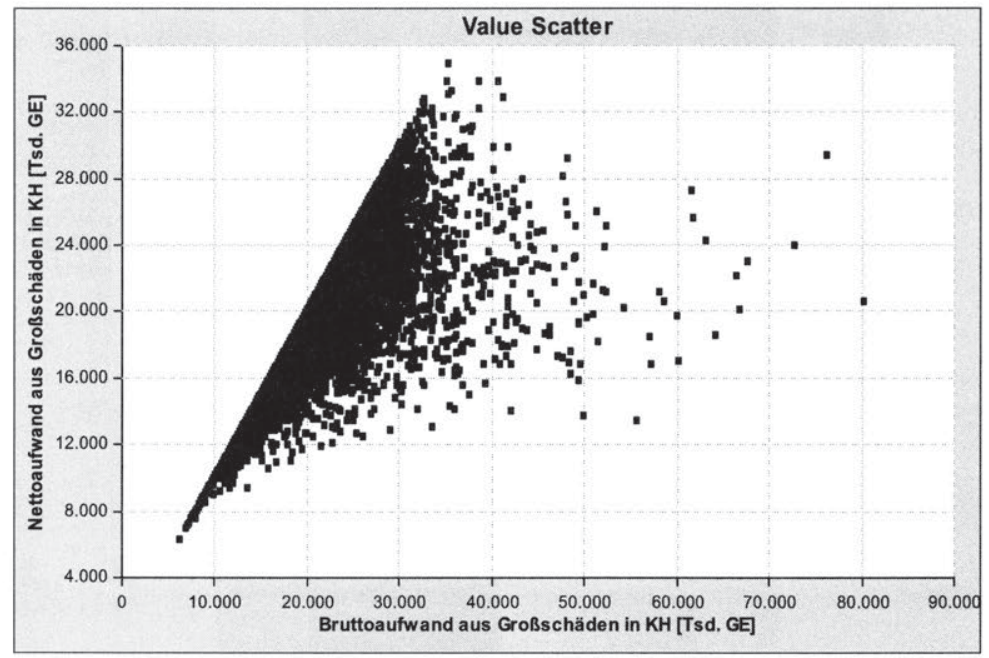

Abbildung 6.25: Streudiagramm der KH-Großschäden im Basisszenario

Das Streudiagramm ist dahingehend zu interpretieren, dass sämtliche Großschadenszenarien, die sich nicht auf der „Winkelhalbierenden“ befinden, durch den XLVertrag erfasst werden. Es wird deutlich, dass der XL-Vertrag aufgrund der vergleichsweise niedrig gewählten Priorität von 1,75 Mio. GE in einer Vielzahl der Simulationsläufe seine Wirkung entfaltet (sog. „Working XL"). ${ }^{1044}$ Ebenso erfahren extreme Schadenszenarien durch die Rückversicherung eine signifikante Entlastung. In einer isolierten Spartenbetrachtung hat dies zur Folge, dass sich der Kapitalbedarf in der Sparte KH von brutto 18,2 Mio. GE auf netto 4,7 Mio. GE reduziert.

\footnotetext{
${ }^{1043}$ In konsistenter Vorgehensweise wird daher eine Kappungsgrenze für Großschäden definiert, so dass die verwendete Schadenhöhenverteilung keine Realisationen größer als 50 Mio. GE erzeugt.

${ }^{1044}$ Vgl. zur Aufteilung nicht-proportionaler Rückversicherungsdeckungen auf verschiedene Haftungsabschnitte (sog. „Layer") PFEIFFER (1994), S. 62, LIEBWEIN (2000), S. 221-222 und SCHWEPCKE (2001), S. 123-124.
} 
Es kann daher als Zwischenergebnis festgehalten werden, dass der XL-Vertrag in der Standalone-Perspektive zweifelsfrei als vorteilhaft zu charakterisieren ist. Offen bleibt hingegen die Frage, welchen Beitrag der XL-Vertrag mit Blick auf die Rendite-RisikoPosition des Gesamtunternehmens leistet. Zur Beurteilung dieses Sachverhalts wird erneut der Netto-RORAC des Gesamtportfolios als Entscheidungskriterium herangezogen. Es wird zunächst der Fall betrachtet, dass das Modellunternehmen in der Sparte KH gänzlich auf Rückversicherungsschutz verzichtet und demzufolge das Großschadenrisiko in voller Höhe im Eigenbehalt trägt. Eine Simulation dieses Sachverhalts liefert folgende Ergebnisse:

\begin{tabular}{|c|c|c|}
\hline [Tsd. GE] & Basisszenario & ohne XL in KH \\
\hline Nettoergebnis (EW) & 9.257 & 9.964 \\
\hline CVaR $_{99,5}$ netto & 51.558 & 52.418 \\
\hline RORAC netto $^{2}$ & $\mathbf{1 8 , 0} \%$ & $\mathbf{1 9 , 0 \%}$ \\
\hline
\end{tabular}

Tabelle 6.14: Nettorisikoposition im Basisszenario und bei Verzicht auf XL-Deckung in KH

Der vollständige Verzicht auf Rückversicherungsschutz in der Sparte KH führt offenbar dazu, dass der Mehrbedarf an Risikokapital auf der Unternehmensebene durch ein verbessertes Nettoergebnis überkompensiert wird und demzufolge der Netto-RORAC ansteigt. Dieses auf den ersten Blick überraschende Ergebnis kann erneut durch die Tail-Dominanz der Naturereignisschäden erklärt werden, denn selbst extreme Bruttoschadenszenarien in „Kraftfahrt-Haftpflicht“ nehmen nur geringen Einfluss auf die Tailstruktur der Gesamtergebnisverteilung. Im Rahmen eines ausschließlich quantitativ ausgerichteten Risiko-Rendite-Kalküls könnte man somit zu der Schlussfolgerung gelangen, dass die Kündigung des XL-Vertrags vorteilhaft sei. Diese Interpretation lässt jedoch qualitative Aspekte außer Acht, welche in dem hier betrachteten Modellansatz nicht abgebildet werden, allerdings in der Praxis ebenfalls beträchtliche Entscheidungsrelevanz besitzen. Würde das Modellunternehmen auf den XL-Vertrag gänzlich verzichten, müsste es nicht nur sämtliche Schäden der Sparte KH im Eigenbehalt tragen, sondern könnte auch keine sonstigen Dienstleistungen des Rückversicherers in Anspruch nehmen. Insbesondere bei der Regulierung von Katastrophenschäden, aber auch im Rahmen der Tarifkalkulation und sonstiger aktuarieller Dienstleistungen kann der Erstversicherer in der Regel erheblich von der Expertise und Erfahrung des Rückversicherers profitieren. ${ }^{1045}$ Ferner gilt es zu beachten, dass die Großschadenentwicklung zwischen den modellierten Versicherungszweigen als unabhängig angenommen wird. Es treten lediglich „natürliche“ Abhängigkeitsstrukturen zwischen denjenigen Sparten auf, die gegenüber Naturgefahren exponiert sind. In dem durchaus vorstellbaren Szenario, dass ein Katastrophenschaden in der Sparte KH zufällig mit einem stark sturmbelasteten Jahr zusammenfällt, würde das ermittelte Nettorisikokapi-

${ }^{1045}$ Vgl. vertiefend LiEBWEIN (2000), S. 47-49. 
tal mit hoher Wahrscheinlichkeit nicht ausreichen, um die angestrebte Unternehmenssicherheit zu gewährleisten. ${ }^{1046} \mathrm{Um}$ insbesondere Spitzen- und Kumulrisiken an den Rückversicherer zedieren zu können, erscheint vor diesem Hintergrund die Fortführung einer XL-Deckung grundsätzlich sinnvoll. Vor dem Hintergrund des oben beschriebenen Sachverhalts gewinnt jedoch die Fragestellung Relevanz, ob ein wirksamer Schutz gegen Spitzenrisiken nicht auch durch die Wahl einer höheren Priorität erreicht werden kann. In Abbildung 6.26 werden die aus alternativen Haftungsstrukturen resultierenden Netto-RORAC-Kennziffern miteinander verglichen. ${ }^{1047}$

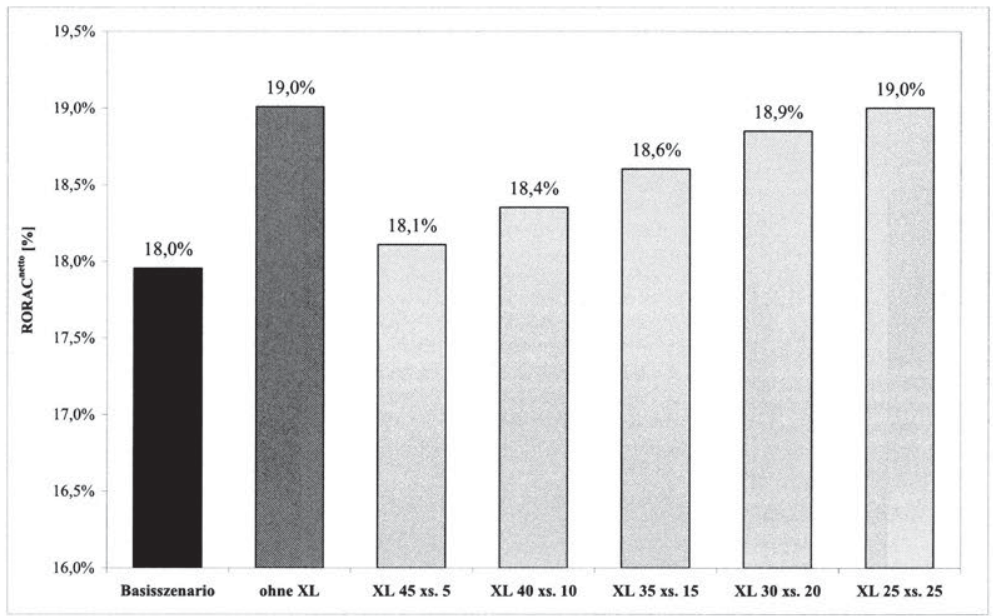

Abbildung 6.26: Auswirkungen unterschiedlicher XL-Prioritäten in „Kraftfahrt-Haftpflicht“

Es ist ersichtlich, dass sich der Netto-RORAC des Modellunternehmens mit wachsender Höhe der XL-Priorität demjenigen Niveau annähert, welches sich bei einem vollständigen Verzicht auf Rückversicherungsschutz einstellen würde. Ein Vergleich mit dem Basisszenario verdeutlicht außerdem, dass die Entlastung des Nettoergebnisses aufgrund eines höheren Eigenbehalts offenbar nicht durch einen überproportionalen Anstieg des Risikokapitalbedarfs kompensiert wird. Die im Basisszenario gewählte Haftungsstruktur eines „Working XL“ ist daher im Diversifikationsumfeld des Gesamtportfolios offensichtlich nicht effizient. Die Beantwortung der Frage, welche Struktur in der Praxis letztendlich durch ein Versicherungsunternehmen gewählt wer-

\footnotetext{
${ }^{1046}$ Als Beispiel für ein derartiges Katastrophenszenario sei hier der Unfall eines Tanklastzuges auf der Wiehlbachtalbrücke am 26.08.2004 genannt. Die hohen Temperaturen der brennenden Tanks verursachten schwerste Verformungen der Brückenstahlträger, so dass ein vollständiger Abriss der Brücke nicht ausgeschlossen werden konnte. Der Gesamtschaden wird auf 33 Mio. Euro geschätzt. Vgl. BERKENKOPF/FROMME (2004).

${ }^{1047}$ Die bereits im Basisszenario festgelegte Haftstrecke von 50 Mio. GE bleibt unverändert bestehen.
} 
den sollte, hängt insbesondere von der Prämienforderung der Rückversicherer sowie der vorherrschenden Wettbewerbsintensität und Kapazitätssituation ab. ${ }^{1048}$ Zusammenfassend kann jedoch festgehalten werden, dass Entscheidungen über die Struktur eines einzelnen Rückversicherungsvertrags immer im Wirkungsgefüge des Gesamtportfolios beurteilt werden sollten. Isolierte Optimierungskalküle dürften allenfalls zufällig zu einer verbesserten Rendite-Risiko-Position des Gesamtunternehmens führen. Neben quantitativen Analysen sollten außerdem auch qualitative Aspekte in den Entscheidungsprozess integriert werden.

\subsubsection{Einfluss der Zeichnungspolitik}

\subsubsection{Sanierung der Sparte „VGV Leitungswasser“}

Neben der Rückversicherungspolitik wurde bereits in Kapitel 3.2.3 die Zeichnungspolitik als zentrales versicherungstechnisches Steuerungsinstrument identifiziert. Die nachfolgend untersuchte Sanierungsstrategie für die Modellsparte „VGV Leitungswasser" ist durch den Sachverhalt motiviert, dass die Leitungswassersparte im deutschen Schaden- und Unfallversicherungsmarkt vielfach durch negative Erfolgsbeiträge bei nur geringer Ergebnisschwankung charakterisiert ist. ${ }^{1049}$ Für die defizitäre Ergebnissituation sind weniger Frostschäden aufgrund außergewöhnlich strenger Winter verantwortlich, sondern primär ein stetig steigender Schadendurchschnitt. Die Schadenhäufigkeit hingegen schwankt um einen langfristig stabilen Mittelwert. ${ }^{1050}$ Die defizitäre Ergebnissituation des Modellunternehmens in der Sparte „VGV Leitungswasser“ ist im Basisszenario angesichts einer Combined Ratio von 118,1\% evident. Aufgrund eines stark negativen und nur schwach volatilen Ergebnisbeitrags ist das Leitungswasserrisiko annahmegemäß am Rückversicherungsmarkt nicht zu akzeptablen Konditionen platzierbar.

Vor dem Hintergrund dieser Problematik werden nachfolgend zwei alternative Vorgehensweisen zur Sanierung der Sparte „VGV Leitungswasser“ simuliert und hinsichtlich ihres Wirkungspotenzials miteinander verglichen. Die nachstehende Analyse konzentriert sich lediglich auf Handlungsalternativen, welche die Zeichnungspolitik betreffen. Von den Möglichkeiten einer restriktiveren Schadenregulierungspraxis und alternativen Maßnahmen des Schadenmanagements wird abstrahiert. ${ }^{1051}$

\footnotetext{
${ }^{1048}$ Vgl. vertiefend SCHRADIN (1998), S. 27-31 und MEHL (1987), S. 31-33.

${ }^{1049}$ Im Durchschnitt der Jahre 1983-2002 betrug der Anteil der Leitungswasserschäden am Gesamtschaden der Verbundenen Gebäudeversicherung 47,5\%. Vgl. hierzu und im Folgenden o.V. (2003), S. 2010.

${ }^{1050}$ Vgl. O.V. (2003), S. 2010.

${ }^{1051}$ Vgl. hierzu detailliert JARA (2000), S. 76-80 und S. 239-276.
} 
Als erste Alternative wird die Einführung einer absoluten Selbstbeteiligung untersucht. ${ }^{1052}$ Diese Form der nicht-proportionalen Risikoteilung zwischen Versicherungsunternehmen und Versicherungsnehmer wird vielfach auch als Abzugsfranchise oder Selbstbehalt bezeichnet. ${ }^{1053}$ Die Einführung einer (Schaden-) Selbstbeteiligung bringt für das Versicherungsunternehmen grundsätzlich zwei wesentliche Vorzüge mit sich. Einerseits werden Kleinschäden bis zur Höhe der Selbstbeteiligung nicht mehr durch die Versicherungsnehmer gemeldet bzw. bei größeren Schäden bis zur Höhe der Franchise eigenständig getragen. Neben einer substanziellen Entlastung des Schadenbedarfs kann hiermit auch eine Reduzierung des Verwaltungs- und Schadenregulierungsaufwands verbunden sein. Aufgrund der typischen Schiefe von Schadenanzahlverteilungen ist die Reduzierung des Schadenaufwandes bisweilen schon für kleine bis mittlere Selbstbehalte beträchtlich. ${ }^{1054}$ Andererseits stellt die Selbstbeteiligung im Falle asymmetrischer Information ein geeignetes Instrument zur Eindämmung des moralischen Risikos dar, weil für den Versicherungsnehmer durch die finanzielle Partizipation am Schadengeschehen ein Anreiz zur Schadenverhütung besteht. ${ }^{1055}$

Im vorliegenden Fall werden die Auswirkungen einer Selbstbeteiligung in Höhe von $250 \mathrm{GE}$ untersucht. Um die Auswirkungen einer derartigen Maßnahme in einem Modell abbilden zu können, müssen in der Praxis hinreichend valide historische Informationen über den zugrunde liegenden Vertragsbestand vorliegen. Der nachfolgenden Analyse werden folgende vereinfachende Annahmen zugrunde gelegt:

- Die Einführung des Selbstbehalts kann zu Beginn des simulierten Anfalljahres durchgeführt werden und erstreckt sich auf sämtliche Policen der Verbundenen Wohngebäudeversicherung, die einen Gefahreneinschluss für Leitungswasserschäden aufweisen.

- Um einer verstärkten Stornoentwicklung entgegenzuwirken, fließen $75 \%$ der erwarteten Schadenentlastung im Wege einer Beitragssenkung an die Versicherungsnehmer zurück.

\footnotetext{
${ }^{1052}$ Vgl. im Folgenden grundlegend zur Wirkung der Selbstbeteiligung unter risikotheoretischen Aspekten STERK (1979) und STERK (1988), S. 775-780.

${ }^{1053} \mathrm{Vgl}$. zu weiteren Formen proportionaler und nicht-proportionaler Risikoteilung MACK (2002), S. 322-325.

${ }^{1054}$ Vgl. auch STERK (1988), S. 776.

${ }^{1055} \mathrm{Vgl}$. vertiefend zu Finanzverträgen und geeigneten Kooperationsdesigns bei asymmetrischer Information HARTMANN-Wendels/Pfingsten/Weber (2004), S. 94-103. Als wesentliche Problemkreise von Versicherungsverträgen mit Selbstbeteilung nennt MACK Inflationseinflüsse, die Gefahr einer Antiselektion und den Informationsverlust hinsichtlich der Kleinschäden. Vgl. vertiefend MACK (2002), S. 355-357.
} 
- Bezüglich der Kostenentwicklung wird unterstellt, dass die Provisionen variablen Charakter haben und sich demzufolge proportional zur Beitragsreduktion vermindern. Die Betriebskosten werden durch die Einführung der Selbstbeteiligung nicht tangiert. ${ }^{1056}$

- Annahmegemäß wurde durch eine Bestandsanalyse ermittelt, dass die Schäden unterhalb der Selbstbeteiligung in der Vergangenheit eine durchschnittliche Schadenhöhe $\mathrm{DS}^{<\mathrm{SB}}$ von $130 \mathrm{GE}$ aufgewiesen haben. ${ }^{1057}$ Der Anteil der Schäden oberhalb der Selbstbeteiligung betrug $\mathrm{s}=75 \%$.

Bei Gültigkeit dieses Prämissenrahmens ergibt sich der Endschadenbedarf $\mathrm{S}^{\mathrm{SB}}$ der Sparte „VGV Leitungswasser“ nach Einführung der Selbstbeteiligung als: ${ }^{1058}$

$$
\begin{aligned}
& \mathrm{S}^{\mathrm{SB}}=\mathrm{S}^{\mathrm{obneSB}}-\underbrace{\text { Schadenanzahl } \cdot\left[\mathrm{s} \cdot \mathrm{SB}+(1-\mathrm{s}) \cdot \mathrm{DS}^{<\mathrm{SB}}\right]}_{\text {Schadenentlastung }} \\
& \text { mit: } \quad \text { SB }=\text { Höhe der Selbstbeteiligung } \\
& \mathrm{S}^{\text {ohne SB }}=\text { Endschadenbedarf vor Einführung der SB } \\
& \text { s } \quad=\text { Anteil der Schäden über der SB [\%] } \\
& \mathrm{DS}^{<\mathrm{SB}}=\text { Durchschnittsschaden der Schäden unter der SB }
\end{aligned}
$$

Eine Simulation des beschriebenen Sachverhalts liefert eine erwartete Schadenentlastung in Höhe von 13,7 Mio. GE. ${ }^{1059}$ Dies entspricht 22,3\% des Endschadenbedarfs vor Einführung der Selbstbeteiligung. Im Zuge der Prämienreduzierung fließen 10,3 Mio. GE $(=75 \%)$ als Franchiserabatt an die Versicherungsnehmer zurück. Die Provisionen reduzieren sich proportional um 1,2 Mio. GE. In Summe resultiert somit aus der Einführung der Selbstbeteiligung eine Ergebnisverbesserung in Höhe von 4,6 Mio. GE, welche einen substanziellen Einfluss auf die in Tabelle 6.15 dargestellte RenditeRisiko-Position des Modellunternehmens ausübt.

\footnotetext{
${ }^{1056}$ Für den Fall, dass das Modellunternehmen durch die Einführung der Selbstbeteiligung eine signifikante Reduzierung der Betriebskosten, insbesondere der Schadenregulierungskosten erwartet, könnte diese zusätzliche Ergebniswirkung über einen Korrekturfaktor berücksichtigt werden.

${ }^{1057}$ Im hier gewählten Anwendungskontext des kollektiven Modells stellt die Modellierung der Schadenentlastung über die Parameter Schadenanzahl und Durchschnittsschaden eine gewisse Vereinfachung dar. Es wäre daher auch denkbar, den Entlastungseffekt des Selbstbehalts mit Hilfe von PML-Bändern zu modellieren.

${ }^{1058}$ Vgl. vertiefend STERK (1988), S. 776-779.

${ }^{1059}$ Vgl. zur Quantifizierung des Entlastungseffekts als Grundlage der Festsetzung von Franchiserabatten MACK (2002), S. 342-349 und zur Prämienkalkulation bei Abzugsfranchisen MACK (2002), S. 351-357.
} 


\begin{tabular}{|c|c|c|c|c|}
\hline \multirow{2}{*}{ Tsd. GE] } & \multicolumn{2}{|c|}{ ohne Selbstbeteiligung } & \multicolumn{2}{c|}{ mit Selbstbeteiligung (250 GE) } \\
\cline { 2 - 5 } & Brutto & Netto & Brutto & Netto \\
\hline AJ-Ergebnis (EW) & 16.369 & 9.257 & 21.063 & 13.959 \\
\hline davon: $V G V L W$ & -11.955 & -11.955 & -7.261 & -7.261 \\
\hline $\mathrm{CVaR}_{99,5}$ & 150.633 & 51.558 & 146.117 & 47.124 \\
\hline RORAC & $\mathbf{1 0 , 8 7 \%}$ & $\mathbf{1 7 , 9 5 \%}$ & $\mathbf{1 4 , 4 2} \%$ & $\mathbf{2 9 , 6 2 \%}$ \\
\hline
\end{tabular}

Tabelle 6.15: Einführung einer Selbstbeteiligung in der Sparte „VGV Leitungswasser“

Aufgrund der Tatsache, dass die Sparte „VGV Leitungswasser“ im Basisszenario ausnahmslos durch negative Erfolgsbeiträge und eine sehr geringe Ergebnisvolatilität charakterisiert ist, führt die durch die Selbstbeteiligung erzielte Ergebnisverbesserung von 4,7 Mio. GE zu einem Rückgang des Risikokapitalbedarfs in vergleichbarer Höhe. Insgesamt kann eine signifikante Verbesserung der RORAC-Kennziffern erreicht werden. Da das Spartenergebnis in „VGV Leitungswasser“ jedoch weiterhin defizitär ist, könnte die Einführung der Selbstbeteiligung durch zusätzliche Maßnahmen, insbesondere durch Leistungsausschlüsse, Schadenverhütungsauflagen ${ }^{1060}$ oder ein restriktiveres Schadenmanagement ${ }^{1061}$ flankiert werden.

Als alternative Maßnahme zur Sanierung der Leitungswassersparte könnte eine Prämienanhebung in Betracht kommen. Im Gegensatz zur oben beschriebenen Einführung einer Selbstbeteiligung stellt die Prämienerhöhung - nach Abzug der Provisionen - eine sichere Ergebniskomponente dar. Dieser Zusammenhang kann daher als Beispiel für das bereits in Kapitel 4.1.2 skizzierte Axiom der Translationsinvarianz angeführt werden, dem das hier verwendete Risikomaß des $\mathrm{CVaR}$ genügt. Wird eine zufallsabhängige Verlustvariable $\mathrm{V}$ um einen sicheren Betrag a vermindert, so ist ein translationsinvariantes Risikomaß durch die Eigenschaft charakterisiert, dass das Risikovolumen - hier operationalisiert durch den Risikokapitalbedarf - genau um diesen Betrag a sinkt. ${ }^{1062}$ Angesichts der oben beschriebenen Auswirkungen einer Selbstbeteiligung ist für das Modellunternehmen von Interesse, welchen Umfang die Prämienerhöhung annehmen müsste, um im Hinblick auf die Rendite-Risiko-Position einen äquivalenten Effekt zu erzielen. Es wird erneut davon ausgegangen, dass die Provisionen variablen Charakter haben und die Betriebskosten von der Prämienanhebung unberührt bleiben. Eine iterative Analyse (durch schrittweises Anheben der Durchschnittsprämie) kommt zu dem Ergebnis, dass das Modellunternehmen das Prämienniveau in der Sparte „VGV Leitungswasser“ um 8,0\% erhöhen müsste, um eine vergleichbare Ergebnisver-

${ }^{1060}$ Vgl. vertiefend auch LiebeLT-WeSTPHAL (1997), MIKOSCH (1991), S. 27-28 und EURICH (2000), S. 177-178.

${ }^{1061}$ Vgl. vertiefend COSTONIS/KNIPP (2000), DIRNBERGER/KNIPP (2003), DIRNBERGER/KNIPP (2004) und BERGER/POSTAI (2004).

${ }^{1062}$ Vgl. vertiefend ARTZNer/DelbaEn/EBer/HEATH (1999), Albrecht (2003), S. 13-14, MAURER (2000), S. 45-47, KORYCIORZ (2004), S. 41-42 sowie die Ausführungen in Kapitel 4.1.2. 
besserung zu erzielen. Diese Berechnung geht von der Prämisse aus, dass durch die Prämienanhebung kein zusätzliches Storno induziert wird, welches sich in der Verbundenen Wohngebäudeversicherung naturgemäß auf sämtliche versicherten Gefahren auswirken würde. Angesichts des ausgeprägten Sturmkumuls und der weiterhin defizitären Ergebnissituation in der Leitungswassersparte ist jedoch zu vermuten, dass sich eine stornobedingte Exposurereduzierung durchaus positiv auf die Risikoexponierung und die Ergebnissituation des Modellunternehmens auswirken könnte (,gewolltes Storno"). Welche Maßnahme letztlich zur Sanierung herangezogen wird, sollte nicht zuletzt auch unter Vertriebs- und Wettbewerbsaspekten diskutiert werden. Es wird jedoch deutlich, dass der entwickelte Modellansatz wichtige Unternehmensentscheidungen eines Versicherungsunternehmens hinsichtlich ihrer potenziellen Auswirkungen auf die Rendite-Risiko-Position quantifiziert und auf eine risikotheoretische Basis stellt. Auf diese Weise wird eine fundierte Entscheidungsfindung erheblich unterstützt.

\subsubsection{Ausbau des industriellen Feuergeschäfts}

Aufgrund der sehr hohen Versicherungswerte wird das Industrieversicherungsgeschäft zumeist in der Form der Mitversicherung betrieben. ${ }^{1063}$ Hierbei zeichnen verschiedene Erstversicherungsunternehmen Anteile an der Originalversicherungssumme bzw. am Original-PML. ${ }^{1064} \mathrm{Da}$ jeder Versicherer nur für seinen Anteil haftet, wird das Originalrisiko auf diese Weise im Markt atomisiert. In der Regel übt ein Unternehmen als Führender die aktive Vertretungsmacht für die übrigen Mitversicherer aus. In Deutschland ist die Industrielle Feuerversicherung seit vielen Jahren durch eine sehr volatile Ergebnisentwicklung gekennzeichnet. ${ }^{1065}$ Dies kann einerseits auf die hohe Anfälligkeit gegenüber Großschäden zurückgeführt werden. Andererseits erodierten in der Vergangenheit die Prämieneinnahmen aufgrund der transparenten, jedoch vielfach ruinösen Wettbewerbsstrukturen auf ein gänzlich unzureichendes Niveau. Als Ergebnis umfangreicher Sanierungsmaßnahmen, wie z.B. Prämien- und Selbstbehaltsanhebungen sowie restriktiver Schadenverhütungsauflagen, erwirtschaftete die Industrielle Feuerversicherung in Deutschland im Jahr 2003 wieder versicherungstechnische Gewinne und erreichte erstmalig auch nettoseitig die Gewinnschwelle. ${ }^{1066}$

Abbildung 6.27 zeigt die Entwicklung der versicherungstechnischen Brutto- und Nettoergebnisse im Zeitraum von 1987 bis 2003.

\footnotetext{
${ }^{1063}$ Vgl. im Folgenden auch KÜHL (1994), S. 34-41 und S. 60 ff. sowie FÜRSTENWERTH, VON/WEIß (2001), S. 440.

${ }^{1064} \mathrm{Vgl}$. zur Bestimmung der Bruttozeichnungsquote in der Industriellen Feuer- und Betriebsunterbrechungsversicherung KÜHL (1994), S. 116-120.

${ }^{1065} \mathrm{Vgl}$. im Folgenden KÜHL (1994), S. 1-6 und BUHK (2001), S. 898-902.

${ }^{1066}$ Vgl. GDV (2004), S. 106.
} 


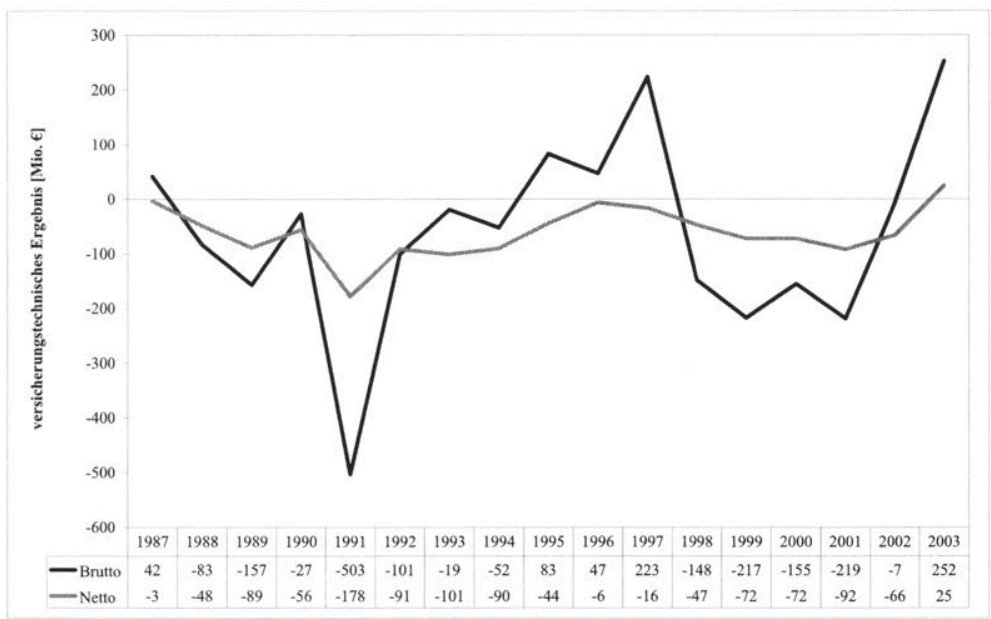

Abbildung 6.27: Versicherungstechnische Ergebnisse der Industriellen Feuerversicherung Quelle: GDV-Branchenstatistik, 2004

In Anbetracht dieser Marktentwicklung soll nunmehr exemplarisch für das Modellunternehmen die Fragestellung analysiert werden, ob bzw. unter welchen Voraussetzungen eine Ausweitung des industriellen Feuergeschäfts zu einer Verbesserung der Rendite-Risiko-Position beitragen könnte. Nachfolgend wird daher untersucht, welche Auswirkungen sich für die Rendite-Risiko-Position des Modellunternehmens ergeben, sofern dieses im Rahmen der bestehenden Geschäftsverbindungen seine Zeichnungsquoten um den Faktor 1,3 erhöht, mithin das Exposure in der Sparte „Feuer Industrie“ um 30\% ausweitet. Es wird von der Annahme ausgegangen, dass das Modellunternehmen aufgrund seiner bislang nur geringen Zeichnungsquoten auch nach der Geschäftsausweitung keine führende Rolle innerhalb eines Versicherungskonsortiums übernimmt und somit keinen unmittelbaren Einfluss auf die Geschäftspolitik des Konsortialführers nehmen kann. ${ }^{1067}$

Im Hinblick auf die Modellierung dieser Zeichnungsstrategie gilt es sich zunächst in Erinnerung zu rufen, dass die Sparte „Feuer Industrie“ über einen obligatorischen Summenexzedentenvertrag rückversichert ist. ${ }^{1068} \mathrm{Um}$ die homogenisierende Wirkung dieser Rückversicherungsdeckung adäquat abbilden zu können, erfolgt die Modellie-

${ }^{1067}$ Vgl. zur Zeichnungs- und Produktpolitik in der Industriellen Feuerversicherung vertiefend KÜHL (1994), S. 60-123.

${ }^{1068}$ Der Summenexzedentenvertrag stellt in der Industriellen Feuerversicherung die häufigste Form der Rückversicherung dar, da sich mit seiner Hilfe insbesondere Portfolios mit stark schwankenden PML-Beträgen nivellieren lassen. Vgl. auch LIEBWEIN (2000), S. 67 und KÜHL (1994), S. 120. 
rung der Einzelrisiken über so genannte PML-Bänder. ${ }^{1069}$ Analog zum Basisszenario liegt den nachfolgenden Ausführungen ein fiktiver Bestand industrieller Feuerrisiken zugrunde. Für jedes Einzelrisiko seien annahmegemäß die Höhe des Original-PML und der jeweilige Anteil des Modellunternehmens (= Zeichnungsquote) bekannt. Mit diesen Informationen kann eine Exposureausweitung realitätsnah abgebildet werden. Während die Anzahl der versicherten Risiken insgesamt unverändert bleibt, hat die Ausweitung der Zeichnungsquoten modelltechnisch zur Folge, dass einige Risiken in das nächst höhere PML-Band wechseln. Als Resultat ergeben sich für jedes Band neue durchschnittliche PML (DPML) und neue Durchschnittsprämien (DP). Die hierdurch bedingte Veränderung der Bestandsstruktur kann Tabelle 6.16 entnommen werden. ${ }^{1070}$

\begin{tabular}{|c|c|}
\hline $\begin{array}{c}\text { Band } \\
\text { Nr. }\end{array}$ & $\begin{array}{c}\text { Bandgrenze } \\
\text { [Tsd. GE] }\end{array}$ \\
\hline 1 & 1.500 \\
\hline 2 & 7.500 \\
\hline 3 & 13.500 \\
\hline 4 & 18.000 \\
\hline
\end{tabular}

\begin{tabular}{|c|c|c|}
\hline \multicolumn{3}{|c|}{ Basisszenario } \\
\hline JE & DPML & DP \\
\hline 272 & 700 & 0,857 \\
\hline 748 & 4.500 & 3,273 \\
\hline 1.092 & 10.500 & 8,735 \\
\hline- & - & - \\
\hline
\end{tabular}

\begin{tabular}{|c|c|c|}
\hline \multicolumn{3}{|c|}{ nach Erhöhung der Zeichnungsquoten } \\
\hline JE & DPML & DP \\
\hline 258 & 886 & 1,067 \\
\hline 723 & 5.590 & 4,103 \\
\hline 587 & 11.697 & 9,763 \\
\hline 544 & 15.227 & 12,710 \\
\hline
\end{tabular}

Tabelle 6.16: Bestandsstruktur „Feuer Industrie“ nach Erhöhung der Zeichnungsquoten [Tsd. GE]

Es wird deutlich, dass nach der Ausweitung der Zeichnungsquoten ein signifikanter Anteil der Risiken (JE) in das höchste PML-Band fällt. Die Anzahl der Risiken in den unteren Bändern reduziert sich zwangsläufig. Die Modellierung der Basisschadenlast erfolgt erneut über die Parameter ,durchschnittlicher Schadengrad“ und „durchschnittlicher PML“ (DPML). ${ }^{1071}$ Während sich der DPML in jedem Band durch die neue Bestandsstruktur erhöht, wird der Schadengrad als konstant angenommen. ${ }^{1072} \mathrm{Im}$ Bereich der Großschäden wird die aus dem Basisszenario bekannte Schadenanzahlverteilung nicht tangiert, da der Bestand keine zusätzlichen Risiken enthält, sondern lediglich ein höherer Anteil an jedem Einzelrisiko gezeichnet wird.

${ }^{1069}$ Vgl. hierzu auch Kapitel 5.4.3.2.1.

${ }^{1070}$ Es sei erneut betont, dass im Rahmen dieser Arbeit bereits Unternehmen mit mehr als 50 Mitarbeitern oder einem Jahresumsatz von mindestens 5 Mio. GE als Industriekunden klassifiziert werden. Dennoch erreichen die versicherten Einzelrisiken im Maximum eine Größenordnung von $1 \mathrm{Mrd}$. GE (Original-PML). Die aus Tabelle 6.16 ersichtlichen durchschnittlichen PML können durch die niedrigen Zeichnungsquoten des Modellunternehmens erklärt werden, die durchschnittlich nur 3\% des Original-PML betragen. Zur Abgrenzung von Spitzenrisiken, Großrisiken, industriellen und gewerblichen Risiken vgl. KÜHL (1994), S. 36-41.

1071 Vgl. zur Modellierung der Basisschadenlast in PML-Sparten auch Kapitel 5.4.3.2.

${ }^{1072}$ Der Schadengrad ist als Quotient aus erwartetem Schadenaufwand und zugehörigem PML definiert. Da im vorliegenden Fall der PML für jedes Einzelrisiko um den Faktor 1,3 ansteigt, impliziert die Annahme eines konstanten Schadengrads, dass sich der erwartete Schadenaufwand ebenfalls um diesen Faktor erhöht. 
Die Verteilungscharakteristik der Schadenhöhen bleibt ebenfalls unverändert, jedoch wird jede simulierte Schadenhöhe mit dem Faktor 1,3 multipliziert. Bezüglich der Kostenentwicklung wird unterstellt, dass die Geschäftsausweitung lediglich eine Erhöhung der Provisionszahlungen an die Vermittler induziert. ${ }^{1073} \mathrm{Da}$ im Rahmen der Zeichnungsstrategie keine zusätzlichen Risiken in das Portfolio aufgenommen werden und die Schadenregulierung annahmegemäß durch den Konsortialführer koordiniert wird, bleiben die Betriebskosten gegenüber dem Basisszenario unverändert. Tabelle 6.17 verdeutlicht die Auswirkungen der skizzierten Zeichnungsstrategie auf die Rendite-Risiko-Position des Modellunternehmens.

\begin{tabular}{|c|c|c|}
\hline [Tsd. GE] & Basisszenario & nach Erhöhung \\
\hline Bruttoergebnis (EW) & 16.369 & 16.624 \\
\hline CVaR $_{99,5}$ brutto & 150.633 & 150.645 \\
\hline RORAC brutto & $\mathbf{1 0 , 8 7 \%}$ & $\mathbf{1 1 , 0 4 \%}$ \\
\hline
\end{tabular}

Tabelle 6.17: Auswirkung der erhöhten Zeichnungsquoten auf die Rendite-Risiko-Position

Das erwartete Bruttoergebnis steigt im Vergleich zum Basisszenario leicht an. Als wesentlicher Grund für die Ergebnisverbesserung kann der Degressionseffekt in Bezug auf die Betriebskosten angeführt werden. Würden die Betriebskosten hingegen proportional zum Prämienvolumen ansteigen, hätte dies eine Verschlechterung des Unternehmensergebnisses zur Folge. Die Prämienerhöhung von 30\% würde in diesem Fall durch zusätzliche Provisionen und Betriebskosten sowie eine signifikant ansteigende Gesamtschadenlast überkompensiert. ${ }^{1074}$ Tabelle 6.17 zeigt darüber hinaus, dass der Risikokapitalbedarf des Gesamtunternehmens trotz einer beträchtlichen Ausweitung des Exposures im industriellen Feuergeschäft nahezu unverändert bleibt. Dieses Phänomen kann im quantitativen Modellkontext erneut durch die Tail-Dominanz der Naturereignisschäden begründet werden. Eine ökonomische Interpretation dieses Sachverhalts führt zu der Erkenntnis, dass das im Basisszenario erforderliche BruttoRisikokapital offenbar ausreicht, um potenzielle Verluste, die aus dem zusätzlich gezeichneten Feuergeschäft erwachsen, mit dem vorgegebenen Sicherheitsniveau von $99,5 \%$ zu decken. ${ }^{1075}$

\footnotetext{
${ }^{1073}$ Im Kontext der Industriellen Feuerversicherung übernimmt der Versicherungsvermittler über die reine Vermittlungstätigkeit hinaus auch zusätzliche Aufgaben im Bereich der Risikoerfassung, Tarifierung, Platzierung, Schadenverhütung und Schadenabwicklung. Vgl. vertiefend KÜHL (1994), S. 41-46 und MiKOSCH (1991), S. 45-48.

${ }^{1074}$ Für den Fall, dass das Prämienniveau im Rahmen der Geschäftsausweitung nicht beibehalten werden kann, würde sich eine substanzielle Verschlechterung des Sparten- und Unternehmensergebnisses ergeben.

${ }^{1075}$ Dieser Interpretation liegt erneut die Annahme zugrunde, dass die Basis- und Großschäden sämtlicher Versicherungszweige stochastisch unabhängig sind. Vgl. in diesem Zusammenhang ausführlich Kapitel 6.2.2.
} 
Wird die Industrielle Feuerversicherung dahingegen isoliert als „Standalone-Unternehmen“ betrachtet, tritt das bislang durch Naturereignisschäden überlagerte Gefährdungspotenzial deutlich zu Tage. Die Erhöhung der Zeichnungsquoten führt dann zu einer deutlichen Zunahme des Bruttokapitalbedarfs von 20,6 Mio. GE auf 25,8 Mio. GE. Offenbar profitiert der Versicherungszweig „Feuer Industrie“ im Portfoliokontext des Gesamtunternehmens von einem erheblichen Diversifikationseffekt.

Mit Blick auf die Rückversicherungsstruktur der Sparte „Feuer Industrie“ wirft diese Beobachtung die Frage auf, bis zu welchem Grad das Modellunternehmen diesen Diversifikationseffekt, den gewöhnlich der Rückversicherer nutzt, für sich selber einsetzen kann, und ob durch eine Anhebung des im Basisszenario gewählten Selbstbehalts (SB) von 1,5 Mio. GE gegebenenfalls die Rendite-Risiko-Position des Gesamtunternehmens verbessert werden könnte. ${ }^{1076}$ Der ohnehin niedrig gewählte Selbstbehalt des Summenexzedentenvertrages würde nach Ausweitung der Zeichnungsquoten vermutlich noch wesentlich häufiger überschritten werden, was zu einer signifikanten Verteuerung des Rückversicherungsschutzes führen würde. Nachfolgend werden daher unterschiedliche Haftungsstrukturen des Summenexzedentenvertrages in Bezug auf den resultierenden Nettokapitalbedarfs des Gesamtunternehmens verglichen. Angesichts der erhöhten Zeichnungsquoten im Versicherungszweig „Feuer Industrie“ wird die Haftstrecke für alle betrachteten Varianten von 13,5 Mio. GE auf 18 Mio. GE erhöht.

\begin{tabular}{|c|c|c|c|c|}
\hline [Tsd. GE] & $\begin{array}{c}\text { SB 1,5 Mio. GE } \\
\text { 11 Maxima }\end{array}$ & $\begin{array}{c}\text { SB 3 Mio. GE } \\
\text { 5 Maxima }\end{array}$ & $\begin{array}{c}\text { SB 6 Mio. GE } \\
\text { 2 Maxima }\end{array}$ & $\begin{array}{c}\text { SB 9 Mio. GE } \\
\text { 1 Maximum }\end{array}$ \\
\hline Nettoergebnis (EW) & 9.315 & 9.424 & 9.624 & 9.784 \\
\hline CVaR $_{99,5}$ netto & 51.807 & 51.823 & 52.090 & 52.285 \\
\hline RORAC netto & $\mathbf{1 7 , 9 8 \%}$ & $\mathbf{1 8 , 1 9 \%}$ & $\mathbf{1 8 , 4 8} \%$ & $\mathbf{1 8 , 7 1 \%}$ \\
\hline
\end{tabular}

Tabelle 6.18: Auswirkungen alternativer Rückversicherungsstrukturen für „Feuer Industrie“

Mit ansteigendem Selbstbehalt verbessert sich das erwartete Nettoergebnis sukzessive, da die eingesparte Rückversicherungsprämie die erhöhte Schadenerwartung überkompensiert. Analog zur Bruttoperspektive üben Großschadenereignisse der Sparte „Feuer Industrie“ im Diversifikationsumfeld des Gesamtunternehmens auch nach Rückversicherungsnahme nur einen geringen Einfluss auf den Kapitalbedarf aus. Auch im Falle hoher Selbstbehalte ist auf der Gesellschaftsebene lediglich ein unterproportionaler Anstieg des Kapitalbedarfs festzustellen, so dass sich der Netto-RORAC leicht verbessert. Die Ergebnisse aus Tabelle 6.18 sind jedoch ausdrücklich im spezifischen Kon-

${ }^{1076}$ Vgl. auch LEYHERR/SCULLY/SOMMERFELD (2003), S. 1795-1797, die in diesem Zusammenhang zutreffend formulieren: „Je größer die Diversifikation einer Sparte mit den anderen Sparten, desto weniger Rückversicherung wird benötigt, da dann Rückversicherung eher das Spartenergebnis als das Gesamtergebnis und somit das Kapital schützt.“ LEYHERR/SCULLY/SOMMERFELD (2003), S. 1796. 
text des Modellunternehmens zu bewerten. Eine Ausweitung des Exposures im industriellen Feuergeschäft um 30\% würde vermutlich in Schadenportfolios mit anderer Risikostruktur (insbesondere geringerer Exponierung gegenüber Naturgefahren) zu abweichenden Effekten führen. Wenngleich sich der Nettokapitalbedarf des Gesamtunternehmens mit steigenden Eigenbehalten nur geringfügig erhöht, tritt die „Gefährlichkeit" einer gekoppelten Strategie aus Exposureausweitung und progressiver Rückversicherungsstruktur umso deutlicher zu Tage, sofern die Sparte „Feuer Industrie“ isoliert betrachtet wird. In Abbildung 6.28 wird für die bekannten Vertragsvarianten jeweils der isoliert ermittelte Nettokapitalbedarf der Sparte „Feuer Industrie“ dem korrespondierenden diversifizierten Kapitalbedarf dieser Sparte gegenübergestellt.

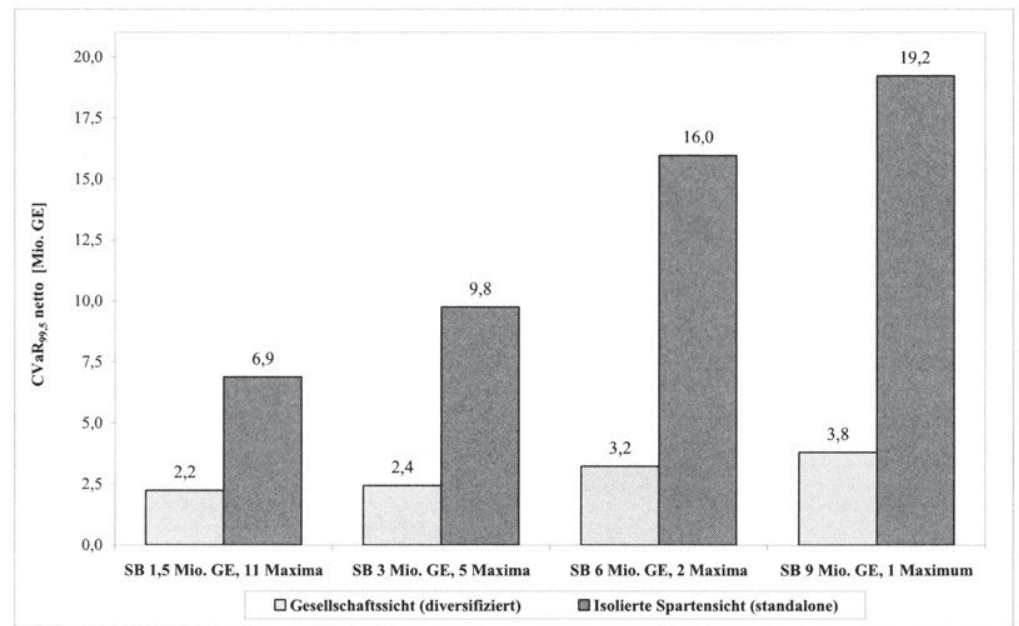

Abbildung 6.28: Auswirkungen alternativer RV-Strukturen für die Sparte „Feuer Industrie“

Im Portfoliozusammenhang zeigt sich, dass der Kapitalbedarf der Sparte „Feuer Industrie" mit wachsendem Eigenbehalt nur geringfügig von 2,2 Mio. GE auf 3,8 Mio. GE zunimmt. Demgegenüber kommt es in der Standalone-Betrachtung annähernd zu einer Verdreifachung des Kapitalbedarfs. Die Sparte „Feuer Industrie“ profitiert an dieser Stelle also - eher zufällig - von einem erheblichen Diversifikationseffekt des Gesamtportfolios, welcher im Rahmen der Kapitalallokation auf die Teilkollektive (hier: Sparten) verteilt wird. Eine Steuerung auf der Basis von spartenspezifischen RORAC-Kennziffern würde somit die Gefahr bergen, das originäre Risikopotenzial des Versicherungszweiges „Feuer Industrie“ zu unterschätzen. Dies verdeutlicht erneut, dass die Vorteilhaftigkeit einer Strategie stets im Wirkungsgefüge des Gesamtportfolios zu beurteilen ist. 
Die Ableitung praktischer Handlungsempfehlungen stößt an dieser Stelle jedoch an Grenzen, da den diskutierten Ergebnissen unverändert eine „technische“ Preisermittlung für den Summenexzedentenvertrag auf Basis des CVaR-Ansatzes zugrunde liegt. Zudem werden qualitative Aspekte der Rückversicherungsnahme nicht abgebildet. Welche Haftungsstruktur letztlich durch ein Versicherungsunternehmen gewählt werden sollte, hängt in der Praxis insbesondere von der Prämienforderung des Rückversicherers sowie der vorherrschenden Wettbewerbs- und Kapazitätssituation ab. Die Festlegung von Zeichnungsquoten sollte im Rahmen expansiver (Markterschließungs-) Strategien stets in enger Abstimmung mit den involvierten Rückversicherern erfolgen. Darüber hinaus kann festgehalten werden, dass strategische Entscheidungen über Rückversicherungsstrukturen nicht aus einer isolierten Sparten- oder Geschäftsbereichsperspektive, sondern stets im Kontext des Gesamtportfolios getroffen werden sollten. ${ }^{1077}$ Dies unterstreicht die zu Beginn des Kapitels formulierte Forderung, strategische Rückversicherungsentscheidungen stets im Aufgabengebiet der zentralen Struktursteuerung anzusiedeln. ${ }^{1078}$ Interne Risikomodelle leisten in diesem Zusammenhang einen risikotheoretisch fundierten Beitrag zur Entscheidungsunterstützung.

\subsection{Bilanzebene}

\subsubsection{Vorbemerkungen}

In den vorangegangenen Kapiteln wurde die Rendite-Risiko-Position des Modellunternehmens durchweg unter betriebswirtschaftlichen Steuerungsaspekten betrachtet. Der Analyse unterschiedlicher Rückversicherungs- und Zeichnungsstrategien lag demzufolge immer der zeitliche Horizont eines Anfall- bzw. Zeichnungsjahres zugrunde. In Kapitel 6.3 wird diese „Steuerungsebene“ verlassen und der wirtschaftliche Einfluss vorangegangener Anfalljahres explizit in die Betrachtung integriert. Auf diese Weise wird ein Übergang auf die handelsrechtliche „Bilanzebene“ ermöglicht. Wenngleich die vorliegende Arbeit vorrangig Fragestellungen der risikoorientierten Steuerung fokussiert, stellt die bilanzielle Sicht auf ein Versicherungsunternehmen dennoch eine "strenge Nebenbedingung" dar. Nachfolgend wird von der realistischen Annahme ausgegangen, dass das Modellunternehmen bereits seit einigen Jahren am Markt agiert. Die Erfolgsrechnung wird daher nicht nur von Schadenereignissen des simulierten Zeichnungsjahres, sondern auch von Schäden vorangegangener Zeichnungsjahre beeinflusst. Auf der Bilanzebene wird die Betrachtung um eben diesen Einfluss erweitert, indem für jeden modellierten Versicherungszweig die Höhe der Schadenrückstellung zu Beginn des simulierten Geschäftsjahres (Eingangsreserve) und ihre Abwicklung im Geschäftsjahr in die Analyse einbezogen wird.

\footnotetext{
${ }^{1077}$ Vgl. übereinstimmend LEYHERR/SCULLY/SOMMERFELD (2003), S. 1796.

${ }^{1078}$ Vgl. hierzu auch die Ausfuhrungen in Kapitel 6.1.
} 
Die Höhe der Brutto- und Netto-Eingangsreserven kann Tabelle 6.19 entnommen werden. ${ }^{1079}$ Wie bereits in Kapitel 5.3.2 erläutert, wurden die Schadenrückstellungen auf ein reduziertes Niveau skaliert, um bilanzielle Rückschlüsse auf das zugrunde liegende Originalunternehmen auszuschließen. Die Struktur der Schadenrückstellungen kann jedoch weiterhin als marktüblich bezeichnet werden.

\begin{tabular}{|c|c|c|c|c|c|c|c|c|c|c|}
\hline [Tsd. GE] & AU & KH & Kasko & FIND & AST & VGV F & VGV LW & VGV ST & VGV EL & SUMME \\
\hline Brutto-Eingangsreserve & 26.912 & 201.102 & 11.646 & 4.727 & 646 & 11.406 & 4.180 & 2.458 & 116 & $\mathbf{2 6 3 . 1 9 4}$ \\
\hline Netto-Eingangsreserve & 17.595 & 175.596 & 11.050 & 1.097 & 93 & 9.999 & 3.543 & 329 & 114 & $\mathbf{2 1 9 . 4 1 5}$ \\
\hline
\end{tabular}

Tabelle 6.19: Netto-Eingangsreserven nach Versicherungszweigen

Aufgrund der besonderen Schadencharakteristik und zumeist langen Abwicklungsdauer bieten HUK-Sparten grundsätzlich die Möglichkeit eines systematischen Aufbaus bilanzieller Haftungsmasse in Form von Schadenrückstellungen. ${ }^{1080}$ Wie in der Versicherungspraxis üblich, entfällt auch im Modellunternehmen ein wesentlicher Anteil der Schadenrückstellungen auf die HUK-Sparten, vor allem auf die Sparte „KraftfahrtHaftpflicht“ (vgl. Tabelle 6.19). Aufgrund des zumeist kürzeren Regulierungszeitraums sind in den Sachversicherungssparten in der Regel deutlich geringere Rückstellungsniveaus zur Bedeckung zukünftiger Auszahlungsverpflichtungen ausreichend. Unbeschadet der Tatsache, dass im Rahmen dieser Arbeit lediglich ausgewählte Versicherungszweige betrachtet werden und insbesondere die in der Regel stark reservierten Sparten „Allgemeine Haftpflicht“ und „Rechtsschutz“ nicht modelliert sind, kann das Verhältnis der Brutto-Schadenrückstellungen zum Bruttoprämienvolumen mit $49 \%$ als unterdurchschnittlich bezeichnet werden. ${ }^{1081}$ Für die Zwecke dieser Arbeit ist jedoch das absolute Niveau der Schadenrückstellungen zunächst von nachgelagerter Bedeutung, da vorrangig strukturelle Effekte analysiert und die zugrunde liegende Ergebnisund Bilanzmechanik offen gelegt werden sollen. Die Bilanz stellt zudem keinen aktiven Parameter der Rendite-Risikosteuerung dar.

Es muss außerdem betont werden, dass die nachfolgend analysierte Bilanz- und Ergebnisstruktur auf der Grundlage einer deterministischen Abwicklung, d.h. unter der

${ }^{1079}$ Auf eine detaillierte Aufspaltung der Eingangsreserven in die zugrunde liegenden Zeichnungsjahre wird an dieser Stelle im Interesse einer übersichtlicheren Darstellung verzichtet. Für die modelltechnische Abwicklung der Vorjahresschadenrückstellungen sind diese Informationen hingegen unverzichtbar.

${ }^{1080}$ Vgl. im Folgenden auch MACK (2002), S. 221-223 und OECKING/SANNER (2004), S. 25-36.

${ }^{1081}$ Lt. BaFin-Geschäftsbericht betrug das Verhältnis von Brutto-Schadenrückstellungen zu verdienten Bruttoprämien für die hier betrachteten 9 Versicherungszweige im Jahr 2003 ca. 113,1\%. Einschränkend ist bei diesem Vergleich jedoch zu berücksichtigen, dass in den Brutto-Schadenrückstellungen die Renten-Deckungsrückstellung und die Rückstellung für noch nicht abgewickelte Rückkäufe, Rückgewährbeiträge und Austrittsvergütungen enthalten sind. Vgl. BAFIN (2005), Tab. 531 und Tab. 541. 
Fiktion nicht schwankender Abwicklungsmuster ermittelt wird. Das Reserverisiko, verstanden als Unsicherheit in Bezug auf die Auszahlungsgeschwindigkeit und Auskömmlichkeit von Schadenrückstellungen vorangegangener Zeichnungsjahre, wird demzufolge nicht abgebildet. ${ }^{1082}$ Ferner ist mit dieser Vorgehensweise die Einschränkung verbunden, dass auch in extrem schlechten Schaden- oder Kapitalmarktszenarien eine unverändert hohe Dotierung der versicherungstechnischen Rückstellungen erfolgt. Dies entspricht jedoch nur eingeschränkt der betrieblichen Praxis, da in diesen Fällen im Interesse eines verbesserten Gesamtergebnisses wahrscheinlich stille Reserven in den Schadenrückstellungen in Form höherer Abwicklungsergebnisse oder Bewertungsreserven in den Kapitalanlagen realisiert würden. Einschränkend ist außerdem zu berücksichtigen, dass die Schwankungsrückstellung im vorliegenden Modell nicht abgebildet wird. ${ }^{1083}$ Die Volatilität des bilanziellen Jahresergebnisses fällt daher höher aus und schlägt unmittelbar auf das Eigenkapital durch. ${ }^{1084}$

Im weiteren Verlauf der Analyse wird stark vereinfachend ein pauschaler Ertragssteuersatz von $50 \%$ unterstellt. Im Falle eines negativen Jahresergebnisses fallen annahmegemäß keine Steuern an. ${ }^{1085}$ Von sonstigen Belastungen in Form von Substanz-, Verbrauchs- oder Verkehrssteuern wird zudem abstrahiert. Um die komplexe Systematik des deutschen Steuerrechts angemessen zu berücksichtigen, sollte in der Praxis ein geeignetes Modul zur Steuerberechnung in das Gesamtmodell integriert werden. Im derzeitigen steuerrechtlichen Kontext der Schaden- und Unfallversicherung sollte dieses insbesondere die differenzierte steuerliche Behandlung von Kapitalanlageerträgen, den Ausgleichsalgorithmus der Schwankungsrückstellungen sowie die realitätsnähere Bewertung von Schadenrückstellungen unterstützen. ${ }^{1086}$ Nachfolgend wird außerdem

\footnotetext{
${ }^{1082} \mathrm{Vgl}$. hierzu auch die Ausführungen in Kapitel 5.4.6.

${ }^{1083}$ Aufgrund des in $\S 29$ sowie Anlage zu $\S 29$ RechVersV formulierten mathematischen Algorithmus ist eine Implementierung jedoch mit vertretbarem Aufwand möglich.

${ }^{1084}$ Durch die Einführung der International Financial Reporting Standards (IFRS) fällt die Möglichkeit zur Bildung einer Schwankungs- bzw. Katastrophenrückstellung für kapitalmarktorientierte Versicherungsunternehmen ab dem Geschäftsjahr 2005 weg. Für Unternehmen, die lediglich Schuldtitel zum Handel am geregelten Markt zugelassen haben oder Konzernabschlüsse nach anderen international anerkannten Standards veröffentlichen, besteht erst ab 2007 eine Umstellungspflicht auf IFRS. Bestehende Schwankungsrückstellungen müssen bis zum 31.12.2005 in das Eigenkapital überführt werden. In Anbetracht dieser Entwicklung ist die Vernachlässigung der Schwankungsrückstellung im vorliegenden Modellansatz ebenso vertretbar wie realistisch. Vgl. auch SwISS RE (2004), S. 14-23.

${ }^{1085}$ Im Kontext eines mehrperiodigen Modells wäre es sinnvoll, für negative Jahresergebnisse einen Steuererstattungsanspruch zu modellieren. Diese Vorgehensweise setzt jedoch voraus, dass die Möglichkeit zur Bildung steuerlicher Verlustvorträge gemäß § $10 \mathrm{~d}$ EStG i.V.m. § 8 Abs. 4 KStG besteht.
}

${ }^{1086}$ Vgl. hierzu vertiefend OECKING/SANNER (2004), S. 30. Die Rechtsgrundlage zur realitätsnäheren Bewertung der Schadenrückstellung ergibt sich aus $\S 6$ Abs. 1 Nr. 3a Buchstabe a EStG i.V.m. $\S 20$ Abs. $2 \mathrm{KStG}$. 
unterstellt, dass die Anteilseigner des Modellunternehmens eine konstante Gewinnausschüttung in Höhe von 3 Mio. GE erhalten. Dies entspricht einer Dividendenrendite von 3\% auf das gezeichnete Kapital. Tabelle 6.20 zeigt die vereinfachte Bilanzstruktur des Modellunternehmens zu Beginn des simulierten Geschäftsjahres. Sowohl die Höhe des gezeichneten Kapitals als auch die strukturelle Verteilung der Aktiva auf die drei Hauptanlageklassen wurden zum Zwecke dieser Arbeit exogen vorgegeben.

\begin{tabular}{|lrc|lrc|}
\hline Aktiva & [Tsd. GE] & {$[\%]$} & Passiva & [Tsd. GE] & {$[\%]$} \\
\hline Aktien & 63.883 & 20,0 & Gezeichnetes Kapital & 100.000 & 31,3 \\
Anleihen & 239.561 & 75,0 & Schadenrückstellungen & 219.415 & 68,7 \\
Geldmarkt & 15.971 & 5,0 & & & \\
\hline Summe & $\mathbf{3 1 9 . 4 1 5}$ & $\mathbf{1 0 0 , 0}$ & Summe & $\mathbf{3 1 9 . 4 1 5}$ & $\mathbf{1 0 0 , 0}$ \\
\hline
\end{tabular}

Tabelle 6.20: Vereinfachte Eröffnungsbilanz

Hinsichtlich derjenigen Haftungsmassen, welche nicht unmittelbar aus der Bilanz abgelesen werden können, werden folgende Annahmen getroffen:

- Zu Beginn der Modellperiode liegt der Marktwert des Aktienbestandes annahmegemäß um 15\% über dem Buchwert. Es ergeben sich mithin Bewertungsreserven in Höhe von 9,6 Mio. GE. Demgegenüber verfügen der Anleihe- und Geldmarktbestand anfänglich über keine Bewertungsreserven.

- Die stillen Reserven in den Schadenrückstellungen, verstanden als Differenz zwischen der bilanziellen Nettoschadenrückstellung und dem jeweils korrespondierenden Endschadenbedarf jedes vorangegangenen (und noch nicht vollständig abgewickelten) Zeichnungsjahres, betragen insgesamt 60,4 Mio. GE.

Neben dem bilanziellen Eigenkapital und den nicht zur Deckung von Provisionen und Betriebskosten benötigten Prämien steht dem Modellunternehmen somit zusätzliches Sicherheitskapital in Höhe von 70,0 Mio. GE zur Verfügung. ${ }^{1087}$

Im nachfolgenden Abschnitt wird zunächst das Basisszenario hinsichtlich seiner bilanziellen Auswirkung dargestellt und analysiert. Daran anschließend werden in Kapitel 6.3.3 ausgewählte Zeichnungs- und Rückversicherungsstrategien unter der Fragestellung beleuchtet, ob die auf der Steuerungsebene als vorteilhaft identifizierten Strategien auch bilanziell zulässige Ergebnisse generieren. Als Beurteilungskriterium werden in diesem Kontext der handelsrechtliche Jahresüberschuss respektive die Veränderung des Eigenkapitals herangezogen.

\footnotetext{
${ }^{1087}$ Vgl. zur Funktion und zu den Komponenten des ökonomischen Sicherheitskapitals detailliert Kapitel 2.3 sowie KORYCIORZ (2004), S. 15-17, FARNY (2000), S. 425 und SCHRADIN (1998), S. $193-198$.
} 


\subsubsection{Bilanzielle Darstellung des Basisszenarios}

Als Ausgangspunkt der Analyse dient zunächst die Gewinn- und Verlustrechnung des simulierten Geschäftsjahres. Unter der Annahme, dass der versicherungstechnische Netto Cashflow ausschließlich in Geldmarktpapiere investiert wird, ergibt sich für das Modellunternehmen im Basisszenario die aus Tabelle 6.21 ersichtliche Ergebnisrechnung. Die Darstellung erfolgt in einem ersten Schritt auf der Grundlage von Erwartungswerten.

\begin{tabular}{|lr|}
\hline Gewinn- und Verlustrechnung & EW in [Tsd. GE] \\
\hline Bruttoprämien & 534.750 \\
Bruttoaufwendungen für GJ-Versicherungsfälle & -441.510 \\
davon: Zahlungen für GJ-Versicherungsfälle brutto & -296.167 \\
Dotierung der Rst. für GJ-Versicherungsfälle brutto & -145.343 \\
Bruttoabwicklungsergebnis & 17.001 \\
davon: Zahlungen für VJ-Versicherungsfälle brutto & -96.593 \\
Veränderung der Rst. für VJ-Versicherungsfälle brutto & 113.594 \\
Bruttoaufwendungen für den Versicherungsbetrieb & -105.277 \\
\hline = versicherungstechnisches Bruttoergebnis & 4.964 \\
Rückversicherungsergebnis & -6.516 \\
\hline = vt. Nettoergebnis (vor Schwankungsrückstellung) & $\mathbf{- 1 . 5 5 2}$ \\
Kapitalanlageergebnis & 13.280 \\
\hline = Jahresüberschuss vor Steuern & $\mathbf{1 1 . 7 2 8}$ \\
Steuern & -6.753 \\
\hline = Jahresüberschuss nach Steuern & $\mathbf{4 . 9 7 5}$ \\
Ausschüttung an Anteilseigner & -3.000 \\
\hline = Gesamtergebnis nach Ausschüttung & $\mathbf{1 . 9 7 5}$ \\
\hline
\end{tabular}

Tabelle 6.21: Gewinn- und Verlustrechnung im Basisszenario

Das versicherungstechnische Bruttogeschäft verläuft in der bilanziellen Betrachtung im Erwartungswert profitabel, wird jedoch durch das negative Rückversicherungsergebnis überkompensiert. Das demzufolge leicht negative Nettoergebnis wird aber durch das Ergebnis aus Kapitalanlagen mehr als ausgeglichen. Nach Abzug von Steuern $^{1088}$ verbleibt im Erwartungswert ein Jahresüberschuss in Höhe von 5,0 Mio. GE, von dem 3,0 Mio. GE als Dividende an die Anteilseigner ausgeschüttet werden. Es wurde bereits darauf hingewiesen, dass das versicherungstechnische Ergebnis im Wege einer Überleitungsrechnung aus dem Anfalljahrergebnis ermittelt werden kann, indem die Veränderung der stillen Reserven in den Schadenrückstellungen in die $\mathrm{Be}$ trachtung einbezogen wird. Das bereits in Kapitel 5.4.7.2 skizzierte Überleitungsschema wird nachfolgend exemplarisch für das versicherungstechnische Bruttogeschäft dargestellt.

${ }^{1088}$ Die in Tabelle 6.21 ausgewiesene Steuerlast entspricht im Erwartungswert nicht 50\% des erwarteten Jahresüberschusses vor Steuern. Dies ist darauf zurückzuführen, dass annahmegemäß nur positive Jahresergebnisse der Besteuerung unterliegen und Jahresfehlbeträge keinen Steuererstattungsanspruch auslösen. 


\begin{tabular}{|c|lr|}
\hline Pos. & Überleitungsrechnung & EW in [Tsd. GE] \\
\hline$(1)$ & Anfalljahresergebnis ${ }^{\text {SimAJ }}$ brutto & 16.369 \\
$(2)$ & - Dotierung der Rst. für GJ-Versicherungsfälle brutto ${ }^{\text {SimAJ }}$ brutto & 145.343 \\
$(3)$ & + Bedarfsreserve $^{\text {SimAJ }}$ SRK brutto & 116.489 \\
$(4)$ & + Bedarfsreserve & 3.806 \\
$(5)$ & - kalkulat. Zinsen aus der Anlage der Bedarfsreserve $^{\text {SimAJ }}$ & 3.358 \\
$(6)$ & + Bruttoabwicklungsergebnis & 17.001 \\
\hline$(7)$ & $=$ versicherungstechnisches Bruttoergebnis & SimGJ \\
\hline
\end{tabular}

Tabelle 6.22: Überleitungsrechnung für das versicherungstechnische Bruttogeschäft

Die Überleitungsrechnung macht transparent, dass im simulierten Geschäftsjahr im Bereich der GJ-Versicherungsfälle insgesamt neue stille Reserven in Höhe von 25,0 Mio. GE gebildet werden. Dies entspricht dem Saldo der Positionen (2) bis (4). Demgegenüber wurden in den Schadenrückstellungen, die vorangegangene Zeichnungsjahre betreffen, stille Reserven in Höhe von 17,0 Mio. GE als Abwicklungsgewinn aufgelöst. Im Saldo verbleibt eine Erhöhung von 8,0 Mio. GE, die unter Vernachlässigung der kalkulatorischen Zinserträge gerade der Differenz zwischen Anfalljahrergebnis und versicherungstechnischem Ergebnis entspricht.

Die bisherige - in Erwartungswerten formulierte - Darstellung lässt noch keine Aussagen über die Schwankungsbreite oder die Eintrittswahrscheinlichkeit der bilanziellen Ergebnisse zu. Die weitere Analyse erfolgt daher durchgängig auf der Basis von Ergebnisverteilungen und bedient sich der bereits bekannten Darstellungsform des Perzentilgraphen. Zunächst werden in Abbildung 6.29 die Verteilungen des versicherungstechnischen Brutto- und Nettoergebnisses miteinander verglichen.

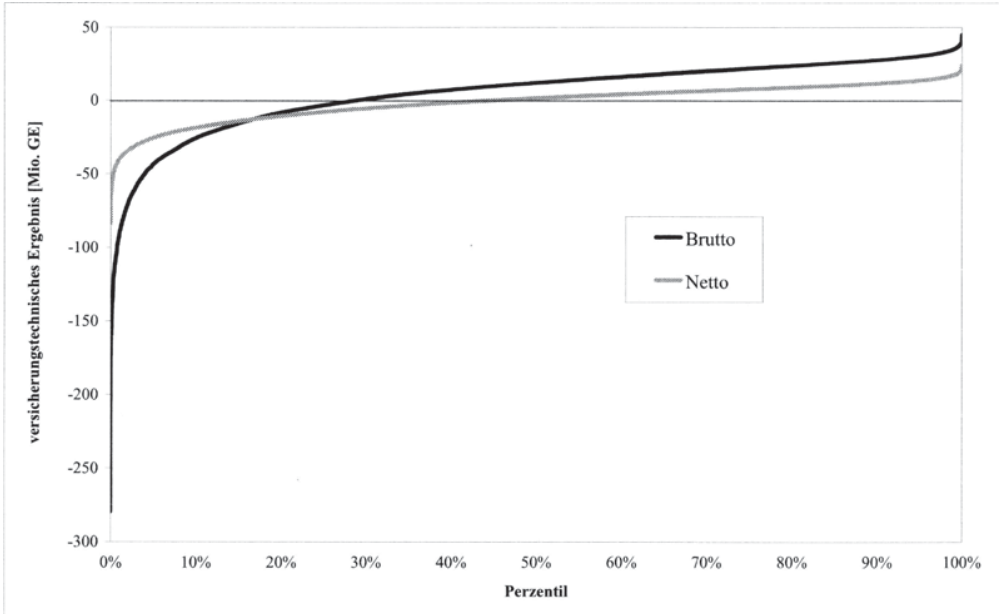

Abbildung 6.29: Versicherungstechnisches Ergebnis (Brutto vs. Netto) 
Es wird deutlich, dass die Rückversicherungsnahme auch in der bilanziellen Betrachtung zu einer signifikanten Reduzierung der Ergebnisvolatilität beiträgt. Das versicherungstechnische Bruttoergebnis weist im Minimum einen Verlust in Höhe von $-279,6$ Mio. GE aus. Demgegenüber beträgt der maximale Nettoverlust lediglich -83,8 Mio. GE. Allerdings wird auch deutlich, dass die Eintrittswahrscheinlichkeit negativer Brutto- bzw. Nettoergebnisse gegenüber der Anfalljahresbetrachtung zugenommen hat. Dieser Effekt ist vorrangig darauf zurückzuführen, dass das Modell auch in katastrophalen Schadenszenarien eine unverminderte (Über-) Dotierung von Schadenrückstellungen vorsieht und außerdem der Ausgleichsmechanismus der Schwankungsrückstellung nicht abgebildet ist. Im Interesse einer realitätsnäheren Darstellung wäre es daher sinnvoll, eine ergebnisabhängige Reservierungsstrategie in das Modell zu implementieren. Eine derartige pfadabhängige Managementregel könnte beispielsweise vorsehen, dass bei Unterschreitung einer zuvor definierten Ergebnisschwelle stille Reserven in den Schadenrückstellungen in Form erhöhter Abwicklungsgewinne realisiert werden, sofern hierdurch nicht die Schadenrückstellungen unter ein ebenfalls festzulegendes Mindestniveau fallen. ${ }^{1089}$

Das versicherungstechnische Ergebnis wird in der bilanziellen Perspektive sowohl durch zahlungswirksame als auch nicht zahlungswirksame Komponenten (insbesondere die Veränderung der Schadenrückstellungen) beeinflusst. Der für Kapitalanlagezwecke nutzbare Zinsträger wird jedoch unterjährig nur durch die liquiditätswirksamen Komponenten erhöht bzw. reduziert. In Abbildung 6.30 werden zunächst die Verteilungen des versicherungstechnischen Brutto und Netto Cashflows einander gegenübergestellt. Ein negativer Brutto Cashflow tritt lediglich in 6\% der Simulationsläufe auf. Die negativen Realisationen sind überwiegend auf Naturgefahrschäden (insbesondere Sturm- und Überschwemmungsschäden) zurückzuführen. Diese treten zwar nur vergleichsweise selten auf, können jedoch ein erhebliches Ausmaß annehmen. Der versicherungstechnische Brutto Cashflow des Modellunternehmens beträgt im schlechtesten Fall -221,9 Mio. GE. Durch das bestehende Rückversicherungsprogramm wird bereits ein signifikanter Teil der Naturgefahr- und Großschäden aufgefangen, so dass ein negativer Netto Cashflow nur noch in ca. 1\% der Fälle zu beobachten ist. Das Minimum der Nettoverteilung liegt lediglich bei -22,3 Mio. GE. Der maximale Liquiditätsbedarf, den das versicherungstechnische Portfolio (in den hier betrachteten 30.000 Simulationsläufen) an das Kapitalanlageportfolio stellt, beträgt somit 22,3 Mio. GE. Dieser Bedarf kann durch die zu Beginn des simulierten Geschäftsjahres vorhandenen kurzfristigen Aktiva (Geldmarktpapiere bzw. Anleihen mit einjähriger Restlaufzeit) gedeckt werden, ohne dass beispielsweise Kursverluste im Aktienbe-

\footnotetext{
${ }^{1089}$ Unter Berücksichtigung des Marktumfeldes könnte die Vorgabe des minimalen Reserveniveaus beispielsweise als Reserve-Beitragsquote oder Reserve-Zahlungsquote formuliert werden.
} 
stand realisiert werden müssen. Bereits aus diesem Sachverhalt wird ersichtlich, dass die Verteilung des versicherungstechnischen Netto Cashflows im betrachteten Datenbeispiel keinen wesentlichen Treiber des Kapitalanlagerisikos darstellt.

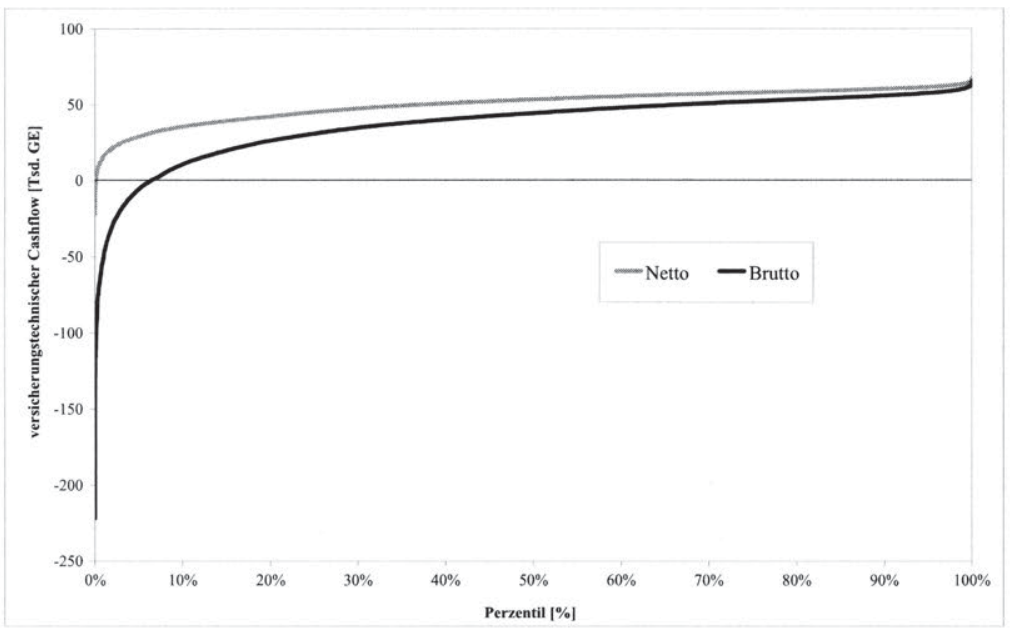

Abbildung 6.30: Perzentilgraph des versicherungstechnischen Cashflows

Zur Beurteilung des Kapitalanlageportfolios wird nachfolgend zwischen dem bilanziellen Kapitalanlageergebnis und dem Total Return differenziert. ${ }^{1090}$ Während das bilanzielle Ergebnis in der Praxis stark durch die Ausübung handelsrechtlicher Bewertungsspielräume sowie den Aufbau bzw. die Realisierung von Bewertungsreserven beeinflusst werden kann, stellt der Total Return als Maßgröße für die Marktwertveränderung des Gesamtportfolios den ökonomisch relevanten Bewertungsmaßstab dar. ${ }^{1091}$ Angesichts der Tatsache, dass das bilanzielle Kapitalanlageergebnis jedoch einen substanziellen Einfluss auf den Jahresüberschuss ausübt und bei Versicherungsnehmern, Ratingagenturen und auch Aufsichtsbehörden unverändert hohe Beachtung findet, ist im Rahmen der Kapitalanlagesteuerung die Einhaltung der bilanziellen Nebenbedingung, beispielsweise in Form einer marktseitig erwarteten Nettoverzinsung erforderlich. ${ }^{1092}$ Darüber hinaus ist festzuhalten, dass die Erreichung bilanzorientierter Zielset-

\footnotetext{
${ }^{1090}$ Vgl. zur Ermittlung dieser Ergebnisgrößen auch Kapitel 5.5.6.

1091 „Nur die Nettoverzinsung III, die dem Versicherer selbst bekannt sein sollte [und in der verwendeten Definition dem Total Return entspricht, Anm. d. Verf.], ist die richtige Meßkenngröße, um die Kapitalanlagepolitik, hier insbesondere die Asset Allocation, sowohl taktisch als auch strategisch beurteilen bzw. bewerten zu können." FORST, VON DER (1998), S. 93. Vgl. zur Bewertungsrelevanz von Bewertungsreserven außerdem UTECHT (2001), S. 539-540.

${ }^{1092}$ Vgl. übereinstimmend GRITZMANN (1998), S. 58-59.
} 
zungen in einer langfristigen Perspektive nicht über bilanzpolitische Maßnahmen, sondern lediglich indirekt über marktwertorientierte Ziele sichergestellt werden kann. ${ }^{1093}$ Eine bestmögliche Erreichung bilanzorientierter Zielsetzungen erfordert daher stets eine konsequente Verfolgung marktwertorientierter Ziele. Abbildung 6.31 stellt dem bilanziellen Kapitalanlageergebnis die Marktwertveränderung des Gesamtportfolios (Total Return) im simulierten Geschäftsjahr gegenüber.

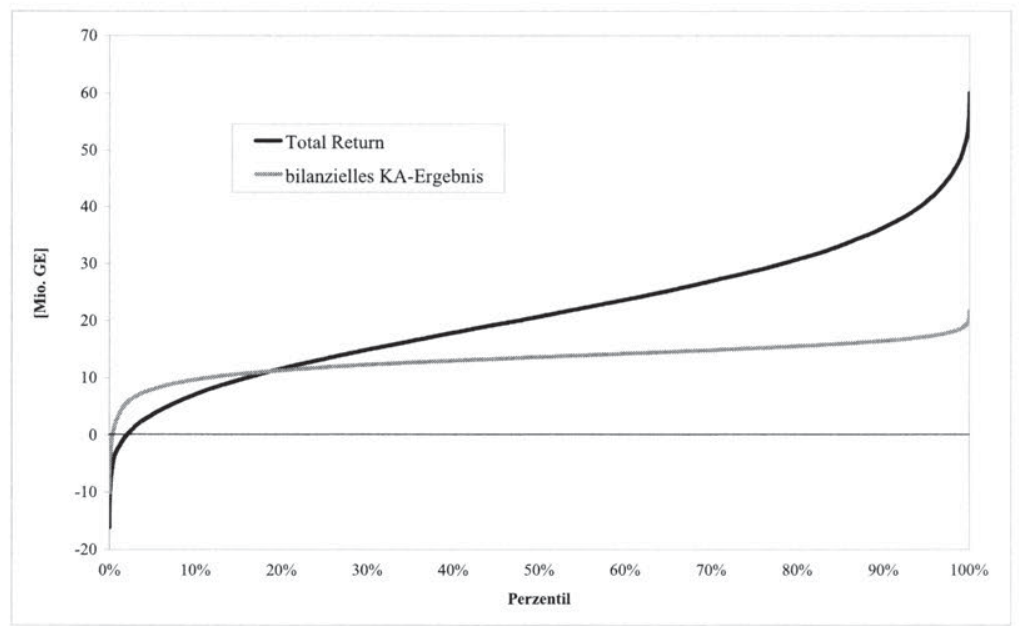

Abbildung 6.31: Kapitalanlageergebnis vs. Total Return

Im Gegensatz zum Total Return weist das bilanzielle Kapitalanlageergebnis eine deutlich geringere Schwankungsbreite auf, da negative Marktentwicklungen durch die zu Periodenbeginn bestehenden Bewertungsreserven abgefedert werden. Aufgrund dieses „Puffereffektes“ treten negative Kapitalanlageergebnisse lediglich mit einer Wahrscheinlichkeit von $0,5 \%$ auf. In denjenigen Szenarien hingegen, in denen die Bewertungsreserven durch stark negative Kapitalmarktentwicklungen vollständig aufgezehrt werden, schlagen darüber hinausgehende Marktwertverluste direkt in Form von Abschreibungen auf das bilanzielle Ergebnis durch. Dieses Risiko kann insbesondere in einem mehrperiodigen Kontext relevant werden, sofern die Kapitalmarktentwicklung in mehreren aufeinander folgenden Perioden negativ verläuft und die Wertpapierbestände bereits teilweise abgeschrieben werden mussten. In diesen Fällen ist der Aufbau eines neuen Ergebnispuffers in Form von Bewertungsreserven erst dann wieder möglich, wenn die Marktwerte ihre historischen Anschaffungskosten erreicht haben.

${ }^{1093}$ Vgl. im Folgenden auch GRITZMANN (1998), S. 58-61. 
Abbildung 6.31 verdeutlicht darüber hinaus, dass durch die Anwendung des strengen Niederstwertprinzips Marktwertsteigerungen, insbesondere Kursgewinne des Aktienbestandes, nicht erfolgswirksam werden, sondern zu einem Aufbau von Bewertungsreserven führen. ${ }^{1094}$ Die intuitive Interpretation, dass in den Szenarien „rechts“ des Schnittpunktes beider Verteilungen Bewertungsreserven aufgebaut werden, ist hingegen nicht zulässig. Aufgrund der Tatsache, dass die Simulationsergebnisse für beide Zufallsvariablen jeweils unabhängig voneinander sortiert sind, stammen die auf ein bestimmtes Perzentil entfallenden Ergebnisrealisationen nicht mehr (oder bestenfalls zufällig) aus demselben Simulationslauf. Eine paarweise Perzentilbetrachtung der Ergebnisgrößen kann daher zu Fehlinterpretationen führen. In Abbildung 6.32 werden die Veränderungen der Bewertungsreserven im simulierten Geschäftsjahr als Perzentilgraph dargestellt.

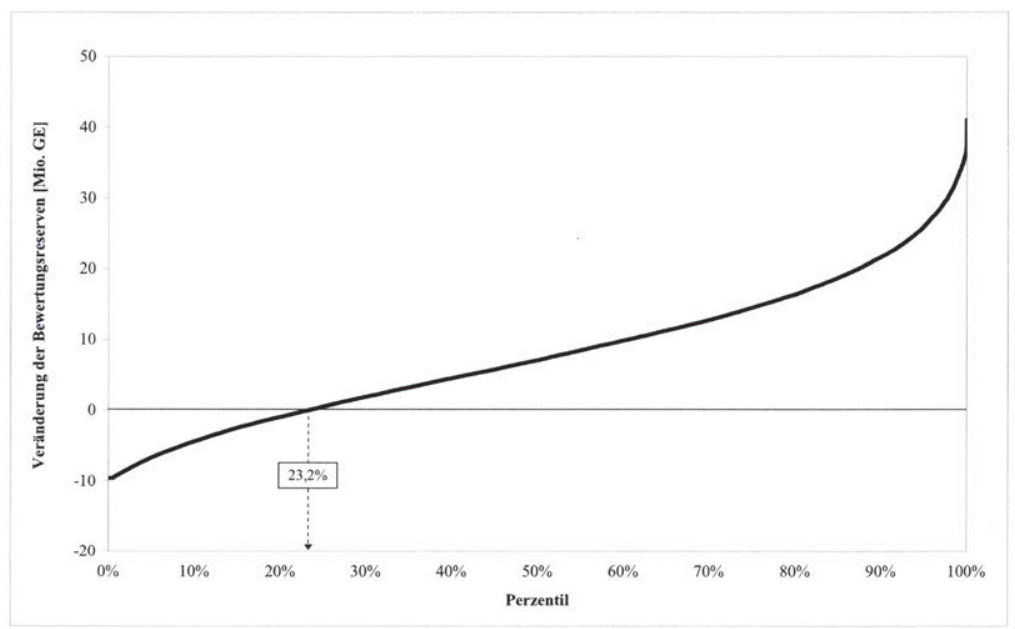

Abbildung 6.32: Veränderung der Bewertungsreserven

Ein vollständiger Verzehr der zu Periodenbeginn vorhandenen Bewertungsreserven in Höhe von 9,6 Mio. GE ist lediglich in 0,5\% aller betrachteten Szenarien zu beobachten. Darüber hinaus zeigt sich, dass das Modellunternehmen in 76,8\% der Fälle zusätzliche Bewertungsreserven aufbauen kann. Mit einer Wahrscheinlichkeit von 23,2\% stehen am Ende des Geschäftsjahres weniger Bewertungsreserven als bilanzieller Ergebnispuffer zur Verfügung. Diese Betrachtungsweise setzt allerdings voraus, dass das Modellunternehmen die im Basisszenario erzielte Nettoverzinsung von 3,77\% als hin-

\footnotetext{
${ }^{1094}$ Aufgrund der Prämisse, dass die historischen Anschaffungskosten der modellierten Wertpapiere mit den Buchwerten übereinstimmen, ist das Auftreten erfolgswirksamer Zuschreibungen nicht möglich.
} 
reichend konkurrenzfähig bewertet und am Ende des Geschäftsjahres keine Bewertungsreserven realisiert, um die Nettoverzinsung optisch zu verbessern. In Analogie zum versicherungstechnischen Geschäft wäre es an dieser Stelle möglich, eine pfadabhängige Entscheidungsregel in den Modellansatz zu integrieren. Diese könnte beispielsweise vorsehen, am Jahresende exakt so viele Bewertungsreserven zu realisieren, wie zur Erreichung einer unternehmensseitig definierten (bzw. von den Marktteilnehmern erwarteten) Ziel-Nettoverzinsung notwendig ist. ${ }^{1095}$

Nach der Aggregation von versicherungstechnischem Nettoergebnis und Kapitalanlageergebnis sowie nach Abzug der Steuerzahlungen verbleibt schließlich der handelsrechtliche Jahresüberschuss nach Steuern. Aus Abbildung 6.33 ist ersichtlich, dass das bilanzielle Eigenkapital von 100 Mio. GE in keinem der betrachteten Szenarien vollständig durch einen Jahresfehlbetrag aufgezehrt wird. Eine Konkursgefährdung aufgrund des Überschuldungstatbestandes ist daher nicht gegeben. ${ }^{1096}$ Allerdings kann der handelsrechtliche Fehlbetrag in den schlechtesten Simulationsläufen durchaus eine Größenordnung von 70 Mio. GE annehmen.

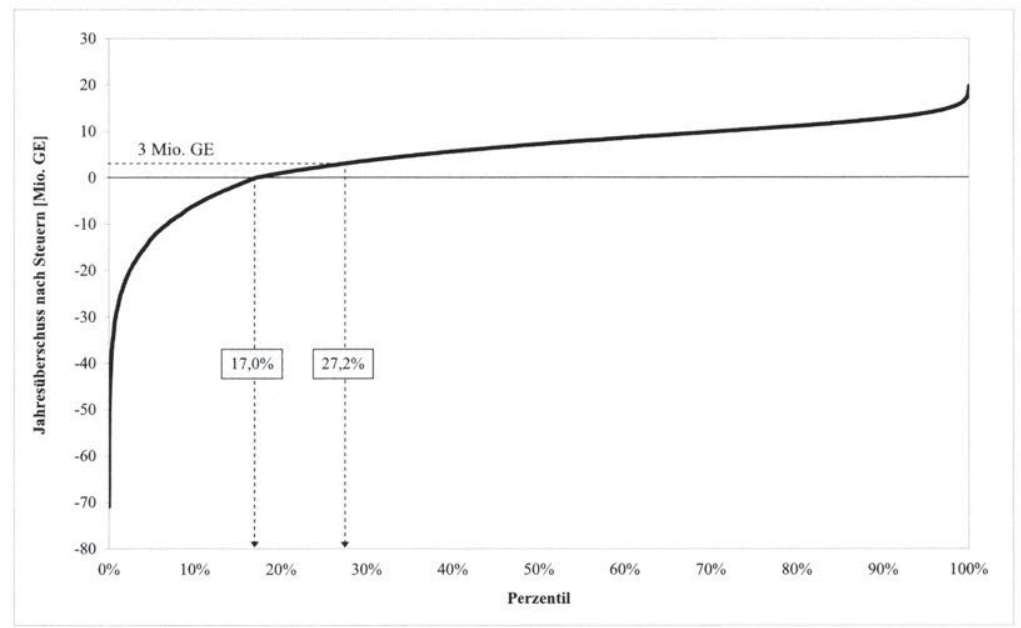

Abbildung 6.33: Jahresüberschuss nach Steuern (Basisszenario)

Eine Analyse dieser extremen Verlustszenarien kommt zu dem Ergebnis, dass sich derartige Fälle ausnahmslos durch hohe versicherungstechnische Verluste auszeich-

\footnotetext{
${ }^{1095}$ Vgl. hierzu vertiefend auch HEINKE (2002a), S. 636.

${ }^{1096}$ Annahmegemäß können negative versicherungstechnische Netto Cashflows jederzeit durch die Aufnahme kurzfristiger Fremdmittel (z.B. Geldmarktkredit) ausgeglichen werden, so dass die Gefahr einer Illiquidität ebenfalls ausgeschlossen ist.
} 
nen, die vereinzelt noch durch negative Kapitalanlageergebnisse verstärkt werden. Als wesentlicher Risikotreiber kann jedoch das versicherungstechnische Kerngeschäft identifiziert werden. Die Wahrscheinlichkeit, dass das bilanzielle Eigenkapital durch ein negatives Jahresergebnis vermindert wird, beträgt $17,0 \%$. Darüber hinaus reicht das Nachsteuerergebnis in 27,2\% der Fälle nicht aus, um die Ausschüttung an die Anteilseigner in Höhe von 3 Mio. GE zu erwirtschaften. Gegebenenfalls müsste die Dividendenzahlung in diesen Szenarien durch eine außerordentliche Realisierung aktivoder passivseitiger Reserven dargestellt werden. ${ }^{1097}$ Die nunmehr beschriebene Struktur des handelsrechtlichen Jahresergebnisses mündet im Basisszenario schließlich in folgender Schlussbilanz:

\begin{tabular}{|lrr|lrr|}
\hline Aktiva & [Tsd. GE] & {$[\%]$} & Passiva & [Tsd. GE] & [\%] \\
\hline Aktien & 63.554 & 16,9 & Eigenkapital & & \\
Anleihen & 236.923 & 63,1 & - gezeichnetes Kapital & 100.000 & \\
Geldmarkt & 68.356 & 18,2 & - Bilanzgewinn & 1.975 & 27,2 \\
Liquiditätskasse & 6.495 & 1,7 & Schadenrückstellungen & 273.353 & 72,8 \\
\hline Summe & $\mathbf{3 7 5 . 3 2 8}$ & $\mathbf{1 0 0 , 0}$ & Summe & $\mathbf{3 7 5 . 3 2 8}$ & $\mathbf{1 0 0 , 0}$ \\
\hline
\end{tabular}

Tabelle 6.23: Vereinfachte Schlussbilanz im Basisszenario (Erwartungswerte)

Aktivseitig ist zunächst festzustellen, dass die Buchwerte des Aktien- und Anleihebestandes nach Ablauf des simulierten Geschäftsjahres leicht rückläufig sind, während der Bestand an Geldmarktpapieren signifikant angestiegen ist. Diese Entwicklung ist darauf zurückzuführen, dass der versicherungstechnische Netto Cashflow unterjährig ausschließlich risikolos am Geldmarkt investiert wurde. Da sich Kurssteigerungen von Aktien und Anleihen lediglich durch einen Anstieg der Bewertungsreserven auswirken, bleiben deren Buchwerte nahezu konstant. ${ }^{1098}$ Der Liquiditätsbestand stellt den Saldo aus laufenden Kapitalanlageerträgen und Kosten der Kapitalanlageverwaltung dar und ist bereits um die Höhe der Steuer- und Dividendenzahlungen vermindert. Infolge der deterministischen Abwicklungssystematik konnte das Modellunternehmen in erheblichem Maße bilanzielle Haftungsmasse in Form versicherungstechnischer Rückstellungen aufbauen. Nach Ausschüttung an die Anteilseigner verbleibt eine Erhöhung der bilanziellen Eigenkapitalbasis um 2,0 Mio. GE. Aufgrund der gestiegenen Bilanzsumme geht die Eigenkapitalquote jedoch im Erwartungswert von 31,3\% auf 27,2\% zurück. Eine Analyse auf der Basis extern verfügbarer Bilanzdaten käme somit zu der Schlussfolgerung, dass sich die finanzielle Solidität des Modellunternehmens im Verlauf des Geschäftsjahres verschlechtert hat.

${ }^{1097}$ In der Praxis kann zur Sicherstellung der Ausschüttungsfähigkeit gegebenenfalls zuvor noch auf Gewinnrücklagen zurückgegriffen werden. Vgl. hierzu auch BAETGE/KIRSCH/THIELE (2003), S. 446-452.

${ }^{1098}$ Die geringfügige Verminderung ist darauf zurückzuführen, dass negative Kapitalmarktszenarien im Falle vollständig aufgezehrter Bewertungsreserven vereinzelt Abschreibungen induzieren, welche die Buchwerte im Erwartungswert leicht reduzieren. 
Werden jedoch darüber hinaus auch außerbilanzielle Risikodeckungsmassen in die Betrachtung einbezogen, ergibt sich folgendes verändertes Bild.

\begin{tabular}{|l|c|c|}
\hline \multicolumn{1}{|c|}{ EW in [Tsd. GE] } & Beginn SimGJ & Ende SimGJ \\
\hline Gezeichnetes Kapital & 100.000 & 100.000 \\
\hline Bilanzgewinn & - & 1.975 \\
\hline Bewertungsreserven & 9.582 & 17.604 \\
\hline Stille Reserven in den Schadenrückstellungen & 60.451 & 68.498 \\
\hline ökonomisches Sicherheitskapital & $\mathbf{1 7 0 . 0 3 4}$ & $\mathbf{1 8 8 . 0 7 7}$ \\
\hline
\end{tabular}

Tabelle 6.24: Ökonomisches Sicherheitskapital im Basisszenario

Als Ergebnis der konservativen Reservierungspolitik erfolgte insbesondere in den HUK-Sparten ein starker Aufbau stiller Reserven in den Schadenrückstellungen. Auf der Kapitalanlageseite führten außerdem Kursgewinne zu einer Erhöhung der Bewertungsreserven. Eine ökonomische Analyse des Geschäftsverlaufs kommt somit zu dem Ergebnis, dass sich die Sicherheitskapitalausstattung des Modellunternehmens im simulierten Geschäftsjahr substanziell verbessert hat. Vergleicht man das verfügbare Sicherheitskapital von 188,1 Mio. GE mit dem in Kapitel 6.2.1.1.2 ermittelten Nettokapitalbedarf in Höhe von ca. 51,6 Mio. GE, so ist eine deutliche Überdeckung festzustellen. Allerdings dient das Sicherheitskapital aus Tabelle 6.24 ausdrücklich nicht nur der Absicherung des versicherungstechnischen Risikos des aktuellen Zeichnungsjahres, sondern muss darüber hinaus auch das Reserverisiko vorangegangener Zeichnungsjahre und das Marktwertrisiko der Kapitalanlagen abdecken.

Auf der Grundlage der bilanziellen Daten kann außerdem die aufsichtsrechtliche Solvabilitätsspanne des simulierten Geschäftsjahres berechnet werden. ${ }^{1099}$ Da keine dreijährige Datenhistorie zur Verfügung steht, können die Parameter „Schadendurchschnitt“ und „Selbstbehaltsquote“ lediglich auf der Basis des simulierten Geschäftsjahres bestimmt werden. Ausgehend von diesen vereinfachenden Annahmen ergibt sich eine Solvabilitätsspanne in Höhe von 90,0 Mio. GE. ${ }^{1100}$ Das gezeichnete Kapital von 100 Mio. GE (= Ist-Solvabilität) reicht demnach im Basisszenario zur Bedeckung der Solvabilitätsspanne aus. Wenngleich sich der auf der Steuerungsebene ermittelte Nettokapitalbedarf von 51,6 Mio. GE lediglich auf das Zeichnungsrisiko des simulierten Anfalljahres bezieht und das Reserve- und Kapitalanlagerisiko zunächst unberücksichtigt bleiben, kann dennoch vermutet werden, dass der Kapitalbedarf durch das derzeitige aufsichtsrechtliche Instrumentarium (Solvency I) überschätzt wird.

\footnotetext{
${ }^{1099} \mathrm{Vgl}$. im Folgenden auch die Ausführungen zur Solvabilitätsspanne in Kapitel 3.1.1.1.

${ }^{1100}$ Der Beitragsindex des simulierten Geschäftsjahres beträgt 78,9 Mio. GE. Angesichts einer bilanziellen Bruttoschadenquote von 79,4\% wird in der aus Beziehung (3.1) bekannten Maximierungsbedingung der Schadenindex in Höhe von 90,0 Mio. GE relevant.
} 
Dies unterstreicht die bereits in Kapitel 3.1.1.2 formulierte Kritik an den volumenbasierten Kenngrößen des Beitrags- und Schadenindexes und verdeutlicht darüber hinaus die Notwendigkeit, die europäischen Solvabilitätsanforderungen im Rahmen von Solvency II auf eine tragfähigere risikotheoretische Grundlage zu stellen.

\subsubsection{Effekte ausgewählter Zeichnungs- und Rückversicherungsstrategien}

Abschließend sollen exemplarisch die bilanziellen Auswirkungen derjenigen Zeichnungs- und Rückversicherungsstrategien untersucht werden, die sich auf der Steuerungsebene als vorteilhaft herausgestellt haben. Es wird daher eine kombinierte Strategie betrachtet, die sowohl den Abschluss einer Stop-Loss-Deckung für die sturmexponierten Sparten „VGV Sturm“ und „Allgemeine Sturm“ als auch die Einführung einer absoluten Schadenselbstbeteiligung in der Sparte „VGV Leitungwasser“ vorsieht. Die Analyse beschränkt sich auf zentrale bilanzielle Effekte und stellt ausschließlich die handelsrechtliche Nettoperspektive in den Mittelpunkt. Abbildung 6.34 visualisiert zunächst die Verteilung des Jahresüberschusses nach Steuern im Perzentilbereich zwischen $0 \%$ und $50 \%$.

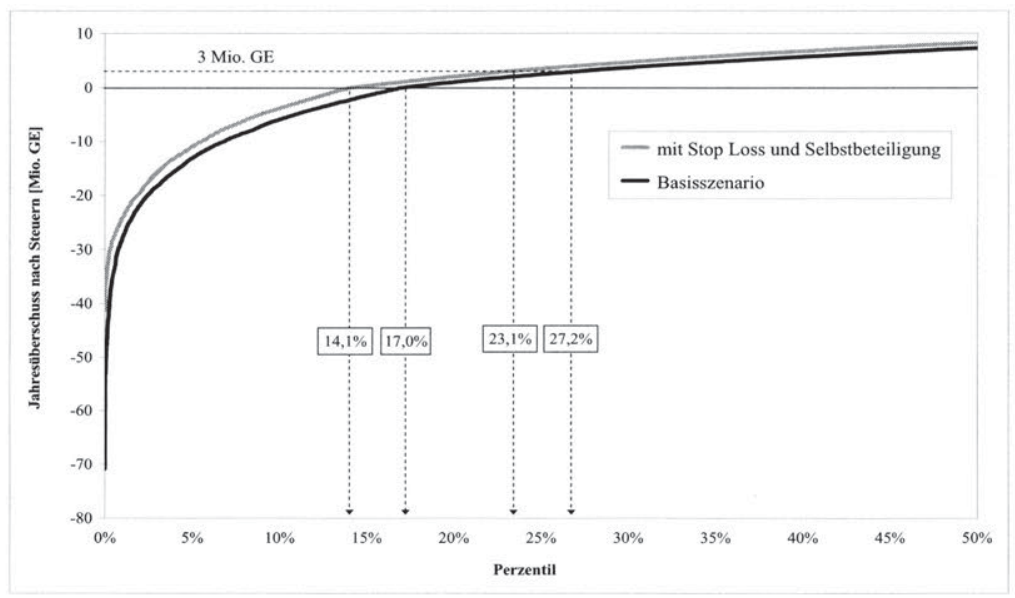

Abbildung 6.34: JÜ nach Steuern mit SL-Deckung (VGV ST, AST) und Selbstbeteiligung (VGV LW)

Der maximale Jahresfehlbetrag reduziert sich von 70,9 Mio. GE im Basisszenario auf nunmehr 44,4 Mio. GE. Der abgeschlossene Stop-Loss-Vertrag wird damit seiner Funktion als „Bilanzschutz“ weitgehend gerecht. Wie bereits auf der Steuerungsebene beobachtet, ermöglicht der SL-Vertrag eine effektive und vergleichsweise kostengünstige Begrenzung des Frequenzrisikos aus Sturmschäden. Die Einführung der Schadenselbstbeteiligung hingegen bewirkt - bildhaft formuliert - eine Parallelverschiebung 
der Ergebnisverteilung nach oben. Im Resultat verbessert sich der erwartete Jahresüberschuss nach Steuern von 2,0 Mio. GE auf 3,3 Mio. GE. Die Wahrscheinlichkeit einer Eigenkapitalminderung reduziert sich von zuvor 17,0\% auf 14,1\%. Zudem sieht sich das Modellunternehmen nach Implementierung der betrachteten Zeichnungs- und Rückversicherungsstrategien nur noch in $23,1 \%$ aller Szenarien der Notwendigkeit ausgesetzt, die Gewinnausschüttung an die Anteilseigner teilweise aus der Unternehmenssubstanz darstellen zu müssen. Tabelle 6.25 zeigt die Auswirkungen der Strategien auf die vereinfachte Bilanzstruktur des Modellunternehmens.

\begin{tabular}{|l|r|r|r|r|r|r|r|}
\hline \multicolumn{1}{|c|}{ Aktiva } & \multicolumn{1}{c|}{ Basis } & \multicolumn{2}{c|}{ mit Strategien } & \multicolumn{2}{c|}{ Passiva } & Basis & \multicolumn{2}{c|}{ mit Strategien } \\
& [Tsd. GE] & {$[$ Tsd. GE] } & \multicolumn{1}{c}{$[\%]$} & & & & \\
[Tsd. GE] & [Tsd. GE] & {$[\%]$} \\
\hline Aktien & 63.554 & 63.554 & 16,9 & Eigenkapital & & \\
Anleihen & 236.923 & 236.923 & 62,9 & - gezeichnetes Kapital & 100.000 & 100.000 & 27,4 \\
Geldmarkt & 68.356 & 70.539 & 18,7 & - Bilanzgewinn & 1.975 & 3.278 & \\
Liquiditätskasse & 6.495 & 5.494 & 1,5 & Schadenrückstellungen & 273.353 & 273.232 & 72,6 \\
\hline Summe & $\mathbf{3 7 5 . 3 2 8}$ & $\mathbf{3 7 6 . 5 1 0}$ & $\mathbf{1 0 0 , 0}$ & Summe & $\mathbf{3 7 5 . 3 2 8}$ & $\mathbf{3 7 6 . 5 1 0}$ & $\mathbf{1 0 0 , 0}$ \\
\hline
\end{tabular}

Tabelle 6.25 Schlussbilanz mit SL-Deckung und Selbstbeteiligung (Erwartungswerte)

Veränderungen gegenüber dem Basisszenario ergeben sich zum einen durch eine Erhöhung des erwarteten Bilanzgewinns und die damit einhergehende leichte Verbesserung der Eigenkapitalquote. Zum anderen ist auf der Aktivseite ein kompensatorischer Effekt zu beobachten. Während der Bestand an Geldmarktpapieren durch die Entlastung des versicherungstechnischen Netto Cashflows ansteigt, reduziert sich die Liquiditätskasse infolge der erhöhten Steuerzahlung. Der Stand der Schadenrückstellungen bleibt aufgrund der starren Reservierungspolitik im Vergleich zum Basisszenario nahezu unverändert. Tabelle 6.26 zeigt die Gesamtentwicklung des ökonomischen Sicherheitskapitals nach Implementierung der Strategien.

\begin{tabular}{|l|c|c|c|}
\hline \multicolumn{1}{|c|}{ EW in [Tsd. GE] } & \multirow{2}{*}{ Beginn SimGJ } & \multicolumn{2}{c|}{ Ende SimGJ } \\
& & Basis & mit Strategien \\
\hline Gezeichnetes Kapital & 100.000 & 100.000 & 100.000 \\
\hline Bilanzgewinn & - & 1.975 & 3.278 \\
\hline Bewertungsreserven & 9.582 & 17.604 & 17.604 \\
\hline Stille Reserven in den Schadenrückstellungen & 60.451 & 68.498 & 70.454 \\
\hline ökonomisches Sicherheitskapital & $\mathbf{1 7 0 . 0 3 4}$ & $\mathbf{1 8 8 . 0 7 7}$ & $\mathbf{1 9 1 . 3 3 6}$ \\
\hline
\end{tabular}

Tabelle 6.26: Ökonomisches Sicherheitskapital mit SL-Deckung und Selbstbeteiligung

Aufgrund des gesunkenen Endschadenbedarfs in der Sparte „VGV Leitungswasser“ ist ein Anstieg der stillen Reserven in den Schadenrückstellungen zu konstatieren. Da die analysierten Strategien keinen Einfluss auf die Asset Allocation des Modellunternehmens ausüben, bleibt die Höhe der Bewertungsreserven unverändert. Insgesamt ist gegenüber dem Basisszenario ein Anstieg des Sicherheitskapitals um weitere 3,3 Mio. GE zu verzeichnen. 
Hinsichtlich der aufsichtsrechtlichen Auswirkungen ist zu konstatieren, dass sich die Solvabilitätsspanne im Vergleich zum Basisszenario von 90,0 Mio. GE auf 87,2 Mio. GE reduziert. Dieser Effekt ist maßgeblich auf die Einführung der Selbstbeteiligung in „VGV Leitungswasser“ zurückzuführen. Der reduzierte Bruttoschadenaufwand in dieser Sparte bedingt insgesamt eine Absenkung des Schadenindexes um 2,8 Mio. GE. Im Ergebnis ist somit festzuhalten, dass eine Durchführung der auf der Steuerungsebene als vorteilhaft identifizierten Strategien sowohl zu einer Verbreiterung der ökonomischen Kapitalbasis als auch zu einer Verbesserung der handels- und aufsichtsrechtlichen Verhältnisse führt. Die Einhaltung der bilanziellen Nebenbedingung ist vor diesem Hintergrund stets gewährleistet.

\subsection{Implikationen der Modellergebnisse für die risikokapitalbasierte Steuerung}

Das in Kapitel 5 entwickelte Modellkonzept wurde in den vorangegangenen Abschnitten am Beispiel eines deutschen Schaden- und Unfallversicherungsunternehmens dargestellt und auf ausgewählte Aspekte der Unternehmenssteuerung angewendet. Abschließend soll nunmehr der Frage nachgegangen werden, welche Schlussfolgerungen die Modellergebnisse im Hinblick auf die risikokapitalbasierte Steuerung nahe legen. Dabei gilt es sich zunächst in Erinnerung zu rufen, dass im Kontext einer integrierten Rendite-Risikosteuerung grundsätzlich zwei Ansätze zur Anwendung kommen können, welche auch in der Literatur kontrovers diskutiert werden. ${ }^{1101}$ Der dezentrale Steuerungsansatz basiert auf der Annahme weitgehend autark agierender Geschäftsbereiche, die auf einem unternehmensinternen Kapitalmarkt um die knappe Ressource Risikokapital konkurrieren. Da die einzelnen Geschäftsbereiche in der Regel anhand risikoadjustierter Performancekennzahlen gesteuert werden, setzt diese Vorgehensweise eine (virtuelle) Allokation von Risikokapital voraus. Im Rahmen eines zentralen Ansatzes wird die Rendite-Risiko-Position hingegen aus der übergeordneten Perspektive des Gesamtunternehmens beurteilt und gesteuert.

Als Ergebnis der Untersuchung ist zunächst festzuhalten, dass Risikoausgleichseffekte einen substanziellen Einfluss auf den Risikokapitalbedarf eines Versicherungsunternehmens ausüben. Angesichts der Tatsache, dass der Ausgleich im Kollektiv die fundamentale Geschäftsgrundlage jeglicher versicherungsspezifischer Geschäftstätigkeit darstellt, überrascht dieses Ergebnis zunächst nicht. Es zeigt sich jedoch, dass die Art und Weise, wie einzelne Teilkollektive im Rahmen der Steuerung zu autarken Einheiten (,Teilunternehmen“) zusammengefasst werden, einen erheblichen Einfluss auf die Höhe des Diversifikationseffektes ausübt. Wenngleich ein geschäftsfeldbasierter Steuerungsansatz unter dem Aspekt einer kundenorientierten und bedarfsgerechten Marktbearbeitung durchaus empfehlenswert erscheint, sollte das Gesamtportfolio zu Steue-

${ }^{1101} \mathrm{Vgl}$. vertiefend hierzu die Ausführungen in Kapitel 3.1.6. 
rungszwecken nicht „künstlich“ in einzelne Teilunternehmen aufgeteilt werden. Segmentübergreifende Ausgleichseffekte, beispielsweise zwischen einzelnen Kundengruppen oder Vertriebswegen, würden anderenfalls unterbunden und die Risikoposition des Gesamtunternehmens auf diese Weise überschätzt. Im Umkehrschluss ist es ebenfalls denkbar, dass risikoerhöhende Abhängigkeitsstrukturen zwischen den Steuerungseinheiten nicht erkannt werden und demzufolge nicht in das Entscheidungskalkül einfließen. In diesem Falle könnte die Risikoposition des Gesamtunternehmens substanziell unterschätzt werden. Es bleibt festzuhalten, dass eine fiktive Kollektivaufspaltung im Rahmen der risikokapitalbasierten Steuerung zu systematischen Fehlanreizen führen und letztlich eine ineffiziente Kapitalnutzung zur Folge haben kann.

Letztgenannter Punkt leitet über zu einem weiteren zentralen Aspekt der risikokapitalbasierten Steuerung. In Kapitel 6.2.2 wurde deutlich, dass stochastische Abhängigkeitsstrukturen zwischen einzelnen Teilkollektiven einen wesentlichen Einfluss auf den Kapitalbedarf eines Versicherungsunternehmens ausüben. Für Portfolios, die nicht gegenüber Naturgefahren exponiert sind, wurde nachgewiesen, dass insbesondere nicht-lineare Abhängigkeiten in den Randbereichen von Schadenverteilungen zu einer gefährlichen Kumulierung von Groß- und Größtschäden führen können. Im realistischeren Fall, dass ein Versicherungsunternehmen auch Produkte mit Naturgefahrdeckungen anbietet, kann ein Großteil stochastischer Verbundeffekte bereits durch „natürliche“ Abhängigkeiten erklärt werden, da Schäden aus Naturgefahrereignissen simultan auf mehrere Steuerungseinheiten wirken. ${ }^{1102}$ Gerade an dem letztgenannten Fall lässt sich ein wesentlicher Schwachpunkt shortfallbasierter Allokationsverfahren und darauf aufbauender dezentraler Steuerungsansätze ausmachen. Im Rahmen der Allokation des Gesamtkapitalbedarfs wird beispielsweise den naturgefahrexponierten Steuerungseinheiten ein sehr hohes Risikokapital zugewiesen, da diese den risikokapitalrelevanten Randbereich der Ergebnisverteilung dominieren und demzufolge nur über ein sehr begrenztes Diversifikationspotenzial verfügen. Andere Geschäftsbereiche werden hingegen durch diesen Effekt (eher zufällig) begünstigt, da ihr originäres Verlustpotenzial durch die Naturgefahrschäden überlagert wird. Diese Steuerungseinheiten würden daher im Kontext eines dezentralen Steuerungsansatzes nur mit vergleichsweise geringen Kapitalkosten belastet. Die Erzielung überdurchschnittlicher Renditen auf das allozierte Risikokapital würde auf diese Weise erleichtert, das eigentliche Risikopotenzial dieser Segmente jedoch unterschätzt. Würden diese Geschäftsbereiche als Ergebnis einer risikoadjustierten Performancesteuerung stark ausgebaut, kann dies eine ungewollte Ausweitung der Gesamtrisikoposition bewirken.

\footnotetext{
${ }^{1102}$ In Abhängigkeit des zugrunde liegenden Spartenmixes lässt sich das Phänomen der TailDominanz natürlich auch auf andere Großschaden- oder Kumulpotenziale wie beispielsweise aus Haftpflicht-, Vermögensschadenhaftpflicht- oder Asbestoseschäden erweitern.
} 
Zu den genannten Kritikpunkten tritt die generelle Problematik risikoadjustierter Performancemaße, auf der Basis einer bekannten Portfoliostruktur auf die Performance einer zukünftigen Portfoliostruktur schließen zu wollen. ${ }^{1103}$ Im Rahmen einer Risiko antizipierenden Steuerungsphilosophie ist daher ein dezentraler Steuerungsansatz über risikoadjustierte Performancemaße mit konzeptionellen Mängeln behaftet.

Die untersuchten Rückversicherungsstrategien legen die Schlussfolgerung nahe, dass isolierte „Optimierungskalküle“ für einzelne Versicherungszweige oder Steuerungseinheiten nicht notwendigerweise, sondern allenfalls zufällig eine effizientere RenditeRisiko-Position des Gesamtunternehmens induzieren. Ausgangspunkt dieser Problematik ist die Erkenntnis, dass das gewählte Rückversicherungsprogramm - in Analogie zur Funktion des Risikokapitals - dem Schutz des Gesamtunternehmens dient und einzelne Vertragsformen nur in Ausnahmefällen (z.B. Aufbauquote) zweifelsfrei einzelnen Teilkollektiven zugeordnet werden können. Im Hinblick auf die mittelfristig erforderliche Schicksalsteilung zwischen Erst- und Rückversicherer und im Interesse eines angemessenen Schutzes des Gesamtunternehmens sind in der Praxis zuweilen bewusste Subventionierungseffekte zwischen einzelnen Rückversicherungsverträgen oder auch Rückversicherungspartnern zu beobachten. Die resultierenden Rückversicherungskosten tragen überwiegend Gemeinkostencharakter und sind demnach sinnvollerweise auf der Unternehmensebene anzusiedeln. ${ }^{1104}$ Darüber hinaus wurde deutlich, dass der Abschluss bestimmter - aus der Sicht des Gesamtunternehmens effizienter - Rückversicherungsdeckungen einen signifikanten Einfluss auf das Allokationsergebnis sämtlicher Teilkollektive nehmen kann. Obwohl sich die Ergebnis- und Risikostruktur der anderen Steuerungseinheiten nicht verändert hat, wird diesen nach Modifizierung der Rückversicherungsstruktur in anderen Geschäftsbereichen ein abweichender Kapitalbedarf zugewiesen. Unter der Zielsetzung einer willkürfreien Steuerung ist dieser Effekt unerwünscht, da die Zielerreichung einzelner Geschäftsbereichs durch zentrale Unternehmensentscheidungen oder auch dezentrale „Optimierungskalküle" anderer Geschäftsbereiche beeinflusst werden könnte. Ein weiteres Problem besteht darin, dass in vielen Versicherungsunternehmen nur spartenspezifische Rückversicherungsdaten vorliegen. Eine direkte Zuordnung von Rückversicherungskosten auf einzelne Steuerungseinheiten erscheint daher nur mit Hilfe von Schlüsselgrößen möglich. Da diese Vorgehensweise jedoch ebenfalls einem erheblichen Ermessensspielraum ausgesetzt ist, muss sie unter Steuerungsaspekten als unangemessen eingestuft werden. Mit Blick auf die Preiskomponenten eines Rückversicherungsprogramms erscheint es sachgerecht, neben der exogenen Vorgabe von Marktkonditionen auch die

\footnotetext{
${ }^{1103} \mathrm{Vgl}$. hierzu auch die kritischen Ausführungen in Kapitel 4.2.2.

${ }^{1104}$ Diese Aussage trifft sowohl auf klassische Rückversicherungsformen als auch (insbesondere) auf moderne Formen der Finanz-Rückversicherung (z.B. Alternativer Risikotransfer) zu, die jedoch in dieser Arbeit nicht thematisiert werden.
} 
Möglichkeit einer modellendogenen Preisermittlung vorzusehen. Ein solches „technisches" Pricing eröffnet zusätzlich die Möglichkeit, für einzelne Rückversicherungsverträge risikoadäquate Prämienraten zu ermitteln. Diese risikotheoretisch „,korrekten“ Preise können im Rahmen der Unternehmenssteuerung unterschiedliche Funktionen erfüllen. Einerseits können die bereits angesprochenen Subventionierungseffekte systematisch aufgedeckt und quantifiziert werden. Andererseits ergeben sich auf der Grundlage risikoadäquater Preise oftmals konkrete Anknüpfungspunkte für Vertragsverhandlungen mit den Rückversicherungspartnern.

Im Hinblick auf potenzielle Zeichnungsstrategien wurde deutlich, dass weitreichende Investitions- oder Desinvestitionsentscheidungen, wie z.B. eine substanzielle Ausweitung der Zeichnungsquoten im industriellen Feuergeschäft, stets im Wirkungsgefüge des Gesamtportfolios beurteilt werden sollten, da nur auf diese Weise sämtliche Risikoverbundeffekte berücksichtigt werden. Um sicherzustellen, dass insgesamt keine existenzgefährdenden Risiken übernommen werden, sollten derartige geschäftspolitische Fragestellungen außerdem immer unter dem Aspekt der Risikotragfähigkeit bewertet werden. Der Abgleich von Risikopotenzial und verfügbaren Risikodeckungsmassen kann jedoch nur auf der Unternehmensebene erfolgen, was einen zentralen Steuerungsansatz in Bezug auf strategische Investitionsentscheidungen nahe legt. Im Gegensatz hierzu sind jedoch auch Entscheidungssituationen oder Handlungsoptionen denkbar, die nur einen begrenzten Einfluss auf die Risikostruktur des Gesamtkollektivs ausüben. Als Beispiel kann die untersuchte Einführung einer Selbstbeteiligung in der Sparte „VGV Leitungswasser“ angeführt werden. Eine dezentrale Steuerung durch die verantwortlichen Marktbereiche stellt in derartigen Fällen eine flexible und zeitnahe Anpassung an Marktveränderungen und Kundenbedürfnisse sicher.

Vor dem Hintergrund der bisherigen Ausführungen kann als Zwischenergebnis festgehalten werden, dass strategische Fragestellungen und geschäftspolitische Entscheidungen, welche unmittelbaren Einfluss auf die Rendite-Risiko-Position eines Versicherungsunternehmens nehmen, einen zentralen Steuerungsansatz erfordern. Nur auf diese Weise können sämtliche Verbund- und Risikoausgleichseffekte in das Entscheidungskalkül einfließen. Als Steuerungskriterium kann beispielsweise der RORAC des Gesamtunternehmens herangezogen werden. Die Risikopräferenz der Unternehmensführung (bzw. der Kapitalgeber) fließt als Sicherheitsniveau in die Quantifizierung des Risikokapitalbedarfs ein und kann sich beispielsweise an einer angestrebten Ratingstufe orientieren. Der Risikokapitalbedarf auf der Gesellschaftsebene berücksichtigt sämtliche Ausgleichseffekte, die innerhalb des Gesamtportfolios wirksam werden. Konkurrierende Handlungsalternativen können anhand des RORAC-Kriteriums bewertet und hinsichtlich ihrer Vorteilhaftigkeit in eine Rangfolge gebracht werden. Um die Einhaltung des Risikotragfähigkeitsgrundsatzes ex-ante zu gewährleisten, kann die RORAC- 
basierte Steuerung zudem um ein geeignetes Risikolimitsystem ergänzt werden. Ein dezentraler Steuerungsansatz über risikoadjustierte Performancemaße wird hingegen angesichts seiner in Kapitel 4.2.2 bereits dargelegten Schwächen aus risikotheoretischer Sicht nicht befürwortet.

Um zu gewährleisten, dass die Ergebnisse der zentralen Unternehmenssteuerung auch durch das Bereichsmanagement umgesetzt werden, bedarf es - insbesondere im Falle asymmetrischer Informationsstrukturen - einer angemessenen Verknüpfung von strategischer und operativer Steuerung. ${ }^{105}$ In diesem Zusammenhang erscheint es opportun, auf das „Duale Steuerungsmodell“ der Bankbetriebslehre zurückzugreifen und dessen Grundidee auf den Anwendungskontext der Schaden- und Unfallversicherung zu übertragen. ${ }^{1106}$ Wie bereits in Kapitel 3.2.4 beschrieben, basiert der duale Steuerungsansatz auf einer expliziten Zuweisung von Entscheidungstatbeständen und Verantwortlichkeiten auf einzelne Steuerungsebenen. ${ }^{1107}$ Auf der Ebene der zentralen Struktursteuerung sind sämtliche Entscheidungen angesiedelt, die nur aus der übergeordneten Perspektive des Gesamtunternehmens beurteilt und verantwortet werden können. Mit Blick auf die oben skizzierten Modellergebnisse fallen hierunter insbesondere Entscheidungen in Bezug auf das angestrebte Sicherheitsniveau, die Rückversicherungspolitik sowie strategische Fragestellungen des Underwritings. Darüber hinaus müssen auf dieser Ebene sowohl aufsichtsrechtliche Solvabilitätsanforderungen als auch bilanzielle Strukturvorgaben (z.B. Eigenkapital- und Reservequote, Ausschüttungsfähigkeit, etc.) als strenge Nebenbedingungen der Steuerung berücksichtigt werden. Auf der Ebene der dezentralen Marktbereichssteuerung sind hingegen Sachverhalte angesiedelt, die geschäftspolitische Einzelentscheidungen betreffen. Hierzu zählen insbesondere die Selektion und Tarifierung von Einzelrisiken sowie andere produktspezifische Entscheidungen, wie z.B. die Einführung von Selbstbeteiligungen oder Fragestellungen des Schadenmanagements. Um sicherzustellen, dass die dezentralen Marktbereiche keine Entscheidungen treffen, welche hinsichtlich ihrer Ergebnis-, Risiko- und Strukturwirkungen aus Sicht des Gesamtunternehmens unerwünscht sind, bedarf es einer Integration beider Steuerungsebenen. ${ }^{1108}$ Als integrative Instrumente kommen im versicherungsspezifischen Kontext insbesondere Zielvereinbarungen, Risikolimite und anreizkompatible Vergütungssysteme in Betracht. Da der Fokus dieser Arbeit auf die strategische Steuerung der Rendite-Risiko-Position gerichtet ist, sollen diese Instrumente nachfolgend nur überblicksartig dargestellt werden.

\footnotetext{
${ }^{1105}$ Vgl. hierzu auch OLETZKY (1998), S. 22-24. Zur Integration des Risikomanagements in das Konzept der Balanced Scorecard vgl. außerdem ROMEIKE/MÜLLER-REICHARD (2005), S. 343-380.

${ }^{1106} \mathrm{Vgl}$. im Folgenden zum „Dualen Steuerungsmodell“ SCHIERENBECK (2001), S. 87-101 und SCHIERENBECK (2003a), S. 293-303 sowie die Ausführungen in Kapitel 3.2.4

${ }^{1107}$ Vgl. auch Abbildung 3.3.

${ }^{1108}$ Vgl. im Folgenden SCHIERENBECK (2003a), S. 298-301.
} 
Zielvereinbarungen sind Bestandteil eines rückgekoppelten Planungs- und Strategieprozesses zwischen zentraler Struktursteuerung und dezentraler Marktbereichssteuerung. ${ }^{1109}$ Im Rahmen einer Planung nach dem Gegenstromverfahren sollen die zentralen Anforderungen der Unternehmenssteuerung mit den Marktpotenzialen und Vorstellungen der dezentralen Geschäftsbereiche abgestimmt und nach Möglichkeit synchronisiert werden. In einem ersten Schritt wird den Marktbereichen eine auf das Unternehmenszielsystem abgestimmte (Teil-) Planung übermittelt. In einem zweiten Schritt werden die Anforderungen und Wünsche der Bereichsverantwortlichen in einem koordinierten Prozess an die zentrale Unternehmenssteuerung zurückgemeldet. Am Ende dieses Prozesses steht idealerweise die Festlegung verbindlicher und hinsichtlich der Unternehmensziele konsistenter Teilpläne für alle Marktbereiche. Im Sinne des Führungsprinzips eines „Management by Objectives“ erhalten die Marktbereiche Ergebnis- und Budgetverantwortung für diejenigen Erfolgskomponenten, die sie unmittelbar beeinflussen können. ${ }^{110}$ Angesichts der oben angesprochenen Problematik, dass eine willkürfreie Zuordnung von Rückversicherungsverträgen auf einzelne Steuerungseinheiten in der Regel nicht möglich ist, bietet sich als relevante Steuerungsgröße das versicherungstechnische Bruttoergebnis des jeweiligen Segments an. Nur für den als ambitioniert einzustufenden Fall, dass die Struktur des Rückversicherungsprogramms und das zur Verfügung stehende Abrechnungs- und Informationssystem eine beanspruchungsgerechte Zuordnung von Rückversicherungskosten und Entschädigungsleistungen auf einzelne Steuerungseinheiten erlauben, erscheint ein Steuerungsansatz auf Nettobasis angemessen.

Als weiteres Integrationsinstrument kommt die Vergabe von Risikolimiten in Betracht. ${ }^{111}$ Ausgehend von dem Postulat der Risikotragfähigkeit begrenzen Risikolimite den Handlungsspielraum der Marktbereiche und stellen auf diese Weise sicher, dass die Übernahme von Risiken in Übereinstimmung mit den verfügbaren Risikodeckungsmassen erfolgt. Neben einer fiktiven Zuweisung von Risikokapital auf einzelne Geschäftsbereiche sind im spezifischen Kontext der Schaden- und Unfallversicherung insbesondere auch explizite Zeichnungslimite und Haftungsbegrenzungen zu nennen. ${ }^{112}$ Unternehmen mit ausgeprägter Exponierung gegenüber Naturgefahren sollten den Marktbereichen darüber hinaus Obergrenzen für die Zeichnung von Naturgefahrhaftungen vorgeben (,Kumulbudgets“). Die Limite sollten durch die zentrale Struktursteuerung formuliert und fortlaufend überwacht werden. Innerhalb der zuteilten Limite erhalten die dezentralen Marktbereiche weitgehende Gestaltungs- und Entscheidungs-

\footnotetext{
${ }^{1109}$ Vgl. im Folgenden auch SCHIERENBECK (2003a), S. 298-299 und ADAM (1996), S. 139-145.

${ }^{1110} \mathrm{Vgl}$. vertiefend zu alternativen Führungsprinzipien BÜHNER (2004), S. 86-92.

${ }^{1111} \mathrm{Vgl}$. auch die Ausführungen in Kapitel 4.2.2.

${ }^{1112}$ Vgl. hierzu und im Folgenden MÜNCHENER RÜCK (2003), S. 133.
} 
freiheit, um flexibel auf Kundenbedürfnisse und Marktveränderungen reagieren zu können. Im Rahmen ihres Kompetenzspielraums sind die Marktbereiche sowohl für das einzelgeschäftsbezogene Risikomanagement (z.B. Annahmepolitik) als auch für die Kalkulation und Durchsetzung risikoadäquater Prämiensätze verantwortlich. Da eine zentrale Vorgabe von Richtkonditionen (z.B. in Form von Mindestprämien) mit einer wesentlichen Schwächung der Ergebnisverantwortung der Marktbereiche verbunden wäre, kann diese Form der Integration von strategischer und operativer Steuerung nicht befürwortet werden. ${ }^{113}$

In Kapitel 4.2.2 wurde bereits auf die Bedeutung anreizkompatibler Vergütungssyste$m e$ eingegangen. Vergütungssysteme setzen für das dezentrale Bereichsmanagement monetäre Verhaltensanreize, die Unternehmensziele aktiv mitzuverfolgen und ihre Bereichsziele auf die übergeordneten Unternehmensziele abzustimmen. ${ }^{114}$ Wesentliche Voraussetzung für die Motivationswirkung variabler Vergütungssysteme ist eine im Hinblick auf die übergeordneten Unternehmensziele konsistente Ausgestaltung des Ziel- und Anforderungskatalogs. Darüber hinaus müssen die formulierten Ziele für die Mitarbeiter erreichbar und im Rahmen der gewährten Handlungsspielräume und Entscheidungskompetenzen beeinflussbar sein. Für die Etablierung eines unternehmenszielkonformen und durch die Mitarbeiter als leistungsgerecht empfundenen Vergütungssystems ist daher eine anspruchsvolle und zugleich realistische Zielformulierung von zentraler Bedeutung. ${ }^{1115}$ Für die Ermittlung der variablen Gehaltsbestandteile kommen unterschiedliche Bemessungsgrundlagen in Betracht. ${ }^{1116}$ Neben dem Erreichungsgrad persönlicher und gemeinsamer Arbeitsziele sollten auch mittelfristig ausgerichtete, mithin strategische Unternehmenskennziffern (z.B. die Entwicklung des Unternehmenswertes bzw. des RORAC auf der Unternehmensebene) in die Bemessung variabler Gehaltskomponenten einfließen.

\footnotetext{
${ }^{1113}$ Vgl. zur Funktion von Richtkonditionen in Kreditinstituten SCHIERENBECK (2001), S. 97-98.

1114 Vgl. im Folgenden RINKER (1997) S. 5-8 und S. 66-71 sowie BÜHNER (2005), S. 170-177.

${ }^{1115}$ Vgl. RINKER (1997), S. 70.

${ }^{1116}$ Vgl. im Folgenden auch BÜHNER (2005), S. 172-177.
} 


\section{$7 \quad$ Zusammenfassung und Ausblick}

Die vorliegende Arbeit ist durch den Sachverhalt motiviert, dass sich Versicherungsunternehmen in zunehmendem Maße sowohl externen als auch internen Anforderungen an die Steuerung ihrer Rendite-Risiko-Position ausgesetzt sehen. Im Wettbewerb um Versicherungsnehmer und Investoren tritt ein Zielkonflikt zwischen diesen Anspruchsgruppen zu Tage, der insbesondere die Höhe des vorzuhaltenden Kapitals betrifft und die Notwendigkeit einer möglichst effizienten Kapitalnutzung in der Mittelpunkt des Steuerungsinteresses rücken lässt. Unter dem Postulat einer wertorientierten Unternehmensführung bedarf es daher eines geeigneten Instrumentariums, welches eine integrierte Steuerung von Chancen und Risiken unterstützt. Interne Risikomodelle können in diesem Spannungsfeld einen maßgeblichen Beitrag zur Unterstützung strategischer Unternehmensentscheidungen leisten, da sie eine systematische Erfassung und risikotheoretisch fundierte Quantifizierung der relevanten Risikofaktoren ermöglichen. Die wissenschaftliche Literatur behandelt bislang vorrangig mathematischanalytische Fragestellungen einer integrierten Rendite-Risikosteuerung. Die in diesem Zusammenhang notwendigen, jedoch sehr restriktiven Modellprämissen bilden die Realität des Versicherungsgeschäfts - insbesondere das Schadengeschehen - nur unzureichend ab, so dass analytische Risikomodelle in der Praxis nur sehr eingeschränkt zu Steuerungszwecken herangezogen werden können.

Die Zielsetzung dieser Arbeit bestand darin, einen konkreten Vorschlag zur Ausgestaltung eines risikokapitalbasierten Steuerungsmodells zu entwickeln. Der Modellansatz sollte die Rendite-Risiko-Position eines Schaden- und Unfallversicherungsunternehmens angemessen abbilden und mit Hilfe der in der Praxis verfügbaren (empirischen) Datenbasis parametrisierbar sein. Im Rahmen einer modellgestützten Risikoanalyse sollten darüber hinaus ausgewählte Unternehmensstrategien untersucht und hieraus Implikationen für die strategische Rendite-Risikosteuerung in der Schaden- und Unfallversicherung abgeleitet werden.

Den Ausgangspunkt der Analyse bildeten in Kapitel 2 zunächst die Definition eines problemadäquaten Risikobegriffs sowie eine Systematisierung versicherungsspezifischer Risikofaktoren. Als wesentliche Risiken des versicherungstechnischen Kerngeschäfts wurden das Zeichnungs- und Reserverisiko herausgestellt. Versicherungstechnische Risiken und Kapitalmarktrisiken führen in ihrer Gesamtheit letztlich dazu, dass ein Versicherungsunternehmen sowohl einem Profitabilitäts- als auch Überschuldungsrisiko ausgesetzt ist. Demgegenüber kommt dem Liquiditätsrisiko nur eine untergeordnete Bedeutung zu. Vor dem Hintergrund des beschriebenen Risikoumfelds der Schaden- und Unfallversicherung wurde im weiteren Verlauf der Begriff des Risikokapitals anhand eines (zunächst abstrakten) Einperiodenmodells auf eine konzeptionelle Grundlage gestellt. 
In Kapitel 3 wurde das Entscheidungsfeld eines Schaden- und Unfallversicherungsunternehmens sowohl aus aufsichtsrechtlicher als auch betriebswirtschaftlicher Perspektive beleuchtet. Die derzeitig auf europäischer Ebene gültigen Solvabilitätsvorschriften sind - wie gezeigt wurde - mit elementaren Mängeln behaftet. Neben der Vernachlässigung von Kapitalanlagerisiken zielt die konzeptionelle Kritik insbesondere auf eine mangelnde risikotheoretische Fundierung des Beitrags- und Schadenindex und die unzureichende Berücksichtung des risikopolitischen Instrumentariums, insbesondere der Rückversicherung ab. Weitere Kritik betrifft die Ermittlung der Ist-Solvabilität sowie die gänzliche Vernachlässigung des Sachverhaltes, dass im Verlustfall nicht alle Vermögensgegenstände gleichermaßen einer sofortigen Verlustverrechnung zugänglich sind (Liquiditätsaspekt). Ausgehend von dieser kritischen Einschätzung wurden die Überlegungen der EU-Kommission zur Neuausrichtung des europäischen Solvabilitätssystems (Solvency II) beschrieben. Solvency II basiert auf einem ganzheitlichen Risiko- und Solvabilitätsverständnis und nimmt insofern eine vorausblickende Aufsichtsperspektive ein. Wesentliche Implikationen für die risikokapitalbasierte Steuerung ergeben sich aus den quantitativen Anforderungen in Bezug auf versicherungstechnische Rückstellungen, Kapitalanlage- und Solvabilitätsvorschriften (Säule 1) sowie aus den qualitativen Anforderungen an den Risikomanagementprozess und die Ausgestaltung interner Risikomodelle (Säule 2). Da die Quantifizierung des aufsichtsrechtlichen Zielkapitalbedarfs zukünftig - unter bestimmten Voraussetzungen - auf der Basis unternehmensinterner Risikomodelle erfolgen kann, kommt deren Entwicklung in der Praxis ein zusätzlicher Stellenwert zu. Es zeichnet sich die wünschenswerte Tendenz ab, dass sich die aufsichtsrechtlichen Rahmenbedingungen zunehmend dem betriebswirtschaftlichen Anforderungskatalog an eine adäquate Steuerung der RenditeRisiko-Position annähern. Im betriebswirtschaftlichen Kontext wurde zunächst die übergeordnete Bedeutung des Sicherheitsziels („Safety First“) begründet. Ausgehend von dem Grundsatz der Risikotragfähigkeit wurde die Notwendigkeit einer integrierten Rendite-Risikosteuerung aufgezeigt sowie die Konzeption risikoadjustierter Performancemaße am Beispiel des RORAC eingeführt und kritisch gewürdigt. Zur Strukturierung des weiteren Untersuchungsverlaufs wurde grundlegend zwischen einer „Steuerungsebene“ und einer „Bilanzebene“ differenziert. Wenngleich der Untersuchungsschwerpunkt dieser Arbeit eindeutig der Steuerungsebene zuzuordnen ist, stellt die Bilanzsicht dennoch eine strenge Nebenbedingung für die Unternehmenssteuerung dar. Als wesentliche Instrumente der Risikosteuerung wurden die Zeichnungs-, Reservierungs- und Rückversicherungspolitik sowie die Schadenregulierungs- und Kapitalanlagepolitik herausgestellt.

Im Mittelpunkt von Kapitel 4 standen die Grundlagen der Risikomessung und Risikokapitalallokation. Hinsichtlich des Einsatzes klassischer Risikomaße wurde zunächst herausgestellt, dass diese unter der Zielsetzung der Risikokapitalsteuerung starker 
konzeptioneller Kritik unterliegen. Im Kontext der Schaden- und Unfallversicherung, welcher in der Regel durch nicht symmetrische Schaden- bzw. Ergebnisverteilungen charakterisiert ist, steht einer Verwendung von klassischen Risikomaßen insbesondere deren symmetrisches Risikoverständnis (z.B. Varianz) und eine nur eingeschränkte ökonomische Interpretierbarkeit (z.B. Schiefe, Wölbung) entgegen. Es wurde außerdem deutlich, dass das BERNOULLI-Prinzip im versicherungsspezifischen Kontext kein adäquates Entscheidungsprinzip unter Unsicherheit darstellt, da es nicht mit der dominanten Stellung des Sicherheitsziels vereinbar ist. Im weiteren Verlauf der Ausführungen wurden wünschenswerte Eigenschaften von Risikomaßen diskutiert. Neben der formalen Kohärenzaxiomatik von ARTZNER/DELBAEN/EBER/HEATH wurden zudem auch ökonomische Anforderungen, insbesondere die Konsistenz zwischen verwendetem Risikomaß und dem postulierten Modellzweck bzw. dem unternehmerischen Zielsystem betont. Im Anschluss daran wurde die Konzeption der Shortfallmaße zunächst in allgemeiner Form anhand der Klasse der Lower Partial Moments eingeführt und dann am Beispiel des Value-at-Risk (VaR) und Conditional Value-at-Risk (CVaR) konkretisiert. Als Schwachpunkt des VaR erwies sich der Sachverhalt, dass der VaR nur unter restriktiven Verteilungsannahmen über die Eigenschaft der Subadditivität verfügt, mithin kollektive Risikoausgleichseffekte nicht generell abzubilden vermag. Darüber hinaus wird das Risikopotenzial des Randbereichs einer Verteilung nicht angemessen berücksichtigt, was gerade in Versicherungsportfolios mit hoher Exponierung gegenüber Natur- oder Kumulgefahren zu einer Unterschätzung des Kapitalbedarfs führen kann. Demgegenüber konnte der $\mathrm{CVaR}$ als kohärentes Risikomaß mit intuitiver ökonomischer Deutungsfähigkeit überzeugen. Auf der Grundlage der skizzierten Erkenntnisse wurden unterschiedliche Zielsetzungen der Risikokapitalallokation diskutiert. Während die Allokation zum Zwecke einer risikoadjustierten Performance- bzw. dezentralen Marktbereichssteuerung konzeptioneller Kritik unterlag, wurde die Zuweisung von Risikokapital im Rahmen von Limitsystemen oder zur Erzielung von Anreiz- und Verhaltenseffekten als zweckmäßig herausgestellt. Als formaler Maßstab zur Beurteilung unterschiedlicher Allokationsverfahren wurde der Kriterienkatalog von DENAULT vorgestellt, welcher für die Realisierung kohärenter Allokationsergebnisse konstituierend ist. Als Ergebnis eines kriteriengeleiteten Vergleichs unterschiedlicher Allokationsverfahren konnte die Überlegenheit des CVaR-Konzepts aufgezeigt werden. Im Rahmen strategischer Fragestellungen über den Auf- und Abbau ganzer Geschäftsbereiche stellte darüber hinaus die inkrementelle Kapitalallokation eine geeignete Vorgehensweise dar.

Kapitel 5 widmete sich der Entwicklung eines praxistauglichen Modellkonzepts zur risikokapitalbasierten Steuerung. Der einperiodige Simulationsansatz zeichnet sich durch seine modulare Struktur und hohe Flexibilität in Bezug auf unternehmensspezifische Modellanforderungen aus. Der Schwerpunkt der Modellierung lag auf einer de- 
taillierten Abbildung des versicherungstechnischen Kerngeschäfts. Unter Rückgriff auf das kollektive Modell der Risikotheorie erfolgte eine separate Abbildung von Basisschadenlast, Großschäden und Schäden aus Naturgefahrereignissen. Es wurde deutlich, dass die gewählte Form der Schadenmodellierung eine unmittelbare Verknüpfung von Schadenaufwand und Bestandsgröße (z.B. gemessen in Jahreseinheiten oder Versicherungspolicen) herstellt und demzufolge eine beliebige Skalierung des Schadenportfolios erlaubt. Dieser Aspekt gewinnt in der Praxis gerade im Kontext strategischer As-If-Analysen an Bedeutung, wenn beispielsweise die Auswirkungen alternativer Unternehmenspolitiken oder Portfoliostrukturen auf die Rendite-Risiko-Position untersucht werden sollen. Die modelltechnische Abbildung des versicherungstechnischen Portfolios in einer Matrixstruktur erlaubt zudem die Durchführung zweidimensionaler Bestands- und Risikoanalysen. Als Analysedimensionen kommen in der Praxis neben der handelsrechtlichen Sparten- bzw. Produktsicht insbesondere Kundengruppen, Vertriebskanäle oder auch Geschäftsregionen in Betracht. Die Rückversicherung stellt das zentrale Instrument zur Gestaltung der durch das Versicherungsunternehmen übernommenen Risikoposition dar. Der entwickelte Modellansatz deckt das gesamte Instrumentarium klassischer Rückversicherungsformen $a b$ und bietet darüber hinaus die Möglichkeit, mit Hilfe eines risikotheoretisch fundierten Pricingansatzes für jeden Rückversicherungsvertrag einen ,technischen“ Preis zu ermitteln. Dieser kann wiederum als Ausgangspunkt für Preisverhandlungen mit den Rückversicherungspartnern dienen. Die Modellierung der Kapitalanlagetätigkeit erfolgte auf der Basis verdichteter Hauptanlageklassen. Empirisch ermittelbare Abhängigkeitsstrukturen zwischen den Assetklassenrenditen konnten mit Hilfe einer Korrelationsmatrix berücksichtigt werden. Für die Abbildung der Interaktion zwischen Versicherungs- und Kapitalanlagegeschäft wurde ein cashflow-orientierter Ansatz gewählt. Auf die Modellierung zusätzlicher Verbundeffekte zwischen Versicherungstechnik und Kapitalanlage (z.B. des Inflationseinflusses) wurde mangels empirischer Belegbarkeit derartiger Wirkungszusammenhänge verzichtet. ${ }^{1117}$ Ein wesentlicher Vorzug des entwickelten Modellkonzepts ist abschließend darin zu sehen, dass dieses sowohl eine risikoorientierte Sicht auf ein Schaden- und Unfallversicherungsunternehmen gestattet als auch die Überleitung in eine handelsrechtliche Perspektive unterstützt. Auf diese Weise können strategische Handlungsalternativen unmittelbar dahingegen überprüft werden, ob die Einhaltung der „bilanziellen Nebenbedingung“ gewährleistet ist.

In Kapitel 6 wurde der entwickelte Modellansatz auf einen (strukturerhaltend modifizierten) Datensatz eines deutschen Schaden- und Unfallversicherungsunternehmens angewendet. In einer modellgestützten Risikoanalyse wurde zunächst die RenditeRisiko-Position des Modellunternehmens im Basisszenario analysiert. Im Anschluss

${ }^{1117}$ Vgl. exemplarisch MAURER (2000), S. 251-253. 
daran wurde exemplarisch der Einfluss stochastischer Abhängigkeitsstrukturen auf den Risikokapitalbedarf aufgezeigt sowie Effekte ausgewählter Rückversicherungs- und Zeichnungsstrategien untersucht. Im Mittelpunkt des Interesses stand durchgängig die ökonomische Rendite-Risiko-Perspektive. Demzufolge wurde als Vorteilhaftigkeitskriterium der auf der Gesellschaftsebene erzielte RORAC herangezogen. Im Anschluss wurde überprüft, welche Wirkung die untersuchten Strategien auf die Bilanzstruktur und Gewinn- und Verlustrechnung des Modellunternehmens, die Entwicklung bilanzieller Risikodeckungsmassen sowie die Solvabilitätskennziffern gemäß Solvency I ausübten. Auf der Grundlage der Modellergebnisse wurden schließlich Implikationen für die risikokapitalbasierte Steuerung abgeleitet, die nachfolgend thesenartig zusammengefasst werden:

(1) Stochastische Abhängigkeitsstrukturen zwischen einzelnen Teilkollektiven üben einen signifikanten Einfluss auf den Risikokapitalbedarf eines Versicherungsunternehmens aus und sollten demzufolge im Rahmen einer integrierten RenditeRisikosteuerung Berücksichtigung finden. Im Falle einer starken Exponierung gegenüber Natur- oder Kumulschadenereignissen kann bereits ein maßgeblicher Anteil stochastischer Verbundeffekte durch ,natürliche“ Abhängigkeitsstrukturen erklärt werden.

(2) Sofern ein Versicherungsunternehmen das versicherungstechnische Kollektiv fiktiv in autarke „Teilunternehmen“ aufteilt und diese isoliert steuert, können Fehlsteuerungsimpulse auftreten, da risikoerhöhende oder risikoreduzierende Verbundeffekte nicht mehr vollständig erfasst werden. Die Art und Weise der Kollektivaufspaltung übt einen erheblichen Einfluss auf die Höhe des Diversifikationseffektes aus.

(3) Im Kontext der Zeichnungspolitik sollten daher strategische Investitions- und Desinvestitionsentscheidungen stets im Wirkungsgefüge des Gesamtportfolios beurteilt werden, da nur auf diese Weise sämtliche Risikoverbundeffekte erfasst werden.

(4) Eine Zuordnung von Kapitalanlageerträgen auf einzelne Steuerungseinheiten erscheint nicht willkürfrei möglich und ist daher unter Steuerungsaspekten abzulehnen. Die Tatsache, dass die Steuerungseinheiten jedoch in unterschiedlichem Ausma $ß$ und für eine unterschiedliche Dauer anlagefähiges Kapital generieren, sollte durch den Ansatz kalkulatorischer Zinsen berücksichtigt werden.

(5) Da im Rahmen der Rückversicherungsnahme in der Regel weder die Kosten noch die Entschädigungsleistungen willkürfrei einzelnen Steuerungseinheiten zugeordnet werden können, induzieren isolierte „Optimierungskalküle“ einzelner Steuerungseinheiten allenfalls zufällig eine effizientere Rendite-Risiko-Position 
des Gesamtunternehmens. Entscheidungen über das Rückversicherungsprogramm sollten daher stets auf der Ebene der zentralen Struktursteuerung angesiedelt sein.

(6) Die obigen Punkte münden in der abschließenden Beurteilung, dass unter der Maxime der Unternehmenswertsteigerung eine direkte Beurteilung von Handlungsalternativen auf der Gesellschaftsebene, z.B. unter Anwendung des RORAC-Kriteriums, einem dezentralen Steuerungsansatz über risikoadjustierte Performancemaße vorzuziehen ist.

Der vorgestellte Modellansatz trifft eine Vielzahl vereinfachender Annahmen, um die Komplexität des Versicherungsgeschäfts strukturerhaltend zu reduzieren. $\mathrm{Zu}$ nennen sind an dieser Stelle insbesondere die Beschränkung auf eine Modellperiode und der Sachverhalt, dass keine pfadabhängigen Entscheidungsregeln implementiert sind. Darüber hinaus wird das Kapitalanlageportfolio lediglich über wenige Hauptanlageklassen abgebildet und das Bonitätsrisiko von Anleiheschuldnern nicht berücksichtigt. Die Wirkungsweise aktivseitiger Sicherungsstrategien mittels derivativer Finanzinstrumente wird bislang nur rudimentär erfasst. Des Weiteren werden qualitative Aspekte nicht modelliert, da sie sich einer Quantifizierung weitgehend entziehen. Im Kontext einer entscheidungsorientierten Unternehmensführung kommt jedoch qualitativen Fragestellungen durchaus hohe Relevanz zu. Als weitere Einschränkung des vorgestellten Ansatzes ist die deterministische Modellierung des Abwicklungsverhaltens der Vorjahresschäden zu nennen. Die Annahme starrer Abwicklungsmuster steht bisher einer Quantifizierung des Reserverisikos entgegen. Ebenfalls wird das differenzierte steuerrechtliche Umfeld eines Versicherungsunternehmens nur sehr pauschal abgebildet.

Durch seinen modularen Aufbau kann das vorgestellte Referenzmodell jedoch sukzessive um die angesprochenen Aspekte erweitert werden, so dass weder die Anwendbarkeit des Modellansatzes in der Praxis noch die generelle Aussagekraft der Ergebnisse in Zweifel gezogen werden müssen. Der entwickelte Ansatz wird somit den zu Beginn dieser Arbeit formulierten generellen und versicherungsspezifischen Anforderungskriterien gerecht. Als positiver „Nebeneffekt" der Modellbildung ist für die Versicherungspraxis festzuhalten, dass bereits die Konzeptionsphase eines internen Risikomodells eine sehr intensive Auseinandersetzung mit der Rendite-Risikoposition des Unternehmens erfordert. Der Modellierungsprozess kann daher als wichtiger ,erster Schritt“ auf dem Weg zu einem erweiterten Risikoverständnis und umfassenden Risikomanagement aufgefasst werden. Interne Risikomodelle können jedoch die gewünschte Wirkung nur dann entfalten, sofern sie in einen unternehmensweiten Steuerungsansatz eingebunden werden, welcher die Integration von operativer und strategischer Unternehmenssteuerung sicherstellt. 
Abgesehen von der Nutzung zu internen Steuerungszwecken bzw. im Rahmen aufsichtsrechtlicher Vorgaben (Solvency II), ergeben sich angrenzende Einsatzgebiete für interne Risikomodelle insbesondere bei der Bewertung versicherungstechnischer Rückstellungen. Die Werthaltigkeit von Schadenrückstellungen ist sowohl für unternehmensinterne Analysen als auch zukünftig im Rahmen einer Zeitwertbilanzierung nach internationalen Rechnungslegungsvorschriften (IFRS) von hoher Bedeutung. Darüber hinaus kann die detaillierte Modellierung des versicherungstechnischen Portfolios für Fragestellungen der Tarifgestaltung und als Ausgangspunkt für Vertragsverhandlungen mit den Rückversicherungspartnern eine wertvolle Entscheidungsunterstützung leisten.

Weiterer Forschungsbedarf besteht insbesondere in der Verfeinerung interner Risikomodelle in Bezug auf die oben skizzierten Sachverhalte. Es sei jedoch betont, dass eine zunehmende Methodenkomplexität stets mit der Gefahr verbunden ist, dass ein vermeintlicher "Sicherheitsgewinn“ in Bezug auf das Zufallsrisiko zumindest teilweise durch ein ansteigendes Modell- bzw. Irrtumsrisiko konterkariert wird. Da erst die quantitative Abschätzung möglicher Fehler einer modellgestützten Entscheidungsfindung die gewünschte Sicherheit verleiht, kommt einer umfassenden Validierung der Inputparameter und Modellergebnisse im Sinne eines „Backtesting“ ebenso hohe Bedeutung zu wie der Umsetzung zusätzlicher Modellanforderungen. Die Entwicklung und Implementierung interner Steuerungsmodelle ist mit einem hohen zeitlichen und finanziellen Aufwand für die Versicherungsunternehmen verbunden. Darüber hinaus werden erhebliche Anforderungen an das aktuarielle Know-how der Mitarbeiter, die EDV-technischen Voraussetzungen und insbesondere den Datenhaushalt der Unternehmen gestellt. Mit Blick auf die Anwendung interner Risikomodelle in der Unternehmenspraxis und deren Anerkennung für aufsichtsrechtliche Zwecke (Zertifizierung) erscheint daher ein Vorgehen sinnvoll, welches die Versicherungsunternehmen schrittweise an komplexe Steuerungsmodelle heranführt. ${ }^{1118}$ Eine praktikable Vorgehensweise der Aufsichtsbehörden könnte darin bestehen, zunächst einen Katalog notwendiger Modelleigenschaften zu definieren und diese im Sinne eines „Meilensteinverfahrens" zeitlich zu priorisieren. Eine Entscheidung über die Anerkennung und Aberkennung interner Risikomodelle könnte schließlich an die Erfüllung dieser Meilensteine geknüpft werden.

${ }^{1118}$ Vgl. im Folgenden auch KRIELE/LIM/REICH (2004), S. 1050-1052. 
Mirko Tillmann - 978-3-631-75327-9

Downloaded from PubFactory at 01/11/2019 06:10:15AM

via free access 


\section{Anhang A:}

\section{Verteilungsannahmen und Parameter der Großschadenmodellierung}

In Kapitel 5.4.3.3 wurden Großschäden als Einzelschäden definiert, welche eine bestimmte (spartenspezifisch festzulegende) Großschadengrenze überschreiten. Zur Ermittlung der Gesamtschadenverteilung aus Großschäden wird auf das kollektive Modell der Risikotheorie zurückgegriffen. Für die Simulation der Schadenanzahl wird durchgängig die Poissonverteilung zugrunde gelegt. Der für jede Sparte-GeschäftsfeldKombination separat ermittelte Poissonparameter $\theta$ kann Tabelle A.1 entnommen werden.

\begin{tabular}{|c|c|c|c|c|c|c|c|}
\hline \multirow{2}{*}{ Sparte } & GS-Grenze & \multicolumn{3}{|c|}{ Poissonparater $\theta$} & \multicolumn{3}{c|}{ Schadenhöhenverteilung } \\
\cline { 3 - 8 } & {$[$ Tsd. GE] } & PRI & GEW & IND & PRI & GEW & IND \\
\hline Allgemeine Unfall & 50 & 17,6 & 5,7 & 0,2 & Lognormal & Lognormal & Lognormal \\
\hline Kraftfahrt-Haftpflicht & 250 & 23,2 & 7,8 & 2,3 & Pareto A & Pareto B & Pareto B \\
\hline Kasko & 50 & 2,3 & 3,5 & 2,1 & Burr & Burr & Burr \\
\hline Feuer Industrie & 250 & - & - & 7,9 & - & - & LogLogistik \\
\hline VGV Feuer & 250 & 12,2 & - & - & Burr & - & - \\
\hline
\end{tabular}

Tabelle A.1: Verteilungsannahmen und Parameter der Großschadenmodellierung

Die Gesamtschadenverteilung wird maßgeblich durch die Wahl einer angemessenen Schadenhöhenverteilung beeinflusst. Als Ergebnis einer Verteilungsanpassung („Fitting") wurde für jedes großschadenbelastete Teilkollektiv eine geeignete Schadenhöhenverteilung ermittelt. Die Vorgehensweise der Verteilungsanpassung wurde in Kapitel 5.5.3.3.3 exemplarisch aufgezeigt. Die in dieser Arbeit verwendeten Schadenhöhenverteilungen für Großschäden sind ebenfalls in Tabelle A.1 aufgeführt. Die Verteilungs- und Dichtefunktionen der verwendeten Wahrscheinlichkeitsverteilungen sind Anhang $\mathrm{C}$ zu entnehmen. 


\section{Anhang B:}

\section{Allokationsergebnisse für unterschiedliche Risikoaggregationsstufen}

\begin{tabular}{|l|r|r|r|r|}
\hline \multicolumn{1}{|c|}{ [Tsd. GE] } & \multicolumn{1}{c|}{ PRI } & \multicolumn{1}{c|}{ GEW } & \multicolumn{1}{c|}{ IND } & \multicolumn{1}{c|}{ SUMME } \\
\hline Allgemeine Unfall & -6.048 & -431 & -314 & $\mathbf{- 6 . 7 9 3}$ \\
\hline Kraftfahrt-Haftpflicht & -5.806 & 4.341 & 6.912 & $\mathbf{5 . 4 4 7}$ \\
\hline Kasko & 3.129 & 234 & 138 & $\mathbf{3 . 5 0 1}$ \\
\hline Feuer Industrie & & & 16.455 & $\mathbf{1 6 . 4 5 5}$ \\
\hline Allgemeine Sturm & & 32.188 & 290 & $\mathbf{3 2 . 4 7 8}$ \\
\hline VGV Feuer & 291 & & & $\mathbf{2 9 1}$ \\
\hline VGV Leitungswasser & 11.972 & & & $\mathbf{1 1 . 9 7 2}$ \\
\hline VGV Sturm & 106.730 & & & $\mathbf{1 0 6 . 7 3 0}$ \\
\hline VGV Elementar & 914 & & & $\mathbf{9 1 4}$ \\
\hline SUMME & $\mathbf{1 1 1 . 1 8 3}$ & $\mathbf{3 6 . 3 3 3}$ & $\mathbf{2 3 . 4 8 1}$ & $\mathbf{1 7 0 . 9 9 7}$ \\
\hline
\end{tabular}

Tabelle A.2: Allokationsergebnis in der Geschäftsfeld-Betrachtung (3 isolierte Teilunternehmen)

\begin{tabular}{|l|r|r|r|r|}
\hline \multicolumn{1}{|c|}{ [Tsd. GE] } & \multicolumn{1}{c|}{ PRI } & \multicolumn{1}{c|}{ GEW } & \multicolumn{1}{c|}{ IND } & \multicolumn{1}{c|}{ SUMME } \\
\hline Allgemeine Unfall & -4.113 & 37 & -304 & $\mathbf{- 4 . 3 8 0}$ \\
\hline Kraftfahrt-Haftpflicht & -4.482 & 17.255 & 5.402 & $\mathbf{1 8 . 1 7 5}$ \\
\hline Kasko & 38.443 & 8.106 & 1.639 & $\mathbf{4 8 . 1 8 8}$ \\
\hline Feuer Industrie & & & 20.648 & $\mathbf{2 0 . 6 4 8}$ \\
\hline Allgemeine Sturm & & 36.695 & 3.543 & $\mathbf{4 0 . 2 3 7}$ \\
\hline VGV Feuer & 5.622 & & & $\mathbf{5 . 6 2 2}$ \\
\hline VGV Leitungswasser & 13.519 & & & $\mathbf{1 3 . 5 1 9}$ \\
\hline VGV Sturm & 109.358 & & & $\mathbf{1 0 9 . 3 5 8}$ \\
\hline VGV Elementar & 25.901 & & & $\mathbf{2 5 . 9 0 1}$ \\
\hline SUMME & $\mathbf{1 8 4 . 2 4 7}$ & $\mathbf{6 2 . 0 9 2}$ & $\mathbf{3 0 . 9 2 7}$ & $\mathbf{2 7 7 . 2 6 7}$ \\
\hline
\end{tabular}

Tabelle A.3: Allokationsergebnis in der Sparten-Betrachtung ( 9 isolierte Teilunternehmen)

\begin{tabular}{|l|r|r|r|r|}
\hline \multicolumn{1}{|c|}{ [Tsd. GE] } & \multicolumn{1}{c|}{ PRI } & \multicolumn{1}{c|}{ GEW } & \multicolumn{1}{c|}{ IND } & \multicolumn{1}{c|}{ SUMME } \\
\hline Allgemeine Unfall & -3.792 & 870 & 159 & $\mathbf{- 2 . 7 6 3}$ \\
\hline Kraftfahrt-Haftpflicht & 4.373 & 21.315 & 14.645 & $\mathbf{4 0 . 3 3 3}$ \\
\hline Kasko & 38.462 & 8.138 & 1.937 & $\mathbf{4 8 . 5 3 7}$ \\
\hline Feuer Industrie & & & 20.648 & $\mathbf{2 0 . 6 4 8}$ \\
\hline Allgemeine Sturm & & 36.695 & 3.544 & $\mathbf{4 0 . 2 3 8}$ \\
\hline VGV Feuer & 5.622 & & & $\mathbf{5 . 6 2 2}$ \\
\hline VGV Leitungswasser & 13.519 & & & $\mathbf{1 3 . 5 1 9}$ \\
\hline VGV Sturm & 109.358 & & & $\mathbf{1 0 9 . 3 5 8}$ \\
\hline VGV Elementar & 25.901 & & & $\mathbf{2 5 . 9 0 1}$ \\
\hline SUMME & $\mathbf{1 9 3 . 4 4 2}$ & $\mathbf{6 7 . 0 1 7}$ & $\mathbf{4 0 . 9 3 4}$ & $\mathbf{3 0 1 . 3 9 2}$ \\
\hline
\end{tabular}

Tabelle A.4: Allokationsergebnis in der Standalone-Betrachtung (16 isolierte Teilunternehmen) 


\section{Anhang C:}

\section{Übersicht der verwendeten Wahrscheinlichkeitsverteilungen}

\section{Burr-Verteilung}

\begin{tabular}{|l|l|l|}
\hline Parameter: & Dichtefunktion: & Verteilungsfunktion: \\
\hline$\alpha \in \mathfrak{R}^{+}$ & $\mathrm{f}(\mathrm{x})=\frac{\alpha \cdot \gamma \cdot(\mathrm{x} / \theta)^{\gamma}}{\mathrm{x} \cdot\left[1+(\mathrm{x} / \theta)^{\alpha+1}\right]}$ & $\mathrm{F}(\mathrm{x})=1-\left(\frac{1}{1+(\mathrm{x} / \theta)^{\gamma}}\right)^{\alpha}$ \\
$\theta \in \mathfrak{R}^{+}$ & $0<\mathrm{x}<\infty$ & $0<\mathrm{x}<\infty$ \\
$\gamma \in \mathfrak{R}^{+}$ & & \\
\hline
\end{tabular}

\section{Inverse Burr-Verteilung}

\begin{tabular}{|l|l|l|}
\hline Parameter: & Dichtefunktion: & Verteilungsfunktion: \\
\hline $\begin{array}{l}\tau \in \mathfrak{R}^{+} \\
\theta \in \mathfrak{R}^{+} \\
\gamma \in \mathfrak{R}^{+}\end{array}$ & $\mathrm{f}(\mathrm{x})=\frac{\tau \cdot \gamma \cdot(\mathrm{x} / \theta)^{\gamma \cdot \tau}}{\mathrm{x} \cdot\left[1+(\mathrm{x} / \theta)^{\gamma}\right]^{\tau+1}}$ & $\mathrm{~F}(\mathrm{x})=\left(\frac{(\mathrm{x} / \theta}{\left.1+(\mathrm{x} /)^{\gamma}\right)^{\gamma}}\right)^{\tau}$ \\
& $0<\mathrm{x}<\infty$ & $0<\mathrm{x}<\infty$ \\
\hline
\end{tabular}

\section{LogLogistik-Verteilung}

\begin{tabular}{|l|l|l|}
\hline Parameter: & Dichtefunktion: & Verteilungsfunktion: \\
\hline $\begin{array}{l}\theta \in \mathfrak{R}^{+} \\
\gamma \in \mathfrak{R}^{+}\end{array}$ & $\mathrm{f}(\mathrm{x})=\frac{\gamma \cdot(\mathrm{x} / \theta)^{\gamma}}{\mathrm{x} \cdot\left[1+(\mathrm{x} / \theta)^{\gamma}\right]^{2}}$ & $\mathrm{~F}(\mathrm{x})=\frac{(\mathrm{x} / \theta)^{\gamma}}{1+(\mathrm{x} / \theta)^{\gamma}}$ \\
& $0<\mathrm{x}<\infty$ & $0<\mathrm{x}<\infty$ \\
\hline
\end{tabular}

\section{Poissonverteilung}

\begin{tabular}{|l|l|l|}
\hline Parameter: & Dichtefunktion: & Verteilungsfunktion: \\
\hline$\lambda \in \mathfrak{R}^{+}$ & $\mathrm{f}(\mathrm{x})=\frac{\lambda^{\mathrm{x}} \mathrm{e}^{-\lambda}}{\mathrm{x} !}$ & $\mathrm{F}(\mathrm{x})=\sum_{\mathrm{k}=0}^{\mathrm{x}} \frac{\lambda^{\mathrm{k}} \mathrm{e}^{-\lambda}}{\mathrm{k} !}$ \\
& $\mathrm{x}=1,2, \ldots \infty$ & $\mathrm{x}=1,2, \ldots \infty$ \\
& $\lambda=\mathrm{E}(\mathrm{X})=\operatorname{Var}(\mathrm{X})$ & $\lambda=\mathrm{E}(\mathrm{X})=\operatorname{Var}(\mathrm{X})$ \\
\hline
\end{tabular}




\section{Normalverteilung}

\begin{tabular}{|l|l|l|}
\hline Parameter: & Dichtefunktion: & Verteilungsfunktion: \\
\hline$\mu \in \Re$ & $\mathrm{f}(\mathrm{x})=\frac{1}{\sigma \sqrt{2 \pi}} \mathrm{e}^{-\frac{(\mathrm{x}-\mu)^{2}}{2 \sigma^{2}}}$ & $\mathrm{~F}(\mathrm{x})=\frac{1}{\sigma \sqrt{2 \pi}} \int_{-\infty}^{\mathrm{x}} \mathrm{e}^{-\frac{(\mathrm{t}-\mu)^{2}}{2 \sigma^{2}}} \mathrm{dt}$ \\
$\sigma \in \Re^{+}$ & $-\infty<\mathrm{x}<\infty$ & $-\infty<\mathrm{x}<\infty$ \\
& $\mu=\mathrm{E}(\mathrm{X})$ & $\mu=\mathrm{E}(\mathrm{X})$ \\
& $\sigma=\sqrt{\operatorname{Var}(\mathrm{X})}$ & $\sigma=\sqrt{\operatorname{Var}(\mathrm{X})}$ \\
& &
\end{tabular}

\section{Lognormalverteilung}

\begin{tabular}{|c|c|c|}
\hline Parameter: & Dichtefunktion: & Verteilungsfunktion: \\
\hline $\begin{array}{l}\mu_{\log N} \in \Re \\
\sigma_{\log N} \in \Re^{+}\end{array}$ & $\begin{array}{l}\mathrm{f}(\mathrm{x})=\frac{1}{\sigma_{\log N}(\mathrm{x}) \sqrt{2 \pi}} \mathrm{e}^{-\frac{\left(\ln \mathrm{x}-\mu_{\log N}\right)^{2}}{2 \sigma_{\log N}^{2}}} \\
x>0 \\
\mu_{\log N}=\ln \left[\frac{\mathrm{E}(\mathrm{X})^{2}}{\sqrt{\mathrm{E}(\mathrm{X})^{2}+\operatorname{Var}(\mathrm{X})}}\right] \\
\sigma_{\log N}=\ln \left[\frac{\operatorname{Var}(\mathrm{X})}{\mathrm{E}(\mathrm{X})^{2}}+1\right]\end{array}$ & $\begin{array}{l}F(x)=\int_{0}^{x} \frac{1}{\sigma_{\log N}(t) \sqrt{2 \pi}} e^{-\frac{\left(\ln t-\mu_{\log N}\right)^{2}}{2 \sigma_{\log N}^{2}}} d t \\
\mu_{\log N}=\ln \left[\frac{E(X)^{2}}{\sqrt{E(X)^{2}+\operatorname{Var}(X)}}\right] \\
\sigma_{\log N}=\ln \left[\frac{\operatorname{Var}(X)}{E(X)^{2}}+1\right]\end{array}$ \\
\hline
\end{tabular}

\section{Negative Binomialverteilung}

\begin{tabular}{|l|l|l|}
\hline Parameter: & Dichtefunktion: & Verteilungsfunktion: \\
\hline $\mathrm{n} \in \mathfrak{R}^{+}$ & $\mathrm{f}(\mathrm{x})=\frac{\Gamma(\mathrm{x}+\mathrm{n})}{\Gamma(\mathrm{x}+1) \Gamma(\mathrm{n})} \mathrm{q}^{\mathrm{x}}(1-\mathrm{q})^{\mathrm{n}}$ & $\mathrm{F}(\mathrm{x})=\sum_{\mathrm{k}=0}^{\mathrm{x}} \frac{\Gamma(\mathrm{k}+\mathrm{n})}{\Gamma(\mathrm{k}+1) \Gamma(\mathrm{n})} \mathrm{q}^{\mathrm{k}}(1-\mathrm{q})^{\mathrm{n}}$ \\
$0<\mathrm{q}<1$ & $\mathrm{x}=1,2, \ldots \infty$ & $\mathrm{x}=1,2, \ldots \infty$ \\
& mit: $\Gamma(\mathrm{x})=\int_{0}^{\infty} \mathrm{t}^{\mathrm{x}-1} \mathrm{e}^{-\mathrm{t}} \mathrm{dt}$ & mit: $\Gamma(\mathrm{x})=\int_{0}^{\infty} \mathrm{t}^{\mathrm{x}-1} \mathrm{e}^{-\mathrm{t}} \mathrm{dt}$ \\
& $\mathrm{q}=1-\frac{\mathrm{E}(\mathrm{X})}{\operatorname{Var}(\mathrm{X})}$ & $\mathrm{q}=1-\frac{\mathrm{E}(\mathrm{X})}{\operatorname{Var}(\mathrm{X})}$ \\
& $\mathrm{n}=\frac{[\mathrm{E}(\mathrm{X})]^{2}}{\operatorname{Var}(\mathrm{X})-\mathrm{E}(\mathrm{X})}$ & $\mathrm{n}=\frac{[\mathrm{E}(\mathrm{X})]^{2}}{\operatorname{Var}(\mathrm{X})-\mathrm{E}(\mathrm{X})}$ \\
\hline
\end{tabular}


Verallgemeinerte Pareto A Verteilung

\begin{tabular}{|c|c|c|}
\hline Parameter: & Dichtefunktion: & Verteilungsfunktion: \\
\hline $\begin{array}{l}\eta \in \mathfrak{R}^{+} \\
\omega \in \Re_{0}^{+} \\
\nu \in \Re\end{array}$ & $\begin{array}{l}\text { Für } \omega=0: \\
f(x)=\frac{1}{\eta} \cdot e^{\left(-\frac{(x-v)}{\eta}\right)} \\
\text { Für } \omega \neq 0: \\
f(x)=\frac{1}{\eta}\left(1+\frac{\omega}{\eta}(x-v)\right)^{-\left(\frac{1}{\omega}+1\right)} \\
x>v\end{array}$ & $\begin{array}{l}\text { Für } \omega=0: \\
F(x)=1-e^{\left(-\frac{(x-v)}{\eta}\right)} \\
\text { Für } \omega \neq 0: \\
F(x)=1-\left[1+\frac{\omega}{\eta}(x-v)\right]^{-\frac{1}{\omega}} \\
x>v\end{array}$ \\
\hline
\end{tabular}

\section{Verallgemeinerte Pareto B Verteilung}

\begin{tabular}{|l|l|l|}
\hline Parameter: & Dichtefunktion: & Verteilungsfunktion: \\
\hline$\alpha \in \mathfrak{R}^{+}$ & $\mathrm{f}(\mathrm{x})=\frac{\Gamma(\alpha+\tau)}{\Gamma(\alpha) \cdot \Gamma(\tau)} \cdot \frac{\theta^{\alpha} \mathrm{x}^{\tau-1}}{(\mathrm{x}+\theta)^{\alpha+\tau}}$ & $\mathrm{F}(\mathrm{x})=\int_{0}^{\mathrm{x}} \frac{\Gamma(\alpha+\tau)}{\Gamma(\alpha) \cdot \Gamma(\tau)} \cdot \frac{\theta^{\alpha} \mathrm{t}^{\tau-1}}{(\mathrm{t}+\theta)^{\alpha+\tau}} \mathrm{dt}$ \\
$\theta \in \mathfrak{R}^{+}$ & mit: $\Gamma(\mathrm{x})=\int_{0}^{\infty} \mathrm{t}^{\mathrm{x}-1} \mathrm{e}^{-\mathrm{t}} \mathrm{dt}$ & mit: $\Gamma(\mathrm{x})=\int_{0}^{\infty} \mathrm{t}^{\mathrm{x}-1} \mathrm{e}^{-\mathrm{t}} \mathrm{dt}$ \\
& $0<\mathrm{x}<\infty$ & $0<\mathrm{x}<\infty$ \\
\hline
\end{tabular}




\section{Anhang D:}

\section{Konvergenzverhalten und Stabilität der Simulationsergebnisse}

Hinsichtlich der Festlegung einer Mindestgröße für den Simulationsumfang ist eine Untersuchung des Konvergenzverhaltens und der Stabilität der Modellergebnisse hilfreich. ${ }^{1119}$ Das Ziel der nachfolgenden Darstellung ist eine visuelle Abschätzung desjenigen Simulationsumfangs, bei dem die Modellergebnisse einen stabilen Zustand (,steady state“) erreichen. Als Referenzgröße wird das (volatil verlaufende) BruttoAnfalljahrergebnis herangezogen. Bezeichne $\mathrm{n}$ die Anzahl der insgesamt durchgeführten Simulationen und $x_{i}$ mit $i=1, \ldots, n$ die Folge der simulierten Brutto-Anfalljahrergebnisse, so sind der (gleitende) Erwartungswert $\bar{\mu}_{\mathrm{i}}$ und die (gleitende) Standardabweichung $\bar{\sigma}_{i}$ bis zum i-ten Element der Folge definiert als:
(A.1)
$\bar{\mu}_{\mathrm{i}}=\frac{1}{\mathrm{i}} \sum_{\mathrm{j}=1}^{\mathrm{i}} \mathrm{x}_{\mathrm{j}}$
$\mathrm{i}=1, \ldots, \mathrm{n}$

$$
\bar{\sigma}_{i}=\sqrt{\frac{1}{i-1} \sum_{j=1}^{i}\left(x_{j}-\bar{\mu}_{i}\right)^{2}}
$$$$
\mathrm{i}=2, \ldots, \mathrm{n}
$$

Die Ergebnisse werden in Abbildung A.1 zunächst für $n=5.000$ graphisch dargestellt.

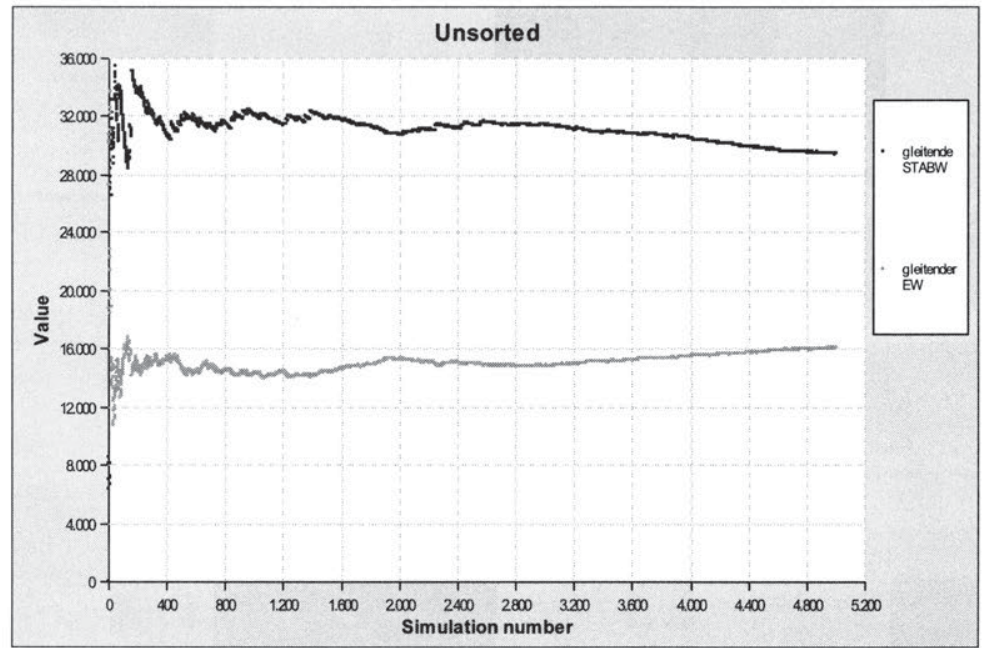

Abbildung A.1: Konvergenzverhalten des Brutto-Anfalljahrergebnisses für $\mathbf{n}=\mathbf{5 . 0 0 0}$

${ }^{1119}$ Vgl. hierzu und im Folgenden LiEBL (1995), S. 156-166 und КоСH (1994), S. 45-54. 
Die Modellergebnisse zeigen in der Anlaufphase eine starke Streuung. Mit wachsendem Simulationsumfang ist eine zunehmende Konvergenz festzustellen. Allerdings weisen die Ergebnisse bei 5.000 Simulationsläufen noch keine hinreichende Stabilität auf. Abbildung A.2 zeigt das Konvergenzverhalten des Modells für eine Simulationsanzahl von $\mathrm{n}=30.000$.

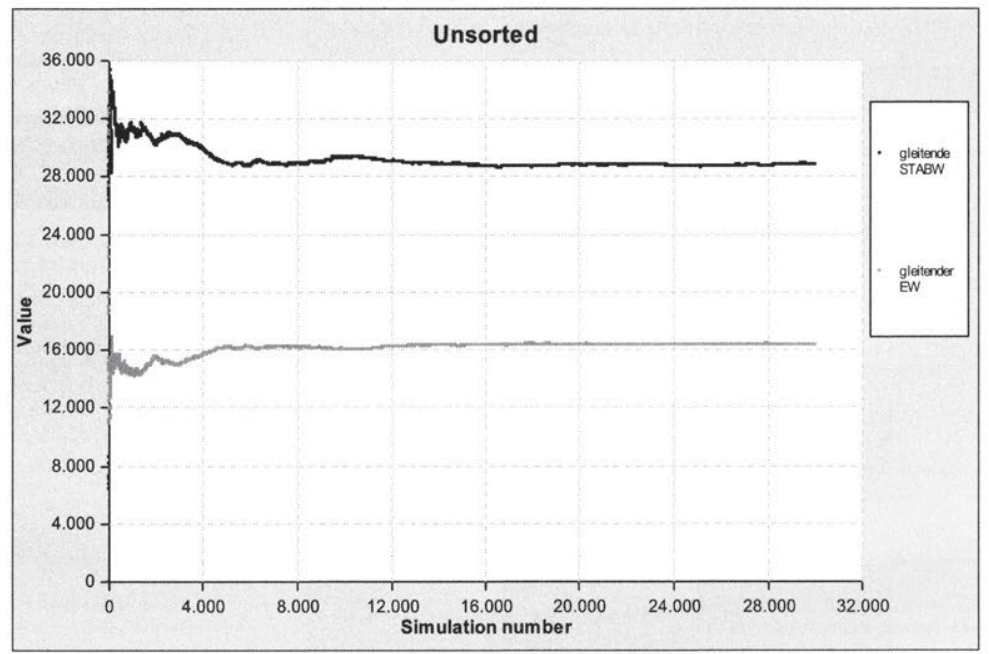

Abbildung A.2: Konvergenzverhalten des Brutto-Anfalljahrergebnisses für $\mathbf{n}=\mathbf{3 0 . 0 0 0}$

Es wird deutlich, dass sich die Ergebnisse bereits bei ca. 16.000 Simulationen weitgehend stabilisiert haben. Der in dieser Arbeit gewählte Simulationsumfang von $\mathrm{n}=30.000$ erscheint vor diesem Hintergrund ausreichend. Mit Blick auf das im Rahmen der Risikokapitalermittlung durchgängig unterstellte Sicherheitsniveau von $1-\varepsilon=99,5 \%$ ist außerdem eine hinreichende Besetzung des Randbereichs (Tails) der Ergebnisverteilung gewährleistet. 


\section{Anhang E:}

\section{Struktur des Randbereichs der Bruttoergebnisverteilung}

Bei der Bestimmung des Conditional Value-at-Risk (CVaR) wird im Rahmen dieser Arbeit ein Sicherheitsniveau von 1- $\varepsilon=99,5 \%$ zugrunde gelegt. Für den gewählten Simulationsumfang von $\mathrm{n}=30.000$ errechnet sich der CVaR demzufolge als Erwartungswert über die 150 schlechtesten Ergebnisszenarien. Nachfolgend soll am Beispiel der Verteilung des Brutto-Anfalljahrergebnisses ausgewählter Versicherungszweige verdeutlicht werden, dass der Brutto-Risikokapitalbedarf des Modellunternehmens maßgeblich durch Schäden aus Naturgefahrereignissen beeinflusst wird. In Abbildung A.3 wird der Randbereich der Bruttoergebnisverteilung szenarioweise zwischen dem $0 \%$ - und $0,5 \%$-Perzentil dargestellt.

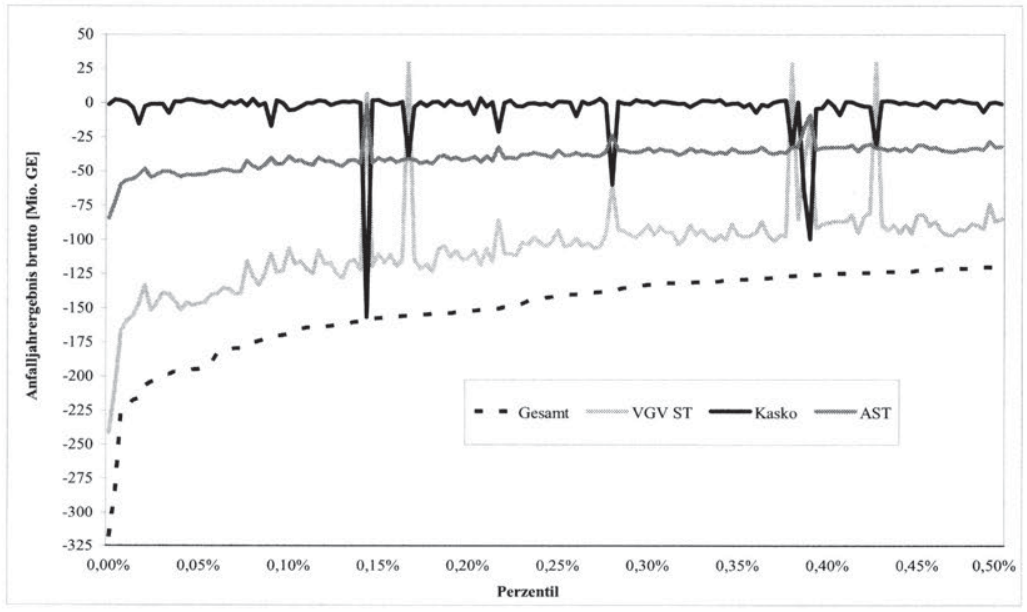

Abbildung A.3: Tailstruktur der Verteilung des Brutto-Anfalljahrergebnisses

Das Gesamtergebnis (gestrichelter Linienzug) wird in den 150 schlechtesten Szenarien stark durch die hierzu korrespondierenden Verluste in den Sparten „VGV Sturm“ und „Allgemeine Sturm“ bestimmt. In denjenigen Simulationsläufen, in denen keine Ergebnisbelastungen durch Sturmereignisse zu verzeichnen sind, wird beispielsweise das Hagelrisiko der Sparte „Kasko“ oder auch das Überschwemmungsrisiko in der Sparte „VGV Elementar" (nicht abgebildet) schlagend. Wenngleich in Abbildung A.3 im Interesse der Übersichtlichkeit nur ausgewählte Versicherungszweige dargestellt sind, wird dennoch deutlich, dass das Verlustpotenzial anderer (volatiler) Versicherungszweige (z.B. „Kraftfahrt-Haftpflicht“" oder „Feuer Industrie“) durch das Schadenpotenzial aus Naturgefahrereignissen überlagert wird. 


\section{Literaturverzeichnis}

Acerbi, C.; Nordio, C.; SirTori, C. (2001): Expected Shortfall as Tool for Financial Risk Management, Arbeitspapier, Abaxbank, Mailand 2001.

ACERBI, C.; TASCHE, D. (2002a): On the coherence of Expected Shortfall, In: Journal of Banking and Finance, 26. Jg. (2002), H. 7, S. 1487-1503.

ACERBI, C.; TASCHE, D. (2002b): Expected Shortfall: A Natural Coherent Alternative to Value at Risk, In: Economic Notes, 31. Jg. (2002), H. 2, S. 379-388.

ADAM, D. (1996): Planung und Entscheidung, 4. Aufl., Wiesbaden (Gabler), 1996.

ALBACH, H. (2001): Shareholder Value und Unternehmenswert - Theoretische Anmerkungen zu einem aktuellen Thema, In: Zeitschrift für Betriebswirtschaft, 71. Jg. (2001), H. 6, S. 643-674.

AlBreChT, P. (1982): Gesetze der großen Zahlen und Ausgleich im Kollektiv - Bemerkungen zu Grundlagen der Versicherungsproduktion, In: Zeitschrift für die gesamte Versicherungswissenschaft, 71. Jg. (1982), S. 501-538.

ALBRECHT, P. (1985): Konstruktion und Analyse stochastischer Gesamtmodelle des Versicherungsgeschäfts auf der Grundlage risiko- und finanzierungstheoretischer Ansätze, Mannheim, 1985 (= zugl. Habil. Univ. Mannheim 1985).

ALBRECHT, P. (1987a): Die Versicherungsproduktion - eine Kuppelproduktion bei Risiko, In: Zeitschrift für Betriebswirtschaft, 57. Jg. (1987), H. 3, S. 316-328.

AlBRECHT, P. (1987b): Ausgleich im Kollektiv und Verlustwahrscheinlichkeit, In: Zeitschrift für die gesamte Versicherungswissenschaft, 76. Jg. (1987), S. 95-117.

ALBRECHT, P. (1991): Kapitalmarkttheoretische Fundierung der Versicherung, In: Zeitschrift für die gesamte Versicherungswissenschaft, 80. Jg. (1991), S. 499-530.

ALBRECHT, P. (1992): Zur Risikotranformationstheorie der Versicherung: Grundlagen und ökonomische Konsequenzen, Karlsruhe (VVW), 1992.

AlBreCHT, P. (1994a): Zur Konzeptualisierung von Risiko und Chance mit den Anwendungen in den Finanz- und Versicherungsmärkten, Aus: Hübner, U.; Helten, E.; Albrecht, P. (Hrsg.): Recht und Ökonomie der Versicherung: Festschrift für Egon Lorenz zum 60. Geburtstag, Karlsruhe (VVW), 1994, S. 1-22.

ALBRECHT, P. (1994b): Dimensionen des versicherungstechnischen Risikos, Aus: Hesberg, D.; Nell, M.; Schott, W. (Hrsg.): Risiko, Versicherung, Markt: Festschrift für Walter Karten zur Vollendung des 60. Lebensjahres, Karlsruhe (VVW), 1994, S. 325-339. 
AlBRECht, P. (1994c): Gewinn und Sicherheit als Ziele der Versicherungsunternehmen: Bernoulli-Prinzip vs. Safety-First-Prinzip, Aus: Schwebler, R. (Hrsg.): Dieter Farny und die Versicherungswissenschaft, Karlsruhe (VVW), 1994, S. 1-18.

AlbreChT, P. (1995): Ansätze eines finanzwirtschaftlichen Portefeuille-Managements und ihre Bedeutung für Kapitalanlage- und Risikopolitik von Versicherungsunternehmen, Karlsruhe (VVW), 1995.

Albrecht, P. (1998): Risikoadjustierte Performancesteuerung in der Schadenversicherung, Aus: Oehler, A. (Hrsg.): Credit Risk und Value-at-Risk Alternativen: Herausforderungen für das Management, Stuttgart (Schäffer-Poeschel), 1998, S. 229257.

Albrecht, P. (2001): Asset Liability Management bei Versicherungen, In: Mannheimer Manuskripte zu Risikotheorie, Portfolio Management und Versicherungswirtschaft, Nr. 134, Mannheim, 2001.

ALBRECHT, P. (2003): Zur Messung von Finanzrisiken, In: Mannheimer Manuskripte zu Risikotheorie, Portfolio Management und Versicherungswirtschaft, Nr. 143, Mannheim, 2003.

AlBRECHT, P.; BÄHRLE, H. F.; KÖNIG, A. (1997): Value-At-Risk: Eine risikotheoretische Analyse der konzeptionellen Grundlagen mit Folgerungen für die Risikokontrolle der Kapitalanlage von Versicherungsunternehmen, In: Zeitschrift für die gesamte Versicherungswissenschaft, 86. Jg. (1997), S. 80-101.

ALBREChT, P.; KLETT, T. (2004): Referenzpunktbezogene risikoadjustierte Performancemaße: Theoretische Grundlagen, In: Mannheimer Manuskripte zu Risikotheorie, Portfolio Management und Versicherungswirtschaft, Nr. 158, Mannheim, 2004.

ALBRECHT, P.; KORYCIORZ, S. (2000): Value-at-Risk für Versicherungsunternehmen: Konzeptionelle Grundlagen und Anwendungen, Aus: Johanning, L.; Rudolph, B. (Hrsg.): Handbuch Risikomanagement Band 2: Risikomanagement in Banken, Asset-Management-Gesellschaften, Versicherungs- und Industrieunternehmen, Bad Soden/Ts. (Uhlenbruch), 2000, S. 1105-1129.

AlBreCht, P.; KoRYCIORZ, S. (2003): Bestimmung des Conditional Value-at-Risk (CVaR) bei Normal- bzw. Lognormalverteilung, In: Mannheimer Manuskripte zu Risikotheorie, Portfolio Management und Versicherungswirtschaft, Nr. 142, Mannheim, 2003.

AlBRECHT, P.; KORYCIORZ, S. (2004): Methoden der risikobasierten Kapitalallokation im Versicherungs- und Finanzwesen, In: Zeitschrift für die gesamte Versicherungswissenschaft, 93. Jg. (2004), S. 123-160.

AlBRECHT, P.; MAURER, R. (2002): Investment- und Risikomanagement: Modelle, Methoden, Anwendungen, Stuttgart (Schäffer-Poeschel), 2002. 
Albrecht, P.; MAURER, R.; RUCKPAUL, U. (2001): Shortfall-Risks of Stocks in the Long Run, In: Financial Markets and Portfolio Manangement, 15. Jg. (2001), H. 4, S. 481-499.

Albrecht, P.; Schradin, H. (1992): Erfolgsorientierte Steuerung des Versicherungsgeschäfts, Aus: Spremann, K.; Zur, E. (Hrsg.): Controlling: Grundlagen Informationssysteme - Anwendungen, Wiesbaden (Gabler), 1992, S. 571-596.

ALBRECHT, P.; SCHWAKE, E. (1988): Risiko, Versicherungstechnisches, Aus: Farny, D.; Helten, E.; Koch, P.; Schmidt, R. (Hrsg.): Handwörterbuch der Versicherung (HdV), Karlsruhe (VVW), 1988, S. 651-657.

AlBRECHT, P.; ZimMERMANN, J. (1992): Risikotheoretische Analyse des Versicherungsgeschäfts auf der Grundlage eines stochastischen Gesamtmodells, In: Transactions of the 24th International Congress of Actuaries (TICA), Montréal, 1992, S. 27-41.

AlfermanN, B.; RADTKE, M.; ReICH, A. (2004): Controlling, Aus: Radtke, M.; Schmidt, K. (Hrsg.): Handbuch zur Schadenresevierung, Karlsruhe (VVW), 2004, S. 65-70.

ANGERER, A. (1994): Zur Abzinsung der Rückstellung für noch nicht abgewickelte Versicherungsfälle, Aus: Schwebler, R. (Hrsg.): Dieter Farny und die Versicherungswissenschaft, Karlsruhe (VVW), 1994, S. 35-44.

AON RÜCK (2004): Solvency II - Anforderungen der Finanzaufsicht an die Versicherungswirtschaft, Hamburg, 2004.

APARICIO, J.; KESKINER, E. (2004): A Review of Operational Risk: Quantitative Methodologies within the Basel-II Framework, Arbeitspapier, Accenture Technology Labs, 2004.

Australian Prudential Regulation Authority (2002): Prudential Standard GPS 210: Liability Valuation for General Insurers, o.O., 2002.

ARNOLDUSSEN, L. (1991): Finanzwirtschaftliche Effekte von Rückversicherungsverträgen in der Schaden- und Unfallversicherung, Teil 1: Textteil, Karlsruhe (Josef Eul), 1991 (= zugl. Diss. Univ. Köln 1990).

ARTZNER, P.; DelBaEN, F.; EBER, J.-M.; HEATH, D. (1997): Thinking Coherently, In: Risk, 10. Jg. (1997), H. 11, S. 68-71.

ARTZner, P.; Delbaen, F.; Eber, J.-M.; Heath, D. (1999): Coherent Measures of Risk, In: Mathematical Finance, 9. Jg. (1999), H. 3, S. 203-228.

ARTZner, P.; Delbaen, F.; Eber, J.-M. (2002): Coherent Multiperiod Risk Measurement, Arbeitspapier, 2002. 


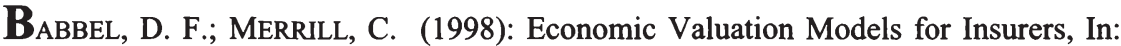
North American Actuarial Journal, July 1998, S. 1-17.

BACH, W. (2003): Erfolgsorientierte Underwritingsteuerung auf Basis von Risikokapitalüberlegungen - ein pragmatischer Modellansatz, In: Zeitschrift für Versicherungswesen, 54. Jg. (2003), H. 21, S. 642-645.

BAETGE, J.; KIRSCH, H.-J.; ThIELE, S. (2003): Bilanzen, 7. Aufl., Düsseldorf (IDW), 2003.

BÄHRLE, H. F. (1997): Risiko-Controlling des Einsatzes derivativer Finanzinstrumente in der Kapitalanlage von Versicherungsunternehmen, Karlsruhe (VVW), 1997 (= zugl. Diss. Univ. Mannheim 1997).

BALCI, O. (1994): Validation, verification, and testing techniques throughout the life cycle of a simulation study, In: Annals of Operations Research, 53. Jg. (1994), S. 121-173.

BAMBERG, G.; DORFLeITNER, G.; GLAAB, H. (2005): Risikobasierte Kapitalallokation in Versicherungsunternehmen unter Verwendung des Co-Semivarianz-Prinzips, Aus: SPREMANN, K. (Hrsg.): Versicherungen im Umbruch: Werte schaffen, Risiken managen, Kunden gewinnen, Berlin u.a. (Springer), 2005, S. 399-414.

BAmBerg, G.; COENENBerG, A. G. (2002): Betriebswirtschaftliche Entscheidungslehre, 11. Aufl., München (Vahlen), 2002.

BAMBERG, G.; SPREMANN, K. (1989): Agency Theory: Information and Incentives, Berlin u.a. (Springer), 1989.

BARTH, M. M. (2000): A Comparison Of Risk-Based Capital Standards under the Expected Policyholder Deficit and the Probability of Ruin Approaches, In: The Journal of Risk and Insurance, 67. Jg. (2000), S. 397-414.

BASLER AUSSCHUSS FÜR BANKENAUFSICHT (2003): Überblick über die Neue Basler Eigenkapitalvereinbarung, Konsultationspapier, Basel, 2003.

BAUMeISTER, A. (2003): Risikoadjustierte Performancemaße, In: Die Betriebswirtschaft, 63. Jg. (2003), H. 2, S. 221-226.

BAWA, V. S. (1982): Stochastic Dominance: A Research Bibliography, In: Management Science, 28. Jg. (1982), S. 698-712.

Beard, R.E.; PentikäInen, T.; Pesonen, E. (1977): Risk Theory - The Stochastic Basis of Insurance, Second Edition, London (Chapman and Hall), 1977.

BEECK, H.; JOHANNING, L.; RUDOLPH, B. (1999): Value-at-Risk-Limitstrukturen zur Steuerung und Begrenzung von Marktrisiken im Aktienbereich, In: OR Spektrum, 21. Jg. (1999), H. 1/2, S. 259-286. 
Berens, W.; DelfMANN, W.; SCHMITTING, W. (2004): Quantitative Planung, 4. Aufl., Stuttgart (Schäffer-Poeschel), 2004.

BERGER, J.; POSTAI, B. (2004): Ertragssteigerung durch besseres Schadenmanagement, In: Versicherungswirtschaft, 59. Jg. (2004), H. 10, S. 759-762.

BERKENKOPF, K.; FROMME, H. (2004): Großschaden mit unbeschränkter Haftung, In: Financial Times Deutschland, 16.11.2004.

BERZ, G. (1999): Naturkatastrophen an der Wende zum nächsten Jahrhundert Trends, Schadenpotentiale und Handlungsoptionen der Versicherungswirtschaft, In: Zeitschrift für die gesamte Versicherungswissenschaft, 88. Jg. (1999), S. 427-442.

BITZ, H. (2000): Risikomanagement nach KonTraG - Einrichtung eines Frühwarnsystems zur Effizienzsteigerung und zur Vermeidung persönlicher Haftung, Stuttgart (Schäffer-Poeschel), 2000.

BLEUEL, H.-H.; SCHMITTING, W. (2000): Konzeptionen eines Risikomanagements im Rahmen der internationalen Geschäftstätigkeit, Aus: Berens, W.; Born, A.; Hoffjan, A. (Hrsg.): Controlling internationaler Unternehmen, Stuttgart (Schäffer-Poeschel), 2000, S. 65-122.

BLEYMÜLLER, J.; GEHLERT, G.; GÜLICHER, H. (2002): Statistik für Wirtschaftswissenschaftler, 13. Aufl., München (Vahlen), 2002.

BoETIUS, J. (1996): Handbuch der versicherungstechnischen Rückstellungen, Handels- und Steuerbilanzrecht der Versicherungsunternehmen, Köln (Dr. Otto Schmidt KG), 1996.

BoHLEY, P. (2000): Statistik, 7. Aufl., München (Oldenbourg), 2000.

BomHaRD, N. von (2004): "Wir wollen in drei Jahren an die Spitze", In: Süddeutsche Zeitung, Nr. 296 vom 21.12.2004, S. 22.

BosSEL, H. (1992): Modellbildung und Simulation - Konzepte, Verfahren und Modelle zum Verhalten dynamischer Systeme, Braunschweig u.a. (Vieweg), 1992.

BRACHINGER, H. W.; WEBER, M. (1997): Risk as a primitive: a survey of perceived risk measures, In: OR Spektrum, 19. Jg. (1997), H. 4, S. 235-250.

BRAEB, P. (1960): Versicherung und Risiko, Wiesbaden (Gabler), 1960.

Braeb, P. (1969): Das Problem der Großschäden unter dem Aspekt der Risikotheorie, In: Zeitschrift für die gesamte Versicherungswissenschaft, 58. Jg. (1969), S. 211224.

BRAMMERTZ, W. (1991): Datengrundlage und Analyseinstrumente fuir das Risikomanagement eines Finanzinstitutes, Eggersriet, 1991 (= zugl. Diss. Univ. Zürich 1991). 
BREUER, W. (1992): Kapitalmarkttheorie und Versicherungswissenschaft, In: Zeitschrift für die gesamte Versicherungswissenschaft, 81. Jg. (1992), S. 617-629.

BROHM, A. (2002): Holistische Unternehmensmodelle in der Schaden- und Unfallversicherung, Karlsruhe (VVW), 2002 (= zugl. Diss. Univ. Mannheim 2001).

BROHM, A.; KÖNIG, A. (2004): Anforderungen an die Abbildung von Versicherungsunternehmen im Rahmen mathematisch-ökonomischer Modelle in der Unternehmenspraxis, In: Zeitschrift für die gesamte Versicherungswissenschaft, 93. Jg. (2004), S. 3-16.

BUHK, A. (2001): Deutsche Industrieversicherung - Wie geht es weiter?, In: Versicherungswirtschaft, 56. Jg. (2001), H. 12, S. 898-902.

BÜHLMANN, H. (1970): Mathematical Methods in Risk Theory, Berlin (Springer), 1970.

BüHLManN, H.; STRAUB, E. (1970): Glaubwürdigkeit für Schadensätze, In: Mitteilungen der Vereinigung Schweizerischer Versicherungsmathematiker, 1970, H. 70, S. 111-133.

BÜHNER, R. (2004): Betriebswirtschaftliche Organisationslehre, 10. Aufl., München u.a. (Oldenbourg), 2004.

BÜHNER, R. (2005): Personalmanagement, 3. Aufl., München u.a. (Oldenbourg), 2005.

BUNDESANSTALT FÜR FINANZDIENSTLEISTUNGSAUFSICHT (2005): Geschäftsbericht 2003, Teil B, Bonn, 2005.

BUSSON, M.; RUB, J.; ZWIESLER, H.-J. (2000): Modernes Asset Liability Management, In: Versicherungswirtschaft, 55. Jg. (2000), H. 2, S. 104-109.

Charpentier, A. (2003): Tail distribution and dependence measures, XXXIV International ASTIN Colloquium, Berlin, 2003.

COENENBERG, A. (2003): Kostenrechnung und Kostenanalyse, 5. Aufl., Stuttgart (Schäffer-Poeschel), 2003.

COPELAND, T.; KOlleR, T.; MURRIN, J. (1998): Unternehmenswert: Methoden und Strategien für eine wertorientierte Unternehmensführung, 2. Aufl., Frankfurt u.a. (Campus), 1998.

CORELl, F.-C. (1998a): Value Based Management (VBM): Teil I: Zielsetzung und Einsatzmöglichkeit in der Versicherungswirtschaft, In: Der Aktuar, 4. Jg. (1998), H. 1, S. 27-34. 
CORELl, F.-C. (1998b): Value Based Management (VBM): Teil II: Fallstudie Schaden- und Unfallversicherer, In: Der Aktuar, 4. Jg. (1998), H. 2, S. 66-78.

CORELL, F.-C. (1999): Risikomanagement und Unternehmenswert von Versicherungen: Die Wertrelevanz der Kapitalanlage, Aus: Johanning, L.; Rudolph, B. (Hrsg.): Handbuch Risikomanagement, Band 2: Risikomanagement in Banken, AssetManagement-Gesellschaften, Versicherungs- und Industrieunternehmen, Bad Soden/Ts. (Uhlenbruch), 2000, S. 1131-1171.

CosTONIS, M.; KNIPP, S. (2000): In der Schadenbearbeitung stecken noch Reserven für mehr Profitabilität, In: Versicherungswirtschaft, 55. Jg. (2000), H. 1, S. 47-50.

CotTIN, C.; KURZ, A. (2003): Asset-Liabilitiy-Management in der Lebensversicherung: Berichte aus Forschung und Lehre, Nr. 16, Fachbereich Mathematik und Technik, Fachhochschule Bielefeld, 2003.

COX, J. C.; INGERSOLL, J. E.; Ross, S. A. (1985a): An Intertemporal General Equilibrium Model of Asset Prices, In: Econometrica, 53. Jg. (1985), S. 363-384.

CoX, J. C.; INGERSOLL, J. E.; Ross, S. A. (1985b): A Theory of the Term Structure of Interest Rates, In: Econometrica, 53. Jg. (1985), S. 385-407.

Cummins, J. D. (2000): Allocation of Capital in Insurance Industry, In: Risk Management and Insurance Review, 3. Jg. (2000), H. 1, S. 7-27.

Cummins, J. D.; Harrington, S. E.; Klein, R. W. (1991): Cycles and Crisis in Property/Casualty Insurance: Causes and Implications for Public Policy, In: Journal of Insurance Regulation, 10. Jg. (1991), S. 50-93.

Daykin, C. D.; PentikäInen, T.; Pesonen, M. (1996): Practical Risk Theory for Actuaries, London (Chapman \& Hall), 1996.

Delbaen, F. (2002): Coherent Risk Measures on General Probability Spaces, Aus: Sandmann, K.; Schönbucher, P. J. (Hrsg.): Advances in finance and stochastics: essays in honour of Dieter Sondermann, Berlin (Springer), 2002, S. 1-37.

DenAult, M. (2001): Coherent allocation of risk capital, In: The Journal of Risk, 4. Jg. (2001), H. 1, S. 1-34.

DHAENE, J.; VANDUfFel, S.; TANG, Q. (2004): Solvency capital, risk measures and comonotonicity: a review, Arbeitspapier, K.U. Leuven, 2004.

DINKELBACH, W. (1977): Modell - ein isomorphes Abbild der Wirklichkeit?, Aus: Grochla, E.; Szyperski, N. (Hrsg.): Modell- und computergestützte Unternehmensplanung, Wiesbaden (Gabler), 1977, S. 152-161. 
DIRNBERGER, E.; KNIPP, S. (2003): Noch viel Einsparpotential in der Schadenabwicklung - Restitutions- und Ersatzmanagement noch wenig entwickelt, In: Versicherungswirtschaft, 58. Jg. (2003), H. 14, S. 1098-1100.

DIRNBERGER, E.; KNIPP, S. (2004): Dem Erfolgsbeitrag des Schadenmanagements auf der Spur, In: Versicherungswirtschaft, 59. Jg. (2004), H. 15, S. 1129-1130.

DOTTERWEICH, A. (2004): Wertorientierte Steuerung von Schadenversicherungsunternehmen, Karlsruhe (VVW), 2004 (= zugl. Diss. Univ. Passau 2004).

DRESEL, T. (2003): Allokation von Risikokapital in Banken, Bad Soden/Ts. (Uhlenbruch), 2003 (= zugl. Diss. Univ. München 2003).

EBerts, E. (2002): Strategische stochastische Investmentmodelle für den Kapitalmarkt, Karlsruhe (VVW), 2002 (= zugl. Diss. Univ. Mannheim 2002).

EFRON, B. (1979): Bootstrap Methods: Another look at the jackknife, In: The Annals of Statistics, 7. Jg. (1979), S. 1-26.

EGBERS, B. (2002): Die Solvabilitätsvorschriften im Banken- und Versicherungsaufsichtsrecht, Frankfurt a. M. (Fritz Knapp), 2002 (= zugl. Diss. Univ. Münster 2002).

EICHHORN, W. (1979): Die Begriffe Modell und Theorie in der Wirtschaftswissenschaft, Aus: Raffée, H.; Abel, B. (Hrsg.): Wissenschaftstheoretische Grundfragen der Wirtschaftswissenschaften, München (Vahlen), 1979, S. 60-104.

ELLER, R.; DeUTSCH, H.-P. (1998): Derivate und Interne Modelle: Modernes Risikomanagement, Stuttgart (Schäffer-Poeschel), 1998.

EMBRECHTS, P.; HöING, A.; JURI, A. (2003): Using Copulae to bound the Value-atRisk for functions of dependent risk, In: Finance and Stochastics, 7. Jg. (2003), H. 2, S. 145-167.

EMBRECHTS, P.; KLÜPPELBERG, C.; MiKosCH, T. (2003): Modelling extremal events for insurance and finance, 4th printing, Berlin u.a. (Springer), 2003.

Embrechts, P.; LINDSKog, F.; MCNeIL, A. (2001): Modelling Dependence with Copulas and Applications to Risk Management, Arbeitspapier, ETH Zürich, Departmentof Mathematics, Zürich, 2001.

Embrechts, P.; MCNeIl, A.; StraumanN, D. (1999): Correlation: Pitfalls and Alternatives, In: Risk, 12. Jg. (1999), H. 5, S. 69-71. 
EMBrechts, P.; MCNeIL, A.; StraumanN, D. (2002): Correlation and Dependence in Risk Management: Properties and Pitfalls, Aus: Dempster, M. A. H. (Hrsg.): Risk Management: Value at Risk and Beyond, Cambridge u.a. (Cambridge University Press), 2002, S. 176-223.

ENGELÄNDER, S.; KÖLSCHBACH, J. (2004): Der Internationale Financial Reporting Standard 4 für Versicherungsverträge, In: Versicherungswirtschaft, 59. Jg. (2004), H. 8, S. 574-579.

ENGLAND, P. D.; VERRALL, R. J. (2002): Stochastic claims reserving in general insurance, Arbeitspapier, Institute of Actuaries, London, 2002.

EURICH, A. (2000): Bestandskundenmarketing von Versicherungsunternehmen, Karlsruhe (VVW), 2000 (= zugl. Diss. Univ. Köln 2000).

EURICH, A.; HÄUSELE, S. (2001): Umsetzung einer Strategie der Marktsegmentierung im Privatkundengeschäft, Aus: Hallmann, T.; Kirchner, W. (Hrsg.): Reader zum Thema Controlling in Versicherungsunternehmen, Band 2, Karlsruhe (VVW), 2001, S. 105-109.

EUROPÄISCHE KOMMISSION (2002a): MARKT/2529/02-DE, Bericht der Arbeitsgruppe Versicherungstechnische Rückstellungen an den VA-Unterausschuss "Solvabilität", Brüssel, 2002.

EUROPÄISCHE KOMMISSION (2002b): MARKT/2535/02-DE, Informationspapier für den Unterausschuss "Solvabilität": Überlegungen zur Form eines künftigen Aufsichtssystems, Brüssel, 2002.

EUROPÄISCHE KOMMISSION (2002c): MARKT/2528/02-DE LV, Bericht der Arbeitsgruppe Lebensversicherungen an den VA-Unterausschuss "Solvabilität", Brüssel, 2002 .

EUROPÄISCHE KOMMISSION (2003a): MARKT/2509/03-DE, Vermerk der Kommissionsdienststellen: Entwurf eines künftigen Aufsichtssystems in der EU - Empfehlungen der Kommissionsdienststellen, Brüssel, 2003.

EUROPÄISCHE KOMMISSION (2003b): MARKT/2539/03-EN, Solvency II - Reflections on the general outline of a framework directive and mandates for further technical work, Brüssel, 2003.

EUROPÄISCHE KOMMISSION (2004a): MARKT/2543/03-EN, Solvency II - Organisation of work, diskussions on pillar I work areas and suggestions of further work on pillar II for CEIOPS, Brüssel, 2004.

EUROPÄISCHE KOMMISSION (2004b): MARKT/2502/04-EN, Solvency II - Further issues for discussion and suggestions for preparatory work for CEIOPS, Brüssel, 2004. 
Fabien, F. (2003): Copula: A new vision for economic capital and application to a four line of business company, XXXIV International ASTIN Colloquium, Berlin, 2003.

FAISST, U.; KovACS, M. (2003): Quantifizierung operationeller Risiken - ein Methodenvergleich, In: Die Bank, 43. Jg. (2003), H. 5, S. 342-349.

FARNY, D. (1966): Unternehmerische Ziel- und Mittelentscheidungen in der Versicherungswirtschaft, In: Zeitschrift für die gesamte Versicherungswissenschaft, 55. Jg. (1966), S. 129-159.

FARNY, D. (1967): Gewinn und Sicherheit als Ziele von Versicherungsunternehmen, In: Zeitschrift für die gesamte Versicherungswissenschaft, 56. Jg. (1967), S. 49-81.

FARNY, D. (1983a): Nichtversicherungstechnische Erträge und Prämienbedarf in der Schaden/Unfallversicherung oder: Versuche und Versuchungen des Cash flowUnderwriting (I), In: Versicherungswirtschaft, 38. Jg. (1983), H. 7, S. 398-403.

FARNY, D. (1983b): Nichtversicherungstechnische Erträge und Prämienbedarf in der Schaden/Unfallversicherung oder: Versuche und Versuchungen des Cash flowUnderwriting (II), In: Versicherungswirtschaft, 38. Jg. (1983), H. 8, S. 476-485.

FARNY, D. (1992): Buchführung und Periodenrechnung im Versicherungsunternehmen, 4. Aufl., Wiesbaden (Gabler), 1992.

FARNY, D. (2000): Versicherungsbetriebslehre, 3. Aufl., Karlsruhe (VVW), 2000.

FEILMEIER, M.; BERTRAM, J. (1987): Anwendung numerischer Methoden in der Risikotheorie, Karlsruhe (VVW), 1987.

FELDBLUM, S. (1993): Risk Loads for Insurers: Authors reply to discussion, In: Proceedings of the Casualty Actuarial Society, 80. Jg. (1993), S. 366-379.

FISCHER, T. (2003): Risk capital allocation by coherent risk measures based on onesided moments, In: Insurance: Mathematics and Economics, 32. Jg. (2003), H. 1, S. 135-146.

FishBuRN, P. C. (1977): Mean-Risk Analysis with Risk Associated with BelowTarget Returns, In: American Economic Review, 57. Jg. (1977), S. 116-126.

FLEMMING, K. (1988): Zur Mathematik der Nichtlebensrückversicherung, Aus: Dienst, H.-R. (Hrsg.): Mathematische Verfahren der Rückversicherung, Karlsruhe (VVW), 1988, S. 98-123.

FÖRTERER, D. J. (2000): Ertrags- und Risikosteuerung von Lebensversicherern aus finanzmarkttheoretischer Sicht: Ein Ansatz zum Asset/Liability Management, Bamberg (Difo-Druck), 2000 (= zugl. Diss. St. Gallen 2000). 
FORST, H. von der (1998): Die Bedeutung des Kapitalanlageergebnisses für die Kapitalanlagepolitik, Aus: Hehn, E. (Hrsg.): Asset Management: Finanzdienstleistungen von und für Versicherungen, Stuttgart (Schäffer-Poeschel), 1998, S. 87-101.

FreY, R.; MCNEIL, A. J. (2002): VaR and expected shortfall in Portfolios of dependent credit risks: Conceptual and practical insights, In: Journal of Banking and Finance, 26. Jg. (2002), H. 7, S. 1317-1334.

Frey, H. C.; NiEBEN, G. (2001): Monte Carlo Simulation - Quantitative Risikoanalyse für die Versicherungsindustrie, München (Gerling Akademie), 2001.

FRIEDMAN, D. G. (1972): Insurance and the Natural Hazards, In: ASTIN Bulletin, 7. Jg. (1972), H. 1, S. 4-58.

Froot, K. A.; StenN, J. C. (1998): Risk Management, Capital Budgeting and Capital Structure Policy for Financial Institutions: an Integrated Approach, In: Journal of Financial Economics, 47. Jg. (1998), S. 55-82.

FÜRSTENWERTH, F. von; WEIB, A. (2001): Versicherungsalphabet, 10. Aufl., Karlsruhe (VVW), 2001.

Fylstra, D.; LASDON, L.; WATSON, J.; WAREN, A. (1998): Design and use of the Microsoft Excel Solver, In: Interfaces, 28. Jg. (1998), H. 5, S. 29-55.

Gabriel, A.; Rockel, W. (2001): Risikomanagement in Schaden- und Unfallversicherungsunternehmen, In: Zeitschrift für die gesamte Versicherungswissenschaft, 90. Jg. (2001), S. 101-135.

GARCHHAMMER, C.; ZAGST, R. (2005): Ein stochastisches Modell zur Ertragsoptimierung bei Versicherungen, Aus: SPREMANN, K. (Hrsg.): Versicherungen im Umbruch: Werte schaffen, Risiken managen, Kunden gewinnen, Berlin u.a. (Springer), 2005, S. 415-442.

Groupe CONSUltatif ACTUARIEL EUROPÉEN (2003): MARKT 2535/02: Considerations on the design of a future prudential supervisory system, o.O., 2003.

GESAMTVERBAND DER DEUTSCHEN VERSICHERUNGSWIRTSCHAFT E.V. (2002a): Aufsichtsmodell für deutsche Schaden-/Unfallversicherer - Stufe 1, Modellbeschreibung (Stand: 01.07.2002), Berlin, 2002.

GESAMTVERBAND DER DEUTSCHEN VERSICHERUNGSWIRTSCHAFT E.V. (2002b): Aufsichtsmodell für deutsche Lebensversicherer - Stufe 1, Modellbeschreibung (Stand: 01.07.2002), Berlin, 2002.

GESAMTVERBAND DER DEUTSCHEN VERSICHERUNGSWIRTSCHAFT E.V. (2004): Jahrbuch 2004, Die deutsche Versicherungswirtschaft, Karlsruhe (VVW), 2004. 
GeSAMTVERBAND DER DEUTSCHEN VERSICHERUNGSWIRTSCHAFT E.V. (2005): Solvency II kompatibles Standardmodell (Säule I) - Modellbeschreibung (Stand: 22.04.2005), Berlin, 2005.

GeRATHEWOHL, K. (1976): Rückversicherung - Grundlagen und Praxis, Band 1, Karlsruhe (VVW), 1976.

GERATHEWOHL, K. (1988): Schicksalsteilung in der Rückversicherung aus heutiger Sicht, Aus: Gerathewohl, K.; Schinzler, H.-J.; u.a. (Hrsg.): Beiträge zur Rückversicherung Horst K. Jannott zum 60. Geburtstag, Karlsruhe (VVW), 1988, S. 13-68.

GERBER, H.-U. (1979): An Introduction to Mathematical Risk Theory, Philadelphia (Huebner Foundation Monograph Nr. 8), 1979.

GoOvaERTS, M. J.; DHAENE, J.; KAAS, R. (2001): Risk Measures, Measures for Insolvency Risk and Economical Capital Allocation, In: Tijdschrift voor Economie en Management, 46. Jg. (2001), S. 545-559.

GOOVAERTS, M. J.; KAAS, R.; DHAENE, J. (2002): Economic capital allocation derived from risk measures, Arbeitspapier, K.U. Leuven, 2002.

Göx, R. (2004): Erfolgsabhängige Gehälter, Belohnung für den Zufall und der Einfluss des Managements auf die Gestaltung seines eigenen Vergütungssystems, In: Zeitschrift für betriebswirtschaftliche Forschung, 2004, Sonderheft Nr. 51, S. 27-56.

GRAUMANN, M. (1997): Ziele der Rückversicherungsnahme, In: Versicherungswirtschaft, 52. Jg. (1997), H. 6, S. 367-370.

GRAUMANN, M.; BAUM, S. (2003): Methoden zur Allokation von Sicherheitskapital, Darstellung und Beurteilung aus Sicht der Unternehmensleitung, In: Zeitschrift für die gesamte Versicherungswissenschaft, 92. Jg. (2003), S. 421-457.

Graumann, M.; HellmaYR, I. (1998): Die Verteilung von Risikokapital auf Geschäftsfelder im Rückversicherungunternehmen, In: Zeitschrift für die gesamte Versicherungswissenschaft, 87. Jg. (1998), H. 4, S. 735-762.

GRÄWERT, A.; STEVENS A.; TADROS, R. (2003): Solvency II: Ein Regulierungsrahmen für risikobasiertes Kapital: Ein Diskussionsbeitrag zum aktuellen Sachstand, In: Versicherungswirtschaft, 58. Jg. (2003), H. 6, S. 394-397.

GRITZMANN, N. (1998): Kapitalanlage-Controlling in Versicherungsunternehmen, Karlsruhe (VVW), 1998 (= zugl. Diss. Univ. Passau 1997).

GROFFMANN, T. (1995): Marktorientierte Steuerung in der Schaden- und Unfallversicherung, Berlin (Duncker \& Humblot), 1995 (= zugl. Diss. Univ. Bonn 1994).

GrossmanN, M. (1967): Sicherheitsstreben und Gewinnstreben in der Versicherungswirtschaft, In: Zeitschrift für die gesamte Versicherungswissenschaft, 56. Jg. (1967), S. 83-99. 
GrossmanN, M. (1977): Rückversicherung - eine Einführung, Bern u.a. (Peter Lang), 1977.

GRÜNDL, H.; SCHMEISER, H. (1999): Asset-Liability Management der Versicherungsunternehmung und Shareholder Value, In: Zeitschrift für die gesamte Versicherungswissenschaft, 88. Jg. (1999), S. 489-514.

GRÜNDL, H.; SCHMEISER, H. (2001): Versicherungswirtschaft, Anlagevorschriften der Versicherungen, Aus: Gerke, W.; Steiner, M. (Hrsg.): Handwörterbuch des Bankund Finanzwesens, 3. Aufl., Stuttgart (Schäffer-Poeschel), 2001, S. 2146-2155.

GRÜNDL, H.; SCHMEISER, H. (2002): Marktwertorientierte Unternehmens- und Geschäftsbereichssteuerung in Finanzdienstleistungsunternehmen, In: Zeitschrift für Betriebswirtschaft, 72. Jg. (2002), H. 8, S. 797-822.

GRÜNDL, H.; SCHMEISER, H. (2004a): Solvency II und interne Risikosteuerungsmodelle, In: Versicherungswirtschaft, 59. Jg. (2004), H. 7, S. 473-474.

GRÜNDL, H.; SCHMEISER, H. (2004b): Zur Problematik der Kapitalallokation in Versicherungsunternehmen, Arbeitspapier, Westfälische Wilhelms-Universität Münster, Oktober 2004.

GRÜNDL, H.; SCHMEISER, H. (2004c): Capital Allocation In Insurance Companies What Good Is It?, Arbeitspapier (revised version 15.09.2004), HumboldtUniversität zu Berlin und Westfälische Wilhelms-Universität Münster, 2004.

Guthoff, A.; Pfingsten, A.; Wolf, J. (1998): Der Einfluss der Begrenzung des Value at Risk oder des Lower Partial Moment auf die Risikoübernahme, Aus: Oehler, A. (Hrsg.): Credit Risk und Value-at-Risk Alternativen: Herausforderungen für das Management, Stuttgart (Schäffer-Poeschel), 1998, S. 111-153.

HAAS, M. (1991): Statistical Methodology for Reliability Studies, In: Journal of Manipulative and Physiological Therapy, 81. Jg. (1991), H. 2, S. 119-132.

Hahn, C.; Pfingsten, A.; Wagner, P. (2002): An Empirical Investigation of the Rank Correlation between different Risk Measures, Diskussionsbeitrag 02-01, Institut für Kreditwesen, Westfälische Wilhelms-Universität Münster Münster, 2002.

HANNOVER RÜCKVERSICHERUNG AG (2003): Geschäftsbericht 2003, Hannover, 2003.

HANSSMANN, F. (1987): Einführung in die Systemforschung, 3. Aufl., München (Oldenbourg), 1987.

Hartmann-Wendels, T.; Pfingsten, A.; Weber, M. (2004): Bankbetriebslehre, 3. Aufl., Berlin (Springer), 2004. 
HARTUNG, T. (2000): Unternehmensbewertung von Versicherungsunternehmen, Wiesbaden (Gabler), 2000 (= zugl. Diss. Univ. München 2000).

HARTUNG, T. (2002): Considerations of the Quantification of Operational Risks, Arbeitspapier, Ludwig-Maximilians-Universität München, 2002.

HARTUNG, T.; HELTEN, E. (2004): Modernisierung versicherungswirtschaftlicher Eigenkapitalnormen durch Solvency II, In: Finanz Betrieb, 2004, H. 4, S. 293-303.

HAX, K. (1969): Das Problem der Großschäden in der Prämienkalkulation der Versicherer, In: Zeitschrift für die gesamte Versicherungswissenschaft, 58. Jg. (1969), S. $187-210$.

HEEP-AltineR, M.; KLEMMSTEIN, M. (2001): Versicherungsmathematische Anwendungen in der Praxis mit Schwerpunkt Kraftfahrt und Allgemeine Haftpflicht, Karlsruhe (VVW), 2001.

HEINKE, V. G. (2000): Asset-Liability-Management mit Spezialfonds bei Lebens- und Sachversicherern, Aus: Kleeberg, J. M.; Schlenger, C. (Hrsg.): Handbuch Spezialfonds: Ein praktischer Leitfaden für institutionelle Anleger und Kapitalanlagegesellschaften, Bad Soden/Ts. (Uhlenbruch), 2000, S. 622-649.

HEINKE, V. G. (2002a): Ein sukzessiver Ansatz für die Asset-Liability-ManagementAnalyse (I), In: Versicherungswirtschaft, 57. Jg. (2002), H. 9, S. 631-636.

HEINKE, V. G. (2002b): Ein sukzessiver Ansatz für die Asset-Liability-ManagementAnalyse (II), Ein Instrument für die strategische Steuerung der Aktivrisiken, In: Versicherungswirtschaft, 57. Jg. (2002), H. 10, S. 722-728.

HeIstermanN, B. (2002a): Vom Müller-Report zu Solvency II, Neue Anforderungen an die finanzielle Ausstattung von Versicherungsunternehmen in Europa (Teil 1), In: Assets \& Liabilities, 2002, H. 3, S. 15-17.

HeistermanN, B. (2002b): Vom Müller-Report zu Solvency II, Neue Anforderungen an die finanzielle Ausstattung von Versicherungsunternehmen in Europa (Teil 2), In: Assets \& Liabilities, 2002, H. 4, S. 15-17.

HEISTERMANN, B. (2004): Risikomanagement und wertorientierte Steuerung im Kontext von Solvency II, In: Assets \& Liabilities, 2004, H. 1, S. 5.

HelbiG, M. (2002): Prämien, Aus: Helbig, M. (Hrsg.): Beiträge zum versicherungsmathematischen Grundwissen, Karlsruhe (VVW), 2002, S. 85-120.

HELTEN, E. (1975): Risikotheorie - Grundlage der Risikopolitik von Versicherungsunternehmen?, In: Zeitschrift für die gesamte Versicherungswissenschaft, 64. Jg. (1975), S. 75-92. 
HeLtEN, E. (1985): Der Einfluss der risikotheoretischen Modellbildung auf die Struktur von Bestands- und Schadendateien, Göttingen (Vandenhoeck \& Ruprecht), 1985.

Helten, E. (1991): Die Erfassung und Messung des Risikos, Aus: Grosse, W.; Müller-Lutz, H.-L.; Schmidt, R. (Hrsg.): Versicherungsenzyklopädie, Band 2, 4. Aufl., Wiesbaden (Gabler), 1991, S. 125-197.

HERI, E. W.; ZIMMERMANN, H. (2001): Grenzen statistischer Messkonzepte für die Risikosteuerung, Aus: Schierenbeck, H.; Rolfes, B.; Schüller, S. (Hrsg.): Handbuch Bankcontrolling, 2. Aufl., Wiesbaden (Gabler), 2001, S. 995-1014.

HERSBERGER, W. (1996): Versicherung und Risikokapital, oder: Die "Value proposition" der Schweizer Rück, Zürich, 1996.

HERZBERGER, J. (1997): Einführung in das wissenschaftliche Rechnen, Bonn (Addison Wesley Longman), 1997.

HESSELAGER, O.; ANDERSSON, U. (2002): Risk sharing and capital allocation, Arbeitspapier, Tryg Insurance, Ballerup (Dänemark), 2002.

HILLIER, F. S.; LIEBERMAN, G. J. (1997): Operations Research: Einführung, München u.a. (Oldenbourg), 1997.

HIPP, C. (1999): Risikomanagement von Naturkatastrophen: helfen mathematische Methoden?, In: Zeitschrift für die gesamte Versicherungswissenschaft, 88. Jg. (1999), S. 443-456.

HIPP, C. (2001a): Risikotheorie 1, Vorlesungsskript, Universität Karlsruhe, 2001.

HIPP, C. (2001b): Risikotheorie: Stochastische Methoden und Statistische Verfahren (Teil 2), Vorlesungsskript, Universität Karlsruhe, 2001.

HIPP, C.; MichEL, R. (1990): Risikotheorie: Stochastische Modelle und Statistische Methoden, Karlsruhe (VVW), 1990.

HIPP, C.; MORLOCK, M. (2001): Schadenversicherungsmathematik, Seminar Grundwissen II, DGVM Seminarunterlagen, Dreieich, 2001.

HoffManN, R.; SANN, M. (2002): Asset Liability Management von Kompositversicherern, Teil 1: Eine Prozessbeschreibung, In: Assets \& Liabilities, 2002, H. 4, S. 12-14.

HOFFMANN, R.; SANN, M. (2003a): Asset Liability Management von Kompositversicherern, Teil 2: Schadenabwicklung und Rückversicherung, In: Assets \& Liabilities, 2003, H. 1, S. 9-12. 
HOFFMANN, R.; SANN, M. (2003b): Asset Liability Management von Kompositversicherern, Teil 3: Die Musterversicherung PRIMUS AG, In: Assets \& Liabilities, 2003, H. 2, S. 7-14.

HöLLER, J. (1997): Versicherungstechnologie: ein Beitrag zur Diskussion theoretischer Grundlagen, Karlsruhe (VVW), 1997 (= zugl. Diss. Univ. Frankfurt a.M. 1997).

HÖLSCHER, R. (2002): Von der Versicherung zur integrativen Risikobewältigung: Die Konzeption eines modernen Risikomanagements, Aus: Hölscher, R.; Elfgen, R. (Hrsg.): Herausforderung Risikomanagement - Identifikation, Bewertung und Steuerung industrieller Risiken, Wiesbaden (Gabler), 2002, S. 3-31.

HOMBURG, C. (1991): Modellgestützte Unternehmensplanung, Wiesbaden (Gabler), 1991.

HOPP, F. W. (2001): Indirekte Investments im Immobiliensektor, Aus: Schwebler, R.; Knauth, K.-W.; Simmert, D. B. (Hrsg.): Kapitalmärkte: Aktuelle Anlage- und Absicherungsmöglichkeiten für Versicherungsunternehmen, Karlsruhe (VVW), 2001, S. 223-246.

Hull, J. C. (2003): Options, futures and other derivatives, 5. Aufl., Upper Saddle River (Prentice Hall), 2003.

HÜRLIMANN, W. (2001): Analytical Evaluation of Economic Risk Capital and Diversification using Linear Spearman Copulas, Arbeitspapier, Value and Risk Management, Winterthur Life and Pensions, Winterthur, 2001.

HÜRLIMANN, W. (2002a): On the Economic Risk Capital of Portfolio Insurance, Arbeitspapier, Value and Risk Management, Winterthur Life and Pensions Winterthur, 2002.

HÜRLIMANN, W. (2002b): Multivariate Fréchet Copulas and Conditional Value-atRisk, Arbeitspapier, Value and Risk Management, Winterthur Life and Pensions Winterthur, 2002.

InTERnational Actuarial Association (2004): A Global Framework for Insurer Solvency Assessment, Research Report of the Insurer Solvency Assessment Working Party, o.O., 2004.

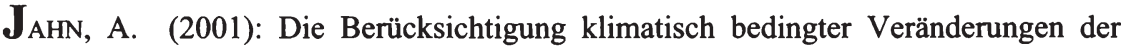
Sturmaktivität in den Entscheidungsprozessen deutscher Erstversicherer, In: Zeitschrift für die gesamte Versicherungswissenschaft, 90. Jg. (2001), S. 389-444. 
JARA, M. K. (2000): Zielorientierte Neugestaltung des Schadenmanagements in Versicherungsunternehmen - Ansätze für die Sach- und Haftpflichtsparten des Privatkundengeschäfts, Gossau (Cavelti), 2000 (= zugl. Diss. Univ. St. Gallen 2000).

JOE, H. (1997): Multivariate Models and Dependence Concepts, London (Chapman \& Hall), 1997.

JOHANNING, L. (1998): Value-at-Risk zur Marktrisikosteuerung und Eigenkapitalallokation, Bad Soden/Ts. (Uhlenbruch), 1998 (= zugl. Diss. Univ. München 1998).

JOHN, O. W. (2002): Das Modell Global CAP-Link zur Simulation des globalen Kapitalmarktes, Aus: Fachausschuss Finanzmathematik der deutschen Aktuarvereinigung (Hrsg.): Investmentmodelle für das Asset Liability Modelling von Versicherungsunternehmen, Abschlussbericht der Themenfeldgruppe Investmentmodelle, Karlsruhe (VVW), 2002, S. 167-191.

JORION, P. (2001): Value at Risk: The New Benchmark for Managing Financial Risk, 2. Aufl., New York u.a. (McGraw-Hill), 2001.

Jost, C. (2000): Das Risk Controlling der Kapitalanlage bei der Helvetia Patria Gruppe, Aus: Schierenbeck, H. (Hrsg.): Risk Controlling in der Praxis: Rechtliche Rahmenbedingungen und geschäftspolitische Konzeptionen in Banken, Versicherungen und Industrie, Stuttgart (Schäffer-Poeschel), 2000, S. 269-295.

JOST, P.-J. (2001): Die Prinzipal-Agenten-Theorie in der Betriebswirtschaftslehre, Stuttgart (Schäffer-Poeschel), 2001.

JPMORGAN; REUTERS (1996): RiskMetrics - Technical Document, New York, 1996.

JUNKER, M.; SCHWARZ, G. (2000): Simultanes Asset Liability Management: Kompetenz für die Altersvorsorge (I), In: Versicherungswirtschaft, 55. Jg. (2000), H. 18, S. 1410-1416.

Karten, W. (1983): Risikopolitik der Versicherer: Grundlagen der Risikopolitik Überblick, In: Zeitschrift für die gesamte Versicherungswissenschaft, 72. Jg. (1983), S. 213-229.

KARTEN, W. (1984): Marginalien zur EG-Solvabilitätskontrolle, Aus: Internationalität der Versicherung, Festgabe für M. Grossmann, St. Gallen, 1984, S. 337-360.

KARTEN, W. (1988): Schwankungsrückstellung, Aus: Farny, D.; Helten, E.; Koch, P.; Schmidt, R. (Hrsg.): Handwörterbuch der Versicherung (HdV), Karlsruhe (VVW), 1988, S. 763-765. 
KERN, M. (1979): Klassische Erkenntnistheorien und moderne Wissenschaftslehre, Aus: Raffée, H.; Abel, B. (Hrsg.): Wissenschaftstheoretische Grundfragen der Wirtschaftswissenschaften, München (Vahlen), 1979, S. 11-27.

KERSTING, G. (2004): Zufallsvariable und Wahrscheinlichkeiten - Eine elementare Einführung in die Stochastik, Frankfurt, 2004.

KINDER, C.; STEINER, M.; WILLINSKY, C. (2001): Kapitalallokation und Verrechnung von Risikokapitalkosten in Kreditinstituten, In: Zeitschrift für Betriebswirtschaft, 71. Jg. (2001), H. 3, S. 281-300.

KING, J. L. (2001): Operational Risk, Measurement and Modelling, Chichester (John Wiley \& Sons), 2001.

Klugman, S.; Panjer, H. H.; Willmot, G. (1998): Loss Models - From Data to Decision, New York (John Wiley \& Sons), 1998.

KNAUTH, K.-W. (1996): Effizienz und Wettbewerb der Aufsichtssysteme für Versicherungsunternehmen und Kreditinstitute, In: Zeitschrift für die gesamte Versicherungswissenschaft, 85. Jg. (1996), S. 232-244.

KNAUTH, K.-W.; SchuberT, T. (2003): Versicherungsaufsicht vor Paradigmenwechsel: Von der Produktgenehmigung zum unternehmerischen Risikomanagement, In: Versicherungswirtschaft, 58. Jg. (2003), H. 12, S. 902-907.

KосH, P. (1988): Versicherungszweige, System und übrige Sparten, Aus: Farny, D.; Helten, E.; Koch, P.; Schmidt, R. (Hrsg.): Handwörterbuch der Versicherung (HdV), Karlsruhe (VVW), 1988, S. 1251-1257.

KoCH, B. H. (1994): Effiziente Simulation in komplexen stochastischen Modellen: Dimensionierung des Simulationsumfangs unter taktischen Aspekten, Hallstadt (Rosch-Buch), 1994 (zugl. Diss. St. Gallen 1994).

KÖNIG, A. (2002a): Stochastische Modelle zur Beschreibung zukünftiger Finanzzeitreihen, Aus: Fachausschuss Finanzmathematik der deutschen Aktuarvereinigung (Hrsg.): Investmentmodelle für das Asset Liability Modelling von Versicherungsunternehmen, Abschlussbericht der Themenfeldgruppe Investmentmodelle, Karlsruhe (VVW), 2002, S. 45-65.

KÖNIG, A. (2002b): Risk Metrics - Ein Überblick, Aus: Fachausschuss Finanzmathematik der deutschen Aktuarvereinigung (Hrsg.): Investmentmodelle für das Asset Liability Modelling von Versicherungsunternehmen, Abschlussbericht der Themenfeldgruppe Investmentmodelle, Karlsruhe (VVW), 2002, S. 243 - 254.

KOPP, U.-C. (1992): Quantitatives Risikomanagement in Banken, St. Gallen (DUV), 1992 (= zugl. Diss Univ. St. Gallen 1992). 
KORYCIORZ, S. (2004): Sicherheitskapitalbestimmung und -allokation in der Schadenversicherung, Eine risikotheoretische Analyse auf der Basis des Value-at-Risk und des Conditional Value-at-Risk, Karlsruhe (VVW), 2004 (= zugl. Diss. Univ. Mannheim 2004).

KPMG DeUTSChE TREuHAND GeSELlSChafT (1994): Rechnungslegung von Versicherungsunternehmen nach neuem Recht, Frankfurt a.M., 1994.

KPMG DeUTSChe TREUHAND GeSEllschaft (2002): Study into the methodologies to assess the overall financial position of an insurance undertaking from the perspective of prudential supervision, Brüssel, 2002.

KRAMER, F. J. (1991): Organisatorische Formen der Risikobewältigung von Banken und Versicherungen, Idstein (Schulz-Kirchner), 1991 (= zugl. Diss. Univ. Frankfurt a.M. 1991).

KRIELE, M.; LIM, G.; REICH, H. (2004): Das Solvabilitätskapital in Solvency II: Ein Diskussionsbeitrag zum Berechnungsrahmen In: Versicherungswirtschaft, 59. Jg. (2004), H. 14, S. 1048-1052.

KROMSCHRÖDER, B. (1994): Cash-Flow Underwriting und kalkulatorische Kapitalkosten in der Schaden/Unfallversicherung, Aus: Schwebler, R. (Hrsg.): Dieter Farny und die Versicherungswissenschaft, Karlsruhe (VVW), 1994, S. 307-334.

KRÜGER. U.-J.; WEHLING, A. (2001): Anlagen am Geldmarkt und in Unternehmensanleihen (Corporate Bonds), Aus: Schwebler, R.; Knauth, K.-W.; Simmert, D. B. (Hrsg.): Kapitalmärkte: Aktuelle Anlage- und Absicherungsmöglichkeiten für Versicherungsunternehmen, Karlsruhe (VVW), 2001, S. 189-223.

KÜHL, H. (1994): Die Geschäftspolitik der Industriellen Feuerversicherung aus der Sicht eines Erstversicherers, Karlsruhe (VVW), 1994.

Lehar, A.; Welt, F.; Wiesmayr, C. (1998a): Risikoadjustierte Performancemessung in Banken, Konzepte zur Risiko-Ertragssteuerung (Teil 1), In: Östereichisches Bankarchiv, 46. Jg. (1998), H. 11, S. 857-862.

LehaR, A.; Welt, F.; WieSMaYR, C. (1998b): Risikoadjustierte Performancemessung in Banken, Konzepte zur Risiko-Ertragssteuerung (Teil 2), In: Östereichisches Bankarchiv, 46. Jg. (1998), H. 12, S. 949-955.

LEHMANN, G. (2002): Statistik - Einführung in die mathematischen Grundlagen für Psychologen, Wirtschafts- und Sozialwissenschaftler, Heidelberg (Spektrum), 2002.

Lehn, J.; WegmanN, H. (1992): Einführung in die Statistik, 2. Aufl., Stuttgart (Teubner), 1992. 
LEYHERR, U.; SCULLY, M.; SOMMERFELD, F. (2003): Wieviel Rückversicherung braucht mein Kapital? Rückversicherungsoptimierung und Analyse des Risikokapitals in der Allianz Gruppe, In: Versicherungswirtschaft, 58. Jg. (2003), H. 22, S. 1795-1797.

LIEBELT-WESTPHAL, U. (1997): Schadenverhütung und Versicherungsvertragsrecht, Frankfurt a.M. (Peter Lang), 1997 (= zugl. Diss. Univ. Hamburg 1997).

LIEBL, F. (1995): Simulation - Problemorientierte Einführung, 2. Aufl., München u.a (Oldenbourg), 1995.

LIEBWEIN, P. (2000): Klassische und moderne Formen der Rückversicherung, Karlsruhe (VVW), 2000 (zugl. Diss. Univ. München 1998).

LIPPE, S. (1983): Integration von Betriebskosten in risikotheoretische Modelle, Karlsruhe (VVW), 1983 (= zugl. Diss. Univ. Mannheim 1982).

LIPPE, S. (1984): Mathematische Grundlagen von Prämienprinzipien, In: Zeitschrift für die gesamte Versicherungswissenschaft, 73. Jg. (1984), S. 133-156.

LISTER, M. (1997): Risikoadjustierte Ergebnismessung und Risikokapitalallokation, Frankfurt a.M. (Fritz Knapp), 1997 (= zugl. Diss. Univ. Basel 1996).

LoCAREK-Junge, H.; STAHL, G. (2001): Value-at-Risk, Aus: Gerke, W.; Steiner, M. (Hrsg.): Handwörterbuch des Bank- und Finanzwesens (HWF), 3. Aufl., Stuttgart (Schäffer-Poeschel), 2001, Sp. 2120-2128.

LONDON GROUP (2002): Prudential Supervision of Insurance Undertaking, SharmaReport, London, 2002.

LUCAS, R. E. (1976): Econometric Policy Evaluation: A Critique, Amsterdam u.a., 1976.

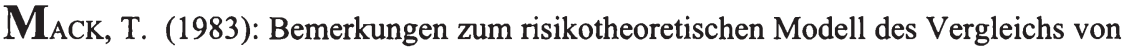
Rückversicherungsformen aus der Sicht der Praxis, In: Zeitschrift für die gesamte Versicherungswissenschaft, 72. Jg. (1983), S. 325-342.

MACK, T. (2002): Schadenversicherungsmathematik, 2. Aufl., Karlsruhe (VVW), 2002.

MANDL, J. (2004): Spieltheoretische Verfahren der Kapitalallokation im Versicherungsunternehmen, In: Mannheimer Manuskripte zu Risikotheorie, Portfolio Management und Versicherungswirtschaft, Nr. 159, Mannheim, 2004.

Markowitz, H. (1952): Portfolio Selection, In: Journal of Finance, 7. Jg. (1952), S. 77-91. 
MARKOWITZ, H. (1959): Portfolio Selection - Efficient Diversification of Investments, New York (John Wiley \& Sons), 1959.

MARLOW, S. (1999): Neuere Aspekte zur Zulässigkeit von Beitragsanpassungsklauseln in Versicherungsverträgen, Aus: Verein zur Förderung der Versicherungswissenschaft an den drei Berliner Universitäten (Hrsg.): Festschrift für Horst Baumann, Karlsruhe (VVW), 1999, S. 209-218.

Matten, C. (2000): Managing Bank Capital: Capital Allocation and Performance Measurement, 2. Aufl., Chichester (John Wiley \& Sons), 2000.

MAURER, R. (2000): Integrierte Erfolgssteuerung in der Schadenversicherung auf der Basis von Risiko-Wert-Modellen, Karlsruhe (VVW), 2000 (zugl. Habil. Univ. Mannheim 2000).

MCCABE, G. M.; WITT, R. C. (1980): Insurance Pricing and Regulation under Uncertainty: A Chance Constraint Approach, In: Journal of Risk and Insurance, 47. Jg. (1980), S. 607-635.

MCNeIL, A. (2000): Extreme Value Theory for Risk Managers, Aus: Embrechts, P. (Hrsg.): Extremes and Integrated Risk Management, London (Risk Books), 2000, S. 3-18.

McNeIl, A.; SAladin, T. (1997): The Peaks over Threshold Method for Estimating High Quantils of Loss Distributions, Arbeitspapier, ETH Zürich, Departement Mathematik, 1997.

MCNeIL, A.; SAladin, T. (2000): Developing Scenarios or Future Extreme Loss Using the Peaks-over-Threshold Method, Aus: Embrechts, P. (Hrsg.): Extremes and Integrated Risk Management, London (Risk Books), 2000, S. 253-267.

MEHL, R. (1987): Risikoadäquate Preisuntergrenzen des Schadenexzedenten-Rückversicherers, Karlsruhe (VVW), 1987 (= zugl. Diss. Univ. Hamburg 1987).

MENTZEL, R. (2004): Rückversicherung und Marktwertorientierung in der Schadenversicherung, Eine theoretische Analyse unter besonderer Berücksichtigung der proportionalen Rückversicherung, Karlsruhe (VVW), 2004 (= zugl. Diss. Univ. Leipzig 2004).

MERTON, R.; PEROLD, A. (1993): Theory of risk capital in financial firms, In: Journal of Applied Corporate Finance, 6. Jg. (1993), H. 2, S. 16-32.

MEYER, M. (1996): Operations-Research - Systemforschung, 4. Aufl., Stuttgart (Fischer), 1996.

MEYER, C. (1999): Value at Risk für Kreditinstitute: Erfassung des aggregierten Marktrisikopotenzials, Wiesbaden (Gabler), 1999 (= zugl. Diss. Univ. München 1998). 
MeYeRS, G. G. (2000): Coherent Measures of Risk: An Exposition for the Lay Actuary, Arbeitspapier, Insurance Services Office, Inc., o.O., 2000.

Mikosch, C. (1991): Industrie-Versicherungen, Ein Leitfaden für nationale und internationale Unternehmen, Wiesbaden (Gabler), 1991.

Mildenhall, S. (2004): A Note on the Myers and Read Capital Allocation Formula, In: North American Actuarial Journal, 8. Jg. (2004), S. 32-44.

MINA, J.; YI XIAO, J. (2001): Return to Risk Metrics: The Evolution of a Standard, New York, 2001.

MuelleR, H. (2003): Economic Capital - Recent Market Developments and Trends, In: Risk and Rewards, Juli 2003, S. 6-11.

MÜLLER, H. (1997a): Aktuelle Entwicklungen der Solvabilität von Versicherungsunternehmen, Versicherungsgruppen und Finanzkonglomeraten aus Sicht der Versicherungsaufsicht, In: Zeitschrift für Versicherungswesen, 48. Jg. (1997), H. 14, S. 382-389.

MÜLlER, H. (1997b): Aktuelle Entwicklungen der Solvabilität von Versicherungsunternehmen, Versicherungsgruppen und Finanzkonglomeraten aus Sicht der Versicherungsaufsicht, In: Zeitschrift für Versicherungswesen, 48. Jg. (1997), H. 15-16, S. 435-438.

MÜLlER, E. (2003): Über das Risiko der Risikobeurteilung, In: Zeitschrift für Versicherungswesen, 54. Jg. (2003), H. 21, S. 655-660.

MÜNCHENER RÜCKVERSICHERUNGS-GESELLSCHAFT AG (1999): Naturkatastrophen in Deutschland: Schadenerfahrungen und Schadenpotenziale, München, 1999.

MÜNCHENER RÜCKVERSICHERUNGS-GESELLSCHAFT AG (2001): 11. September 2001, München, 2001.

MÜNCHENER RÜCKVERSICHERUNGS-GESELLSCHAFT AG (2003): Geschäftsbericht der Münchener-Rück-Gruppe 2003, München, 2003.

MÜNCHENER RÜCKVERSICHERUNGS-GESELLSCHAFT AG (2004): IFRS - Neue Rechnungslegung, Überschwemmungsrisiken, Schwere Personenschäden, Topics 2/2004, München, 2004.

Mulvey, J. M.; Thorlacius, A. E. (1998): The Towers Perrin Global Capital Market Scenario Generation System, Cambridge, 1998.

MYERS, S.; READ, J. (2001): Capital allocation for insurance companies, In: The Journal of Risk and Insurance, 68. Jg. (2001), H. 4, S. 545-580. 


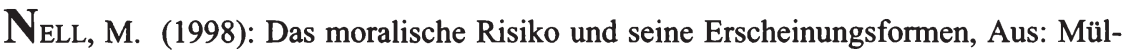
ler, W.; Stöhr, J. (Hrsg.): Frankfurter Vorträge zum Versicherungswesen, Karlsruhe (VVW), 1998, S. 7-34.

NELSEN, R. (1999): An Introduction to Copulas, New York (Springer), 1999.

NeSLEHOVÁ, J. (2004): Dependence of Non-Continuous Random Variables, Aachen (Shaker), 2004 (= zugl. Diss. Univ. Oldenburg 2004).

Neubauer, W. (1994): Statistische Methoden: Ausgewählte Methoden für Wirtschaftswissenschaftler, München (Vahlen), 1994.

NeUmanN, J. von; MORGENSTERn, O. (1973): Spieltheorie und wirtschaftliches Verhalten, 3. Aufl., Würzburg (Physica), 1973.

NeUSSER, K. (2000): Maximum Likelihood (ML) Schätzung, Universität Bern, Vortragsskript zur Mikroökonemetrie, WS 2000/01, Bern, 2000.

NierhaUS, F. (1990): Der Preis für proportionale Vertrags-Rückversicherung - besonders in der Sachversicherung, In: Versicherungswirtschaft, 45. Jg. (1990), H. 19, S. 1158-1162.

NÖHRBASS, G. (2001): Der Schock, der die Versicherungswirtschaft verändern wird, In: Versicherungswirtschaft, 56. Jg. (2001), H. 19, S. 1572-1573.

Oecking, S.; SANNer, A. (2004): Bilanzierung und Rechnungslegung, Aus: Radtke, M.; Schmidt, K. (Hrsg.): Handbuch zur Schadenresevierung, Karlsruhe (VVW), 2004, S. 25-36.

OLETZKY, T. (1998): Wertorientierte Steuerung von Versicherungsnternehmen: Ein Steuerungskonzept auf der Grundlage des Shareholder-Value-Ansatzes, Karlsruhe (VVW), 1998 (= zugl. Diss. Univ. Hannover 1998).

OletZKY, T.; SchulenbURG, J.-M. Graf von der (1998): Shareholder Value Management Strategie in Versicherungsunternehmen, In: Zeitschrift für die gesamte Versicherungswissenschaft, 87. Jg. (1998), S. 65-93.

OSETROVA, A.; SCHMEISER, H. (2005): Solvency II: Interne Risikomodelle aus wissenschaftlicher Sicht, Arbeitspapier, Westfälische Wilhelms-Universität Münster.

OverBeCK, L. (2000): Allocation of Economic Capital in loan portfolios, Aus: Franke, J.; Härdle, W.; Stahl, G. (Hrsg.): Measuring Risk in complex stochastic systems, New York u.a. (Springer), 2000, S. 1-17.

O.V. (2003): Selbst Sachversicherungen von Solvency II tangiert, In: Versicherungswirtschaft, 58. Jg. (2003), H. 24, S. 2010-2011. 
o.V. (2004): Dow Jones STOXX Index Guide, Zürich, 2004.

Panjer, H. H. (2001): Measurement of risk, solvency requirements and allocation of capital within financial conglomerates, Arbeitspapier, Waterloo (Canada), 2001.

PANJER, H. H.; WiLlmot, G. (1992): Insurance Risk Models, Schaumburg (Illinois), 1992.

PaUl, S. (2001): Risikoadjustierte Gesamtbanksteuerung, Bern (Paul Haupt), 2001 (= zugl. Diss. Univ. Basel 2001).

PERRIDON, L.; STEINER, M. (2004): Finanzwirtschaft der Unternehmung, 13. Aufl., München (Vahlen), 2004.

PFAFF, D. (2004): Performancemessung und Vertragsfunktion - Zu Bedeutung und Eigenschaften von Performancemaßen bei vermögensbeschränkten Managern, In: Zeitschrift für betriebswirtschaftliche Forschung, 2004, Sonderheft 51, S. 1-26.

PFEIFER, D. (2000): Wissenschaftliches Consulting im Rückversicherungsgeschäft: Modelle, Erfahrungen, Entwicklungen, In: Zeitschrift für Versicherungswesen, 51. Jg. (2000), H. 21, S. 771-777.

PFEIFER, D. (2003): Möglichkeiten und Grenzen der mathematischen Schadenmodellierung, In: Zeitschrift für die gesamte Versicherungswissenschaft, 92. Jg. (2003), S. 665-696.

PFEIFER, D. (2004): Solvency II: Neue Herausforderung an Schadenmodellierung und Risikomanagement, Aus: Albrecht, P.; Lorenz, E.; Rudolph, B. (Hrsg.): Risikoforschung und Versicherung, Festschrift für Elmar Helten, Karlsruhe (VVW), 2004, S. 467-481.

PfeifER, D.; NESLEHOVÁ, J. (2003a): Modeling and generating dependent risk processes for IRM and DFA, XXXIV International ASTIN Colloquium, Berlin 2003.

PFEIFER, D.; NESLEHOVÁ, J. (2003b): Modeling dependence in finance and insurance: the copula approach, In: Blätter der DGVFM, 2003, H. 2, S. 177-191.

PFEIFFER, C. (1994): Einführung in die Rückversicherung, 4. Aufl., Wiesbaden (Gabler), 1994.

Pfingsten, A.; Homölle, S.; RIESo, S. (2001): Risikomaße, Aus: Gerke, W.; Steiner, M. (Hrsg.): Handwörterbuch des Bank- und Finanzwesens, 3. Aufl., Stuttgart (Schäffer-Poeschel), 2001, S. 1869-1879. 
PfLUG, G. C. (2000): Some Remarks in the Value-at-Risk and the Conditional Valueat-Risk, Aus: Uryasev, S. (Hrsg.): Probalistic Contrained Optimization: Methodology and Applications, Netherlands (Kluwer Academic Publishers), 2000, S. 272291.

PHILBRICK, S. W. (1991): Discussion of Paper "Risk Loads for Insurers" by S. Feldblum, Discussion by S. W. Philbrick, In: Proceedings of the Casualty Actuarial Society, 57. Jg. (1991), S. 56-63.

Philbrick, S. W.; PAINTER, R. A. (2001): DFA Insurance Company Case Study, Part II: Capital Adequacy and Capital Allocation, Aus: Casualty Actuarial Society (Hrsg.): Casualty Actuarial Society Forum, Spring 2001, Including the Dynanic Financial Analysis Call Paper, Arlington (Virginia), 2001, S. 99-151.

PICKANDS, J. (1975): Statistical inference using extreme order statistics, In: The Annals of Statistics, 3. Jg. (1975), H. 1, S. 119-131.

Poddig, T.; Dichtl, H.; Petersmeier, K. (2001): Statisitk, Ökonometrie, Optimierung, Methoden und ihre praktische Anwendung in Finanzanalyse und Portfoliomanagement, 2. Aufl., Bad Soden/Ts. (Uhlenbruch), 2001.

PohlHausen, R. (1999): Gedanken zur Überschwemmungsversicherung in Deutschland, In: Zeitschrift für die gesamte Versicherungswissenschaft, 88. Jg. (1999), S. 457-467.

POPPER, K. R. (1963): Conjectures and Refutations: The growth of scientific knowledge, New York (Harper), 1963.

POPPER, K. R. (1994): Logik der Forschung, 10. Aufl., Tübingen (J.C.B. Mohr), 1994.

PORTMANN, T. (1999): Lower Partial Moments: Unter besonderer Berücksichtigung ihres Zeithorizontverhaltens, Bern (Paul Haupt), 1999 (= zugl. Diss. Univ. St. Gallen 1999).

PRÖHLSS, J. (1988): Gebäudeversicherung, Aus: Farny, D.; Helten, E.; Koch, P.; Schmidt, R. (Hrsg.): Handwörterbuch der Versicherung (HdV), Karlsruhe (VVW), 1988, S. 211-218.

QuARG, G. (2003): The Munich Chain Ladder - a claims reserving technique that closes the gap between paid and incurred based IBNR-estimates, Arbeitspapier, o.O., 2003.

RADTKE, M. (2004): Separationsverfahren, Aus: Radtke, M.; Schmidt, K. (Hrsg.): Handbuch zur Schadenreservierung, Karlsruhe (VVW), 2004, S. 183-188. 
RADTKE, M.; SCHMIDT, K. (Hrsg.) (2004): Handbuch zur Schadenreservierung, Karlsruhe (VVW), 2004.

RAFFÉE, H. (1995): Grundprobleme der Betriebswirtschaftslehre, 9. Aufl., Göttingen (Vandenhoeck \& Ruprecht), 1995.

RAFFÉE, H.; ABEL, B. (1979): Aufgaben und aktuelle Tendenzen der Wissenschaftstheorie in den Wirtschaftswissenschaften, Aus: Raffée, H.; Abel, B. (Hrsg.): Wissenschaftstheoretische Grundfragen der Wirtschaftswissenschaften, München (Vahlen), 1979, S. 1-10.

RAPPAPORT, A. (1986): Creating Shareholder Value: the new standard for business performance, New York u.a. (The Free Press), 1986.

RAPPAPORT, A. (1999): New Thinking on How to Link Executive Pay with Performance, In: Harvard Business Review, 77. Jg. (1999), S. 91-101.

RAUSCher, M.; TeMPLER, A. (2004): Rebalancing - Ein Konzept zur Steuerung von Kapitalanlagen, Institut für Betriebswirtschaftliche Risikoforschung und Versicherungswirtschaft, Manuskript Nr. 47, Ludwig-Maximilian-Universität München, 2004.

ReichmanN, T. (2001): Controlling mit Kennzahlen und Managementberichten: Grundlagen einer systemgestützten Controlling-Konzeption, München (Vahlen), 2001.

RiEBEL, P. (1994): Einzelkosten- und Deckungsbeitragsrechnung, 7. Aufl., Wiesbaden (Gabler), 1994.

RIEPER, B. (1992): Betriebswirtschaftliche Entscheidungsmodelle, Berlin (NWB), 1992.

RINKER, A. (1997): Anreizsysteme in Kreditinstituten: Gestaltungsprinzipien und Steuerungsimpulse aus Controllingsicht, Frankfurt a.M. (Fritz Knapp), 1997.

RitTMANN, M.; ROCKEL, W. (2004): Rechnungslegung und Aufsicht von Versicherungsunternehmen - Zur Vereinbarkeit von IFRS und Solvency II, In: Zeitschrift für die gesamte Versicherungswissenschaft, 93. Jg. (2004), S. 441-476.

RoCKEL, W. (2004): Fair Value-Bilanzierung versicherungstechnischer Verpflichtungen - eine ökonomische Analyse, Wiesbaden (DUV), 2004 (= zugl. Diss. Univ. München 2004).

ROCKEL, W.; SAUER, R. (2004): IFRS für Versicherungsverträge: Inhalte, Problemfelder und Auswirkungen auf Solvency II, Institut für Betriebswirtschaftliche Risikoforschung und Versicherungswirtschaft, Manuskript Nr. 48, Ludwig-MaximilianUniversität München, 2004. 
Romano, C. (2002): Applying Copula Function to Risk Management, Arbeitspapier, Banca di Roma, Rom, 2002.

ROMEIKE, F.; MÜLLER-REICHARD, M. (2005): Risikomanagement in Versicherungsunternehmen, 1. Aufl., Weinheim (Wiley-VCH), 2005.

RoTHSCHILD, M.; STIGLITZ, J. (1970): Increasing Risk: A Definition, In: Journal of Economic Theory, 2. Jg. (1970), H. 3, S. 225-243.

S ACHS, L. (2002): Angewandte Statistik, 10. Aufl., Berlin u.a. (Springer), 2002.

SAITA, F. (1999): Allocation of Risk Capital in Financial Institutions, In: Financial Management, 28. Jg. (1999), H. 3, S. 96-111.

SANN, M. (2001): Asset Liability Modellierung von Kompositversicherern, In: Assets \& Liabilities, 2001, H. 4, S. 10-13.

SANN, M.; Justen, P.; OTTEN, U.; Fromme, S. (2003): Asset Liability Management für Kompositversicherer: Ganzheitliches Risikomanagement in der Schaden-/Unfallversicherung, In: Capital Aspects, 2003, H. 4, S. 1-24.

SAUER, R. (2004): Eigenkapital im Versicherungsunternehmen, Institut für Betriebswirtschaftliche Risikoforschung und Versicherungswirtschaft, Manuskript Nr. 53, Ludwig-Maximilian-Universität München, 2004.

SCHENK, P. (1995): Rückversicherungsentscheidungen von Schaden- und Unfallversicherungsunternehmen: Ergebnisse eines empirischen Forschungsprojekts, In: Versicherungswirtschaft, 50. Jg. (1995), S. 363-369.

SCHENK, P. (1998): Derivate Finanzinstrumente: Teil einer integrativen Risikopolitik in Versicherungsunternehmen, Wiesbaden (Gabler), 1998 (= zugl. Diss. Univ. München 1997).

SCHERER, B. (2002): Das Markowitz-Kalkül und seine Erweiterungen, Aus: Fachausschuss Finanzmathematik der deutschen Aktuarvereinigung (Hrsg.): Investmentmodelle für das Asset Liability Modelling von Versicherungsunternehmen, Abschlussbericht der Themenfeldgruppe Investmentmodelle, Karlsruhe (VVW), 2002, S. 93126.

SCHIERENBECK, H. (1999): Risikokalküle im Ertragsorientierten Bankenmanagement, Aus: Schierenbeck, H.; Rolfes, B.; Schüller, S. (Hrsg.): Handbuch Bankcontrolling, 2. Aufl., Wiesbaden (Gabler), 2001, S. 718-757.

SCHIERENBECK, H. (2001): Das Duale Steuerungsmodell, Aus: Schierenbeck, H.; Rolfes, B.; Schüller, S. (Hrsg.): Handbuch Bankcontrolling 2. Aufl., Wiesbaden (Gabler), 2001, S. 87-101. 
SCHIERENBECK, H. (2003a): Ertragsorientiertes Bankmanagement, Band 1: Grundlagen, Marktzinsmethode und Rentabilitätscontrolling, 8. Aufl., Wiesbaden (Gabler), 2003.

SCHIERENBECK, H. (2003b): Ertragsorientiertes Bankmanagement, Band 2: RisikoControlling und integrierte Rendite-/Risikosteuerung, 8. Aufl., Wiesbaden (Gabler), 2003.

SCHIERENBECK, H.; LISTER, M. (2001): Value Controlling - Grundlagen wertorientierter Unternehmensführung, München (Oldenbourg), 2001.

SCHIERENBECK, H.; LISTER, M. (2002): Risikomanagement im Rahmen der wertorientierten Unternehmenssteuerung, Aus: Hölscher, R.; Elfgen, R. (Hrsg.): Herausforderung Risikomanagement - Identifikation, Bewertung und Steuerung industrieller Risiken, Wiesbaden (Gabler), 2002, S. 181-203.

SCHINZLER, H.-J. (1988): Gedanken zur Rentabilität in der Rückversicherung, Aus: Gerathewohl, K.; Schinzler, H.-J.; u.a. (Hrsg.): Beiträge zur Rückversicherung, Horst K. Jannott zum 60. Geburtstag, Karlsruhe (VVW), 1988, S. 73-131.

SCHMALENBACH, E. (1909): Über Verrechnungspreise, In: Zeitschrift für handelswissenschaftliche Forschung, 3. Jg. (1909), S. 165-185.

SCHMEISER, H. (1997): Risikotheoretisch fundierte Ansätze zur Neugestaltung des europäischen Solvabilitätssystems für Schadenversicherer, Karlsruhe (VVW), 1997 (= zugl. Diss. Univ. Passau 1997).

SCHMEISER, H. (1998): Solvabilitätsanalyse von Schadenversicherungsunternehmen, In: Zeitschrift für die gesamte Versicherungswissenschaft, 87. Jg. (1998), S. 95-123.

SCHMEISER, H. (1999): Rückversicherung und Kapitalanlage simultan optimieren, In: Versicherungswirtschaft, 54. Jg. (1999), H. 2, S. 91-95.

SCHMEISER, H. (2001): Risikomanagement von Versicherungsunternehmen nach KonTraG, In: Zeitschrift für die gesamte Versicherungswissenschaft, 90. Jg. (2001), S. 139-159.

SCHMEISER, H. (2004a): New Risk-Based Capital Standards In The European Union: A Proposal Based On Empirical Data, In: Risk Management and Insurance Review, 7. Jg. (2004), H. 1, S. 41-52.

SCHMEISER, H. (2004b): Interne Risikosteuerungsmodelle und Solvency II, Arbeitspapier (18.11.2004), Westfälische Wilhelms-Universität Münster, 2004.

SCHMIDT, K. (2001): Versicherungsmathematik, Vorlesungsskript, Technisches Universität Dresden, Lehrstuhl für Versicherungsmathematik, Dresden, 2001.

SCHMIDT, K. (2002): Versicherungsmathematik, Berlin u.a. (Springer), 2002. 
SCHMIDT, K. (2004a): Abwicklungsmuster, Aus: Radtke, M.; Schmidt, K. (Hrsg.): Handbuch zur Schadenresevierung, Karlsruhe (VVW), 2004, S. 15-20.

SCHMIDT, K. (2004b): Chain-Ladder Verfahren, Aus: Radtke, M.; Schmidt, K. (Hrsg.): Handbuch zur Schadenresevierung, Karlsruhe (VVW), 2004, S. 55-64.

SCHMIDT, K. (2004c): Kollektives Modell, Aus: Radtke, M.; Schmidt, K. (Hrsg.): Handbuch zur Schadenresevierung, Karlsruhe (VVW), 2004, S. 111-114.

SCHNEEWEIB, C. (1984): Elemente einer Theorie betriebswirtschaftlicher Modellbildung, In: Zeitschrift für Betriebswirtschaft, 54. Jg. (1984), H. 5, S. 480-505.

SCHRADIN, H. (1994): Erfolgsorientiertes Versicherungsmanagement: Betriebswirtschaftliche Steuerungskonzepte auf risikotheoretischer Grundlage, Karlsruhe (VVW), 1994 (= zugl. Diss. Univ. Mannheim 1993).

SCHRADIN, H. (1998): Finanzielle Steuerung der Rückversicherung - Unter besonderer Berücksichtigung von Großschadenereignissen und Fremdwährungsrisiken, Karlsruhe (VVW), 1998 (= zugl. Habil. Univ. Mannheim 1998).

SCHRADIN, H. (2000): Risikoadäquate Kapitalallokation im Versicherungskonzern, Aus: Britzelmaier, B.; Geberl, S. (Hrsg.): Wandel im Finanzdienstleistungssektor, 1. Liechtensteinisches Finanzdienstleistungs-Symposium, Heidelberg, 2000, S. 101110 .

SCHRADIN, H. (2003): Entwicklung der Versicherungsaufsicht, In: Zeitschrift für die gesamte Versicherungswissenschaft, 92. Jg. (2003), S. 611-664.

SCHRADIN, H.; ZoNS, M. (2002): Determination and allocation of risk-adequate equity capitalization for performance measurements, Arbeitspapier, Institut für Versicherungswissenschaft, Universität Köln, 2002.

SCHRÖTER, K. J. (1995): Verfahren zur Approximation der Gesamtschadenverteilung: Systematisierung, Techniken und Vergleiche, Karlsruhe (VVW), 1995 (zugl. Diss. Univ. Karlsruhe 1994).

SChUbert, T.; Griebmann, G. (2004a): Solvency II - Die EU treibt die zweite Phase des Projektes voran, In: Versicherungswirtschaft, 59. Jg. (2004), H. 7, S. 470-472.

Schubert, T.; Grießmann, G. (2004b): Solvency II - Die EU treibt die zweite Phase des Projektes voran (II), In: Versicherungswirtschaft, 59. Jg. (2004), H. 10, S. 738739.

SCHÜRLE, M. (1998): Zinsmodelle in der stochastischen Modellierung, Bern (Haupt), 1998 (= zugl. Diss. Univ. St. Gallen 1998).

SCHULENBURG, GRAF VON DER, J.-M. (2005): Versicherungsökonomik - Ein Leitfaden für Studium und Praxis, Karlsruhe (VVW), 2005. 
SCHULTE, M. (1998): Bank-Controlling 2: Risikopolitik in Kreditinstituten, 3. Aufl., Frankfurt a.M. (Bank-Akademie), 1998.

SCHWAKE, E. (1988): Das versicherungstechnische Risiko als arteigenes Risiko der Versicherungsunternehmen?, In: Zeitschrift für die gesamte Versicherungswissenschaft, 77. Jg. (1988), S. 61-81.

SCHWEBLER, R. (1991): Vermögensanlage und Anlagevorschriften der Versicherungsunternehmen, Aus: Schwebler, R. (Hrsg.): Vermögensanlagepraxis in der Versicherungswirtschaft: Kommentare, Gesetze, Rundschreiben, Karlsruhe (VVW), 1991, S. 15-90.

SCHWEIZER, B.; SKLAR, A. (1983): Probabilitic Metric Spaces, New York (NorthHolland), 1983.

SCHWEIZERISCHE RÜCKVERSICHERUNGS-GESELLSCHAFT (1997a): Proportionale und nichtproportionale Rückversicherung, Die wesentlichen Unterschiede zwischen den beiden Rückversicherungs-Deckungsarten - Eine Besprechnung mit anschaulichen Beispielen, In: Swiss Re Technical Publishing, Zürich, 1997.

SCHWEIZERISCHE RÜCKVERSICHERUNGS-GESELLSCHAFT (1997b): Alternativer Risiko-Transfer durch Finite Risk-Rückversicherung: Ein wirksamer Beitrag zur Stabilität der Versicherungswirtschaft, In: sigma, H. 5, 1997, Zürich.

SCHWEIZERISCHE RÜCKVERSICHERUNGS-GESELLSCHAFT (1999): Vom Risiko zum Kapital, Überlegungen aus der Versicherungsperspektive, In: Swiss Re Integrated Risk Financing, Zürich, 1999.

SCHWEIZERISCHE RÜCKVERSICHERUNGS-GESELLSCHAFT (2000): Die Eigenkapitalausstattung der Nichtlebenversicherer im Spannungsfeld von Sicherheit und Renditeanforderungen, In: sigma, H. 1, 2000, Zürich.

SCHWEIZERISCHE RÜCKVERSICHERUNGS-GESELLSCHAFT (2001): Rentabilität der Nichtleben-Versicherungswirtschaft: Zurück zum Underwriting, In: sigma, H. 5, 2001, Zürich.

SCHWEIZERISCHE RÜCKVERSICHERUNGS-GESELLSCHAFT (2002a): Management des Unternehmenswertes - So schaffen Versicherer Shareholder-Value, In: Swiss Re Technical Publishing, Zürich, 2002.

SCHWEIZERISCHE RÜCKVERSICHERUNGS-GESELLSCHAFT (2002b): Die globale Nichtleben-Versicherung in einer Zeit der Kapazitätsknappheit, In: sigma, H. 4, 2002, Zürich.

SCHWEIZERISCHE RÜCKVERSICHERUNGS-GESELLSCHAFT (2003): Alternativer Risikotransfer - eine Bestandsaufnahmen, In: sigma, H. 1, 2003, Zürich. 
SCHWEIZERISCHE RÜCKVERSICHERUNGS-GESELLSCHAFT (2004): Die Auswirkungen der IFRS auf die Versicherungswirtschaft, In: sigma, H. 7, 2004, Zürich.

SCHWEPCKE, A. (2001): Rückversicherung - Grundlagen und aktuelles Wissen: Ein Leitfaden zum Selbststudium, Karlsruhe (VVW), 2001.

SHAPLEY, L. S. (1971): Cores of Convex Games, In: International Journal of Game Theory, 1. Jg. (1971), H. 1, S. 11-26.

SiebEN, G.; SchILdBACH, T. (1994): Betriebswirtschaftliche Entscheidungslehre, 4. Aufl., Düsseldorf (Werner), 1994.

SIMON, S. (2004): Neues Hochwassermodell für Deutschland, In: Versicherungswirtschaft, 59. Jg. (2004), H. 17, S. 1356-1357.

SimON, L. J.; BAILEY, R. A. (1960): Two Studies in Automobile Insurance Ratemaking, In: ASTIN Bulletin, 1. Jg. (1960), S. 192-217.

SimON, S.; GROLlmanN, T. (2002): Flutkatastrophen - Boten des Klimawandels?, In: Zeitschrift für Versicherungswesen, 53. Jg. (2002), H. 11, S. 682-689.

SMITH, A. (1997): Modelling Global Returns on Assets with Fat Tails, Actuaries Investment Conference, Edinburgh, 1997.

SoBOL, I. M. (1991): Die Monte-Carlo-Methode, 4. Aufl., Berlin (Deutscher Verlag der Wissenschaften), 1991.

SpellmanN, F. (2002): Gesamtrisiko-Messung von Banken und Unternehmen, Wiesbaden (Gabler), 2002 (= zugl. Diss. Univ. Bamberg 2001).

STANDARD \& POOR'S (2000): Ratingdefinitionen für Insurer Financial StrengthRatings, o.O., 2000.

STEINER, M.; BRUNS. C. (2002): Wertpapiermanagement, 8. Aufl., Stuttgart (SchäfferPoeschel), 2002.

STEINMÜllER, H. (1988): Bedeutung, Volkswirtschaftliche der Versicherung, Aus: Farny, D.; Helten, E.; Koch, P.; Schmidt, R. (Hrsg.): Handwörterbuch der Versicherung (HdV), Karlsruhe (VVW), 1988, S. 49-53.

STEPHAN, T. G. (1995): Strategische Asset Allocation in Lebensversicherungsunternehmen, Karlsruhe (VVW), 1995 (= zugl. Diss. Univ. Mannheim 1995).

STERK, H.-P. (1979): Selbstbeteiligung unter risikotheoretischen Aspekten, Karlsruhe (VVW), 1979 (= zugl. Diss. Univ. Mannheim 1979).

STERK, H.-P. (1988): Selbstbeteiligung, Aus: Farny, D.; Helten, E.; Koch, P.; Schmidt, R. (Hrsg.): Handwörterbuch der Versicherung (HdV), Karlsruhe (VVW), 1988, S. 775-780. 
StOER, J. (1993): Numerische Mathematik 1, 6. Aufl., Berlin (Springer), 1993.

StOUGHTON, N.; ZeCHNER, J. (2000): Konzepte zur Risiko-Ertragssteuerung in Kreditinstituten, Aus: Johanning, L.; Rudolph, B. (Hrsg.): Handbuch Risikomanagement Band 2: Risikomanagement in Banken, Asset-Management-Gesellschaften, Versicherungs- und Industrieunternehmen, Bad Soden/Ts. (Uhlenbruch), 2000, S. 879-902.

STOUGHTON, N.; ZECHNER, J. (2004): Optimal capital allocation using RAROC ${ }^{\mathrm{TM}}$ and EVA ${ }^{\circledR}$, CEPR Discussion Paper No. 4169, 2004.

STRAUß, J. (1988): Die Rückversicherungsverfahren in der Praxis, Aus: Dienst, H.-R. (Hrsg.): Mathematische Verfahren der Rückversicherung, Karlsruhe (VVW), 1988, S. 7-34.

T Asche, D. (2002): Expected shortfall and beyond, In: Journal of Banking and Finance, 26. Jg. (2002), H. 7, S. 1519-1533.

TAYLOR, G. C. (1991): An Analysis of Underwriting Cycles and their Effects on Insurance Solvency, Aus: Cummins, J.D.; Derrig, R.A. (Hrsg.): Managing the Insolvency Risk of Insurance Companies: proceedings of the Second International Conference on Insurance Solvency, Boston (Kluwer Academic Publishers), 1991, S. 376.

TIJS, S. H.; DRIESSEN, T. S. (1986): Game Theory and Cost Allocation Problems, In: Management Science, 32. Jg. (1986), H. 8, S. 1015-1027.

ULRICH, P.; HILL, W. (1979): Wissenschaftstheoretische Grundlagen der Betriebswirtschaftslehre, Aus: Raffée, H.; Abel, B. (Hrsg.): Wissenschaftstheoretische Grundfragen der Wirtschaftswissenschaften, München (Vahlen), 1979, S. 161-190.

URBAN, M. (2002): Allokation von Risikokapital auf Versicherungsportfolios, Diplomarbeit, Technische Universität München, Zentrum Mathematik, 2002.

UTECHT, T. (2001): Shareholder Value - Ein praktischer Ansatz für Schaden- und Unfallversicherer, In: Zeitschrift für die gesamte Versicherungswissenschaft, 90. Jg. (2001), S. 527-581.

V ALDEZ, E.; CHERNIH, A. (2003): Wang's capital allocation formula for elliptically contoured distributions, In: Insurance: Mathematics and Economics, 32. Jg. (2003), S. 517-532. 
VARAIN, T. C. (2004): Ansatz und Bewertung versicherungstechnischer Verpflichtungen von Schaden- und Unfallversicherungsunternehmen nach IAS/IFRS, Lohmar (Eul), 2004 (= zugl. Diss. Univ. Göttingen 2003).

VASICEK, O. A. (1977): An Equilibrium Characterization of the Term Structure, In: Journal of Financial Economics, 1977, H. 5, S. 177-188.

Venter, G. G. (2002): Allocating Surplus - Not!, In: The Actuarial Review, 29. Jg. (2002), S. 5-6.

Venter, G. G. (2003): Discussion of "Capital Allocation for Insurance Companies" by Stewart C. Myers and James R. Read Jr., Arbeitspapier, CAS-Forum, 2003.

VENTER, G. G. (2004): Capital Allocation Survey with Commentary, In: North American Actuarial Journal, 8. Jg. (2004), H. 4, S. 96-107.

VERRALL, R. J. (1994): A method for modelling varying run-off evolutions in claims reserving, In: ASTIN Bulletin, 24. Jg. (1994), H. 2, S. 325-332.

Vose, D. (1998): Quantitative Risk Analysis: A Guide to Monte Carlo Simulation Modelling, Chichester u.a. (John Wiley \& Sons), 1998.

W AGNER, F. (1992): Solvabiltätspolitik als Unternehmenspolitik von Kompositversicherungsunternehmen, Berlin (Duncker \& Humblot), 1992 (= zugl. Diss. Univ. Köln 1991).

WAGNER, F. (2000): Riskmanagement im Erstversicherungsunternehmen: Modelle, Strategien, Ziele, Mittel, Karlsruhe (VVW), 2000.

WAGNER, F.; WARMUTH, W. (2005): Wertorientierte Bepreisung im Versicherungsgeschäft, Karlsruhe (VVW), 2005.

WAKKeR, P. P.; ThALER, R. H.; TVERSKY, A. (1997): Probabilistic Insurance, In: Journal of Risk and Uncertainty, 15. Jg. (1997), S. 7-28.

WANG, S. S. (2002): A Set of New Methods and Tools for Enterprise Risk Capital Management and Portfolio Optimization, Aus: Casualty Actuarial Society (Hrsg.): Casualty Actuarial Society Forum 2002, Including the Dynamic Financial Analysis Papers, Arlington (Virginia) 2002, S. 43-77.

WeBER, J. (2004): Einführung in das Controlling, 10. Aufl., Stuttgart (SchäfferPoeschel), 2004.

WeIGEL, H.-J. (1991): Die Vermögensanlage in Wertpapieren, Aus: Schwebler, R. (Hrsg.): Vermögensanlagepraxis in der Versicherungswirtschaft: Kommentare, Gesetze, Rundschreiben, Karlsruhe (VVW), 1991, S. 141-220. 
WENGERT, H. M. (2000): Gesamtunternehmensbezogenes Risikomanagement bei Lebensversicherungsunternehmen, Ulm (IFA), 2000 (= zugl. Diss. Univ. Ulm 2000).

WiesehaHN, A. (2001): Ausgestaltung der Prozesskostenrechnung in Versicherungsunternehmen, Aus: Hallmann, T.; Kirchner, W. (Hrsg.): Reader zum Thema Controlling in Versicherungsunternehmen, Band 2, Karlsruhe (VVW), 2001, S. 397404.

WILKIE, D. A. (1995): More on a stochastic asset model for actuarial use, In: British Actuarial Journal, 1995, H. 1, S. 777-964.

WINTER, R. v. (2001): Risikomanagement und interne Kontrollen beim Sachversicherer im Sinne des KonTraG (Gesetz zur Kontrolle und Transparenz im Unternehmensbereich), Karlsruhe (VVW), 2001.

WIRCH, J. L.; HARDY, M. R. (1999): A synthesis of risk measures for capital adequacy, In: Insurance: Mathematics and Economics, 25. Jg. (1999), H. 3, S. 337-347.

WÖHLE, C. (2003): Modellanalytische Bilanzstrukturoptimierung unter Rendite-/ Risiko-Kriterien im Rahmen des Dualen Steuerungsmodells, In: Kredit und Kapital, 36. Jg. (2003), H. 1, S. 82-119.

Y AmaI, Y.; Yoshiba, T. (2002): On the Validity of Value-at-Risk: Comparative Analysis with Expected Shortfall, In: Monetary and Economic Studies, 20. Jg. (2002), H. 1, S. 57-85.

ZIELKE, C. (2004): Ohne Risiko kein Gewinn, In: Versicherungswirtschaft, 59. Jg. (2004), H. 22, S. 1716-1723.

ZIELKE, C. (2005): IFRS für Versicherer: Hintergründe und Auswirkungen, Wiesbaden (Gabler), 2005.

ZIETSCH, D. (2005): Rückversicherung als Instrument des Financial Engineering, Aus: SPREMANN, K. (Hrsg.): Versicherungen im Umbruch: Werte schaffen, Risiken managen, Kunden gewinnen, Berlin u.a. (Springer), 2005, S. 351-373.

ZimmermanN, C.; BaCh, C.; Raub, J. (2004): Von der Pflicht zur Kür im Risikomanagement (II), In: Versicherungswirtschaft, 59. Jg. 2004, H. 5, S. 299-303.

ZWEIFEL, P.; EISEN, R. (2003): Versicherungsökonomie, 2. Aufl., Berlin u.a. (Springer), 2003. 


\section{Beltrăge zum Controlling}

\section{Herausgegeben von Wolfgang Berens}

Band 1 Wolfgang Berens / Joachim Strauch: Due Diligence bei Unternehmensakquisitionen - eine empirische Untersuchung. Unter Mitarbeit von Thorsten Behrens und Julia Lescher. 2002.

Band 2 Andreas Siemes: Marktorientierte Kreditrisikobewertung. Eine empirische Untersuchung mittels Künstlicher Neuronaler Netze. 2002.

Band 3 Karl Christoph Heinen: Die Berücksichtigung von Kosten in der Konkurrenzanalyse. 2002.

Band 4 Thomas Mosiek: Interne Kundenorientierung des Controlling. 2002.

Band 5 Vera Südmeyer: Wettbewerbsvorteile durch strategisches Betriebsformenmanagement. Ein dynamischer Bezugsrahmen für Einzelhandelsunternehmen. 2003.

Band 6 Wolfgang Berens / Walter Schmitting (Hrsg.): Controlling im E-Business. Rückkehr zur Rationalităt. 2004.

Band 7 René Bertelsmann: Entwicklung einer Controlling-Konzeption im verallgemeinerten Neuen Steuerungsmodell für Trägerorganisationen der gesetzlichen Unfallversicherung. 2005.

Band 8 Mirko Tillmann: Risikokapitalbasierte Steuerung in der Schaden- und Unfallversicherung. Konzeption einer modellgestützten Risikoanalyse. 2005.

www.peterlang.de 


\section{Chancen- und \\ Risiko-Controlling}

\section{Erklärungsansatz zur Wirkungsweise von Chancen und Risiken im Controlling sowie dem unternehmensspezifischen Aufbau seiner Instrumente}

Frankfurt am Main, Berlin, Bern, Bruxelles, New York, Oxford, Wien, 2005.

XXVIII, 444 S., 129 Abb.

Controlling und Management.

Herausgegeben von Thomas Reichmann und Martin K. Welge. Bd. 30

ISBN 3-631-53422-1 · br. € 74.50*

Unternehmen, ihre Entwicklung und ihr Erfolg sind Einflüssen ausgesetzt, die aus ihrem Umfeld und aus ihren inneren Strukturen entstehen. Diese Einflüsse sind vielfältiger Herkunft, verschiedenartig nach Art und AusmaB ihrer Konsequenzen und unterliegen solch komplexen Wirkungszusammenhängen, daß eine systematische und umfassende Bewältigung zunächst kaum möglich erscheint. Chancen und Risiken als neue Wertkategorien und Maßstäbe des mittel- und langfristigen Unternehmenserfolgs dienen als Ansatzpunkte zur Systematisierung und schrittweisen Identifikation, Analyse, Steuerung und Überwachung von Einflüssen und Wirkungszusammenhängen.

Aus dem Inhalt: Anhaltspunkte zur Einordnung der Begriffe von Chance und Risiko in allgemeine betriebswirtschaftliche Zusammenhänge und die speziellen des Controlling · Hilfestellung zur Einordnung seiner Instrumente und ihrer Funktion in den erweiterten Steuerungskontext des Chancen- und Risikomanagements sowie die dazu erforderliche Führungsunterstützung durch das Controlling

Frankfurt am Main - Berlin - Bern - Bruxelles - New York - Oxford · Wien Auslieferung: Verlag Peter Lang AG

Moosstr. 1, $\mathrm{CH}-2542$ Pieterlen

Telefax 0041 (0) $32 / 3761727$

*inklusive der in Deutschland gültigen Mehrwertsteuer Preisänderungen vorbehalten

Homepage http://umw.peterlang.de 